\title{
DESIGN AND DEVELOPMENT OF THE \\ HYPERLOOP DEPLOYABLE WHEEL SYSTEM
}

\author{
by \\ Graeme P.A. Klim \\ Bachelor of Engineering, Ryerson University (2015)
}

\author{
A thesis \\ presented to Ryerson University \\ in partial fulfillment of the \\ requirements for the degree of \\ Master of Applied Science \\ in the program of \\ Aerospace Engineering
}

Toronto, Ontario, Canada, 2018

(C) Graeme P.A Klim 2018 


\section{AUTHOR'S DECLARATION FOR ELECTRONIC SUBMISSION OF A THESIS}

I hereby declare that I am the sole author of this thesis. This is a true copy of the thesis, including any required final revisions, as accepted by my examiners.

I authorize Ryerson University to lend this thesis to other institutions or individuals for the purpose of scholarly research.

I further authorize Ryerson University to reproduce this thesis by photocopying or by other means, in total or in part, at the request of other institutions or individuals for the purpose of scholarly research.

I understand that my thesis may be made electronically available to the public. 


\title{
DESIGN AND DEVELOPMENT OF THE HYPERLOOP DEPLOYABLE WHEEL SYSTEM
}

\author{
Graeme P.A Klim
}

Master of Applied Science, Aerospace Engineering, Ryerson University, Toronto (2018)

\section{Abstract}

In 2013 Elon Musk inspired engineers and entrepreneurs with his idea for a $5^{\text {th }}$ mode of transportation: the Hyperloop. Using large near-vacuum tubes as a medium, Musk envisioned sending humans and cargo in levitating pods from Los Angeles to San Francisco California in 35 minutes or less. Consisting of multiple subsystems, these pods would use magnetic or air-bearing technology for primary levitation to accommodate speeds approaching $700 \mathrm{mph}$. To address Musk's call for a traditional deployable wheel system to provide added safety and low-speed mobility for the pods, a patent-pending Hyperloop Deployable Wheel System (HDWS) was developed. This report details the author's contribution to the design and development of the award-winning HDWS and examines the constraints and limitations imposed by the Hyperloop concept: small operational space, near-vacuum low-pressure conditions, high-speed use and smooth ride requirements. 


\section{Acknowledgements}

I would like to sincerely thank Dr. Seyed M. Hashemi for supporting and guiding me throughout the entirety of my university education. Your attentive attitude towards my educational and personal ambitions has been greatly appreciated.

I would like to give a huge "shout-out" to the student team members of Ryerson's International Hyperloop Team - Tayo Shonibare, Whintta Ghebreiyesus, Jesse Brito, Min Adhikari and Moeid Elahi - for their contributions to the present work. I am indebted to the project's academic and industrial advisors: Steve Amberg, Clement Rodrigues, Primoz Cresnik, Faizan Rehematullah and Seyed M. Hashemi who spent a great deal of their personal time supporting the development of the Hyperloop Deployable Wheel System.

I would like to thank the large "team" of supporters at Safran Landing Systems Canada Inc. who provided me with access to development funding, engineering expertise, test facilities and even provided me a desk onsite for the duration of the project. By the same token, the assistance of Ryerson University, from the president's office to the lab technicians, was unwavering and greatly appreciated. The additional financial support from the Natural Sciences and Engineering Research Council of Canada (NSERC) through an ENGAGE grant is also acknowledged.

I would like to extend a deep sense of gratitude to Mr. Pete Cooper who spent a great deal of his 2016 calendar year manufacturing the structural components for the Hyperloop Deployable Wheel System and its test fixtures. Pete's commitment to manufacturing excellence ensured that the final product not only functioned well, but looked great too!

Finally, I would like to thank my friends and family, whose love and encouragement has enabled me to complete this work. Thank you to my partner, Kelly Firth, for always encouraging and supporting me throughout this project. Thank you to my Mom and Dad, and my grandparents, Alex (2010) and Blanche (2015) Klim, Clarence and Walterina Mountain, for all your love and support throughout the years.

Without the support of all these people and organizations, the Hyperloop Deployable Wheel System would have remained a great idea yet to be realized. 


\section{Table of Contents}

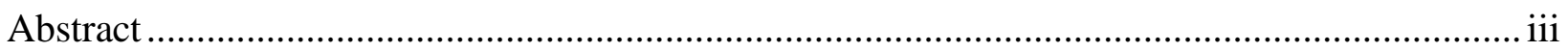

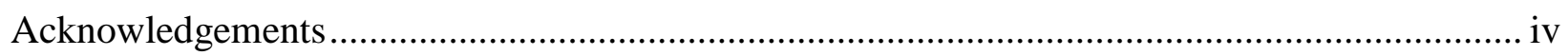

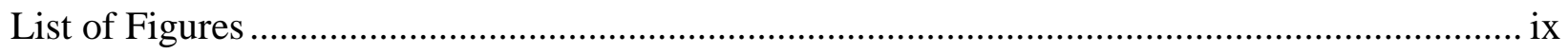

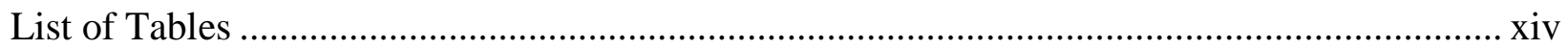

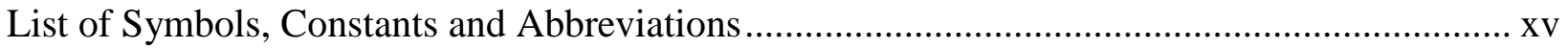

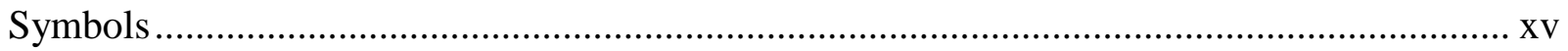

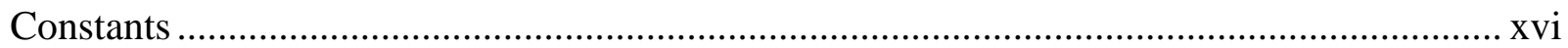

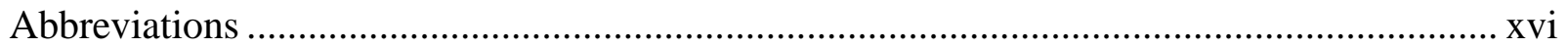

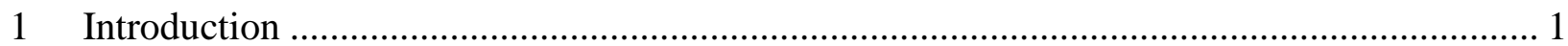

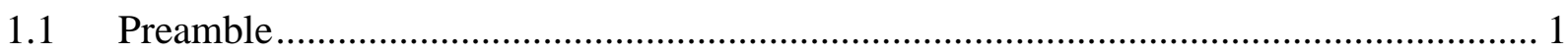

1.2 The SpaceX Hyperloop Competition .............................................................. 1

1.3 Ryerson's International Hyperloop Team ............................................................ 2

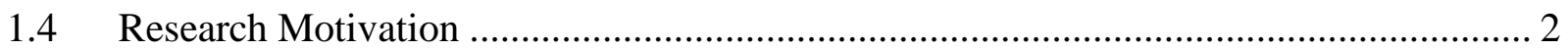

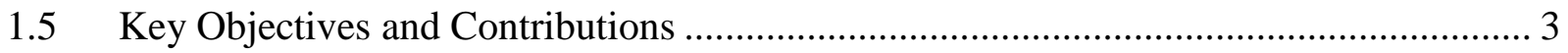

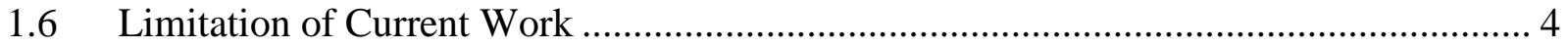

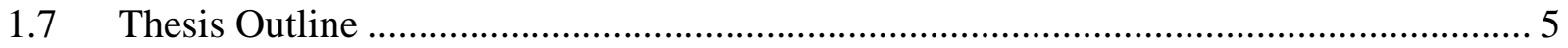

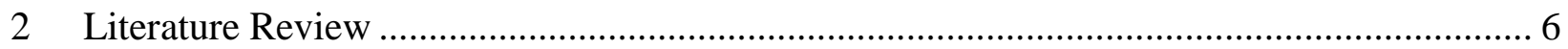

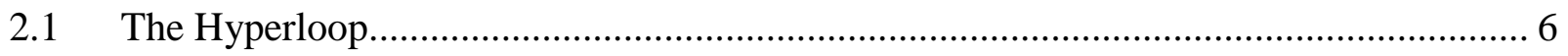

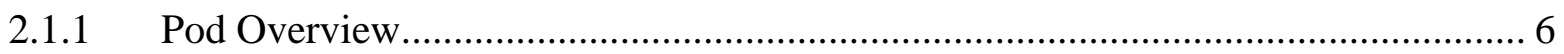

2.1.2 Major Subsystems Breakdown …............................................................ 7

2.1.3 The Tube System .......................................................................................... 9

2.1.4 SpaceX Hyperloop Tube System ............................................................. 10

$2.2 \quad$ Hyperloop Pod Operation Environment........................................................... 11 


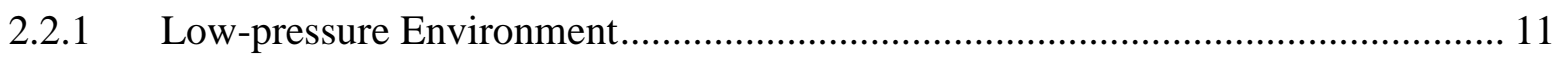

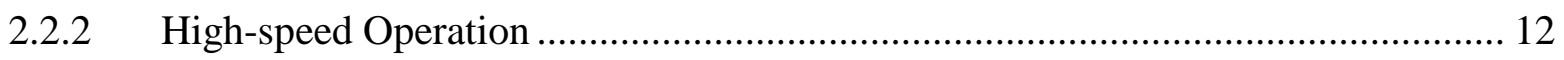

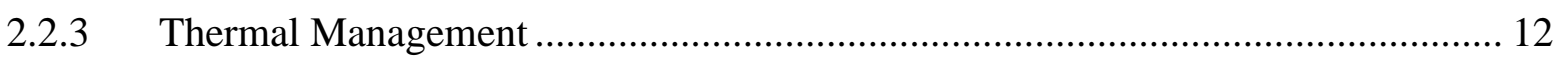

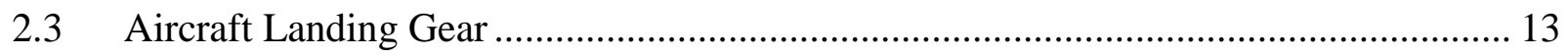

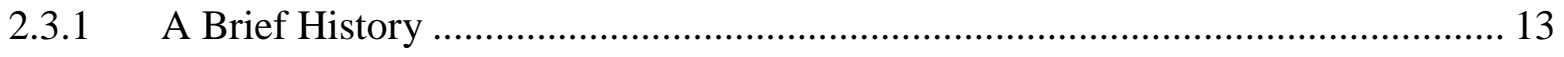

2.3.2 Key Components and Configurations .................................................................. 14

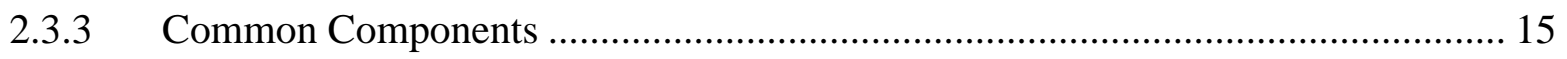

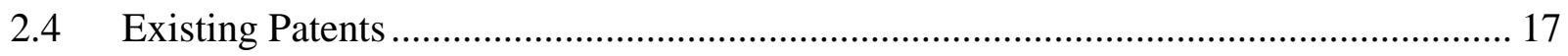

3 Product Definition and Preliminary Design ................................................................... 23

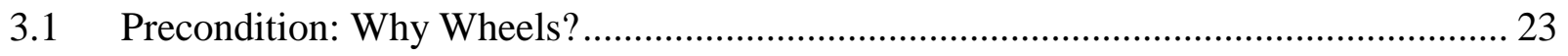

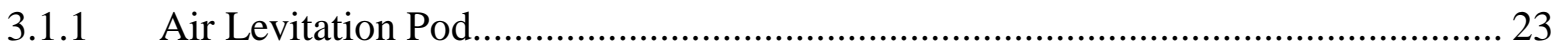

3.1.2 Magnetic Levitation Pod ................................................................................. 24

3.2 Former University of Toronto Partnership ............................................................... 25

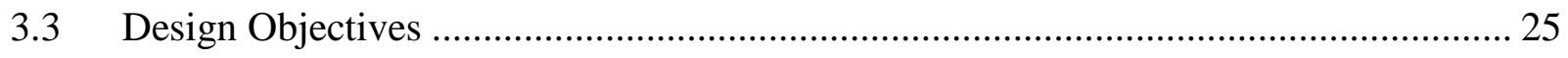

3.4 Product Development Guidelines and Constraints...................................................... 26

3.4.1 Design Constraints ......................................................................................... 27

3.4.2 Performance Constraints and Guidelines .......................................................... 28

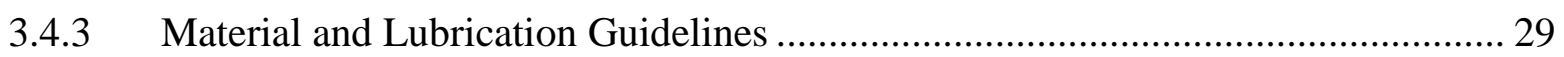

3.5 HDWS Architecture and Kinematics ........................................................................ 30

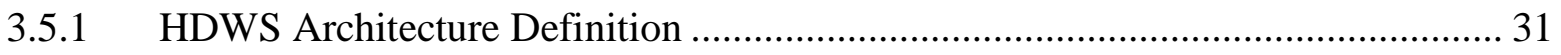

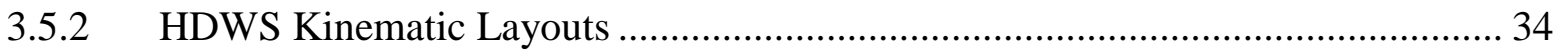

3.6 Component Definition, Product Structure and Process Scope …….................................. 38

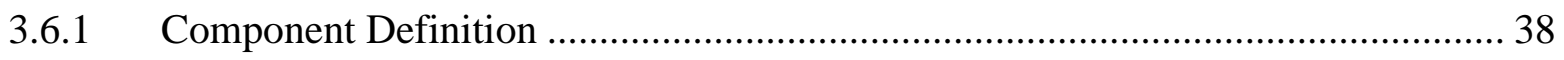

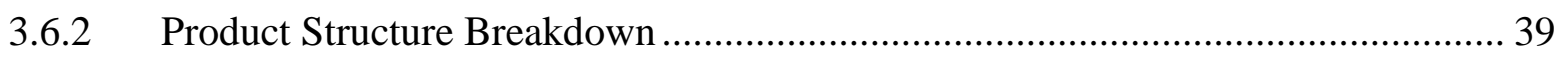

3.6.3 Design and Development Process Scope ............................................................ 40 


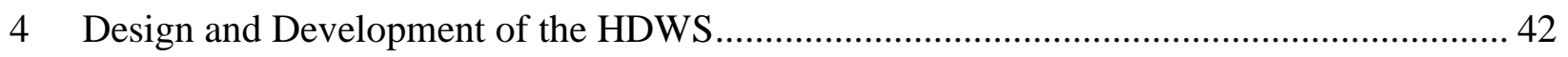

4.1 HDWS Dressed Assembly (P/N 10-1000-00)_........................................................... 42

4.1.1 HDWS Undressed Assembly (P/N 20-1000-00) .................................................... 43

4.1.2 Electric Motor Assembly (P/N 20-2000-00)....................................................... 44

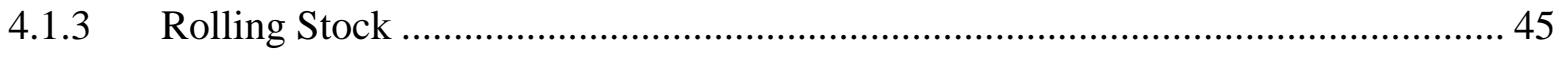

4.1.4 Electrical Dressings and Hardware ...................................................................... 48

4.2 HDWS Undressed Assembly Components .............................................................. 50

4.2.1 Trailing Arm Sub-assembly (P/N 30-1100-00) ……………………………...... 50

4.2.2 Shock Strut Sub-assembly (P/N 30-1200-00).................................................... 53

4.2.3 Retraction Sub-assembly (P/N 30-1300-00) ..................................................... 56

4.2.4 Torque Link Sub-assembly (P/N 30-1400-00) …………………………........... 57

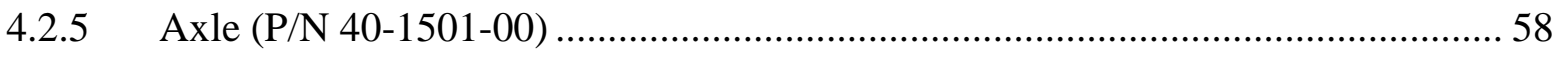

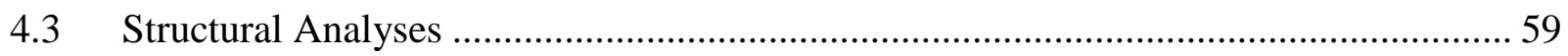

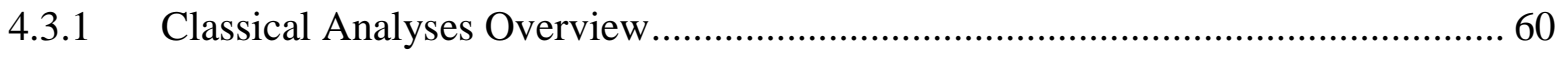

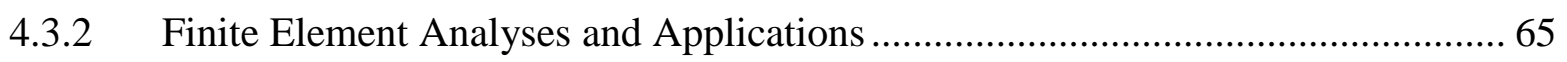

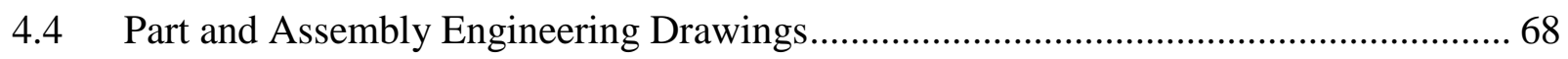

4.5 Manufacturing and Treatment Processes ..................................................................... 70

4.5.1 Subtractive Manufacturing.................................................................................. 71

4.5.2 Protective Finishes and Processes................................................................... 72

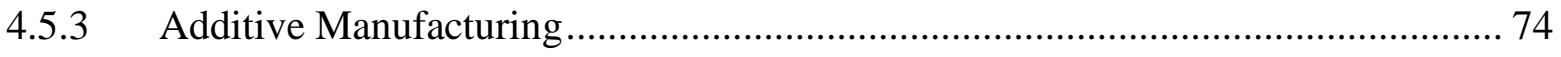

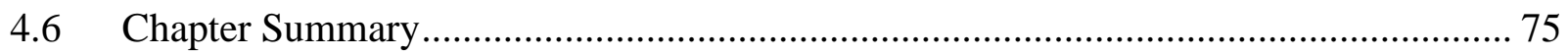

$5 \quad$ The Deployable and Retractable Shock Strut ......................................................................... 76

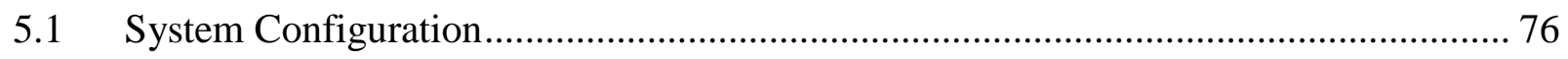

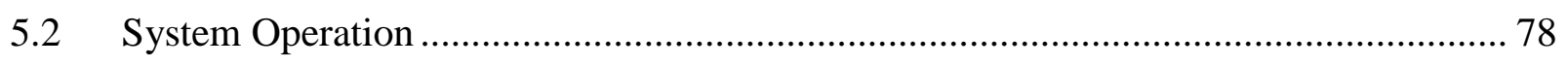

5.3 HDWS Shock Absorber Design ............................................................................. 79 
5.3.1 Oleo-Pneumatic Shock Absorber Introduction ............................................ 79

5.3.2 Shock Absorber Gas Spring Preliminary Design......................................... 81

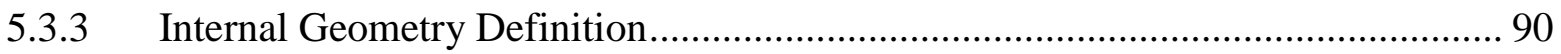

5.3.4 Detailed Gas Spring Design ................................................................... 94

5.3.5 Shock Absorber Detail Design................................................................. 98

5.4 HDWS Actuation Mechanism Design ............................................................. 103

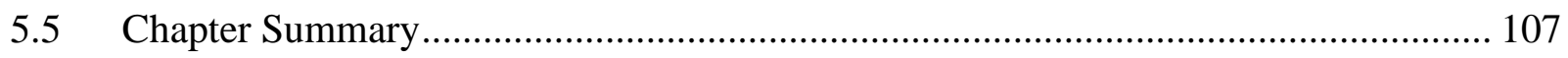

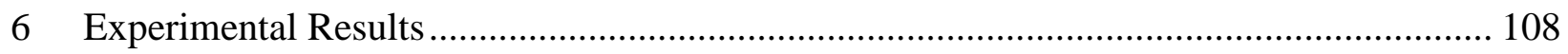

6.1 Polyurethane Wheel Performance Testing ......................................................... 108

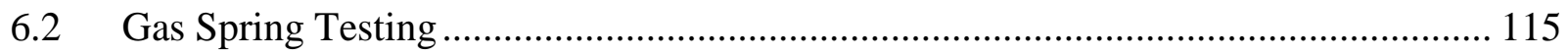

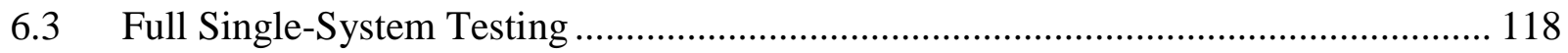

6.4 Dummy Pod Test (4 Units) ........................................................................ 123

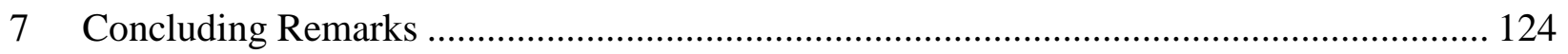

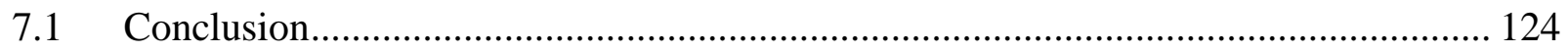

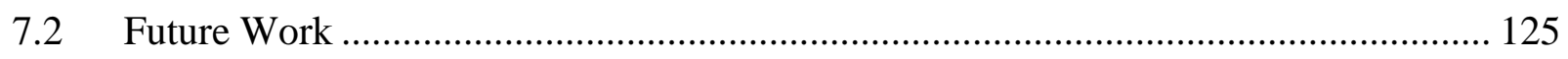

Appendix A: HDWS Component Drawings .............................................................. 126

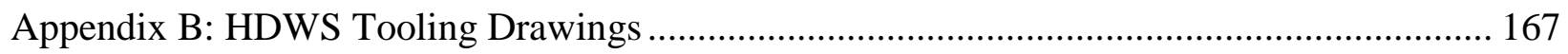

Appendix C: HDWS Test Fixture Drawings ............................................................. 172

Appendix D: Gas Spring Test Result Data ................................................................... 183

Appendix E: Retractable Shock Strut Concept Art......................................................... 189

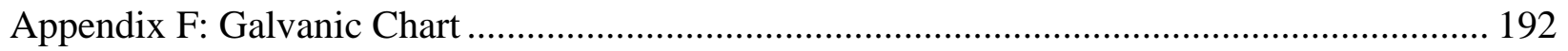

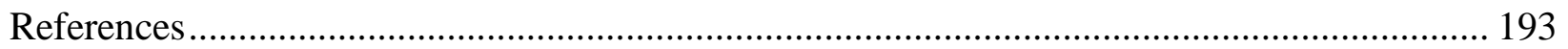




\section{List of Figures}

Figure 2.1: Example of a Hyperloop passenger pod, subsystem notional locations (not to scale) [1]

Figure 2.2: Hyperloop One's test "sled" is shown to include (1) magnets for levitating and (2) a

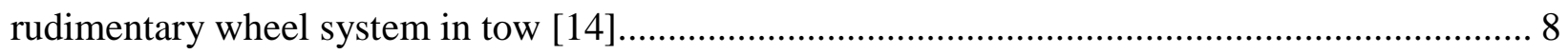

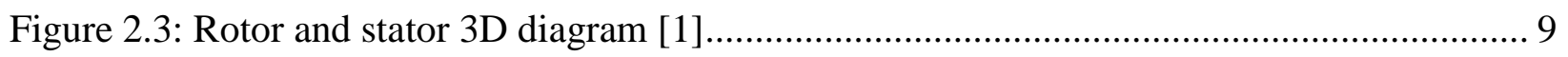

Figure 2.4: Artist rendering of a Hyperloop tube cutaway equipped with solar arrays [1] ......... 10

Figure 2.5: Image of the SpaceX Hyperloop sub-scale test track, image taken January 2017 ..... 11

Figure 2.6: Tail wheel configuration landing gear on a STOL Maule MX-7-235 Super Rocket. 14

Figure 2.7: General arrangement of a retractable MLG Trailing arm configuration [23] ........... 15

Figure 2.8: Sharples kinematic retraction configuration (left) with a cutaway of the contractive shock strut (right), shown as items 11 and 25 in the kinematic configuration (left) [25]........... 18

Figure 2.9: An illustration provided in Veaux et al. illustrating a motor and gearbox assembly embedded into the lower half of a piston to lengthen the strut [26] ........................................ 19

Figure 2.10: An illustration of the design proposed by Currey and Renshaw [27] .................... 20

Figure 2.11: An image (left) illustrating key components and with the advantageous kinematic architecture displayed (right), proposed by Waide [8] ....................................................... 21

Figure 2.12: Two possible configurations of a retractable shock strut, proposed by Waide [8] .. 21

Figure 3.1: Normalized force of the EDS Maglev plotted against translational velocity [29] ..... 25

Figure 3.2: A 2D side view of a Hyperloop pod with the HDWS positioned, not to scale ......... 27

Figure 3.3: Partial cross-section view of the SpaceX Hyperloop sub-track interface, dimensions

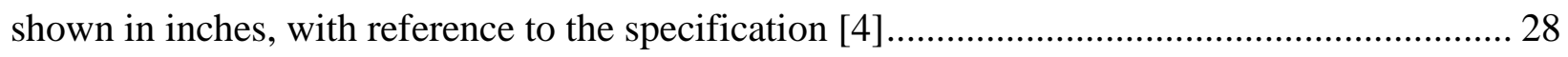

Figure 3.4: The HDWS preliminary configuration, stick diagram, not to final scale................. 32

Figure 3.5: The HDWS Hybrid actuator concept illustration ............................................... 33

Figure 3.6: The HDWS Hybrid actuator concept \#2 illustration ............................................ 34

Figure 3.7: An example of a preliminary HDWS kinematic layout, retracted, shown in CATIA ${ }^{\circledR}$

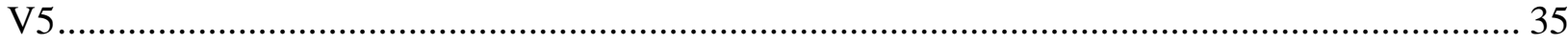

Figure 3.8: U of T HDWS kinematic layout, retracted position (left) and WOW position (right)37 Figure 3.9: U of T HDWS kinematic layout, sample static stroke (left) and sample static stroke + pod height adjustment of 0.5 in $(12.7 \mathrm{~mm})$ from the reference position (right) ...................... 37 
Figure 3.10: The HDWS product structure tree. 39

Figure 3.11: The HDWS design and development process scope overview .......................... 40

Figure 4.1: The HDWS Dressed assembly, CATIA ${ }^{\circledR}$ V5 rendering .......................................... 42

Figure 4.2: The HDWS Undressed assembly, CATIA ${ }^{\circledR}$ V5 rendering .................................... 43

Figure 4.3: The HDWS Electric Motor assembly, installed on the Retract sub-assembly .......... 44

Figure 4.4: CATIA $^{\circledR}$ V5 renderings of the final HDWS Wheel Assembly (left), and alternate wheel concept as designed with input from the author (right) ..................................................... 45

Figure 4.5: A Wheel and Tire assembly, 3D cross-sectional view, as drawn by Khan [45] ....... 47

Figure 4.6: The rolling stock as installed on the HDWS Dressed ......................................... 47

Figure 4.7: Sensors, brackets, electrical and hardware mount (orange) shown on the HDWS

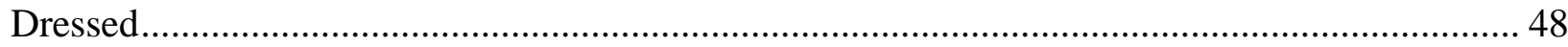

Figure 4.8: Sensors, brackets, electrical and hardware labeled (some features hidden for clarity)

Figure 4.9: The Trailing Arm sub-assembly, parts labeled …............................................. 51

Figure 4.10: The Trailing Arm sub-assembly cross-sectional view ....................................... 52

Figure 4.11: The Trailing Arm axle installation pin ....................................................... 53

Figure 4.12: The HDWS Shock Strut sub-assembly, CATIA ${ }^{\circledR}$ V5 rendering ........................... 53

Figure 4.13: Full cross-section view of the Shock Strut sub-assembly, Find Numbers shown.... 54

Figure 4.14: Partial cross-section view of the shock strut sub-assembly, Find Numbers shown 54

Figure 4.15: Retract sub-assembly 3D view (left) and cross section (right), Find Numbers shown 57

Figure 4.16: Torque Link sub-assembly (left) and exploded view (right), Find Numbers shown 58

Figure 4.17: The axle component as equipped to the trailing arm sub-assembly ......................59

Figure 4.18: Section cut, illustrating a typical double shear loading on the HDWS .................. 61

Figure 4.19: Section cut, illustrating a shear stress around the circumference of the bolt head... 63

Figure 4.20: Flanged bush (left), illustrating a projected bearing area (right) used for the

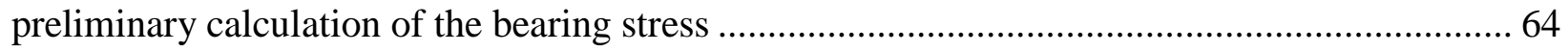

Figure 4.21: Flanged bush illustrating the features involved in a bearing length refinement ...... 64

Figure 4.22: Sample FEA result from ANSYS ${ }^{\circledR}$, trailing arm lug analysis [40] ...................... 65

Figure 4.23: HDWS motor mount bracket, machine from solid variant (maroon colored) [17].. 66

Figure 4.24: Final bracket design, produced from an FEA based optization [17].................... 67 
Figure 4.25: Example of a bracket displacement analysis, gravity load applied [17] ............... 67

Figure 4.26: Sample of a typical HDWS engineering drawing, key elements encircled............. 68

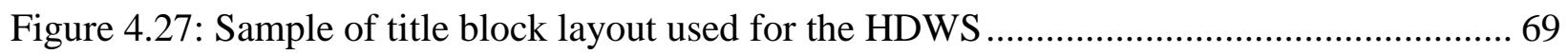

Figure 4.28: Typical design notes used for the HDWS aluminum parts ................................. 70

Figure 4.29: Images depicting from (1) through (3) the stages in the turning process performed for the manufacturing of the shock absorber outercylinder ACME 3G thread ............................. 71

Figure 4.30: Images (1) through (3) depict the milling process starting from a solid metal block, and image (4) depicts the internal thread screw cut performed on a lathe ............................. 72

Figure 4.31: Trailing arm pictured after undergoing blue anodizing.................................... 73

Figure 4.32: A shock strut outer cylinder shown with black oxide surface treatment................ 74

Figure 4.33: Images (1) through (2) depict the DMLS process used to create the final (3) AMM

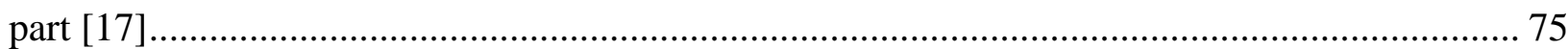

Figure 5.1: Deployable and retractabe shock strut system configuration, partial cross-section ... 77

Figure 5.2: HDWS extension and retraction process, some parts hidden for clarity .................. 78

Figure 5.3: An example of an oleo-pneumatic shock strut with oil and gas free to mix [5] ........ 80

Figure 5.4: Example of a hypothetical pod layout, loads applied to the HDWS in a static position 82

Figure 5.5: The HDWS preliminary configuration, stick diagram ..................................... 83

Figure 5.6: Illustration of oleo-pneumatic shock strut key operational states .......................... 86

Figure 5.7: Graphic representation of the isothermal oleo-pneumatic gas spring curve produced

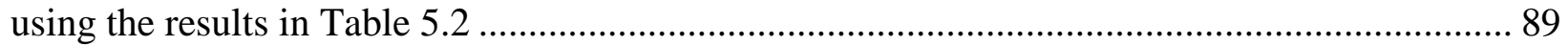

Figure 5.8: A simplified oleo-pneumatic strut, key preliminary design components labeled for

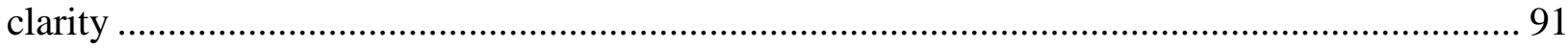

Figure 5.9: An example of an oleo-pneumatic shock strut highlighting preliminary geometric features

Figure 5.10: Piston rod gland design, key dimensions labeled, as defined by Greene Tweed [59]

Figure 5.11: Dynatool ${ }^{\odot}$ HDWS gas spring curve (static and dynamic), load vs. stroke............. 96

Figure 5.12: Dynatool ${ }^{\odot}$ HDWS gas spring curve (static and dynamic), pressure vs. stroke ....... 96 Figure 5.13: Cross-section view showing the shock strut while fully compressed at servicing, total oil volume (red) $6.426 \mathrm{in}^{3}$ 97 
Figure 5.14: Cross-section view showing the total volume when extended (purple) $9.174 \mathrm{in}^{3}$, gas+oil. 97

Figure 5.15: Cross-section view of the shock strut trapped air volume (white) $0.106 \mathrm{in}^{3}$, max piston compression . 97

Figure 5.16: Cross-section, lower bearing, key design details labeled.... 98

Figure 5.17: 3D cross-section of the filler tube, key design details labeled 100

Figure 5.18: 3D cross-section view of the filler tube with trapped gas illustrated 101

Figure 5.19: 3D view of the piston, spherical bearing installed, key features labeled 101

Figure 5.20: 3D cross-sectional view of the shock strut outer cylinder, key features labeled.... 102

Figure 5.21: 3D cross-sectional view of the retract assembly, trunnion pins labeled 103

Figure 5.22: Retract housing (bushed), key design features labeled 104

Figure 5.23: 3D cross-section view, ACME retract nut, key design features labeled 105

Figure 5.24: Retract assembly 3D exploaded view, key design features labeled 106

Figure 6.1: Wheel test configuration 109

Figure 6.2: Reflector tape positioning and wheel temperature measurement region shown ...... 110 Figure 6.3: Plot illustrating Test \#1, the pre-spin of the spin-up machine, engaging the stationary pod mounted wheel at $180 \mathrm{mph}$, and braking to stop at a decceleration rate of $0.6 \mathrm{~g}$. 111

Figure 6.4: Localized wear marking, followed by a trail-off effect, observed after Test \#1 ...... 112 Figure 6.5: Thin polyurethane deposited on the aluminum fly-wheel observed after Test \#2, wheel deterioration increased under same loading and speeds 112 Figure 6.6: Shifting of the reflective tape caused by the expanding and contracting outer diameter of the wheel after disengagement from the spin-up machine at $180 \mathrm{mph}$ 113

Figure 6.7: Grease splatter and roller rotation evident as traced by the orange anti-rotation paint 114

Figure 6.8: Graph displaying the average load measured by the load cell as applied by the spin machine, load spikes at initial contact encircled in red 114

Figure 6.9: Shock absorber gas spring verification initial, initial setup 115

Figure 6.10: Shock absorber gas spring verification test, final setup .... 116

Figure 6.11: HDWS full system test configuration (temperature sensors out of view). 118

Figure 6.12: Example of torque paint (orange) applied, used to track loosening of the hardware 
Figure 6.13: General pod mission profile inidcating key regions/events of interest (not to scale) 120

Figure 6.14: Graph of the data recorded during Test Case \#4, with the HDWS subjected to $2.4 \mathrm{~g}$ acceleration and decceleration, key events labeled for clarity.....

Figure 6.15: An example of typical wheel wear as shown after full systems testing 122 Figure 6.16: Dummy pod test bench, control system testing fixture with $200 \mathrm{lbm}$ mobile dummy mass to simulate shifts in $\mathrm{CG}$. 123 


\section{List of Tables}

Table 3.1: Representative SpaceX pusher acceleration values (2016) [4]............................... 28

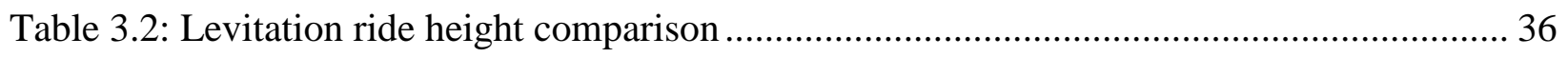

Table 4.1: Electric Motor assembly breakdown summary .................................................. 44

Table 4.2: Electric Motor assembly breakdown summary .................................................. 50

Table 4.3: Trailing Arm sub-assembly breakdown summary .............................................. 51

Table 4.4: Shock Strut sub-assembly breakdown summary ................................................... 55

Table 4.5: Retraction sub-assembly component breakdown summary ................................... 56

Table 4.6: Torque Link sub-assembly component breakdown summary ............................... 58

Table 5.1: Shock strut design standards and specifications referenced .................................... 79

Table 5.2: Shock strut design tool developed by the author using equations derived in Currey [5]

Table 5.3: Key shock strut stroke position outputs (ideal gas law, isothermal assumption) ....... 85

Table 5.4: Shock strut gas spring design features and definitions per Currey [5] ..................... 87

Table 5.5: Shock strut mechanical design features and definitions ...................................... 91

Table 5.6: Piston detail design template, as defined in the author's shock strut design tool....... 93

Table 5.7: Piston rod dynamic seal gland dimensions, per Greene Tweed [59], based on SAE

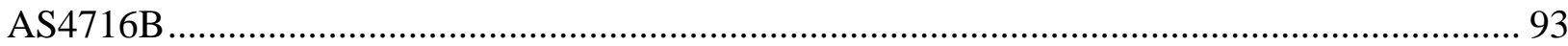

Table 5.8: Sample set of the Dynatool ${ }^{\odot}$ program interface inputs required from the user.......... 95

Table 6.1: Gas spring test verification results vs. Dynatool ${ }^{\circledR}$ theoretical data.......................... 117 


\section{List of Symbols, Constants and Abbreviations}

\section{Symbols}

\begin{tabular}{|c|c|}
\hline$A_{B}$ & Bearing area (projected) \\
\hline$A_{T}$ & Tensile area \\
\hline$A_{p}$ & Piston area \\
\hline$A_{S C}$ & Shear area \\
\hline$F_{A}$ & Force applied or Axial force \\
\hline$F_{T}$ & Tensile Force \\
\hline$F_{S}$ & Static force (at static piston stroke) \\
\hline$L_{B}$ & Bearing length \\
\hline$P_{B}$ & Bearing pressure \\
\hline$P_{S}$ & Static pressure (at static piston stroke) \\
\hline$\sigma_{T}$ & Tensile stress \\
\hline$\tau_{s}$ & Shear stress \\
\hline$A$ & Area \\
\hline $\mathrm{CH}$ & Chamfer length \\
\hline$F$ & Force \\
\hline$I D$ & Inner Diameter \\
\hline$M$ & Moment \\
\hline$O D$ & Outer Diameter \\
\hline$P$ & Pressure \\
\hline$V$ & Volume \\
\hline$W$ & Weight \\
\hline$g$ & Gravity Force \\
\hline$m$ & Mass \\
\hline$r$ & Radius \\
\hline$t$ & Thickness \\
\hline
\end{tabular}



$x \quad$ Cartesian coordinate
$y \quad$ Cartesian coordinate

\section{Constants}

$\begin{array}{ll}\pi & 3.141592654 \\ g & 9.81 \mathrm{~m} / \mathrm{s}^{2} \text { or } 32.2 \mathrm{ft} / \mathrm{s}^{2}\end{array}$

\section{Abbreviations}

$2 \mathrm{D}$

3D

AFT

AIA

$\mathrm{Al}-\mathrm{Br}$

AlSi10Mg

AM

AMM

ANSI

ASME

BLDC

CAD

CG

CRES

DC

DMLS

EDS

EMS

FEA

FWD
Two Dimensional

Three Dimensional

Aft (rear) of an aircraft

Aerospace Industries Association

Aluminum Bronze

Aluminum Silicon 10 Magnesium

Additive Manufacturing

Additive Metal Manufacturing

American National Standards Institute

American Society of Mechanical Engineers

Brushless Direct Current

Computer Aided Design

Center of Gravity

Corrosion Resistant (Steel)

Direct Current

Direct Metal Laser Sintering

Electrodynamic Suspension

Electromagnetic Suspension

Finite Element Analysis

Forward 


$\begin{array}{ll}\text { GD\&T } & \text { Geometric Design and Tolerancing } \\ \text { HDWS } & \text { Hyperloop Deployable Wheel System } \\ \text { ID } & \text { Inner Diameter } \\ \text { INBD } & \text { Inboard } \\ \text { ISO } & \text { International Standards Organization } \\ \text { LOI } & \text { Letter of Intent } \\ \text { LTL } & \text { Lower Torque Link } \\ \text { MLG } & \text { Main Landing Gear } \\ \text { MMPDS } & \text { Metallic Materials Properties Development and Standardization } \\ \text { NAS } & \text { National Aerospace Standards } \\ \text { NLG } & \text { Nose Landing Gear } \\ \text { OD } & \text { Outer diameter } \\ \text { OML } & \text { Outer Mold Line } \\ \text { P/N } & \text { Part Number } \\ \text { QTY } & \text { Quantity } \\ \text { RIHT } & \text { Ryerson's International Hyperloop Team } \\ \text { RPM } & \text { Rotations per minute } \\ \text { SAE } & \text { Society of Automotive Engineers } \\ \text { SS } & \text { Stainless Steel } \\ \text { STOL } & \text { Short Takeoff and Landing } \\ \text { TPIS } & \text { Tire Pressure Indication System } \\ \text { U of T } & \text { University of Toronto } \\ \text { UTL } & \text { Upper Torque Link } \\ \text { VDC } & \text { Voltage-Direct Current } \\ \text { WOW } & \text { Weight on Wheels } \\ & \end{array}$




\section{Introduction}

\subsection{Preamble}

In the summer of 2013, Elon Musk (Tesla, SpaceX) released a white paper proposing a 5th mode of transportation: the Hyperloop [1]. He imagined levitating pods traveling through a low-pressure, near-vacuum tube capable of achieving speeds more than $700 \mathrm{mph}(1126 \mathrm{kph})$ while free of significant air resistance. Consisting of multiple subsystems, Hyperloop pods are expected to include either magnetic or air-bearing levitation as a primary suspension system during high-speed travel, with the magnetic method leading most proposed designs [2]. For added safety and lowspeed travel, 100mph (160 kph), the Hyperloop pods should include a traditional deployable wheel system, similar to an aircraft landing gear [1]. When realized, the Hyperloop is expected to be the fastest form of ground transportation on Earth.

\subsection{The SpaceX Hyperloop Competition}

On June $15^{\text {th }} 2015$, Musk and company announced the SpaceX Hyperloop Competition I. The first of its kind, the competition was created to accelerate the development of the Hyperloop but also succeeded in generating public interest. The competition was divided into three main categories: Design and Build (Pod Team), Conceptual Design Only (Pod Team) and Subsystem (Subsystem Team). In support of the competition, SpaceX organized a design weekend (January 2016, Texas A\&M) and hosted the final pod race at their headquarters (January 2017, Hawthorne California). To facilitate the races and testing of key technologies, SpaceX built the world's first Hyperloop test track outside of their headquarters, a near vacuum tube running approximately $1.5 \mathrm{~km}$ (1 mile) in length [3], [4]. Out of over 1000 student teams from all over the world, 124 were invited to the Design Weekend at Texas A\&M. One year later, in January 2017, 34 teams, comprised of competitors and exhibitors, descended on SpaceX headquarters for the final pod races and product demonstrations. 


\subsection{Ryerson's International Hyperloop Team}

In September 2015, Ryerson's International Hyperloop Team (RIHT), led by the author, submitted a letter of intent (LOI) to compete in the SpaceX Hyperloop Competition I. By October 2015, our team comprised of three graduate students and three advisors (industrial and academic), committed to compete in the Subsystem category of the competition. In January 2016, RIHT joined 124 student teams from over 20 countries at the Texas A\&M design weekend where we presented our concept for a Hyperloop Deployable Wheel System (HDWS). Selected by the judges for the "Subsystem Innovation Award", the team spent the entirety of 2016 building a network of 20 sponsors, enabling the development, manufacturing, marketing and testing of our design. To facilitate the increase in workload and to address the need for additional expertise, the team expanded to 6 students and 5 advisors throughout 2016. In January 2017, our team presented 5 completed prototypes at the final phase of the SpaceX Hyperloop Competition in Hawthorne California.

\subsection{Research Motivation}

The author's motivation to develop a deployable wheel system spun out of text in Musk's Alpha Document that reads the Hyperloop pod "may also include traditional deployable wheels similar to aircraft landing gear for ease of movement at speeds under $100 \mathrm{mph}(160 \mathrm{kph})$ and as a component of the overall safety system" [1]. Considering the operational requirements, environment, power sources available, and hypothetical geometrical constraints of the Hyperloop transportation system, it became apparent that an-off-the-shelf landing gear or the like would not be sufficient for this unique application. To use aspects of existing technologies and lessons learned for a new design, a detailed literature and patent review of landing gear systems was conducted. The works of Currey [5] and Conway [6], experts in the field of landing gear design, provided many starting points for a new Hyperloop-suited design, particularly in the area of oleopneumatic shock absorber design. However, these reviews also left many questions unanswered (e.g. performance impacts of a system in near-vacuum conditions, active control stability, compact design for small operations spaces). These items are not typically addressed in landing gear design (although the author acknowledges the existence of the Space Shuttle landing gears). 
One key challenge, as it relates to the Hyperloop, is associated with the kinematics required by the design while ensuring performance requirements (e.g. smooth ride operations) are met. It is well understood that some aircraft, helicopters and Hyperloop pods, have very limited space for housing a deployable wheel system and/or landing gear. Prior patent art includes systems that attempt to solve the problem by compressing the shock strut element of a landing gear prior to retraction, and, thus, for the duration of stowage, such as presented in Churchill et al. [7]. These are commonly referred to as shrink or contracting struts [5]. The closest viable solution came from Waide [8], who proposed an electrically contracted shock strut, using a coaxial motor. However, in the configuration proposed by Waide, the shock absorption portion of the system is impacted and does not allow for desired energy dissipation (the purpose of such). Additionally, operational space required by both Waide and Churchill et al.'s designs is likely too large to accomplish the space saving goals imposed by the pod geometry.

To address the gap in available solutions, the Hyperloop Deployable Wheel System (HDWS) was designed for emergency and low-speed travel, a necessary addition to future Hyperloop transportation systems. Using an innovative, patent-pending deployable and retractable shock strut [9], the system is operable within small spaces and employs advanced technologies and software that enables the system to provide active height stabilization control and can be fitted with wheel motors as well as regenerative braking capabilities [9].

\subsection{Key Objectives and Contributions}

The objective of the present thesis was to design and develop a deployable wheel system proposed for Hyperloop pods. Using the functional requirements defined within Musk's Hyperloop Alpha document as a baseline, alongside historical and modern landing gear developments, the HDWS was designed and developed. This process demonstrates how the many disciplines of engineering, such as: strength of materials; statics; dynamics; compressible adiabatic $\&$ isothermal gas theory; stress analysis; galvanic corrosion \& material compatibility; tribology; cost versus weight; computer aided optimization and program management, interact in a highly iterative design process to develop the HDWS. The key objectives and contributions are to:

1. Investigate what requirements must be considered to develop a Hyperloop subsystem 
2. Investigate the guidelines, requirements and constraints necessary to develop a deployable wheel system for the Hyperloop and apply those to the creation of the HDWS

3. Develop a baseline system architecture of a deployable wheel system for the Hyperloop

4. Address and discuss technical design challenges imposed by the environment of the Hyperloop as they pertain to the developed HDWS system architecture

5. Provide a detail design overview of the developed HDWS system architecture for the SpaceX Hyperloop Competition I

6. Present key tests performed on prototypes developed under the HDWS system architecture and discuss future challenges and opportunities for future improvements

The author's contribution through the present research and development has led to a patentpending application, for the design of a "Deployable and Retractable Shock Strut," filed with the US Patent Office on April 26th, 2017. The application was sponsored by Safran Landing Systems Canada Inc., the assignee.

\subsection{Limitation of Current Work}

The current work is bound by the following limitations:

1. The five prototype Hyperloop Deployable Wheel Systems (HDWS) were not tested in a near vacuum low-pressure environment due to logistical reasons.

2. Time constraints within the framework of the SpaceX Hyperloop Competition often forced prototype development shortcuts. Whenever present, short comings due to the time constraints are addressed within this report.

3. Budget constraints typically drove a design-to-cost methodology. Consequently, components were not optimized for weight savings, although savings were often realized because of a system performance requirement or a key technology demonstration, such as additive metal manufacturing.

4. For prototyping purposes of proving the basic operational concepts, production quality materials and hardware were not used unless required to meet key performance drivers.

5. The interface architecture was limited by available information (Fall 2015) on pod geometry, layout etc. 


\subsection{Thesis Outline}

This thesis report is divided into seven chapters, outlined as follows:

Chapter 1 provides the backstory and motivation of this thesis. The contributions that are discussed throughout the thesis are summarized and the limitations of the current work are stated.

Chapter 2 contains a literature review of the relevant background information and theory that was used to develop the present HDWS. Background information on the Hyperloop is presented to lay the ground work for the interfacing systems and operational environment challenges. Research on potential solutions is discussed, followed by a brief history of aircraft landing gears. Following the literature review, Chapter 3 provides the product definition for the HDWS by highlighting key functional requirements and performance goals, and defining the prototype's development scope within the SpaceX Hyperloop Competition framework.

Chapter 4 develops the HDWS solution and breaks it down to the subsystem and component level subject to the system architecture defined and highlighted in Chapter 3. Following the detailed system overview, the detailed design of the deployable and retractable shock strut, a complex interdisciplinary concept, is presented in Chapter 5. Chapter 6 outlines the experimental tests performed on the HDWS prototypes. Qualitative and quantitative results are provided where available.

Finally, Chapter 7 concludes with a summary of the current work and proposed future work to be considered. The author's contributions, including co-authored conference papers and presentations, are highlighted in list form. The appendices provide readers interested in design details access to the associated part and assembly drawings, selected concept art, and a sample of raw test data. 


\section{Literature Review}

This chapter is intended to familiarize the reader with the Hyperloop concept, its major components and the medium of pod transportation. The operational environment and constraints imposed on the system are discussed. A brief section is dedicated to the fundamentals of aircraft landing gear, providing necessary background terminology. Finally, background information on the field of advanced aircraft landing gear system design is presented. Relevant information discussed in this chapter, required to design the Hyperloop Deployable Wheel System (HDWS), is then utilized in Chapter 3.

\subsection{The Hyperloop}

There currently exists only four fundamental forms of transportation: planes, trains, ships and automobiles. These modes of transport use air, rail, water and roads as a medium for operation. Proposed as the $5^{\text {th }}$ mode of transport in 2013, the Hyperloop is conceptualized as a new, very high speed, intercity transportation system [1], [2], [10]. At its core, the Hyperloop transportation system consists of: a set of near-vacuum tubes running between cities, pods traveling through the tubes transporting cargo and humans, and stations at the beginning and end of each route.

As a result of sending pods through a self-contained low-pressure tube, the Hyperloop transportation system is immune to wind, rain, ice and fog - weather elements that cause delays or cancelations for every other form of transportation [1], [2], [10]. Acknowledging the potential benefits of quiet supersonic air travel for longer distances, the proposal is intended for corridors in the 900-mile $(1500 \mathrm{~km})$ range, such as the now infamous Los Angeles to San Francisco route [1]. As an open-sourced idea first proposed in 2013, various companies such as Hyperloop One [11], Hyperloop Transportation Technologies [12] and Transpod [13] are working to bring the Hyperloop to life in parallel to SpaceX's Hyperloop Competition.

\subsubsection{Pod Overview}

Key to the Hyperloop architecture is the use of pod structures to transport humans and cargo through the near-vacuum low-pressure environment. Current configurations, as proposed by Musk 
and developers, expect to transport up to 28 passengers per pod [1]. Because of the environment, the pods are imagined by most, particularly in the aerospace industry, to have an aircraft fuselage frame structure best suited for the cyclic loading induced by traveling between standard (station) and low-pressure environments (tube). A typical pod shall use either passive magnets or airbearings for the primary suspension (or levitation) system. Both suspension systems have been demonstrated. Hyperloop One has conducted successful tests on the passive magnetic system [14], and student teams have recently demonstrated air-bearing levitation in the SpaceX Hyperloop Competition II [3]. As originally proposed by Musk, current pod designs also use wheels as a secondary mobility system for loading and unloading at stations as well as low-speed travel [14].

\subsubsection{Major Subsystems Breakdown}

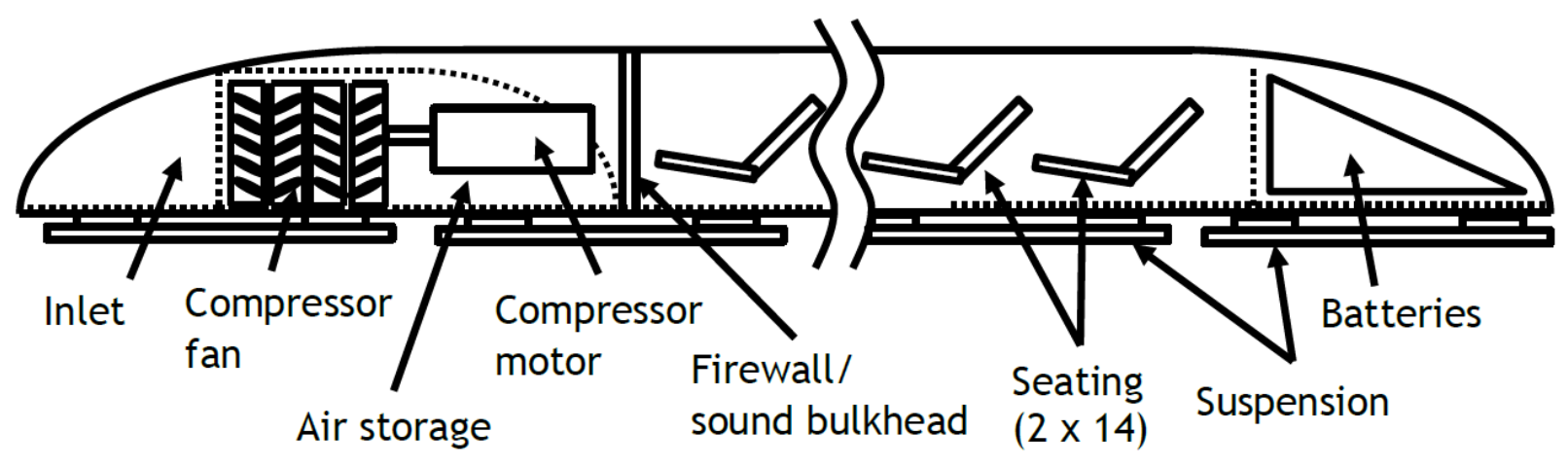

Figure 2.1: Example of a Hyperloop passenger pod, subsystem notional locations (not to scale) [1]

A typical Hyperloop pod architecture, as illustrated in Figure 2.1, is considered to include, but is not limited to, the following subsystems:

1) Life Support System: Pressurized air, collected and/or stored in onboard tanks, is required for pressurized cargo and passengers. The design of pressurized section of the pod will be similar to that of aircraft fuselage and or spacecraft [1]. A life support system should also be capable of circulating air and provide a method of regulating temperature.

2) Onboard Power: The pods are proposed to run exclusively on electrical power. The various capsule configurations are to include batteries, like the type used onboard the Tesla Model S, for energy storage, which can be quickly swapped out and charged at each station [1]. 
3) High-speed Levitation System: To achieve the high speeds proposed within the Hyperloop architecture, the pod shall traverse the track using a low-friction interface to reduce the energy required to sustain the speeds and provide a smooth ride [1]. The initial proposal called for airbearing (or air-cushion) based levitation, like an air-hockey table. However, more recent concepts, like the one shown in Figure 2.2, have opted to use a more familiar magnetic levitation source with the added penalty of larger drag forces at low speeds [15], [16].

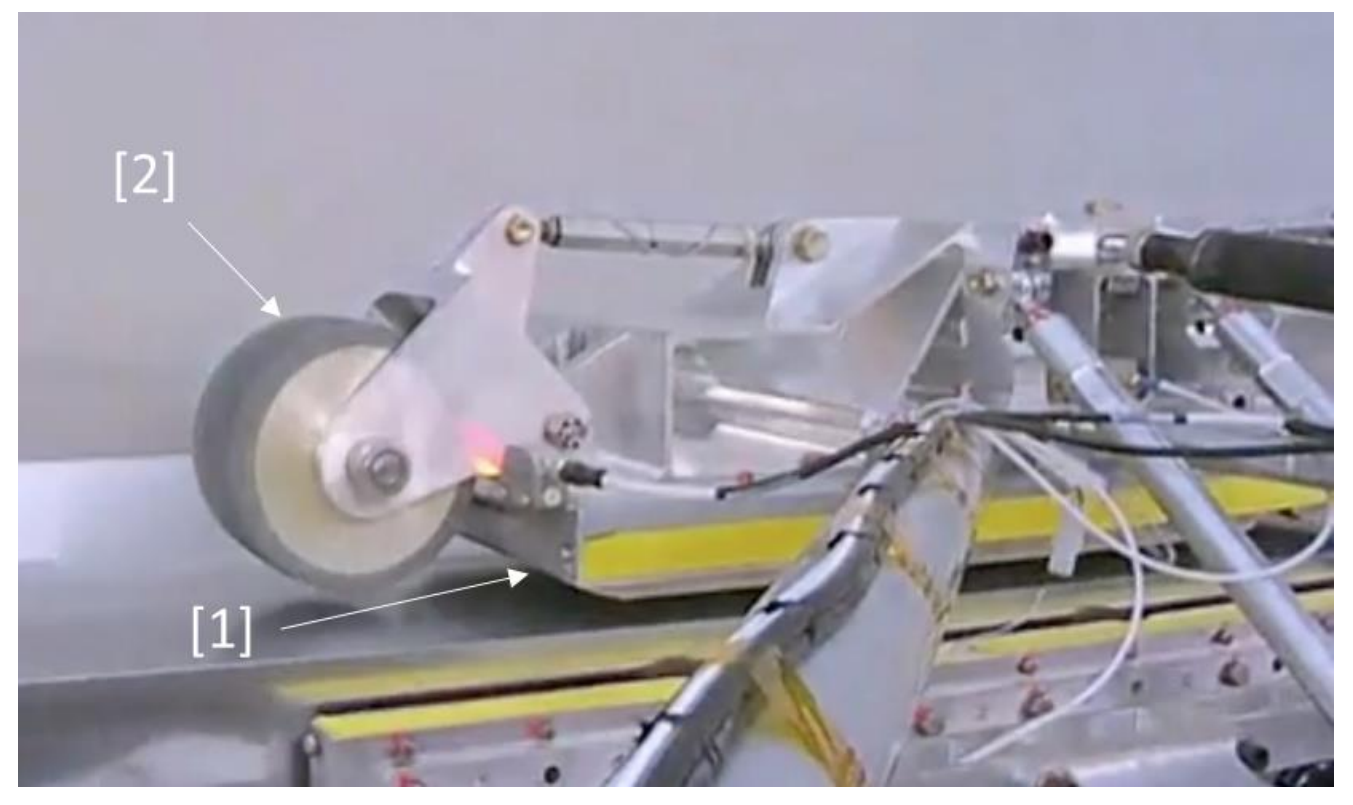

Figure 2.2: Hyperloop One's test "sled" is shown to include (1) magnets for levitating and (2) a rudimentary wheel system in tow [14]

4) Low-speed Levitation System: For low-speed travel less than $100 \mathrm{mph}(160 \mathrm{kph})$, loading passengers and cargo at stations, and as a component of the overall safety system, a deployable wheel system, similar to an aircraft landing gear is included [1], [17]. As recently shown in July 2017, Hyperloop One's test sled (Figure 2.2) is shown to incorporate a rudimentary retractable wheel system.

5) Propulsion System: The Hyperloop will use an advanced linear motor, suitable for operation within a vacuum and capable of dissipating generated heat. The linear motor proposed by Musk [1] is being developed to accelerate the pod at speeds approaching $760 \mathrm{mph}(1220 \mathrm{kph})$ at a maximum of $1 \mathrm{~g}$ acceleration to ensure passenger comfort. Musk proposes to equip the rotor (or moving element) on the pod, and the stator (stationary motor element) to the tube structure. 
Hyperloop One has created a similar "proprietary electric propulsion system" that was first successfully demonstrated in May 2016 [2].

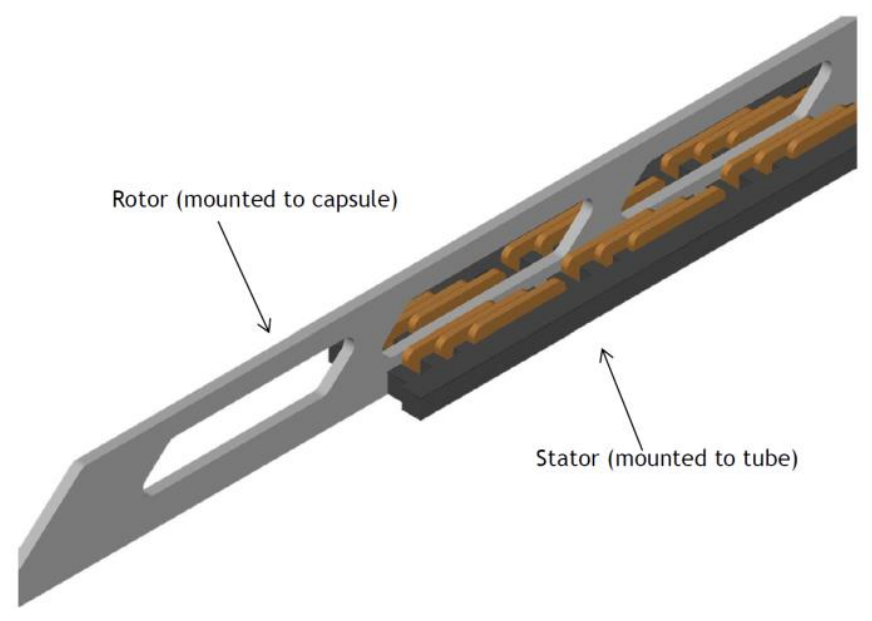

Figure 2.3: Rotor and stator 3D diagram [1]

6) Braking System: Current pod designs propose the use of permanent magnets actuated by electrical mechanical actuators, as a primary braking source. No clear secondary braking system has been proposed, although this requirement has been recognized by those in the industry for safety and future certification activities [2]. The author proposes that a secondary, wheel-mounted braking system, for lower speeds, should be investigated.

To design a subsystem for integration to a pod, it is important to understand the interdisciplinary couplings inherent to the Hyperloop System [18]. Thus, a non-exhaustive list of key subsystems has been presented. Information gathered from the present summary, such as the pod's proposed reliance on electrical power, begs one to consider the available resources at hand when proposing a Hyperloop wheel system design.

\subsubsection{The Tube System}

The tube system, shown in Figure 2.4, is comprised of large, partially evacuated (0.015 psi or 100 $\mathrm{Pa}$ ) steel tubes mounted on support pillars. Steel has been the common choice among Hyperloop tube developers for its low cost and weldability. Although two Hyperloop tube systems are currently in operation, no "final size" for the tube has been selected. A recent study in 2015, 
conducted by a group of researchers at NASA's Glen Research Center, indicated that the required tube size is highly dependent on the pod geometry required and the intended speeds to travel [18]. Thus, this may be a route-specific decision.

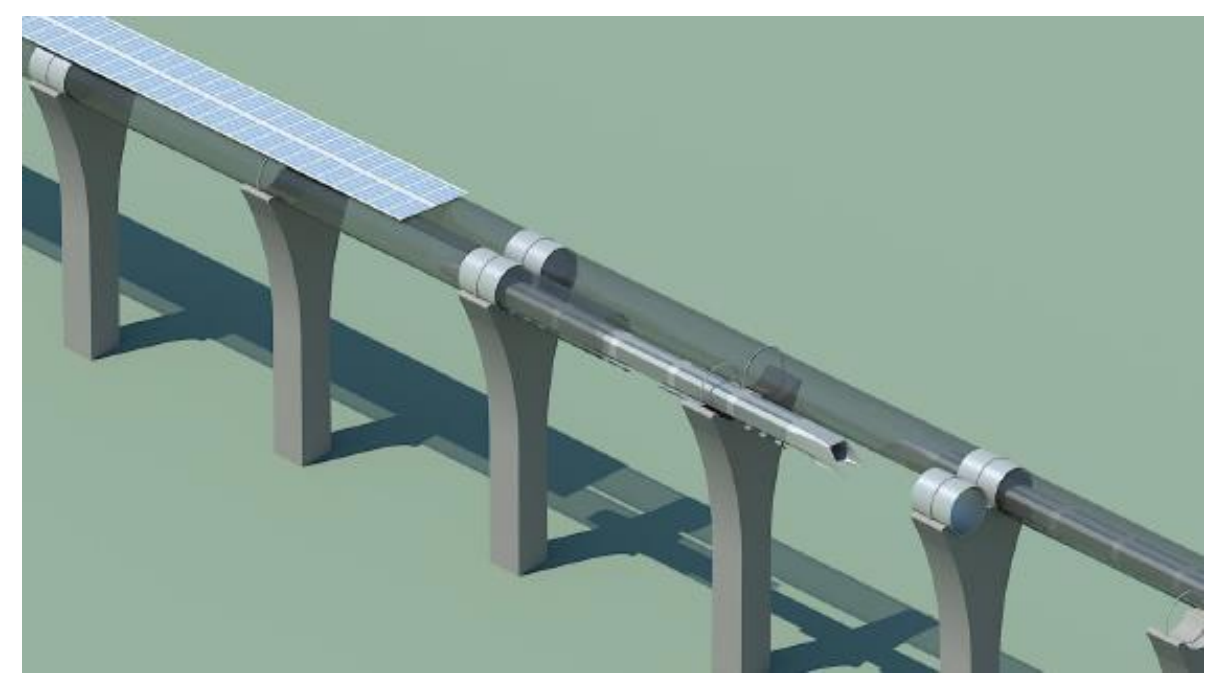

Figure 2.4: Artist rendering of a Hyperloop tube cutaway equipped with solar arrays [1]

The tube support columns, to be made of reinforced concrete, will be equipped with specially designed slip joints to combat thermal expansion [1]. Hyperloop One recently announced that they will be incorporating "isolators and dampers" to limit the effects of earthquakes [2].

\subsubsection{SpaceX Hyperloop Tube System}

In support of the SpaceX Hyperloop Competition, SpaceX built a one-mile long, six-foot in diameter Hyperloop tube system (see Figure 2.5), located at SpaceX headquarters in Hawthorne, California. The tube is lined with concrete base on which aluminium (Al 6101) track plates and an aluminium I-beam are fixed [3]. SpaceX claims that the Hyperloop system developed is approximately half scale, when compared to the eventual production size [19]. Since the present work was originally designed at scale for the SpaceX Competition, the specifications of the tube/track, referenced for the author's design in Chapter 3, correspond to the SpaceX Hyperloop tube system. 


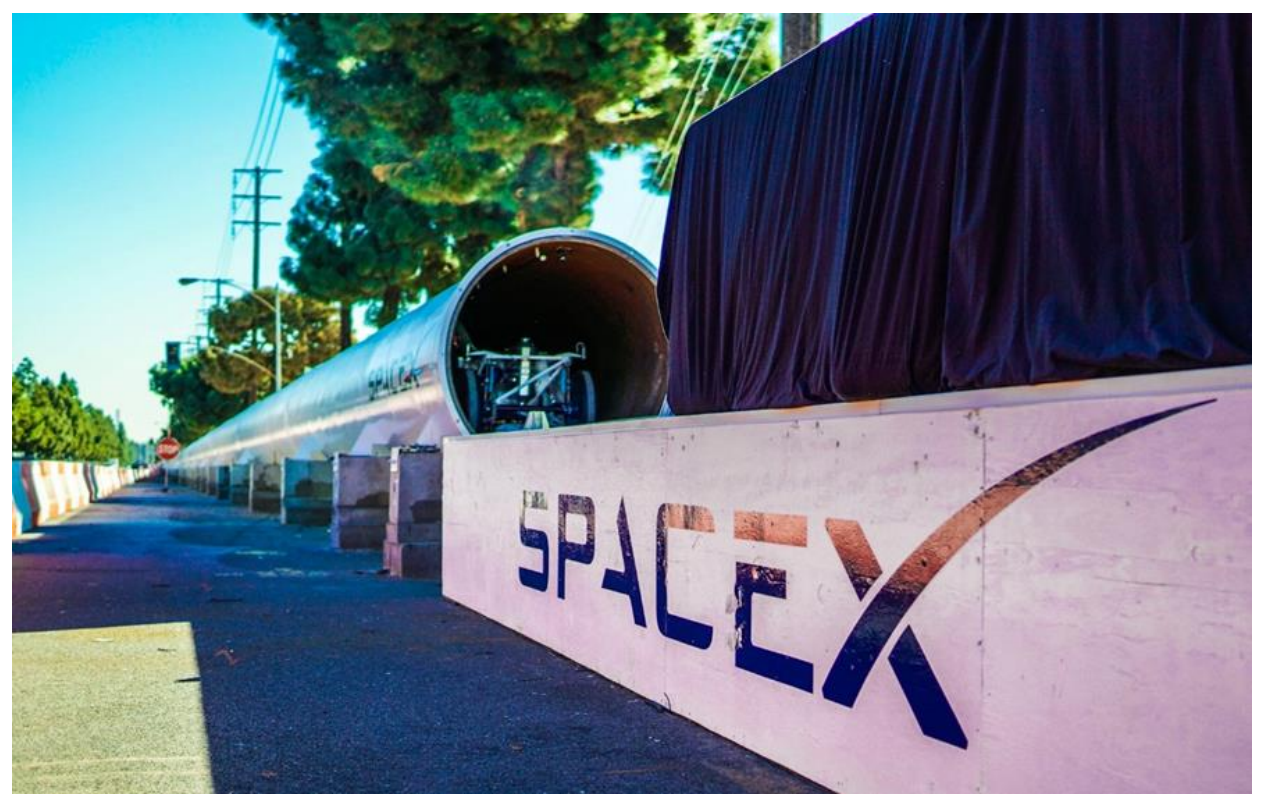

Figure 2.5: Image of the SpaceX Hyperloop sub-scale test track, image taken January 2017

\subsection{Hyperloop Pod Operation Environment}

In addition to understanding the interdisciplinary interactions between subsystems within the pod architecture while developing a deployable wheel system, an understanding of the external operational environment must be understood and quantified when necessary.

\subsubsection{Low-pressure Environment}

The pod will be subjected to cyclic pressure loading as it transitions from the pressurized stations $(14.7 \mathrm{psi}$ or $101.3 \mathrm{kPa})$ to the low-pressure tube (0.015 psi, or $100 \mathrm{~Pa})$. In emergency situations, the rate of depressurisation, due to a tube breach or leak, would be uncontrolled. Regardless, it will be necessary to design the pod frame to withstand this pressure loading. If a deployable wheel system is to use a typical pneumatic tire/wheel configuration, the pressure changes must be accounted for or an alternate "tire" material, perhaps unpressurized, must be selected. However, the loading due to pressure is only one consequence of a low-pressure tube.

Another critical factor imposed on the design of the pod and its non-pressurized subsystems, like a deployable wheel system, is the effects of outgassing. Outgassing is the release or evaporation of gases that were trapped, dissolved or absorbed in a material [20]. Thus, the design engineer 
must be cautious of selected lubricants, adhesives and even materials that can be prone to outgassing. Gases omitted from materials within the vacuum have the potential to contaminate and sometimes cloud optical sensor equipment. The tendency of a material to evaporate is defined by its volatility [20]. This becomes a critical design element when considering the lubrication of joints and the seizing thereof after a long period of outgassing. The design of the HDWS proposes methods to reduce and eliminate the possibility of seizure at all rotational wear surfaces, as discussed further in Chapter 3.

\subsubsection{High-speed Operation}

The Hyperloop pod will theoretically travel at speeds of up to $760 \mathrm{mph}(1,220 \mathrm{kph}$, Mach 0.99 at $68^{\circ} \mathrm{F}$ or $20^{\circ} \mathrm{C}$ ) [1]. These speeds, not yet achieved during Hyperloop testing [14], are capable of being reached in part due to the passive magnetic or air bearing levitation systems. That is, at high speeds, conventional wheel and axle systems, like on a car or landing gear, become impractical due to high frictional losses and dynamic instability [1]. A reader familiar with aircraft landing gear design can appreciate that the instability of a wheeled system at high speeds can result in shimmy loading [5]. Liu et al. [21], published authors in the art of Maglev train design, have also indicated the extreme wear and tear from the frictional loading between wheels on rails at high speeds. This may also lead to accelerated equipment deterioration.

Since a wheel system designed for the Hyperloop may be required in high-speed emergency situations, a system shall be designed with consideration of all the above aspects. Particularly, one can now appreciate another reason for the retractability of a wheeled system. That is, a fixed system, constantly cycling through touch-and-go loading due to changes in magnetic and air levitation distances, may cause premature wear, especially at high speeds.

\subsubsection{Thermal Management}

As noted by Musk [1] and Chin et al. [18], each pod passing through the tube adds energy to the surrounding low-pressure air in the form of heat. When the frequency of pod launches is considered, the heating of the overall environment becomes non-trivial. Thus, in the absence of normal atmospheric pressure, and the circulation thereof (convective cooling), a significant 
medium for heat dissipation is lost [1]. Consequently, when designing subsystems, such as a deployable wheel system, the thermal management must be considered and addressed.

\subsection{Aircraft Landing Gear}

\subsubsection{A Brief History}

Landing gears support the entire weight of the aircraft during takeoff, landing and ground operations [22]. They are designed to endure the harsh loading conditions at touchdown and ensure a soft and safe landing. Because of this, one author refers to landing gears as "the essential intermediary between the aeroplane and catastrophe" [6].

According to Currey [5], the first aircraft to use wheeled landing gears appeared shortly after the Wright Brothers' first flight in 1903. However, it wasn't until World War II that aircraft began to adopt a more recognizable architecture. The addition of shock absorbers, a key component of modern landing gear, became necessary as aircraft weight and rate of descent (sink) speeds increased while runways surfaces became harder and wartimes demanded performance on uneven terrain. These shock absorption designs included modern oleo-pneumatic (oil and gas) struts in addition to less common rubber blocks, compression springs, and liquid springs, to name a few [5]. In parallel, as aircraft technologies advanced and enabled higher flight speeds, the retractable landing gear became a popular method to improve aerodynamic efficiency.

Nowadays, modern aircraft utilize state-of-the-art landing gears equipped with numerous and typically complex systems such as steering, electrically operated composite brakes, hydraulic extension and retraction actuators in addition to complex electrical sensing and control instrumentation.

It is important to note that not all landing gears use wheels - some use skis, while others that land on water use pontoons. However, the subject of this thesis is closely related to retractable wheeled landing gears so other concepts are omitted for brevity. For more detailed information regarding the history of landing gear, wheeled and otherwise, reference [5], [6] and [22] provide a great starting point. 


\subsubsection{Key Components and Configurations}

Landing gear architecture is constantly evolving to meet the needs of each new aircraft's unique performance requirements. Early configurations, such as those seen in World War I, made use of a tail gear arrangement (Figure 2.6) with the main landing gear (MLG), fixed, or, in later years, retractable, located forwards of the aircrafts center of gravity (CG). Consequently, this required support in the tail from a third wheel [22]. Later, during World War II, as aircraft achieved higher speeds, the retractable landing gear in a tricycle configuration became a popular method to reduce parasite drag and improve aerodynamic efficiency at high speeds. These configurations typically consisted of a single nose landing gear (NLG) at the front and at least two MLGs slightly aft of the aircraft CG [5]. In all cases, landing gears are attached to rigid primary structural elements typically wing spars, and the fuselage structure (nose and mid) are primary candidates. The most relevant designs to the current subject are wheeled configurations.

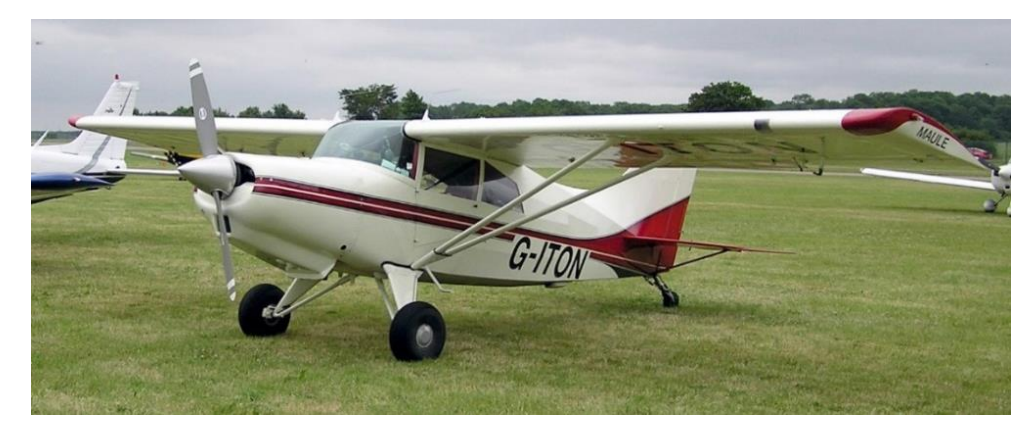

Figure 2.6: Tail wheel configuration landing gear on a STOL Maule MX-7-235 Super Rocket

The development of landing gear kinematics and part geometries were discovered to be interdependent during the design process. Since landing gear designs are heavily centered on their retraction and extension capabilities, landing gear was used as a baseline for the HDWS conceptualization, rather than attempting to adapt fixed wheel/tire configurations on cars and trains. After reviewing existing architectures, the author chose to closely mimic that of an aircraft trailing arm MLG, an example of which is shown in Figure 2.7. However, a distinct feature of this concept is the requirement of an inboard swinging action for retraction - an action that would be logistically difficult within a Hyperloop pod design space, if only because of its proximity to the ground (sub-track). 


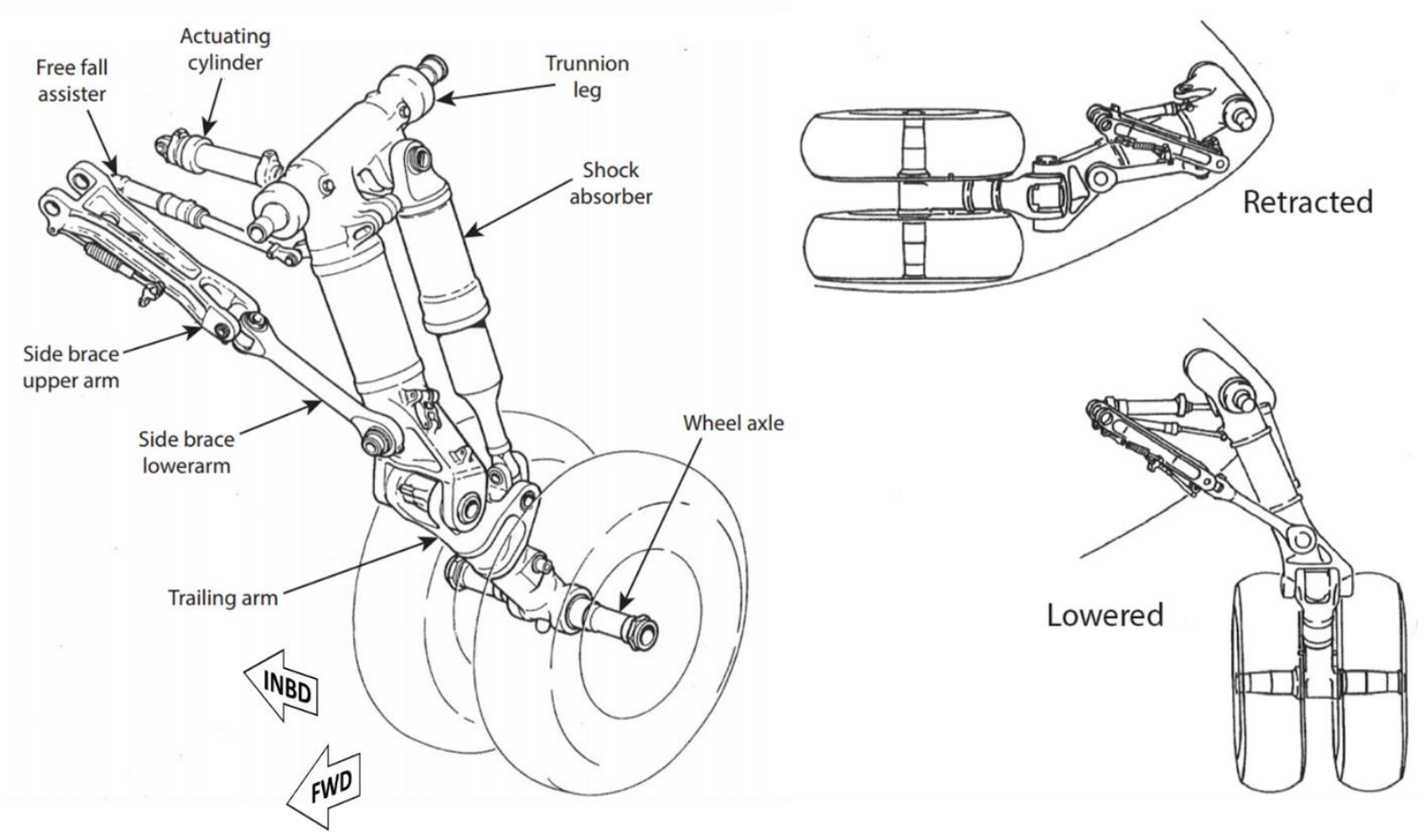

Figure 2.7: General arrangement of a retractable MLG Trailing arm configuration [23]

This thesis presents a novel solution to retain the benefits of a trailing arm configuration, shown in Figure 2.7, while at the same time achieving better retraction architecture for the Hyperloop pod. To appreciate the new approach, common landing gear components are briefly defined next to equip the reader with basic landing gear terminology.

\subsubsection{Common Components}

It is fair to say that most landing gears, at least within similar classes of aircraft, use very similar design features and components. Landing gears typically consist of the following key components [22]:

Shock Absorber - Referred to by Currey as "usually the most complex part of the landing gear," the shock absorber (or shock strut) is designed to dissipate energy during taxi, landing and takeoff [5]. The most common modern configuration is oleo-pneumatic with damping to limit the piston's rate of motion and avoid spring back effects [24]. The shock absorber is typically used as a main structural member, interfacing with the aircraft frame in NLG configurations, subject to bending 
loads, or as an axially loaded member of the MLG trailing arm configuration, shown in Figure 2.7. The design of the shock absorber for the present work is covered in Chapter 5.

Main Fitting - In MLG configurations, the main fitting or "trunnion leg" as shown in Figure 2.7 is the primary interface between the landing gear and the aircraft. A main fitting is typically rotationally mounted via oppositely disposed trunnion pins as can be seen at the top left section of Figure 2.7.

Trailing Arm - In MLG configurations, the trailing arm acts as a lever arm, connected to both the shock strut assembly and main fitting through pinned sockets or lugs. Typically, trailing arms are subject to tension loading and are designed to resist torsion induced at landing, during taxi and otherwise. Generally, the trailing arm is designed to ensure only axial loading is transferred to the attached shock strut. It is further noted, as added value to the author's present work, that the trailing arm configuration is highly stable at landing and taxiing due to its lever arm configuration. Geometrically, referring to Figure 2.7, the vertical wheel axle travel (VAT) and the travel (stroke) of the shock absorber piston are not the same. This allows for energy absorption through relatively large displacements at the axle, compared to the direct 1:1 relationship in cantilever shock struts like the NLG configuration previously discussed.

Drag and/or Side Brace Arm - Drag and/or side brace arms are often required as additional load bearing members. Some larger aircraft with higher loading and longer main fitting/shock strut members tend to incorporate both drag and side braces (see Figure 2.7) in their design for added stability and load carrying capacity.

Steering Systems - Used almost exclusively on NLG architectures, landing gears can be equipped with either rack and pinion or tandem actuator steering subsystems. As a steering system was not considered in the present work interested readers are referred to Currey [5] who provides an excellent detailed overview into steering system designs and their operation.

Wheels and Brakes - Landing gear are typically equipped with specially designed pneumatic aircraft tires. Most gears, typically the MLG, contain carbon or steel brakes, actuated either by hydraulic or electrical means.

Retraction Mechanism- The retraction of a landing gear is generally accomplished, in part or wholly, by a retraction actuator disposed between the landing gear structure and the airframe. 
These systems typically operate using hydraulic fluid supplied throughout the aircraft. As can be found in various patents the design of electric or hybrid retraction actuators is an ongoing subject of interest.

Feedback Sensor Equipment - Weight on Wheel (WOW) sensors are common main stay alongside tire pressure indication systems (TPIS) and steering feed-back transducers. As landing gears become more and more electric, there is likely to be a demand for increased sensor presence, including health monitoring.

\subsection{Existing Patents}

A patent search was conducted to find state-of-the-art landing gear retraction systems, shock strut designs, and kinematic layouts suited for tight spaces. The review revealed that the subject of "contracting" the shock absorber prior to retraction, but retaining a separate traditional retract actuator, is frequently addressed. These "shortening" or "contracting" mechanisms frequently provided excellent solutions for cantilever type landing gears, such as an NLG and canted MLG configuration. In some cases, as presented by Sharples [25], designs enable a kneeling function that is useful for helicopters and military transports. Mostly, however, the goal is to ensure the landing gear would fit inside the allotted stowage volume in the aircraft. Churchill et al. [7] presented a configuration that is capable of retracting and extending the shock strut piston into the outer cylinder by transferring pressurized hydraulic fluid into a shrink chamber, resulting in an overall lengthening and shortening of the shock strut. Although this provides a potential means for saving space when stowed, it relies on external pressurized hydraulic fluid sources, which would not be available on Hyperloop pods.

Sharples [25] presents a tempting solution (Figure 2.8) to not only shrink the effective length of the shock strut, but also to retract it into the aircraft using a ball screw and ball nut guide, both externally mounted. The design uses both electric power (for retraction) and hydraulic fluid pressure (for contraction) ported from the aircraft. The adaptation and detail design of the proposed architecture would require the addition of a hydraulic reservoir and a significant portion of vertical space in a pod frame design. The use of a screw and nut for retraction will eventually be adopted, in another form, by the author. 

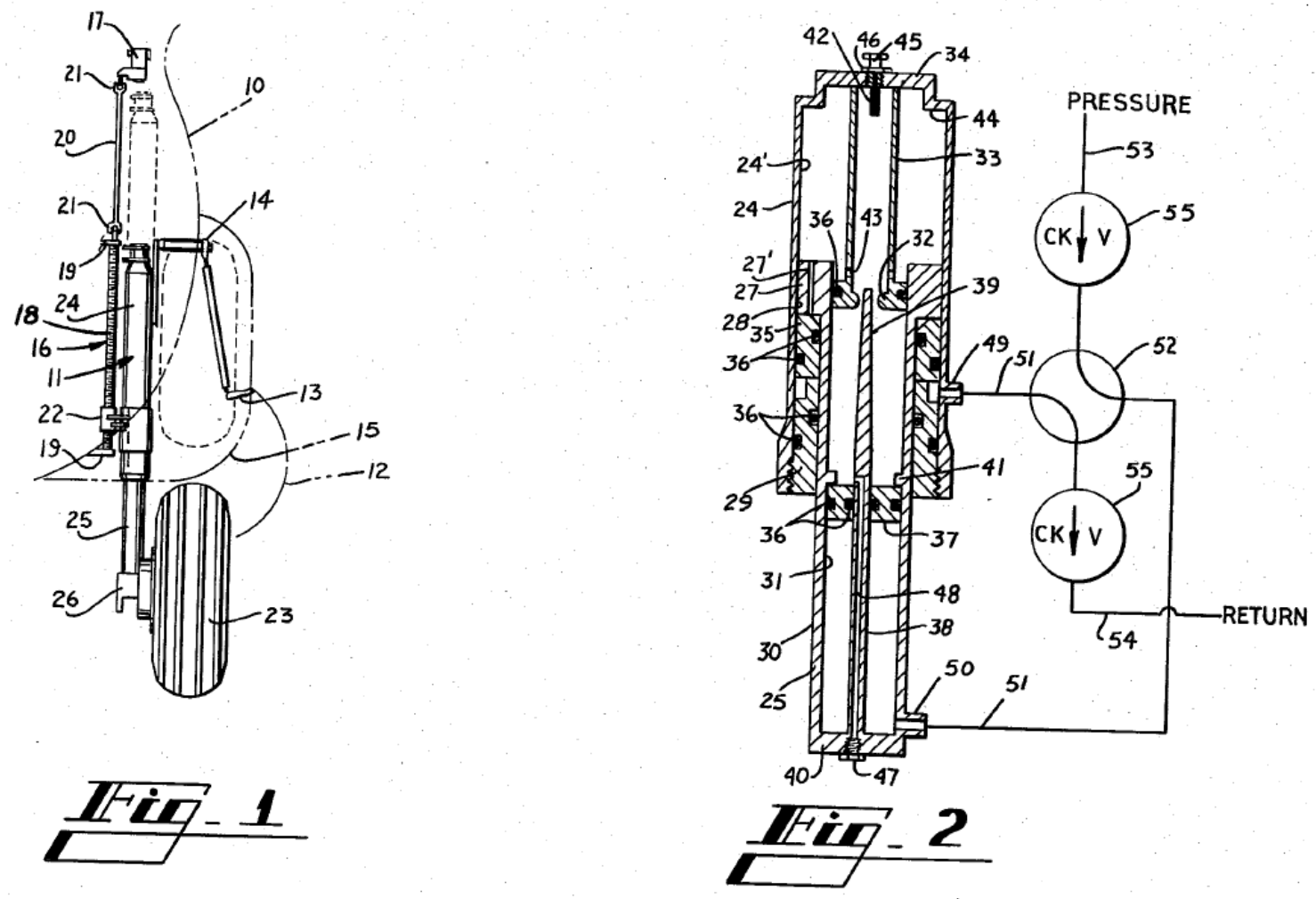

Figure 2.8: Sharples kinematic retraction configuration (left) with a cutaway of the contractive shock strut (right), shown as items 11 and 25 in the kinematic configuration (left) [25]

In another patent, Veaux et al. [26] provided a different approach, internalizing the contraction mechanism of the shock strut. In one embodiment, shown in Figure 2.9, the design integrates an electric motor into the bottom of a modified shock strut piston. Using a rotating internal ball screw shaft and a fixed internal ball nut, the total length of the landing gear strut can be adjusted to modify the attitude of the airplane. This procedure is to be done when stationary or at low speeds without affecting internal gas spring properties. In its current embodiment, this concept does not aid in the act of retraction, nor in any appreciable shortening and may in fact add to the overall length of such strut by design. Furthermore, a large internal diameter is required to house the motor, the size of which is typically a function of the required torque output. 


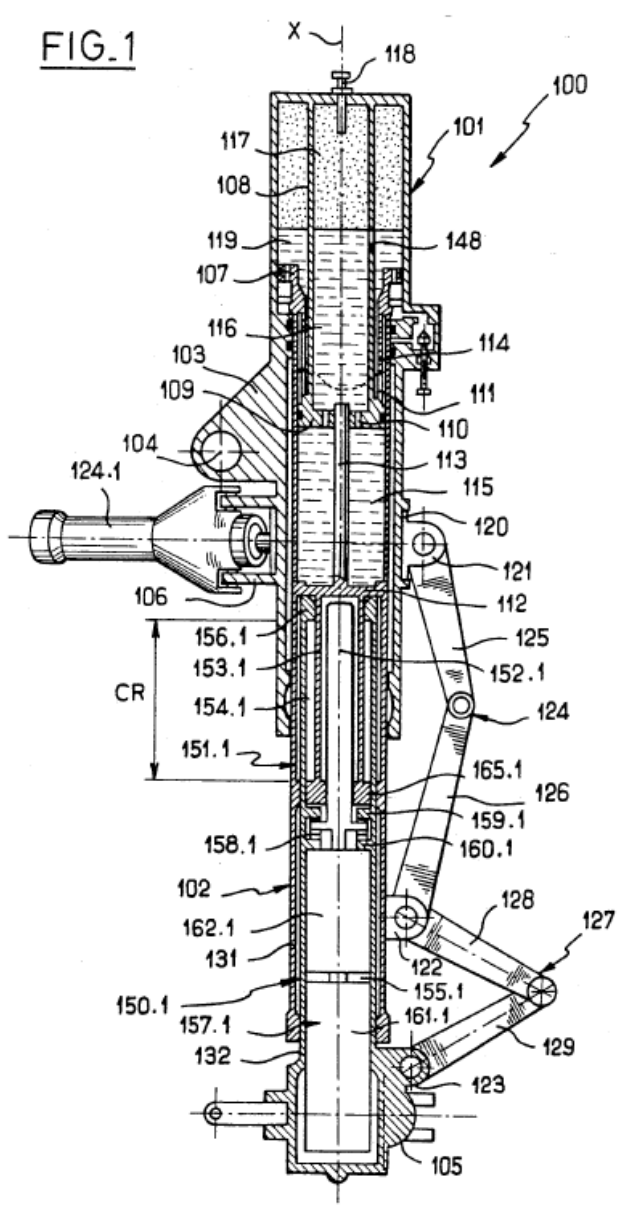

Figure 2.9: An illustration provided in Veaux et al. illustrating a motor and gearbox assembly embedded into the lower half of a piston to lengthen the strut [26]

Another variant on an internally integrated screw shaft, nut and motor system is proposed by Currey and Renshaw [27]. This configuration, proposed for short takeoff and landing (STOL) vehicles, allows for a considerable amount of lengthening and shortening and thus provides a means for vehicle attitude adjustment. As with the design of Veaux et al. [26], Currey and Renshaw's design is not capable of providing a decreased storage length. It does however include a clever variance in the design - a rotating ball nut and a fixed lead screw by means of an adjacent torque rod, shown in Figure 2.10. 


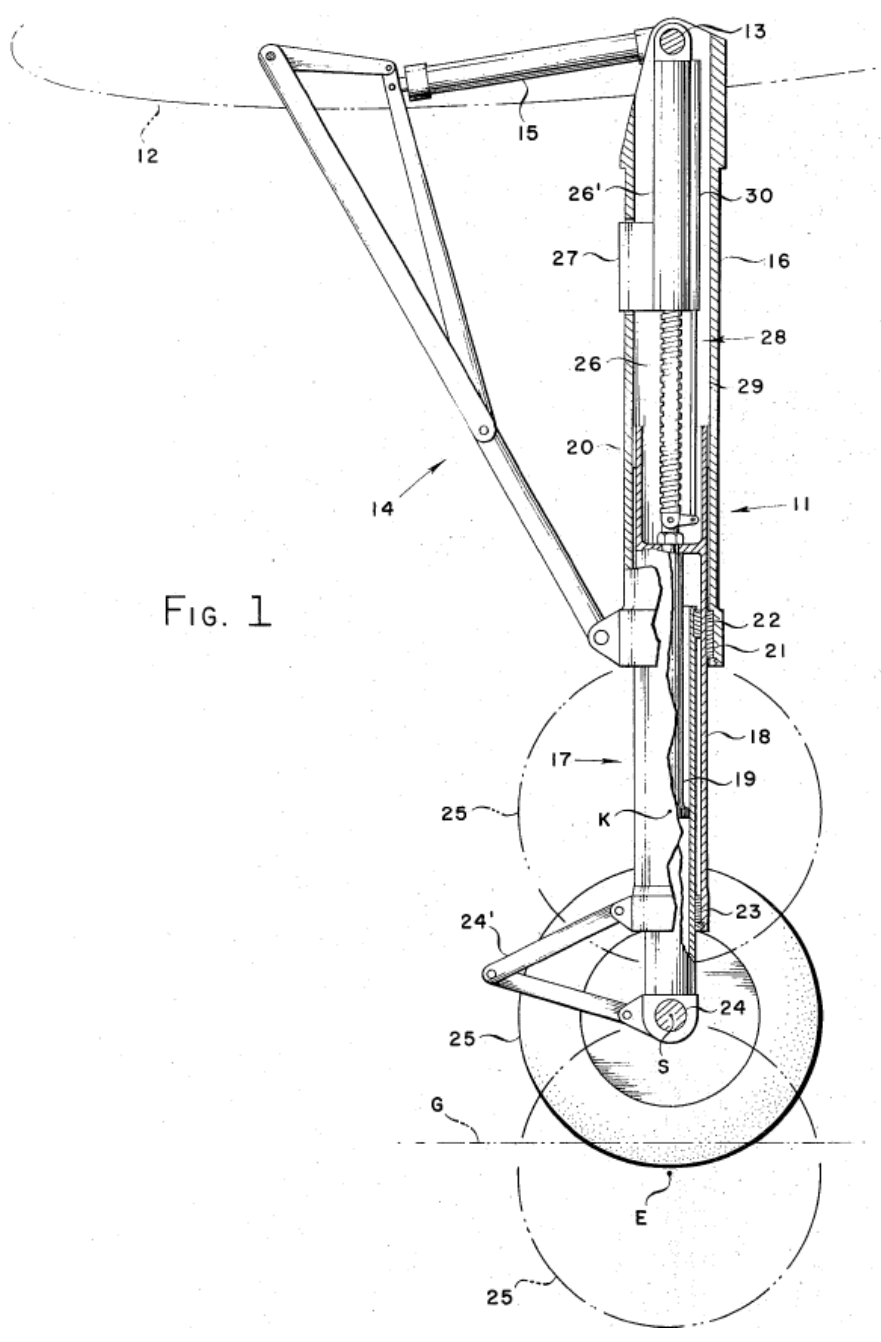

Figure 2.10: An illustration of the design proposed by Currey and Renshaw [27]

Waide [8] presents a landing gear, developed for use in helicopters, that enables small space operation, planner motion (advantageous for the Hyperloop design), electrical operation and even active stabilization capabilities. Although the overall kinematic system of the design by Waide, shown in Figure 2.11 is far more advantageous than the traditional inward swinging MLG retraction, the gear still occupies a considerable vertical stowage space within the aircraft body. One familiar with the art of shock strut design will note that the system exhibits a questionable interference with the internal shock strut geometry, due to the space allocated for the lead screw rod, shown in Figure 2.12, that would prohibit adequate gas spring properties (mainly pressure over area), drive a large internal shock strut diameter, and limit the integration of dampening features. 


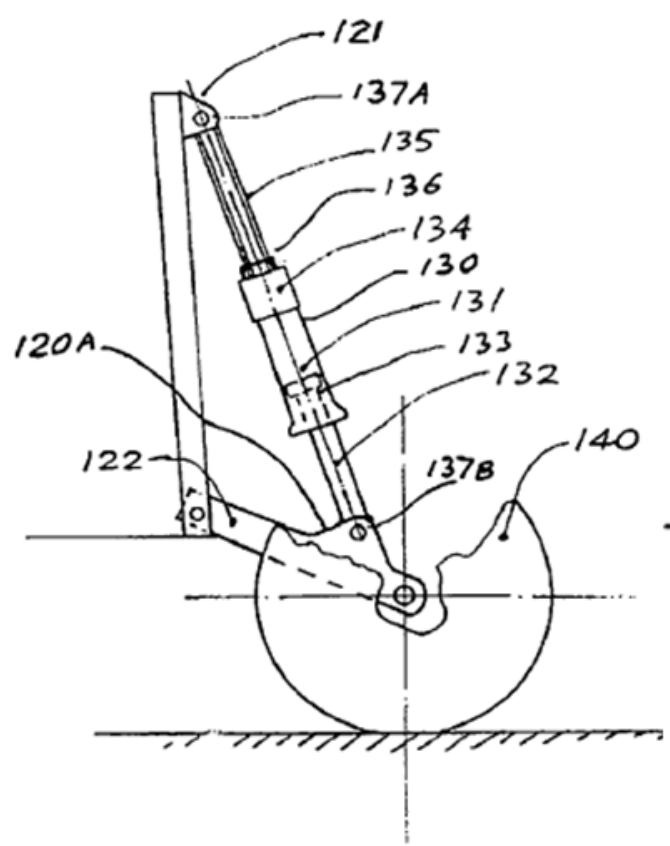

Fig. 3

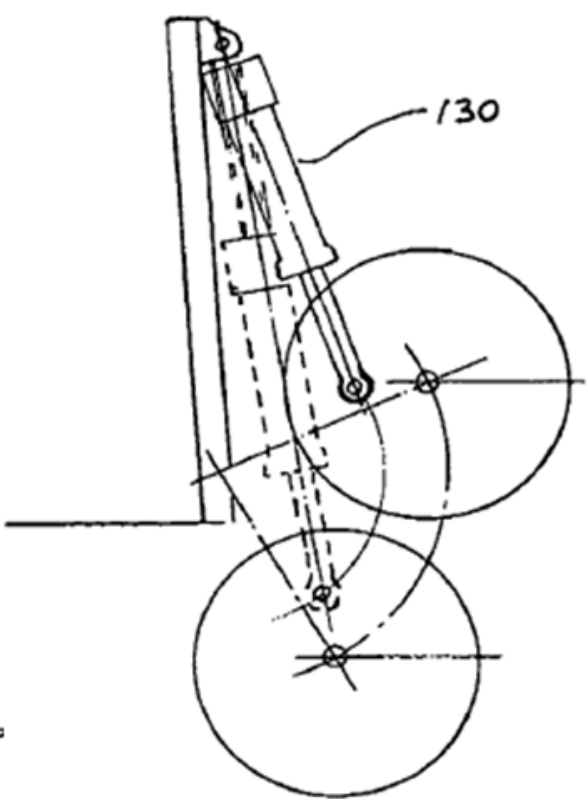

Fig. 4

Figure 2.11: An image (left) illustrating key components and with the advantageous kinematic architecture displayed (right), proposed by Waide [8]

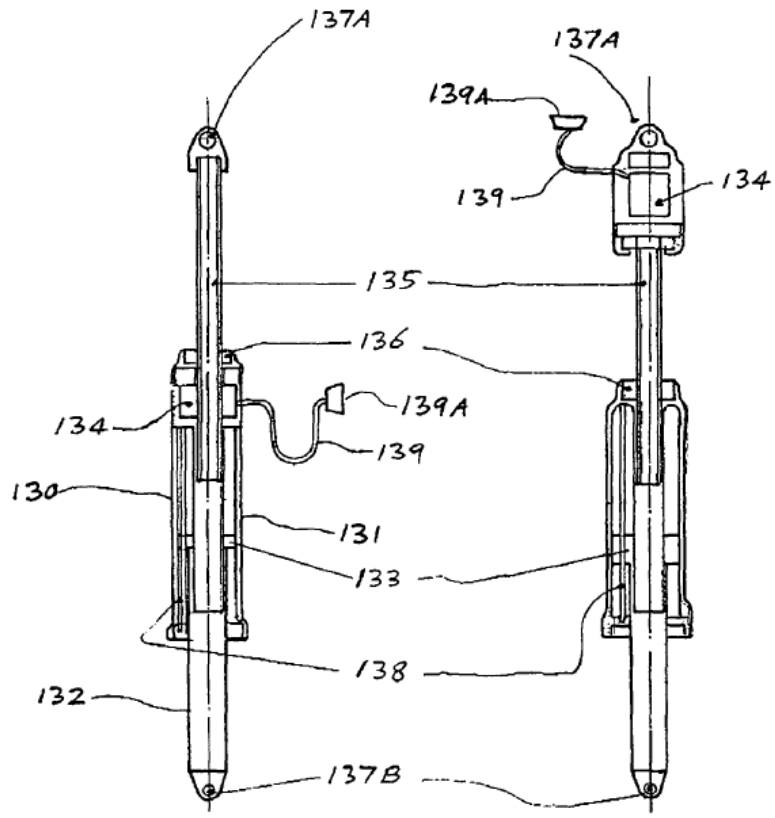

Fig. 5

Fig. 6

Figure 2.12: Two possible configurations of a retractable shock strut, proposed by Waide [8] 
Generally, the design proposed by Waide [8] introduces undesirable load paths highlighted by:

- A long, slender, loaded lead screw rod, that when subject to axial loading, could tend to buckle

- A configuration that allows direct axial load transfer through the motor gear shaft

- Anti-rotation using an internal sliding rod linked directly to the shock strut piston, fixed to a trailing arm with a spherical bearing (this is not a good design, spherical bearings cannot react torque)

The designs' clear optimization of shock strut dead length (the limiting of unused volume), kinematic simplicity and reduction in part count (no side brace, no separate retraction actuator) are acknowledged.

The proposed shock strut configuration for the design of the HDWS, to be discussed in subsequent chapters, addresses the specific volumetric constraints and retraction/extension requirements imposed by Hyperloop pod and tube geometry and thus provides a clear benefit over the configurations mentioned here. 


\section{Product Definition and Preliminary Design}

In Chapter 2 the reader was introduced to the Hyperloop transportation system concept and received a breakdown of its major components and subsystems. The author's review and understanding of the operational environment along with its challenges were addressed and will be frequently referenced throughout the remainder of this thesis. Further, a brief overview of aircraft landing gear and a patent search regarding innovative retraction and shock absorbing configurations were presented. As will be discovered in the present chapter, these subjects together provide a necessary background to appreciate the author's Hyperloop Deployable Wheel System (HDWS) design.

This chapter outlines the product definition for the HDWS, including an overview of the system architecture and kinematic arrangement. This will provide the basis of knowledge required to appreciate the detailed component discussions in Chapter 4 and Chapter 5. A component definition scheme is presented and partially illustrated using a product structure tree - an essential tool in organization of a design project. Lastly, the design process followed for the present development is summarized.

\subsection{Precondition: Why Wheels?}

As discussed in Chapter 2, the Hyperloop pod may utilize magnetic or air-bearing levitation to enable high speed travel without frictional losses. The necessity of an additional system, in the form of a wheeled system, will now be affirmed.

\subsubsection{Air Levitation Pod}

A Hyperloop pod relying on air-bearing levitation, as defined by Musk [1], uses compressed air ejected through pod-mounted skis to enable the pod levitation. During a typical journey, the pod replenishes onboard air storage tanks with compressed air received through the intake compressor mounted at the front of the pod. However, when at rest, such as in a station, it is not reasonable for a pod to use the stored air to maintain levitation as it awaits passengers and cargo. Further, if airlevitation is to be used because of the efficiencies at high speed (no rolling friction or excessive 
wear), then why not utilize a wheeled system for low-speed and tight turns, as proposed by Musk. Further, if a pod is to rely on compressed air for levitation, what happens if there is a failure in the air supply system? As proposed by Musk [1] in the Alpha document, the Hyperloop shall utilize a retractable wheel system to increase the safety of the pod.

\subsubsection{Magnetic Levitation Pod}

Hyperloop pods using passive magnetic levitation have been successfully demonstrated in the SpaceX Hyperloop Competition [3] and by industry leader Hyperloop One [14]. This arrangement uses permanent magnets fixed to the Hyperloop pod, typically on externally mounted pod skis, which when translated along a conductive sheet at high speeds (aluminum sub-track) induces a current in the sub-track and generates an opposing magnetic field. The magnetic field generated by the relative motion between the pod and the conductive sub-track causes a repulsive force, lifting the vehicle. This method, however, is not novel and falls under a specific category of Maglev known as electrodynamic suspension (EDS) which has been demonstrated by engineers in Japan [28]. The permanent magnetic EDS system is passive and thus does not require a constant power source like the most common Maglev technology, electromagnetic suspension system (EMS). Using a Maglev based EDS system would ensure that a failure in the pod's propulsion system would not cause the pod to fall onto the track instantly. This contrasts with EMS that requires a constant power to maintain levitation and would require a back-up power source to ensure levitation is maintained in case of a power failure [21].

A drawback to the EDS passive magnetic approach is that the induced current on the conductive sub-track results in a magnetic drag force. The drag force has been recorded in literature to increase with speed until it reaches a peak value and is then slowly decreased as the speed increases [29]. A plot of the normalized force from the EDS Maglev is shown in Figure 3.1 with respect to translational velocity. As an added result, the passive EDS system cannot levitate the Hyperloop pod at a station and can only levitate the pod at sufficiently high speeds, about $94 \mathrm{mph}$ (150 kph) [21]. Consequently, a Hyperloop pod equipped with passive magnetic levitation should use wheels at rest and when accelerated, like an aircraft, as suggested for EDS Maglev configurations by He et al. [28] and Paudel [29]. 


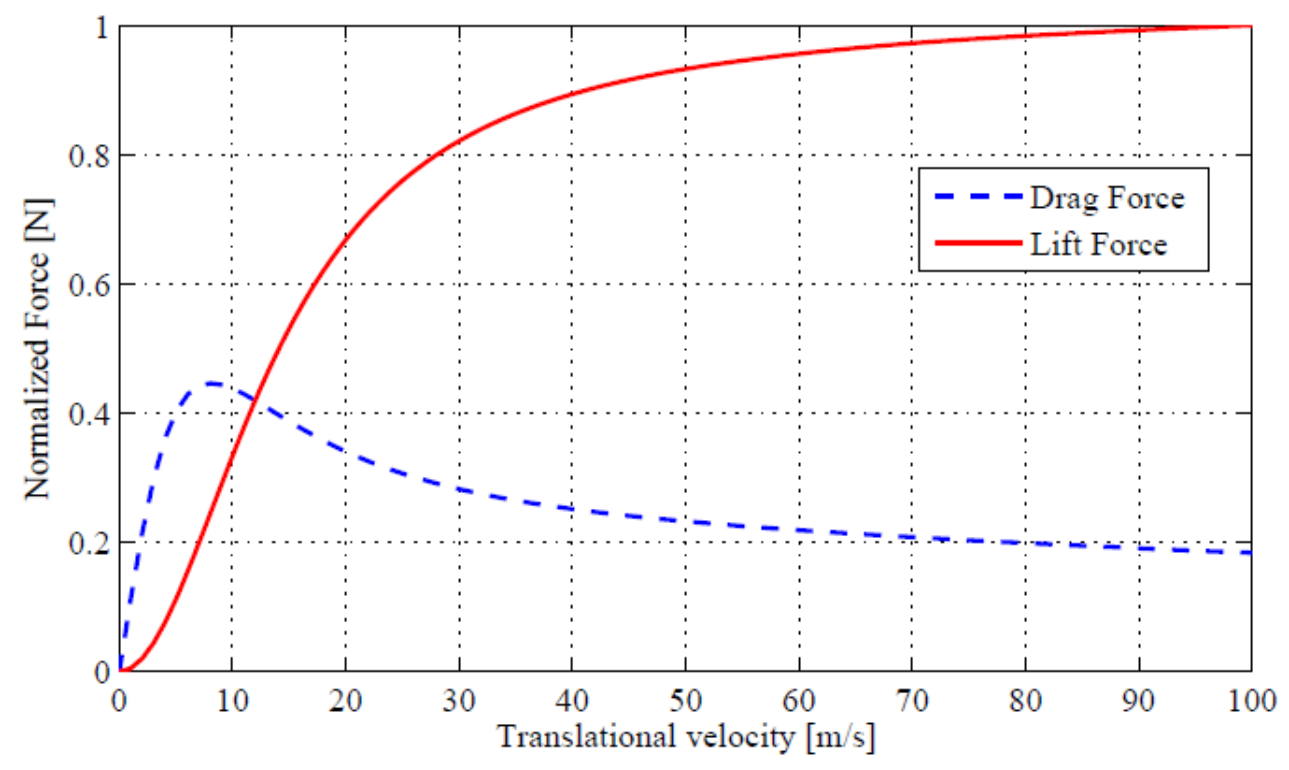

Figure 3.1: Normalized force of the EDS Maglev plotted against translational velocity [29]

\subsection{Former University of Toronto Partnership}

In Spring 2016 the author established a partnership with the University of Toronto (U of T) Hyperloop pod team to install four deployable wheel systems. Therefore, the original design, submitted to SpaceX in January 2016, was scaled down in size to better suit the U of $\mathrm{T}$ configuration. Thus, a large portion of the work presented in the following sections has been completed utilizing information provided by the $\mathrm{U}$ of $\mathrm{T}$ team, which influenced the design of the system's kinematics (Section 3.5.2) and shock strut gas spring design (Chapter 5). In the summer of 2016, the $\mathrm{U}$ of $\mathrm{T}$ withdrew from the competition but the author's present embodiment of the HDWS remains developed with information exchanged throughout that time.

\subsection{Design Objectives}

The main objective of this work was to design and develop a wheeled suspension system that operates in tandem with the proposed air bearing or passive magnetic levitation systems. The system shall be primarily designed to operate at low speeds within the tube environment defined in Chapter 2, be retractable to minimize drag, and act as a component of the overall safety system. 
A goal was to consider as many key design drivers as possible throughout development to ensure a scalable and complete solution is presented. Using the functional requirements defined within Musk's Hyperloop Alpha document as a baseline, alongside historical and current landing gear development literature, the HDWS was designed and developed.

The specific objectives targeted throughout the design and development of the HDWS are as follows:

1) Develop a prototype wheeled system capable of operating nominally at $180 \mathrm{mph}$ (290 $\mathrm{kph})$ to ensure that it can operate at predicted max speeds, in case of emergency, within the SpaceX pod competition framework (lower max speeds)

2) Ensure that the deployment of the wheel system limits the direct transfer of load and vibrations throughout the vehicle using a shock absorber (+ damper if required)

3) Develop a system with advantageous kinematics that is scalable and operable in the least amount of volume possible

4) Develop a system that is electrically powered (no requirement for the porting of pressurized fluids) and thus compatible with known pod power supplies (e.g. lithium-ion batteries)

5) Develop a system that is simple to integrate, preferably two mounting points

6) Ensure that a wheel system is capable of retracting and extending quickly, a key feature in an emergency scenario

7) Address the issues presented by near-vacuum operation (e.g. outgassing)

8) Design a common wheel system that can be duplicated and distributed throughout the pod with minimal modifications required for left-hand, right-hand, front and back installations

9) Identify and investigate key weight saving opportunities since a wheeled system is "dead weight" during most of regular vehicle operation

\subsection{Product Development Guidelines and Constraints}

At present time, no regulatory body has been established to govern the certification requirements for the Hyperloop and its various subsystems. Consequently, the author's present work has used a combination of aircraft landing gear industry design standards, examples of performance 
guidelines found in literature, and the information contained within the SpaceX competition tube specifications [4] and competition rules manual [19].

\subsubsection{Design Constraints}

The HDWS was subject to extreme space constraints imposed by the $\mathrm{U}$ of $\mathrm{T}$ pod configuration. That is, the HDWS was to operate within a 20-in by 20-in box, as imposed on a side view of a pod layout, shown in Figure 3.2. Furthermore, the wheel system was to be fully compatible with the SpaceX test track as outlined in the SpaceX Tube Specification [4] and partially illustrated in Figure 3.3. Based on the width of the aluminum sub-track, shown Figure 3.3, the HDWS was designed to fit within the maximum sub-track engagement width of 12-in.

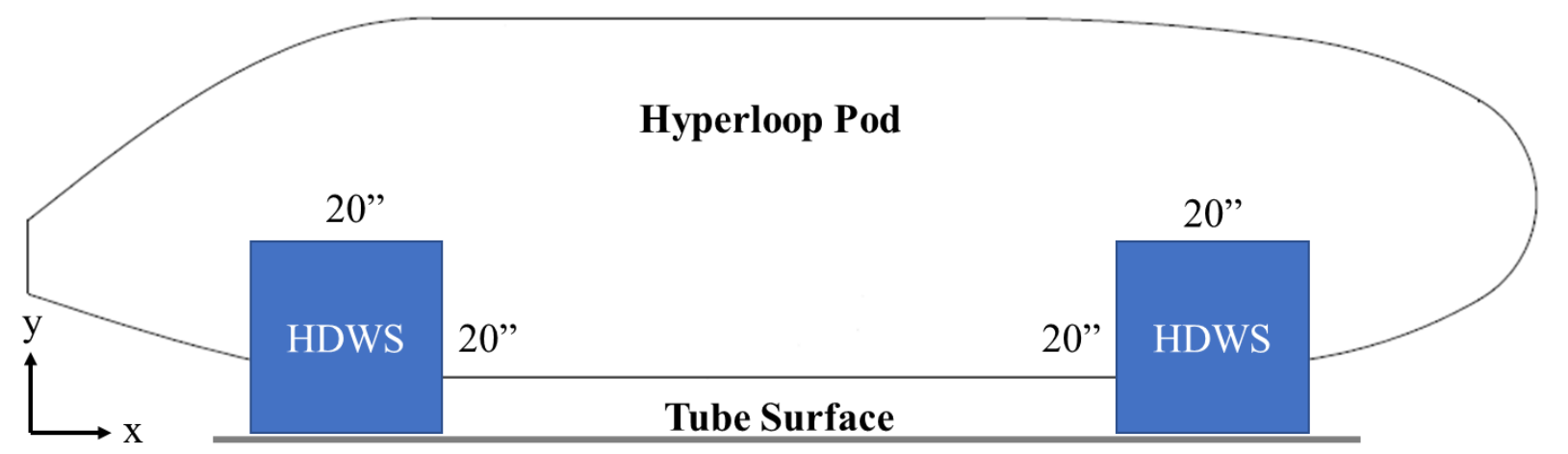

Figure 3.2: A 2D side view of a Hyperloop pod with the HDWS positioned, not to scale

The following design constraints and guidelines are presented as follows:

1) The HDWS shall be operable within the volume provided by the mating pod, using approximated values selected by the author in consultation with the $\mathrm{U}$ of $\mathrm{T}$ Pod team $(\mathrm{L}=20$ in, $\mathrm{W}=12$-in, $\mathrm{H}=20$-in).

2) Designs shall be compatible with the SpaceX tube (sub-track) structural tolerances, defined by the SpaceX Hyperloop tube specification [19].

3) All components shall maintain structural safety factors (SF). SpaceX specifies a SF of 2.0 for reasonable load cases (e.g. acceleration, deceleration, pressure) [19].

4) All compressed gas shall be limited to pressures of 4,000 psi (27.6 MPa) [19].

5) All high-pressure gas systems shall have vent and relief valves [19]. 
6) Flow directions through vent and relief valves shall be oriented for the safest possible outcome in the event of a pressure burst [19].

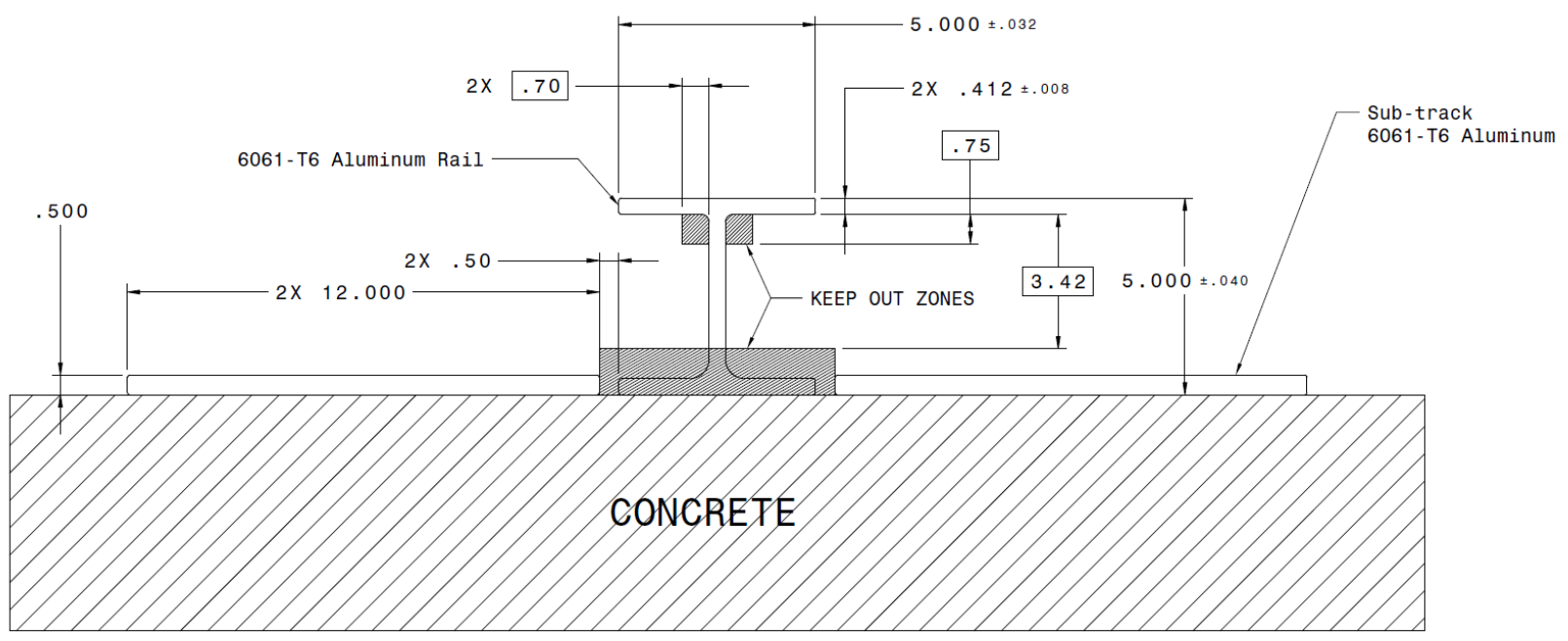

Figure 3.3: Partial cross-section view of the SpaceX Hyperloop sub-track interface, dimensions shown in inches, with reference to the specification [4]

\subsubsection{Performance Constraints and Guidelines}

1) The HDWS shall be designed to operate under the accelerations highlighted in Table 3.1, provided by SpaceX, for the purposes of the Hyperloop competition. The HDWS shall be tested under these conditions, where possible, prior to initial full pod testing and integration.

Table 3.1: Representative SpaceX pusher acceleration values (2016) [4]

\begin{tabular}{|c|c|c|}
\hline Pod Mass (kg) & Pod Mass (lbm) & Pod Acceleration (g) \\
\hline 250 & 550 & 2.4 \\
\hline 500 & 1100 & 2.0 \\
\hline 750 & 1650 & 1.7 \\
\hline 1000 & 2200 & 1.5 \\
\hline 1500 & 3300 & 1.2 \\
\hline 2000 & 4400 & 1.0 \\
\hline 2500 & 5500 & 0.9 \\
\hline 3000 & 6600 & 0.8 \\
\hline 4000 & 8800 & 0.6 \\
\hline 5000 & 11000 & 0.5 \\
\hline
\end{tabular}


2) A wheeled system shall perform nominally when subject to the Hyperloop system operating pressures ranging from $14.7 \mathrm{psi}(101.3 \mathrm{kPa})$ to $0.15 \mathrm{psi}(100 \mathrm{~Pa})$ as per the alpha document [1] and tube specifications [4].

3) A wheeled system shall be nominally operable, at a minimum, at sustained speeds between 0$100 \mathrm{mph}(0-160 \mathrm{kph})$ as specified per the alpha document [1].

4) While deployed, a wheeled system's operation shall ensure that the pod and any externally mounted features (e.g. air-bearing skis) do not contact the SpaceX tube sub-track

5) A wheel system should deploy to an agreed upon position (agreed between the supplier and pod manufacturer) within a "reasonable time" to assist in a "levitation loss" emergency scenario. The retraction speeds shall be determined in the same manor and the following discussion points are now offered.

a. A "reasonable time" shall be defined based on future pod emergency scenario analysis

b. As a reference, Currey [5] notes that U.S. Navy aircraft require that the gear "shall be retractable in no more than $10 \mathrm{~s}$ " and "extensible in less than $15 \mathrm{~s}$ "

\subsubsection{Material and Lubrication Guidelines}

The material and lubrication used for the HDWS were based on standard material and tribology practices, specifically those defined in the aircraft design industry. Based on this information, and the information presented in Chapter 2 regarding the effects of outgassing, the following requirements and guidelines were established:

1) Material strength properties, specified by part suppliers and the Metallic Materials Properties Development and Standardization (MMPDS-07) handbook [30], shall be observed, including:

a. static yield pressures $\sigma_{Y S}$

b. metallic bearing allowable pressures $\sigma_{Y B}$

c. self-lubricating bearing liner allowable pressures, dynamic and static [31]

2) Material compatibility, utilizing the galvanic tables found in industry literature [32] shall be enforced to ensure compatible materials were selected for joints, or:

3) The materials shall be treated for corrosion protection, considering:

a. anodization, all over exposed aluminum components for corrosion resistance (anodization is also used in spacecraft design with a low-risk of outgassing [33], [34]) 
b. cadmium plating, applied to outer diameter of off-the-shelf bushings (Note: best avoided if possible because of its susceptibility to outgassing) [35]

c. installation and assembly of matting components at joints shall be lubricated [5] using grease (preferably vacuum certified, such as Dow Corning ${ }^{\circledR}[36]$ high-vacuum silicone grease)

d. passivation shall be performed on all corrosion resistant steel (CRES) components [37]

e. other methods of surface protection, as available, shall be used to satisfy material compatibility as per the galvanic tables [32] and to protect from natural corrosion

4) Material selection shall be selected based on the desired end-form and function (e.g. a bronze based material shall be used in a rotating and wear surface due to its low friction interaction with aluminum and steel) [5].

5) Wherever possible, corrosion resistant steel, passivated, shall be the preferred choice of steel, especially for pins and sealing surfaces [5].

6) Bushings shall be procured and designed using CRES or bronze based materials only, as is the typical standard in the industry [5].

7) Lubrication used shall be certified for use in a vacuum, ensuring lubricative properties are retained. Consideration shall be given to lubrication forms such as:

a. Dow Corning ${ }^{\circledR}$ high-vacuum silicone grease [36]

b. Self-lubricating liners, such as KamaticsRWG's KAron ${ }^{\circledR}[31]$

As a final note, it is important to recognize that above guidelines should be satisfied for the development of a production grade HDWS product. However, the present work, due to budgetary reasons, could not always use the optimal materials to meet the above guidelines.

\subsection{HDWS Architecture and Kinematics}

A key design consideration for the development of a deployable wheel system for the Hyperloop pod is that its wheel system be deployable and retractable. This creates minimal impact to the aerodynamic efficiency of the pod outer mold line (OML) and insures that drag, produced at high speeds within the enclosed near-vacuum tube diameter, is kept to an absolute minimum [5], [18]. Of initial importance was ensuring that the HDWS's geometry allowed for operation within the small space 
provided by the typical Hyperloop competition pod. Thus, a key element in the design of the deployable wheel system, like landing gear, was the development of a product architecture and kinematic layout. Since the end goals - retractability, extensibility and operation at high speeds are all common elements in aircraft landing gear design, this work focused on evolving these designs to fit the needs of the Hyperloop.

\subsubsection{HDWS Architecture Definition}

Canted landing gears are found to require a large forward sweep area for retraction and extension, without any shortening mechanism [5]. A stowed landing gear with the shock strut in an initial extended position (no load applied), as is common during flight, requires an unrestricted sweep volume to be successfully swung into "landing" position from a FWD, AFT or INBD direction. After successfully deploying, the landing gear would touch the ground with an unloaded shock strut, and compress to a static shock absorber stroke thereafter. In the context of the Hyperloop pod, the pod would "land" on the tube sub-track and begin to compress the stroke using the extension actuator. For an optimized shock strut to reach a typical static stroke, as discussed by Currey [5], it would require at least $70 \%$ of the piston stroke, meaning that the pod will have surely "touched down" on the tube surface through its magnetic or air-bearing skis prior to the strut reaching a statically loaded position capable of maintaining a steady pod's weight. Thus, a canted gear without shortening prior to touchdown or without lengthening capabilities at touchdown (fully extended piston stroke) is not considered feasible for the present application. Further, should a canted gear be sized and shrinkable to an appreciable stowage size, the design still requires the addition of a drag brace member and retraction mechanism, with retraction classically accomplished using a hydraulic actuator.

Considering the canted gear's stated deficiencies for the current application, an MLG trailing arm architecture was adopted to the benefit of stability and the possibility of kinematic alterations. Specifically, the elimination of the inboard swinging element, requiring a retraction actuator and typically a side brace member, was proposed in favor of an "all-in-one" shock strut and electric retraction solution.

Of interest for preliminary 2D kinematic analysis are the stowage compartment dimensions imposing constraints on the space in which the HDWS must operate. It was decided, based on 
these constraints, that a fully planar 2D HDWS operation best suited the deployment characteristics desired. However, this posed a problem with space requirements due to the nature of gear storage in the retracted position. The hybrid actuator concept was proposed to address this issue, as will be described in the present section.

An initial proposal for a preliminary system architecture is shown in Figure 3.4. This diagram depicts a stowage frame (approximated by a 2D box as drawn), the tube surface, of which the HDWS will be deployed onto, and a familiar trailing arm architecture.

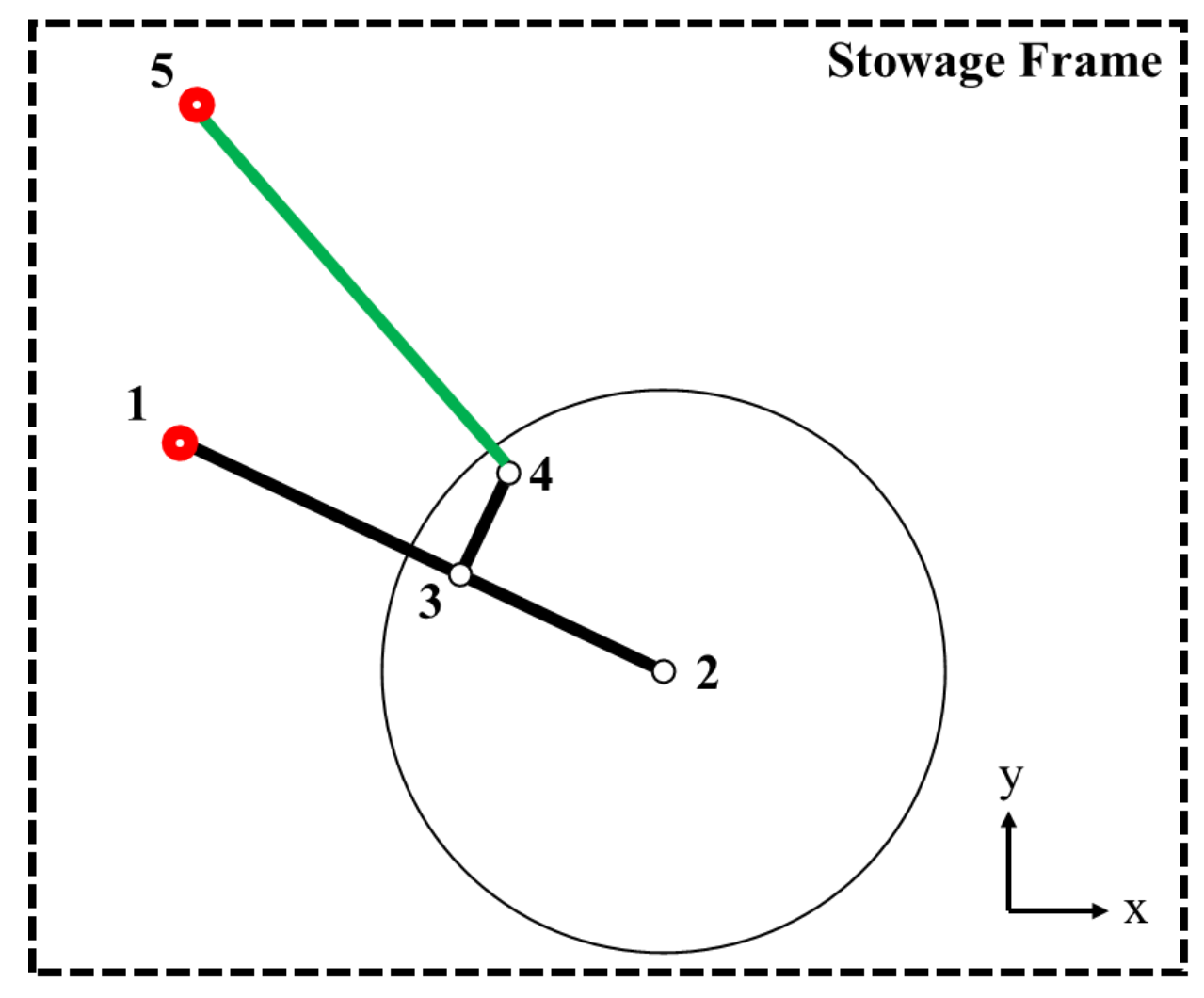

Tube Surface

Figure 3.4: The HDWS preliminary configuration, stick diagram, not to final scale

For the initial configuration, five key points were proposed to define the geometry of the system. In this early configuration, referring to Figure 3.4, a trailing arm, comprised of points (1) - (4) is attached to a pod-fixed point (1) consisting of a revolute joint, and thus allowing the trailing arm 
to rotate. The trailing arm is equipped with a wheel at an axle (2). As is typical in an landing gear trailing arm configuration, a shock strut, comprised of points (4) - (5) is proposed and fixed at point (4) to the trailing arm by a 2D revolute joint (later a spherical joint in 3D) and by a revolute joint, fixed to the pod frame at point (5). Thus, in the current description, a force applied at (2) in the vertical y-direction would cause the trailing arm to pivot counterclockwise about point (1) and the shock strut member disposed between points (4) and (5), consisting of a piston and cylinder, to contract in length. This is a desirable result; however, the present description (Figure 3.4) does not yet propose a method for extending and retracting the system from the present state.

Now, consider a member (4) - (5), shown in Figure 3.5, further comprised of a piston, shock strut cylinder and lengthening device of unknown design, co-axially arranged, mounted between points (4) and (5) in Figure 3.4. In such a configuration, it becomes conceivable to develop kinematics that allow for the new member (4) - (5) to provide both extension, retraction, shock absorbing and possibility active height adjustment during operation to adjust the pod height above the tube surface as required.

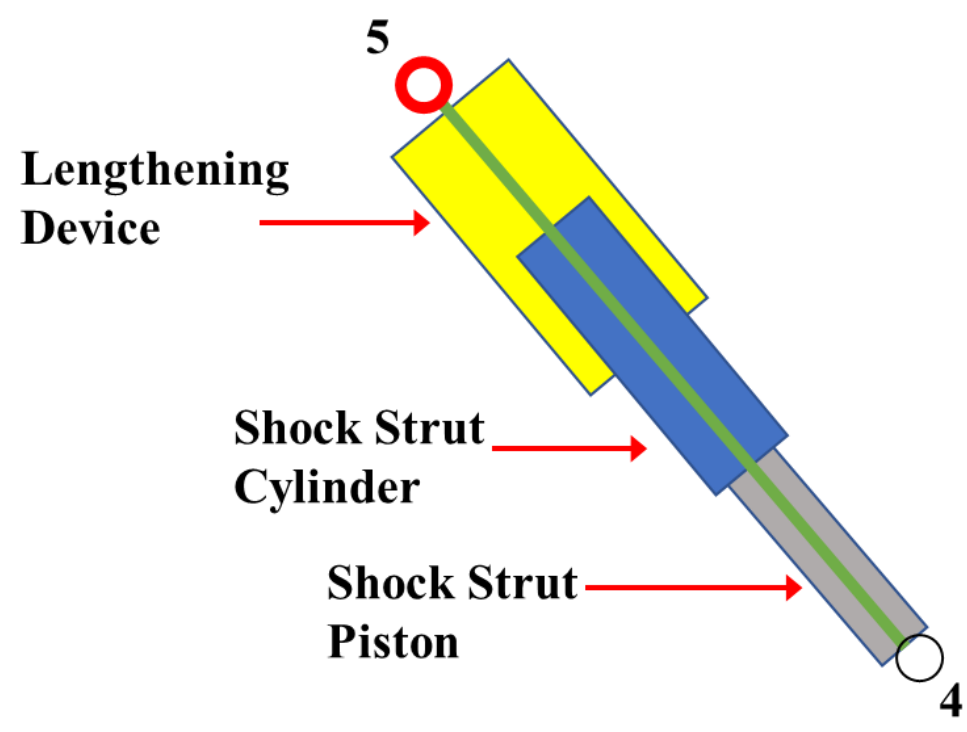

Figure 3.5: The HDWS Hybrid actuator concept illustration

Such a system could be designed and developed using hydraulic fluid, supplied by a reservoir, to pressurize the area within the "lengthening device" and cause the configuration shown in Figure 3.4 to extend and contract. A solution like this, however, poses two main problems: the 
requirement for an externally supplied hydraulic line (hydraulic systems are not in the current pod architectures) and the mounting point (5) would need to be placed relatively far vertically inside the pod structure to accommodate a mechanism long enough to provide adequate lengthening at static stroke (pod attitude adjustment) and extension/retraction. If one was to utilize this configuration with an electrical deployment and retraction, a design such as that proposed by Waide [8] and in Conway and Renshaw [27] could be explored to similar space and operational limitations.

A new configuration is shown in Figure 3.6. This configuration includes the same key elements of Figure 3.5, arranged so that the point (5), which the lengthening device is mounted and the point at which the member (4) - (5) pivots, is movable during the design process. Such a configuration could be accomplished at point (5) by utilizing oppositely disposed trunnion pins, on each side of the lengthening device. The author therefore proposes a design in the spirit of Figure 3.6 and will provide kinematic layouts based on this concept in the following section.

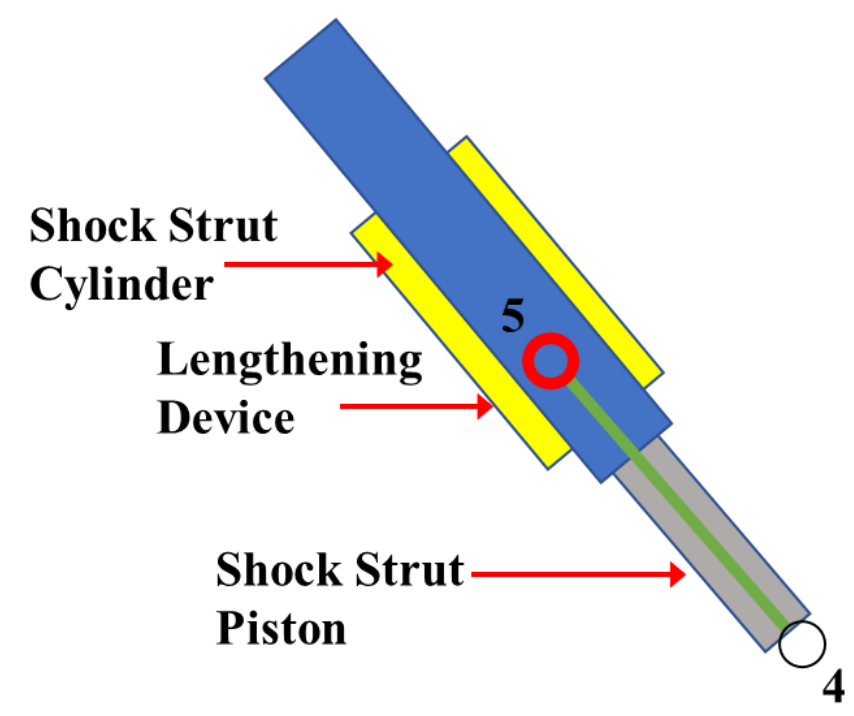

Figure 3.6: The HDWS Hybrid actuator concept \#2 illustration

\subsubsection{HDWS Kinematic Layouts}

This section illustrates the kinematic arrangement of the HDWS using this work's deployable and retractable shock absorber. Its configuration will be shown in Chapter 4 and discussed in greater detail within Chapter 5. Based on the basic architecture defined in Figure 3.4, a kinematic mock- 
up was drawn using CATIA ${ }^{\circledR}$ V5 [38]. As a preliminary example, the mock-up is shown in Figure 3.7 with the HDWS in a stowed (retracted) position and bound within boxed dimensions: L= 20in and $\mathrm{W}=14$-in. The pod frame is shown to be 3.75-in above the surface of the sub-track.

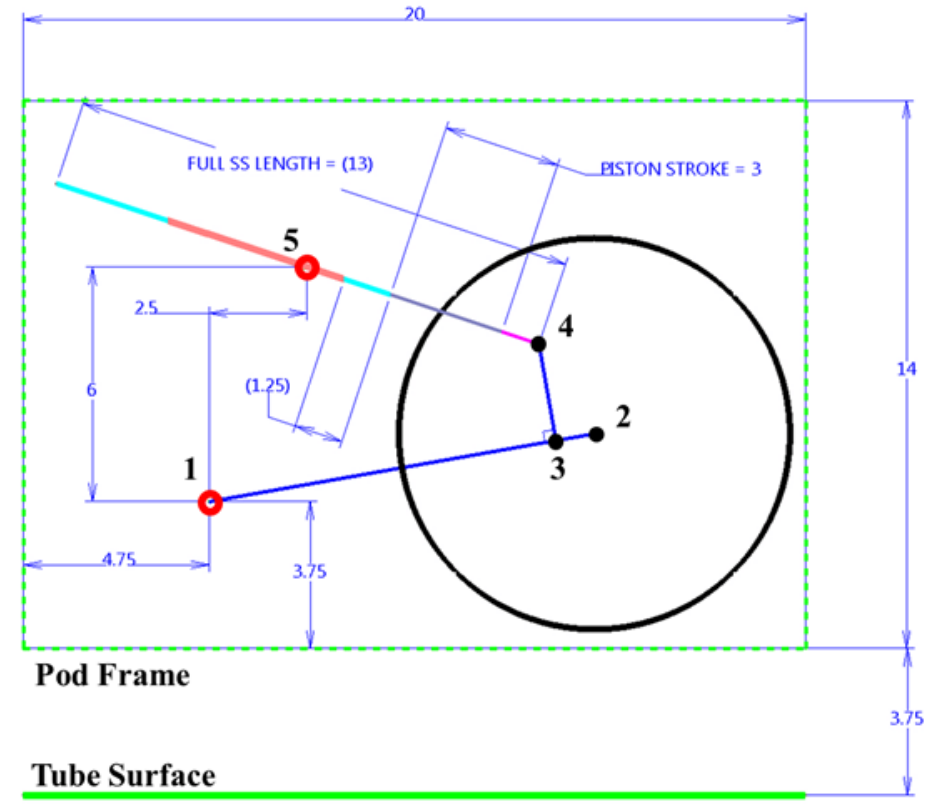

Figure 3.7: An example of a preliminary HDWS kinematic layout, retracted, shown in CATIA ${ }^{\circledR}$ V5

During deployment, the wheels reach a point where they touch the bottom of the tube, typically referred to as weight on wheels (WOW). At this point, the HDWS system must use its retractable and deployable shock strut to further deploy the wheels until the static load is reached. The static load is the piston stroke at which the total forces in each of the shock struts, produced by the internal pressure times the piston area, are equal to the total weight of the Hyperloop pod distributed proportionally across the wheel systems. At this point, it is suggested that the Hyperloop pod should be raised further above the tube surface using the same actuation mechanism.

The adjustment of the pod ride height ("ride height maintenance") is believed to be especially important since the air-bearings or magnetic skis which the vehicle rides on throughout the journey, are known to have extremely small operational clearances, as detailed in Table 3.2. Per Janzen [39], the air-bearing levitation system is particularly sensitive to small irregularities in the Hyperloop tube sub-track. Janzen suggests that a momentary loss in levitation can "cause the [air] 
bearing to slam down on the [sub-track] and risk self-damage." Based on the above discussion, and tabulated data in Table 3.2, the decision was made to enforce a minimum height adjustment of 0.5 in $(12.7 \mathrm{~mm})$ after static stroke is achieved in order to combat the risk of damaging a pod's levitation feature that evidently requires a low operational clearance.

Table 3.2: Levitation ride height comparison

\begin{tabular}{|c|c|c|}
\hline \multirow{2}{*}{ Levitation Source } & \multicolumn{2}{|c|}{ Levitation Gap } \\
\cline { 2 - 3 } & {$[\mathbf{i n}]$} & {$[\mathbf{m m}]$} \\
\hline Air bearing [1] & $0.020-0.050$ & $0.5-1.3$ \\
\hline Magnetic (Passive) [21] & $0.31-0.39$ & $8-10$ \\
\hline
\end{tabular}

Ideally, the HDWS will lift the vehicle, relative to the bottom of the air or magnetic levitation skis, by a distance that enables the shock strut, when deployed, to compress and extend freely (i.e. absorb and react to disturbances during operation) without the pod touching the tube sub-track surface. The minimum height adjustment value selected was directly related to the stiffness of the shock absorber gas spring. That is, a stiffer gas spring, as will be discussed in Chapter 5, requires less height maintenance versus a softer gas spring that will allow the pod to compress greater distances under loads. Based on the anticipated loading during braking and acceleration that was analyzed by Ryerson's International Hyperloop Team [40], the gas spring model for the HDWS required a gap of at least $0.5 \mathrm{in}(12.7 \mathrm{~mm})$ to ensure the system remains clear of sub-track in all cases. In addition to the pre-emptive raising of the pod to allow for load oscillations, a desired gap between the pod and the sub-track is monitored and controlled by the HDWS's active control and leveling capabilities based on a program written and designed by Shonibare [16].

The pod kinematic attachment points, (1) and (5), as selected for the pod originally proposed by $U$ of T, are shown in Figure 3.8 and Figure 3.9. These kinematic illustrations, designed in CATIA ${ }^{\circledR}$ V5, use dimensional characteristics that were selected based on an iterative trial and error method cycling between the required kinematic ranges of motion, the anticipated part geometry (e.g. lug sizing, member lengths, etc.), and clearance with the pod based on the anticipated part geometry. The final number of HDWS's integrated to a pod, and their distribution throughout the pod's length, is a function of the available interface geometry and the distribution of the pod weight like aircraft landing gear distribution methodologies [30]. 


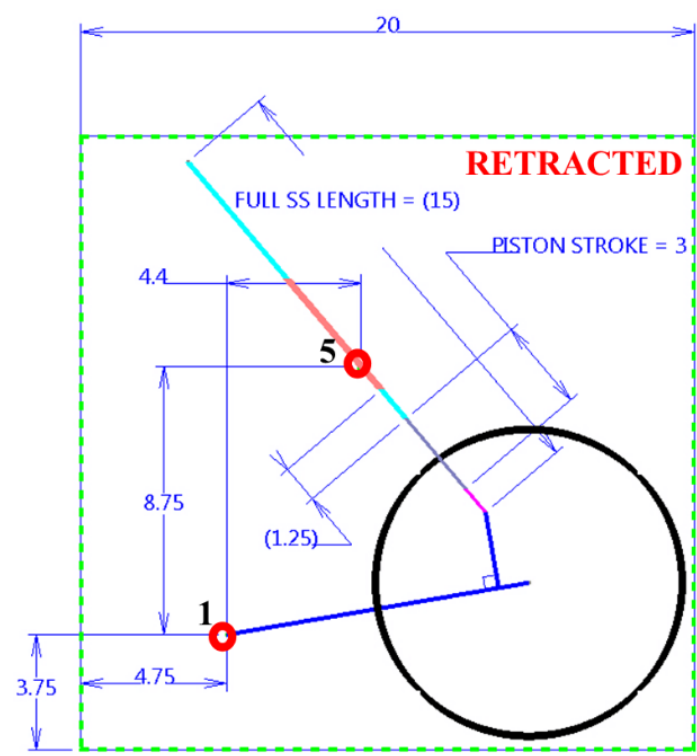

Pod Frame

Tube Surface

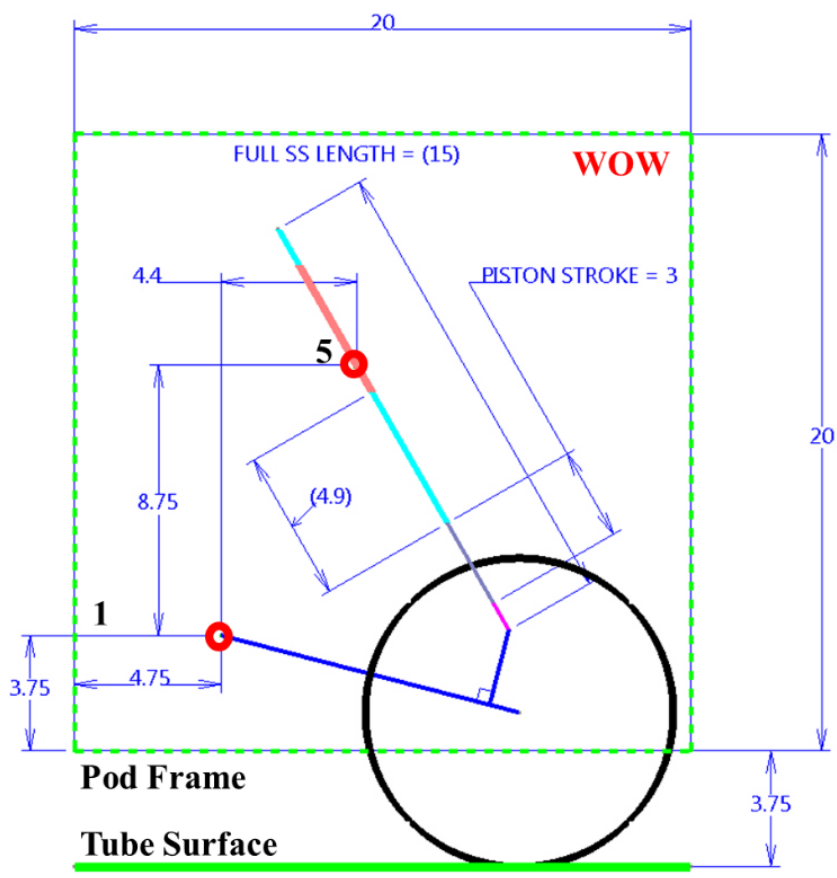

Figure 3.8: U of T HDWS kinematic layout, retracted position (left) and WOW position (right)
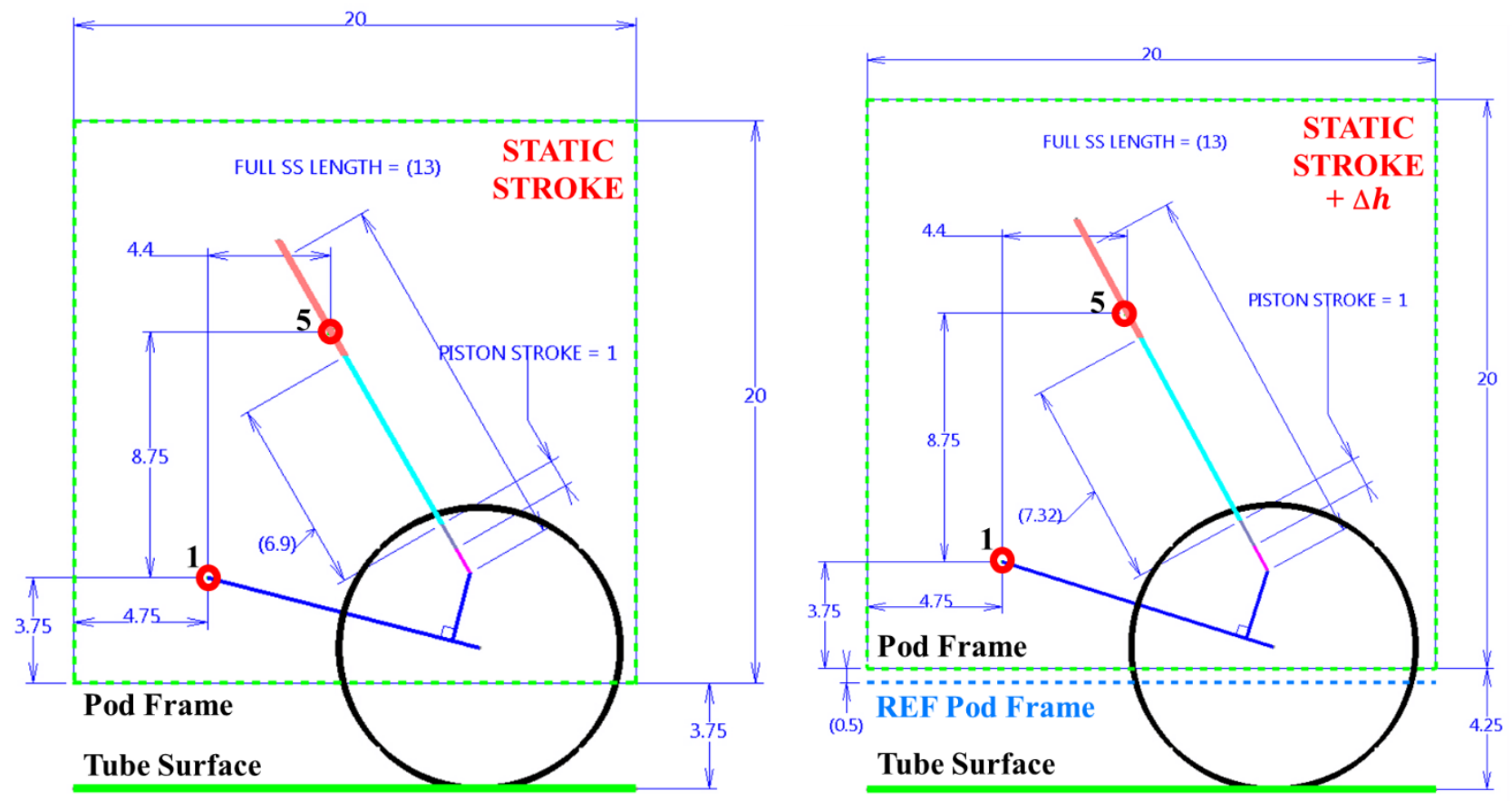

Figure 3.9: U of T HDWS kinematic layout, sample static stroke (left) and sample static stroke + pod height adjustment of $0.5 \mathrm{in}(12.7 \mathrm{~mm})$ from the reference position (right) 


\subsection{Component Definition, Product Structure and Process Scope}

\subsubsection{Component Definition}

A part numbering system was developed for the HDWS to manage the various parts, assemblies and configurations. The present scheme was developed alongside the product structure configuration tree presented in Section 3.6.2. The scheme operates on five levels, with 10representing the complete assembly at level one, and 40-, level five, representing the numbering for individual parts and thus encompassing the HDWS product from the top-level assembly down to each standard nut and bolt. As is standard practice in the industry, custom components designed by the author were assigned individual part numbers and standard hardware retained their corresponding part numbers. Standard components sourced from other industry partners such as the Greene Tweed [41] seals, SKF [42] roller bearings, and KamaticsRWG [43] bushings also retained their respective supplier part numbers.

\section{Part Numbering Scheme}

\section{AA-BXYY-ZZ}

AA - Component Level (10, 20, 30, 35 or 40)

B - (1 Undressed Assembly, 2 Electric Motor Assembly, 3 Rolling Stock)

X - Parent Sub-assembly (see Figure 3.10)

YY - Individual Part Identification Number

ZZ - Revision number of the part/product (ie. Initial release (00), Rev 1 (01), Rev 2 (02), etc.)

\section{Example:}

40-1101-01 - Corresponds to a level 5 part (40, lowest), will assemble into the undressed assembly (1), falls under the sub-assembly "Trailing Arm" (1), carries the first part number in that subassembly (01), and is at revision 1 (01). 


\subsubsection{Product Structure Breakdown}

For organizational purposes, a product structure tree was developed (Figure 3.10) to establish the HDWS component layout. The top-level model on the product structure tree, "HDWS Dressed", encompasses the entire structure, motor, mounted electrical equipment (sensors, wires) and rolling stock. Chapter 4 provides a detailed pictorial view of the components described here in Figure 3.10 .

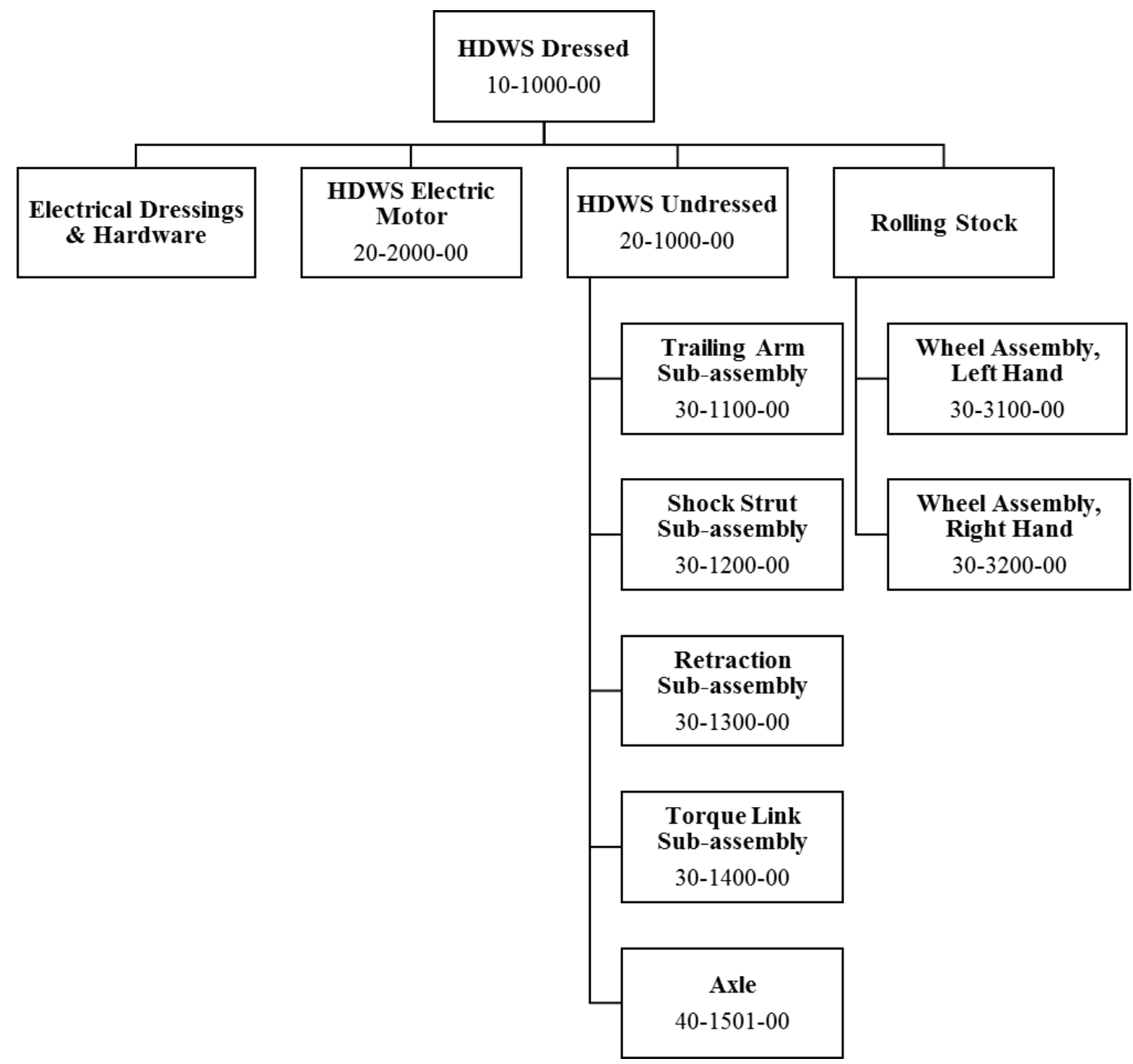

Figure 3.10: The HDWS product structure tree 


\subsubsection{Design and Development Process Scope}

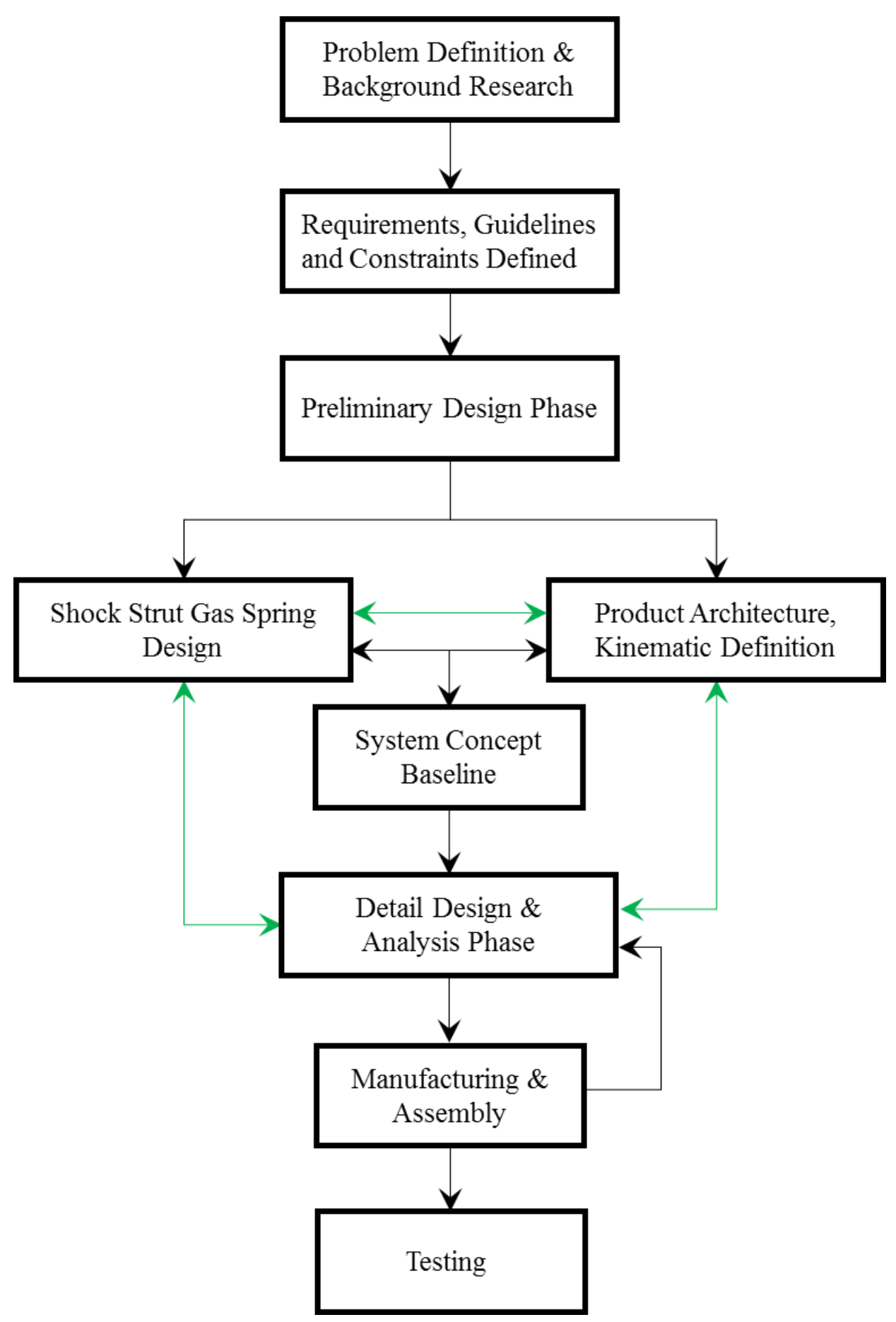

Figure 3.11: The HDWS design and development process scope overview 
The scope of the HDWS's design and development, as illustrated in Figure 3.11, was highly iterative in nature and generally followed a typical engineering development project architecture. The early stages of the project consisted of the background literature survey and problem definition. Here, the need for a wheeled system was investigated and key design drivers (e.g. it must be sufficiently compact) were defined. After conducting this review, the constraints, guidelines and possible requirements that would apply to a wheeled subsystem for the Hyperloop were defined.

Using the information collected in the early stages, a preliminary design phase commenced. During this time, the general architecture (e.g. shock absorber + wheel(s)) was chosen and the initial sizing of a shock strut gas spring (see Chapter 5) was defined. The goal at this phase was to develop a system baseline concept that could be demonstrated as manufacturable and scalable. The development of the product architecture and kinematics (Chapter 3) and the design of a functioning shock strut become interdependent at this stage in the development. Trade studies were performed on the fly, varying key dimensions to retain the desired kinematic motion yet allow for the required geometry to design a functioning $3 \mathrm{D}$ product within a $2 \mathrm{D}$ kinematic wireframe.

After a baseline model was developed and 3D printed using plastics for demonstration purposes, the detailed design phase commenced. At this point, the structural design was scaled to meet the needs of the prospective Hyperloop pod partner, the $U$ of $T$. Although the adherence to the baseline design remained firm, during the detail design stage, the scaling of shock absorber internal geometries required adjustments to the gas spring design while manufacturability constraints drove additional changes to the product geometry and kinematic definition. Once the baseline was fully adapted to the needs of the prospective pod partner, the $2 \mathrm{D}$ drawings generated using CATIA ${ }^{\circledR}$ V5 were submitted for manufacturing.

Although not discussed in the present thesis, the development of the electronic control system architecture began during the detailed design phase of the present work. After being defined by the author, the features of the control system were developed by dedicated electronic and control system actors. Once integrated with the structural elements of the HDWS developed by the author, the electronics and control system became the driving force behind the kinematic operation capabilities of the HDWS. 


\section{Design and Development of the HDWS}

This chapter provides an overview of the main assemblies that make up the Hyperloop Deployable Wheel System (HDWS). The system Undressed (structural) assembly is subsequently broken into its components and key functions and features are described. Following the system description, the various design activities that were carried out (including a discussion on the drawing arrangement, common structural analysis exercises, and component optimization) are outlined. This chapter concludes with a brief discussion of manufacturing.

\subsection{HDWS Dressed Assembly (P/N 10-1000-00)}

The HDWS Dressed assembly (Figure 4.1) is the top-level assembly containing the primary structure (HDWS Undressed), electric motor, assembly hardware, sensors and the rolling stock. Since the HDWS design consists of many sub-assemblies and parts, as shown in Figure 4.1, defining a top-level assembly was important to realise a smooth integration of all subassemblies that ultimately made up the complete system.

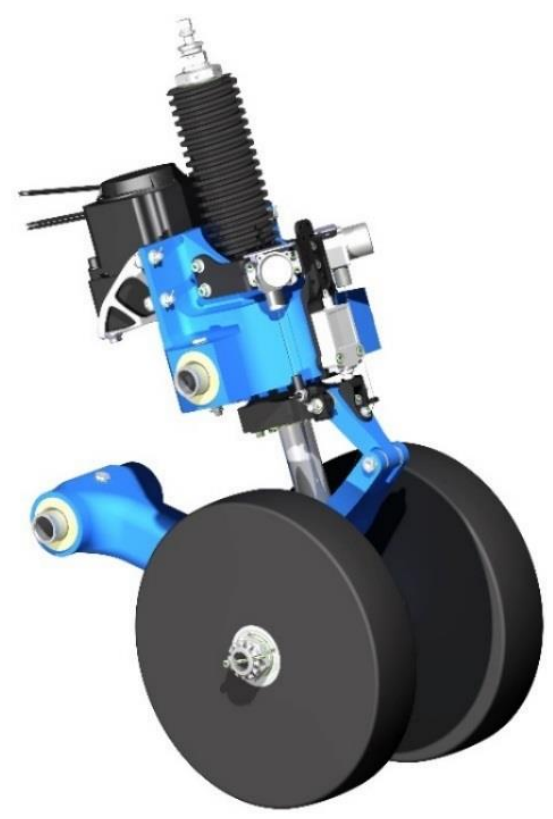

Figure 4.1: The HDWS Dressed assembly, CATIA $^{\circledR}$ V5 rendering 
At the HDWS Dressed level, the system is fully connected to its kinematic skeleton defined in Chapter 3. Using the kinematic operations within CATIA ${ }^{\circledR}$ V5, the HDWS could be cycled through all operational positions and checked for any clashing of components on the HDWS and any external interference with a mating pod structure.

\subsubsection{HDWS Undressed Assembly (P/N 20-1000-00)}

The HDWS Undressed Figure 4.2 is the first major sub-assembly of the HDWS Dressed and contains five key sub-assemblies and most of the major HDWS structural components. The Undressed assembly includes the axle, the Trailing arm sub-assembly, Retraction sub-assembly, Torque link sub-assembly and Shock absorber sub-assembly. Thus, main structure "dressings" such as the electric motor, including its sensors and wiring as well as the rolling stock (wheels) are covered as standalone sub-assemblies that are integrated to the Undressed assembly to create the final Dressed assembly.

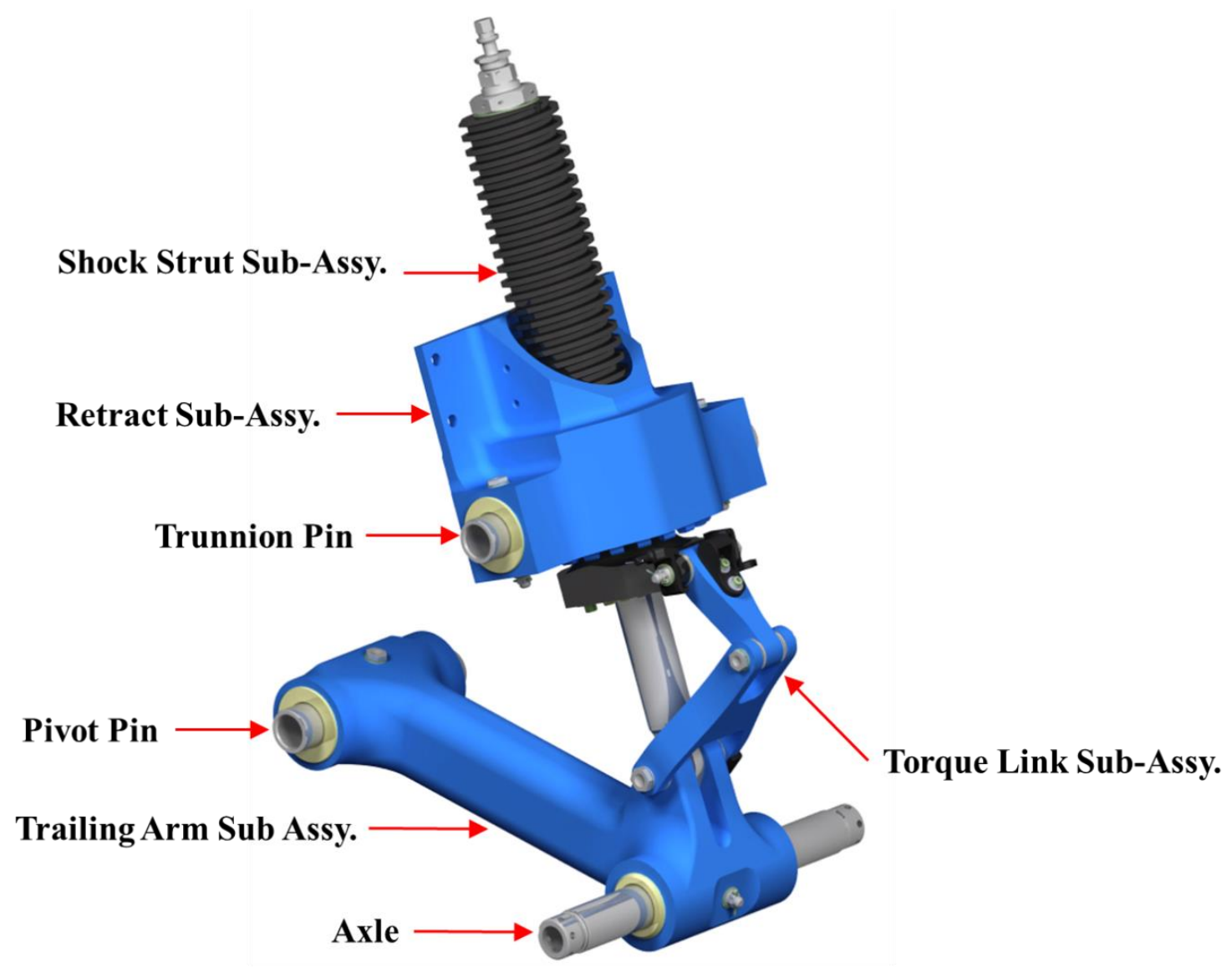

Figure 4.2: The HDWS Undressed assembly, CATIA ${ }^{\circledR}$ V5 rendering 


\subsubsection{Electric Motor Assembly (P/N 20-2000-00)}

The HDWS Electric Motor assembly, shown in Figure 4.3, is equipped to the HDWS Dressed assembly and is the driving force that enables the extension, retraction and active leveling capabilities of the HDWS. This assembly is comprised of the motor (1) with integrated electrical wiring (2), attached to a motor mounting bracket (3) that is fixed to the Retract sub-assembly using standard NAS bolts. The motor shaft is equipped with a pinion spur gear (4) which interfaces with the retract assembly leading to the actuation of the system further discussed in Chapter 5 .

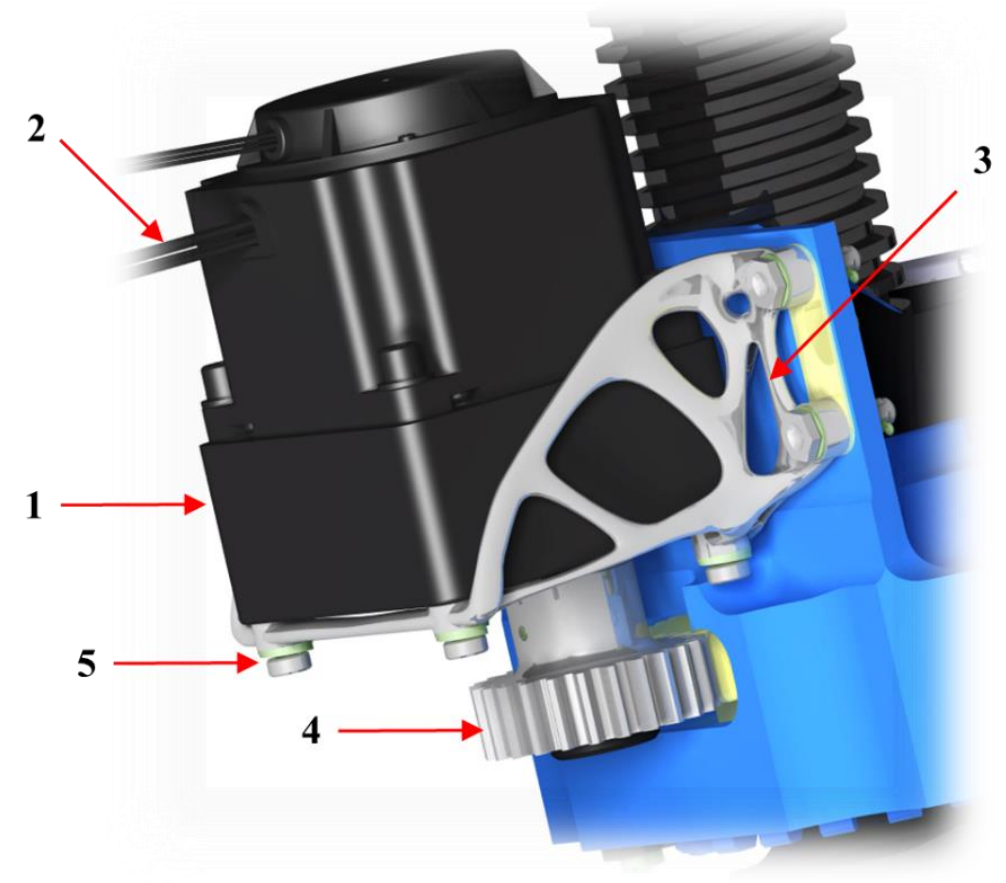

Figure 4.3: The HDWS Electric Motor assembly, installed on the Retract sub-assembly

Table 4.1: Electric Motor assembly breakdown summary

\begin{tabular}{|c|c|c|c|c|}
\hline Find \# & P/N & Part Description & Material & QTY \\
\hline 1 & $40-2101-00$ & BLDC Motor \& Gearbox (48 VDC, 3200 RPM) & Various & 1 \\
\hline 2 & --- & Electrical wiring (power, signal transfer) & Various & --- \\
\hline 3 & $40-2102-00$ & Motor mount bracket (AMM) & AlSi10Mg & 1 \\
\hline 4 & $40-2103-00$ & Custom designed Spur Gear pinion & 303 SS & 1 \\
\hline 5 & M6T25L & M6 Screws & CRES 316 & 4 \\
\hline
\end{tabular}




\subsubsection{Rolling Stock}

The rolling stock are the last components integrated to the HDWS dressed assembly. In the current embodiment, the rolling stock is comprised of two wheel assemblies, identical for the left and right mounting on the axle. The wheel assembly shown in the left image of Figure 4.4 was designed and produced to meet the needs of the $\mathrm{U}$ of $\mathrm{T}$ Hyperloop Pod. To enable a quick turnaround time, a wheel configuration was produced from a solid polyurethane 95A durometer disk that was balanced and equipped with roller bearings at the axle interface. Per the manufacturer, Sunray Inc. [44], polyurethane is proposed to outperform a standard rubber tire in terms of wear-resistance and abrasion. However, the manufacturer notes that at temperatures above $90^{\circ} \mathrm{C}\left(195^{\circ} \mathrm{F}\right)$, rubber becomes a preferred option.
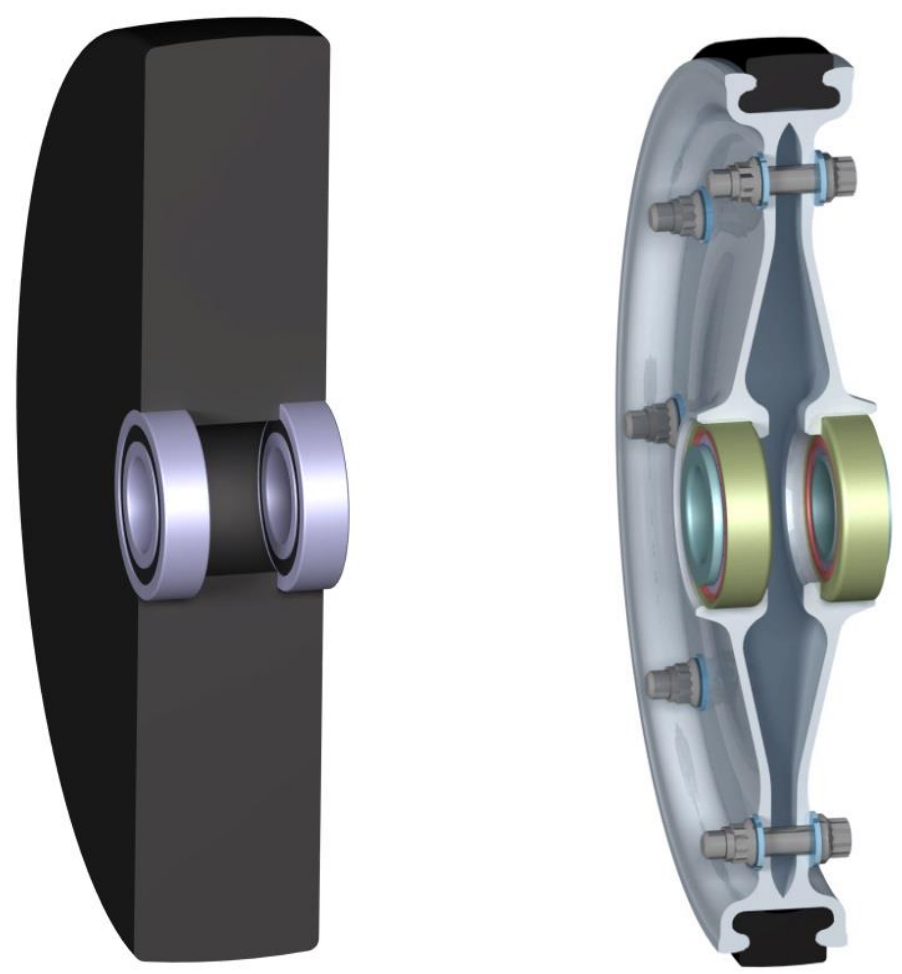

Figure 4.4: CATIA $^{\circledR}$ V5 renderings of the final HDWS Wheel Assembly (left), and alternate wheel concept as designed with input from the author (right)

In another embodiment, a more traditional framed wheel concept was conceived, consisting of a solid polyurethane or rubber lining trapped between an aluminum frame, shown in Figure 4.4. The advantage of this design over the one currently used for the HDWS is the light-weight aluminum 
frame, which also provides added structural stability. A significant challenge however comes in designing a reliable retention for the externally mounted polyurethane (or rubber) liner, shown in black, on the outer diameter of the wheel frame (right image, Figure 4.4). In the proposed concept, the external liner could tend to rotate within the aluminum frame under high speeds and loads (even with a pre-squeeze) and the liner could tend to lift from the outer aluminum frame under high rotational speeds. To solve this, adhesives could be used in conjunction with a mechanical lock as drawn. However, after consulting with design advisors in support of the HDWS project, it was deemed too high-risk and complex to develop this design within the project timelines. As will be discussed in the testing results, Chapter 6, such a design will need to be investigated to best address the bearing dislodgment due to radial wheel growth at high speeds that was identified during test.

In a first iteration, a wheel and tire configuration as used on aircraft and cars was conceived (Figure 4.5). Originally sized for a first HDWS iteration circa January 2016, a wheel was designed and developed by Khan [45] and fit with a standard aircraft tire capable of withstanding the high speeds and loads. Although a typical pneumatic tire was investigated in the early stages, there were uncertainties associated with using such a system in a low-pressure environment. It was hypothesized that the application of such a design would require an active pressurization control unit to ensure the tire was adequately inflated during all external conditions (e.g. low-pressure, sudden tube de-pressurization and waiting in a pressurized station). After a review of wheel and tire architecture it was found that most pneumatic tire configurations were developed to absorb irregular operating surface conditions, such as pot holes, and in the case of landing gear, the high loads (due to the rate of decent on impact) and speeds at landing [5]. Since the Hyperloop will operate inside a highly-controlled environment without large sub-track misalignments but it is instead subjected to the varying environmental pressure discussed in Chapter 2, the present solid polyurethane design was explored, as shown in Figure 4.4. This design draws inspiration from roller coasters, which also operate at relatively high speeds on a highly-controlled track surface. The design proposed by Khan [45] was presented as part of the SpaceX Hyperloop Competition design package but was ultimately not used for the final HDWS configuration which is shown in Figure 4.6. 


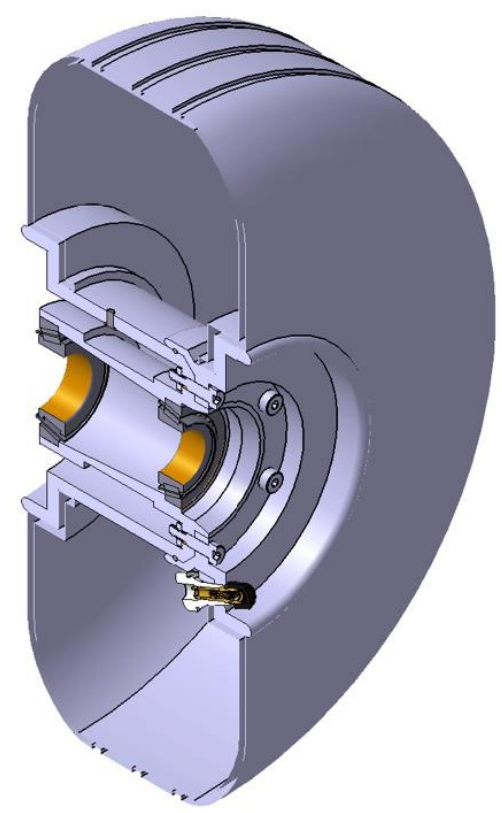

Figure 4.5: A Wheel and Tire assembly, 3D cross-sectional view, as drawn by Khan [45]

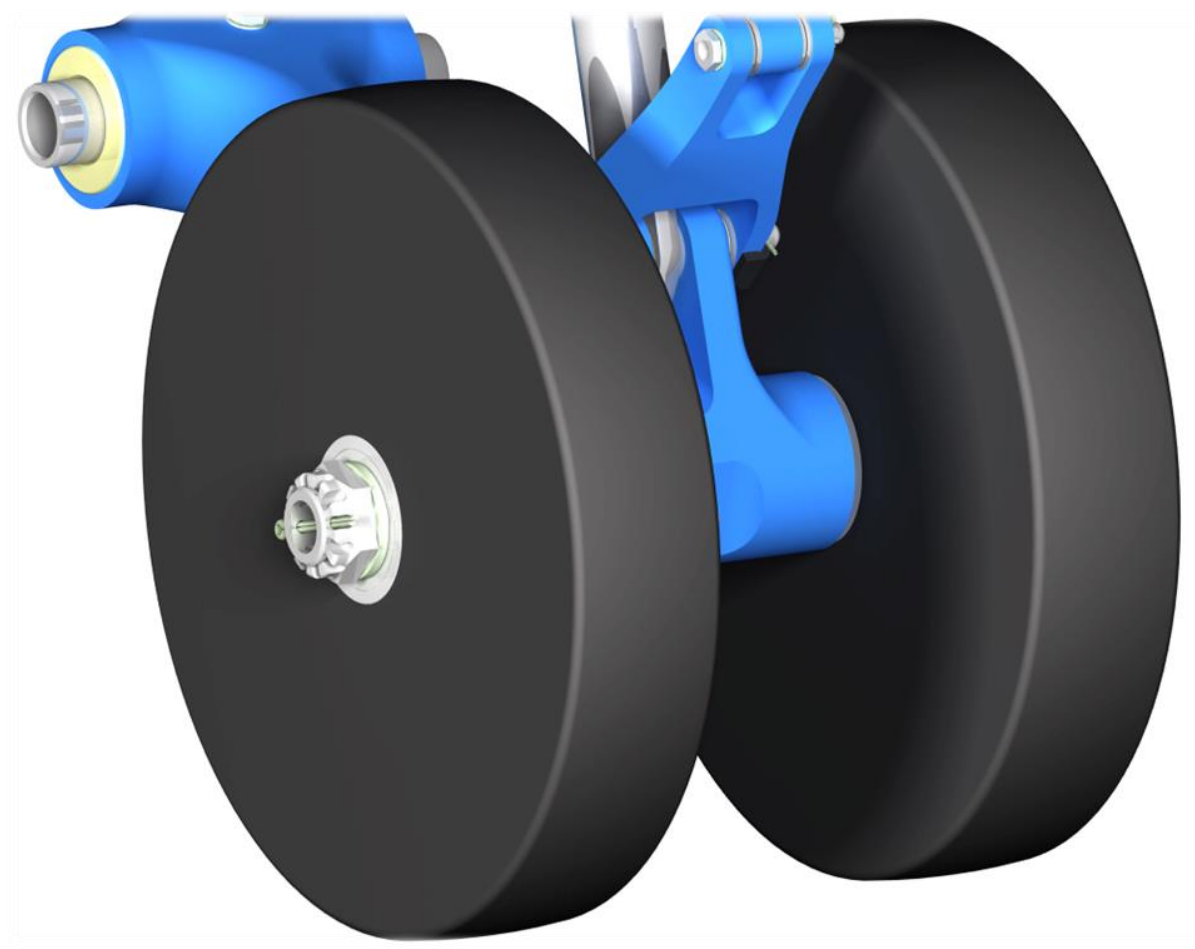

Figure 4.6: The rolling stock as installed on the HDWS Dressed 


\subsubsection{Electrical Dressings and Hardware}

The HDWS is not only capable of extending and retracting into a Hyperloop Pod, but can also operate as an active suspension system. Using externally mounted sensors shown in Figure 4.7, the HDWS uses real time data to control the position of the Hyperloop pod frame relative to the tube sub-track. The sensor data is filtered through a closed-loop control system, developed by Shonibare [16]. The sensors provide the signal and the motor performs the active adjustments, retraction and extension using the sensor feedback and commands from the external controller.

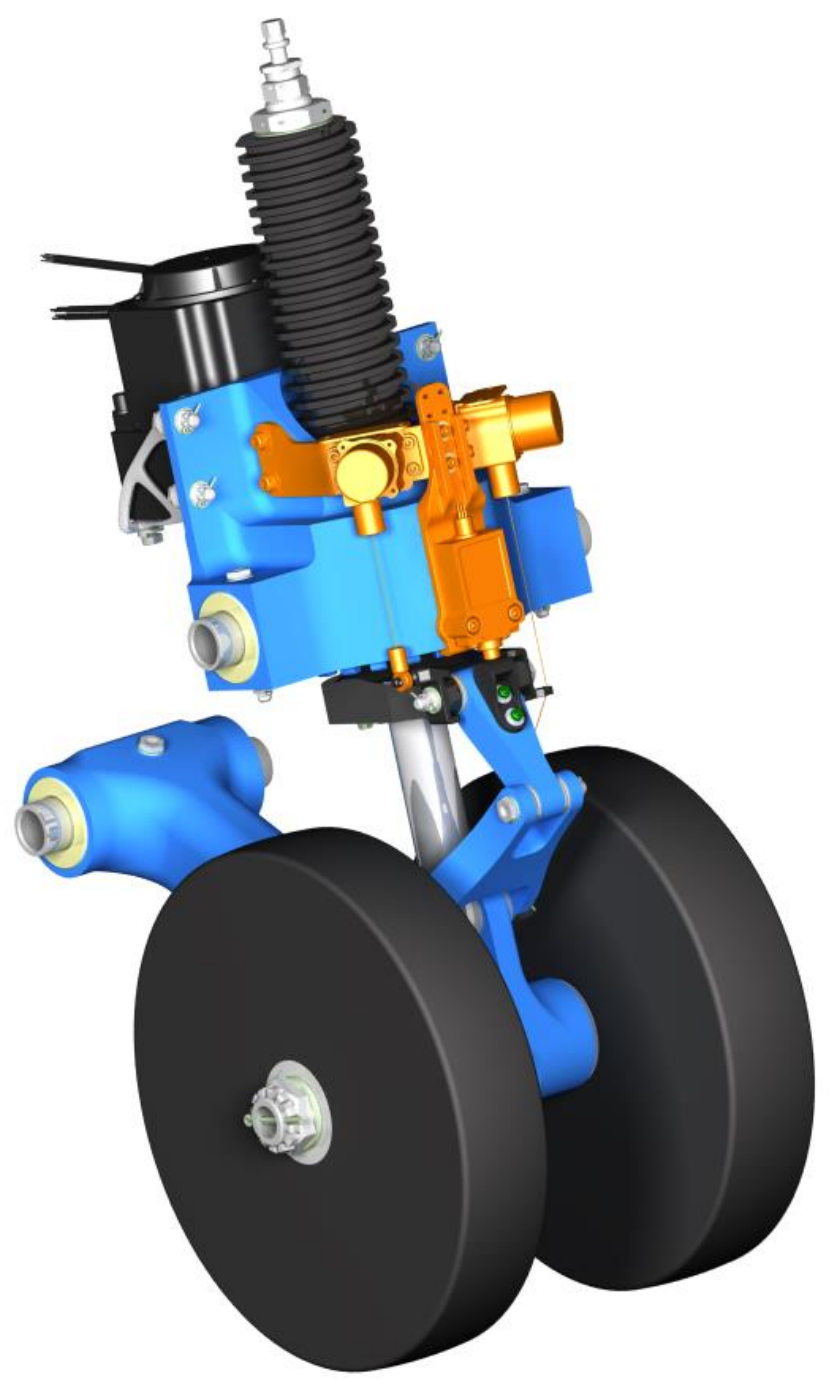

Figure 4.7: Sensors, brackets, electrical and hardware mount (orange) shown on the HDWS Dressed 
To accomplish the preceding tasks, the HDWS Dressed Assembly is equipped (Figure 4.8) with two externally mounted cable potentiometers (1) and (2) that monitor the translation of the shock strut cylinder and shock strut piston stroke respectively. The potentiometers are fixed to a sensor bracket (3) via four cap screws (4), per potentiometer, and locked using spring back washers. The bracket (3) is itself fixed to the outer frame of the Retraction sub-assembly via four NAS cap screws (5), and locked using lock-wire at install (not shown here). The shock strut cylinder potentiometer cable is attached to an eyelet receiver bracket (6) and locked on installation using a cotter pin. The piston stroke potentiometer cable is routed via a transfer bracket (7) and attached at the piston rod-end eyelet receiver bracket (8).

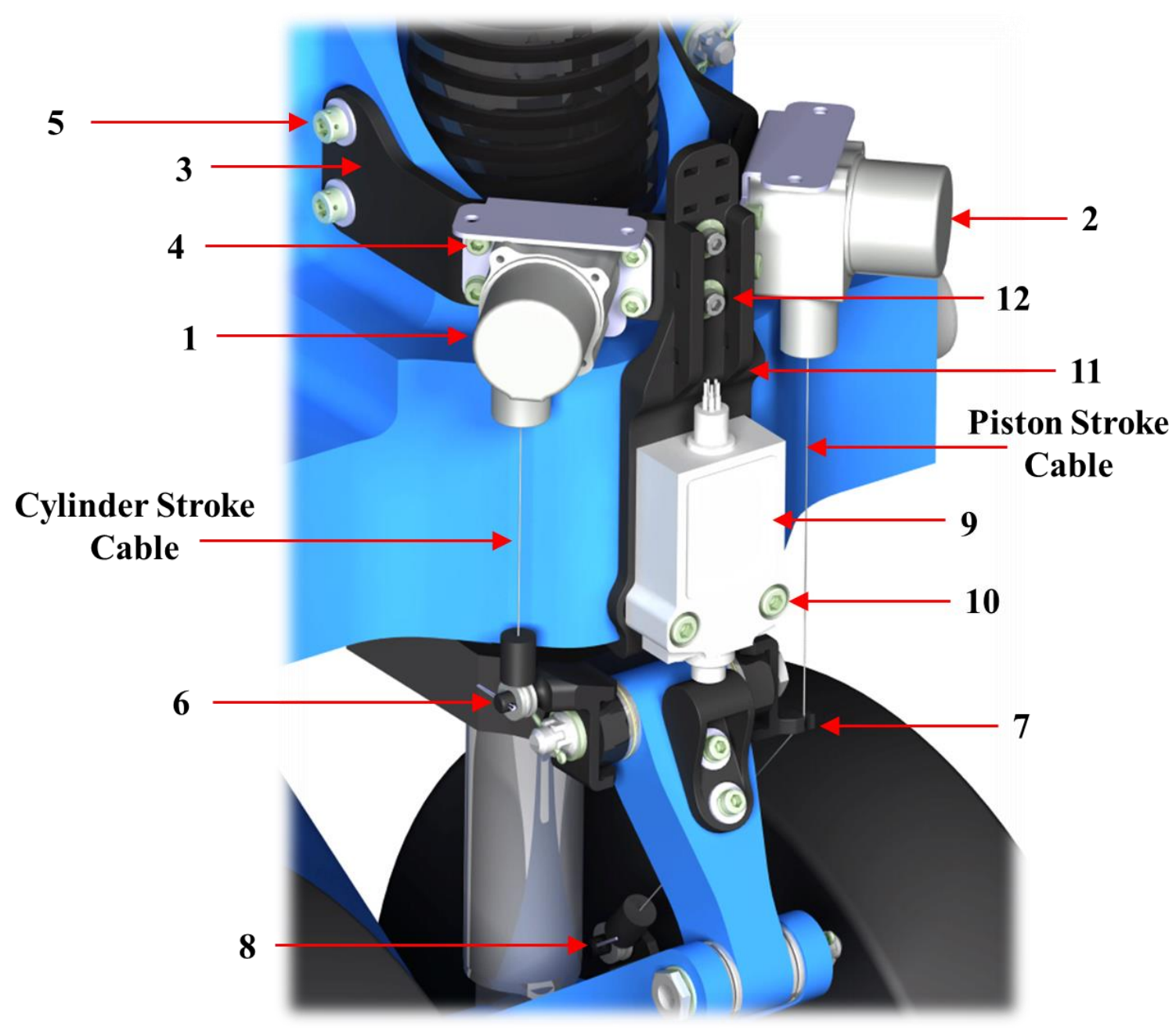

Figure 4.8: Sensors, brackets, electrical and hardware labeled (some features hidden for clarity) 
Table 4.2: Electric Motor assembly breakdown summary

\begin{tabular}{|c|c|c|c|c|}
\hline Find \# & P/N & Part Description & Material & QTY \\
\hline 1 & SM2-12.5 & Miniature String Potentiometer (12.5-in range) & Various & 1 \\
\hline 2 & SM2-25 & Miniature String Potentiometer (25-in range) & Various & 1 \\
\hline 3 & $40-1310-01$ & Bracket, Cable Potentiometers & ABS & 1 \\
\hline 4 & NAS1352C06-6 & NAS Cap Screw & CRES & 8 \\
\hline 5 & NAS1351C3H8 & Cap Screw, Drilled Head & CRES & 4 \\
\hline 6 & $40-1211-00$ & Eyelet bracket, ACME Potentiometer & ABS & 1 \\
\hline 7 & $40-1212-00$ & Eyelet transfer bracket, Piston Potentiometer & ABS & 1 \\
\hline 8 & $40-1404-00$ & Eyelet bracket, Oleo Potentiometer & ABS & 1 \\
\hline 9 & NGCMB10AX01B & Limit Switch & Various & 1 \\
\hline 10 & NAS1352C08-8 & Cap Screw & CRES & 2 \\
\hline 11 & $40-1311-00$ & Bracket, Limit Switch & CRES & 2 \\
\hline 12 & NAS1352C06-8 & Cap Screw & & 1 \\
\hline
\end{tabular}

Once reaching a fully retracted state, a limit switch (9), attached via two NAS cap screws (10) to a mounting bracket (11) is triggered by a bumper on the upper torque link indicating a "fully retracted" system state and subsequently shuts down the motor. The bracket (11) is mounted to the Retraction sub-assembly via two NAS cap screws (12).

\subsection{HDWS Undressed Assembly Components}

\subsubsection{Trailing Arm Sub-assembly (P/N 30-1100-00)}

The Trailing Arm sub-assembly (Figure 4.9) was designed as a primary load-carrying member of the HDWS. The design of the trailing arm was sized principally on anticipated side loads and dynamic braking loads (external source braking) that the system could face during operation within the SpaceX Hyperloop Competition. Table 4.3 details the individual components that make up the trailing arm sub-assembly that is itself part of the HDWS Undressed assembly. 


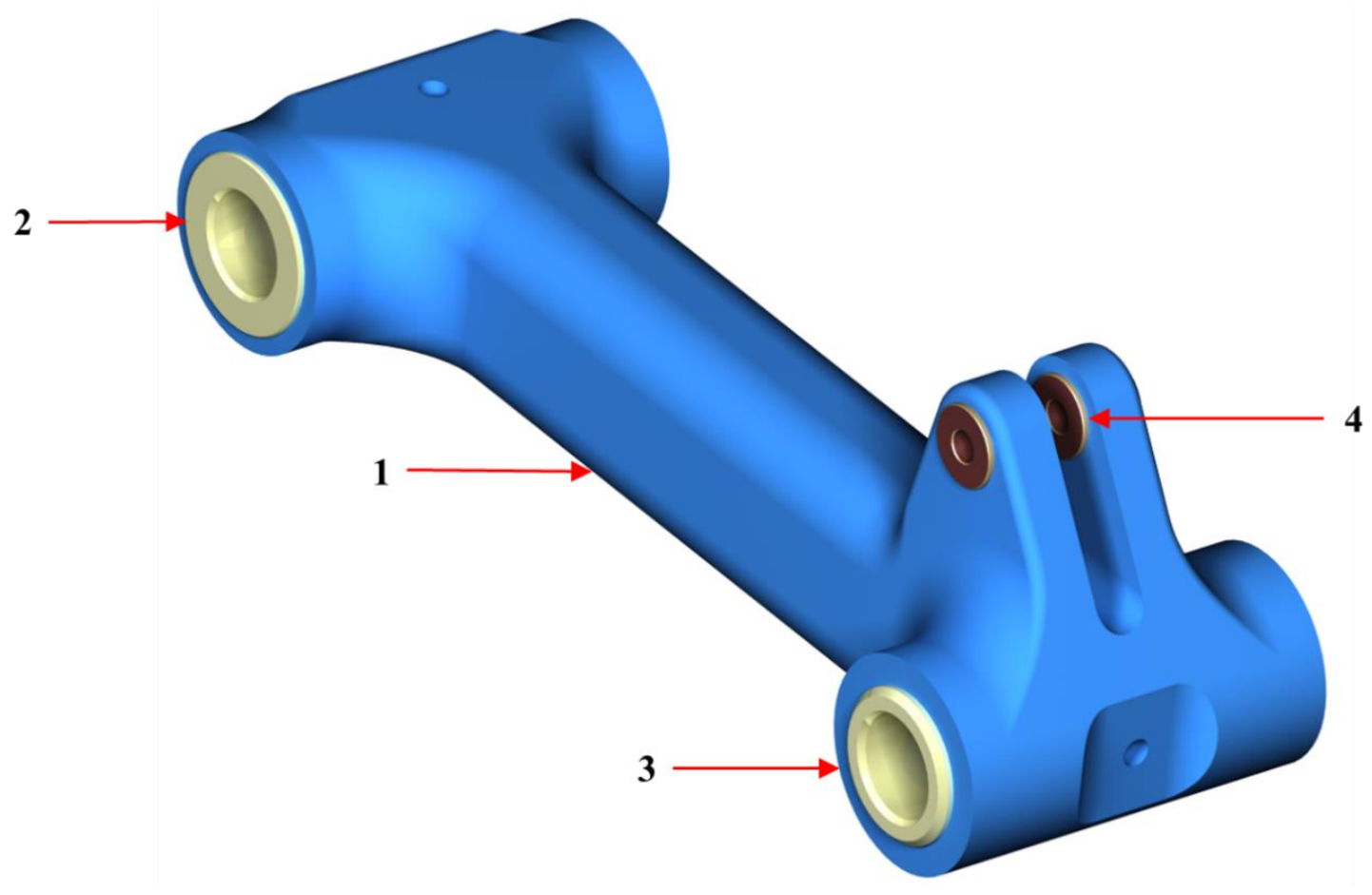

Figure 4.9: The Trailing Arm sub-assembly, parts labeled

In Figure 4.9, Find Numbers (used to identify key parts of the system), corresponding to the information in Table 4.3, can be found. Find Number (1) consists of the machined from solid trailing arm component. This part, made from aluminum (Al) 6061-T6, was anodized blue for aesthetic appearance and proactively controls and initiates the natural oxidization for increased corrosion protection. The flanged bushings at the trailing arm pivot socket (2) are machined from aluminum bronze (Al-Br) and installed using an interference press fit method. The bushings (2) material choice and design protect the aluminum trailing arm structure by providing a stronger bearing surface, resisting wear and acting as a sacrificial repair component that enable the protection of the main structure.

Table 4.3: Trailing Arm sub-assembly breakdown summary

\begin{tabular}{|c|c|c|c|c|}
\hline Find \# & P/N & Part Description & Material & QTY \\
\hline 1 & $40-1101-66$ & Trailing Arm & $\mathrm{Al} 6061-\mathrm{T6}$ & 1 \\
\hline 2 & $40-1105-00$ & Bush, Pivot Pin, Trailing Arm & $\mathrm{Al-Br}$ & 2 \\
\hline 3 & $40-1106-00$ & Bush, Axle, Trailing Arm & $\mathrm{Al-Br}$ & 2 \\
\hline 4 & KRJ5-UDSVC-008 & KAron ${ }^{\circledR}$ Bush, Piston Clevis & CRES 17-4PH & 4 \\
\hline
\end{tabular}


The flanged bushings (3) for the axle interface socket are designed specifically to ensure contact with the inner-race of the wheel mounted bearings. In contrast, if the bushings at (2) were used for the axle interface socket, the wheel bearing's inner and outer race would contact the bush flange face causing undesirable friction and wear to the roller bearings and trailing arm bushings. The bushings at (2) and (3) are typically greased prior to pin installation [5], especially when the joint is subject to rotation. In this application, the bushings at (2) and (3) will contain a pin and axle respectively, locked in rotation and axial translation by a standard NAS bolt, washer and nut set. The locations of the two bores housing the bolt, washer and nut sets are shown in Figure 4.11.

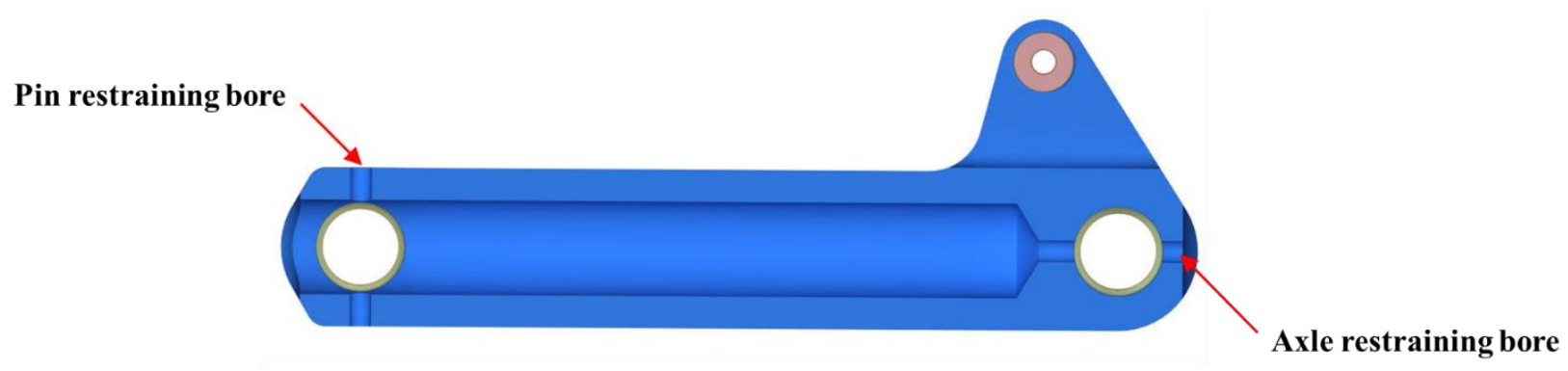

Figure 4.10: The Trailing Arm sub-assembly cross-sectional view

As discussed in Chapter 2, since the HDWS operates in a near vacuum, typical greasing methods are unacceptable due to outgassing and the evaporation of oil based lubricants within a vacuum. To combat the issue, self-lubricated bushings were sourced from Kamatics Speciality Bearings (subsidy of KAMAN Aerospace [43]), suppliers of the patented KAron ${ }^{\circledR}$ dry-lubricated bearings. These bearings are specially designed to operate within a vacuum environment and are used on various spacecraft and satellites. These self-lubricated bushings (4) are installed on the trailing arm at the piston lug interfaces and lower torque-link interfaces. The KAron ${ }^{\circledR}$ lubrication was particularly required on both the flange and inner diameter of the bushing to provide lubrication for the rotating parts and NAS bolt fitted through the ID of the part. The bushings (4) were installed using a press fit method at laboratory temperature, $68^{\circ} \mathrm{F}\left(20^{\circ} \mathrm{C}\right)$.

In an earlier concept of the trailing arm, illustrated in Figure 4.11, the trailing arm had two small lugs machined on the end. These small lugs enabled the easy locking of a thread-less eye-bolt, using a dowel pin, shown in Figure 4.11. This feature did not end up on the final design due to the 
added manufacturing cost associated with these small features. However, for a future production variant, such a system should be considered for the quick removal and attachment of the axle.

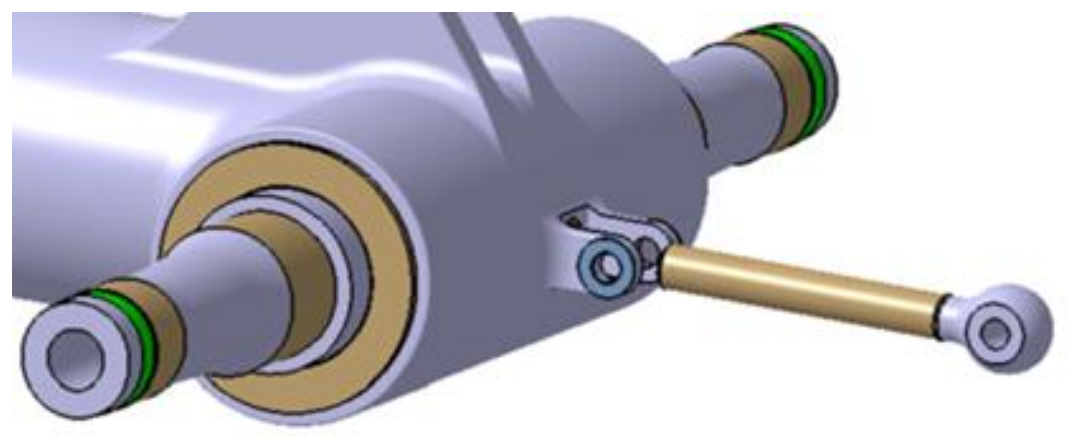

Figure 4.11: The Trailing Arm axle installation pin

\subsubsection{Shock Strut Sub-assembly (P/N 30-1200-00)}

The Shock Strut sub-assembly (Figure 4.12) is one of the most important sub-assemblies of the HDWS and was included to ensure a smooth ride for the Hyperloop pod and its payload. The shock absorber was designed as an oleo-pneumatic type, a typical choice for aircraft landing gear due to its high efficiency and relatively low mass [5]. The detailed design and performance characteristics, principally characterized by the sizing of the gas spring, is one of the most complex features of the HDWS and will be presented in Chapter 5.

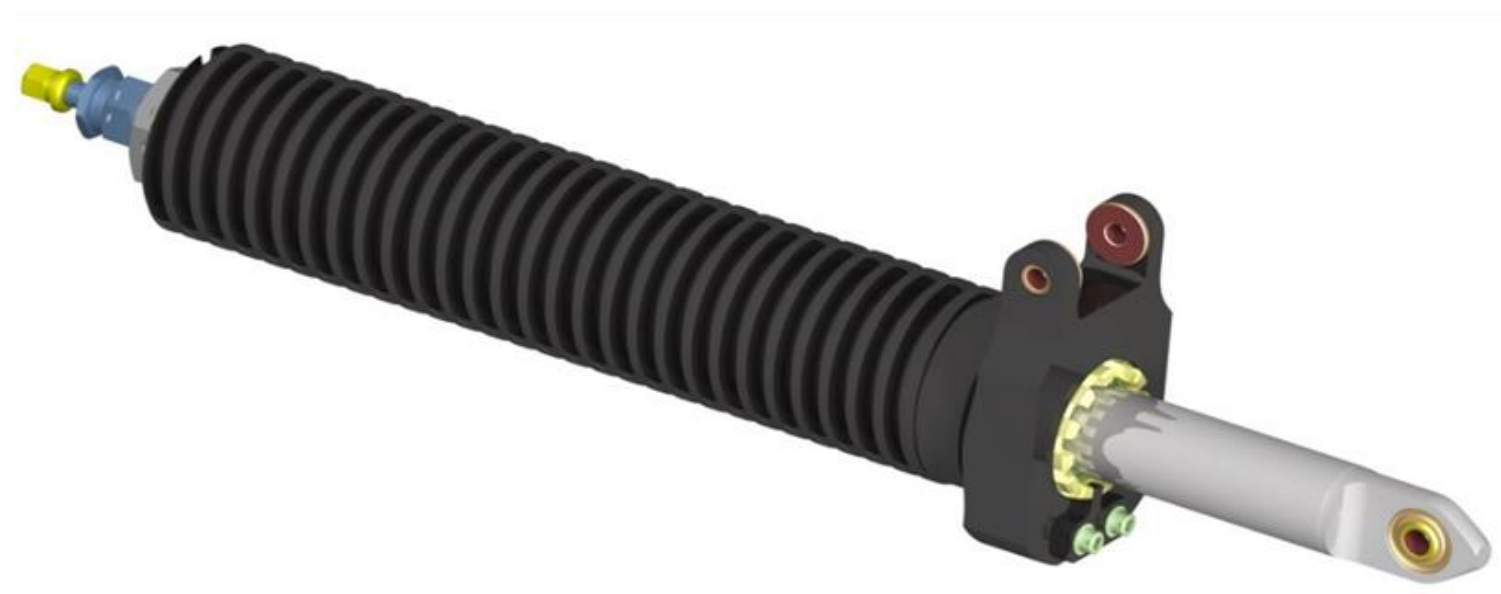

Figure 4.12: The HDWS Shock Strut sub-assembly, CATIA ${ }^{\circledR}$ V5 rendering 


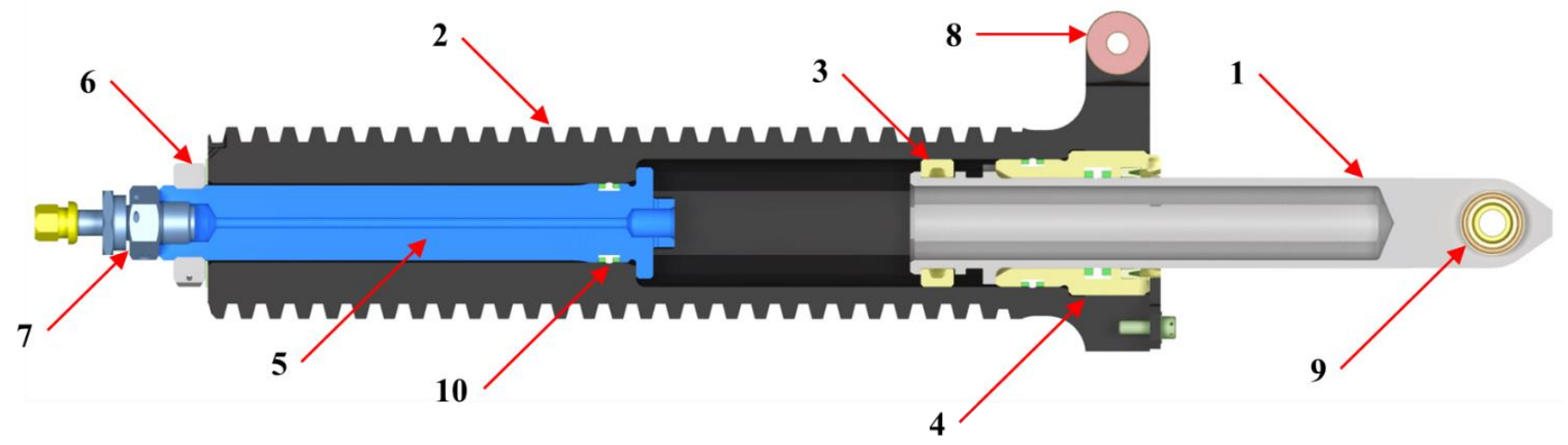

Figure 4.13: Full cross-section view of the Shock Strut sub-assembly, Find Numbers shown

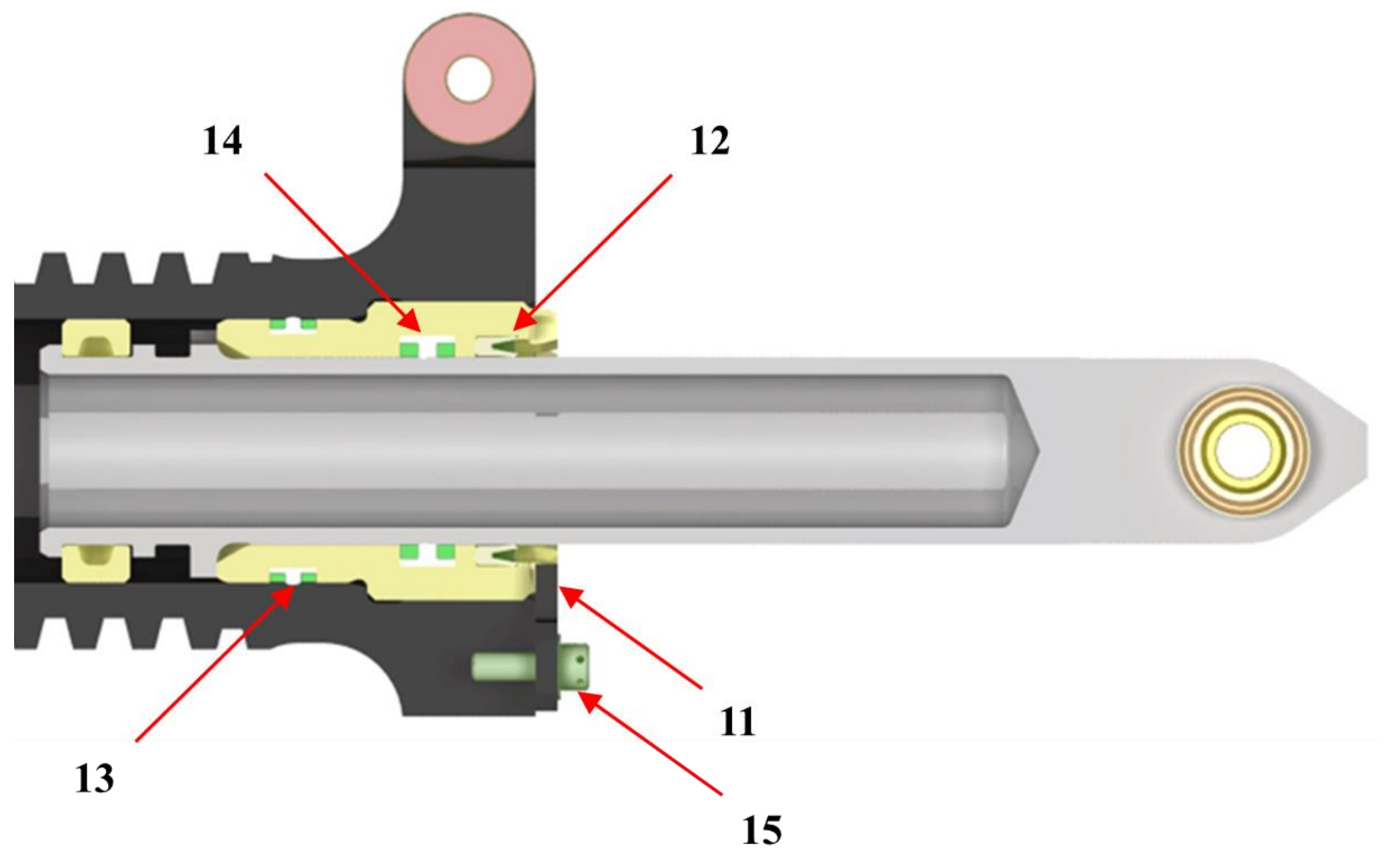

Figure 4.14: Partial cross-section view of the shock strut sub-assembly, Find Numbers shown 
Table 4.4: Shock Strut sub-assembly breakdown summary

\begin{tabular}{|c|c|c|c|c|}
\hline Find \# & P/N & Part Description & Material & QTY \\
\hline 1 & $40-1201-00$ & Piston & 303 SS & 1 \\
\hline 2 & $40-1202-00$ & Cylinder & AISI 12L14 & 1 \\
\hline 3 & $40-1205-00$ & Piston Upper Bearing & $\mathrm{Al-Br}$ & 1 \\
\hline 4 & $40-1207-00$ & Gland Nut (Lower Bearing) & $\mathrm{Al-Br}$ & 1 \\
\hline 5 & $40-1209-00$ & Support Tube & $\mathrm{Al} 6061-\mathrm{T} 6$ & 1 \\
\hline 6 & NAS1423-14 & Jam Nut (Hex, Drilled Head) & 4340 & 1 \\
\hline 7 & MS28889-2 & Charge Valve (Gas/Oil) & CRES & 1 \\
\hline 8 & KRJ4-UDSVC-008 & KAron ${ }^{\circledR}$ Bush, UTL Lug & CRES 17-4PH & 2 \\
\hline 9 & KR5-CNGBP & Spherical Bearing & CRES 17-4PH & 1 \\
\hline 10 & 711 D6MTE-161-P15 & AGT Ring (Static Seal) & --- & 1 \\
\hline 11 & $40-1208-00$ & Lock tab & AISI 12L14 & 1 \\
\hline 12 & R2361-215E344 & MSE Scraper & --- & 1 \\
\hline 13 & 7125 MT-964-P15 & AGT Ring (Static Seal) & --- & 1 \\
\hline 14 & $7215 F T-964-P 17$ & AGT Ring (Dynamic Seal) & --- & 1 \\
\hline 15 & NAS1352C08H8 & Cap Screw (Drilled Head) & CRES & 2 \\
\hline
\end{tabular}

As illustrated in Figure 4.13 and Figure 4.14 and described in Table 4.4, the assembly consists of a piston (1) and outer cylinder (2). The outer cylinder (2) is finished on the exterior with 3G ACME threads to interface with the retraction assembly. A set of bushed lugs protruding at the base of the cylinder mates with the upper torque link (UTL) as seen in Figure 4.2. 303 SS (stainless steel) was used to manufacture the piston (1) with its outer diameter (OD) ground to a surface roughness specified by the seal manufacturer to ensure excellent sealing characteristics. The piston is locked inside the outer cylinder by a gland nut (4), retained by a lock tab (11), and standard NAS drilled head cap screws (15) locked in place using lock-wire (not shown). The piston is attached to the trailing arm lugs using a KAron ${ }^{\circledR}$ lined spherical bearing (9) and NAS bolt (not shown, equipped at Undressed level).

The piston itself is equipped with an upper bearing (3) which maintains the contact (acts as an axial bearing) of the piston with the outer cylinder wall during stroke operation and functions as a restrictive flow path for the oil. The shock absorber is charged with nitrogen and filled with oil using the filler support tube (5), fastened using a jam nut (6) and fitted with a standard MS charge valve (7). All pathways to the outside environment are sealed using static and dynamic seals. A 
Green Tweed AGT static seal (13) (with 2 back-up rings) is used to seal the gland nut to the cylinder inner diameter (ID). A Green Tweed AGT ${ }^{\circledR}$ dynamic seal (14) is equipped in the ID of the gland nut (4) and is used to seal the piston OD. Lastly, a static seal (10) is fit between the support tube (5) and the shock strut outer cylinder ID, completing the sealing of the inner chamber.

\subsubsection{Retraction Sub-assembly (P/N 30-1300-00)}

The Retraction sub-assembly, as shown in Figure 4.15, plays a primary role in the successful operation of the HDWS. The sub-assembly converts the externally mounted BLDC motor's rotational motion (torque) into translational motion (at the shock strut), enabling the retraction and extension of the HDWS. By the same token, this sub-assembly, whose components are defined in Table 4.5, provides the translational motion for the active height control of the pod using feedback from various sensors mounted to the retraction sub-assembly at the dressed level. The retraction housing (1) is connected to the pod using two oppositely disposed pins (not shown) at the bearings (5) and locked in translation and rotation using NAS shear bolts. A large flange at the back of the housing (1) is used to mount the BLDC electric motor, as seen in Figure 4.3.

The internally mounted lead screw retract nut (2), which is lined on its OD with standard spur gear teeth, is mounted inside the housing (1) and held in place by two deep groove ball bearings (6). The nut and thrust bearings are packed into the housing via a large castellated packing nut (3) and locked from rotation via a lock tab (4), fastened to the housing (1) via two standard NAS cap screws (7). The two cap screws (7) are lock-wired on assembly, lock-wire not shown here.

Table 4.5: Retraction sub-assembly component breakdown summary

\begin{tabular}{|c|c|c|c|c|}
\hline Find \# & P/N & Part Description & Material & QTY \\
\hline 1 & $40-1301-02$ & Retract Housing & $\mathrm{Al} \mathrm{6061-T6}$ & 1 \\
\hline 2 & $40-1302-02$ & Retraction Nut & $\mathrm{Al-Br}$ & 1 \\
\hline 3 & $40-1303-01$ & Packing Nut & $\mathrm{Al} 6061-\mathrm{T} 6$ & 1 \\
\hline 4 & $40-1304-00$ & Lock Tab, Retract Housing & AISI 12L14 & 1 \\
\hline 5 & $40-1308-00$ & Trunnion Bush, Trunnion Pin & $\mathrm{Al-Br}$ & 2 \\
\hline 6 & $61813-2 \mathrm{RZ}$ & Deep Groove Ball Bearing & CRES & 2 \\
\hline 7 & NAS1352C08H4 & Cap Screw, Drilled Head & CRES & 2 \\
\hline
\end{tabular}



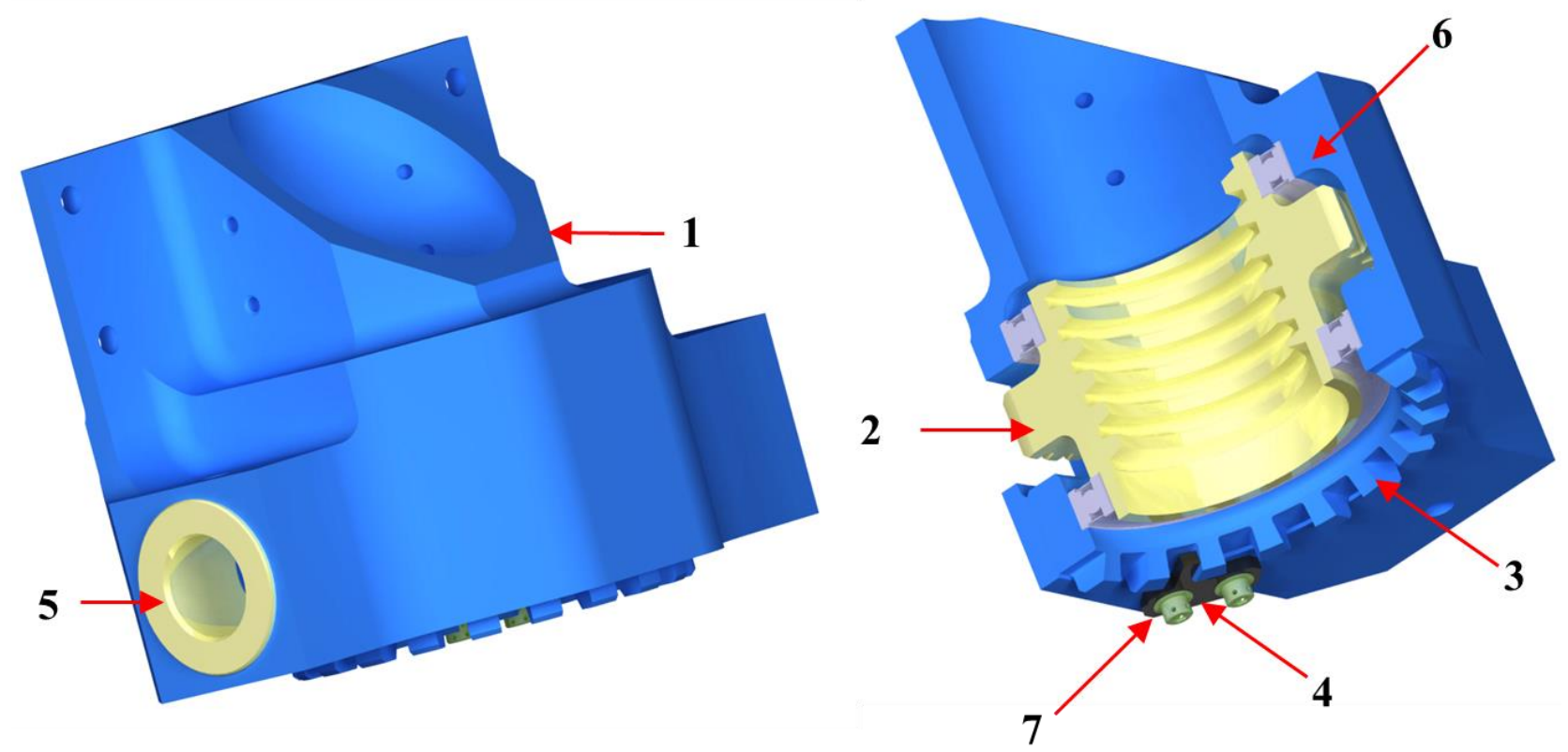

Figure 4.15: Retract sub-assembly 3D view (left) and cross section (right), Find Numbers shown

\subsubsection{Torque Link Sub-assembly (P/N 30-1400-00)}

As shown in Figure 4.16, the Torque Link sub-assembly is composed of two major components, the upper (1) and lower (2) torque links. The torque links are required to react the loads induced by the friction between the ACME thread retract nut, as shown in Figure 4.15, and the shock strut cylinder, as shown in Figure 4.12. The reaction of these loads enables the Shock Strut subassembly to translate axially and cause the HDWS to extend, retract, and operate in an actively controlled manner. Since the torque links will be under rotation and under load (as the shock absorber strokes), the joints are equipped with $\mathrm{KAron}^{\circledR}$ lined CRES bushings (4, 5 and 6) providing lubrication in the low-pressure environment and a tough wear surface protecting the Al 6061-T6 torque link bores. The upper torque link (UTL) and the lower torque link (LTL) are connected at the apex joint using a standard NAS bolt (7). The LTL is attached to the trailing arm piston lug and the UTL is attached to the shock strut cylinder lug (see Figure 4.2). Lastly, a limit switch bumper (3) is equipped to the UTL using two standard NAS Cap screws (8). Upon reaching a fully retracted position, the UTL bumper triggers a limit switch sensor, signaling that the HDWS is now retracted. 


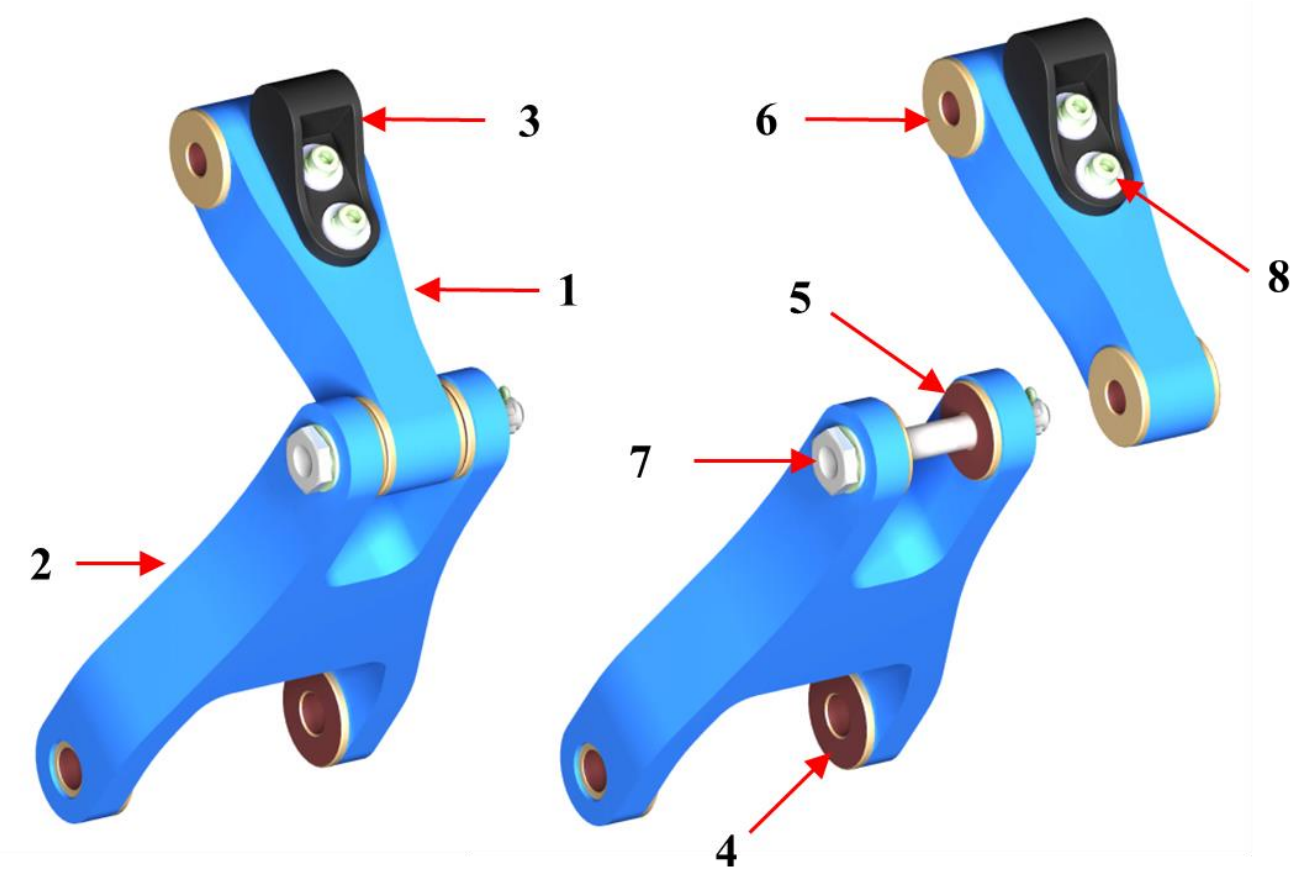

Figure 4.16: Torque Link sub-assembly (left) and exploded view (right), Find Numbers shown

Table 4.6: Torque Link sub-assembly component breakdown summary

\begin{tabular}{|c|c|c|c|c|}
\hline Find \# & P/N & Part Description & Material & QTY \\
\hline 1 & $40-1401-00$ & Upper Torque Link & Al 6061-T6 & 1 \\
\hline 2 & $40-1402-02$ & Lower Torque Link & Al 6061-T6 & 1 \\
\hline 3 & $40-1403-00$ & Limit Switch Bumper & ABS (AM) & 1 \\
\hline 4 & KRJ5-UDSVC-008 & KAron ${ }^{\circledR}$ Bush, LTL Lug & CRES 17-4PH & 2 \\
\hline 5 & KRJ4-UDSVC-008 & KAron ${ }^{\circledR}$ Bush, LTL Apex Lug & CRES 17-4PH & 2 \\
\hline 6 & KRJ4-USVC-008 & KAron ${ }^{\circledR}$ Bush, UTL & CRES 17-4PH & 4 \\
\hline 7 & NAS6204-22D & NAS Bolt & 4340 & 1 \\
\hline 8 & NAS1352C06-06 & NAS Cap Screw & CRES & 2 \\
\hline
\end{tabular}

\subsubsection{Axle (P/N 40-1501-00)}

The axle component (Figure 4.17) is an integral part of the Undressed assembly. The axle was manufactured from $303 \mathrm{SS}$ and designed with a 1-in $(25.4 \mathrm{~mm})$ shaft OD. It is equipped, as shown in Figure 4.17, to the Trailing Arm sub-assembly through the axle bushings located at the back of 
the arm. The axle is further constrained from rotation and translation using a standard NAS shear bolt equipped at the Undressed level of the assembly and features externally threaded ends with locking features for the axle nut, used to retain the wheels at installation (see Figure 4.6).

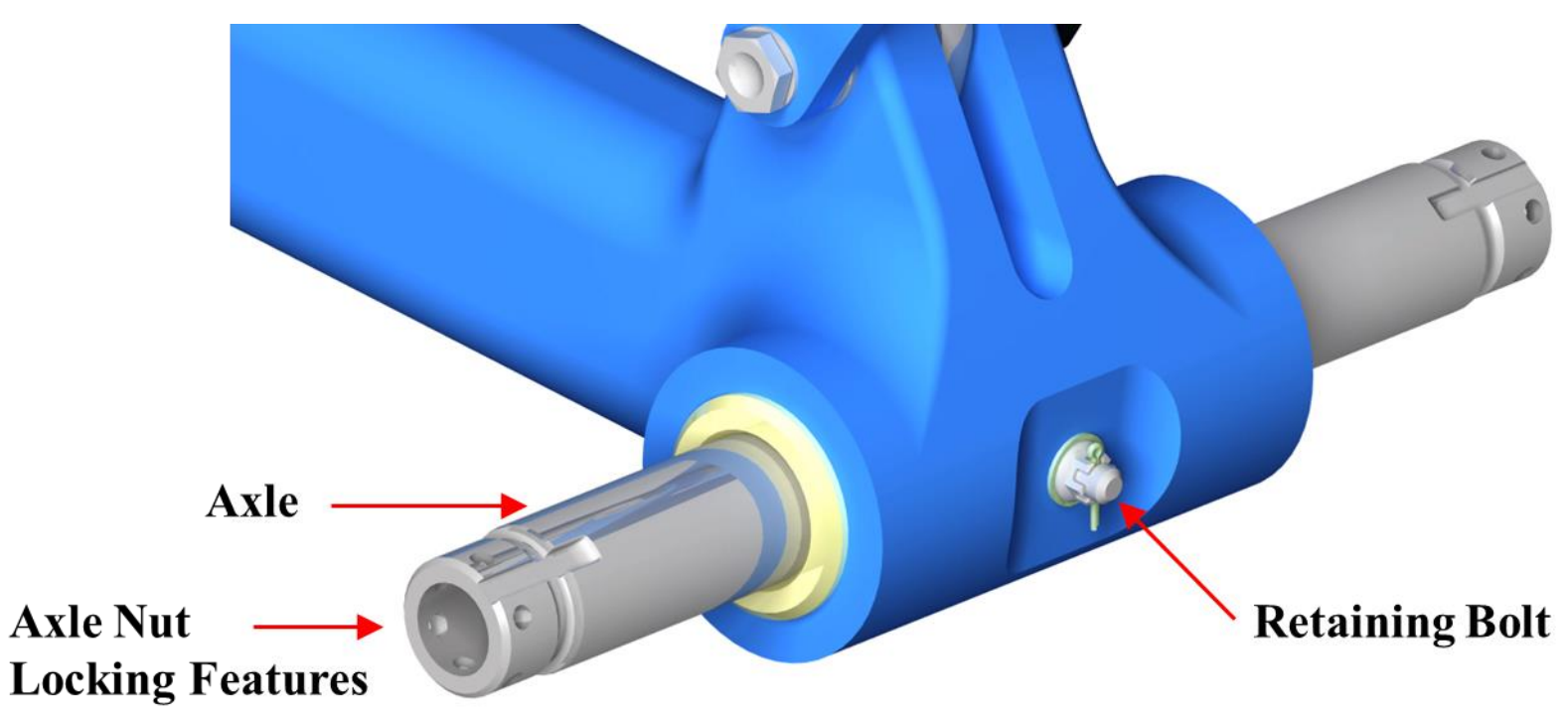

Figure 4.17: The axle component as equipped to the trailing arm sub-assembly

\subsection{Structural Analyses}

The structural analysis of the HDWS components was principally split into two broadly defined areas: classical hand calculation analysis and finite element analysis (FEA). The common use cases for each broad method of analyses are discussed here using applicable examples to illustrate. It shall be stated that the HDWS project had the benefit of a dedicated structural analyst, Moeid Elahi, hereby referred to as the "actor", who was the primary source of the project's official FEA and classical hand calculations. Wherever the work of the actor is shown or discussed, a differentiation will be made between the actor's contribution and the author's. In all cases, the minimum SF requirement ( $\mathrm{SF}=2$ ), defined by SpaceX [19], was achieved by comparing the stresses in the component to its relevant material allowable. The static yield and bearing allowable stresses for all materials utilized on the HDWS can be found in the MMPDS-07 handbook [30] or obtained from the part and material suppliers. 


\subsubsection{Classical Analyses Overview}

Classical hand calculations rely on the fundamental strength of materials and statics equations that can be found in standard engineering textbooks, such as those authored by Hibbeler [46]. Classical hand calculations were performed whenever possible on simple features and cross sections. During the design process these fundamentals were frequently used to perform initial sizing calculations, two common samples of which are discussed in this section.

\section{Bolts Analysis:}

Depending on the application, bolts can be subjected to a variety of loading including shear, tension, bending or a combination.

As an initial sizing exercise the author used estimated loading values provided by a system beam model, developed by the actor with help from the project advisors. Using this information, simple calculation checks were performed, some of which are described here.

When a bolt was acted on in shear (typically double shear as shown in Figure 4.18 in this application) the following formulae were applied [46]:

\section{Bolt, Single Shear:}

$$
\tau_{S}=\frac{F_{S}}{A_{S}}
$$

Here, $\tau_{S}$ is the shear stress on the bolt, $F_{S}$ is the force transmitted through the joint, and $A_{S}$ is defined as the shear area for bolt. For the loading condition shown in Figure 4.18, the joint is subjected to double shear through the pin diameter. Thus, the equation for $A_{S}$ becomes:

$$
A_{S}=\pi \frac{d_{b}^{2}}{4}
$$

where the area subjected to shear is defined as the pin diameter area with diameter defined by $d_{b}$. Note that the value for $d_{b}$ shall be selected as the nominal bolt diameter, as defined in the part specification. 
When the pin is under double shear loading conditions, illustrated in Figure 4.18, the equation for the shear stress in the pin is defined by:

\section{Bolt, Double Shear:}

$$
\tau_{S}=\frac{F_{S} / 2}{A_{S}}
$$

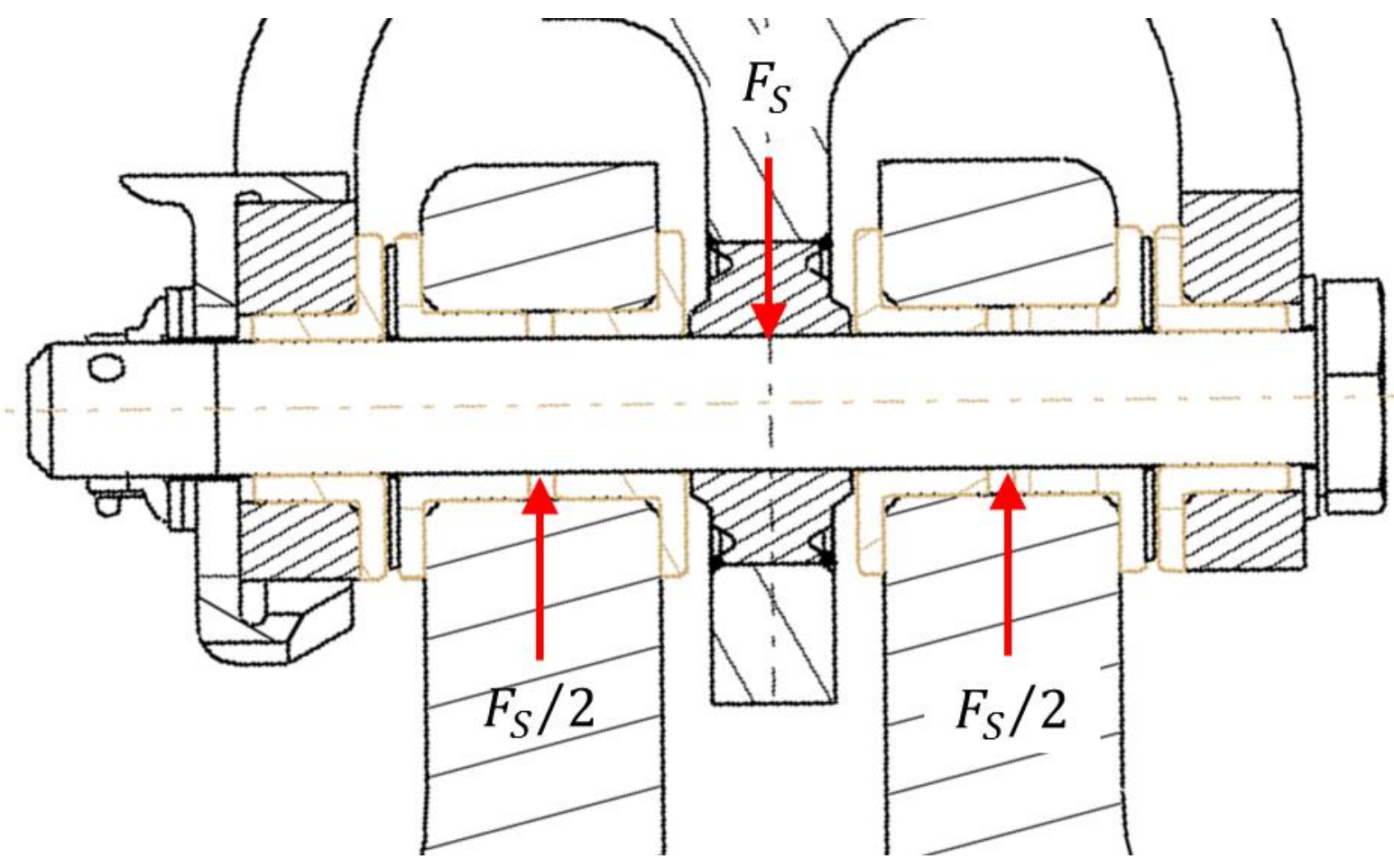

Figure 4.18: Section cut, illustrating a typical double shear loading on the HDWS

Here the loading is simplified, assuming equal distribution acting through the centre of each of the lugs. For the piston lug, where the axial load $F_{S}$ is transmitted through a single spherical bearing, this loading definition can be approximated as linear. However, in practice, and as can be more clearly recognized at the trailing arm lug interface, where the loading is reacted between the two lugs, the load can be more appropriately defined by taking into account the deformation of the pin. Such considerations were applied in the detailed analyses performed by the noted stress actor and were not the subject of attention for the author during the pre-sizing of joints. 
Bolts that were subjected to tensile loading were checked for tensile stresses within the bolt diameter using the following equation [46]:

\section{Bolt Under Tensile Loading:}

$$
\sigma_{T}=\frac{F_{T}}{A_{T}}
$$

Here, $\sigma_{T}$ is the tensile stress on the bolt, $F_{T}$ is the tensile force, typically due to bolt pre-loading applied at assembly, and $A_{T}$ is defined as the tensile area of the bolt. In practice, the portion of the pin with the smallest $A_{T}$ will be the likely source of failure in a bolt under tension. These minimized areas are typically found at the head of the pin, where a small undercut is typically applied at the design phase to allow for a larger transition radius, reducing stress concentrations. For the standard NAS bolts used on this project, however, the bolt head is designed to mate with a large ID washer and thus does not lose bolt area close to the head. So, for a conservative initial calculation, the minor diameter of the bolts thread was selected as the smallest area $A_{T}$ and therefore subject to the highest tensile stress, $\sigma_{T}$.

Lastly, a final check of the shear stress produced in the bolt head, while the bolt is subject to tensile loading, as shown in Figure 4.19, was conducted. The eqution is defined as follows:

$$
\tau_{S}=\frac{F_{T}}{A_{S C}}
$$

where $A_{S C}$, the shear area of the pin head under tensile loading, is defined by:

$$
A_{S C}=2 \pi r t
$$

Here the value for $r$ was typically chosen as $d_{b} / 2$, that is, the circumferential location where the shear force is maximum, and the thickness $t$ of the bolt head as defined by the bolt manufacturers. When combined, the value for $r$ and $t$ define shear area of the pin head, as shown in Figure 4.19. 


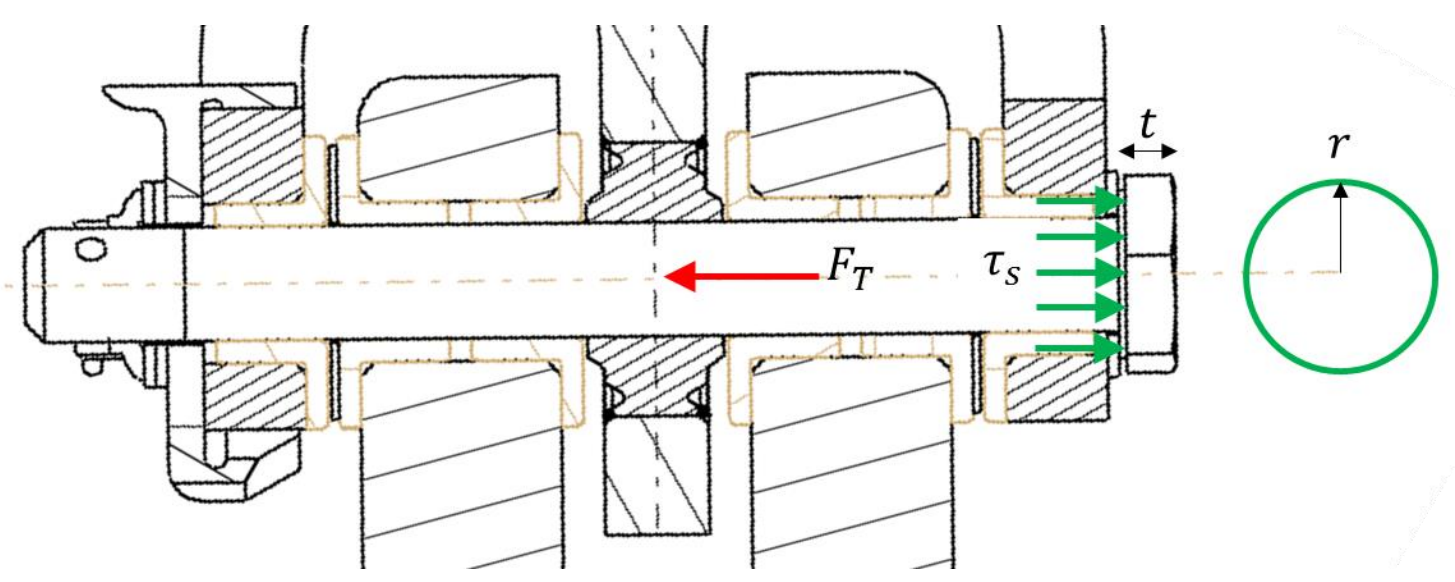

Figure 4.19: Section cut, illustrating a shear stress around the circumference of the bolt head

\section{Bush and Bearing Sizing:}

As discussed in Chapter 4, the flanged bushings and spherical bearings that were used for all rotational joints were of the self-lubricating type. As a preliminary sizing exercise, the guidelines illustrated in the Kamatics engineering product catalogue were used [47].

Both spherical and flanged bushings use a "projected area" method, widely used in the engineering industry when defining the initial bearing pressure $P_{B}$. As per Kamatics, the equation for $P_{B}$ can be written as [47]:

$$
P_{B}=\frac{F_{A}}{A_{B}}
$$

Here $F_{A}$ is defined as the applied load on the bearing originating from a pin or bolt. The force $F_{A}$ is then assumed to be distributed linearly across the bearing ID. As recommended by Kamatics, the value of bushing area $A_{B}$ is defined by the projected area (see Figure 4.20) using the equation [47]:

$$
A_{B}=L_{B} x I D_{B}
$$



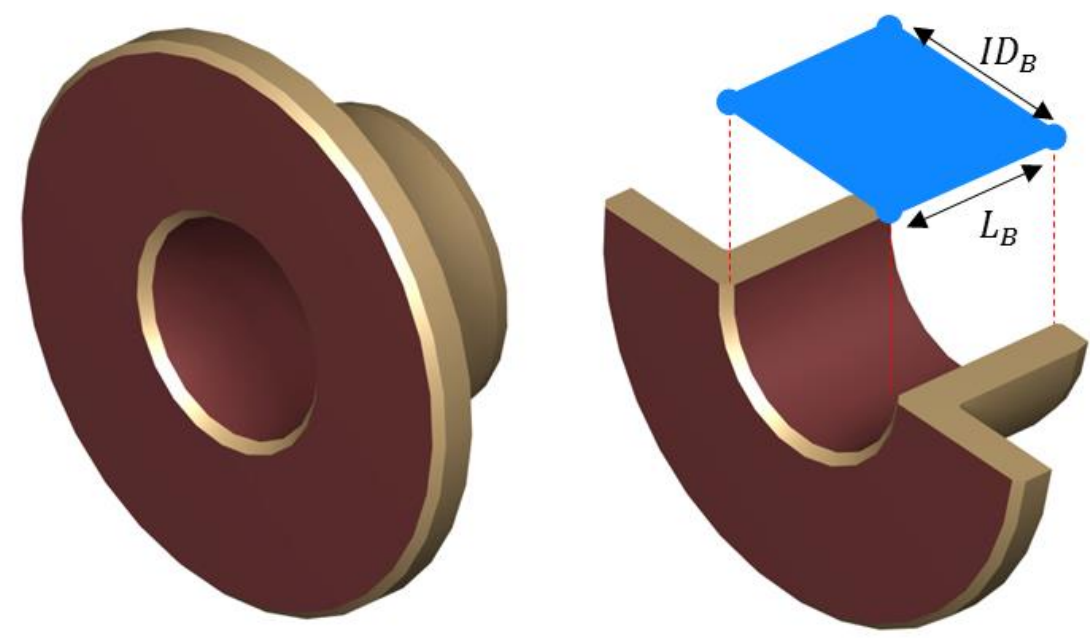

Figure 4.20: Flanged bush (left), illustrating a projected bearing area (right) used for the preliminary calculation of the bearing stress

Following insights from the HDWS project industrial advisors and taking note of the discussions found in reference [47], equation (4.8) is further refined to remain conservative. That is, the useable length of the bearing $L_{B}$ is reduced by the machining of chamfers at each end of the bushing ID. Referencing Figure 4.21, the equation for $A_{B}$ becomes:

$$
A_{B}=\left(L_{B}-C H_{1}+C H_{2}\right) x I D_{B}
$$

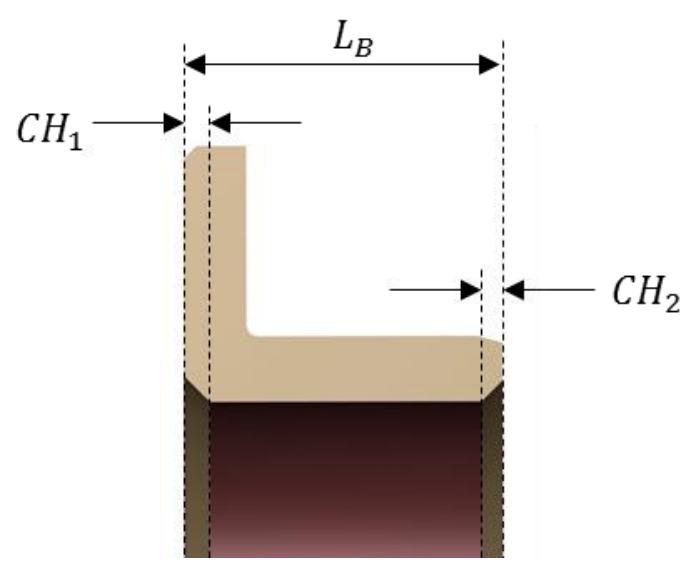

Figure 4.21: Flanged bush illustrating the features involved in a bearing length refinement 
The approach used here for the bearing pressure calculations is valid; however, it is a simplified approach. A wide variety of factors contribute to a more refined bearing pressure calculation, which is known to be a non-linear distribution along the length, $L_{B}$, principally due to pin bending. Other elements of bearing design and selection, such as the pressure on the flange face, the pressure induced by the fitting of the bushing into a housing, and thermal stresses induced on the fit as a product of the thermal dilatation coefficients are known and were checked using proprietary design guide tools provided by Safran Landing Systems Canada Inc. [48]. For this reason, they are not available for outside distribution.

\subsubsection{Finite Element Analyses and Applications}

FEA was used for components with more complex geometrical features and complex combined loading. The software used by the author and actor for the FEA was performed using the analysis

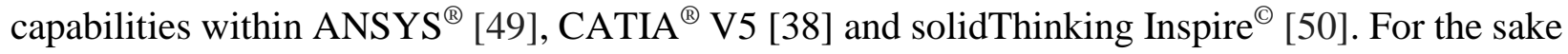
of brevity and to remain focused on the author's contributions to the HDWS, only a sample of the actor's FEA is presented in Figure 4.22 below and the rest are not discussed in this thesis.

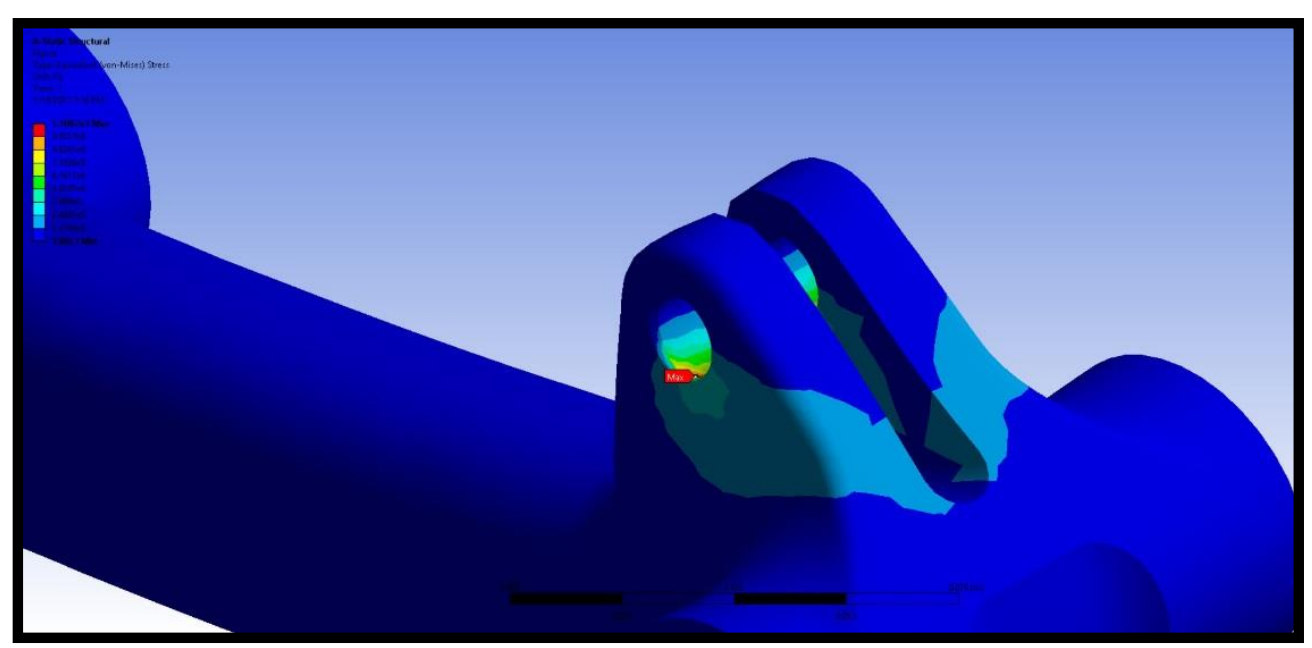

Figure 4.22: Sample FEA result from ANSYS ${ }^{\circledR}$, trailing arm lug analysis [40]

The author had the opportunity to use the analysis and design software solidThinking Inspire ${ }^{\odot}$ that enabled an FEA-based topology optimization study on the BLDC motor mounting bracket as 
shown in Figure 4.23. The bracket was to be machined from a solid aluminum block in its baseline configuration; however, the application of topology optimization enabled the production of a design concept that was additive manufacturing (AM) friendly and highly mass optimized. The finished topology optimized design concept for the motor bracket is shown in Figure 4.24 and a sample of the FEA analysis is shown in Figure 4.25.

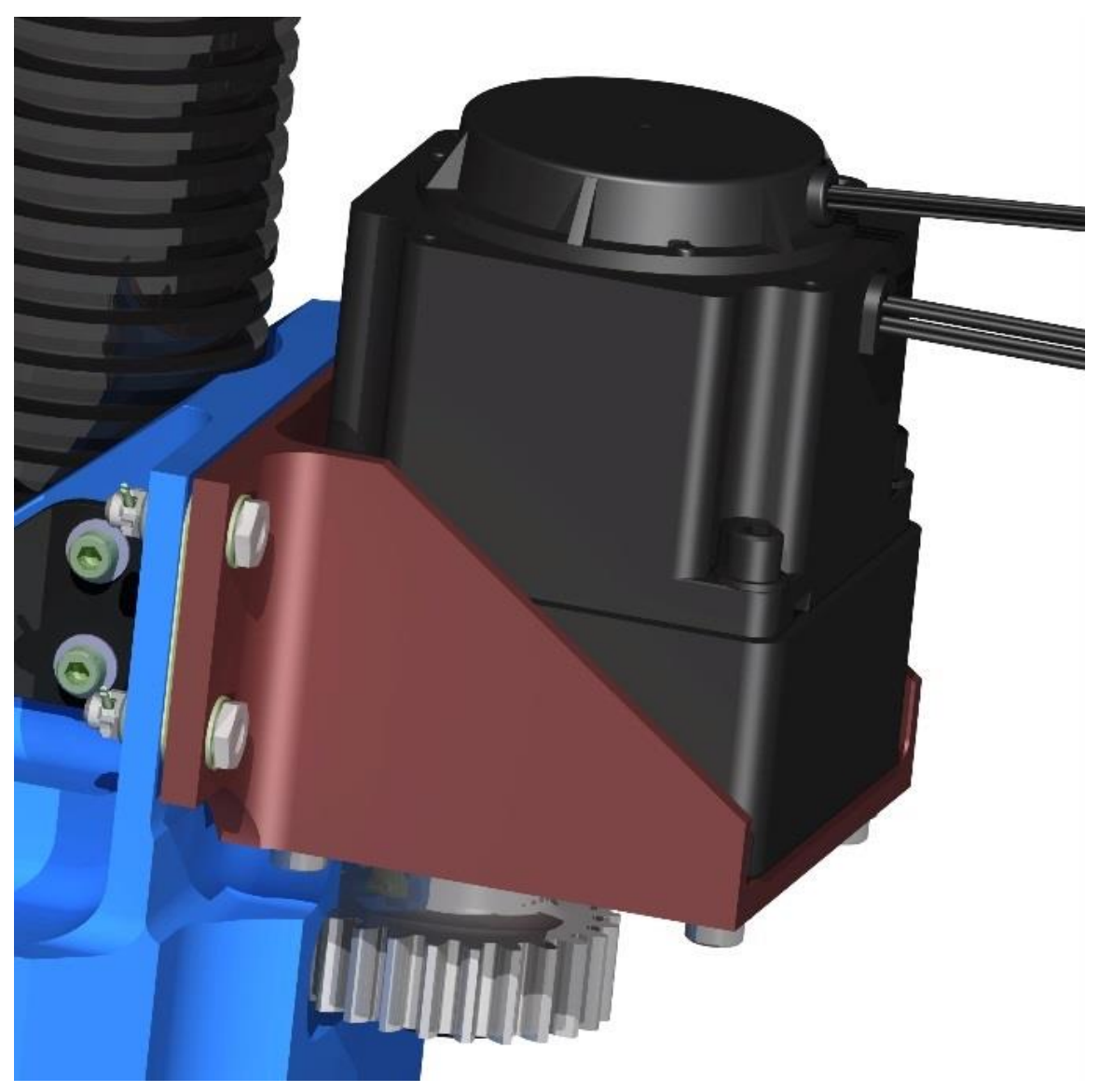

Figure 4.23: HDWS motor mount bracket, machine from solid variant (maroon colored) [17]

The optimized bracket was subsequently manufactured using an additive metal manufacturing (AMM) process. The process and the finished product are shown in Section 4.5.3. Readers interested in further details regarding the topology optimization methodology (design for addictive manufacturing) used in the present work may refer to reference [17]. 


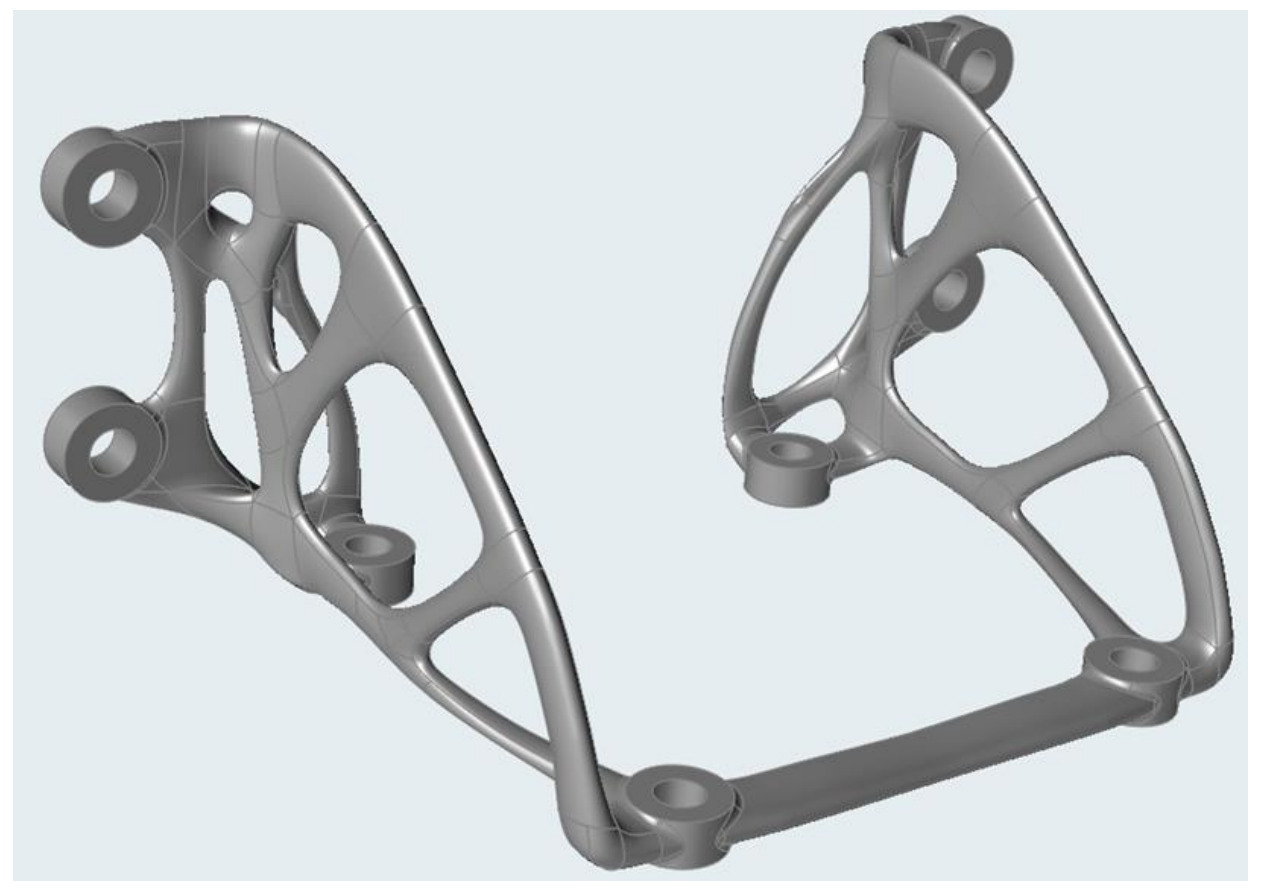

Figure 4.24: Final bracket design, produced from an FEA based optization [17]

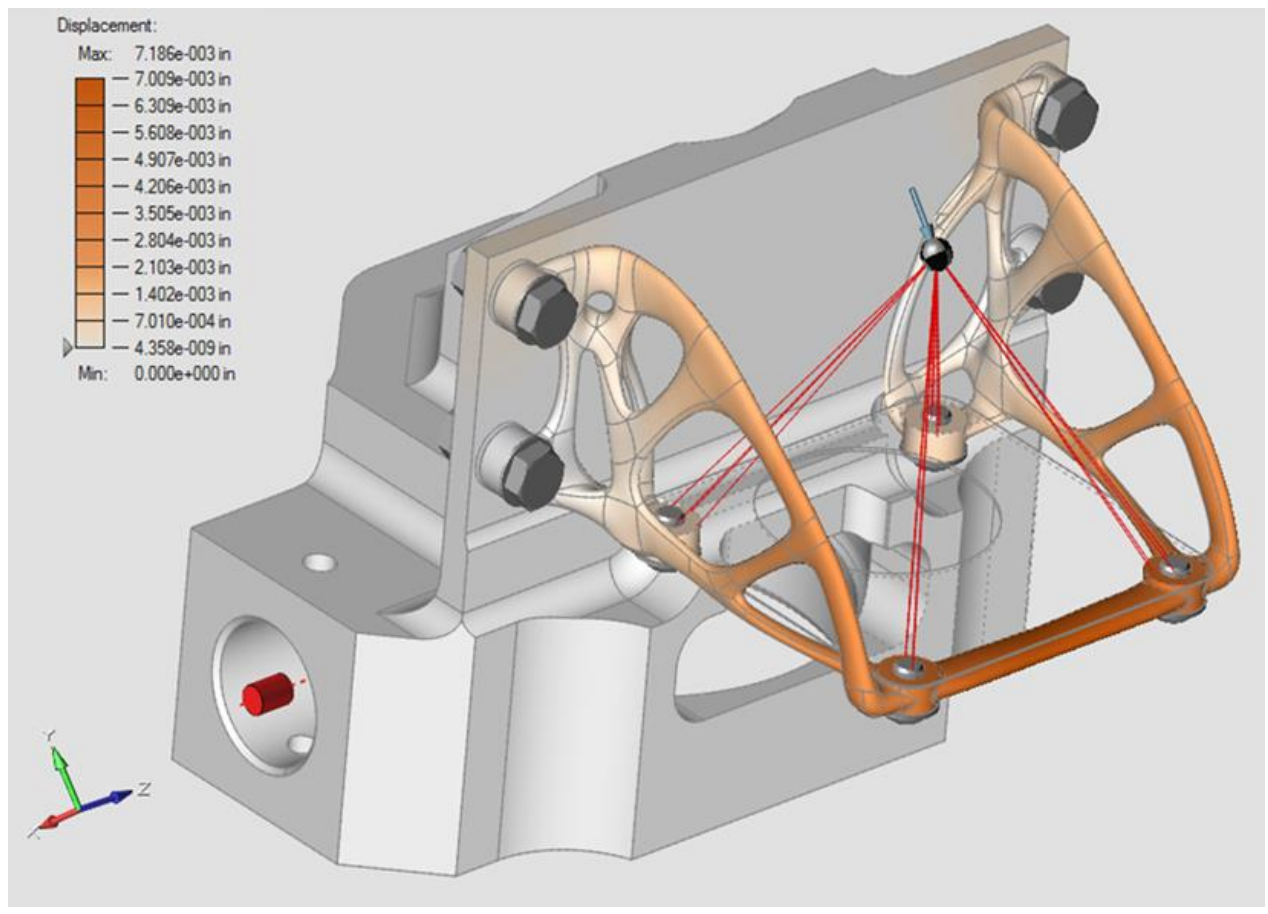

Figure 4.25: Example of a bracket displacement analysis, gravity load applied [17] 


\subsection{Part and Assembly Engineering Drawings}

The HDWS detail design process followed a standard industry design approach were concept level sketches, typically hand-drawn, are first drafted for visualization purposes and then transferred to computer aided design (CAD) software. CATIA ${ }^{\circledR}$ V5, widely used in the aerospace and automotive industry, was used for the design of the HDWS. As per modern design methodology, 3D models of the HDWS parts, sub-assemblies and final assembly were produced using CATIA ${ }^{\circledR}$ V5. After the 3D models were finished the models were drawn using CATIA ${ }^{\circledR}$ V5's Drafting Workbench. In order to follow the latest industry standards and best practices as they pertain to draftsmanship, the ANSI/ASME Y14.5M-1994 Geometric Dimensioning and Tolerancing (GD\&T) [51] standard was followed where applicable.

Ensuring an industry standard level of quality for the finished drawings, the author utilized an industry based template, developed with assistance from Safran Landing Systems Canada Inc. [48], for the HDWS's drawings. An example of the drawing template is shown in Figure 4.26.

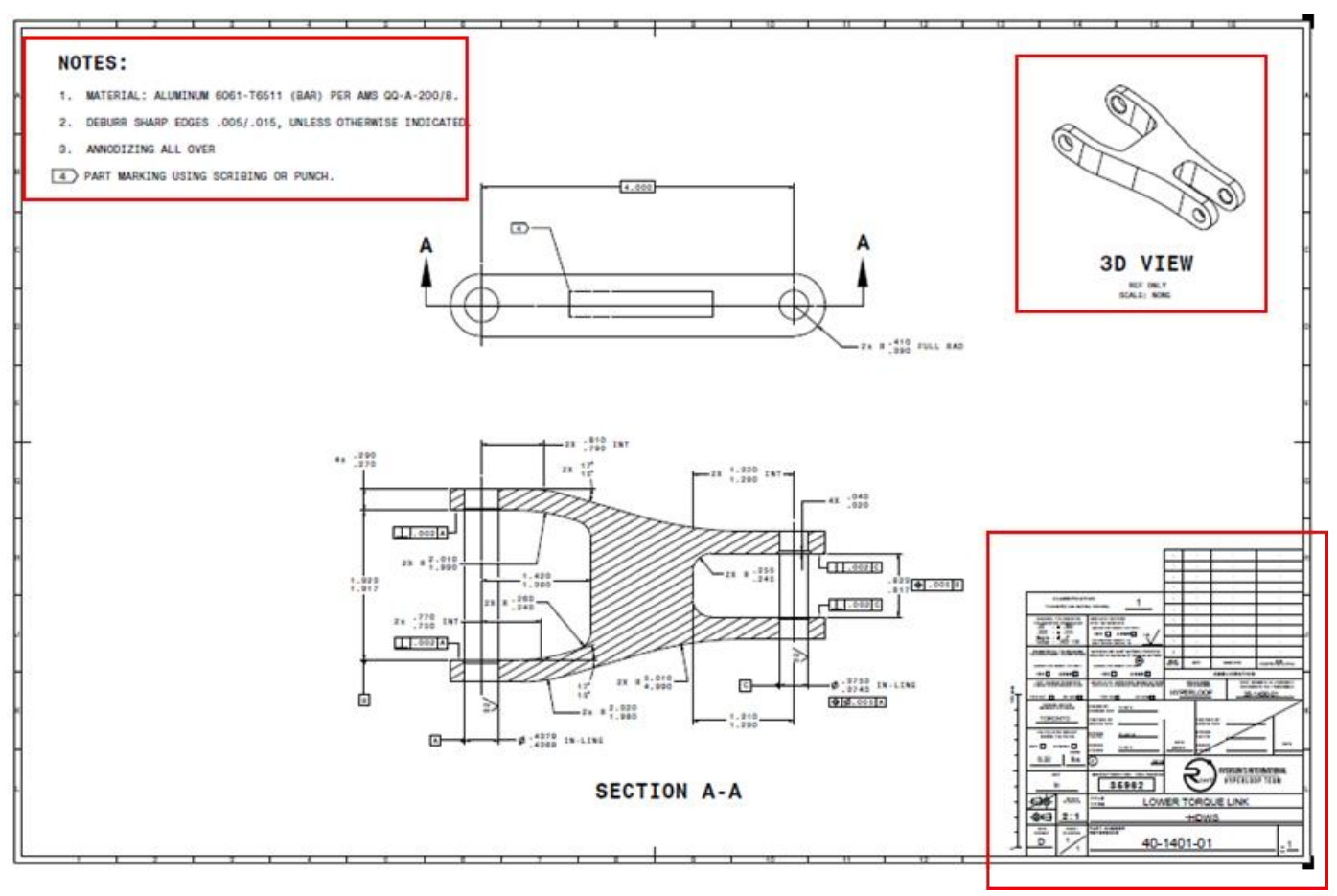

Figure 4.26: Sample of a typical HDWS engineering drawing, key elements encircled 
The template features the basic elements required to adequately communicate the design to a reviewer and most importantly the manufacturer. In particular, the HDWS drawing template, shown in Figure 4.26, incorporates: part specific design notes (typically included in the top left corner); a drawing title block (bottom right); 3D view(s) to provide an overview of the finished component or assembly and the body of the drawing containing views only as is required to clearly convey the design features. An example of the drawing title block and typical part notes can be seen in Figure 4.27 and Figure 4.28, respectively.

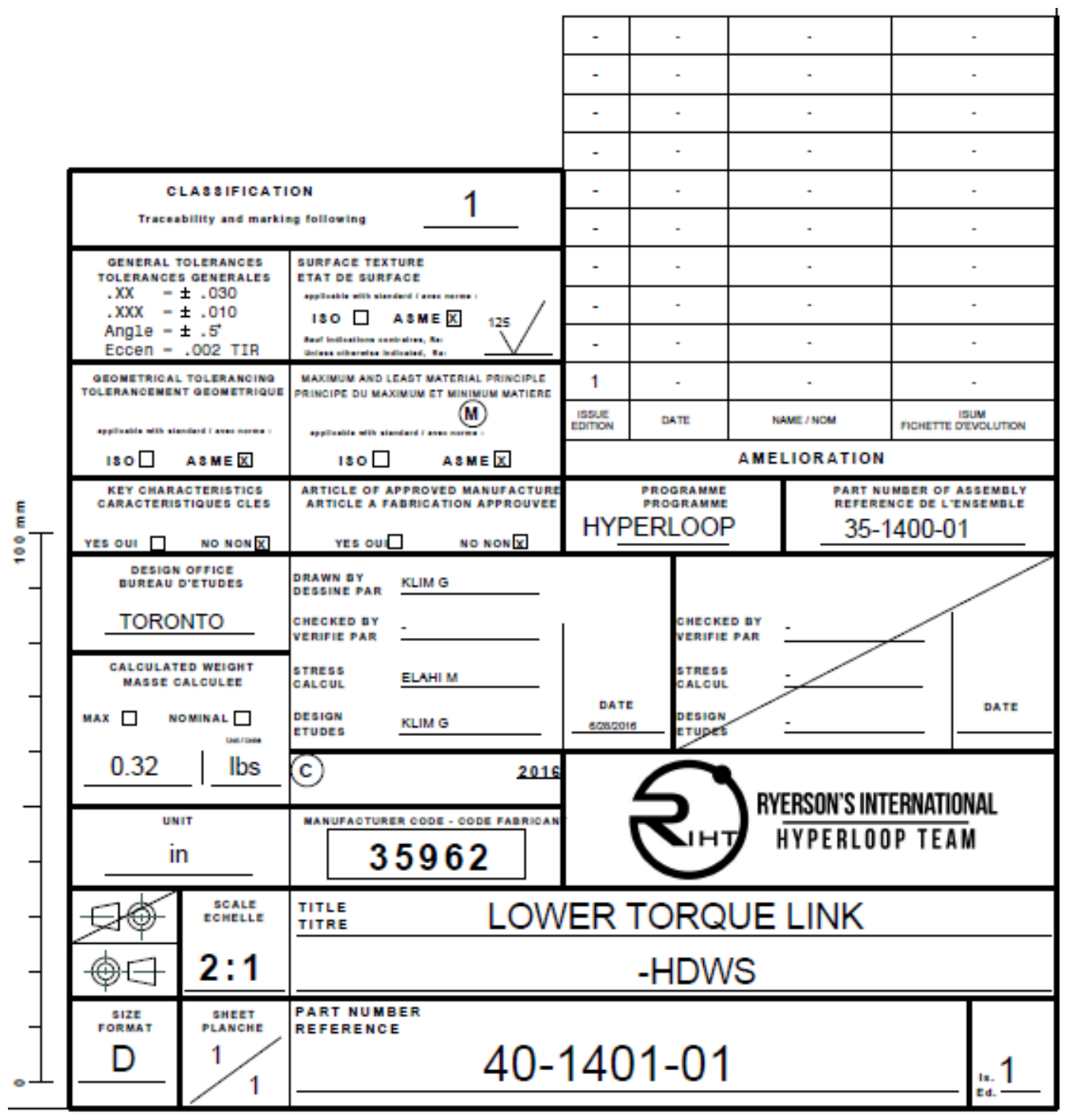

Figure 4.27: Sample of title block layout used for the HDWS 


\section{NOTES:}

1. MATERIAL: ALUMINUM 6061-T6511 (BAR) PER AMS QQ-A-200/8.

2. DEBURR SHARP EDGES .005/.015, UNLESS OTHERWISE INDICATED.

3. ANNODIZING ALL OVER

4 PART MARKING USING SCRIBING OR PUNCH.

Figure 4.28: Typical design notes used for the HDWS aluminum parts

After being submitted through a formal drawing check process conducted by project advisors, the drawings produced by the author for the HDWS's detail parts were transmitted to the manufacturing company contracted for the project, Durham Precision Machine CNC Inc. For this project, the 2D drawings were considered the master plan for manufacturing and the 3D models, if supplied to the manufacturer, were for reference use only. This practice is still very common in the industry today to ensure that the notes on the drawing, material choice, surface finishes and tolerances are closely followed. However, the author is aware of a new model-based definition (MBD) design concept [52], where no 2D drawing is produced and all the typical drawing notes, tolerancing and feature identifiers are defined in the 3D model.

The assembly drawings produced by the author were used for the assembly of the HDWS, the installation of bushings and the pressure seals. All drawings used in the manufacturing and assembly of the HDWS for the components discussed throughout the entirety of Chapter 4 are included in the Appendix A.

\subsection{Manufacturing and Treatment Processes}

The following presents a summary of the different manufacturing processes used to create the HDWS product. It is important to note that each component of the HDWS was designed with the end manufacturing process in mind, e.g. cutting tool radii, tool paths, and number of operation setups. 


\subsubsection{Subtractive Manufacturing}

According to Kalpakijian and Schmid [53], subtractive manufacturing covers a wide variety of manufacturing process including: $\mathrm{CNC}$ milling, turning of parts on a lathe, drilling operations and grinding. Each of these manufacturing methods were used in creating the HDWS and several examples are described in Figure 4.29 and Figure 4.30.
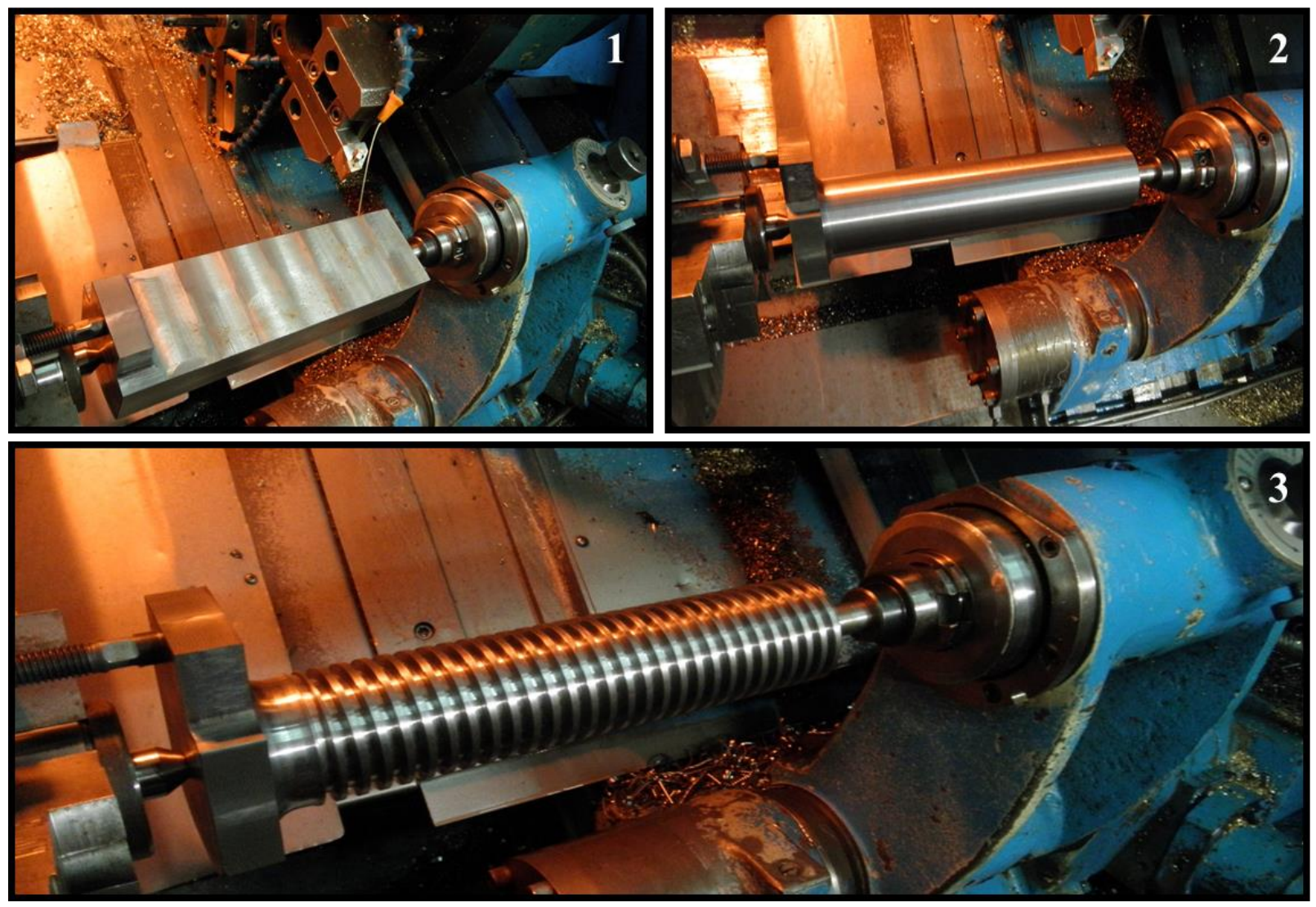

Figure 4.29: Images depicting from (1) through (3) the stages in the turning process performed for the manufacturing of the shock absorber outercylinder ACME 3G thread 

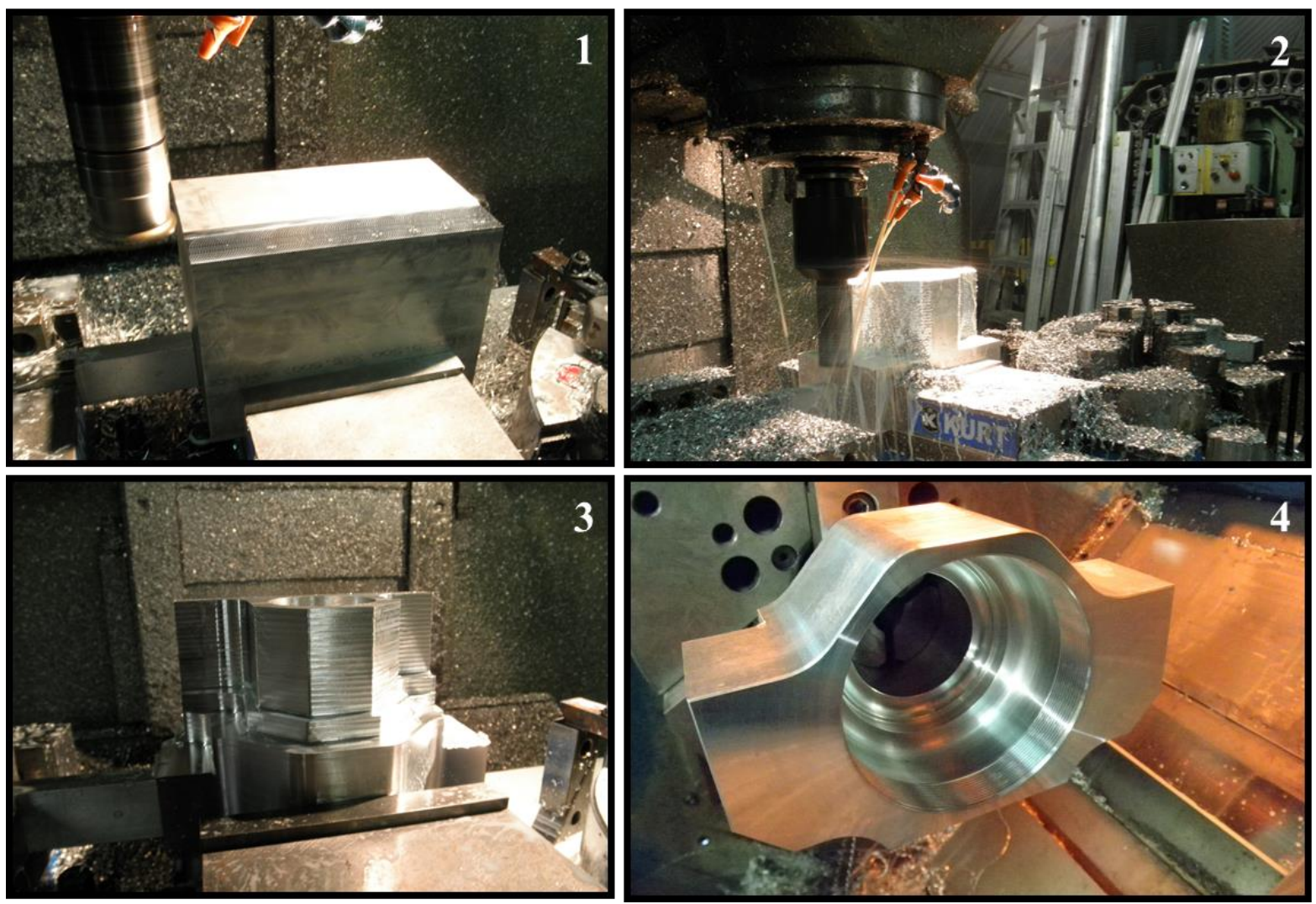

Figure 4.30: Images (1) through (3) depict the milling process starting from a solid metal block, and image (4) depicts the internal thread screw cut performed on a lathe

\subsubsection{Protective Finishes and Processes}

After the manufacturing process was completed, the components that required protections, mainly the aluminum and steel components, underwent protective treatment processes. In most cases these protections were for external environmental effects (natural corrosion) and in others to prevent galvanic corrosion caused by dissimilar metals [5]. The processes used for the present work are briefly described and illustrated where possible with examples.

\section{Aluminum Parts:}

Although aluminum does not rust like steel, aluminum does react naturally with oxygen and overtime forms an external layer of aluminum oxide. This external layer of aluminum oxide represents a protective surface with much higher corrosion and abrasion resistance than freshly machined aluminum. The electrochemical process of anodizing pre-emptively oxidizes the surface of the aluminium part providing a finished part with a more durable and corrosion-resistant finish. 
During the anodizing process the parts can be coloured (typically for aesthetic purposes) using organic dyes [54]. Figure 4.31 below shows the result of anodizing (blue) one of the trailing arm components.

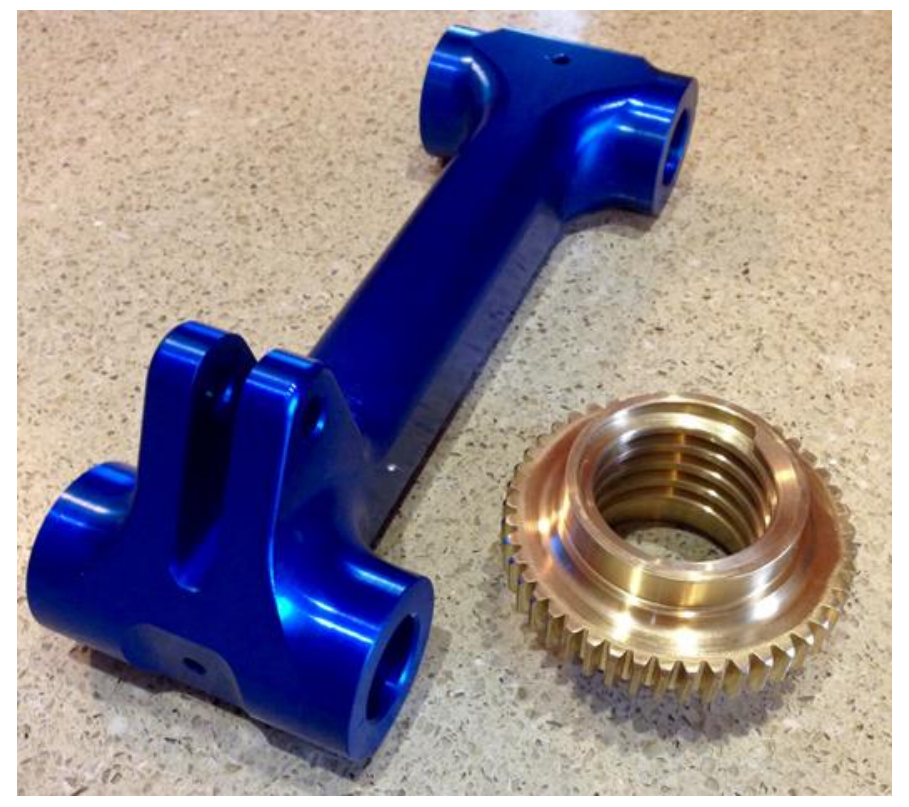

Figure 4.31: Trailing arm pictured after undergoing blue anodizing

\section{Carbon Steel:}

For ease of manufacturing the external ACME thread and for overall cost savings, the prototype HDWS's shock absorber outer cylinder was machined from AISI 12L14 carbon steel. Being noncorrosion resistant by nature, the steel required post treatment to protect against corrosion (rusting). Since the part was required to maintain its tight operating tolerances internally for the sealing surfaces and externally for the ACME threaded engagement, black oxide conversion coating was selected for its minimal build up (thickness) and superior abrasion resistance when compared to paint. The abrasion resistance was particularly important to consider along the ACME threaded surface, where the black oxide was expected to deteriorate overtime [55]. Figure 4.32 shows the shock strut outer cylinder assembly after going through the conversion coating process. 


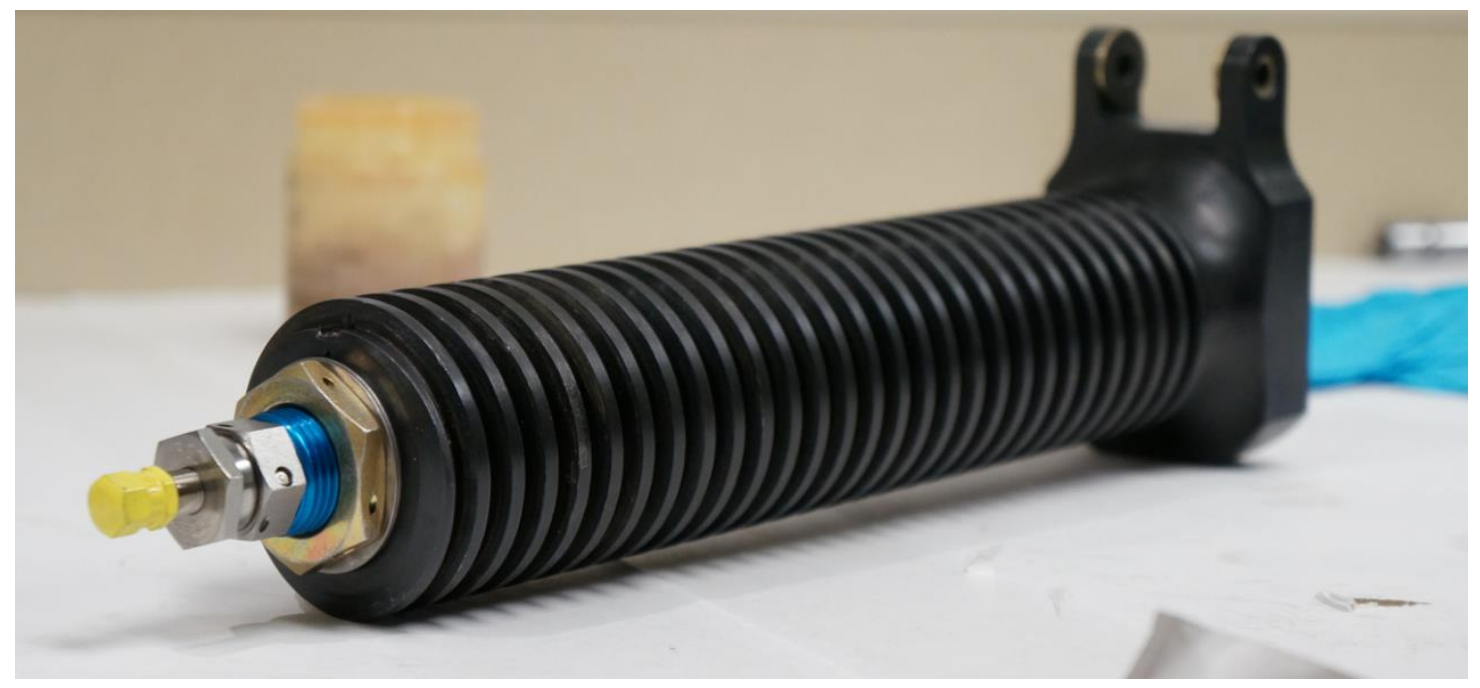

Figure 4.32: A shock strut outer cylinder shown with black oxide surface treatment

\section{Corrosion Resistant Steel (CRES):}

The HDWS prototype used two different types of stainless steel-303L for the piston and pins, and 17-4PH CRES for the KAron ${ }^{\circledR}$ [47] lined bushings. A common form of post-fabrication protection for stainless steels is passivation. As explained by Debold and Martin [56], it is a process that enhances the inherent corrosion resistant properties of stainless steels, such as 303L. In practice, the need for passivation after manufacturing the stainless part comes from the micro-deposits of iron left behind from the grinding or cutting tools and is deposited on the surface. Although microscopic, Debold and Martin notes that this frequently causes a fine layer of rust over extended periods of time [56]. For these reasons, all the HDWS 303L components were passivated.

The 17-4PH bushings were treated with cadmium plating on their installation surfaces (the outer diameter) to protect the bushing and mounting part from galling (stainless on stainless) and galvanic effects [32].

\subsubsection{Additive Manufacturing}

From the resulting topology optimization discussed in Section 4.3.2, the motor mounting bracket was selected for AMM. Thanks to Burloak Technologies Inc. [57], seven motor brackets were 
printed from AlSi10Mg using state-of-the-art direct metal laser sintering (DMLS) processes [17]. The DMLS process uses a high-power laser to sinter the part, layer by layer, in an additive fashion [58]. After each layer is sintered, a fresh layer of metal powder is swept over the previously sintered layer, as illustrated in Figure 4.33. This process is repeated until the final part geometry has been achieved. The result, after post processing (shot peening and build support removal), is shown in Figure 4.33.
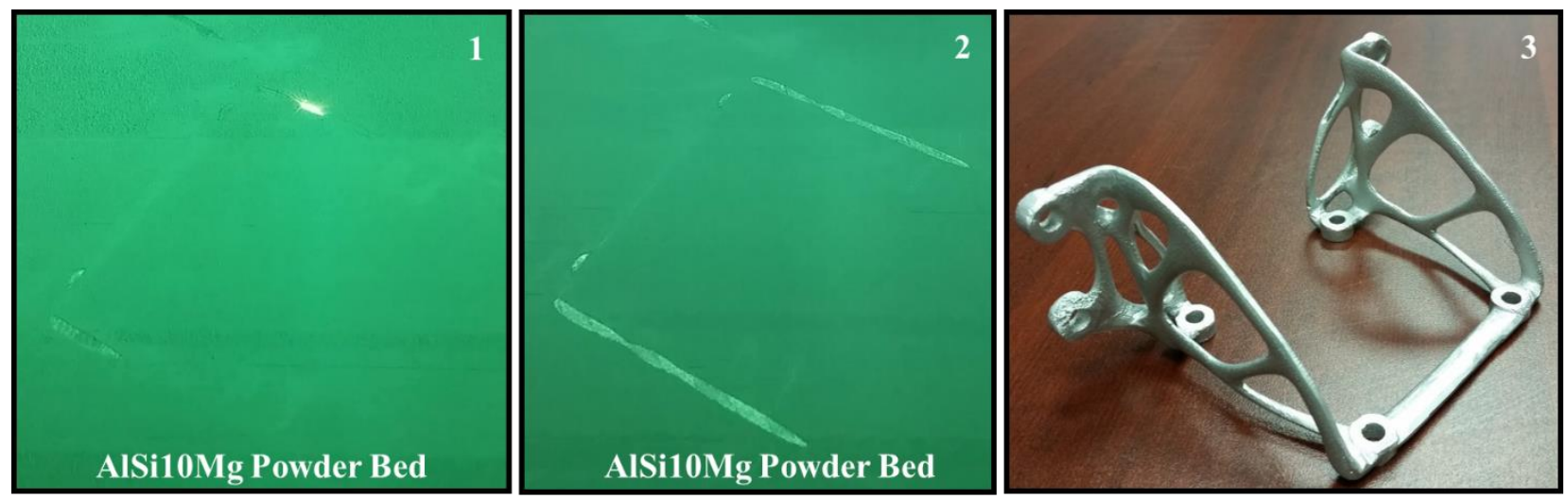

Figure 4.33: Images (1) through (2) depict the DMLS process used to create the final (3) AMM part [17]

\subsection{Chapter Summary}

The present chapter provided a detailed overview of the HDWS components and discussed some of their basic functions. After discussing the primary top-level (dressed) assemblies, the assemblies that make up the main structure (undressed assembly) were described. As it was mentioned earlier in Section 4.2.2, the detailed design and performance characteristics of the shock strut, fitted within the retraction assembly, is one the most complex components on the HDWS. For this reason, the detailed design of the shock strut and its operation within the retraction and extension framework, defined in Section 3.5, will be the primary subject of Chapter 5. 


\section{The Deployable and Retractable Shock Strut}

Following the definition of the kinematic layout, the HDWS concept was broken down into its key subsystems in Chapter 4, to familiarize the reader with the baseline concept design. As explained in Chapter 4, a significant portion of the HDWS design is comprised of the Shock Strut subassembly (P/N 30-1200-00) and the Retraction sub-assembly (P/N 30-1300-00). Due to their complex nature, their specific detail design and functions, separately and as a combined unit, are discussed in this chapter.

\subsection{System Configuration}

The Deployable and Retractable Shock Strut [9] configuration is itself the most important component of the HDWS and was designed to address the unique kinematic requirements of the Hyperloop, as defined within Chapter 3. Utilizing the present design, it is now possible to achieve shock absorption, retraction and extension capabilities within one combined unit (Figure 5.1), also proven through testing.

By design, the system includes a retract housing (fixed to the pod and free to rotate), where at least one motor is attached, and a classic oleo-pneumatic shock absorber internal configuration is retained but has its exterior modified to define a power lead screw, specifically ACME based. The benefit here, as compared to Waide [8] and Sharples [25], is that the shock absorber can linearly translate through the trunnion pin mounted retract housing, using an ACME gear nut mounted between two bearings and free to rotate. This generates translational motion and allows the system to fold into a vertically compact stowed position.

As is designed here, the system can be optimally configured (kinematically) when utilized with a classic trailing arm configuration, requiring only two total pod interface points (at the retract housing and trailing arm) and an electrical connection to the pod. The use of torque links here to react the rotational forces generated by friction in the ACME threads is particularly robust when compared to the proposal discussed in Chapter 2 by Waide [8]. As an added benefit, using externally mounted sensors and those typically pre-installed within a BLDC motor (hall sensor), the position of the system can be monitored and managed in real time. 

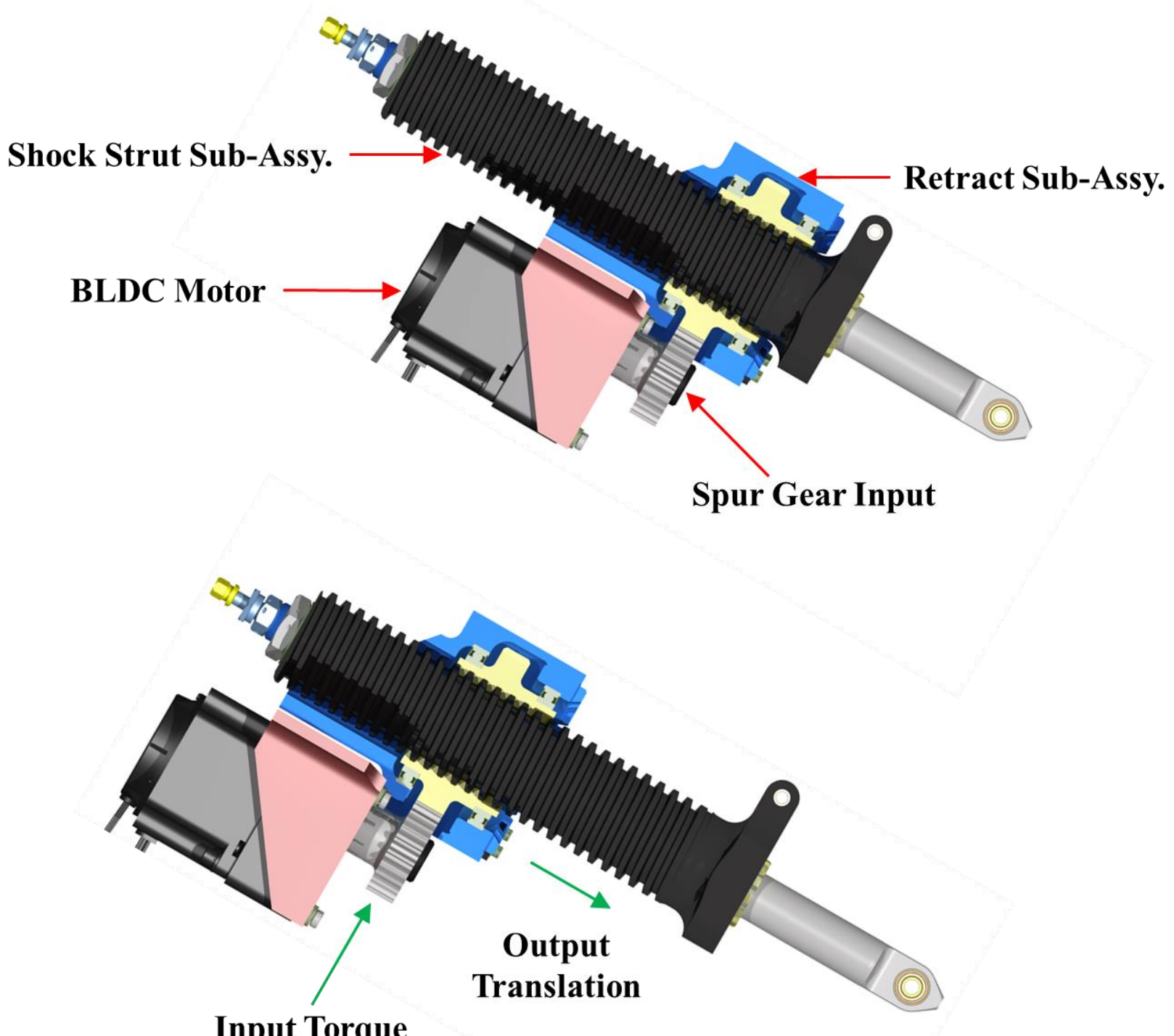

Figure 5.1: Deployable and retractabe shock strut system configuration, partial cross-section

This electrically operated arrangement came as an alternate solution to a standard hydraulic fluid system, typically used in the extension of landing gear, as discussed in Chapter 2. The current electric based design requires only a power supply (pre-existing on a pod) and avoids the use of a hydraulic system, which would require pumps, reservoirs, and hydraulic lines to be located throughout the pod. 


\subsection{System Operation}

Using the deployable and retractable shock strut configuration discussed in Section 5.1, the kinematic architecture as defined in Chapter 3, Section 3.5.2, was achieved. The extension (deployment) of the system, as shown in Figure 5.2, was accomplished in-part using an electronics and control system developed by Shonibare [16] and Adhikari [40]. The same system was used for the retraction of the system, by reversing the torque on the motor shaft, and performing height maintenance activities. By utilizing two linear potentiometers and four pod mounted lasers (one in each corner), the deployable and retractable shock strut was demonstrated to provide active feedback response, when the pod mass was disturbed by external sources (i.e. the change of the pod CG, for testing purposes, using a moving mass).

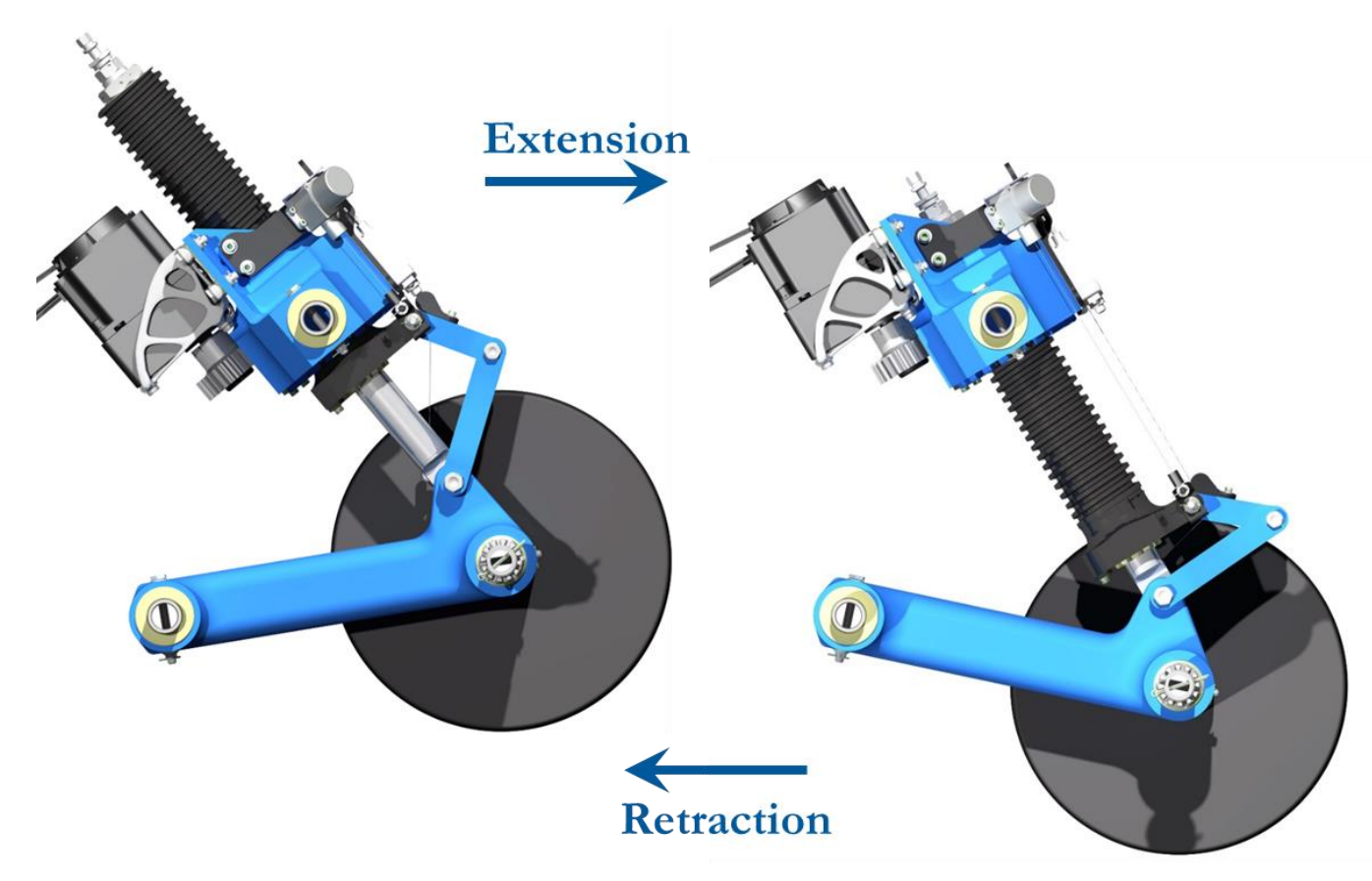

Figure 5.2: HDWS extension and retraction process, some parts hidden for clarity

Now that the reader is familiar with the overall HDWS concept and has been introduced to the deployable and retractable shock strut concept, the detail design considerations of the primary elements will be discussed. 


\subsection{HDWS Shock Absorber Design}

This section presents the preliminary and detail design of the HDWS shock absorber (or shock strut) component. The shock strut provides energy dissipation on extension (at touchdown) and ensures no loads are directly transferred to the Hyperloop pod vehicle frame. An oleo-pneumatic shock strut architecture was selected, which is the typical choice for aircraft landing gear due to their light-weight design and superior energy dissipation characteristics [5]. The preliminary design of the shock strut, particularly the design of an oleo-pneumatic shock strut, is covered by the works of Currey [5] and Conway [6] and was used here for design reference. Industry specifications and standards were used for the detail design process, the most applicable of which are tabulated here in Table 5.1 for convenience.

Table 5.1: Shock strut design standards and specifications referenced

\begin{tabular}{|l|l|}
\hline Standard/Specification & Document Description \\
\hline ASME/ANSI B1.5-1997 & General Purpose Acme Thread Form \\
\hline SAE AIR1362B & Aerospace Hydraulic Fluid Physical Properties \\
\hline SAE AS28889A & Valve, Air, High Pressure Charging, 5000 PSI \\
\hline SAE AS8934/2 & Bearing, Sleeve, Flanged, Self-lubricating \\
\hline SAE AS8879D & Screw Threads - UNJ Profile, Inch \\
\hline SAE AS4716B & Gland Design, O-ring and Other Elastomeric Seals \\
\hline SAE AS5857A & Gland Design, O-ring and Other Elastomeric Seals, Static Applications \\
\hline MIL-L-8552C & Landing Gear, Aircraft Shock Absorber (Air-Oil Type) \\
\hline MIL-T-6053C & Tests, Impact, Shock Absorber Landing Gear, Aircraft \\
\hline MIL-PRF-5606H & Hydraulic Fluid, Petroleum Base (Red Oil) \\
\hline
\end{tabular}

\subsubsection{Oleo-Pneumatic Shock Absorber Introduction}

Oleo-pneumatic shock absorbers consist of oil, typically petroleum based as specified by MILPRF-5606H, and a gas, typically dry nitrogen. Although it is possible to use air as the gas, modern designs use dry nitrogen gas due to its inert properties, therefore inhibiting corrosion [5]. An example of a single acting shock absorber, typically comprised of a piston, shock absorber cylinder and orifice, is shown in Figure 5.3. In this design, when the shock strut is compressed, oil is forced through a controlled area (typically a dampening orifice) and into the pressurized gas spring to dissipate energy in a controlled manor. In most designs, the shock absorber's rate of motion under 
dynamic loading is limited by the damping action induced by the oil flowing through a controlled area [24]. As shown in Figure 5.3, there are many ways in which a designer can configure the internals of a shock strut to achieve the desired performance characteristics, with no one way being the best solution in all conditions.

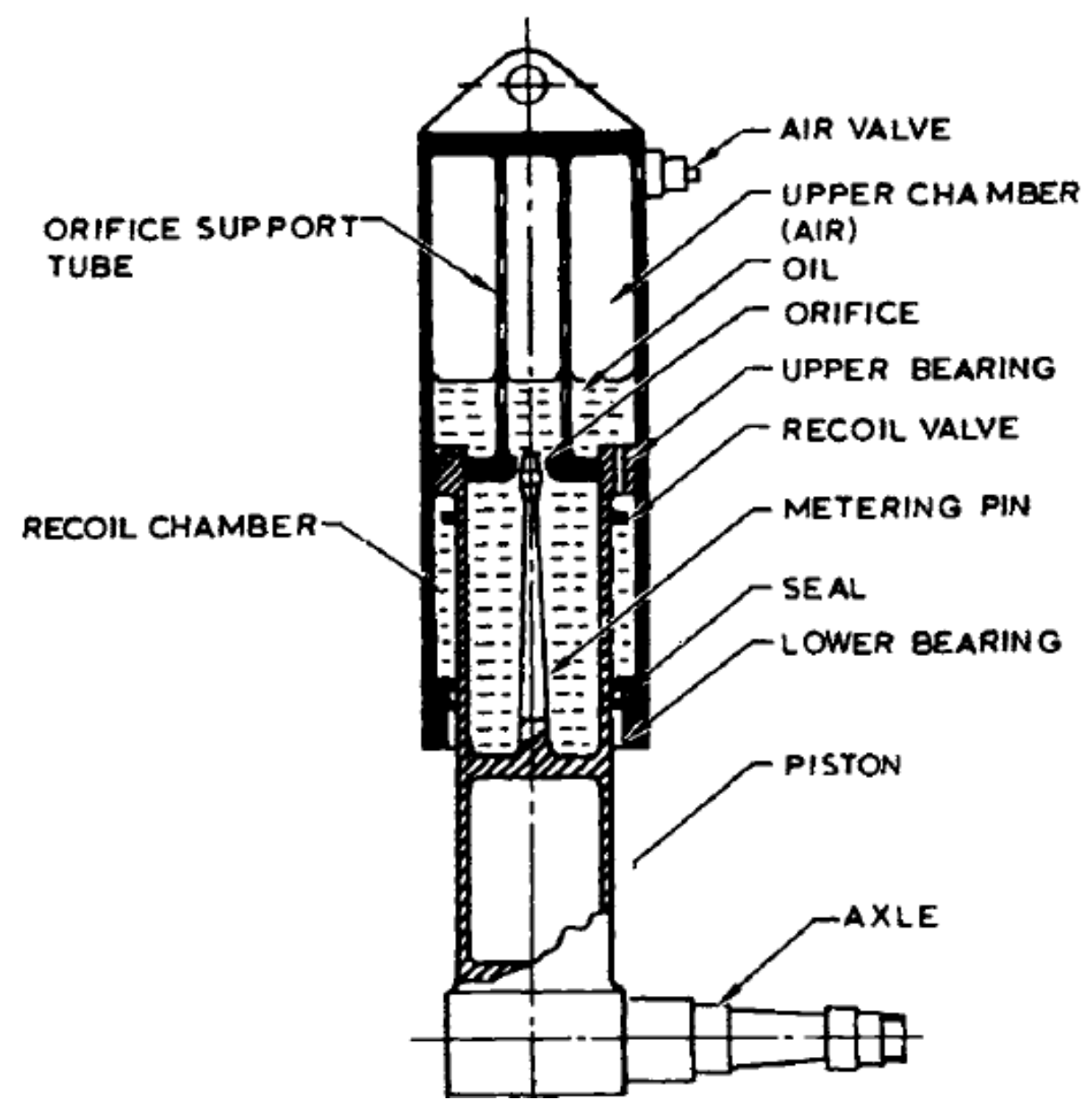

Figure 5.3: An example of an oleo-pneumatic shock strut with oil and gas free to mix [5]

The first stage in the shock absorber design process is typically to understand the energy dissipation requirements at landing. This is ordinarily the scenario in which an aircraft shock strut sees its worst-case loading and requirement for energy dissipation. This provides a rough estimate for the required landing stroke (distance through which the kinetic energy is dissipated). As is demonstrated by Currey [5], this preliminary exercise usually considers, at minimum, a rate of descent (sink speed) of $6 \mathrm{ft} / \mathrm{s}(1.8 \mathrm{~m} / \mathrm{s})$ for aircraft. In the case of the HDWS, which is deployed in a controlled manor onto the sub-track surface, the equivalent sink speed provided by the 
deployment actuation unit is approximately $0.11 \mathrm{ft} / \mathrm{s}(0.035 \mathrm{~m} / \mathrm{s})$. For this reason, it was expected that this method would be unsuccessful for the HDWS design. Hence, other preliminary design methods and assumptions outlined by Currey were used in its place [5].

In this case, a mix of gas spring properties, preliminary design guidelines and geometric constraints imposed by the prescribed operational space were applied. For the design of a shock absorber gas spring for a wheeled vehicle, the effects of tire compression under load should also theoretically be considered [5], however, the polyurethane wheel discussed in Chapter 4 was assumed to be sufficiently rigid and its compression was subsequently ignored for this work.

\subsubsection{Shock Absorber Gas Spring Preliminary Design}

After a general layout of the shock strut arrangement was created for reference, the next step was to begin assigning values to variables defined in Table 5.5. The process for selecting the final numbers was highly iterative but began first, as recommended in Currey [5], by defining a pressure at static stroke. Turning to statics, the following equation is defined [46]:

$$
P_{S}=\frac{F_{S}}{A_{P}}
$$

where $P_{S}$ is defined as the pressure at static piston stroke, $F_{S}$ is the static axial loading on the shock strut and $A_{P}$ is the area of the piston. The equation for the area of the piston is circular and is defined by the piston rod outer diameter, $O D_{P R}$. This is used to determine the total volume change within the shock absorber throughout stroking operations.

At this point, there are three unknowns in equation (5.1). A key piece of information required from the pod manufacturer is an estimate of the entire system's final weight. Based on initial estimates of the $\mathrm{U}$ of $\mathrm{T}$ pod architecture, a pod mass was selected that enabled the determination of the force, $F_{S}$, at a static stroke. Using Figure 5.4 as an example, assume a pod exists with a known mass $m$ and CG, an optimal positioning of the HDWS's within the pod frame can be determined.

For the purposes of the present discussion an equal distribution of the pod mass, $750 \mathrm{lbm}(340 \mathrm{~kg})$ was assumed. Note that if detailed pod CG and inertia was known, the distribution of the HDWS's 
throughout the pod could be determined for best performance and stability during all projected loading scenarios (e.g. dynamic braking, accelerating). This process would normally be done in practice at the early stages of HDWS subsystem design and pod frame design.

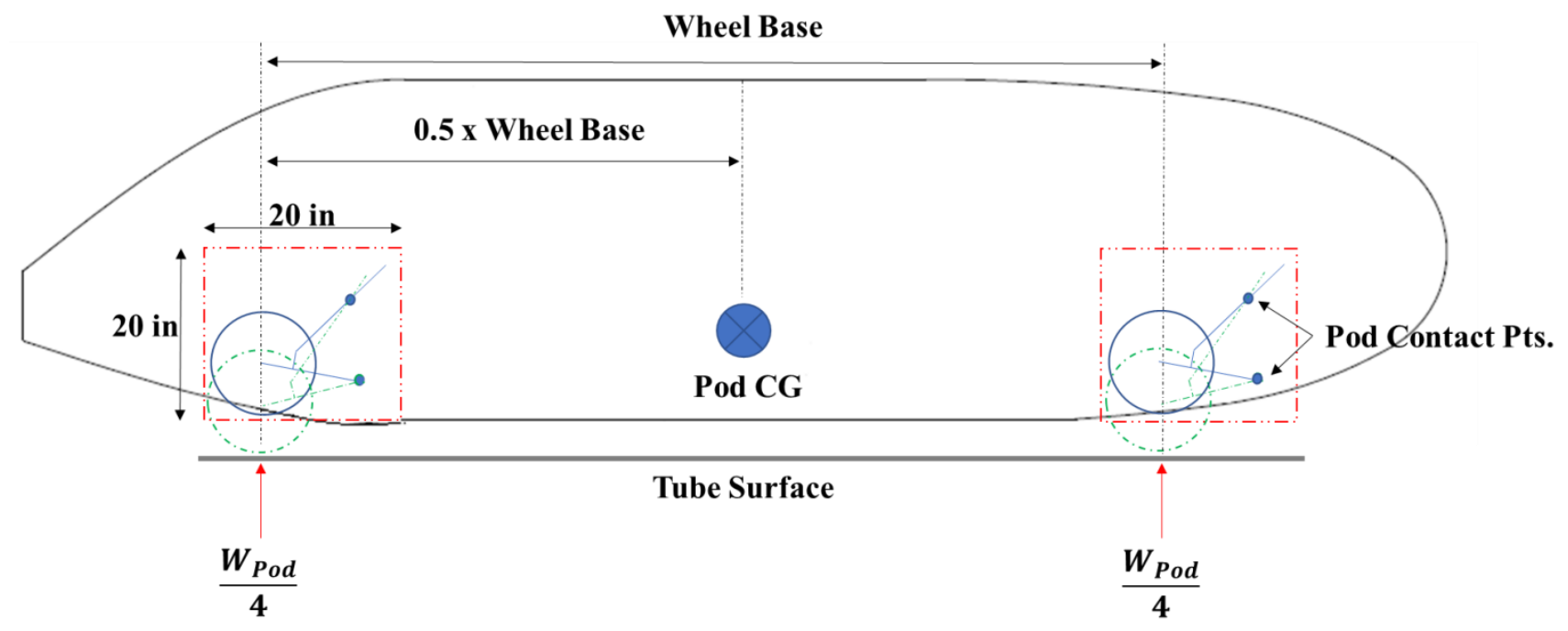

Figure 5.4: Example of a hypothetical pod layout, loads applied to the HDWS in a static position

As shown in Figure 5.4, the HDWS reaches a static stroke when the vertical load transferred through the trailing arm axle is equivalent to the weight of the pod divided equally among the systems. Since the system architecture is of the trailing arm type, the load through the shock strut is not equivalent to the load at the wheel axle. In Figure 5.5 the reference key points for the HDWS kinematics are shown in its Cartesian coordinates as defined in Chapter 3. These key points - (1 through 5) - are used to mathematically determine the force in shock strut member (4) - (5), defined as $F_{45}$.

As shown in Figure 5.5, the loading in $F_{45}$ was calculated by finding the sum of the moments about point (1). To do so, the perpendicular distance (shortest distance) between member (4) - (5) and pivot point (1) was determined. By the same token, the horizontal distance (shortest distance) between point (1) and line of action of the force at (2), where a portion of the pod weight is reacted, was also determined. 


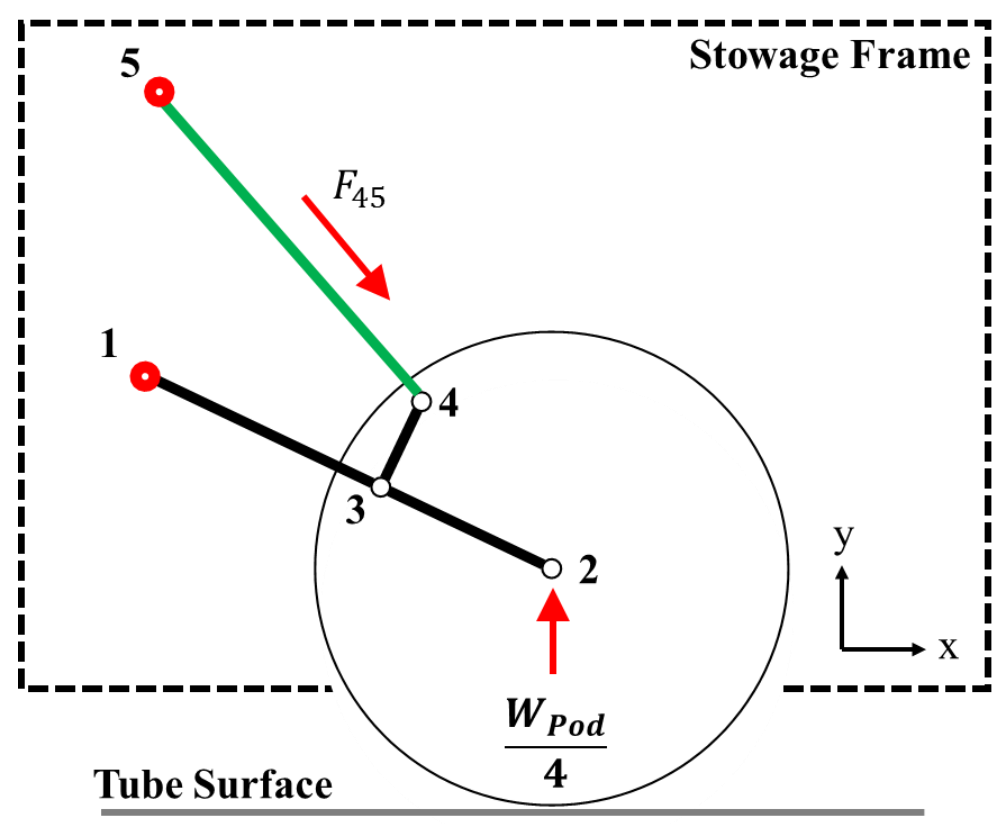

Figure 5.5: The HDWS preliminary configuration, stick diagram

It was found that the moment arm distances for each force about point (1) were best determined from the kinematic models within CATIA ${ }^{\circledR}$ V5. This was advantageous in the early stages of design when the positions of the key points defined in Figure 5.5 were subject to change. The mathematical definition is shown here for reference.

Let $a$ be the horizontal distance along the x-axis between points (1) and (2) such that:

$$
a=\left|x_{2}-x_{1}\right|
$$

where $x_{1}$ and $x_{2}$ are the $x$-coordinates relative to a specific reference point, namely point (1).

Let $b$ be the perpendicular distance from the force vector $F_{45}$ defined as:

$$
b=\frac{\left|\left[\left(x_{4}-x_{5}\right)\left(y_{4}-y_{1}\right)\right]-\left[\left(x_{4}-x_{1}\right)\left(y_{5}-y_{4}\right)\right]\right|}{\sqrt{\left(x_{4}-x_{5}\right)^{2}+\left(y_{4}-y_{5}\right)^{2}}}
$$

Finally, the force in the shock strut $F_{45}$ can be determined. In this case, the value for $F_{S}$ is desired to assist in the initial shock strut sizing. Thus, the values utilized for the $x$ and $y$ coordinates of the points (2) and (4) shall be those approximating the position of static stroke. As a first approximation, Currey [5] provides a data table consisting of historic static stroke positions as a 
percentage of maximum stroke while Conway [6] suggested that a maximally loaded vehicle should have a compressed stroke position of not more than two-thirds total stroke. Acknowledging the preceding, the HDWS's unique kinematic motion taking place between the retracted state (shock strut fully extended, stroke equal to zero) and the static stroke state has no effect on the values of $a$ and $b$ defined in equations (5.2) and (5.3). It can now be stated that the values of $a$ and $b$ change with the extension and retraction of the HDWS, rotating about point (1), up to WOW (touchdown). After WOW is achieved, the system compresses the oleo-pneumatic shock strut piston until reaching the static load of the pod. During the compression, the motion of the shock strut is linear - that is, the perpendicular distance defined by $b$ remains the same. The assumption here holds true if the pod is levitating at a planned altitude throughout the extension operation.

Furthermore, when the HDWS performs a height adjustment of the pod relative to the ground, the values of $a$ and $b$ defined in equations (5.2) and (5.3) change as the pod is raised. This can be easily understood by imagining points (1) and (5), fixed to a pod raising in the vertical direction, while point (4) swings CW about point (5) and point (2) remains "fixed" at a radial distance about the tube sub-track. Bearing all the preceding information in mind, it can be appreciated that by modifying the component geometry it is possible to control the value for $F_{45}$ while maintaining the kinematic arrangement within the specified area. Thus, the equation governing the value for $F_{45}$, referencing Figure 5.5, is defined by:

$$
+C C W \sum M_{A}=0 ; \quad\left(\frac{W_{P o d}}{4}\right)(a)-F_{45} b=0
$$

Solving equation (5.4) for $F_{45}$ :

$$
F_{45}=\frac{\left(\frac{W_{P o d}}{4}\right)(a)}{b}
$$

As discussed earlier in this report, equation (5.5) is only valid for solving the force $F_{45}$ at the static stroke of the piston and at any new static stroke defined by a height adjustment thereafter.

Since the present calculations were based on a pod mass of $750 \mathrm{lbm}(340 \mathrm{~kg})$, a value for $F_{45}$ can be determined using the kinematic arrangements determined in Chapter 3. 
Referring to equation (5.1), two terms remain unknown - the area of the piston $A_{P}$ and the pressure at static stroke $P_{S}$. It was decided that the minimum permissible pressure required would be used to overcome the seal friction (sticksion) stated by Currey [5] to be $60 \mathrm{psi}(413.7 \mathrm{kPa})$. As noted by Currey, this initial pressure assures that the shock absorber can fully extend when unloaded. Thus, using 60 psi as the initial pressure, a spread sheet partially shown in Table 5.2 was created by the author based on the ideal gas law (isothermal) formulae presented by Currey [5]. This tool enabled the calculation of an initial gas spring utilizing the preliminary compression ratios defined by Currey and based on the kinematic geometry required to achieve the static force required to balance the pod weight, that is $F_{S}=F_{45}\left(W_{P o d}, a, b\right)$. The initial key results of the iteration, as displayed in the shock strut design excel sheet, are shown in Table 5.2 and Table 5.3 for reference.

Table 5.2: Shock strut design tool developed by the author using equations derived in Currey [5]

\begin{tabular}{|c|c|}
\hline \multirow{2}{*}{ LEGEND } & Input \\
\hline & Output \\
\hline Required Info/Design Parameter & Value \\
\hline Pod Max Weight (lb) & 750.000 \\
\hline No. of Struts & 4.000 \\
\hline Load per Strut, Static Case (lb) & 187.500 \\
\hline Length, Trailing Arm (in) & 10.000 \\
\hline Postion, SS/T-Arm (in) & 9.000 \\
\hline Radial offset of SS/T-Arm Lug (in) & 2.500 \\
\hline Static Trailing Arm Position (rad) & -0.305 \\
\hline Static SS Position (rad) & 2.069 \\
\hline Static Axial Loading (lb) & 222.249 \\
\hline Static to Extended Ratio & 4.200 \\
\hline Compressed to Static Ratio & 5.000 \\
\hline Total Stroke (in) & 3.000 \\
\hline Charge Pressure(psi) & 60.000 \\
\hline Total Gas Displacement (V1-V3) (in3) & 2.642 \\
\hline
\end{tabular}

\begin{tabular}{|c|c|c|c|}
\hline \multicolumn{4}{|c|}{ Gas Spring (Isothermal) } \\
\hline Stroke (in) & V (in3) & P (psi) & Load (lb) \\
\hline 0 & 2.775 & 60.000 & 52.849 \\
\hline 0.5 & 2.334 & 71.321 & 62.820 \\
\hline 1 & 1.894 & 87.907 & 77.429 \\
\hline 1.5 & 1.453 & 114.545 & 100.893 \\
\hline 2 & 1.013 & 164.348 & 144.759 \\
\hline 2.1 & 0.925 & 180.000 & 158.546 \\
\hline 2.2 & 0.837 & 198.947 & 175.235 \\
\hline 2.3 & 0.749 & 222.353 & 195.850 \\
\hline 2.4 & 0.661 & 252.000 & 221.964 \\
\hline 2.5 & 0.573 & 290.769 & 256.112 \\
\hline 2.6 & 0.484 & 343.636 & 302.678 \\
\hline 2.7 & 0.396 & 420.000 & 369.940 \\
\hline 2.8 & 0.308 & 540.000 & 475.637 \\
\hline 2.9 & 0.220 & 756.000 & 665.892 \\
\hline 3 & 0.132 & 1260.000 & 1109.819 \\
\hline
\end{tabular}

Table 5.3: Key shock strut stroke position outputs (ideal gas law, isothermal assumption)

\begin{tabular}{|c|c|c|c|c|c|c|c|c|c|}
\hline Extended Load (lb) & 52.9 & P1 (psi) & 60 & V1 $\left(\mathrm{in}^{3}\right)$ & 2.775 & Stroke (in) & 0.00 & $\%$ Stroke & 0.00 \\
\hline Static Load (lb) & 222.2 & P2 (psi) & 252 & V2 $\left(\mathrm{in}^{3}\right)$ & 0.661 & Stroke (in) & 2.40 & $\%$ Stroke & 80.00 \\
\hline Compressed Load (lb) & 1111.2 & P3 (psi) & 1260 & V3 $\left(\mathrm{in}^{3}\right)$ & 0.132 & Stroke $(\mathrm{in})$ & 3.00 & $\%$ Stroke & 100.00 \\
\hline
\end{tabular}


Using the theory presented by Currey [5] as a preliminary calculation, the initial gas spring calculation and its results shown in Table 5.2 and Table 5.3 were generated based on an isothermal simplification of the ideal gas law (thermodynamics). The equations used to generate the results shown in Table 5.2 and Table 5.3 are now defined with reference to Figure 5.6.

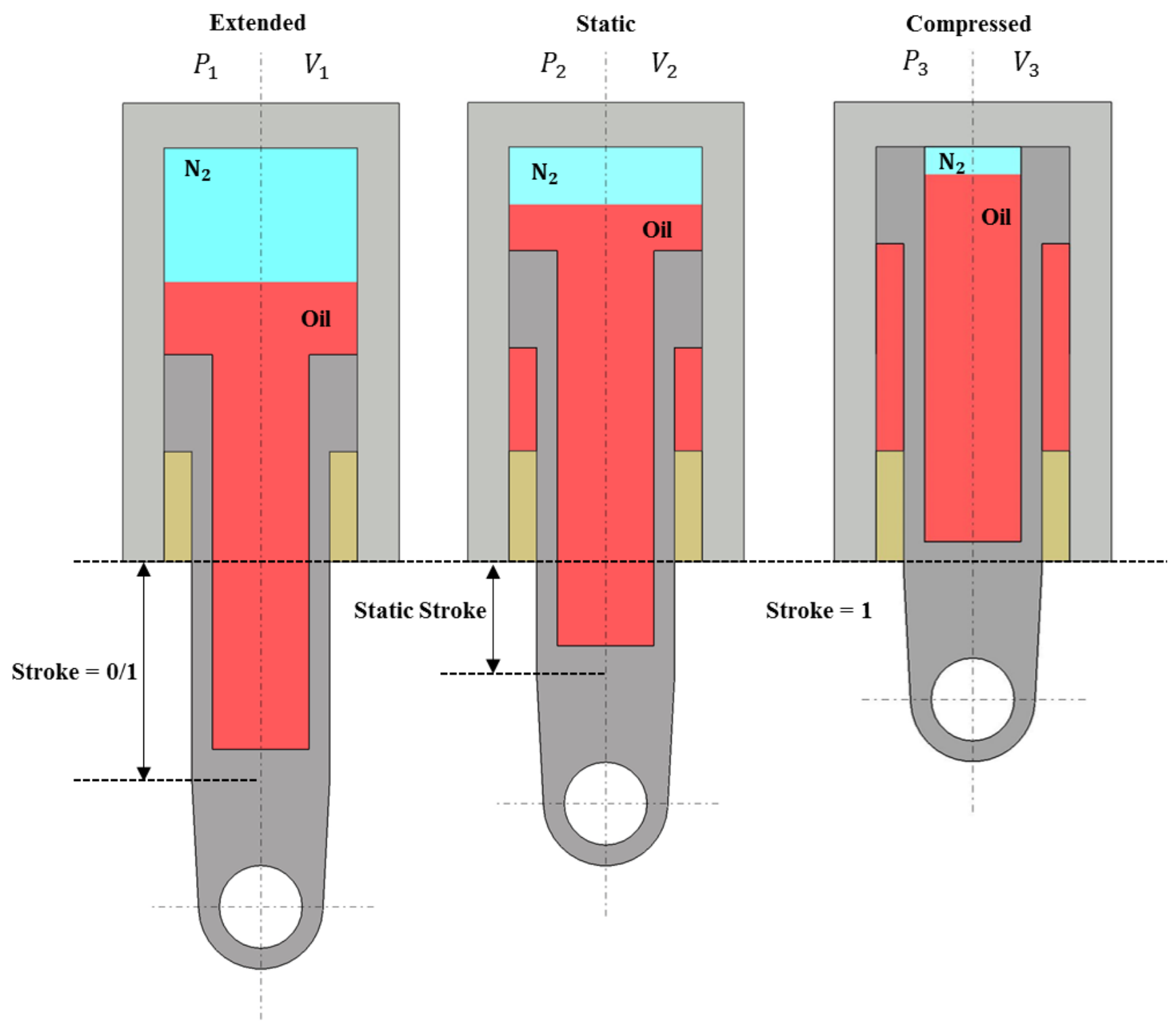

Figure 5.6: Illustration of oleo-pneumatic shock strut key operational states 
As an approximation of the static gas spring behaviour at the states illustrated in Figure 5.6, the ideal gas law is applied under isothermal assumptions, where the temperature $T$ is assumed constant such that $T_{1}=T_{2}=T_{3}$ resulting in the relationship:

$$
P_{1} V_{1}=P_{2} V_{2}=P_{3} V_{3}
$$

Referencing equation (5.6) and Figure 5.6, the following definitions apply:

Table 5.4: Shock strut gas spring design features and definitions per Currey [5]

\begin{tabular}{|c|c|}
\hline Symbol & Description \\
\hline$P_{1}$ & Gas pressure at full extension, 0\% stroke \\
\hline$P_{2}$ & Gas pressure at static stroke under static load \\
\hline$P_{3}$ & Gas pressure in the compressed position, 100\% stroke \\
\hline$V_{1}$ & Gas volume at full extension, 0\% stroke \\
\hline$V_{2}$ & Gas volume at static stroke under static load \\
\hline$V_{3}$ & Gas volume in the compressed position, $100 \%$ stroke \\
\hline
\end{tabular}

Since $P_{1}$ was selected to be 60 psi the next step was to determine $P_{2}$ and $P_{3}$ using the recommended pressure ratios provided by Currey [5]. These ratios were selected to help ensure floor height variation between static and compressed positions during dynamic operation is kept to a minimum. This was an important design factor to consider when designing the gas spring that ensured the pod's primary levitation systems or any part of the pod frame would not crash into the sub-track. For preliminary sizing purposes, the ratios utilized per Currey [5] were:

$$
\begin{gathered}
\text { Static to extended }=4 / 1 \\
\text { Compressed to static }=3 / 1
\end{gathered}
$$

Note: the above compression ratios should be used with caution as they do not account for the compressibility of the oil [5]. 
Based on the ratio defined by (5.7) the pressure $P_{2}$ is found by:

$$
\frac{4}{1}=\frac{P_{2}}{P_{1}} \rightarrow P_{2}=4 P_{1}
$$

Similarly, the pressure at the compressed position (maximum stroke) $P_{3}$ is estimated using (5.8) and found by:

$$
\frac{3}{1}=\frac{P_{3}}{P_{2}} \rightarrow P_{3}=3 P_{2}
$$

Now, using a known value of $F_{S}$ and by substituting the result of equation (5.9), the area of the piston is determined as follows:

$$
P_{2}=4 P_{1}=\frac{F_{S}}{A_{P}}
$$

Re-arranging equation (5.11) for the piston area $A_{P}$ gives:

$$
A_{P}=\frac{F_{S}}{4 P_{1}}
$$

This enabled a preliminary sizing for the shock strut piston outer diameter, $O D_{P R}$, which was solved by:

$$
O D_{P R}=\sqrt{\frac{4 A_{P}}{\pi}}
$$

Note: The significance of equation (5.13) will not be discussed here but will play a key role in determining the final gas spring properties of the shock strut and will thus be referred to at the end of this section prior to defining the internal geometries at a later stage.

Knowing the area $A_{P}$ by which the internal volume of the shock strut is compressed, the total change in volume from extended stroke to compressed stroke can be found by:

$$
\Delta V=V_{3}-V_{1}=A_{P} S_{\text {Max }}
$$


where $S_{\operatorname{Max}}$ is defined as the total permissible stroke of the shock strut. At this stage, it was necessary to define a maximum stroke which was selected based on the kinematic studies highlighted in Chapter 3. For the present work, $S_{\operatorname{Max}}=3$.

In general, solving equation (5.14) for $V_{3}$ and substituting the result into equation (5.6) gives:

$$
V_{1}=\frac{V_{3} P_{3}}{P_{1}}=\frac{P_{3} A_{P} S_{M a x}+V_{1} P_{3}}{P_{1}}
$$

After solving for $V_{1}$, the ideal gas law was used to find $V_{2}$ and $V_{3}$. Using these results a shock absorber load-stroke curve shown in Figure 5.7 was produced.

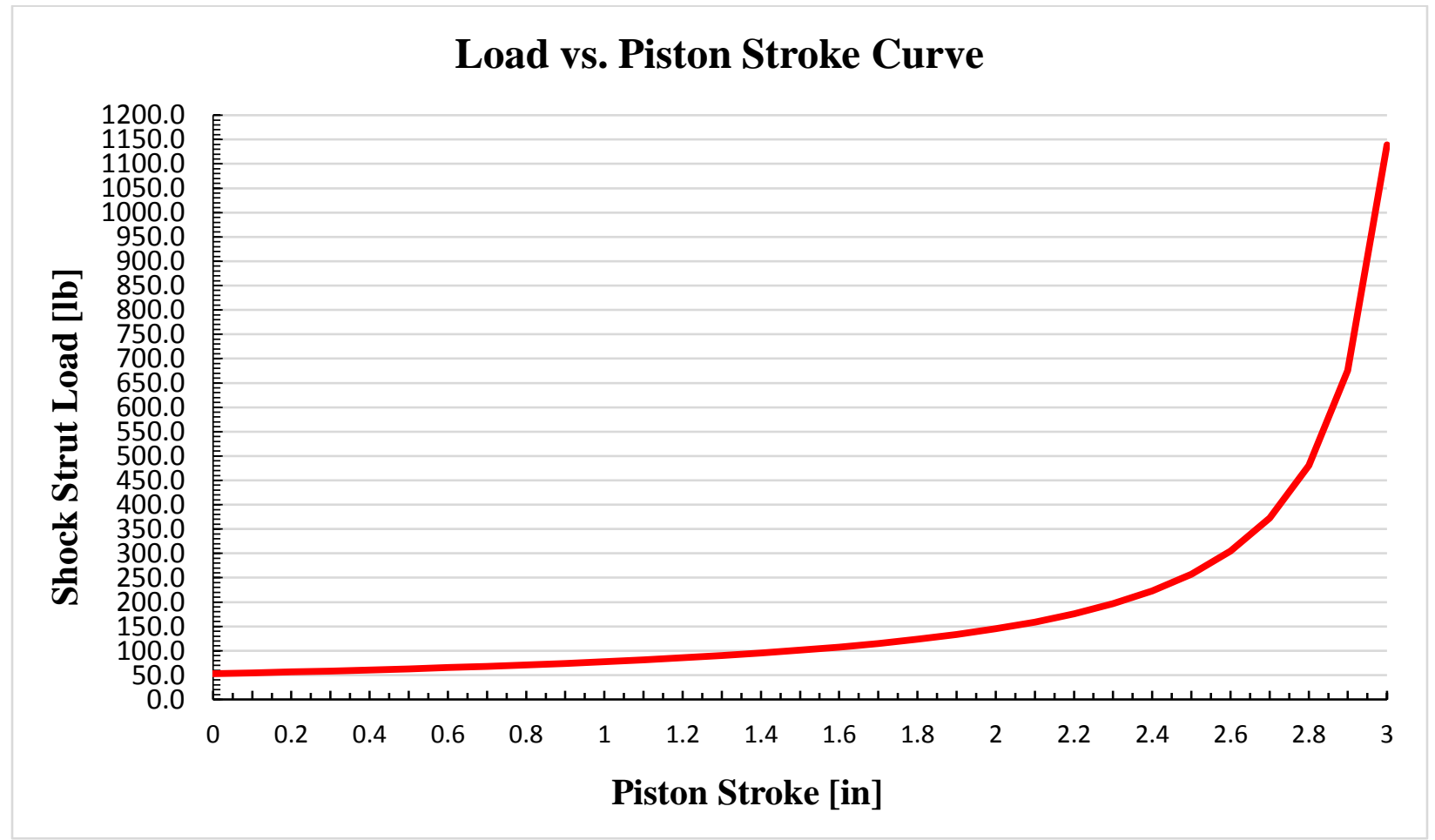

Figure 5.7: Graphic representation of the isothermal oleo-pneumatic gas spring curve produced using the results in Table 5.2

By examining Figure 5.7 it can be said that a shock strut whose static stroke is in the range of 2.2 to 3 inches will be best able to resist the effects of dynamic loading during pod travel. This is an 
important consideration for the HDWS shock strut design to ensure that any dynamic loading, which by design causes the shock strut to compress, does not allow rapid and large displacements that could damage the pods exterior elements close to the tube sub-track.

As the details of the shock strut internals were further defined geometrically the final internal gas and oil volumes were determined with the assistance of CATIA ${ }^{\circledR}$ V5. Before moving to the next section on the geometric sizing of the shock strut, it is important to recognize the following points, summarized in list form, to be addressed in the detailed gas spring design.

1) The preceding gas spring design was developed under isothermal assumptions. It is known that as substance compresses, particularly at high speeds and pressures, that the effect of temperature should not be ignored.

2) By the same token, the present process has not yet considered the compressibility of the fluid utilized, industry standard MIL-H-5606 red mineral oil. The bulk modulus of the fluid, as defined by AIR 1362B, was used to determine the final gas spring.

3) The present gas spring has only considered static loading, which, per Currey [5], is sufficient for normal, low-speed disturbances. The final gas spring will consider both dynamic and static loading using an isentropic (adiabatic) gas coefficient suitable for a mixed oil and gas shock strut configuration, defined by $\gamma$.

4) The initial pressure ratios defined by (5.7) and (5.8) were iteratively varied to meet geometric requirements, specifically for the sealing surface on the piston outer diameter $\left(O D_{P R}\right)$ defined by equation (5.13). Using the ratio specified by Currey [5] as a guideline, the ratio was varied, keeping a 60-psi charge pressure constant, to converge on a value for $O D_{P R}$ near a standard sealing OD, specified by SAE AS4716B for dynamic sealing surfaces.

\subsubsection{Internal Geometry Definition}

This section will present an overview of the design factors from a mechanical and performance point of view. Figure 5.8 highlights the fundamental shock absorber components whose relevant geometric features are described in Table 5.5 and further defined in Figure 5.9. Table 5.5 provides definitions for the key geometrical elements that were considered during the design phase and later used to size the final detail parts. 


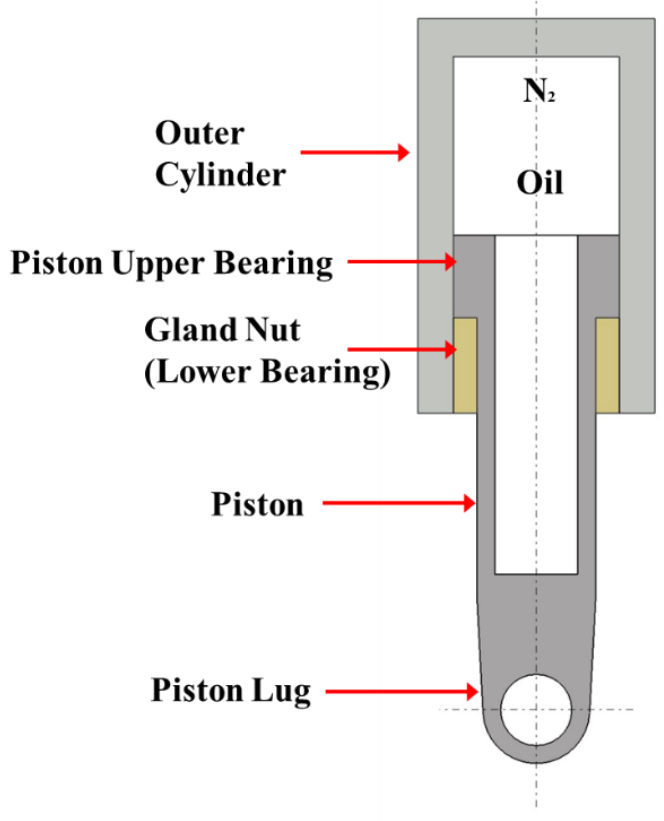

Figure 5.8: A simplified oleo-pneumatic strut, key preliminary design components labeled for clarity

Table 5.5: Shock strut mechanical design features and definitions

\begin{tabular}{|c|l|}
\hline Symbol & \multicolumn{1}{|c|}{ Description and Sizing Considerations } \\
\hline$R_{P L}$ & $\begin{array}{l}\text { Piston Lug Radius - defined to ensure an adequate lug thickness, typically }<\mathrm{OD}_{\mathrm{PR}} \text { for lower } \\
\text { bearing assembly purposes. }\end{array}$ \\
\hline$I D_{P L}$ & $\begin{array}{l}\text { Piston Lug Inner Diameter - typically sized based on the interfacing pin connection, or bush } \\
\text { install (in this case, a spherical bearing). This joint diameter was sized for a swaged spherical } \\
\text { bearing that was installed with a small interference fit and swaged after installation. }\end{array}$ \\
\hline$O D_{P R}$ & $\begin{array}{l}\text { Piston Rod Outer Diameter - defined as the running surface against the dynamic seals located } \\
\text { within the lower bearing (gland nut), typically adjusted to meet closest standard seal sizing per } \\
\text { AS4716B after preliminary pressure/volume sizing. }\end{array}$ \\
\hline$D_{P R}$ & $\begin{array}{l}\text { Piston Rod Inner Diameter - is sized preliminarily based on required wall thicknesses to } \\
\text { withstand hoop stress and compression stresses (buckling) at bottoming. The inner diameter can } \\
\text { be further refined based on internal configuration requirements, e.g. the addition of an orifice } \\
\text { support tube with externally mounted dynamic seal (Figure 5.3). }\end{array}$ \\
\hline$O D_{P U B}$ & $\begin{array}{l}\text { Piston Upper Bearing } \text { Outer Diameter - typically sized in conjunction with the lower bearing } \\
\text { gland nut and the outer cylinder inner diameter (ID } \\
\text { additional flow regulation, as will be shown in the detail design discussion. }\end{array}$ \\
\hline$L_{P U B}$ & $\begin{array}{l}\text { Piston Upper Bearing Length - preliminarily sized based on the total bearing overlap length } \\
\left(L_{B O}\right) \text { and then further refined based on the bearing pressures and material selection (typically a } \\
\text { bronze for good wear resistance and lubricity). }\end{array}$ \\
\hline
\end{tabular}




\begin{tabular}{|c|c|}
\hline$L_{L B}$ & $\begin{array}{l}\text { Lower Bearing Length - the final sizing of the lower bearing length is a highly dependent on } \\
\text { the utilized engagement method (e.g. threading to the ID or OD of the outer cylinder) and the } \\
\text { quantity and size of the seals installed within its volume. }\end{array}$ \\
\hline$L_{B O}$ & Total Bearing Overlap Length $-\left(L_{B O}>1.25 x O D_{P R}\right.$ per MIL-L-8552C and Currey [5] $)$ \\
\hline$I D_{O C}$ & $\begin{array}{l}\text { Outer Cylinder, Inner Diameter - typically sized based on required piston head area for optimal } \\
\text { P/A load bearing capabilities. The inner diameter typically directly engages with the Piston } \\
\text { upper bearing outer diameter }\left(O D_{P U B}\right) \text { and thus shall be sized in parallel with the outer diameter } \\
\text { of the cylinder }\left(O D_{O C}\right) \text { based on the required wall thickness. }\end{array}$ \\
\hline$O D_{O C}$ & $\begin{array}{l}\text { Outer Cylinder, Outer Diameter - the outer cylinder is typically sized to minimize cylinder } \\
\text { growth, possible at high pressures, and to withstand bending loads. The present outer cylinder } \\
\text { was sized based on the minor diameter of the ACME } 3 \mathrm{G} \text { thread machined onto its OD. }\end{array}$ \\
\hline$S_{\text {Max }}$ & $\begin{array}{l}\text { Max Piston Stroke - the max stroke is the total amount of length adjustment a shock strut piston } \\
\text { can undergo during operation. Typically, pre-sized based on the energy dissipation requirements } \\
\text { at touchdown. After an initial sizing, typically from expected landing loads, the max stroke is } \\
\text { tweaked to ensure adequate ground clearance while deployed and meet kinematic requirements. } \\
\text { It is important to note, as mentioned by Currey [5], that the piston stroke is not a function of the } \\
\text { supporting structure (e.g. Hyperloop pod) weight. }\end{array}$ \\
\hline$L_{P L O}$ & $\begin{array}{l}\text { Piston Lug Centre Offset - this feature is typically defined in the detail design phase to ensure } \\
\text { that the piston OD is clear of the interfacing geometry at the piston/trailing arm lug. The offset } \\
\text { is typically at least equal to the Piston Lug Radius }\left(R_{P L}\right) \text {. }\end{array}$ \\
\hline
\end{tabular}

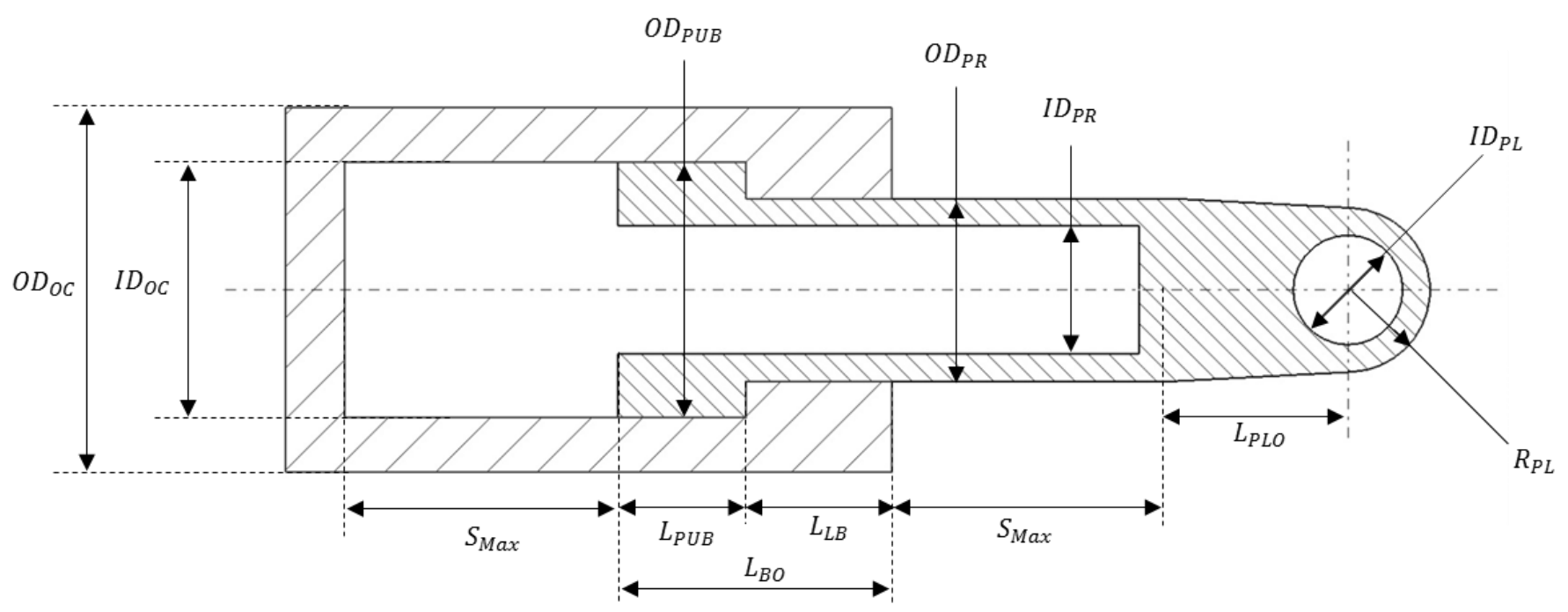

Figure 5.9: An example of an oleo-pneumatic shock strut highlighting preliminary geometric features 
Based on the preliminary gas spring calculations performed and discussed in Section 5.3.2, the sizing of the piston rod outer diameter $\left(O D_{P R}\right)$ is used to determine the behavior of the gas spring. For this reason, the sizing of $O D_{P R}$ was incorporated into a shock strut design spread sheet, as shown in Table 5.6, and calculated from the piston area, $A_{P}$, determined by the ratio of 4:1 for static to extended, as defined by Currey [5]. Utilizing a spread sheet format with key terms defined enabled quick iterations to be performed. The result of one calculation, shown in Table 5.6, indicates that the dimension for $O D_{P R}$ was to be 1.086-in. Using this result, the available sealing sizes were referred to and helped to refine the calculated value, if necessary.

Table 5.6: Piston detail design template, as defined in the author's shock strut design tool

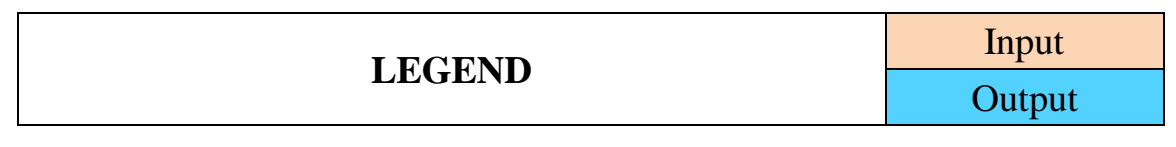

\begin{tabular}{|l|c|}
\hline Detail Design of Piston & Value \\
\hline Piston D/t (for steel) (<15) & 10.000 \\
\hline Calculated Piston Wall Thickness (in) & 0.109 \\
\hline Resulting Piston Area (in2) & 0.926 \\
\hline Resulting Piston Outer Diameter (in) & 1.086 \\
\hline Required Piston Rod OD (see gland table), B (in) & 1.059 \\
\hline Required Piston Area (in2) & 0.881 \\
\hline Estimated Piston Inner Diameter (in) & 0.869 \\
\hline Required ID (see gland table), A (in) & 0.864 \\
\hline Piston Thickness (based on Gland Req.) (in) & 0.098 \\
\hline
\end{tabular}

As shown in Table 5.7, the closest possible piston rod bore dimension (B), defined by the sealing design specification SAE AS4716B, called for a $O D_{P R}$ of 1.059-in. To accommodate the standard fitting size based on the Rod Bore dimension shown in Table 5.7, the ratio of static to extended stroke, defined by equation (5.7), was modified.

Table 5.7: Piston rod dynamic seal gland dimensions, per Greene Tweed [59], based on SAE AS4716B

\begin{tabular}{|c|c|c|c|c|}
\hline \multicolumn{5}{|c|}{ Piston Dynamic Seal } \\
\hline GLAND \# & Rod Bore, (B) [in] & Tolerance [in] & Gland ID [in] & Gland Width, G [in] \\
\hline-215 & 1.06 & $(-) 0.002$ & 1.302 & 0.304 \\
\hline
\end{tabular}


For the reference of the reader, Figure 5.10 illustrates an example of a typical gland design, the feature that is used to house a seal. A similar design trade-off process, as was discussed here, was followed for all sealing surfaces during the design of the shock strut.

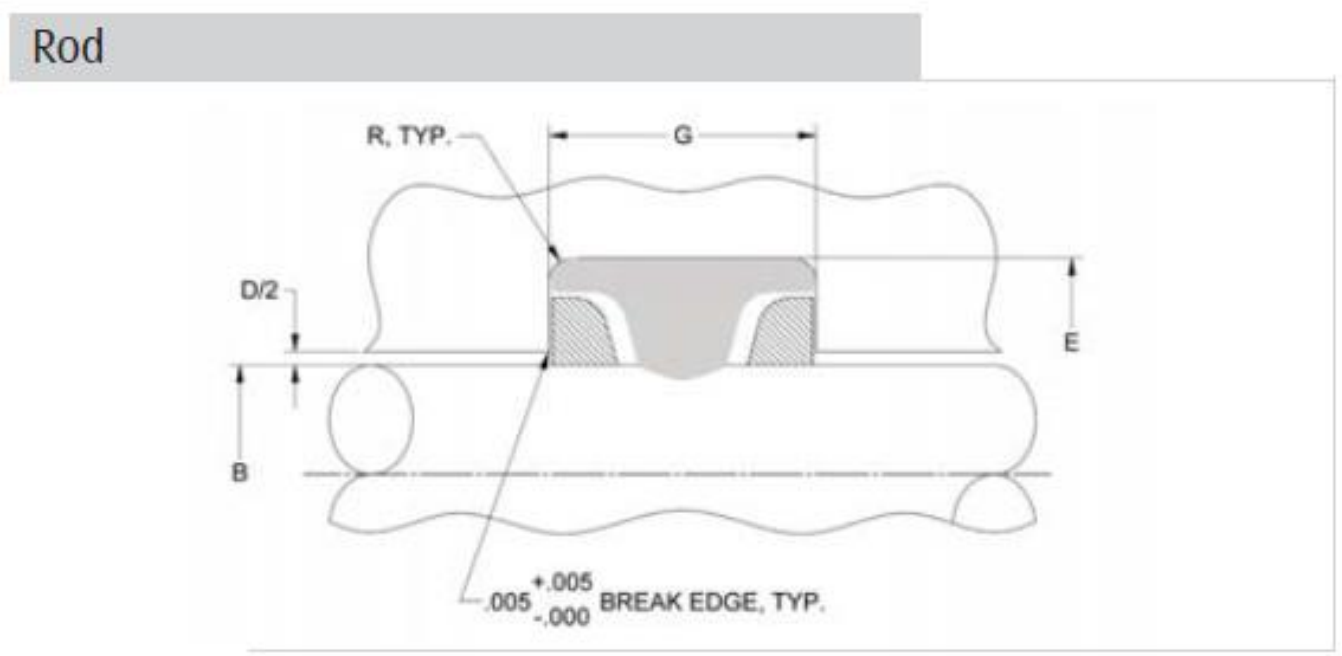

Figure 5.10: Piston rod gland design, key dimensions labeled, as defined by Greene Tweed [59]

\subsubsection{Detailed Gas Spring Design}

The calculation to determine the final gas spring was accomplished using a Safran Landing Systems Inc. proprietary gas spring software, Dynatool ${ }^{\circ}$ Version 2.1 [60]. Fundamentally, Dynatool ${ }^{\odot}$ provides a means to:

1) Apply the compressible polytropic (adiabatic) and isothermal ideal gas theory to produce dynamic and static gas spring curves using nitrogen specific volume data presented by Din [61] on the behaviour of real gases

2) Account for the compressibility of the fluid (oil) by incorporating the fluids bulk modulus, defined in AIR 1362B [62] as the ability of a fluid to resist changes in volume under pressure, and provided a means to account for the dynamic effects by utilizing the bulk modulus as a function of pressure and temperature (in both isothermal and adiabatic calculations).

3) Account for the thermal expansion of the oil using the coefficient of thermal expansion $\alpha$ 
4) Account for the mechanical behaviour of the structural elements, i.e. the piston and cylinder components and their respective tendencies expand and stretch under pressure.

Finally, considering points 1) through 4) noted above, the tool obeys the continuity equation (no oil or gas is destroyed) such that:

$$
0=V_{c}-V_{c o}-A_{P} S-\left(V_{g}-V_{g o}\right)-\left(V_{f}-V_{f o}\right)
$$

where: $V_{c}$ is the total volume of the shock strut at operating temperature and pressure; $V_{c o}$ is the total volume of shock strut at its initial servicing (filling) conditions; $A_{P}$ is the area of the piston found using equation (5.12); $S$ is defined as the stroke of the shock strut piston; $V_{g}$ is defined as the volume of nitrogen gas at operating temperature and pressure; $V_{g o}$ is the volume of nitrogen gas at servicing conditions; $V_{f}$ is the volume of oil at operating temperature and pressure; and $V_{f o}$ is the initial volume of oil supplied at shock strut servicing conditions.

The results of the Dynatool ${ }^{\odot}$ gas spring curve are shown in Figure 5.11 and Figure 5.12 representing the load without friction vs. stroke and pressure vs. stroke respectively. A sample of the output data is also shown in Appendix D.

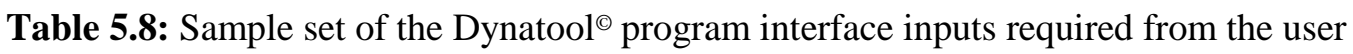

\begin{tabular}{|c|c|c|c|}
\hline Input Description & Value & Input Description & Value \\
\hline Cylinder ID (in) & 1.492 & Nitrogen Charge Pressure, P1 (psi) & 60 \\
\hline Cylinder OD (in) & 1.709 & Final (Servicing) Volume, V1 (in $\left.{ }^{3}\right)$ & 0.106 \\
\hline Cylinder Poisson Ratio & 0.32 & Dynamic Polytropic Exponent & N/A \\
\hline Cylinder Elastic Modulus (psi) & 290000 & Max Piston Stroke (in) & 3 \\
\hline Piston ID (in) & 0.864 & Cylinder Load at Static (lb) & 222.5 \\
\hline Piston OD (in) & 1.059 & Operating Temperature $\left({ }^{\circ} \mathrm{C}\right)$ & 23 \\
\hline Piston Poisson Ratio & 0.32 & Oil Bulk Modulus (psi) & 260000 \\
\hline Piston Elastic Modulus (psi) & 290000 & $\begin{array}{l}\text { Change in Bulk Modulus with pressure } \\
\text { (adiabatic) (psi/psi) }\end{array}$ & 6.1 \\
\hline $\begin{array}{l}\text { Total Volume (oil+gas) at fully } \\
\text { extended stroke }\left(\mathrm{in}^{3}\right)\end{array}$ & 9.174 & Oil Thermal Expansion Coefficient $\left(1 / 1 /{ }^{\circ} \mathrm{C}\right)$ & 0.000864 \\
\hline Servicing Temperature $\left({ }^{\circ} \mathrm{C}\right)$ & 23 & Static Stroke (est.) & 2.26 \\
\hline $\begin{array}{l}\text { Oil Bulk Modulus, isothermal } \\
(\mathrm{psi})\end{array}$ & 200000 & $\begin{array}{l}\text { Change in Bulk Modulus with pressure } \\
\text { (isothermal) (psi/psi) }\end{array}$ & 5.1 \\
\hline
\end{tabular}


HDWS Gas Spring: Load vs. Stroke

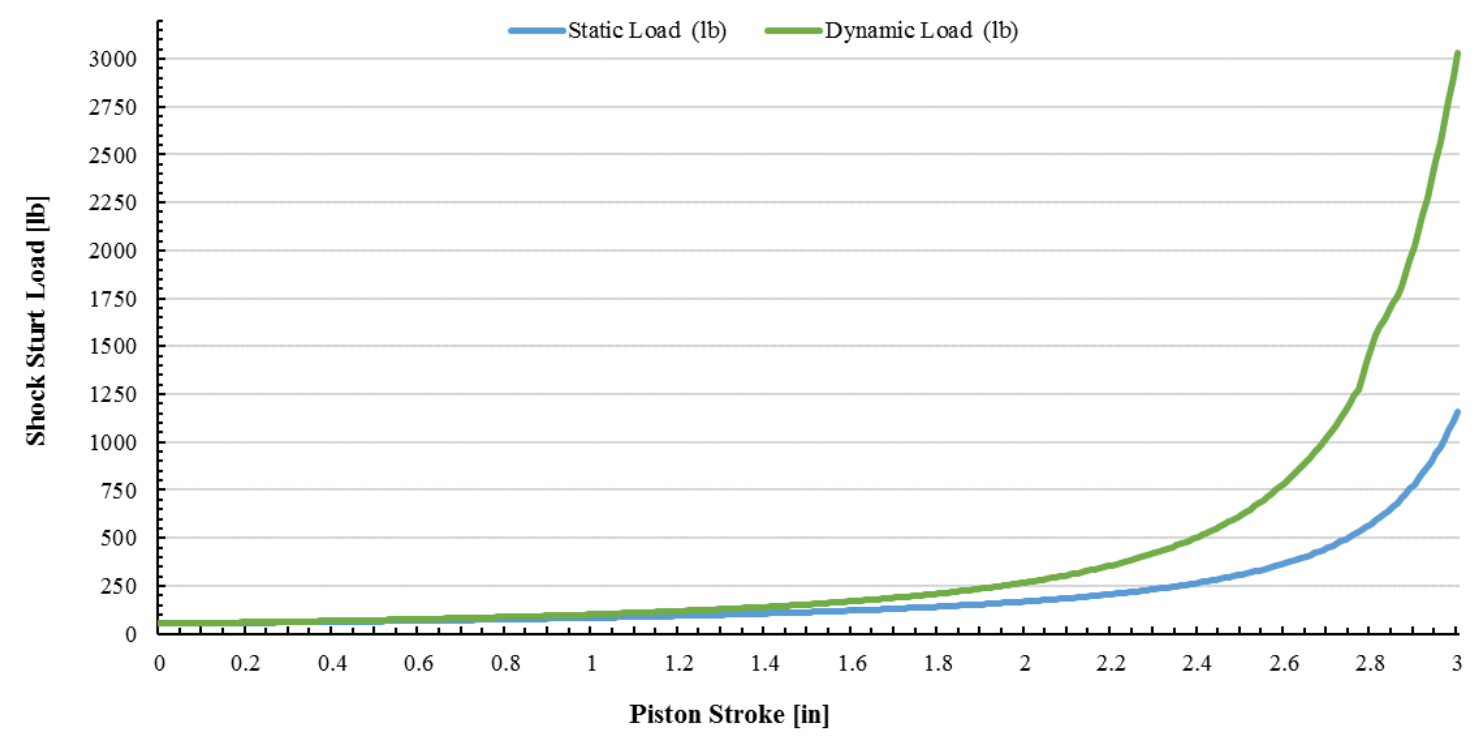

Figure 5.11: Dynatool ${ }^{\odot}$ HDWS gas spring curve (static and dynamic), load vs. stroke

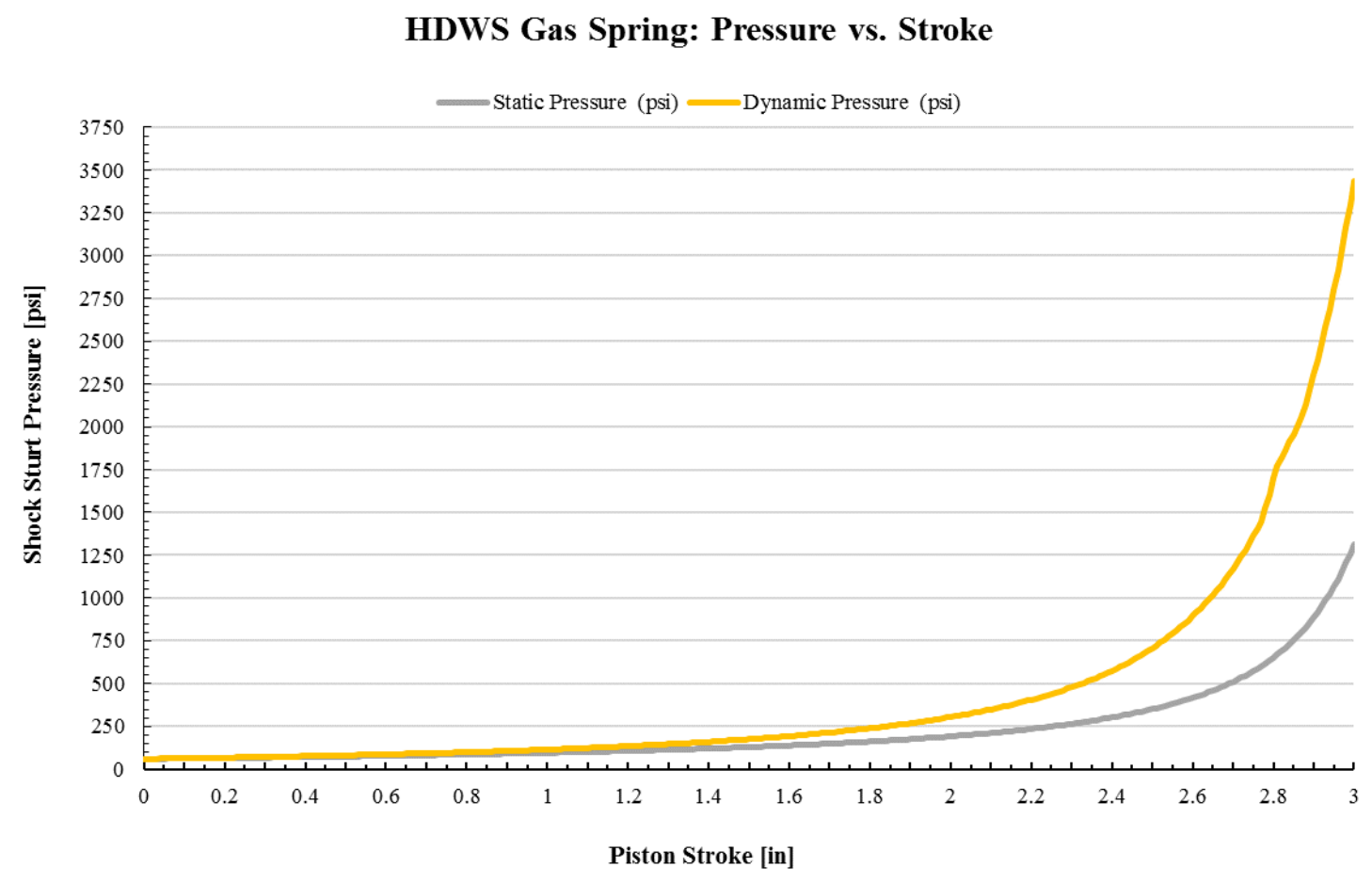

Figure 5.12: Dynatool ${ }^{\odot}$ HDWS gas spring curve (static and dynamic), pressure vs. stroke 
To provide the most accurate possible values for the input volumes required to run Dynatool ${ }^{\odot}$, the CATIA $^{\oplus}$ V5 Part Design Workbench was used and the results are shown in Figure 5.13 through Figure 5.15.

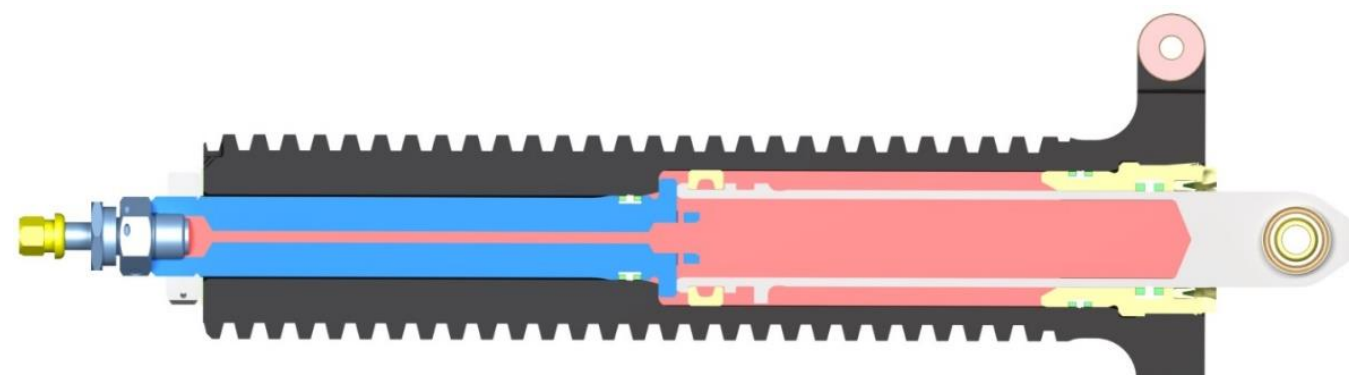

Figure 5.13: Cross-section view showing the shock strut while fully compressed at servicing, total oil volume (red) $6.426 \mathrm{in}^{3}$

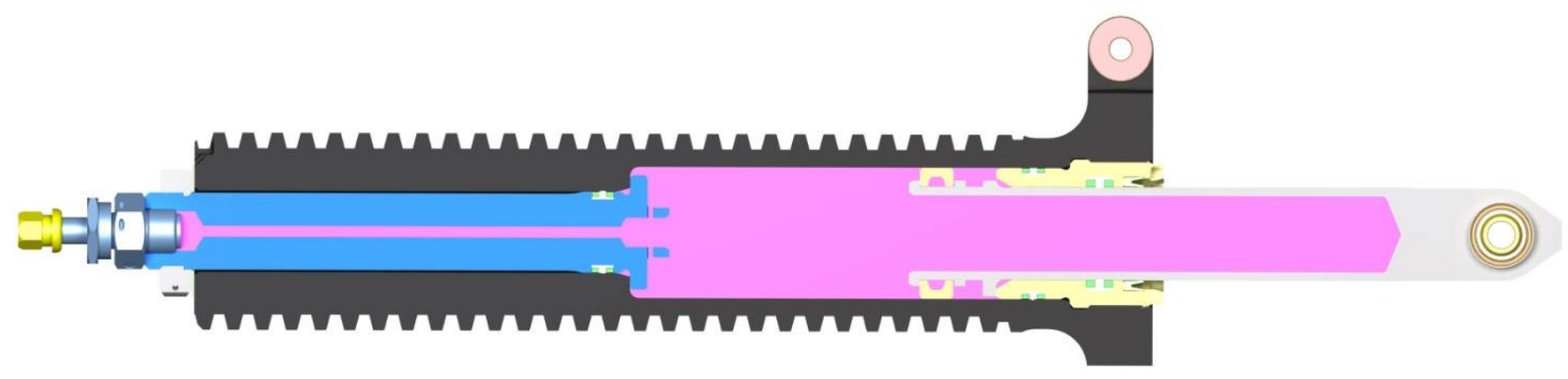

Figure 5.14: Cross-section view showing the total volume when extended (purple) $9.174 \mathrm{in}^{3}$, gas+oil

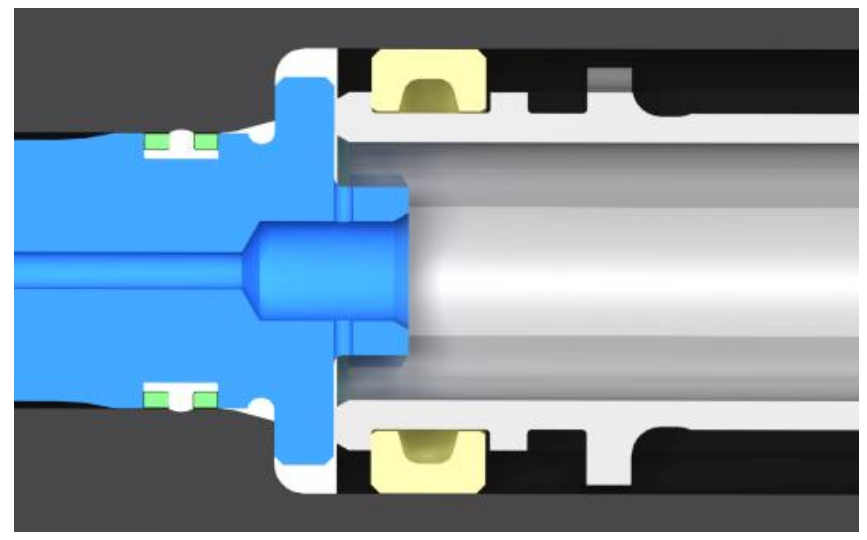

Figure 5.15: Cross-section view of the shock strut trapped air volume (white) $0.106 \mathrm{in}^{3}$, max piston compression 


\subsubsection{Shock Absorber Detail Design}

After determining the basic internal dimensions discussed in Section 5.3.2 and Section 5.3.3 required to create a functioning shock absorber, the detail geometric design process commenced. In reality, the close relationship between the seal gland sizing and interfacing surfaces forced the consideration of design details throughout the preliminary design phase. Some of the key details are now briefly discussed.

\section{Lower Bearing (Gland Nut):}

The shock strut lower bearing (Figure 5.16) was designed to serve three purposes: to act as a seal carrier for the dynamic, scraper and static seals; to act as a bearing surface keeping the piston rod axially aligned with the shock strut during stroke; and to act as an out-stop surface retaining the piston while under extended pressure (60 psi).

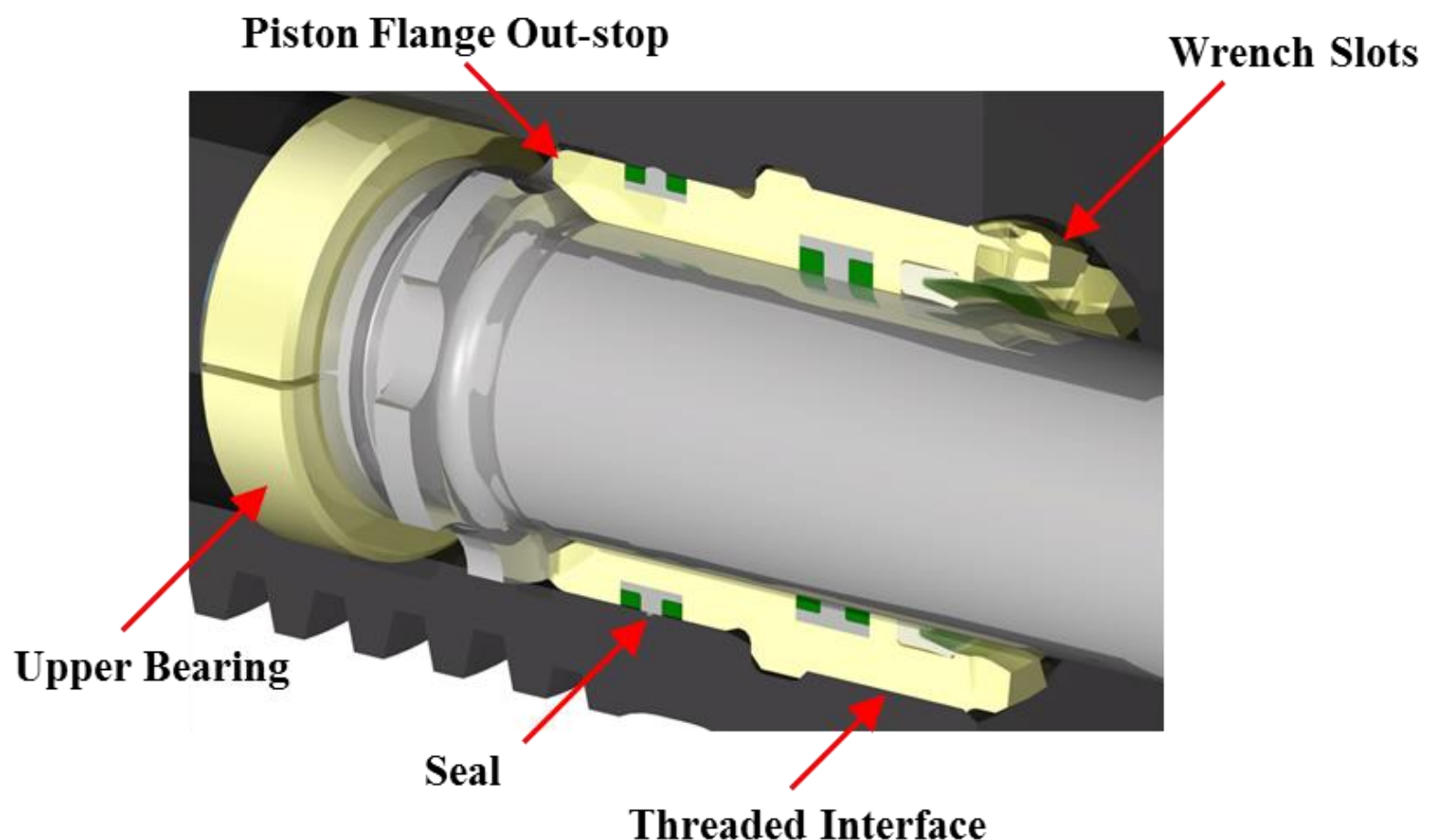

Figure 5.16: Cross-section, lower bearing, key design details labeled 
The gland nut was fitted with wrench slots, as defined in MIL-S-8552 and recommended by Currey [5], for ease of assembly. Specifically, this enabled the use of standard spanner wrench tooling. The sizes of the wrench slots were defined with reference to MIL-S-8552. The same wrench slots also provided an interface for a lock tab, labeled in Figure 4.14, prohibiting the gland nut from unscrewing. Furthermore, the threads on the outer diameter of the lower bearing were sized to handle at least two times the maximum pressure loading induced by oil and gas volume at maximum compression. In order prevent damage to the static seal during assembly, the threading was sufficiently offset to guarantee seal protection on assembly.

As alluded to earlier, the seal glands were sized with reference to the SAE AS4176B and AS5857A specifications for the dynamic and static seal gland designs respectively. Custom installation tooling was designed to protect the seals during installation and, in some cases, provide a presqueeze to enable a smoother installation with a mating part. The tool drawings can be found in Appendix B.

\section{Upper Bearing, Piston Head:}

The piston head upper bearing, as can be seen in Figure 5.16, was designed to serve two purposes: to act as a flow regulator under fast compressions speeds, in theory providing additional dampening, by limiting flow rate of the oil during stroke; and to act in tandem with the lower bearing keeping the piston rod axially aligned with the shock strut during stroke. The design is a split ring configuration, attached on assembly within grooves machined into the shock strut piston outer diameter, prior to install into the shock strut outer cylinder.

\section{Filler Tube and Servicing Valve:}

The shock strut filler tube (Figure 5.17) was designed to serve two purposes: to act as a seal carrier for the static seal interfacing between the outer diameter of the filler tube and inner diameter of the cylinder; to enable the filling of the shock strut volume with oil and gas through the use of a standard valve designed per AS28889A in accordance with MIL-S-8552 [5]. As can be deduced

from observing Figure 5.17 the filler tube must be assembled through the bottom of the shock strut 
prior to installing the piston and lower bearing. The flange head within the interior of the cylinder utilizes a machined hexagonal socket interface for installation and to enable secure fitting of the filler tube through the tightening of the jam nut disposed below the AS28889A valve. The valve is mounted to the inner diameter of the filler tube using threads designed by the AS8879D UNJF thread specification per the matting requirements specified in AS28889A.

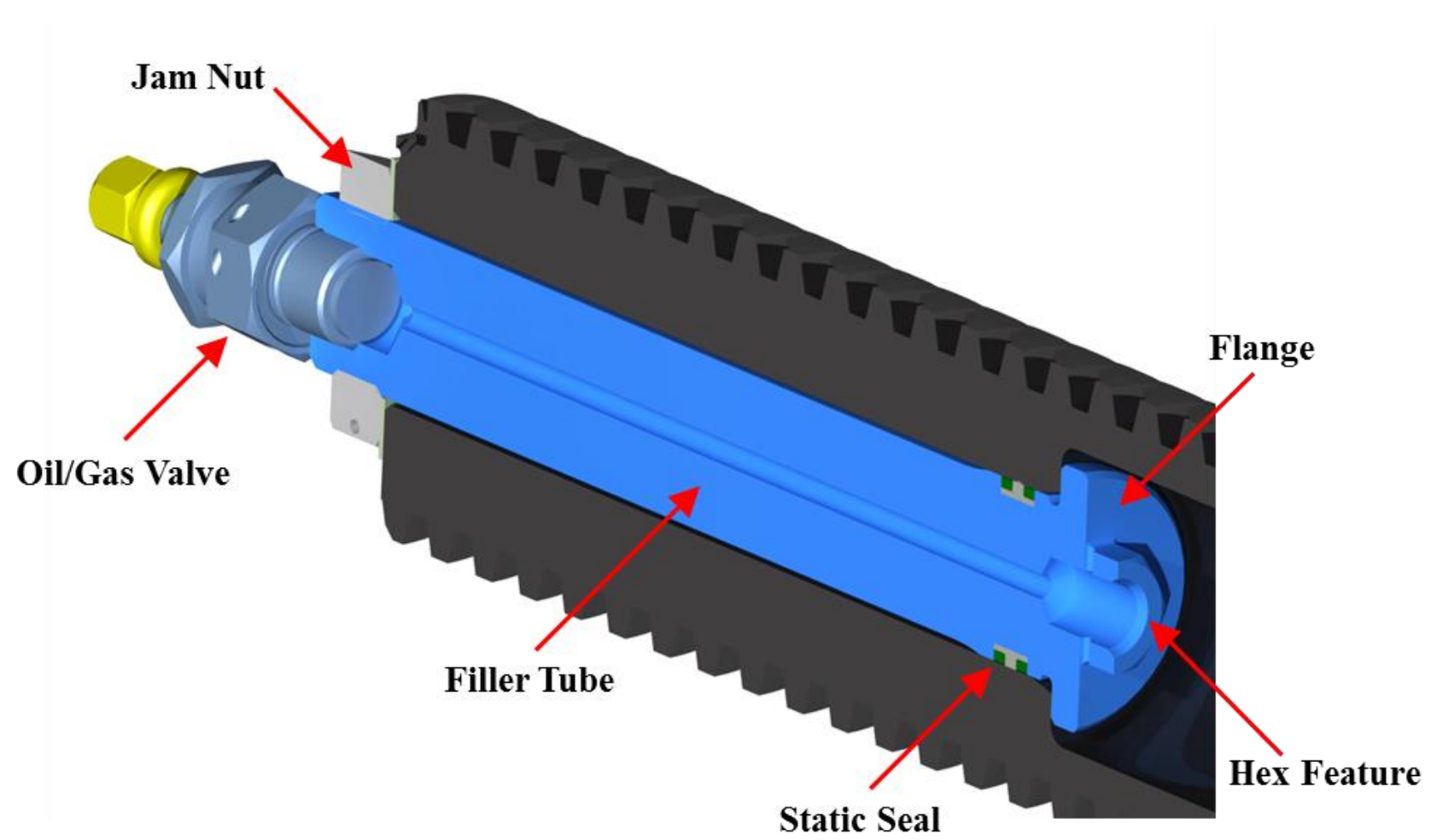

Figure 5.17: 3D cross-section of the filler tube, key design details labeled

As can be seen in Figure 5.17, the filler tube flange head was sized to act as a stoppage feature when the piston reaches maximum stroke (max compression). An additional important feature, highlighted in Figure 5.18, is the small bore machined perpendicular to the main filler bore axis. The positioning of this bore helps to regulate the amount of trapped gas in the shock strut during compression and thus plays a role in determining the stiffness of the gas spring. As can be seen in Figure 5.18, when the shock strut is compressed, the gas (colored in turquoise) tends to become trapped within the volume available above the small bore. The positioning of this feature allows for the softening of the gas spring, that is, it directly relates to the positioning of the static stroke along a given gas spring curve. 


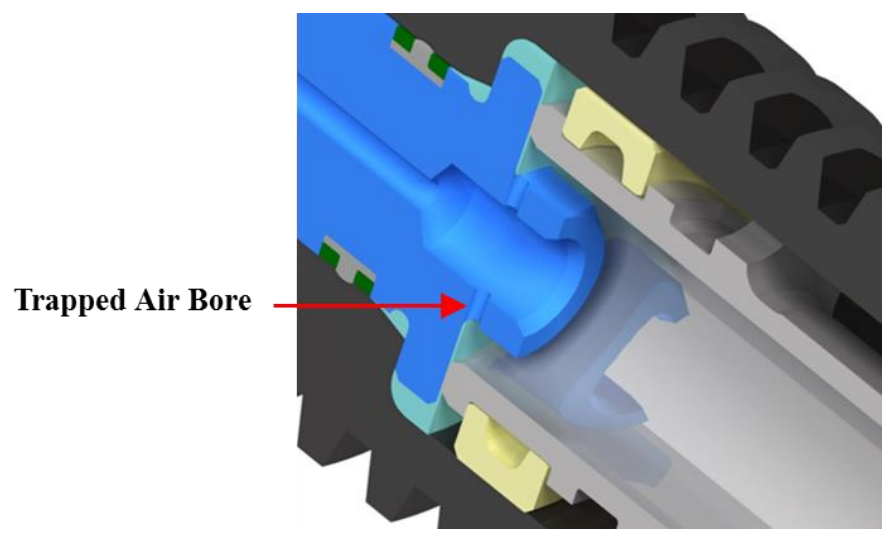

Figure 5.18: $3 \mathrm{D}$ cross-section view of the filler tube with trapped gas illustrated

\section{Piston:}

The piston (Figure 5.19) was manufactured from 303L structural stainless steel for corrosion resistance and to obtain a tough running surface for the seals. To meet specifications provided by the seal manufacturer, the outer diameter of the piston rod was ground to a specified surface roughness of $12 \mu \mathrm{in} \mathrm{Ra}$ or better. The thickness of the piston was pre-sized using diameter to thickness (D/t) ratios provided for reference by Safran Landing Systems Canada Inc. [48], not available for outside distribution. Finally, the piston was fit with a spherical bearing at the lug rodend to limit the loading transferred from the trailing arm to axial loads only. The lug at the rodend was subsequently sized to ensure that it could be passed through the lower bearing during installation.

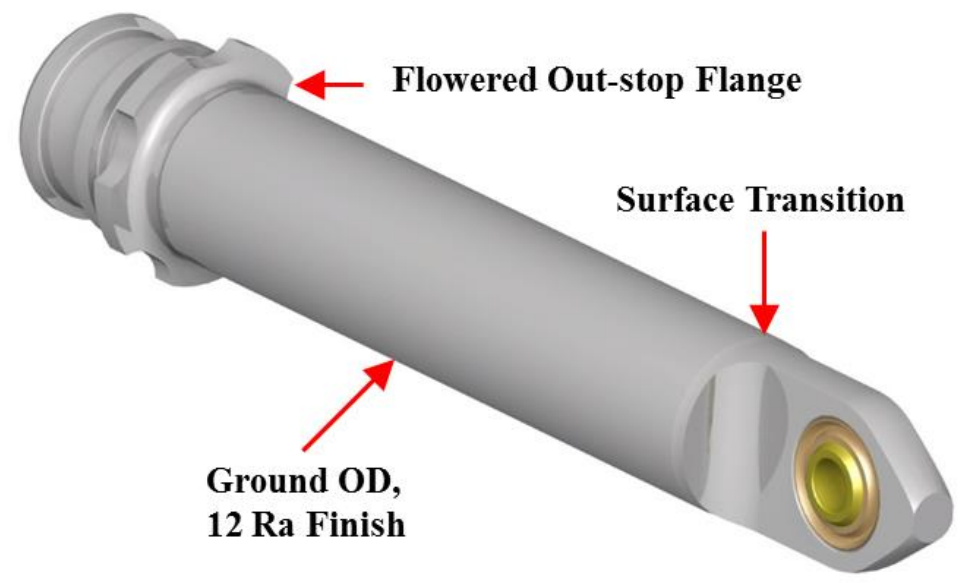

Figure 5.19: 3D view of the piston, spherical bearing installed, key features labeled 


\section{Outer Cylinder:}

The shock absorber outer cylinder (Figure 5.20) was designed to enable the use of typical internal shock absorber components, as discussed in the present chapter. In addition, the shock absorber for the HDWS is also designed to enable the extension and retraction of the HDWS system when equipped to the Retraction sub-assembly housing through the ACME teeth on its exterior.

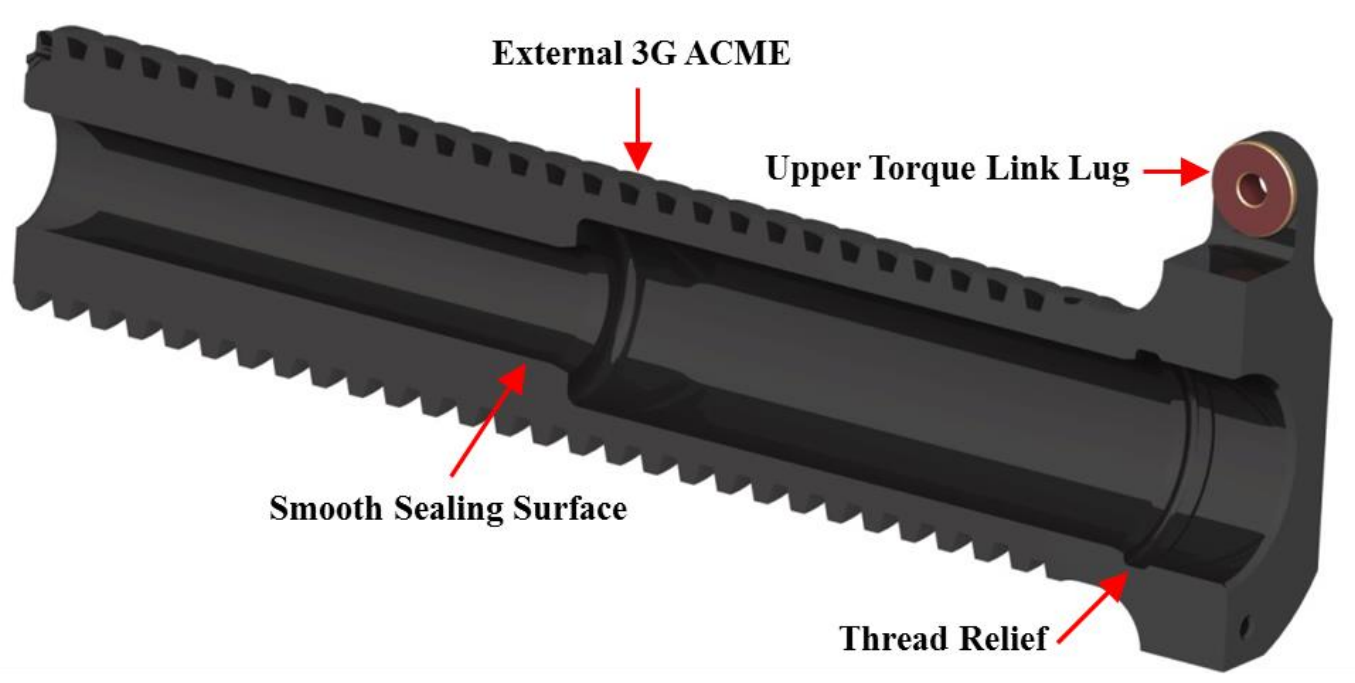

Figure 5.20: 3D cross-sectional view of the shock strut outer cylinder, key features labeled

In particular, the thread was designed per the ASME/ANSI B1.5-1997 [63] General Purpose Acme thread form design specification. Per the information provided by Oberg et al. [64] within the Machinery's Handbook, General Purpose threads are classified into three classes: 2G, 3G, and 4G. These three classes define the pitch diameter tolerances of the thread form. Due to the tight control required to maintain the height of the pod and reduce gaps in the actuation that could allow for vibration movement, a 3G ACME thread was selected, as recommended in [64], for use in cases where less backlash or "end-play" is desired.

Based on the geometrical stack up imposed on the design by the sizing of the shock strut gas spring and internal features described here, a 2.250-3 ACME-3G external thread was selected for the shock strut. This size provided a sufficient outer cylinder wall thickness, which was closely monitored due to the stress concentration prone zones at the thread root. The sizing here played a key role in sizing the mating ACME nut, discussed in the next section. 
The last feature of interest is the set of lugs (partially hidden in Figure 5.20) protruding from the bottom of the shock strut outer cylinder. These lugs, when attached with the mating torque link assembly routed through the trailing arm, prevents the shock strut from rotating with the spur gear driven ACME retraction nut by removing the torque due to friction and enables the translational motion of the shock strut.

\subsection{HDWS Actuation Mechanism Design}

The Retraction sub-assembly was produced to provide a trunnion mounted configuration, with pod mounting pins disposed on either side of the retraction housing component, allowing the space between the mounting points to be utilized for functional features as can be seen in Figure 5.21. The Retraction sub-assembly performs three major functions that enable the HDWS's operation. These functions include: acting as a pod interface structure by transmitting the load from the shock strut directly to the pod frame; housing an ACME geared retract nut responsible for transmission of the motor torque to the shock strut ACME threaded outer cylinder; and acting as the support structure for the mounting of the BLDC motor and sensor equipment described in Chapter 4's Sections 4.1.2 and 4.1.4 respectively.

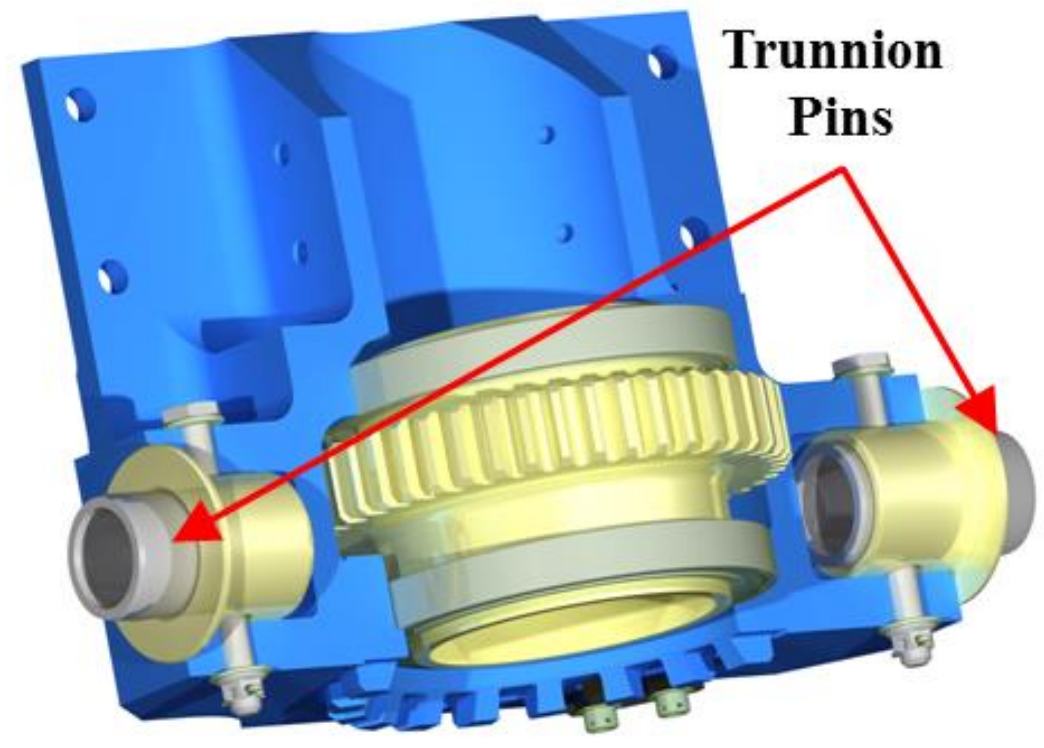

Figure 5.21: 3D cross-sectional view of the retract assembly, trunnion pins labeled 


\section{Retract Housing (Bushed):}

Fabricated from light-weight 6061-T6 aluminum the retract housing (Figure 5.22) is the primary structural element of the Retraction sub-assembly housing all the transmission components within its inner volume. The housing was fitted with AS8879D UNJF threads on the center bore, for engaging with a packing nut (not shown in Figure 5.22) during assembly. The body was machined from solid and includes a flange for mounting the motor bracket, using four 0.25 -in bolt holes, and provided threaded pickup points for the cable potentiometer bracket, described in Section 4.1.4.

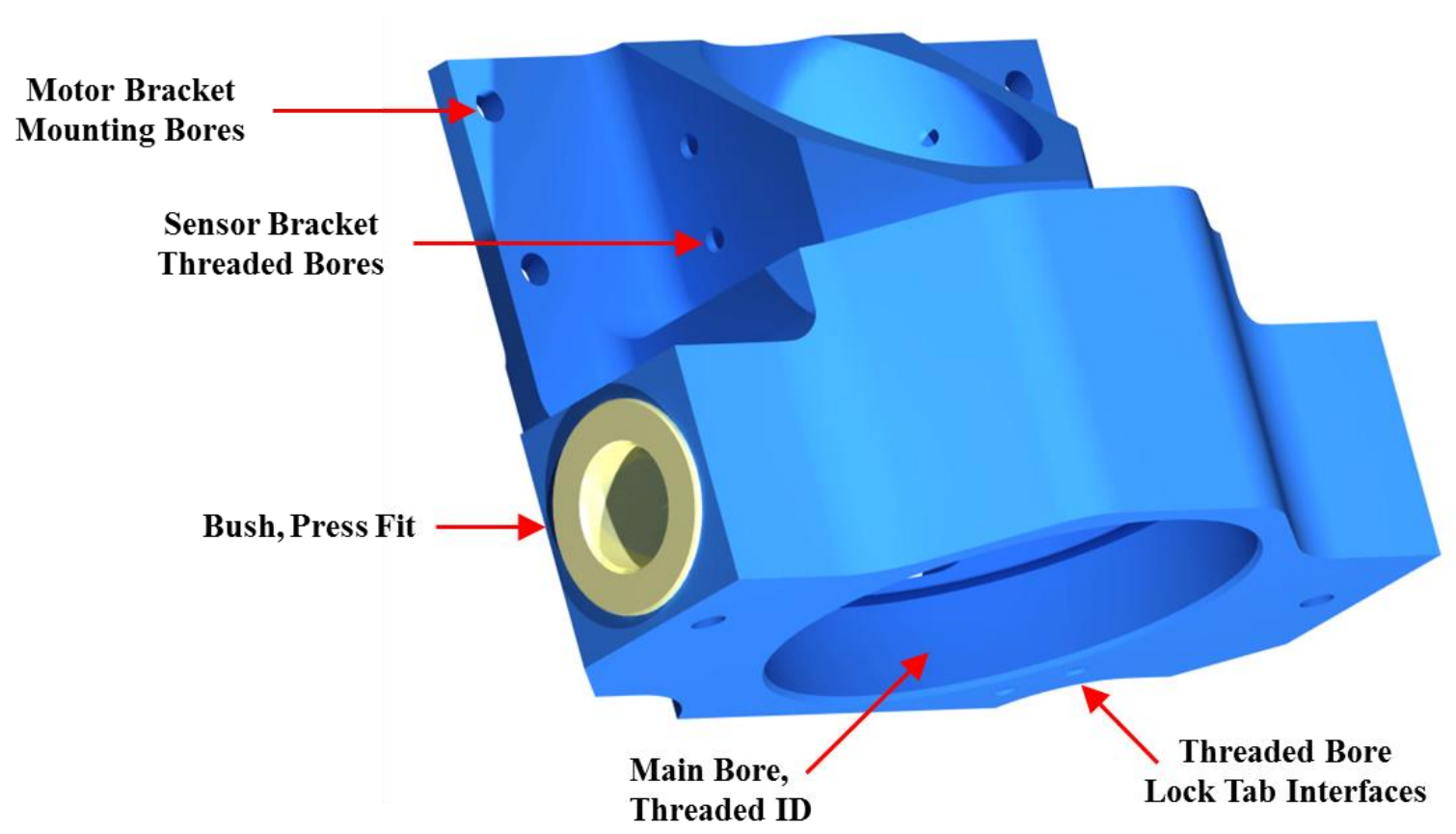

Figure 5.22: Retract housing (bushed), key design features labeled

To provide a bearing surface with excellent wear properties, the trunnion bores (Figure 5.21) were fitted with custom aluminum bronze (Al-Br) flanged bushings. To fix the rotation of the pin within the housing trunnion bore, the bushings and housing frame utilize cross bolts as an anti-rotation feature for the installed trunnion pins shown in Figure 5.21. Because of the anti-rotation feature, the inner diameter of the flanged bush (press-fit) does not require lubrication after initial installation since there is no relative rotation between the pin and the bushing. Lastly, the wear due 
to low speed rotation is limited to the bushing outer flange, sized to mate with self-lubricating KAron ${ }^{\circledR}$ lined bushings at the pod interface.

\section{ACME Retract Nut:}

The ACME retract nut as shown in Figure 5.23 was designed to interface with the shock absorber outer cylinder, as shown in Figure 5.20. Consequently, the inner diameter of the rotatable nut was fitted with a 2.250-3 ACME-3G internal thread as specified by the ASME/ANSI B1.5-1997 [63] General Purpose Acme thread form design specification. To ensure no clashing or thread-end binding occurs between the transition radius (shown above the lugs in Figure 5.20) of the mating external ACME thread and the ACME nut, the nut's design incorporated a modest thread offset, shown in Figure 5.23, providing clearance between the ACME nut and the cylinder lug transition feature.

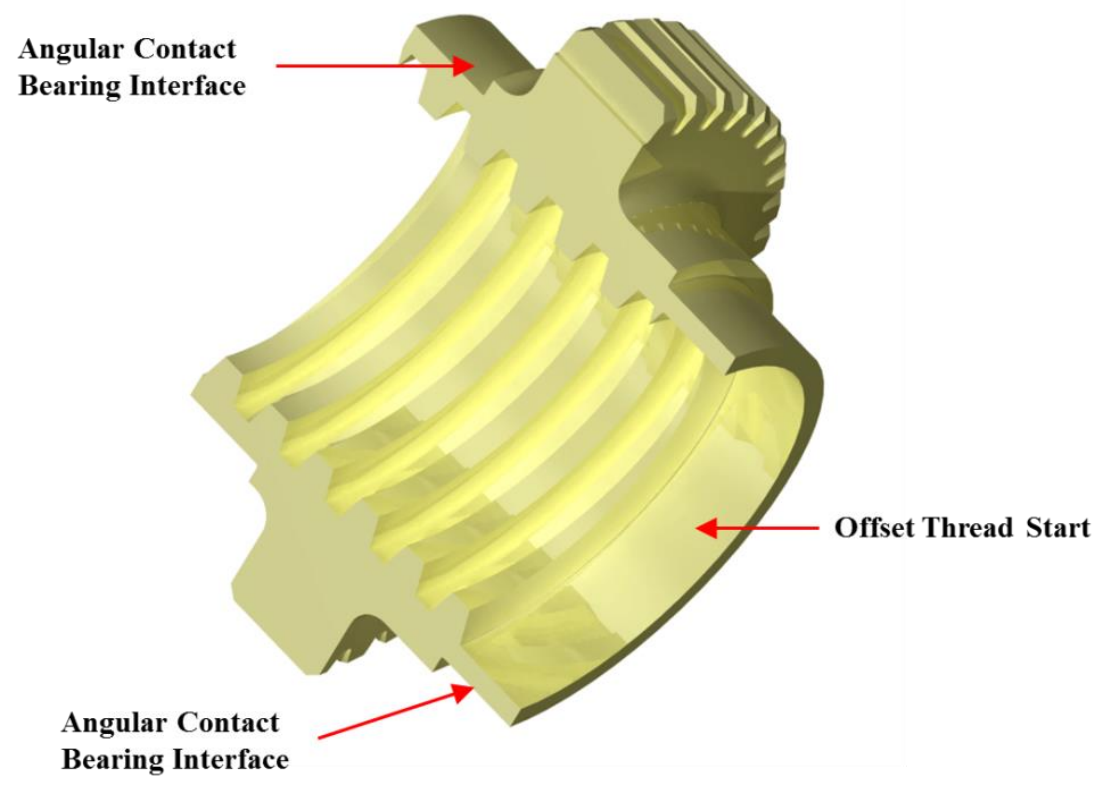

Figure 5.23: 3D cross-section view, ACME retract nut, key design features labeled

By design, the use of ACME threads provides a self-locking characteristic [65] that is considered to be a built-in safety feature for the HDWS. As an example, an extended and loaded HDWS will not retract (back-drive) if a power failure occurs on the pod since its locked position is not held in place by torque from the BLDC motor. Instead, the high friction of the threads is used, which does result in low thread efficiency [65]. The efficiency is then maximized in operation using $\mathrm{Al}-\mathrm{Br}$ 
(good friction and wear characteristics) as the ACME gear nut material and by applying vacuum grease lubrication (Dow Corning ${ }^{\circledR}$ [36] high-vacuum silicone grease) to provide a well-lubricated interface between the $\mathrm{Al}-\mathrm{Br}$ nut and the steel cylinder.

The ACME nut shown in Figure 5.23 has two machined interface grooves sized for an interference fit with mating deep groove angular contact bearings, supplied by SKF [42]. The bearings are installed on the outer diameter of the ACME nut, shown in Figure 5.24, prior to installation into the retract housing. The type of interference fit was selected based on the recommendations from the supplier and ensured that the inner race (ring) of the ball bearing was fixed from rotating relative to the ACME nut but that the internals of the ball bearing were free to rotate. Since angular contact bearings are specifically designed for this type of combined loading (thrust and radial) they were selected in place of thrust bearings.

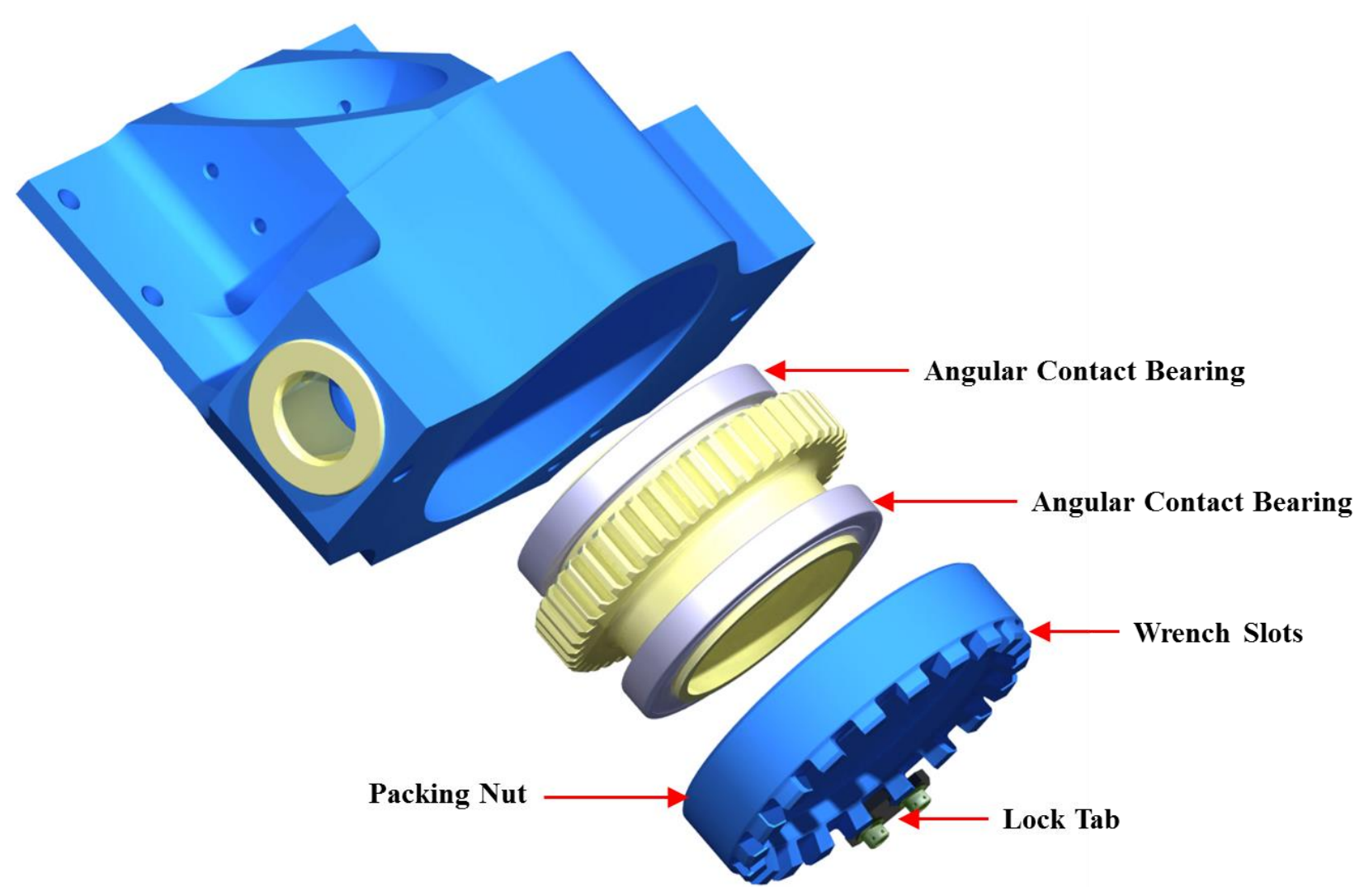

Figure 5.24: Retract assembly $3 D$ exploaded view, key design features labeled 


\section{Packing Nut:}

Finally, with reference to Figure 5.24, a packing nut was designed equipped with wrench slots, as defined in MIL-S-8552 for ease of assembly. Specifically, this enabled the use of standard spanner wrench tooling. The same wrench slots also provided an interface for a lock tab, which is mounted to the retract housing via two NAS drilled head cap screws which are locked on assembly with lock-wiring (not shown).

\subsection{Chapter Summary}

In this chapter, the Deployable and Retractable Shock Strut, as equipped to the HDWS, has been presented and its operation defined. The key elements of the Deployable and Retractable Shock Strut have been discussed and a detail design summary for the shock strut has been provided. After completing the detail design phase, five HDWSs were manufactured and assembled for testing and demonstration purposes. The next chapter will briefly discuss these tests and provide insights into both observed and measured results. 


\section{Experimental Results}

This chapter provides a brief overview of the testing that took place on the HDWS's starting in August of 2016 and concluding in January 2017. The testing of the HDWS was split up into component testing and full system testing. Using the testing capabilities and facilities provided by aircraft landing gear manufacturer Safran Landing Systems Canada Inc. [48] testing was performed on: the polyurethane wheel subject to high speeds and loading, the shock strut gas spring to verify the gas spring performance, and a full HDWS system.

\subsection{Polyurethane Wheel Performance Testing}

As discussed in Chapter 4, the HDWS prototypes used 95A polyurethane wheels for initial proof of concept testing and to meet the development deadlines set by SpaceX [3]. Testing was initiated in August 2016 to confirm the viability of the wheel proposed by Sunray Inc. [44]. The purpose of these tests was to confirm the safety and performance of the polyurethane wheel. The tests were designed to submit the polyurethane wheel to the expected Hyperloop competition speeds and projected loading conditions, based on the $\mathrm{U}$ of $\mathrm{T}$ pod concept.

For the test to be deemed a success, the wheel was required to demonstrate that its performance (including wheel balance, roundness and resistance to wear) will not deteriorate under the specified loads and rotational speeds. To perform this test, a fixture was designed to house a single polyurethane wheel, the detail design drawings of which are included in the Appendix C. A wheel spin-up machine, consisting of an electric motor, drive shaft, and fly-wheel, developed by Safran Landing Systems Canada Inc. [48], was used to simulate the required testing speed of $180 \mathrm{mph}$ (290 kph). The spin-up machine was linearly retractable and extendable, capable of engaging and disengaging with the fixed polyurethane wheel. The spin-up machine was positioned at engagement to exert an average load of $200 \mathrm{lb}$, close to the load seen in the designed $\mathrm{U}$ of $\mathrm{T}$ configuration, and was measured using a load cell. The typical test setup, prior to engagement, is shown in Figure 6.1. 


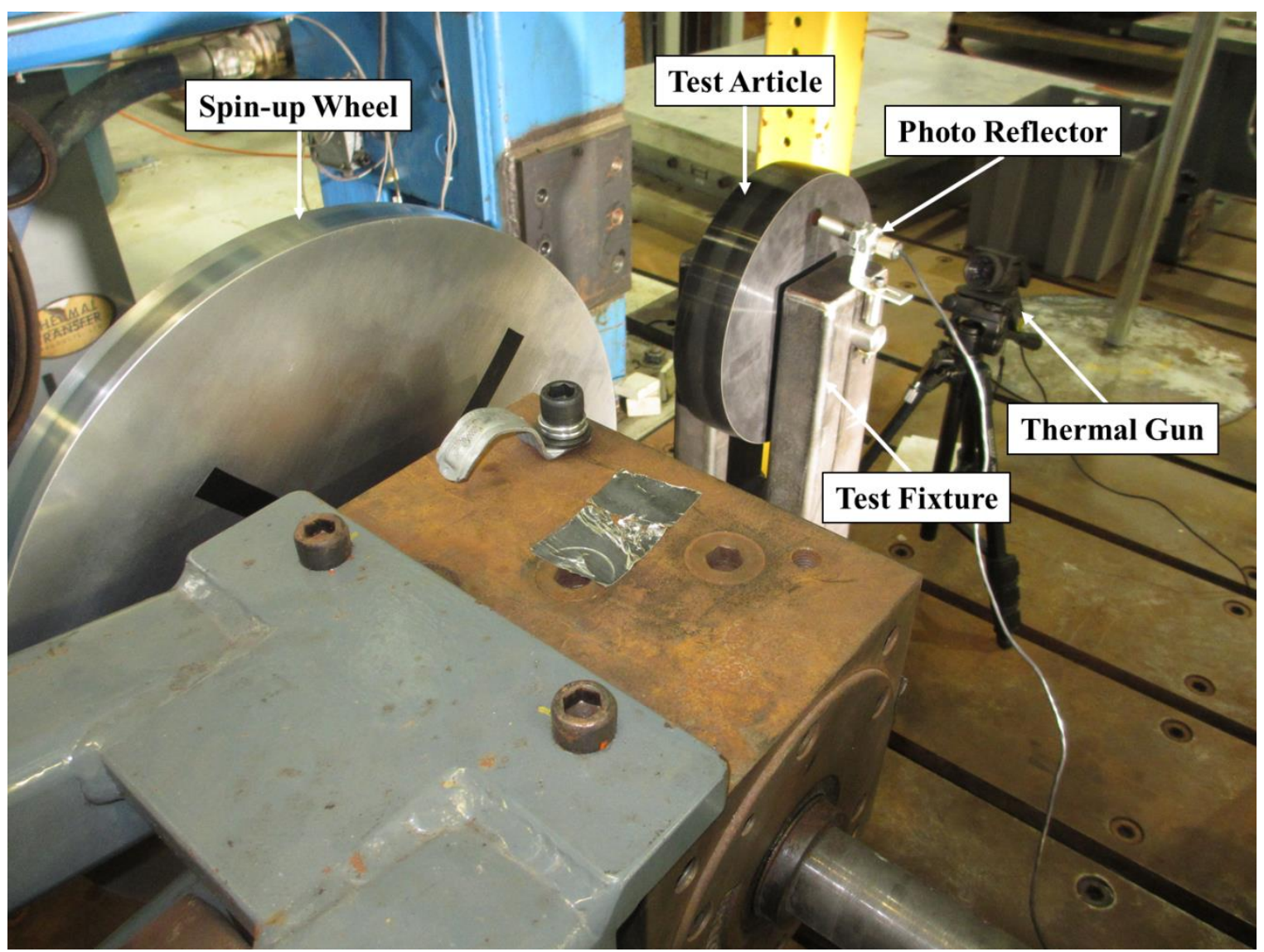

Figure 6.1: Wheel test configuration

The testing procedure used is generally summarized here.

\section{Test Summary:}

1) The wheel (test article) was installed on the test fixture frame

2) The test fixture frame was fitted with an photo reflector to measure the speed (RPM) of the test article

3) The test article was equipped with reflective tape used to trigger the photo reflector sensor and provide feedback on the wheel RPM (Figure 6.2)

4) A thermal gun (Figure 6.1.) was used to measure in real-time the temperature of the wheel surface, placed 180 degrees from the contact point, clear of the engaging spin-up fixture (Figure 6.2) 
5) Prior to engagement with the test article, the spin-up machine accelerated its Al 6061-T6 fly wheel to approximately 3400RPM, simulating a contact speed equivalent to a pod traversing the sub-track at $180 \mathrm{mph}$.

6) The spin-up machine engaged the test article with a relative velocity of $180 \mathrm{mph}$, simulating a "touchdown" loading condition at contact spin-up machine contact, shown at 15s in Figure 6.3.

It was determined that this test would provide a worst case loading condition, applying approximately $200 \mathrm{lb}$ plus high speeds instantaneously. In reality, the shock strut will begin to slowly compress at "touch-down" and the load will gradually increase on the wheel from $0-200$ $\mathrm{lb}+$ over time. This more accurate loading case was achieved during full-system testing. Test data, as recorded by the data acquisition unit, is shown in Figure 6.3. Here, the RPM of the fly-wheel (blue) is plotted along with the RPM as measured by the photo reflector of the polyurethane wheel (red). For convenience, the RPM of the polyurethane wheel was also shown as a linear velocity (green), defined as the pod speed (mph).

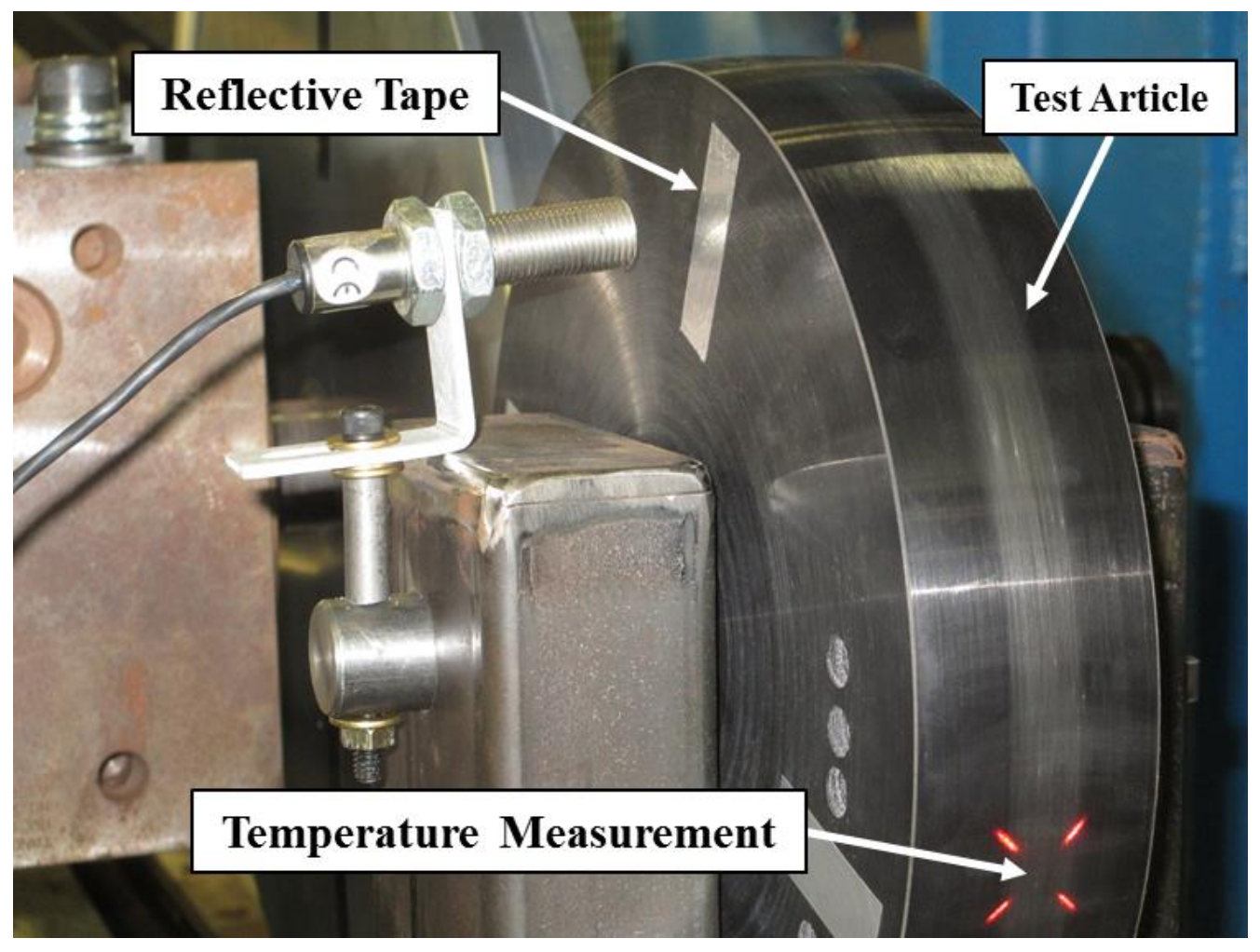

Figure 6.2: Reflector tape positioning and wheel temperature measurement region shown 


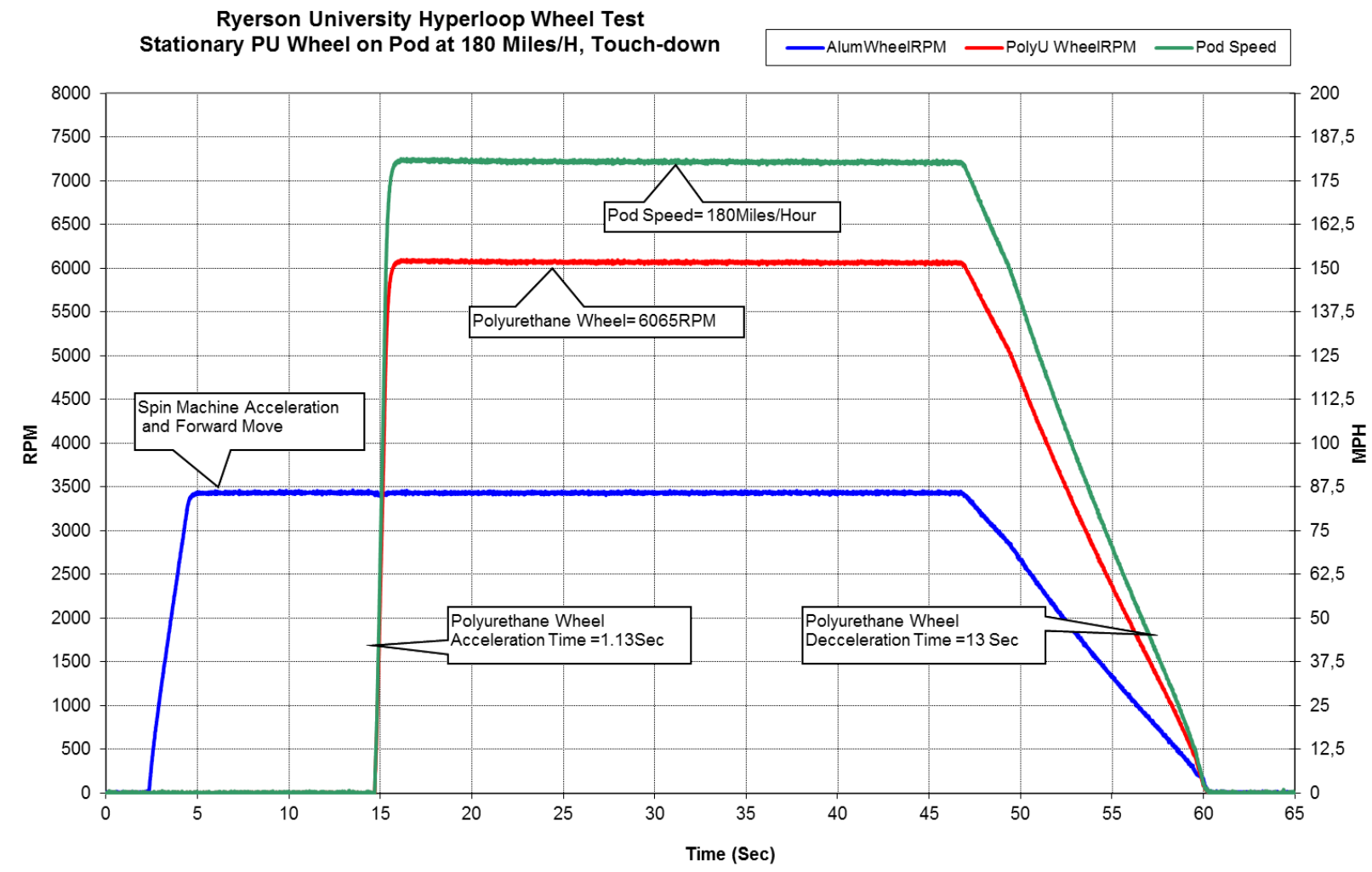

Figure 6.3: Plot illustrating Test \#1, the pre-spin of the spin-up machine, engaging the stationary pod mounted wheel at $180 \mathrm{mph}$, and braking to stop at a decceleration rate of $0.6 \mathrm{~g}$

Because of the aggressive acceleration, shown graphically in Figure 6.3, and instantaneous loading conditions described above, the following results were observed:

1) Examining the wheel outer diameter before and after engagement it was noticed that localized, and "trailing" wear marking appeared. These markings appeared to be most significant at one localized point, and trailed off across the rest of the wheel diameter, shown in Figure 6.4. This would seem to indicate the wear is caused at the point of touchdown, when the relative velocities are furthest apart. This localized "spot" wearing is likely to have been caused in part by the "instantaneous" application of $2001 \mathrm{~b}$ on the wheel outer diameter. It was noted that the temperature reading on the outer diameter, as measured by the thermal gun, indicated a peak temperature of over $176^{\circ} \mathrm{F}\left(80^{\circ} \mathrm{C}\right)$, close to the $195^{\circ} \mathrm{F}\left(90^{\circ} \mathrm{C}\right)$ operational performance limit of the material specified by Sunray Inc. [44].

2) After an initial marking was formed, the tests were repeated, and it was found that after the first surface damage had occurred, the wear on the wheel outer diameter significantly 
increased. This was observed during the second test when a thin layer of polyurethane was "shed" from the surface of the wheel and deposited on the spin-up fixture's aluminum flywheel, shown in Figure 6.5. This event was also observed using a high-speed camera.

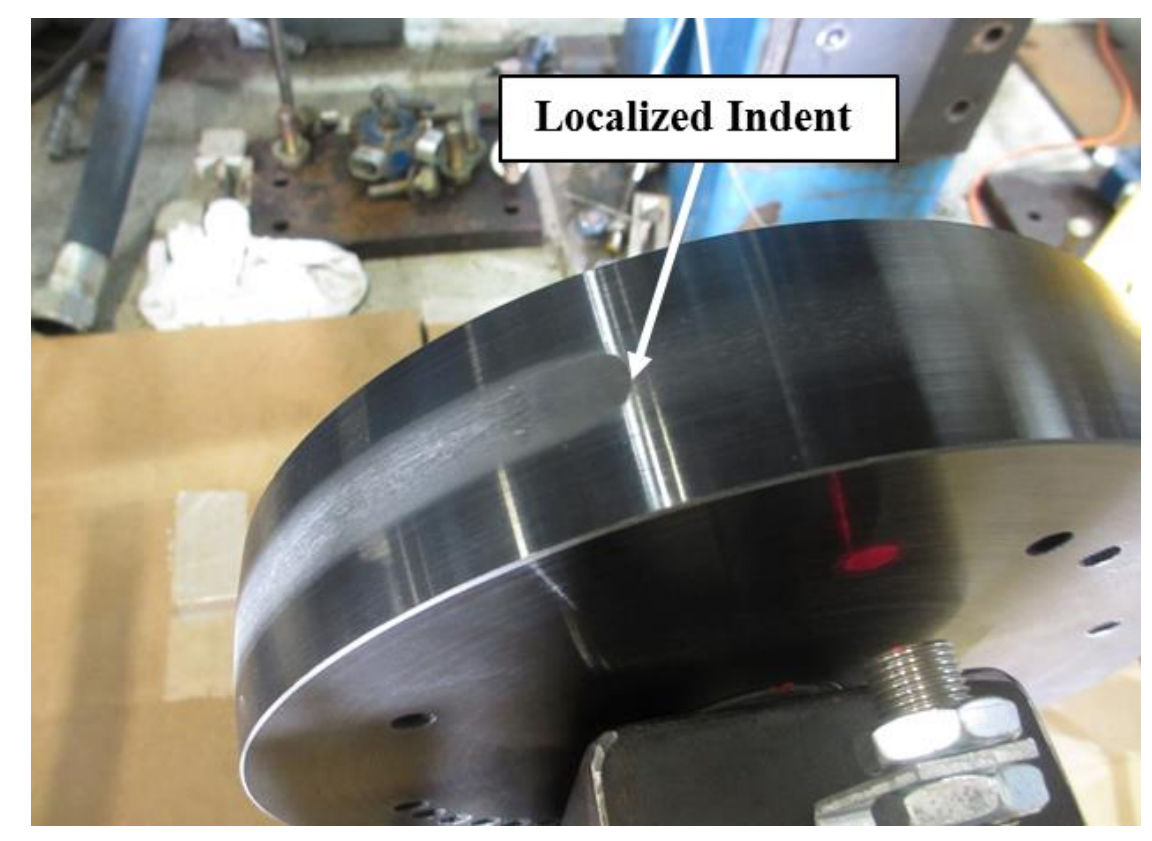

Figure 6.4: Localized wear marking, followed by a trail-off effect, observed after Test \#1
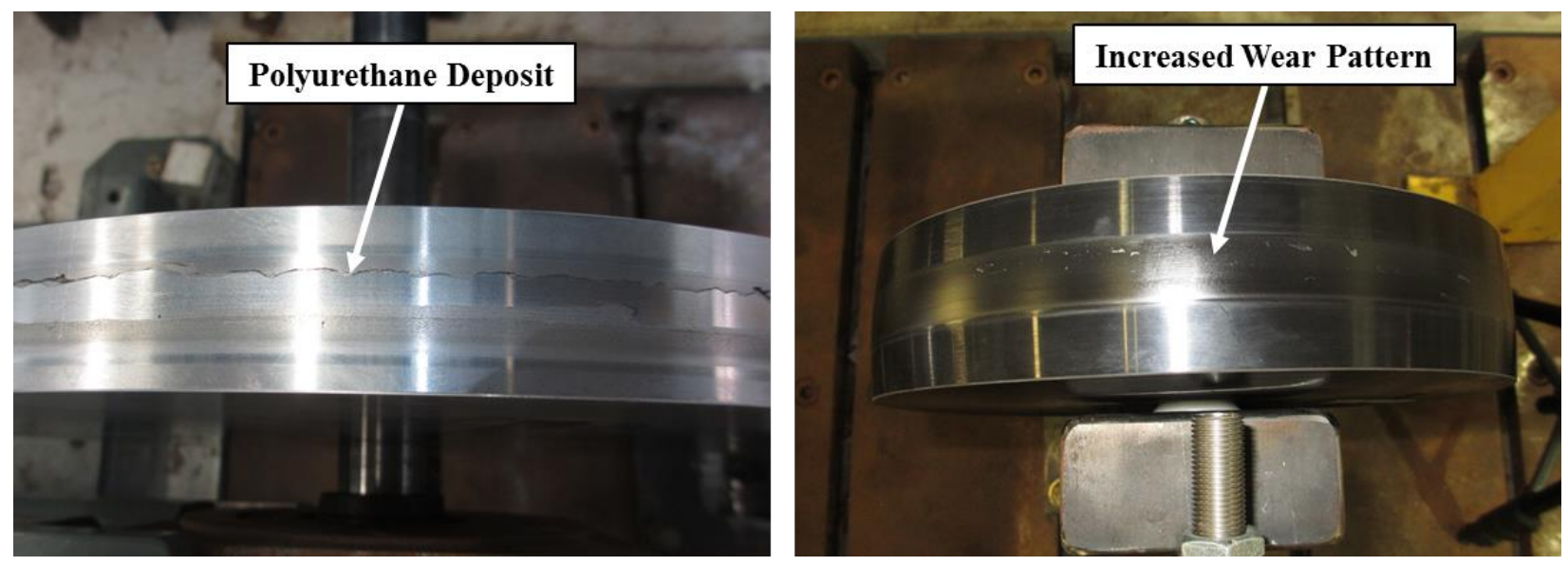

Figure 6.5: Thin polyurethane deposited on the aluminum fly-wheel observed after Test \#2, wheel deterioration increased under same loading and speeds 
3) In subsequent testing, the wheel was accelerated in the same fashion as Figure 6.3 and after reaching $180 \mathrm{mph}$, the spin-up machine was "instantaneously" disengaged from the polyurethane wheel. This was meant to simulate the wheel "lifting off" from the pod sub-track once the pod has reached the usable speed of its primary levitation system. The test showed that the sudden disengagement caused the wheel to ovalize immediately after the disengagement of the load, and expand and contract in an oscillatory manor. This was recorded via the use of a high-speed camera and its effects were further documented through before and after photos, shown in Figure 6.6, indicating an expansion and contraction had occurred. Furthermore, subsequent tests of the same nature, but at speeds of $220 \mathrm{mph}$, showed signs of roller bearing rotation within its housing and loss of grease through the bearing seal cover due to the high rotation speeds of the bearing, shown in Figure 6.7.
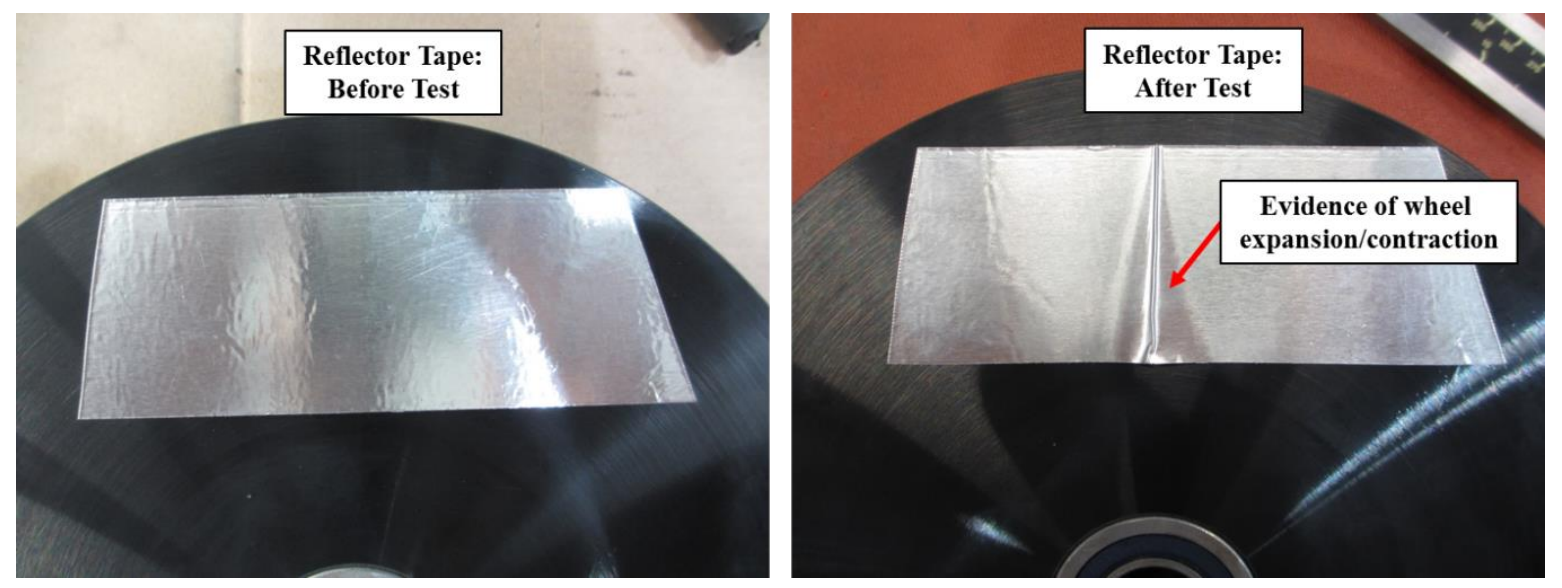

Figure 6.6: Shifting of the reflective tape caused by the expanding and contracting outer diameter of the wheel after disengagement from the spin-up machine at $180 \mathrm{mph}$

In conclusion, the testing performed on the polyurethane wheel suggested that the solid polyurethane frame was unstable at high speeds and moderate loading. The disengagement at retraction should not cause the wheel to oscillate violently and cause bearing dislodgement from its housing frame, in this case, the solid polyurethane frame. Hence, the aluminum framed wheel discussed in Section 4.1.3, should be considered for future designs.

On the other hand, a decision regarding the wear properties was not made at this time, as it was believed the addition of the shock absorbing unit would reduce the wear at touchdown caused by 
a rigidly fixed wheel acted on by an "instantaneous" contact load. Furthermore, tests conducted on the spin-up machines load application, graphed in Figure 6.8, indicate the possibility of a large load spike at initial contact due to the spin-up machines forward inertia, before stabilizing at 200 lb.

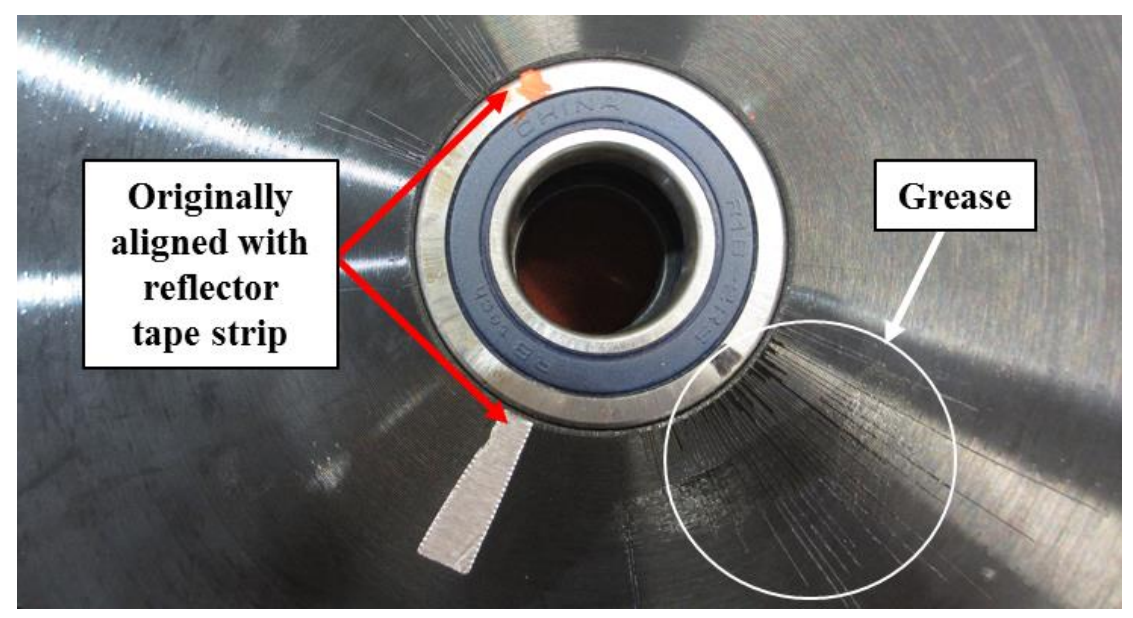

Figure 6.7: Grease splatter and roller rotation evident as traced by the orange anti-rotation paint

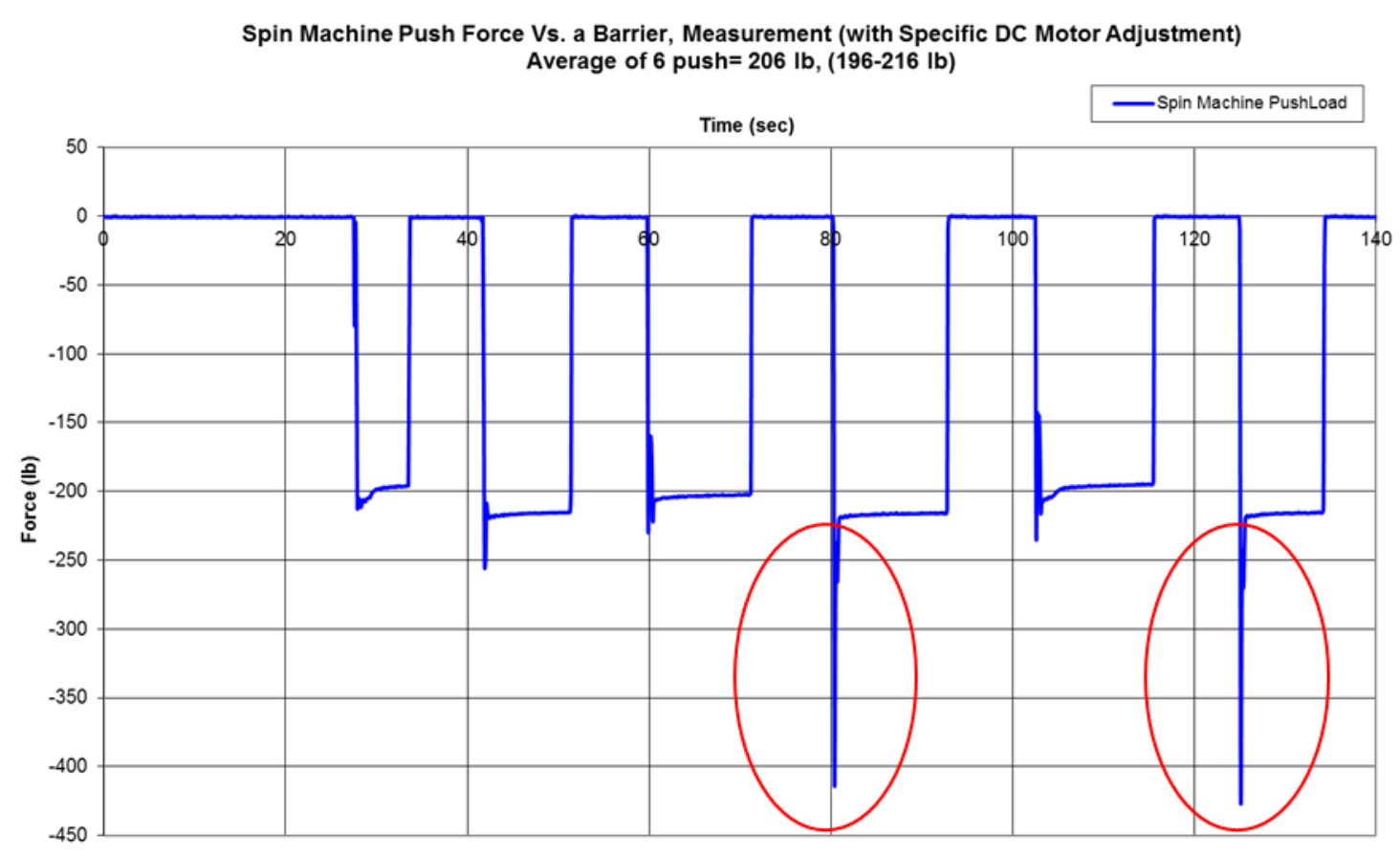

Figure 6.8: Graph displaying the average load measured by the load cell as applied by the spin machine, load spikes at initial contact encircled in red 


\subsection{Gas Spring Testing}

Testing was performed on the shock strut to verify the behaviour of its gas spring, as defined using Dynatool $^{\odot}$ and the preliminary isothermal calculations discussed in Chapter 5. The testing was performed a full day after servicing (filling the strut with oil and nitrogen) to allow the gas and oil to mix and settle. The internal pressure was measured using a pressure transducer mounted at the top of the shock strut through its filler valve prior to testing. In each case, for all five shock absorbers (HDWS\#1 through HDWS\#5), the measured values were compared to the theoretical values generated by Dynatool ${ }^{\odot}$. The test fixture was configured as shown in Figure 6.9 and included a hydraulic actuator to apply the compressive force on the shock strut measured through a load cell mounted between the shock strut and the hydraulic actuator rod-end. The test setup, as shown in Figure 6.9, was re-oriented vertically, as shown in Figure 6.10 for the final testing to ensure that the oil remained at the lower half of the strut and the gas in the upper half.

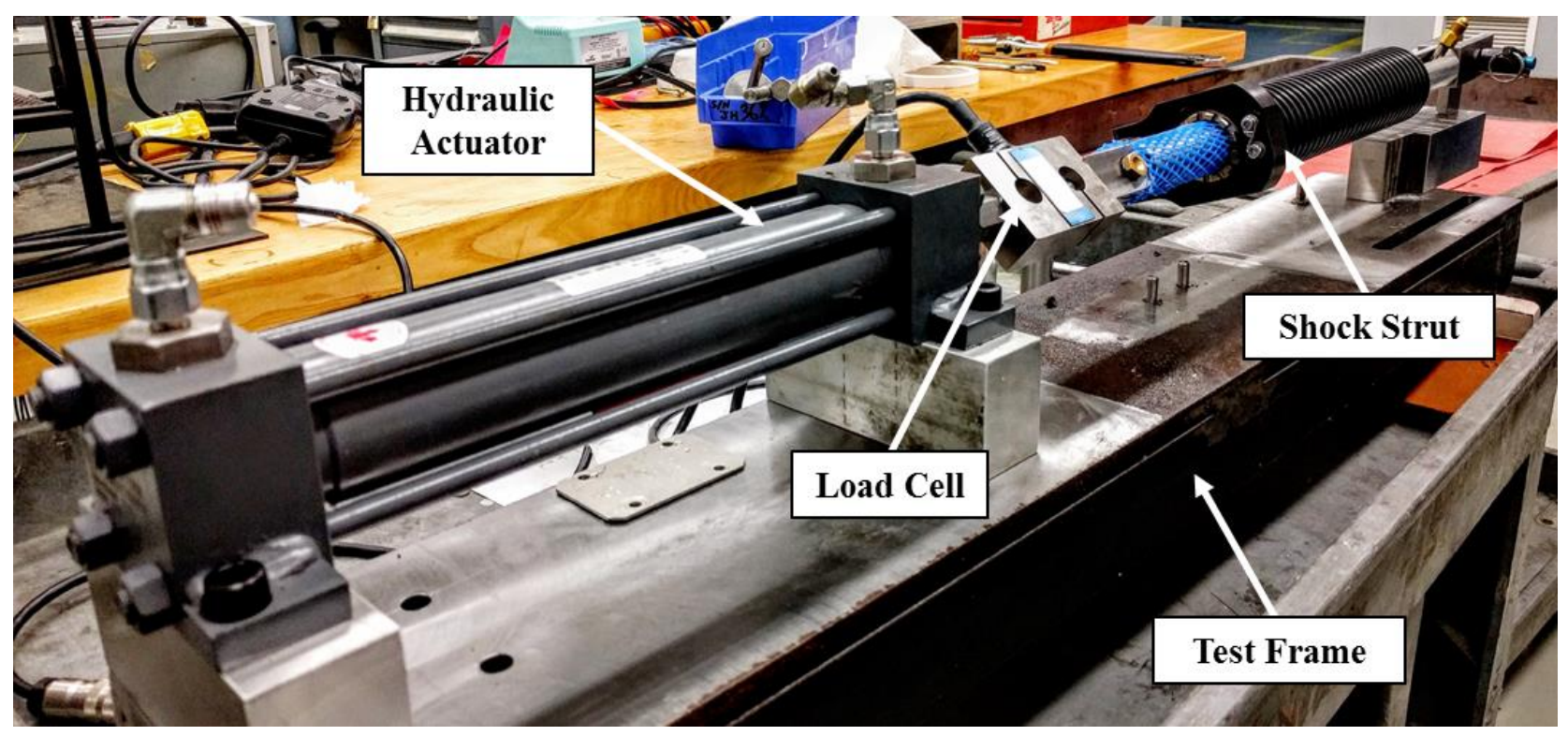

Figure 6.9: Shock absorber gas spring verification initial, initial setup 


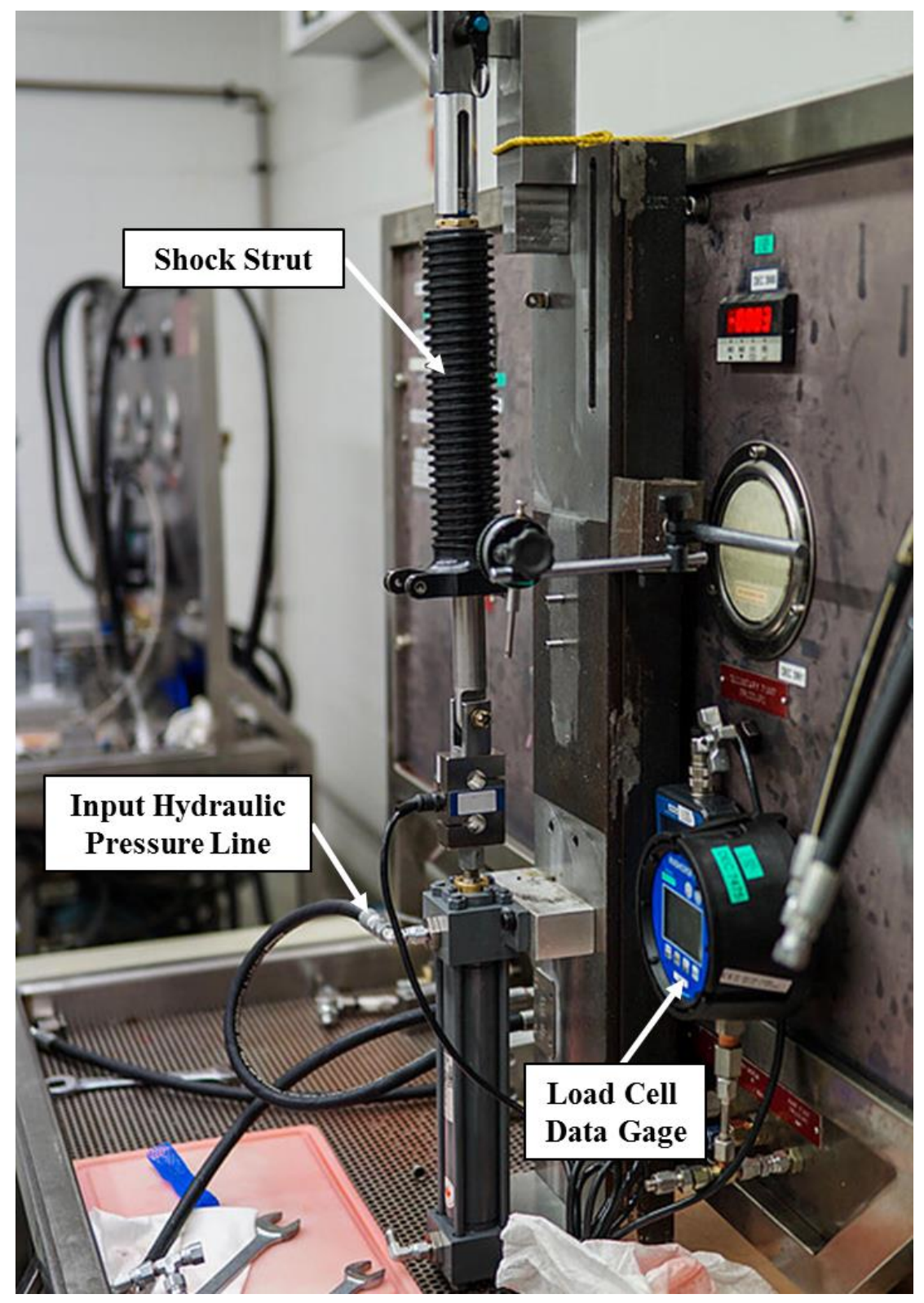

Figure 6.10: Shock absorber gas spring verification test, final setup

Using a pressure control valve on the hydraulic load input actuator, the piston was compressed and the load values as a function of stroke were measured and recorded. Since the measuring operation was done manually, using a digital Vernier caliper and reference points on the bottom of the shock strut cylinder and top of the load cell, only specific strokes were identified to be measured in the interest of time. These key strokes are defined in Table 6.1 and their data for HDWS \#4 is recorded 
for reference. As can be seen by the comparison of the collected data and the theoretical data, some variation exists.

It was observed that the vertical configuration of the test stand, shown in Figure 6.10, induces a pre-load on the load cell equal to the weight of the shock strut, filled with oil and nitrogen, was measured to be $9.385 \mathrm{lbm}$ for HDWS \#4. This adjustment in the load is accounted for in Table 6.1 and its value is compared to the theoretical data produced in Dynatool ${ }^{\odot}$.

Table 6.1: Gas spring test verification results vs. Dynatool ${ }^{\odot}$ theoretical data

\begin{tabular}{|c|c|c|c|c|}
\hline \multirow[b]{2}{*}{ Stroke (in) } & \multicolumn{2}{|c|}{ HDWS \# 4} & \multirow[b]{2}{*}{$\begin{array}{c}\text { Static curve, } \\
\text { Dynatool@, (lb) }\end{array}$} & \multirow[b]{2}{*}{ \% Deviation } \\
\hline & $\begin{array}{c}\text { Measured } \\
\text { Load (lb) }\end{array}$ & $\begin{array}{l}\text { Adjusted Load } \\
\text { (lb) }\end{array}$ & & \\
\hline 1 & 97 & 87.615 & 83.7 & 4.468412943 \\
\hline 2 & 189 & 179.615 & 168.8 & 6.021212037 \\
\hline 2.1 & 202 & 192.615 & 186.2 & 3.330477896 \\
\hline 2.2 & 219 & 209.615 & 207.3 & 1.104405696 \\
\hline 2.3 & 248 & 238.615 & 236.3 & 0.970182092 \\
\hline 2.4 & 277 & 267.615 & 266.3 & 0.491377539 \\
\hline 2.5 & 314 & 304.615 & 309.2 & 1.505178668 \\
\hline 2.6 & 364 & 354.615 & 367.2 & 3.54891925 \\
\hline 2.7 & 429 & 419.615 & 449.6 & 7.145836064 \\
\hline 2.8 & 516 & 506.615 & 574.4 & 13.37998283 \\
\hline 2.9 & 663 & 653.615 & 780.7 & 19.44340323 \\
\hline 3 & 1077 & 1067.615 & 1159.9 & 8.644033664 \\
\hline
\end{tabular}

It can be said that the deviation in the measured values to that of the theoretical one is largely related to the human error caused by the manual measuring of the stroke using a Vernier caliper. This is a reasonable assumption when one examines the initial and final measurements and recognize the relatively small percent deviation. Another source of error can be related to the servicing process. During this process, it is imperative that the strut be filled with oil and it be serviced so that all trapped air is removed from the cylinder. This is especially important here since the impact of a discrepancy in the initial volume of nitrogen vs. oil can have a significant effect when dealing with the relatively small-scale of the design. Lastly, the theoretical gas spring curve utilizes nominal geometrical values for each of the input features, so a part used with a dimension 
other than nominal, but still within the specified tolerances, could in theory produce a slightly different gas spring curve.

For further reference, the measured data is recorded in the Appendix D for the reader's interest. This data is in an "as-recorded" state and has not been adjusted to account for the weight of the shock strut which, as mentioned previously, creates an initial offset in the load cell due to the vertical testing position.

\subsection{Full Single-System Testing}

The purpose of the full single-system testing, shown in Figure 6.11, was to confirm the performance of the HDWS when subjected to high-speeds and representative loading conditions. To perform this, it was necessary to impose a typical Hyperloop pod mission profile as defined within the framework of the SpaceX Hyperloop Competition, for which the present prototype was designed. The goal of these tests was to verify: the performance of the Deployable and Retractable Shock Strut; that the wear on the polyurethane wheels would be reduced when used in combination with the shock strut; and that the sensor hardware bracketry was adequately designed to support the equipment under vibration loading.

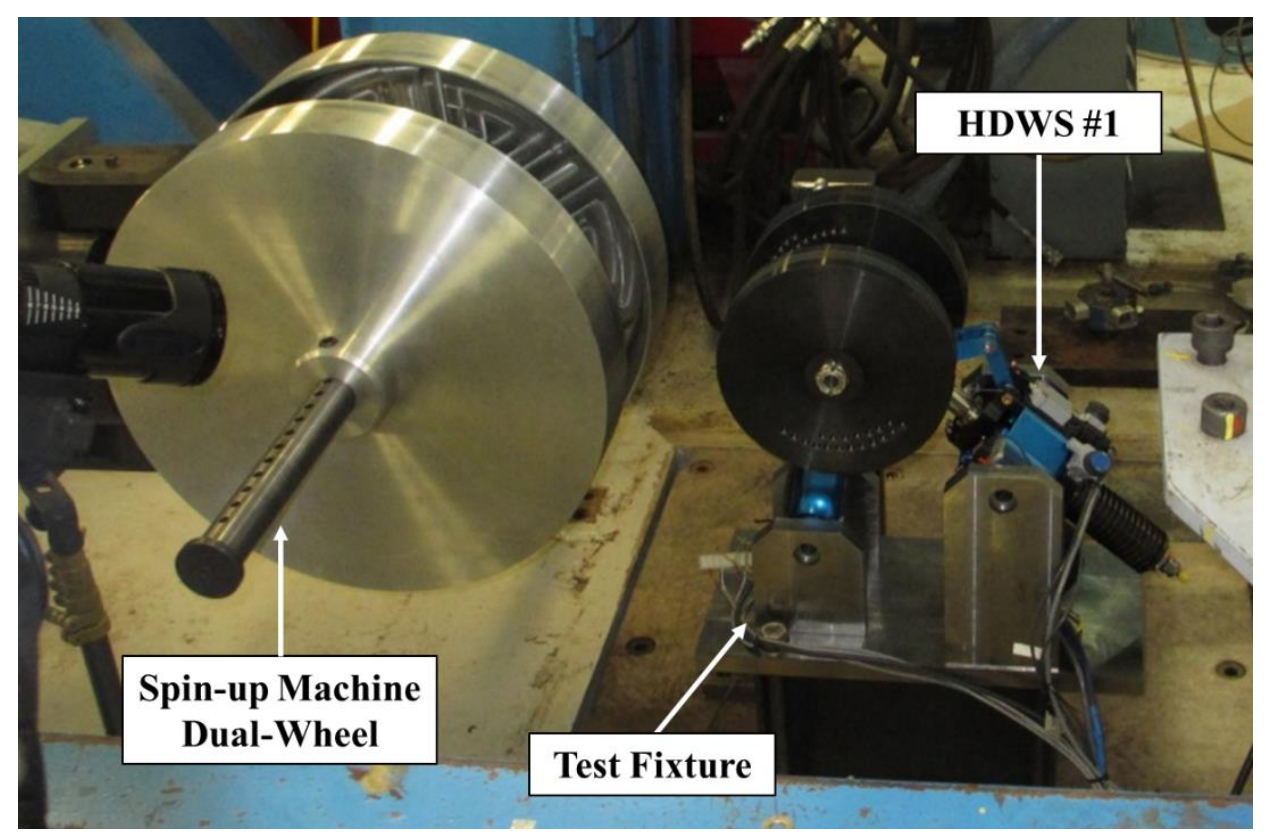

Figure 6.11: HDWS full system test configuration (temperature sensors out of view) 
The testing was further defined by the following success criteria:

\section{Polyurethane Wheel Inspection:}

Polyurethane wheel inspection indicating minor distributed wear was deemed acceptable. Any wear causing a visual rotational imbalance to the polyurethane wheel when spun, or surface temperatures exceeding $195^{\circ} \mathrm{F}\left(80^{\circ} \mathrm{C}\right)$, during nominal mission profile conditions, causing the polyurethane wheel to melt, was considered failure. The wheel assemblies bearing-wheel interface was to be examined and documented prior to each test to monitor the unseating of the bearing from the wheel bore shown in the wheel component tests.

\section{Fastener Inspections:}

Visual inspections were performed and documented at the start and completion of every test. Fasteners, locked and marked with torque seal (orange paint marked, Figure 6.12), were monitored exclusively. If an inspection reveals that a fastener has come lose then that fastener must be documented with the data corresponding to that test (pod speed, G's, loading).

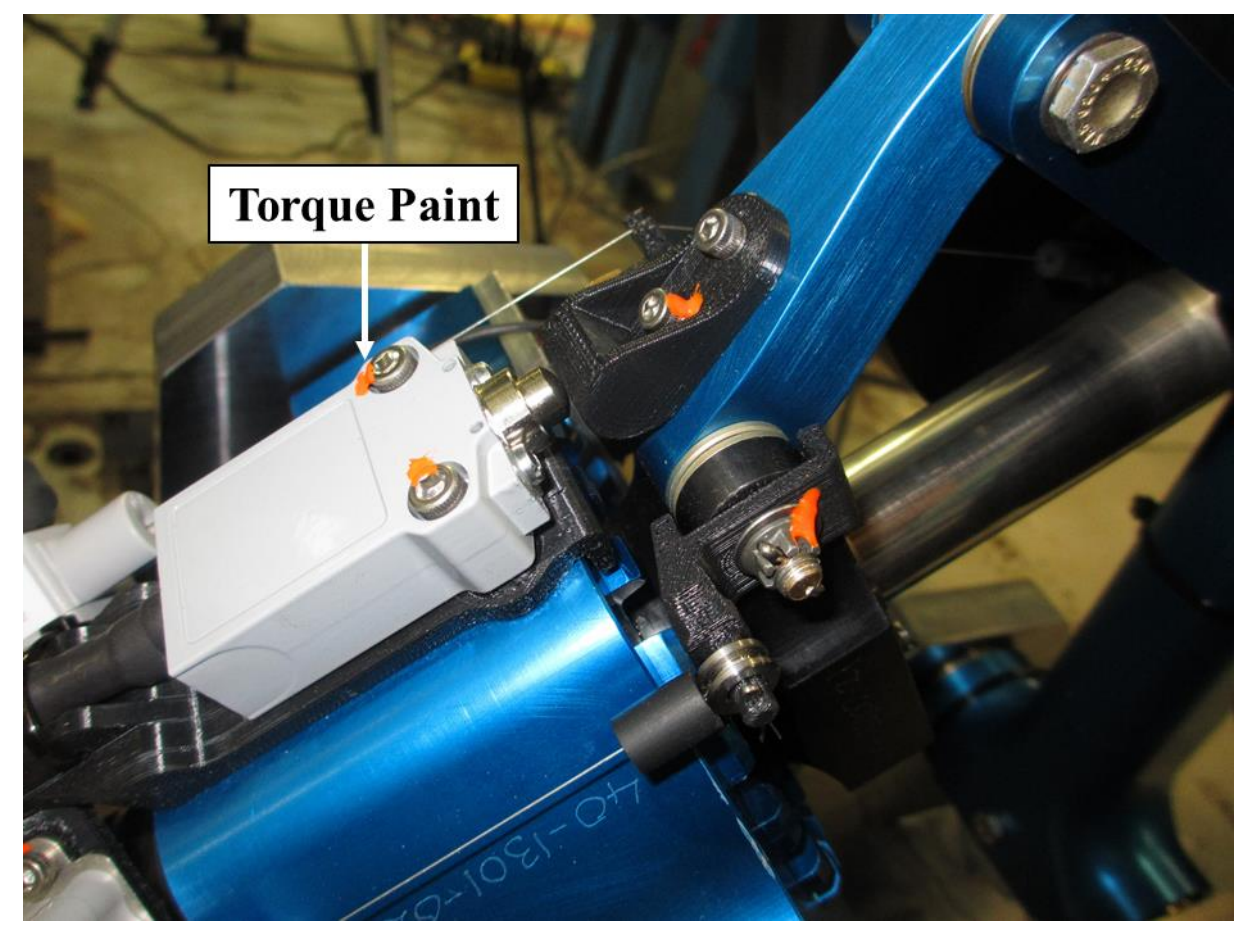

Figure 6.12: Example of torque paint (orange) applied, used to track loosening of the hardware 


\section{External Bracketry Inspections:}

Visual inspections were preformed and documented at the start and completion of every test. Brackets used to mount and route sensor equipment (potentiometers, limit switch) were inspected for visual damage (e.g. delamination, thread shearing).

With all the above-mentioned success criteria in mind, the testing mission profile is now defined as was designed for the testing. Referencing Figure 6.13, the mission profile consists of an acceleration phase (A), cruise phase (C) and deceleration phase (D) which ends with successful stoppage of the pod's forward motion. The HDWS begins the mission profile in a deployed and locked position defined by static stroke. As the pod moves through the mission profile the HDWS goes through a retraction phase $(\mathbf{R})$, that occurs part way through the acceleration and an extension phase (E) during deceleration. The HDWS remains deployed and locked at static load stroke at completion of the mission profile.

The major phases of the mission profile, described above, were further defined in the testing procedures to include: variations of the acceleration and declaration rate, varying the load applied to the wheel system (generated by the wheel system compressing to a desired stroke correlating with a theoretical load as found on the gas spring curve), and varying the pod speed at which the HDWS would extend and retract to and from the sub-track (in this case aluminum fly-wheels as shown in Figure 6.11). For the sake of brevity, further details are omitted here and a sample is shown.

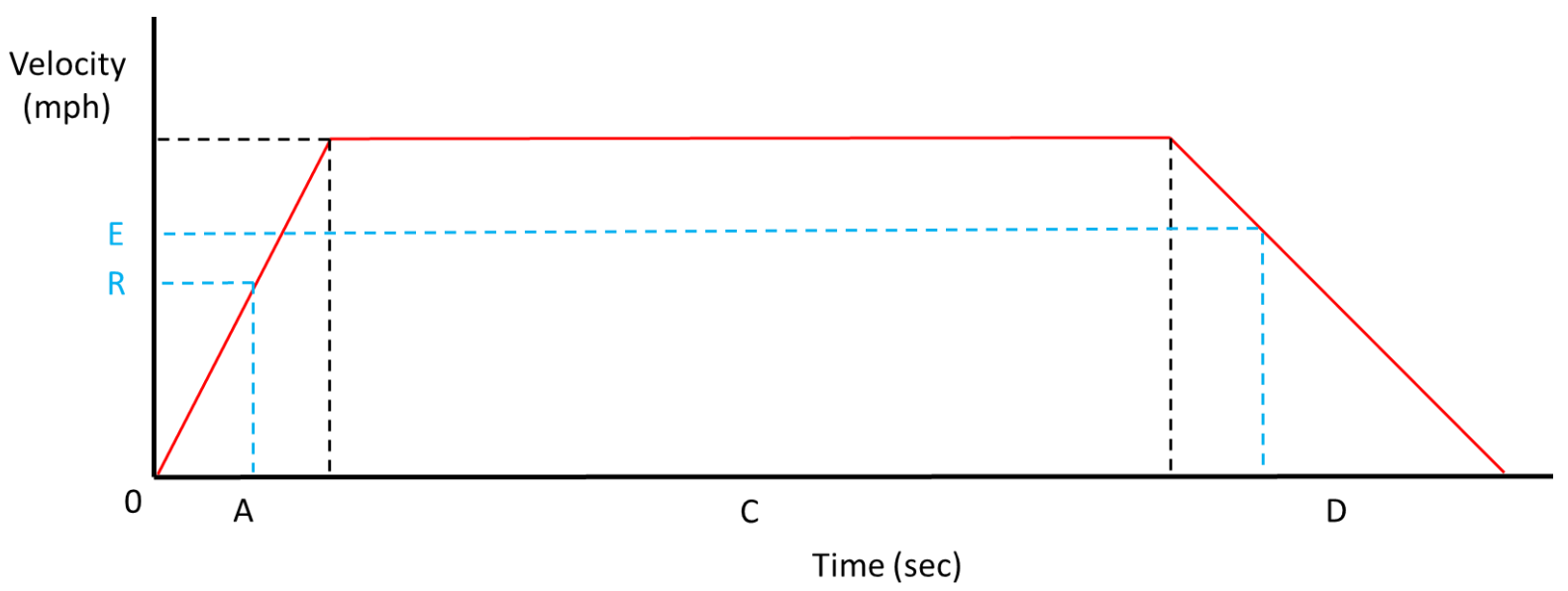

Figure 6.13: General pod mission profile inidcating key regions/events of interest (not to scale) 
As an example, a test result from the HDWS single unit test, Test Case \#4, is shown in Figure 6.14. The graph illustrates the input command from the spin-up machine's command module, shown in the graph legend as CMD (black). This represents the "commanded" profile for the spin-up machine to follow, where as the actual profile followed by the spin-up machine is defined by SM (purple). The difference between the commanded CMD and the actual SM can be caused by data lag but was likely to have been caused by the inertia of the motor and fly-wheel assembly. The graph shows that the HDWS (red) accelerated, approximately linearly, up to a pod speed (blue) of approximately $130 \mathrm{mph}$ and proceeded to retract from the sub-track (fly-wheels) while the pod accelerated up to approximately $190 \mathrm{mph}$, before beginning to decelerate at 18s. At approximately $78 \mathrm{mph}$ the HDWS touched down on the sub-track (aluminum fly-wheels) and was decelerated while engaged until braking.

Hyperloop Deployable Wheel System:

Full System Test - Test Case \#4: $2.4 \mathrm{~g}$ Acceleration and Deceleration

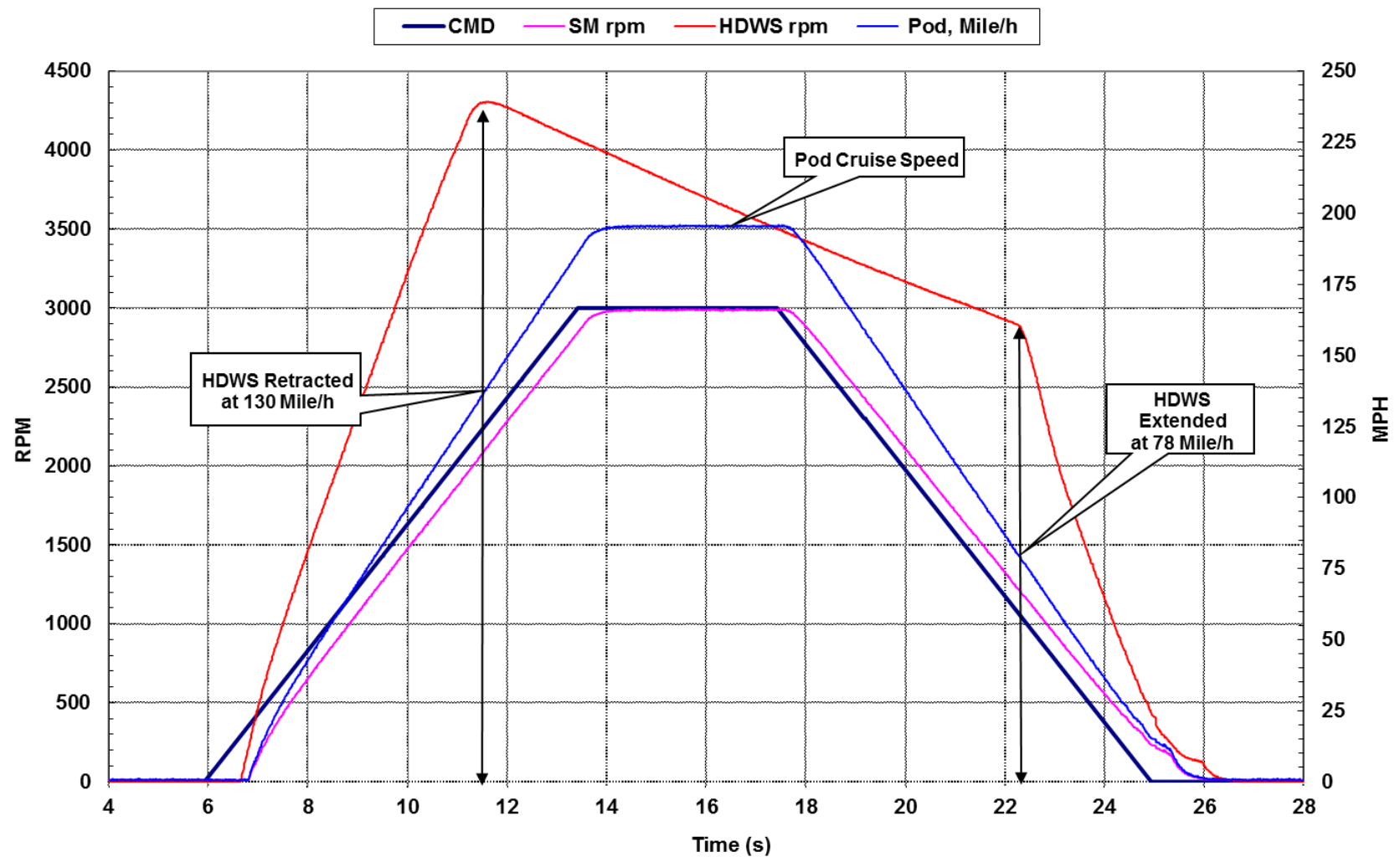

Figure 6.14: Graph of the data recorded during Test Case \#4, with the HDWS subjected to $2.4 \mathrm{~g}$ acceleration and decceleration, key events labeled for clarity 
As was hypothesized, the wear on the wheels (Figure 6.15) was reduced to a slight "polish" due to the "gradual" loading and unloading capabilities of the retracting and extending shock strut. The temperature, monitored on the outer diameter of the wheel, was found to peak $79.2^{\circ} \mathrm{F}\left(26.2^{\circ} \mathrm{C}\right)$ from a laboratory temperature of $70.5^{\circ} \mathrm{F}\left(21.4^{\circ} \mathrm{C}\right)$. This was a significant change from the $176^{\circ} \mathrm{F}$ $\left(80^{\circ} \mathrm{C}\right)$ peek temperature measured during the wheel component testing without the shock absorber.

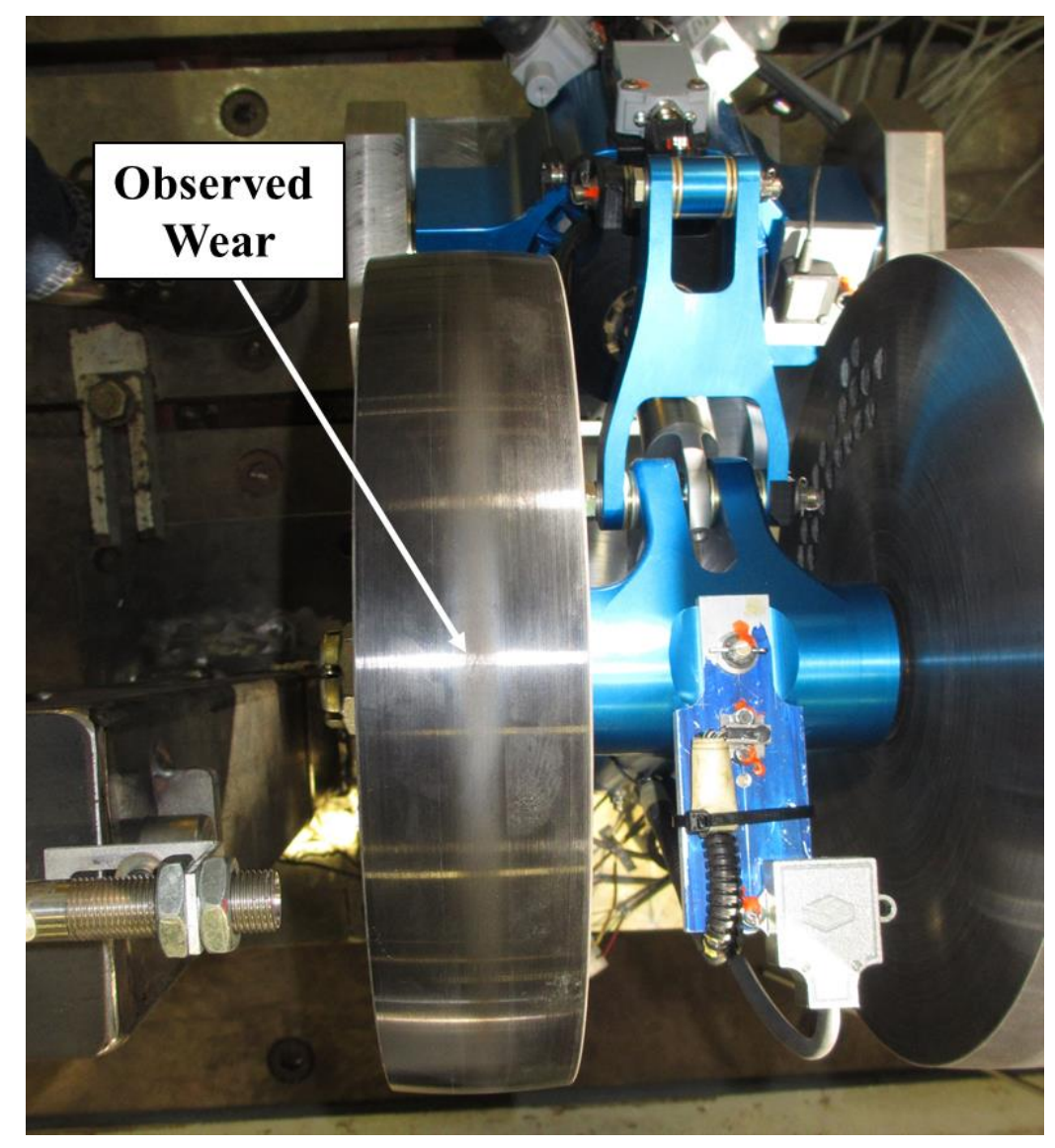

Figure 6.15: An example of typical wheel wear as shown after full systems testing

In all cases, it was shown that the spinning of the unloaded polyurethane wheel after being disengaged from the fly-wheel, caused a rotation of the wheel bearings relative to its installed position in the wheel housing. This confirmed that future considerations shall include the design of a custom wheel with a metallic frame but has not ruled out the use of a polyurethane liner on the exterior of a custom wheel, as proposed in Chapter 4. 


\subsection{Dummy Pod Test (4 Units)}

To address the need for a demonstration pod after the $\mathrm{U}$ of $\mathrm{T}$ departure, a testing chassis or "dummy pod" was developed. The dummy pod was equipped with mounting points for four HDWSs and provided a flat bed (at the top of the dummy pod) where a $200 \mathrm{lbm}$ dummy mass was located. This dummy mass, equipped with wheels, was traversable on the flat bed providing the effect of CG imbalance and thus altering the distribution of the pod loading. The testing pod was further equipped with four laser sensors distributed at each corner of the pod. These sensors provided accurate and active feedback for the pod height above the dummy sub-track. The dummy pod's features enabled the successful development and testing of the HDWS electronic and control system as conceived by Adhikari [40] and developed Shonibare [16].

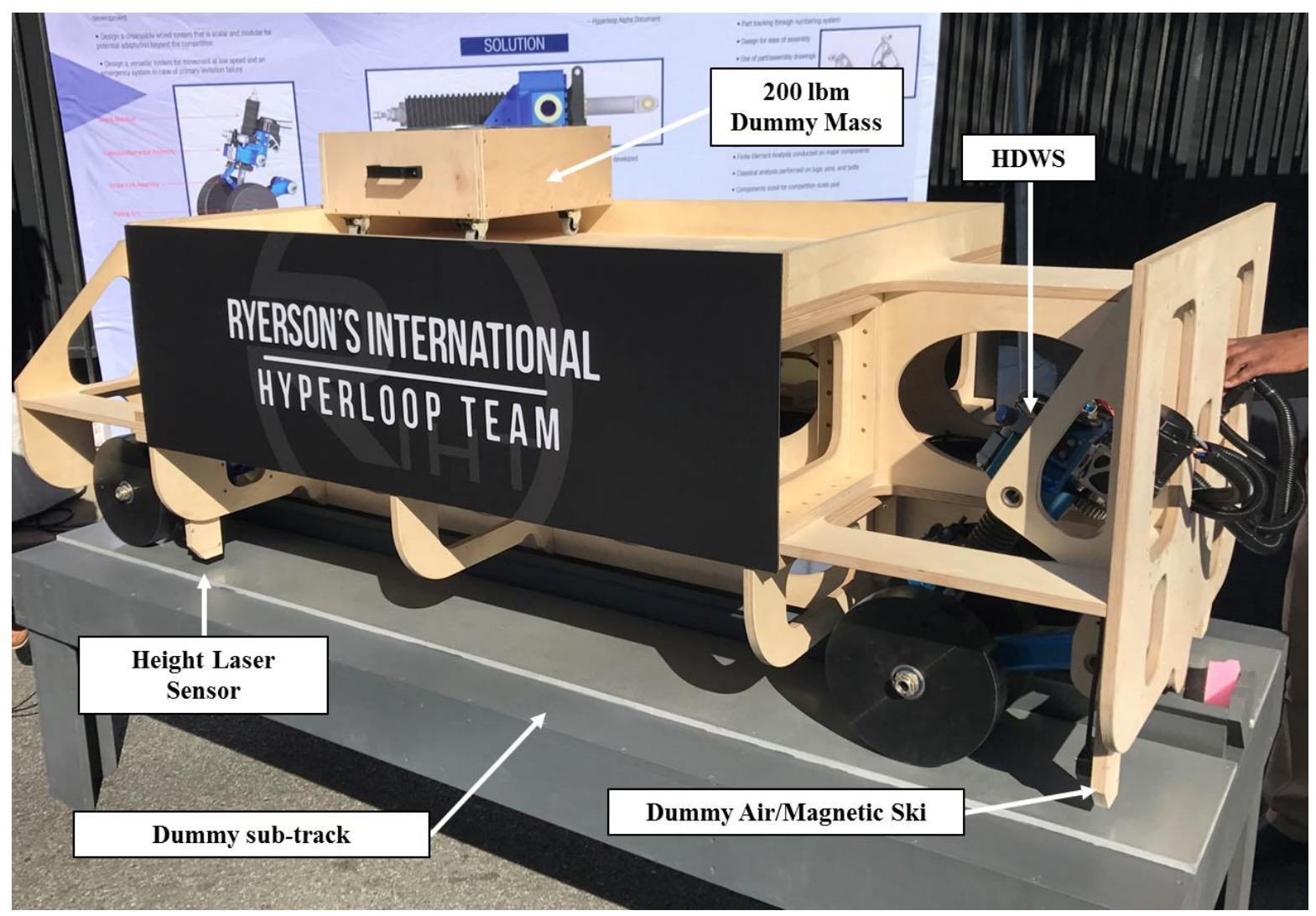

Figure 6.16: Dummy pod test bench, control system testing fixture with $200 \mathrm{lbm}$ mobile dummy mass to simulate shifts in $\mathrm{CG}$ 


\section{Concluding Remarks}

\subsection{Conclusion}

The present work set out to design and develop a deployable wheel system for Elon Musk's proposed high-speed transportation system, the Hyperloop. Since modern wheeled systems of the retractable and extendable type operate within relatively large spaces, the challenge was to develop a new method of performing these operations, electrically, within a Hyperloop pod framework. In doing so, the requirements, guidelines and constraints imposed on the design of a Hyperloop subsystem were explored. These subjects were then addressed using the most up-to-date Hyperloop research and, where applicable, the knowledge gap was filled by the author with applicable aerospace engineering design and development practices and standards. By applying key aspects of landing gear design theory to the Hyperloop, a unique, electrically based, system architecture was proposed to address the gap in existing retractable and extendable wheel system design.

In addressing the technical design challenges associated with the development of a Hyperloop wheel system architecture, a highly iterative engineering design process was employed demonstrating the multi-disciplinary aspects of the present engineering problem. Engineering design management was used to establish a product organization structure, defining the baseline numerical configuration and enabling the mixing and matching of components while tracing the configuration with ease - extremely important in a research and development project. The detail design of the system's shock absorbing mechanism utilized compressible adiabatic and isothermal gas theory, in combination with geometric, material selection and structural analysis considerations, all resulting in the fabrication of a complete working product.

This work led to an innovative, patent-pending deployable and retractable shock strut, built for the

author's Hyperloop Deployable Wheel System baseline architecture and capable of addressing the need for small volume operation, utilizing only electrical power to operate, and incorporating active control features enabling active height stabilization. 


\subsection{Future Work}

Ideas for areas of further development and prototype research can be defined within three broad categories: Wheels and brakes, Hybrid Extension and Retraction, and Electronics and Controls.

\section{Wheels and Brakes}

1. Investigate the possibility of integrating an electric braking system directly into the wheels

2. Design a customized wheel/tire for Hyperloop track and operation conditions

3. Investigate the possibility of direct drive technology integration into the axle, enabling low speed propulsion

4. Green power generation methods for a sustainable power mode, utilizing regenerative breaking Hybrid Extension and Retraction System

1. Investigate the custom design of a compact, dual redundancy motor for safety and reliability

2. Re-design the retract housing for integrated sensors, increasing sensor robustness, for measuring stroke in real-time, indicating full retraction, stowage, WOW, etc.

3. Explore the addition of a contractible and extendable bellow sleeve, as demonstrated by Bradford [66], to protect the ACME cylinder from dust and debris.

\section{Electronics and Controls}

1. Refine and develop a commercially viable control and electronics system for single unit and multi-unit operation

2. Design electronic systems for operation in a near-vacuum environment (utilize pre-existing methods, standards, etc.) 


\section{Appendix A: HDWS Component Drawings}

This page was intentionally left blank 


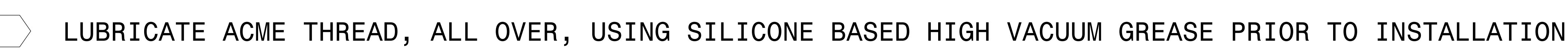

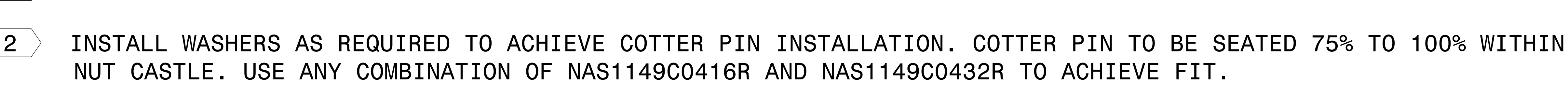

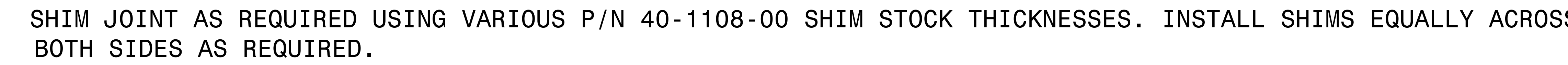

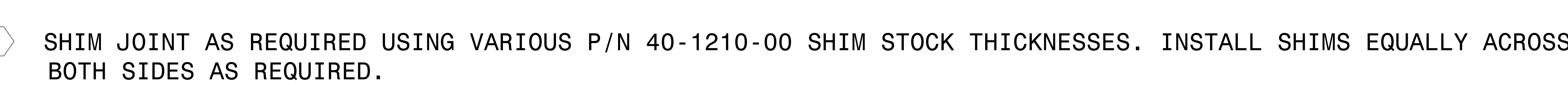

DEFöM COTTER PIN AFTER InSTALAATION.

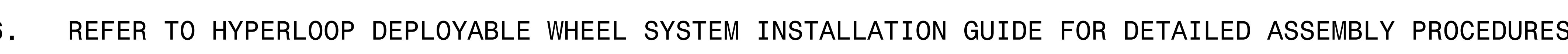
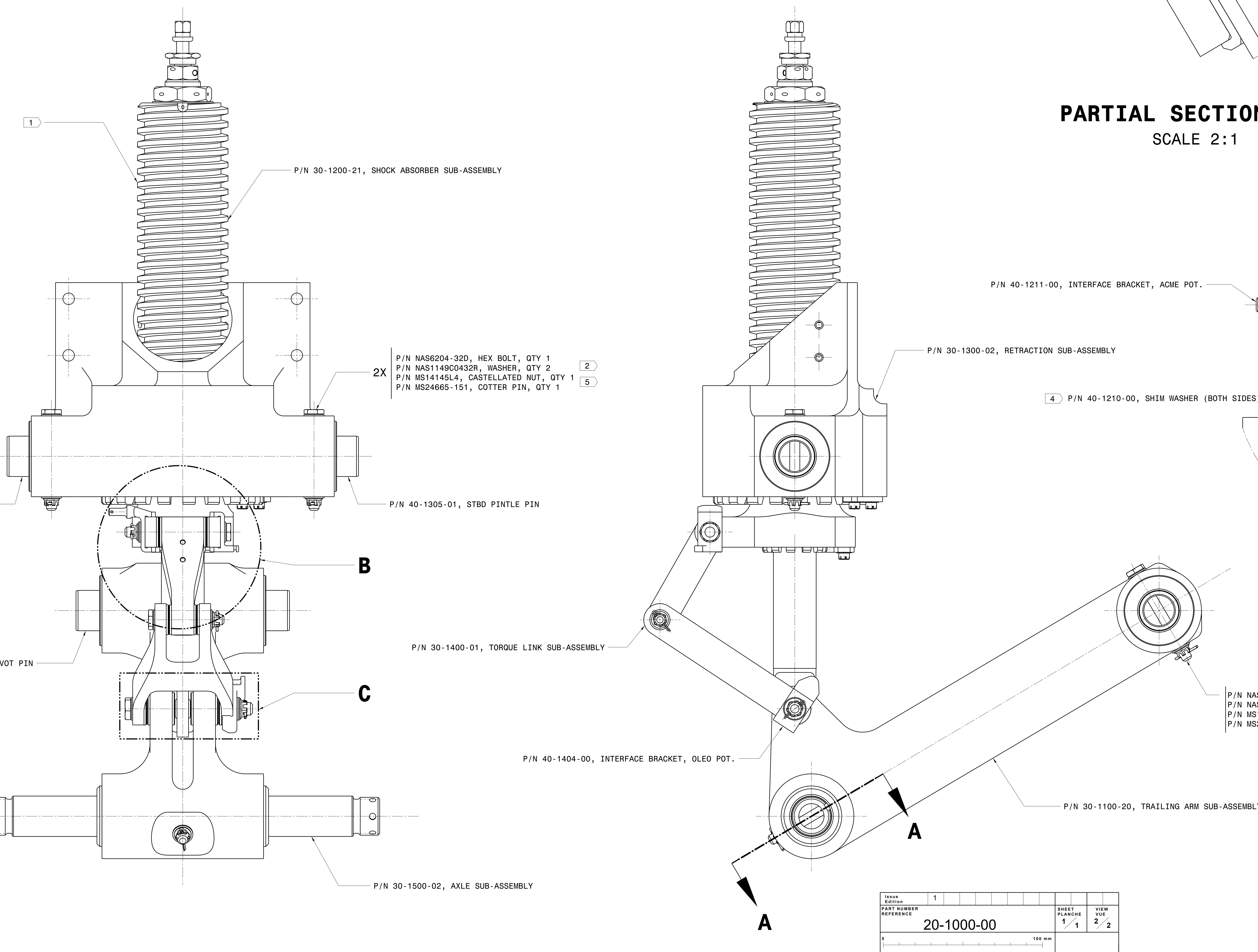

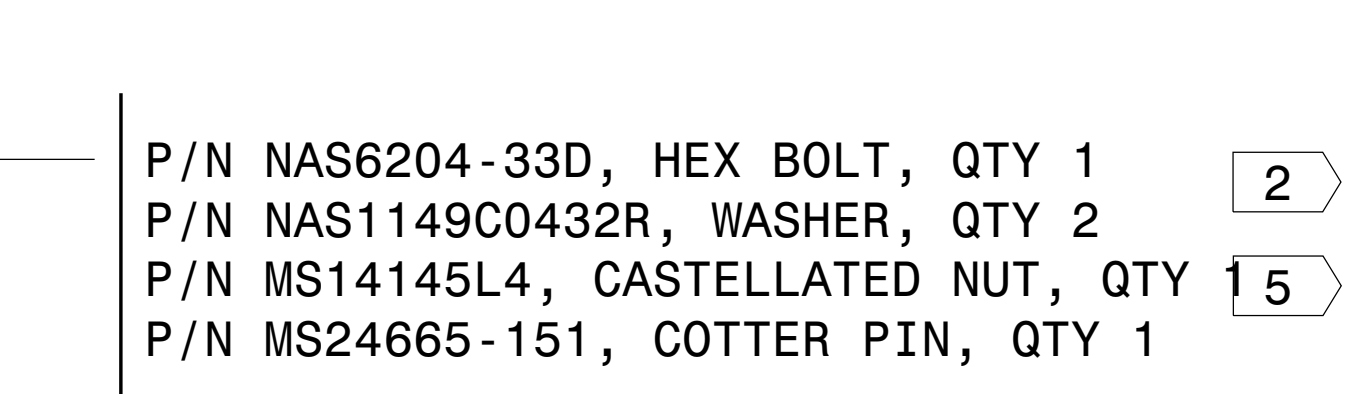

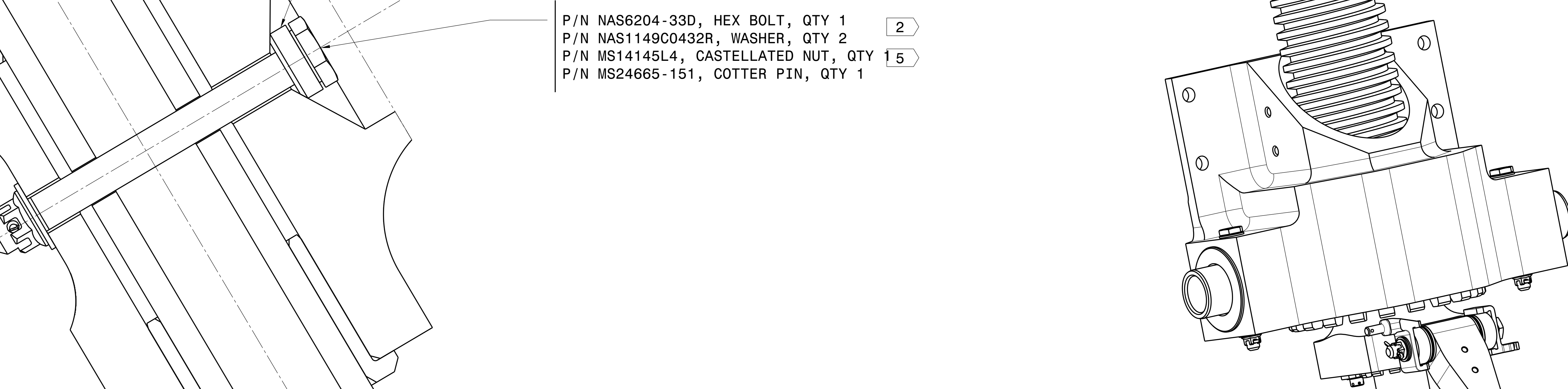

a) (D)

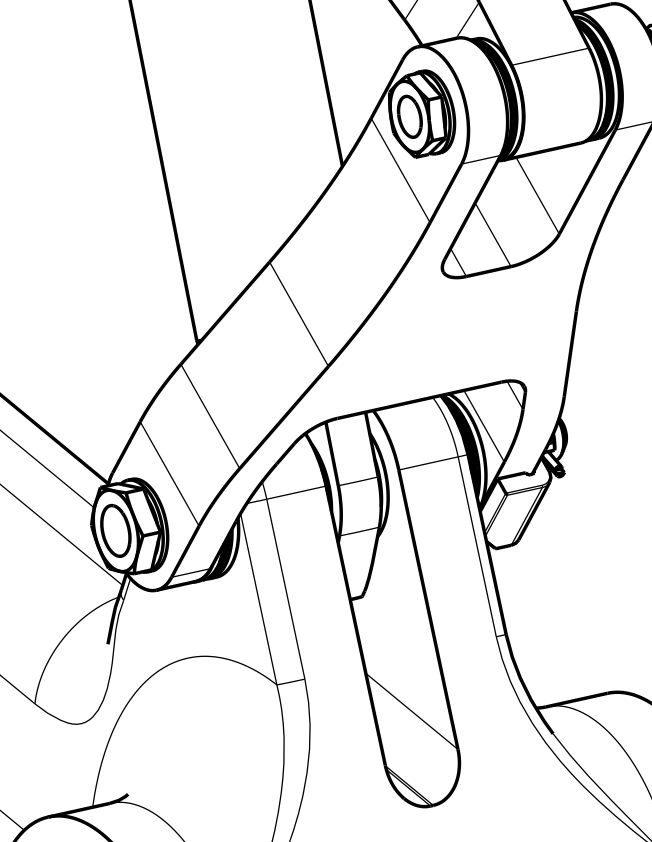

(1)

3D VIEW 1

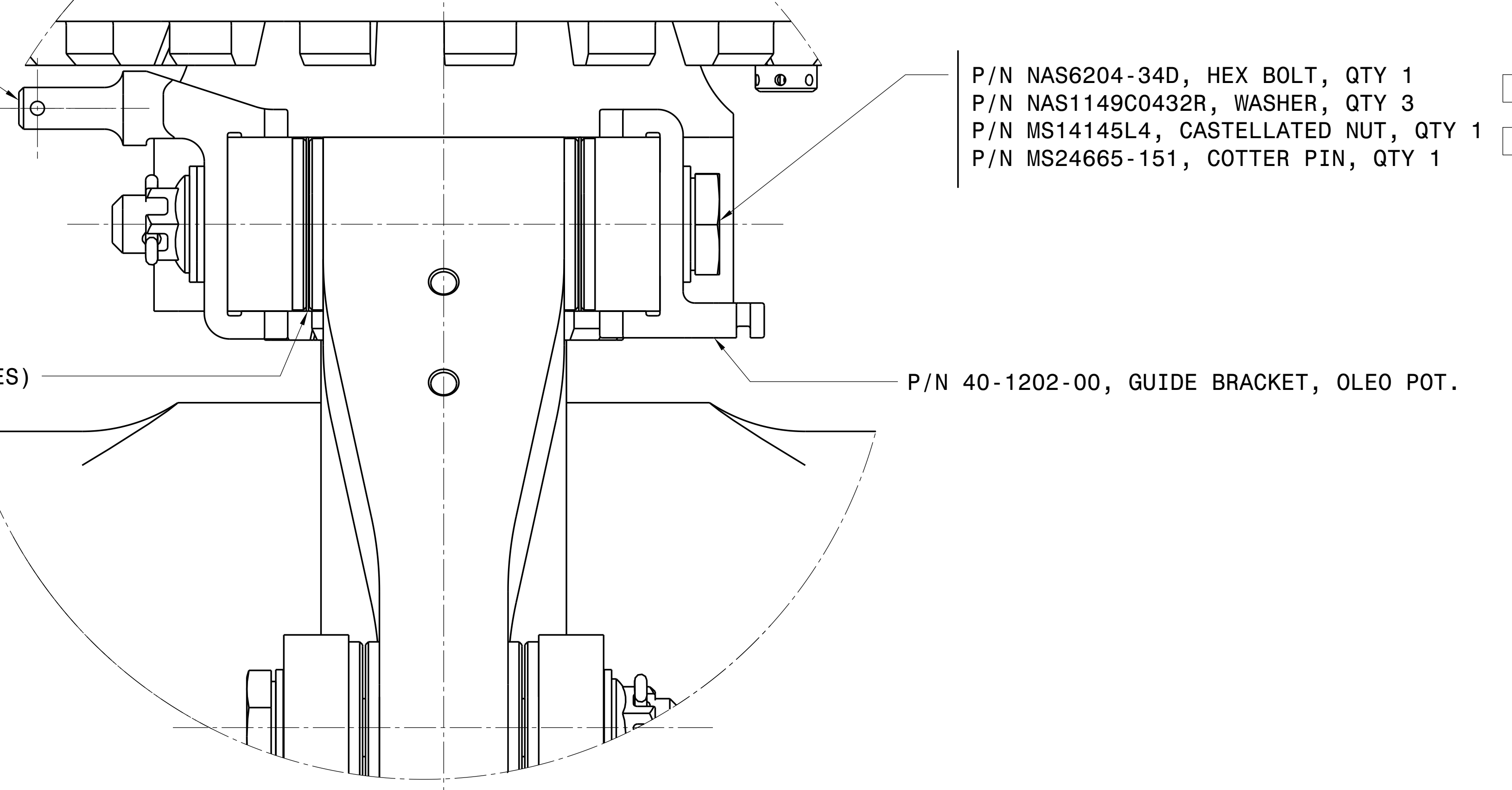

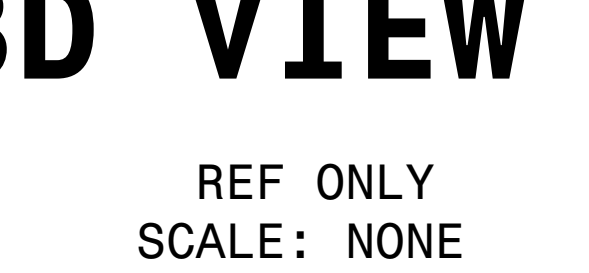

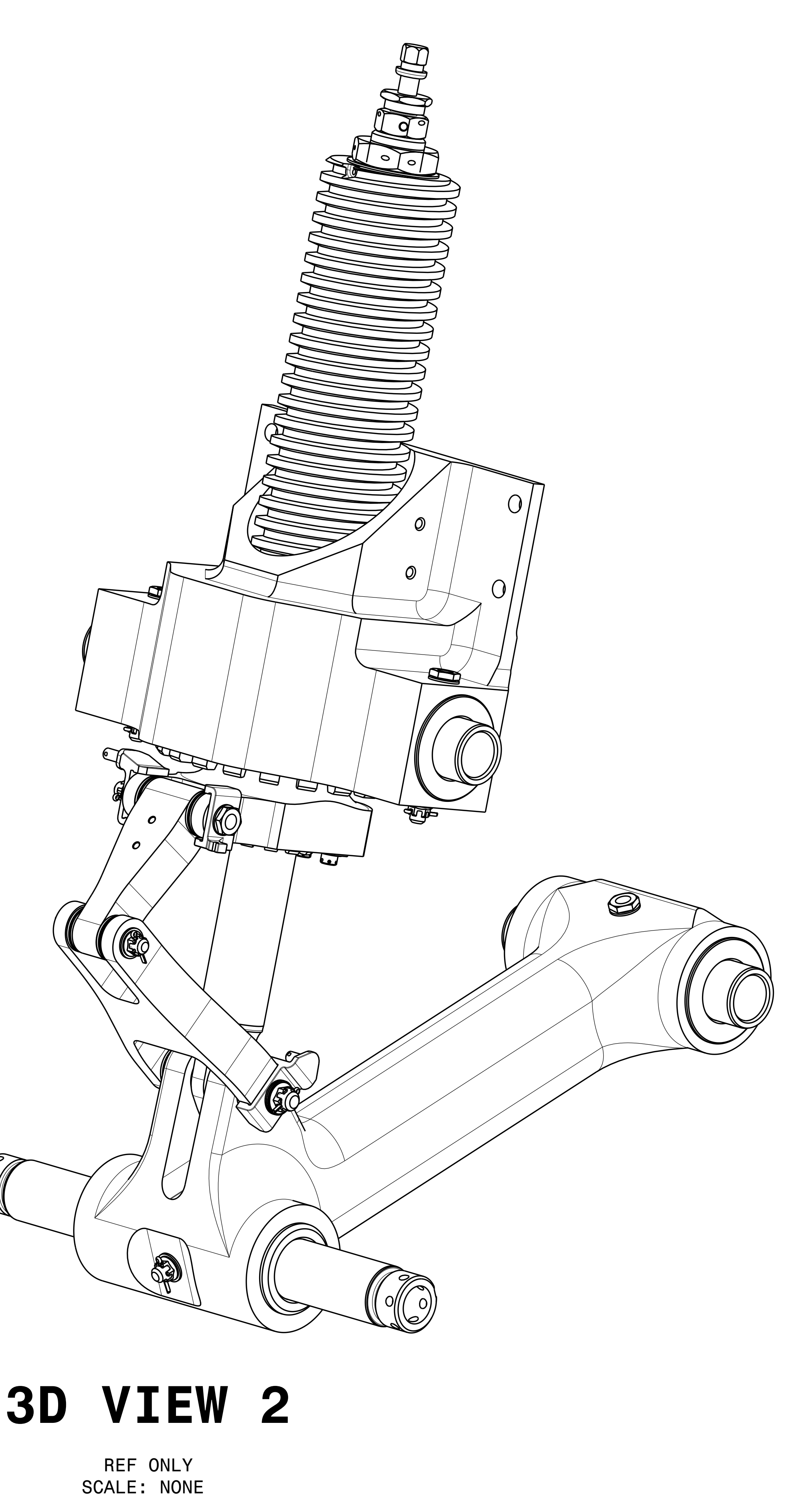

DETAIL B

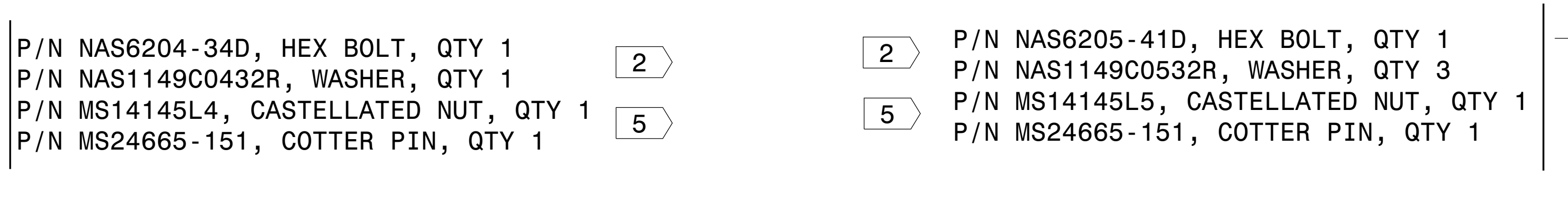
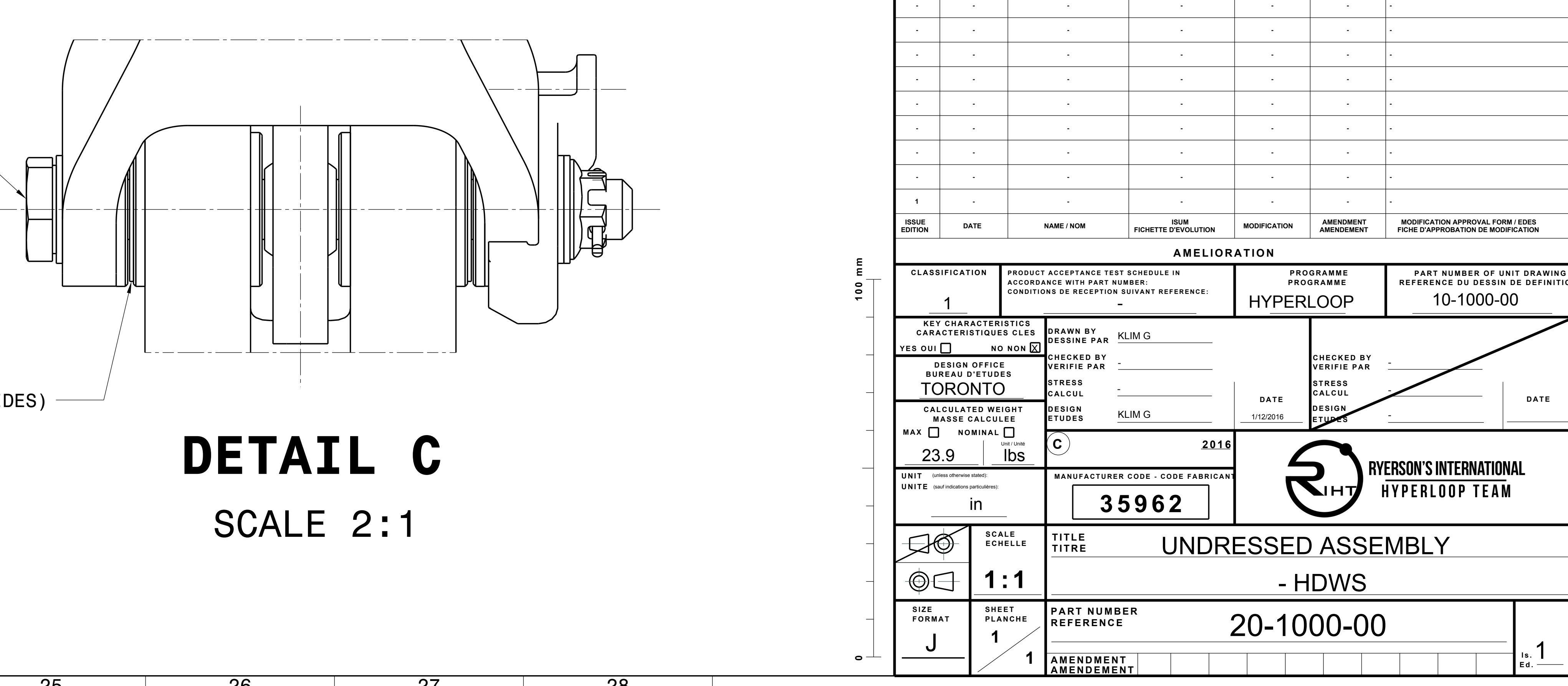


\section{NOTES:}

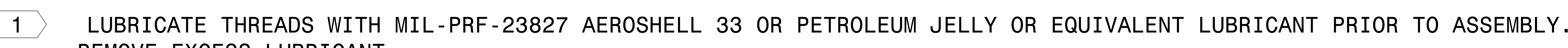
REMOVE EXCESS LUBRICANT.

2 APPLY LOCTITE (THREADLOCKER) OR EQUiVALENT TO THREAD SURFACE PRIOR TO INSTALLATION.

3 TORQUE TIGHT, ADD $1 / 4$ TURN.

4 APPLY SUPERGLUE ADHESIVE OR EQUIVALENT.

5. REFER TO HYPERLOOP DEPLOYABLE WHEEL SYSTEM ASSEMBLY GUIDE FOR DETAILED ASSEMBLY PROCEDURES.

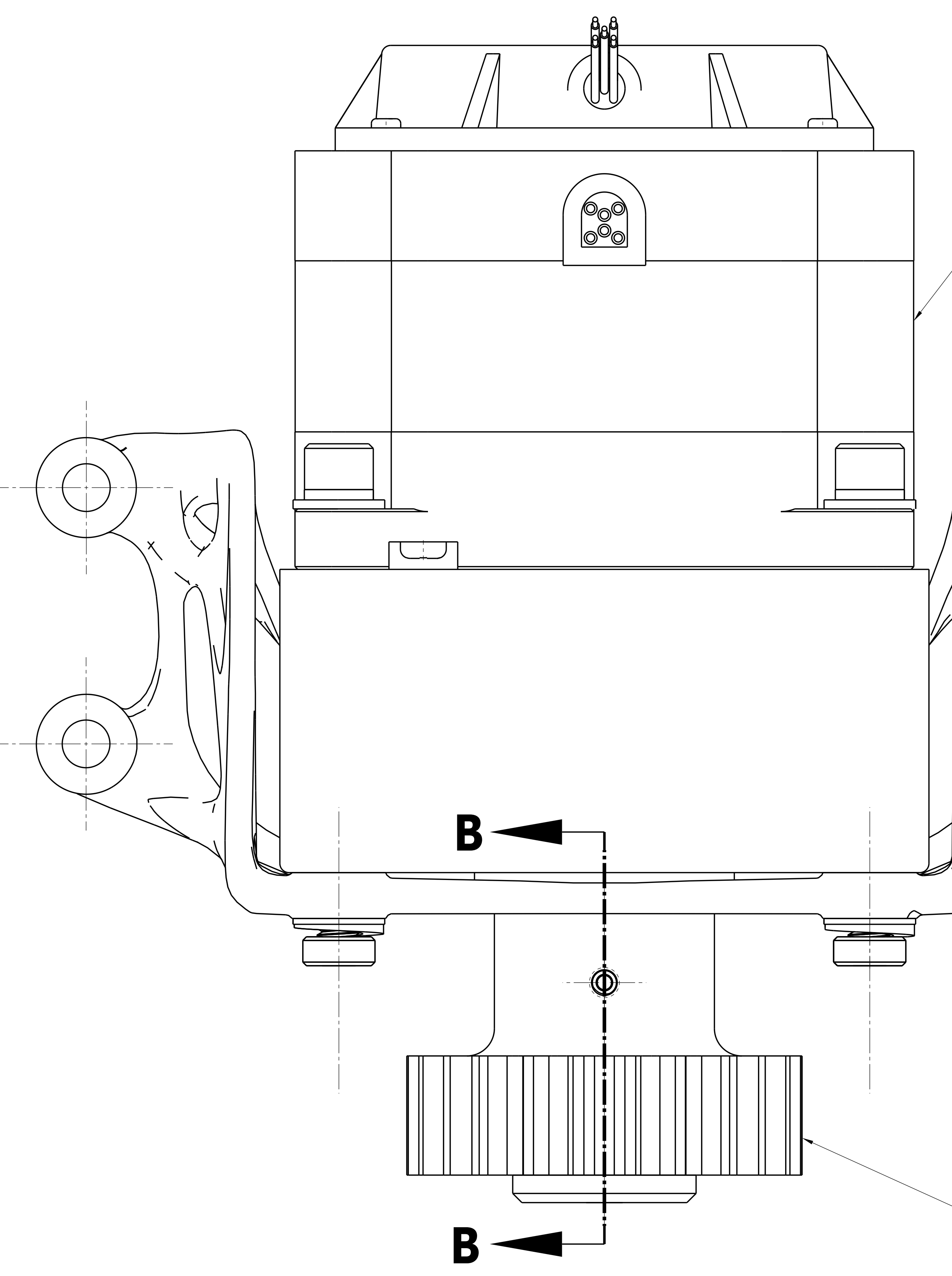

P/N 40-2102-00, PINNION GEAR (SPUR)
-P/N 40-2101-00, BLYSG34 BRUSHLESS DC MOTOR

P/N 40-2103-03, MOTOR MOUNT BRACKET (AMM)

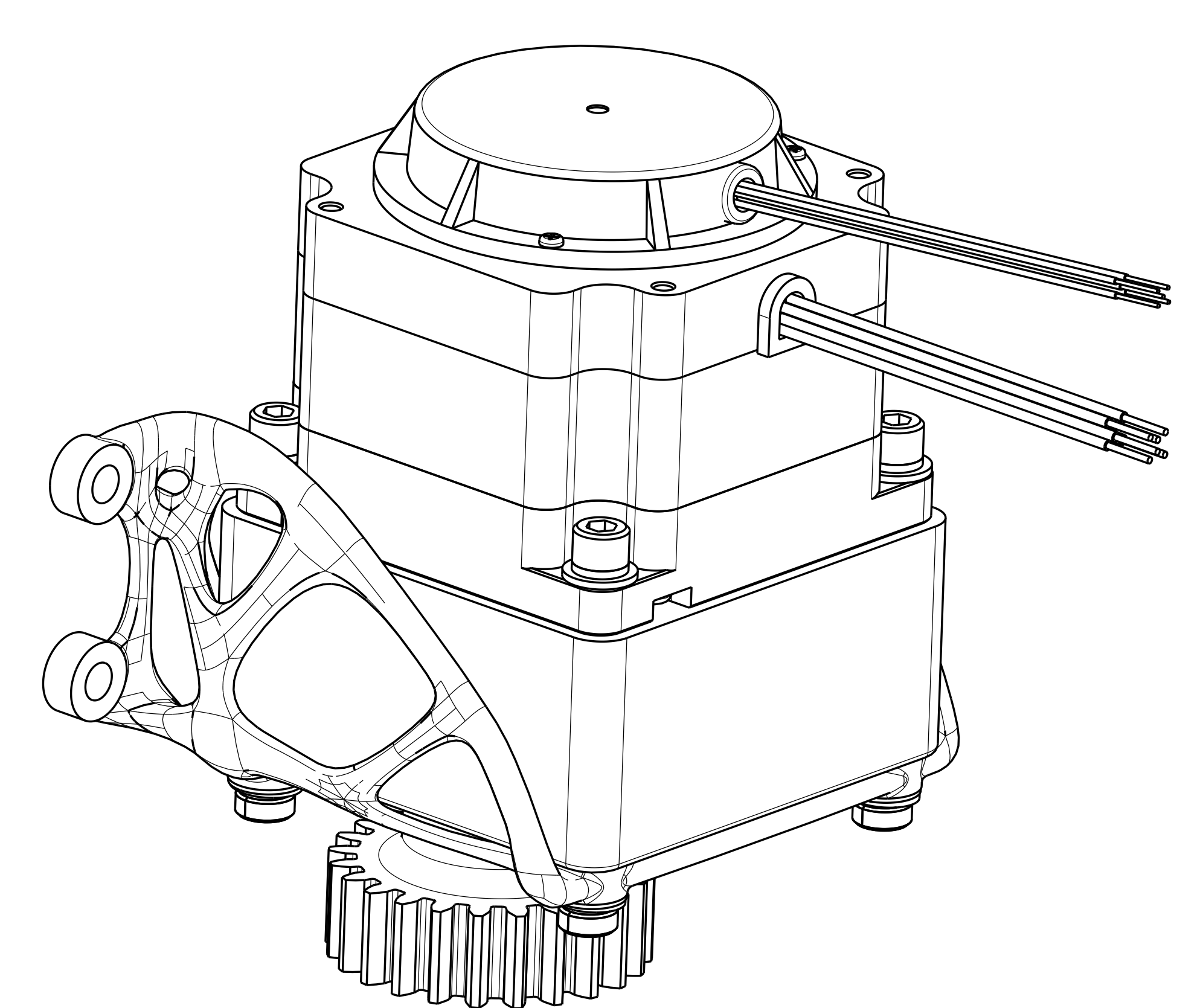

3D VIEW

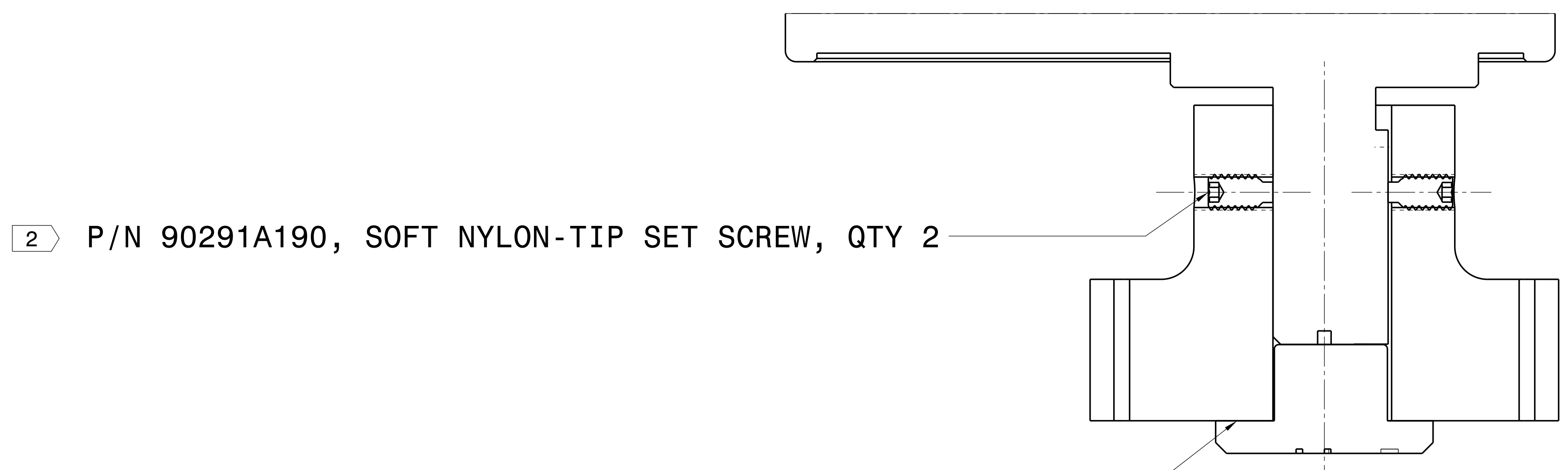

PARTIAL SECTION B-B

P/N M6X1-25, LOW-PROFILE SS CAP SCREWS (METRIC) P/N NAS1149C0432R, FLAT WASHER
P/N MS35338-139, SPLIT LOCK WASHER

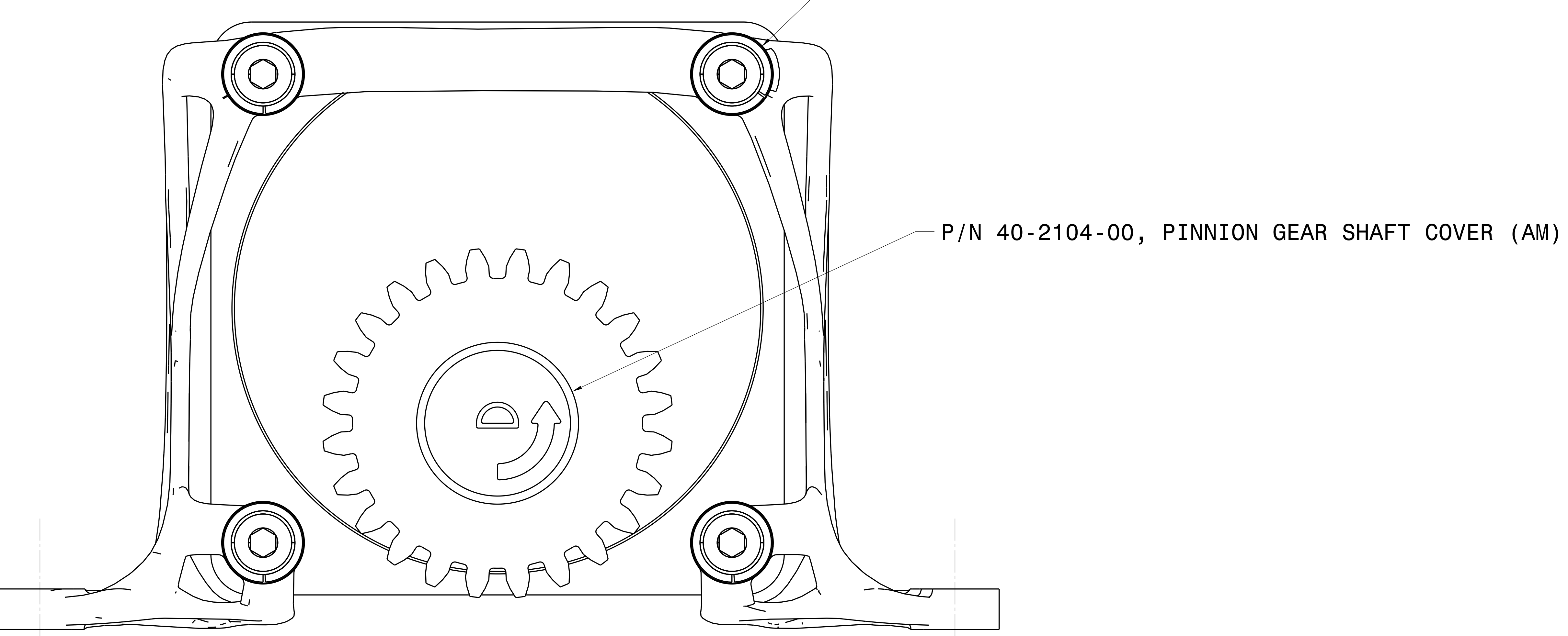




\section{NOTES:}

1 Dimension applies after installation. hone or hand ream onLy.

2 INSTALL CUSTOM BUSHINGS USING PRESS FIT METHOD ONLY.

3 INSTALL KAMATICS BUSHINGS USING PRESS Fit OR COOLING METHOD.

4. Bushings to be installed after anNodizing of trailing arm Component.

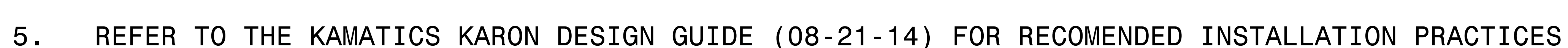

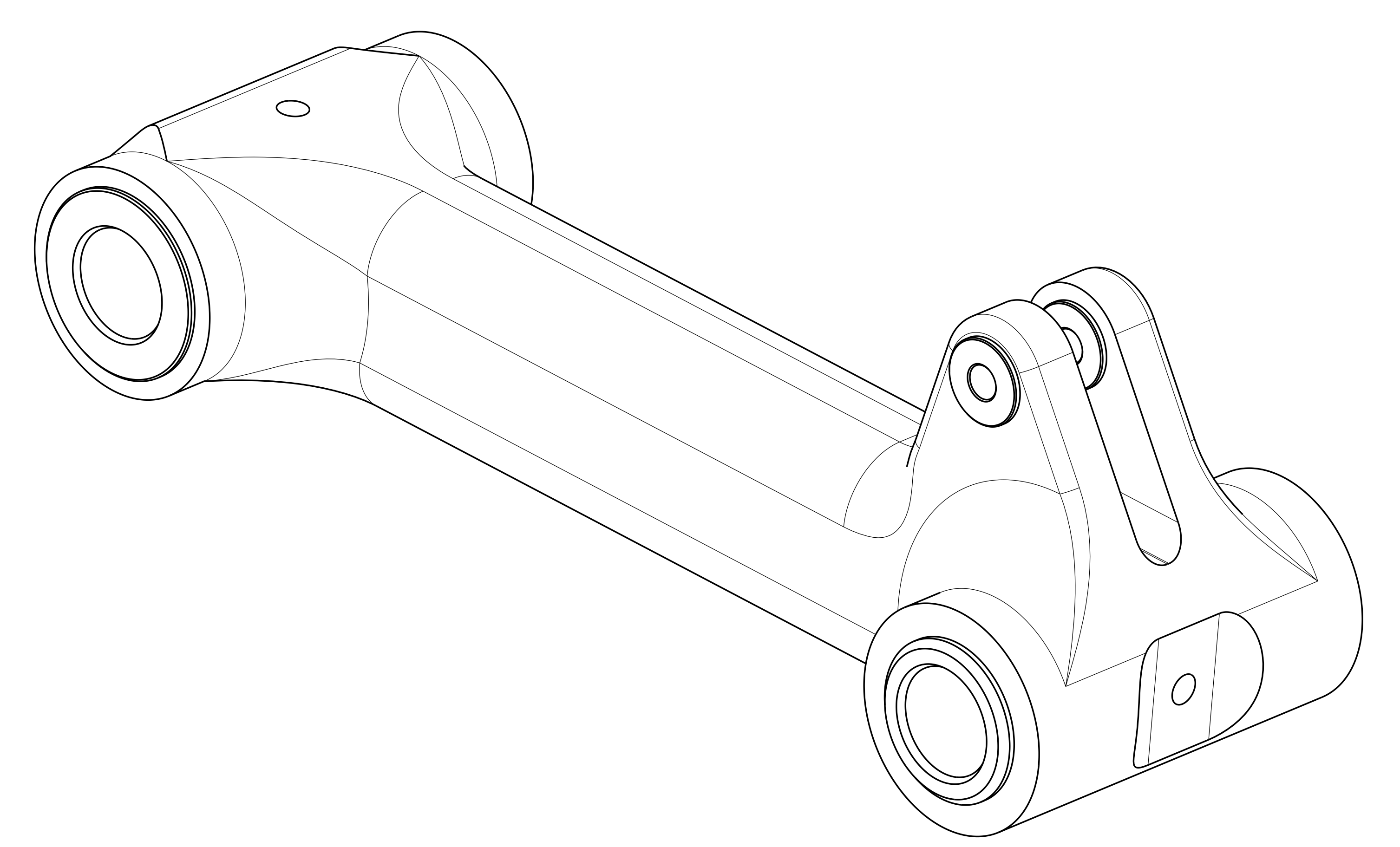

3D VIEW

SCAEE: ONLY
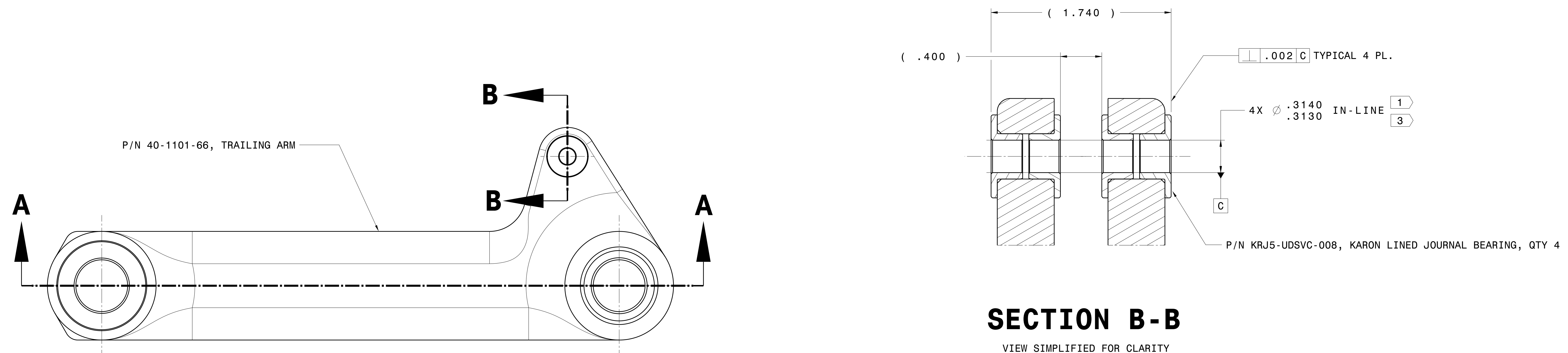

\section{SECTION B-B}

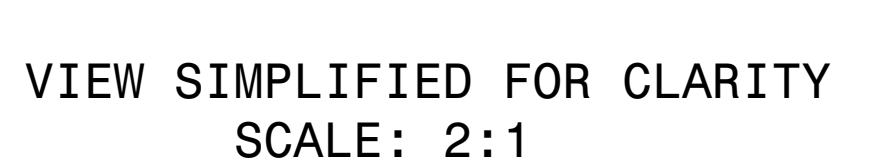
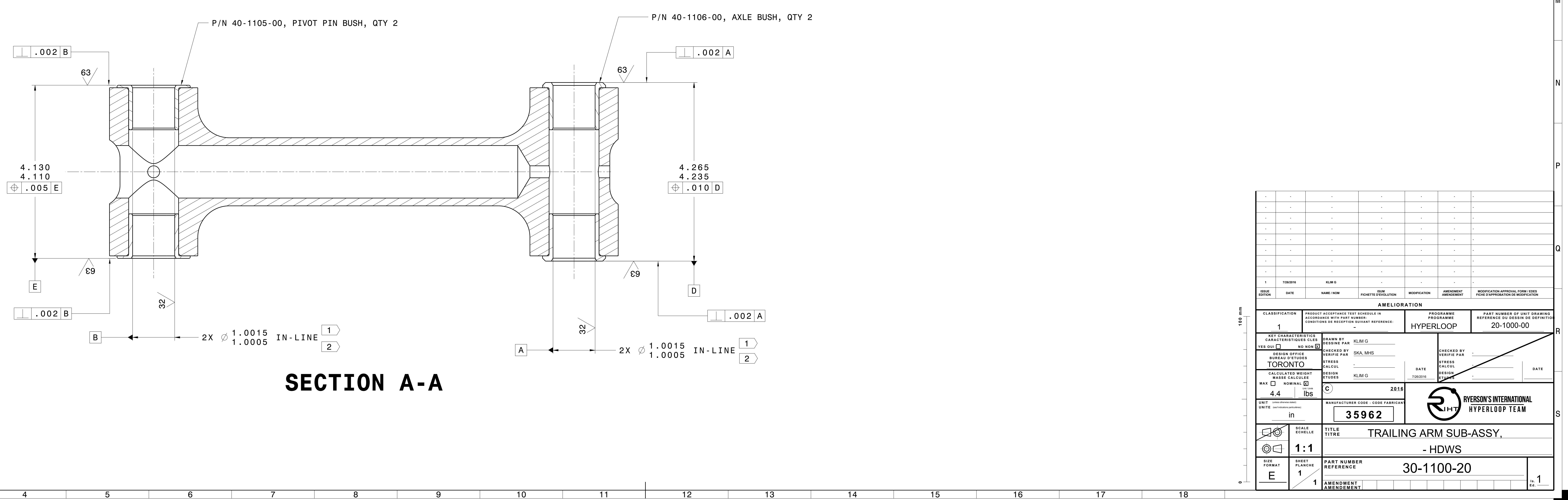


\section{NOTES :}

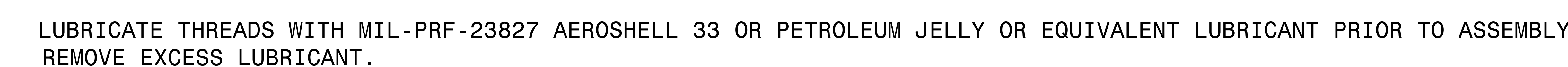

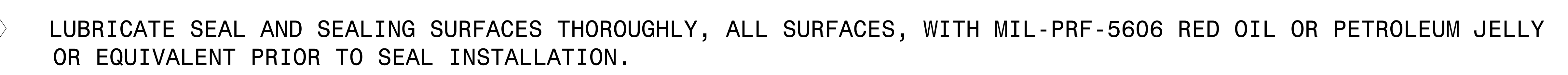

TOROUE GLAND NUT TIGHT, BACK OFF TO NEABEST CASTELLATION OF PISTTON GLAND NUT AND LOCK WITH LOCKKNG TAB.

TOHOUE CAPSCREN TIGH, ADO T/4 TUAN, INSTALL LOCKNIRE,

5) TOROUE JAMUUT AND CHAAGE VALVE TIGHT, ADD 1/4 TURN, LOCKNIRE FROM SHOCK ABSORBERR TO JAM NUT,

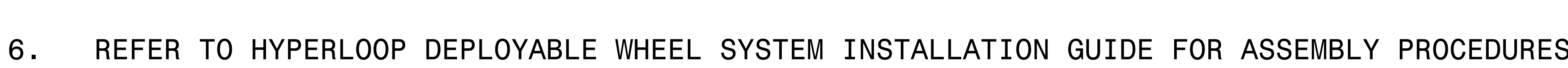

P/N 35-1202-21, SHOCK ABSOABER CYLINNER SUB-ASEEWBLY

6. REEER TO HYPELLOOP DEFLOXYABLE WHEE
1. 5 P P M MS28889-2, CHAAGE VALVE-

A B

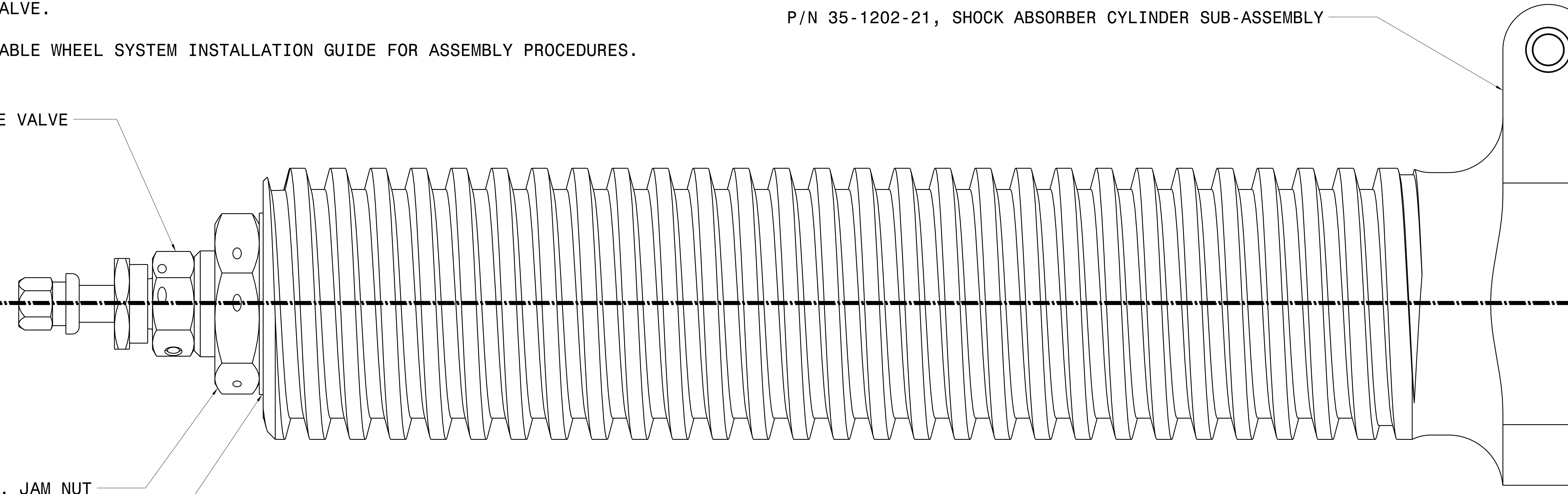

1. 5 P/N NAS1423-14, JAM NUT

PIN 40-1205-02, PISTON UPEER BEARTNG (SPLIT RTNG)

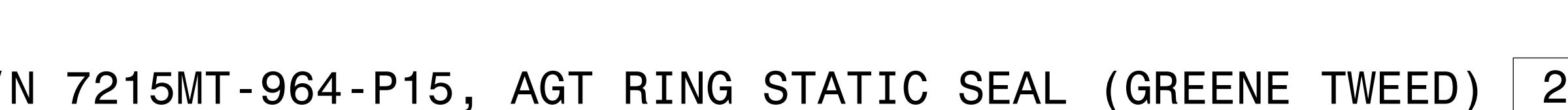

PIN NAS1 149C1 1432R, WASHER
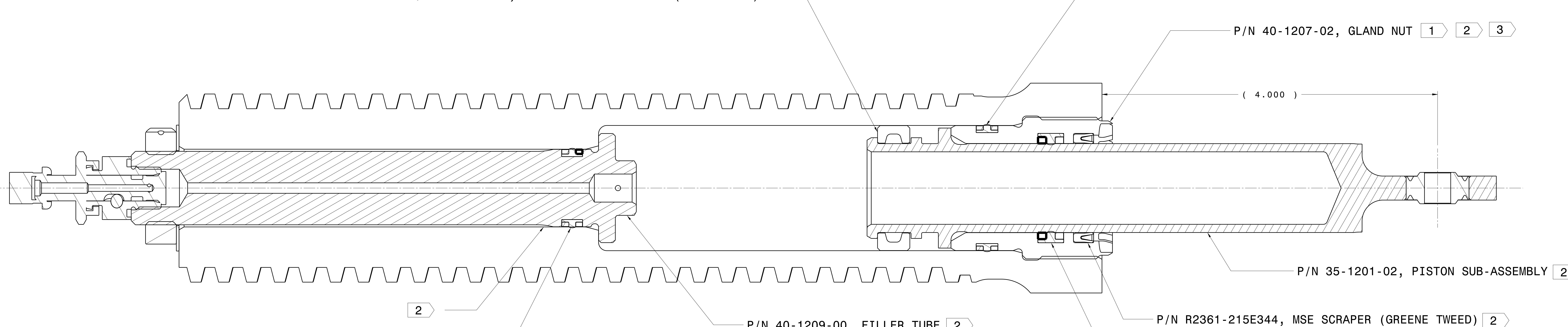

2. P/N 7110BWTE-161-P15, AGT RING STATIC SEAL (GREENE TUEED)

SECTION A-A

P/N 7215FT-964-P17, AGt RING DYNAMIC SEAL (GREENE TWEED) 2

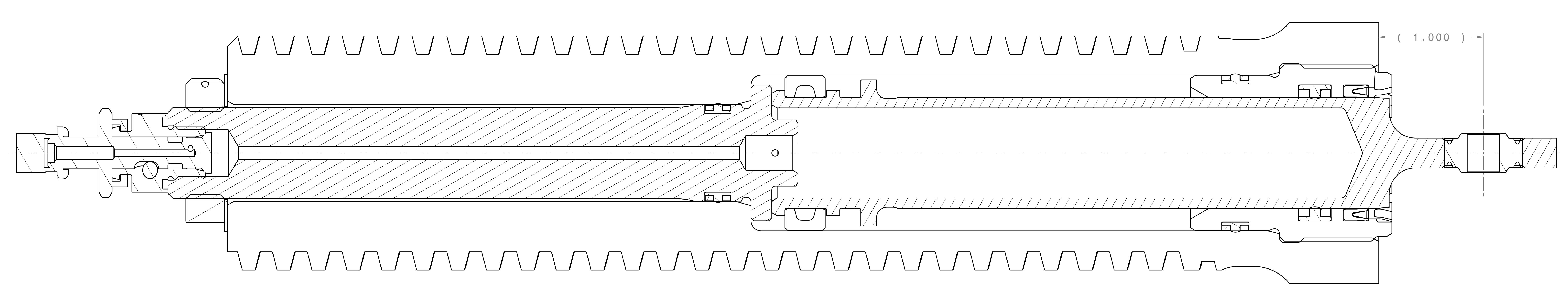

SECTION B-B

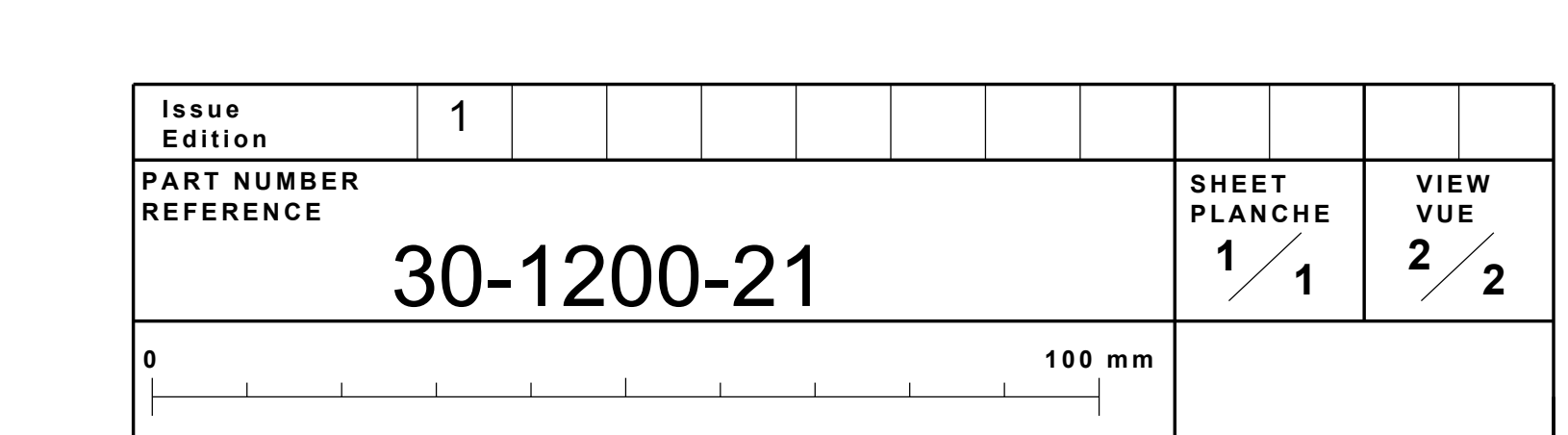
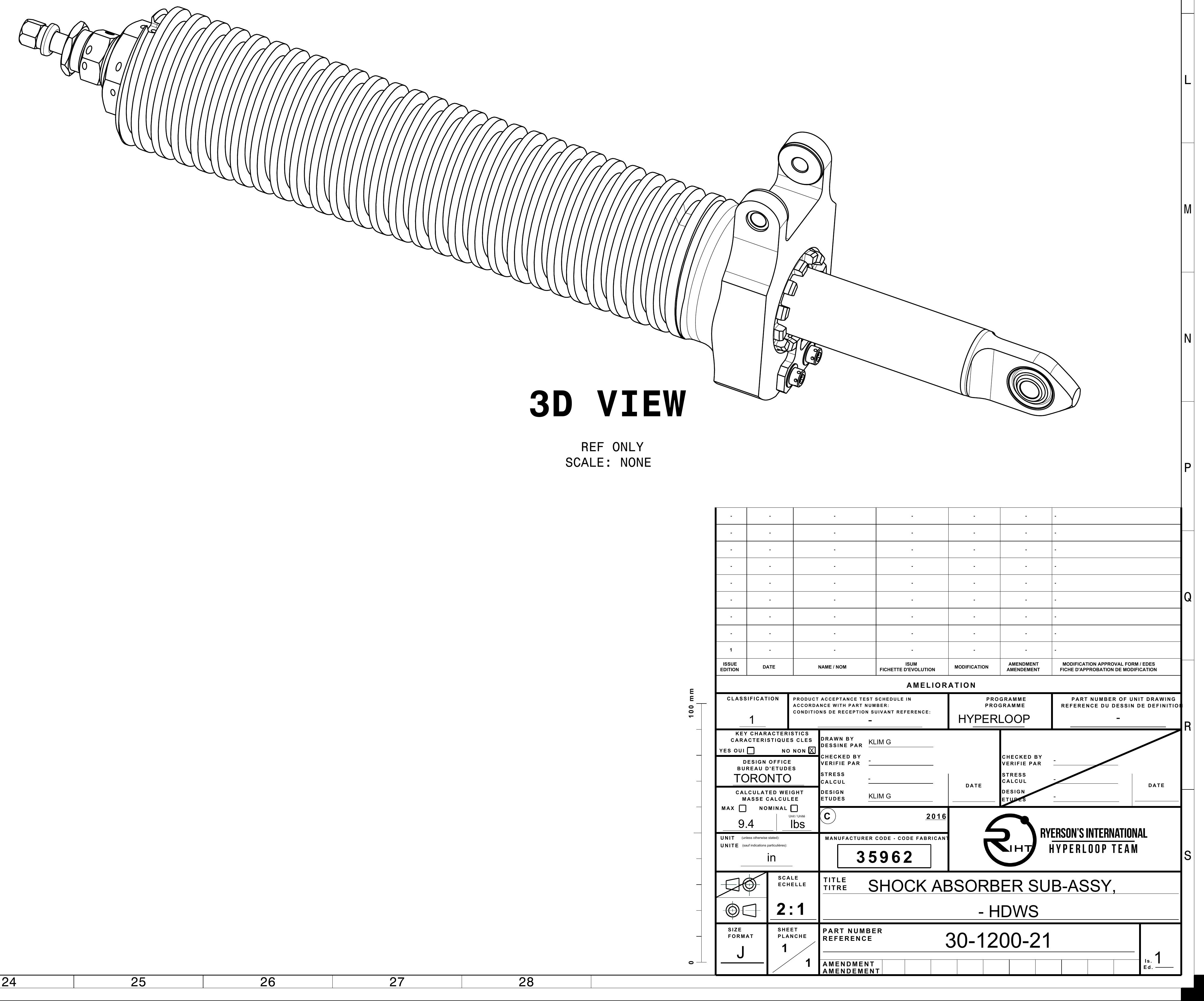


\section{NOTES:}

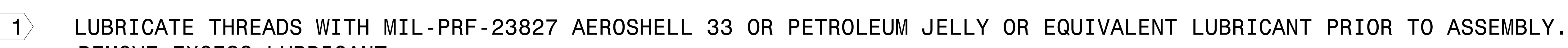
REMOVE EXCESS LUBRICANT.

2. LUBRICATE BEARING, INNER AND OUTER RACE DIAMETER FACES, WITH MIL-PRF-23827 AEROSHELL 33 OR PETROLEUM JELLY OR

3) install using custom bearing installation tool.

4 ToRque packing NUT Tight, BACK OfF tO NEAREST CASTELLATION AND LOCK WITH LOCKING TAB,

5. ensure retract nut rotates freely Without binding after torquing the packing nUt.

6. TORQUE CAPSCREW TIGHT, ADD 1/4 TURN, INSTALL LOCKWiRE.

7. REFER to HYPERLOOP DePLOYABLE WHEEL SYStem ASSembly GUIDE FOR DETAILED ASSEMBLY PROCEDURES.
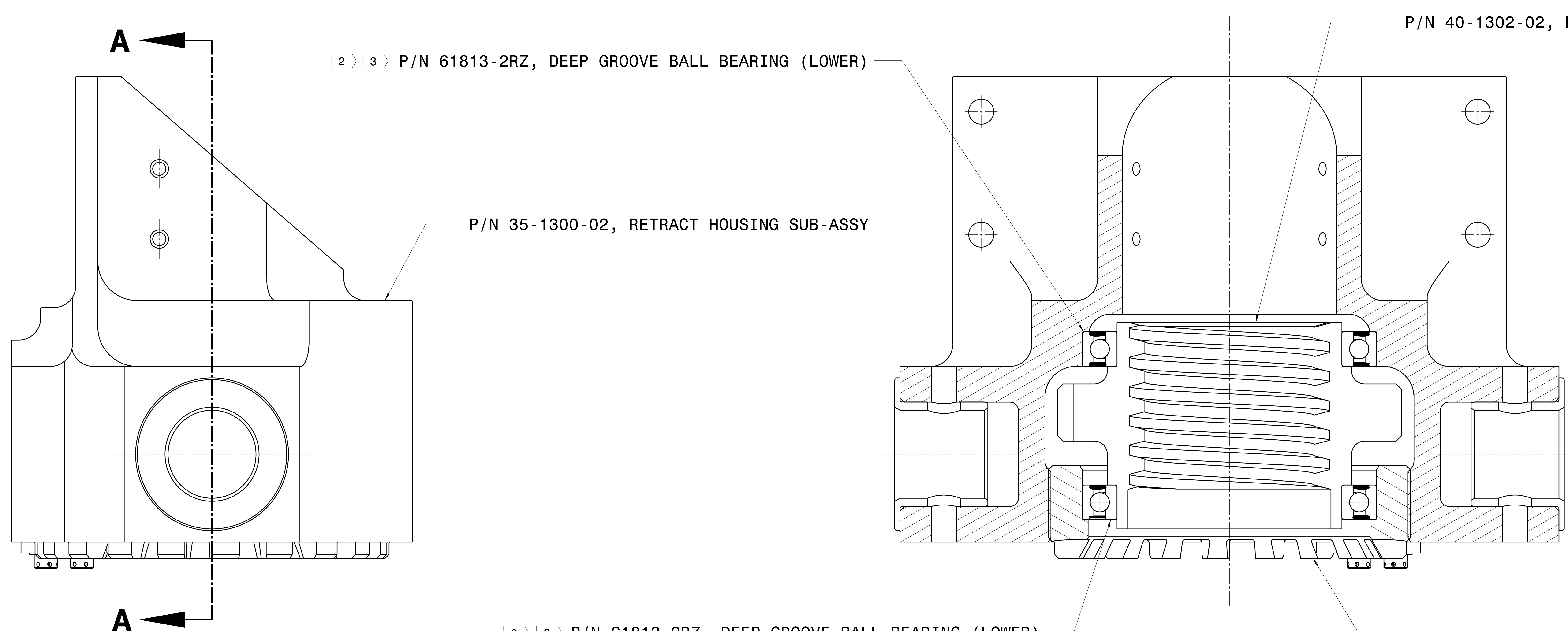

2) 3. P/N 61813-2RZ, DEEP GRoove BALl BeARing (LOWER)

P/N 40-1303-01, PACKING NUT 1

\section{SECTION A-A}
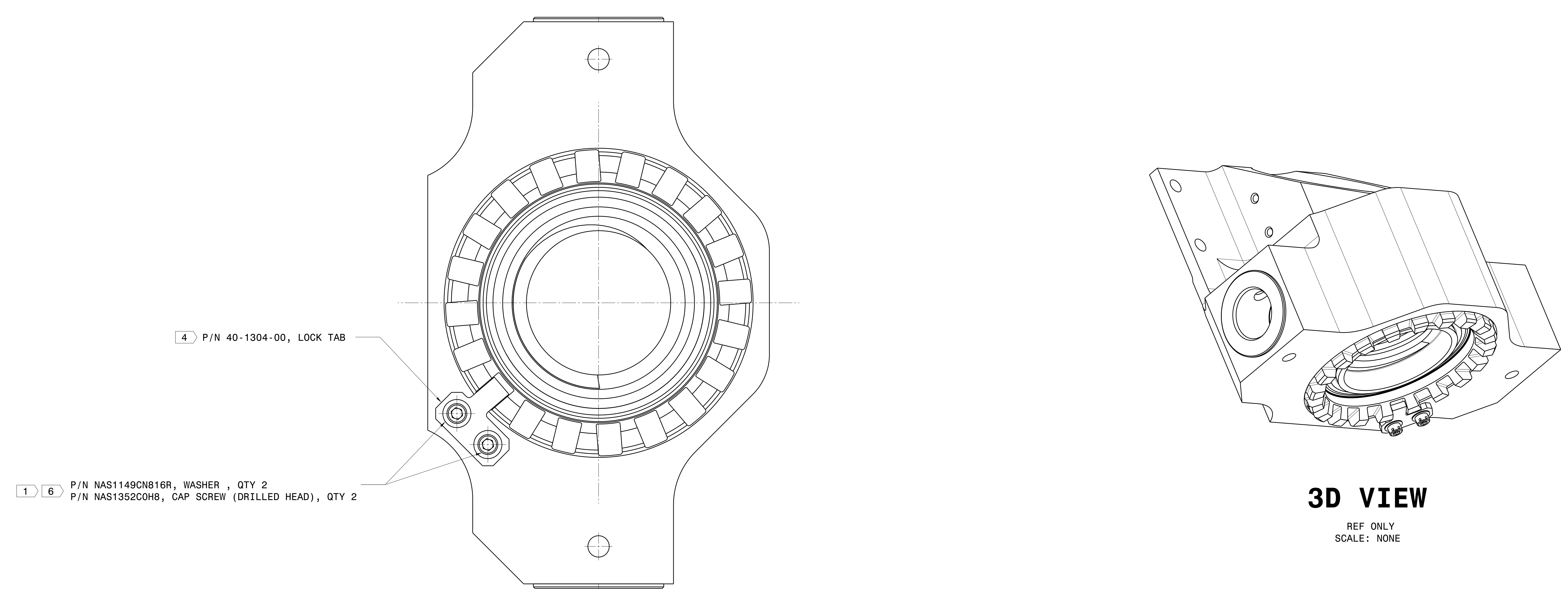

3D VIEW

SoEE ONLY

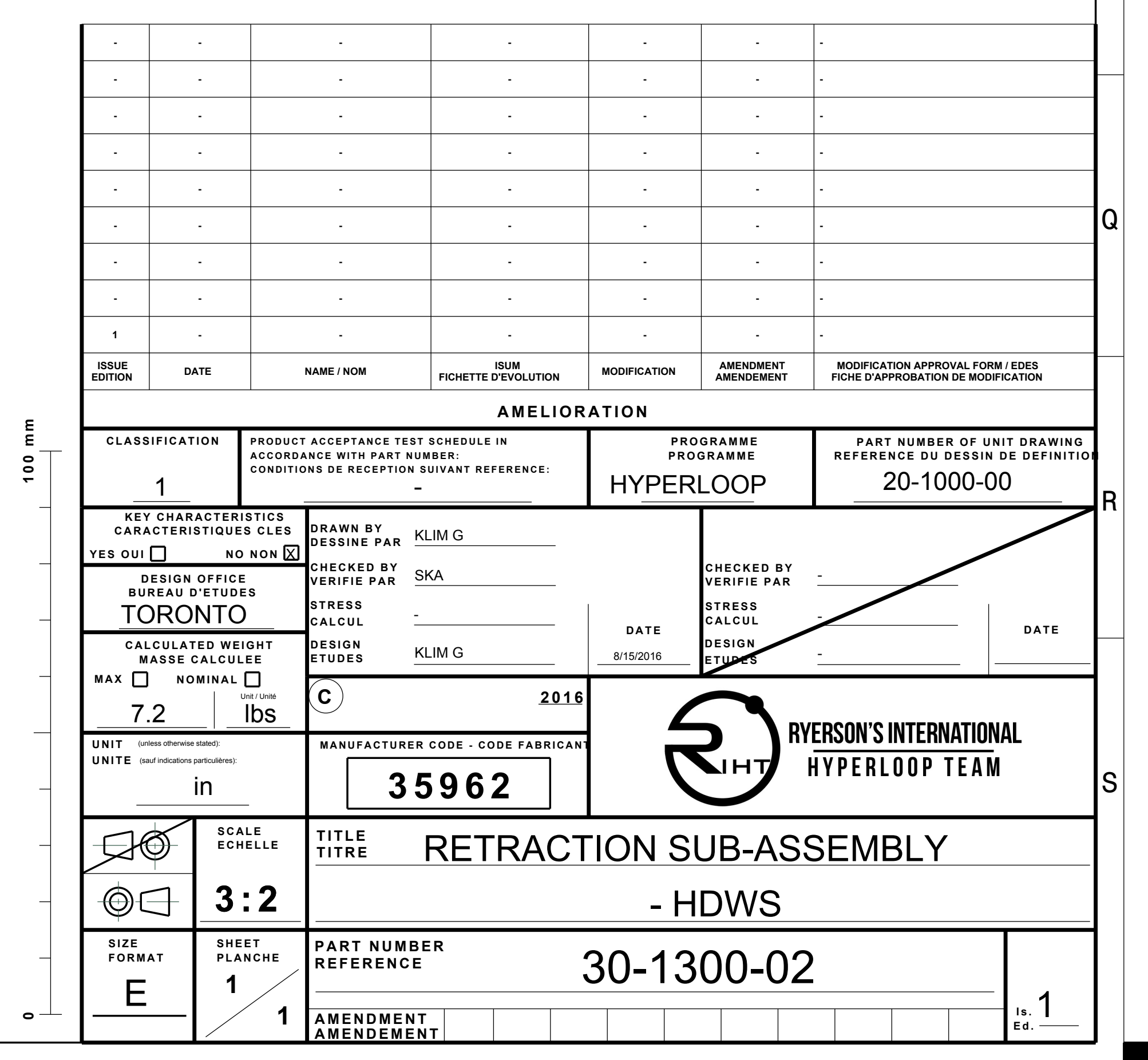




\section{NOTES:}

1) INSTALL WASHERS AS REQUiRed TO ACHIEVE COTTER PIN INSTALLATION. COTTER PIN TO BE SEATED 75\% T0 100\% WITHIN NUT CASTLE. USE ANY COMBINATION OF NAS1149C0416R AND NAS1149C0432R TO ACHIEVE FIT.

2. Deform COtTer Pin after installation.

3. REFER tO hYPERLOOP DEPLOYABLE WHEEL SYSTEM INSTALLATION GUIDE FOR DETAILED ASSEMBLY PROCEDURES.

P/N 35-1402-01, UPPER TORQUE LINK SUB-ASSEMBLY

P/N 35-1401-01, LOWER TORQUE LINK SUB-ASSEMBLY

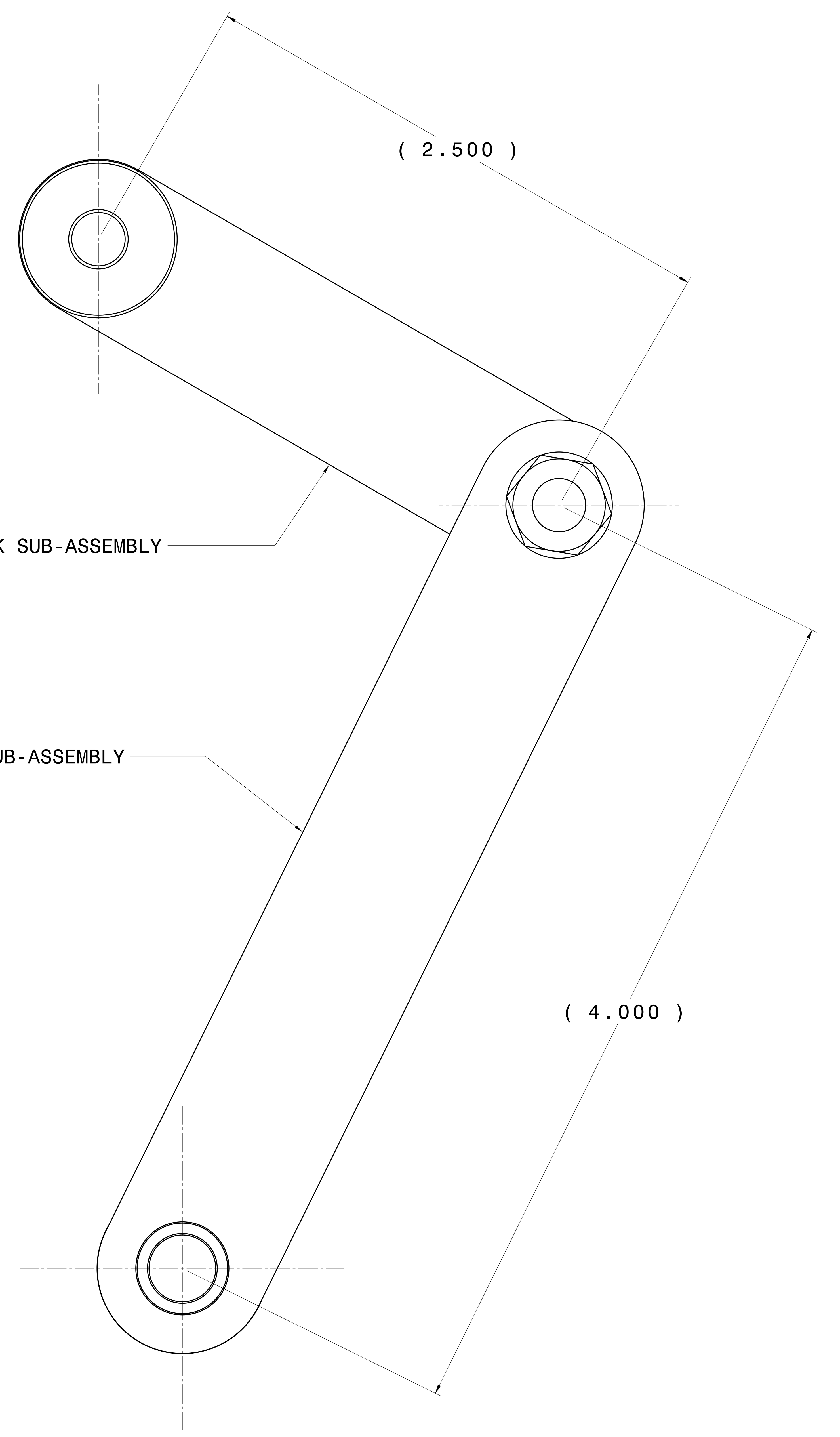

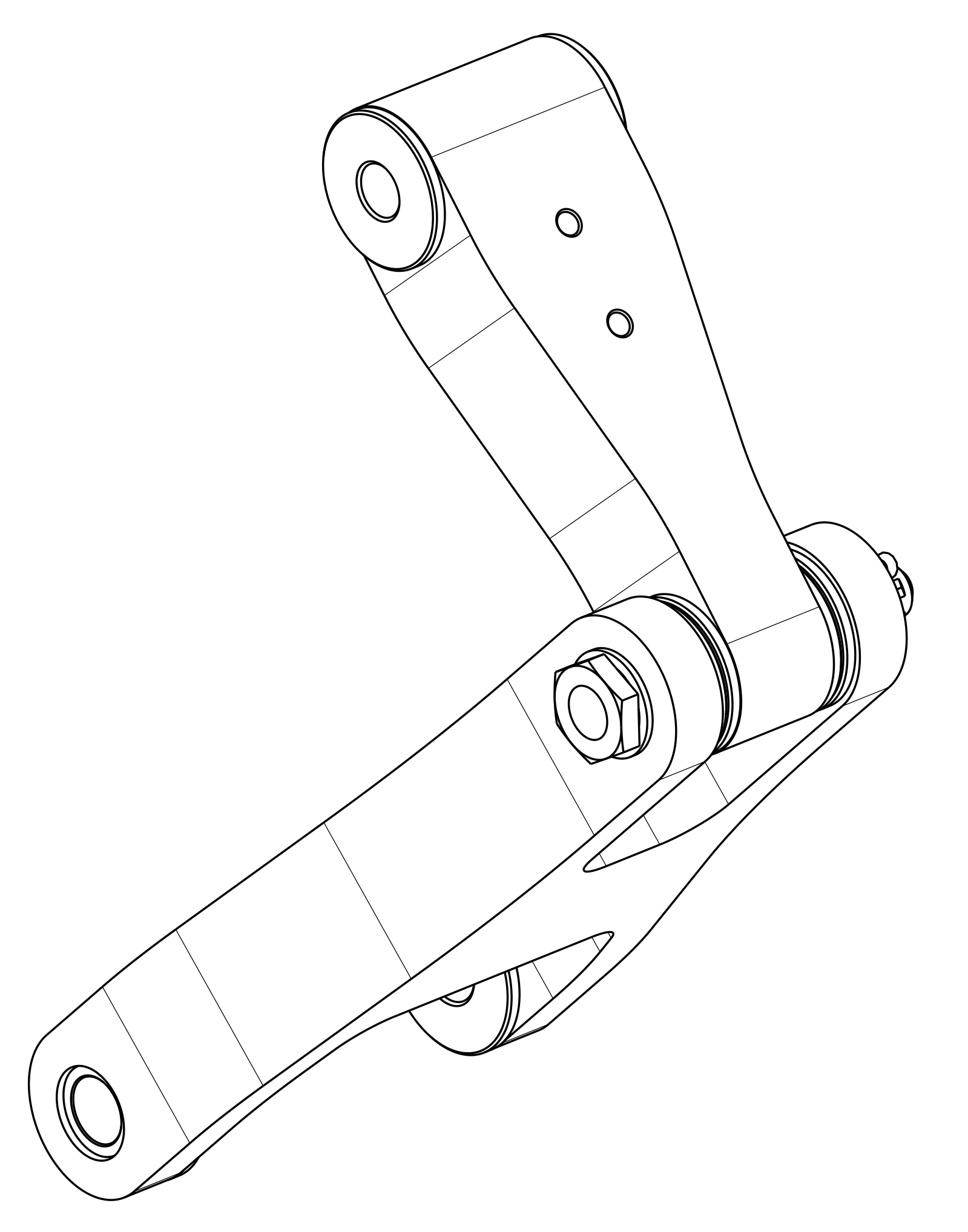

3D VIEW

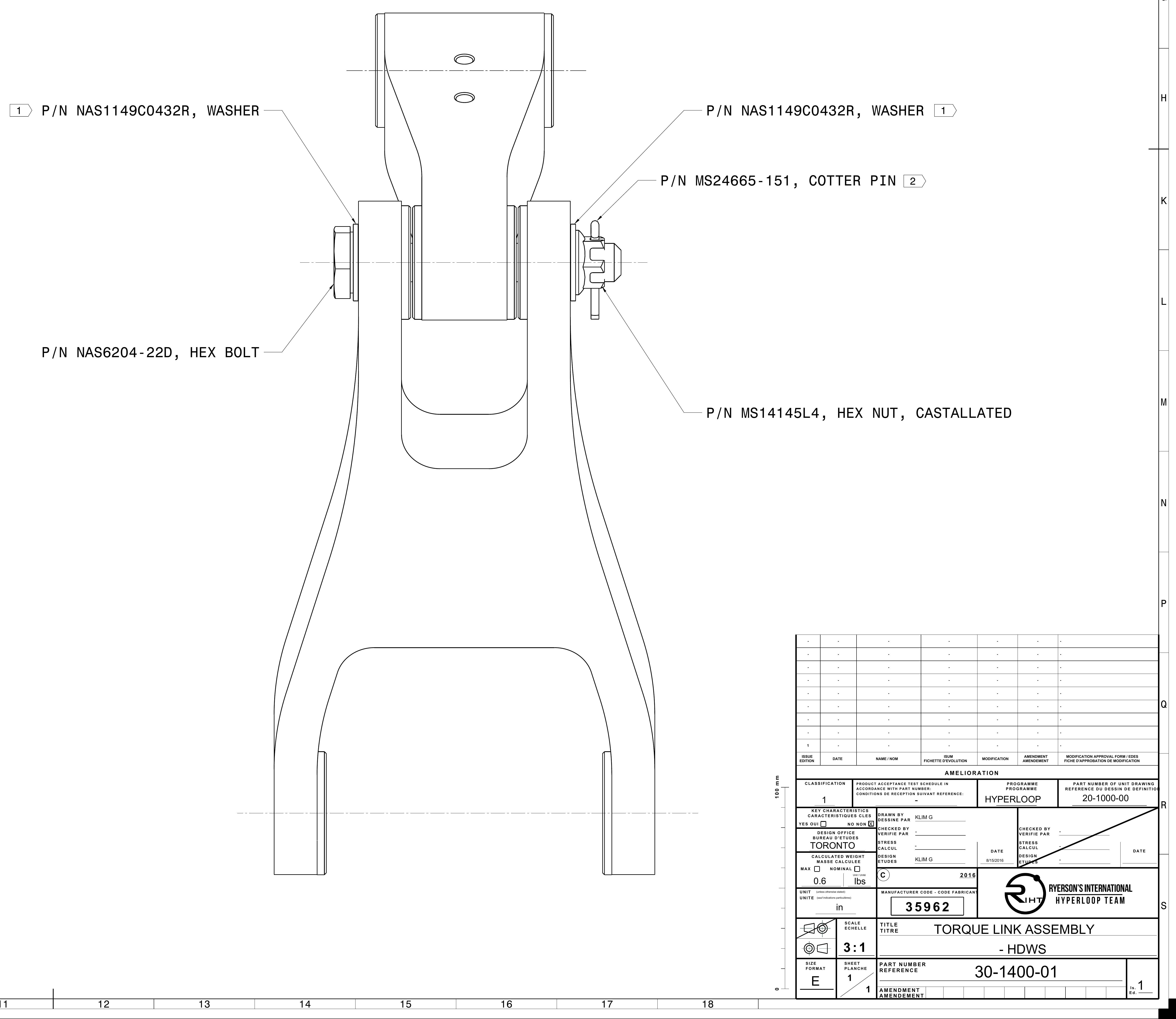




\section{NOTES:}

1. CUSTOM WHEEL/BEARING CONFIGURATION PROCURED FROM SUNRAY INC.

2. Wheel material: polyurethane 95A, black.

3. BEARINGS INSTALLED BY SUNRAY INC, SUPPLIED BY AST.

4. WHEEL BALANCED BY SUNRAY INC TO 6000 RPM OR $178 \mathrm{MPH}$.
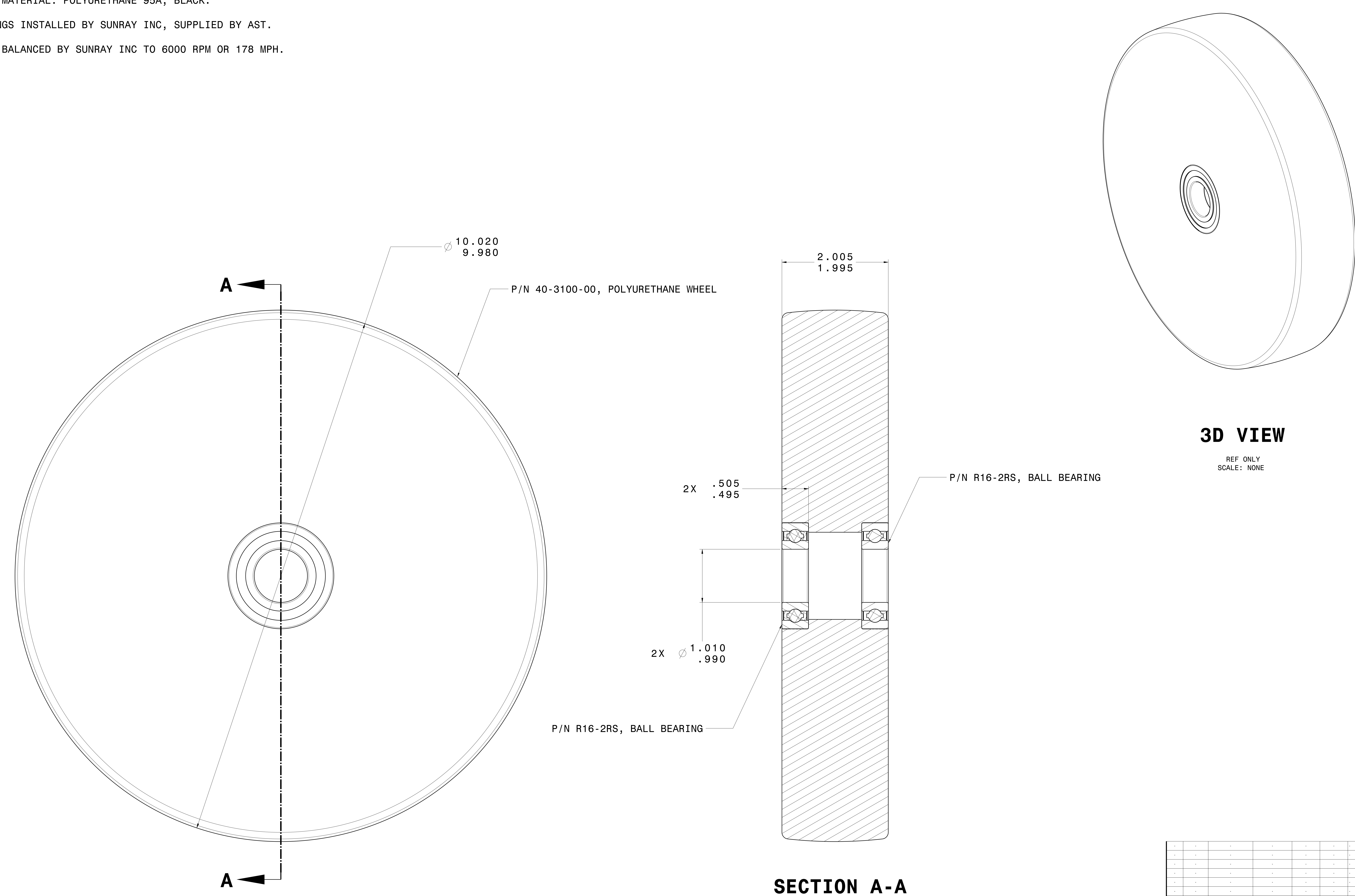

3D VIEW

REF ONLY
SCALE: NONE

\section{SECTION A-A}

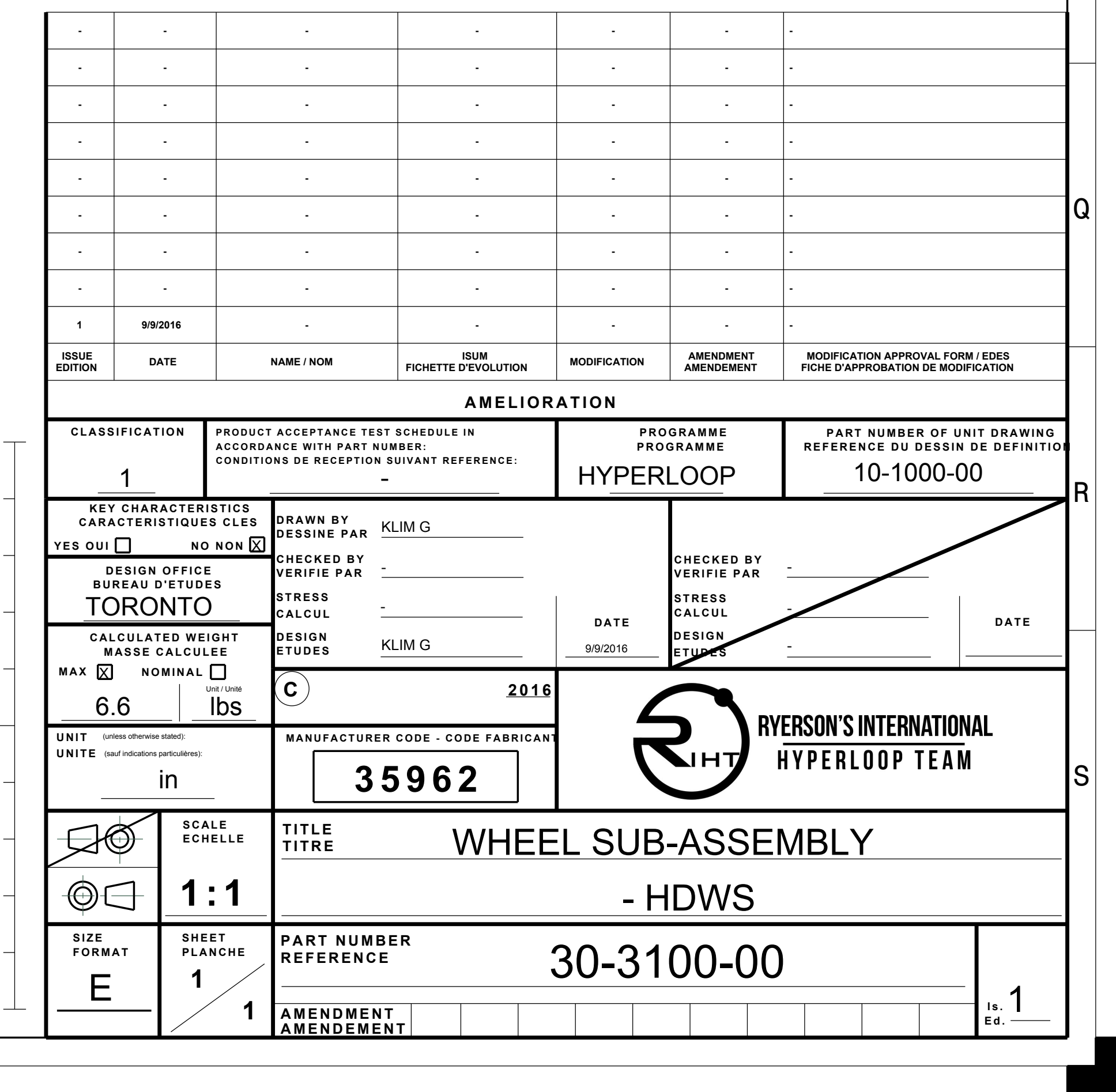




\section{NOTES:}

1 Dimension APPLies after installation. HONE OR haNd REAM OnLy.

2 INSTALL KaMATICS BUSHings USing PRESS Fit OR COOLING METHOD.

3. BUSHINGS TO BE INSTALLED AFTER ANNODIZing OF MATING COMPONENT.

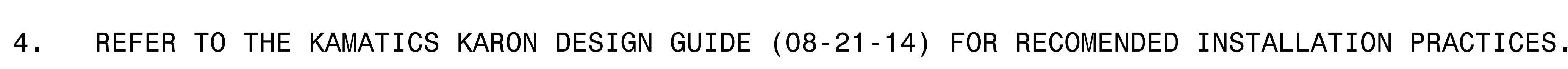

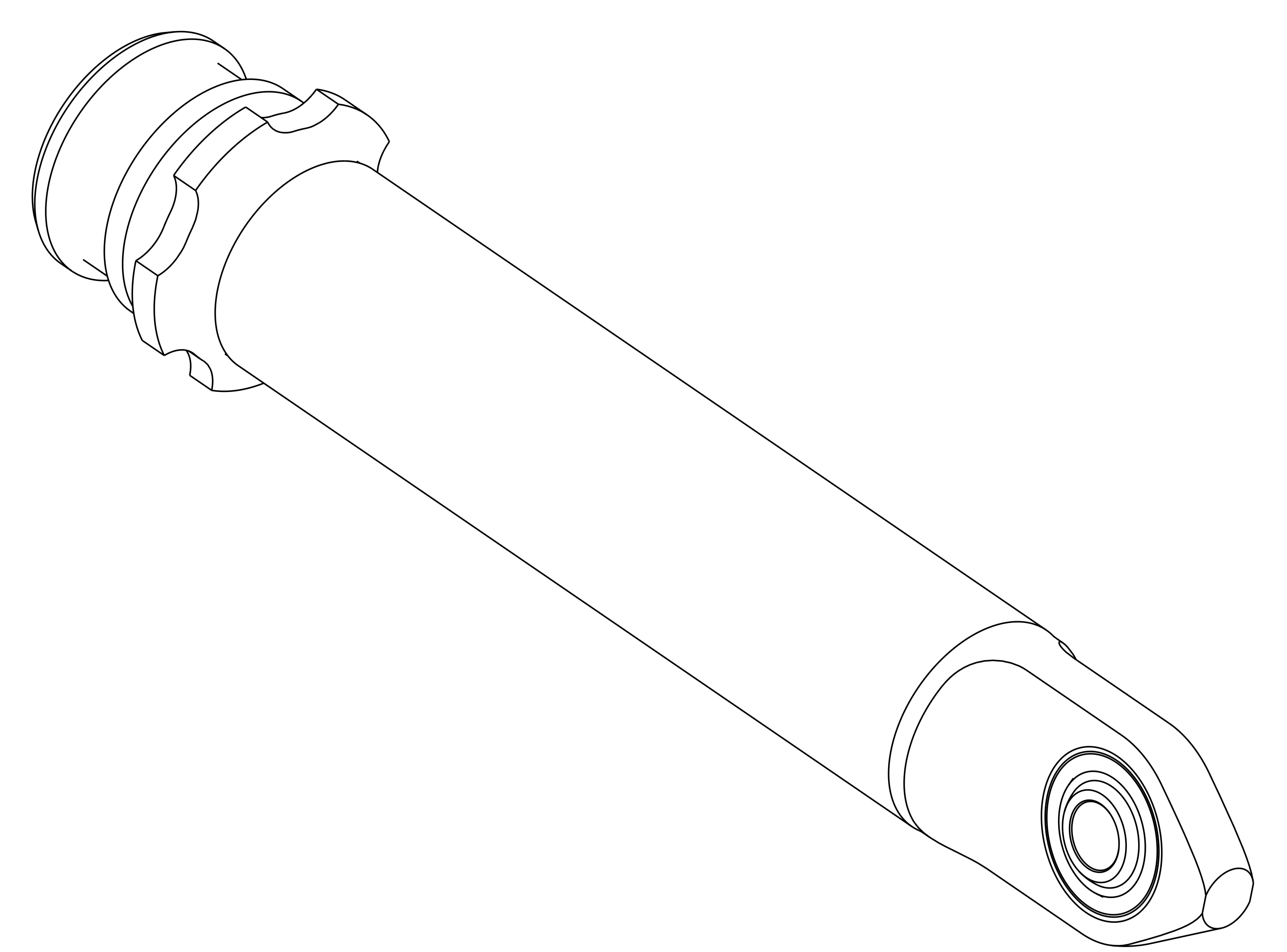

3D VIEW

BEE OOMY
SCCALE : NONE

P/N 40-1201-02, SHOCK ABSORBER PISTON

A

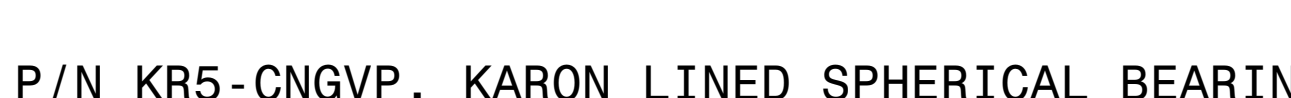

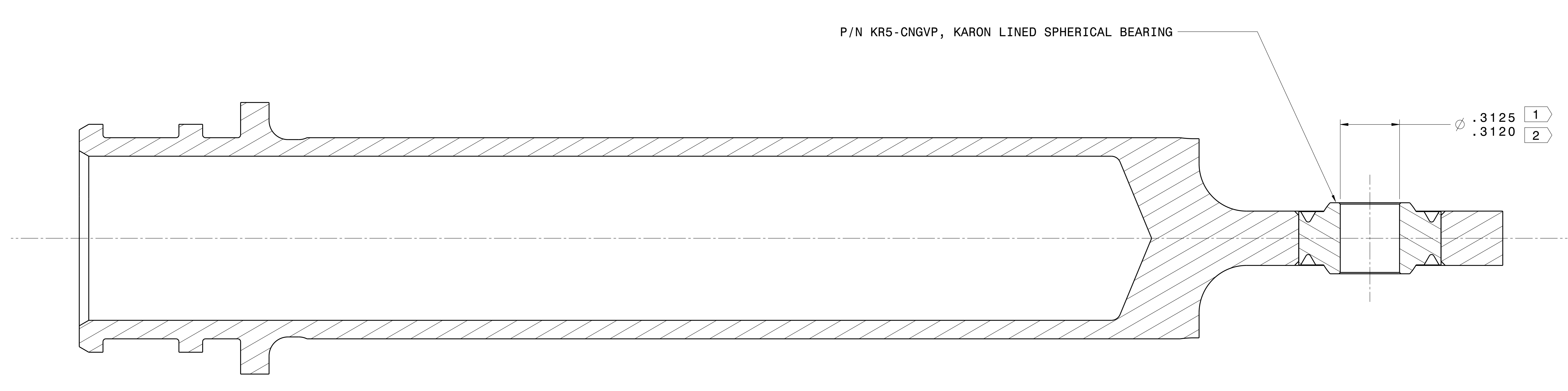

SECTION A-A

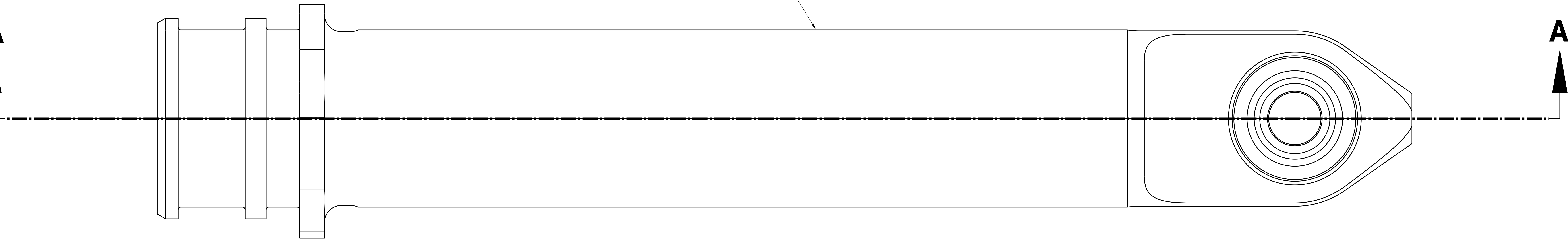

$\Lambda$

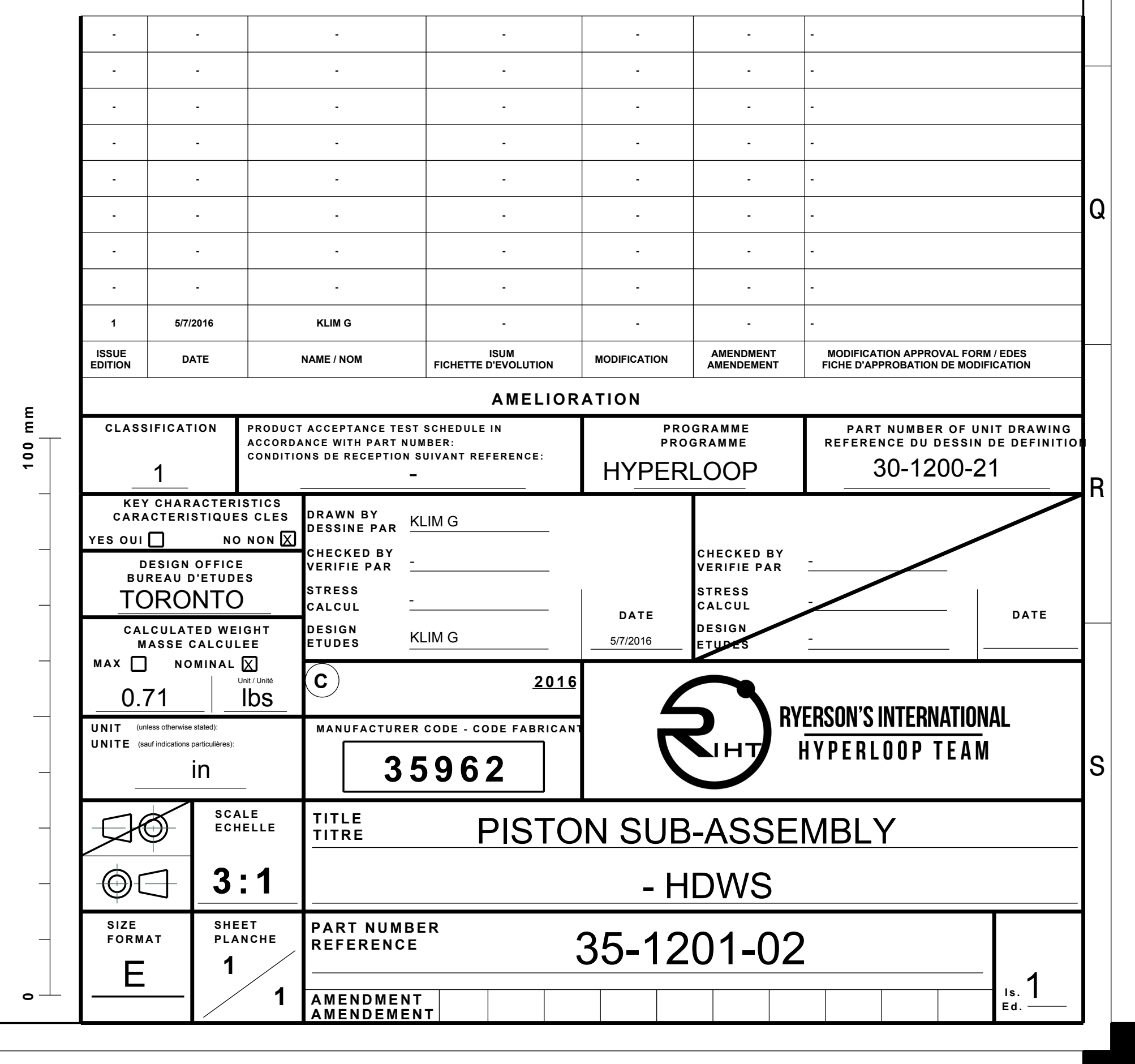




\section{NOTES:}

1 Dimension applies after installation. hoNe OR hand ream onLy.

2 INSTALl KAMATICS BUSHINGS USing PRESS Fit OR COOLING METHOD..

3. BUSHings tO BE INSTALLED AFTER ANNODIZing OF MATING COMPONENT.

4. Refer to the kamatics Karon design Guide (08-21-14) FOR ReCOMEnded installation Practices.

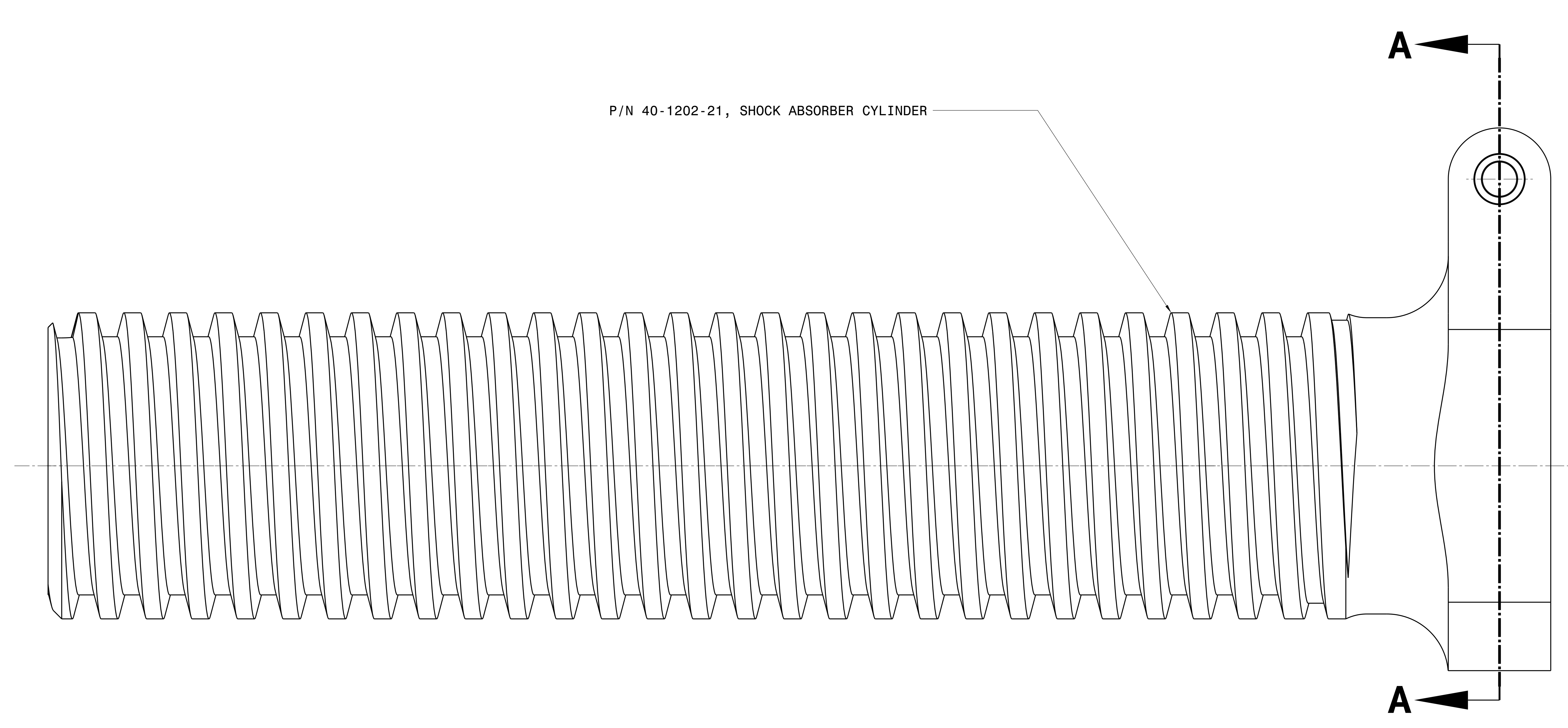

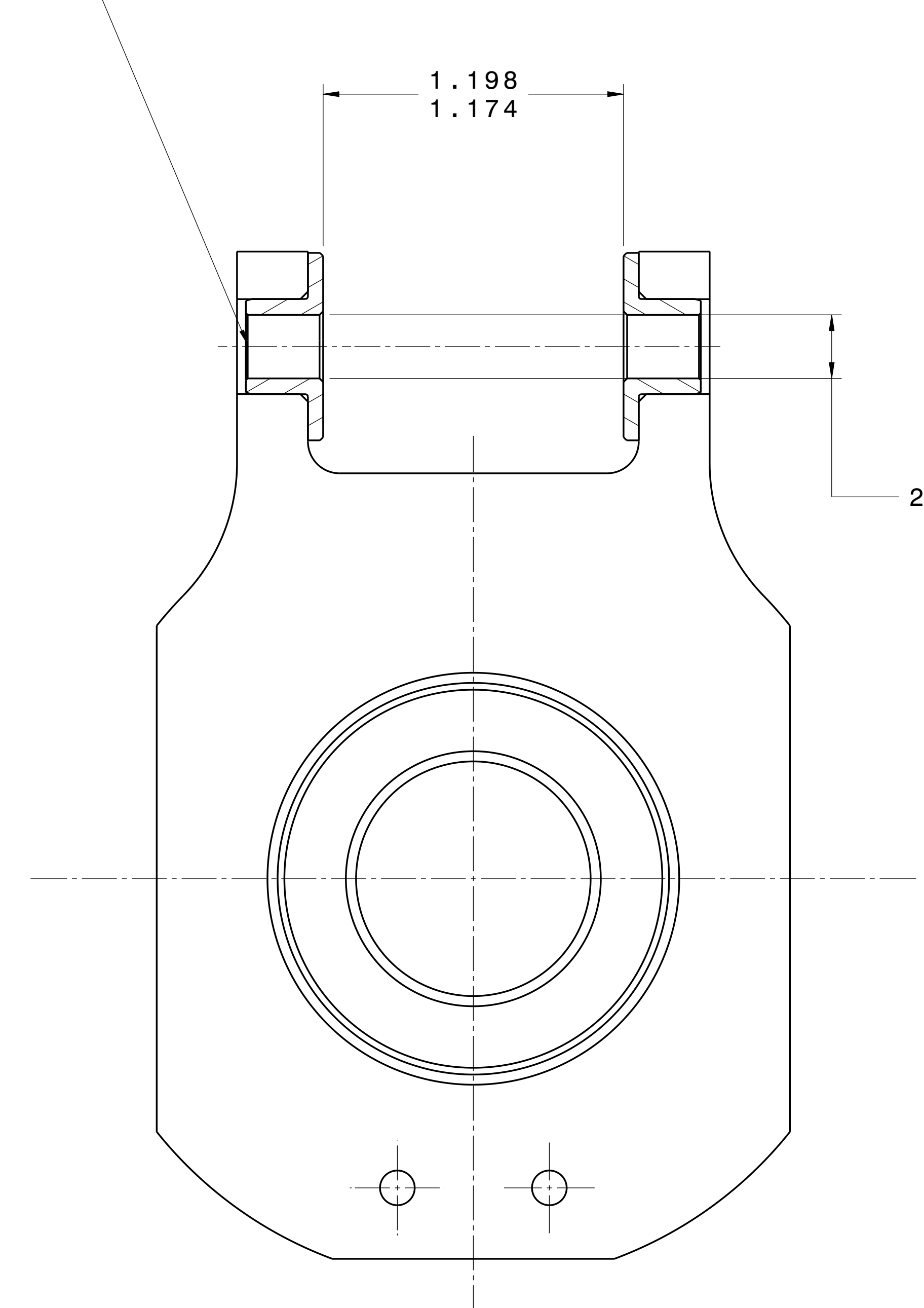

SECTION A-A
BeE ONYY 


\section{NOTES:}

1 Dimension APPLIES AFTeR INSTALLATION. HONE OR HAND REAM ONLY.

2 INSTALl CUSTOM BUSHINGS USing PRESS Fit METHOD OR EQUiVALENT.

3. BUSHINGS TO BE INSTALLED AFTER ANNODIZING OF RETRACT HOUSing COMPONENT.

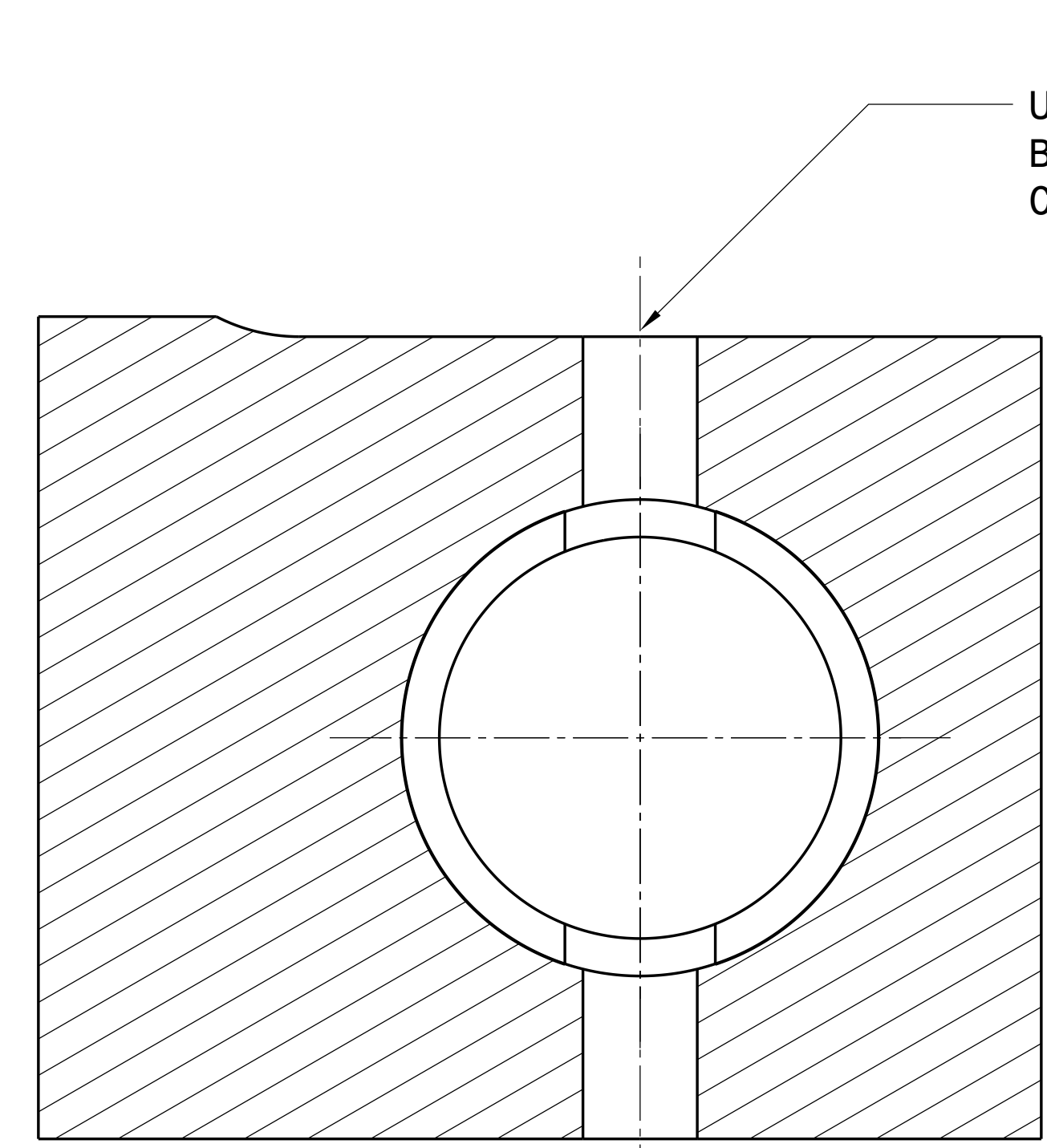

SECTION B-B

PAATTAL VIEN ONLY

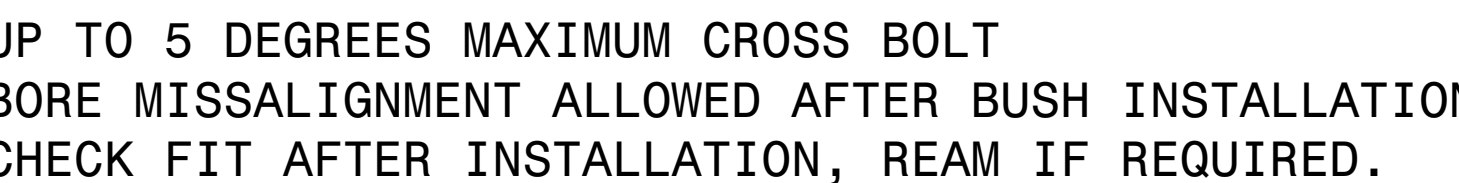

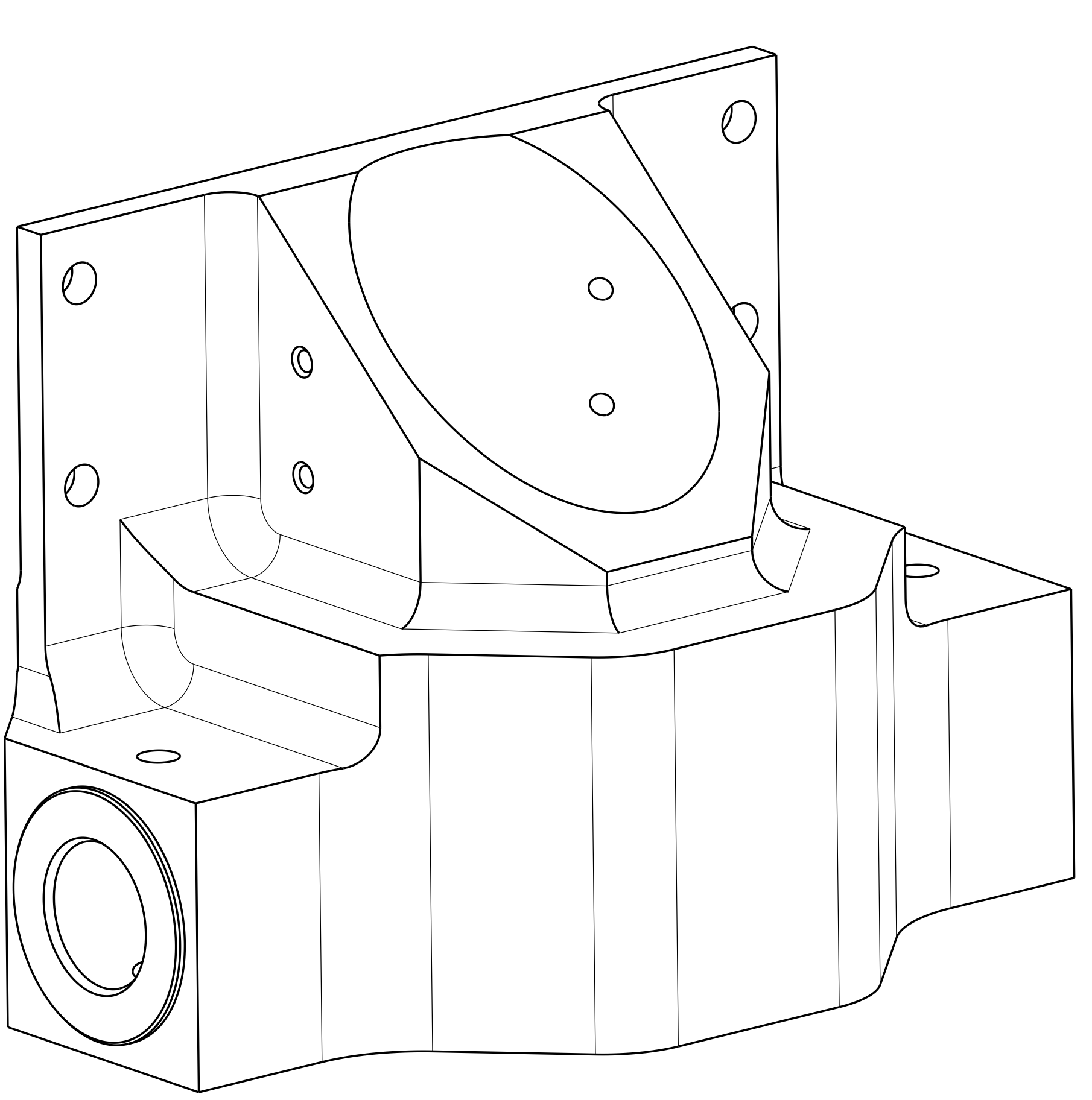

3D VIEW

REE ONLY
SCALE N NOI

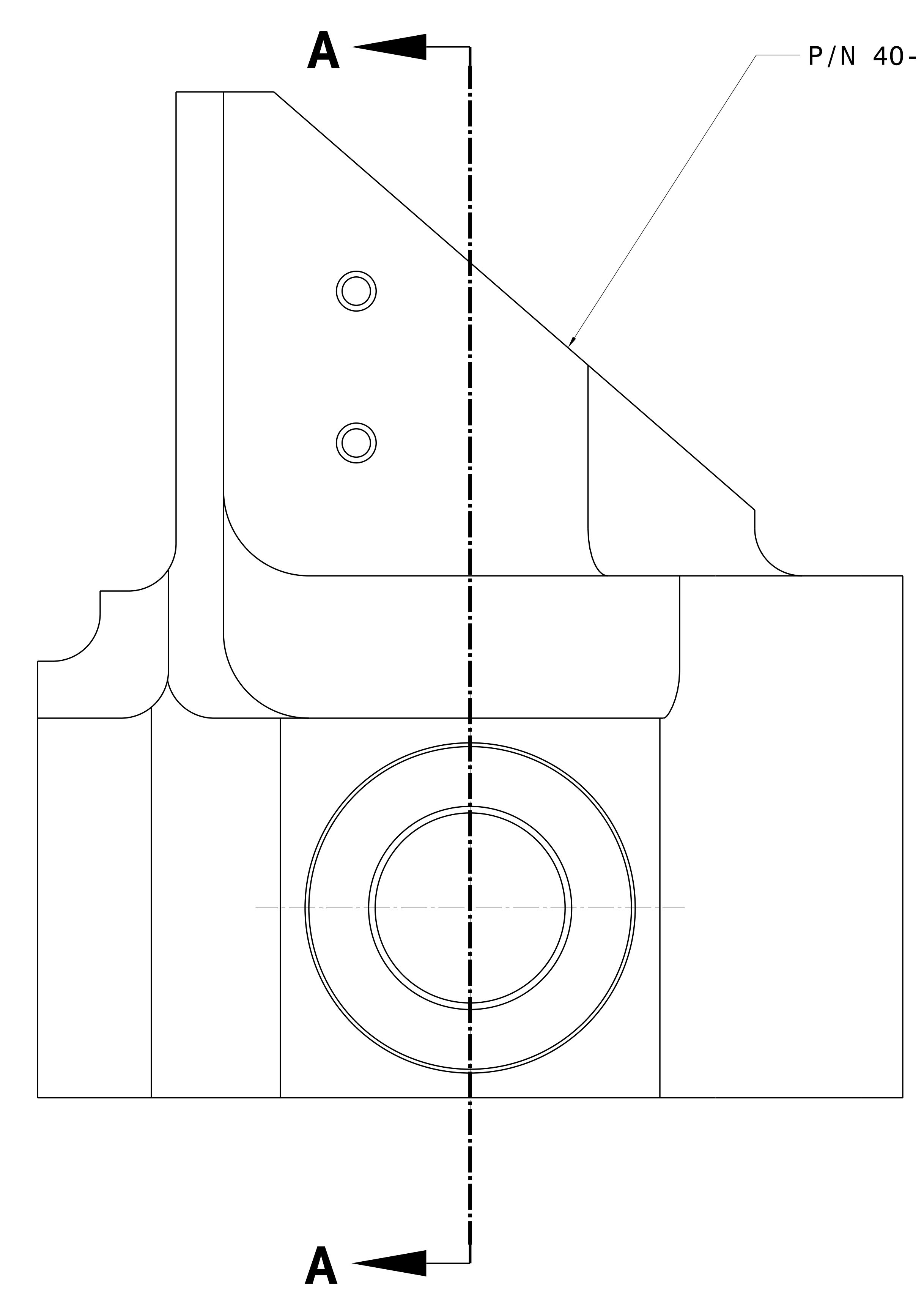

PN 40-1308-00, TRUNION BUSH, QTY 2-

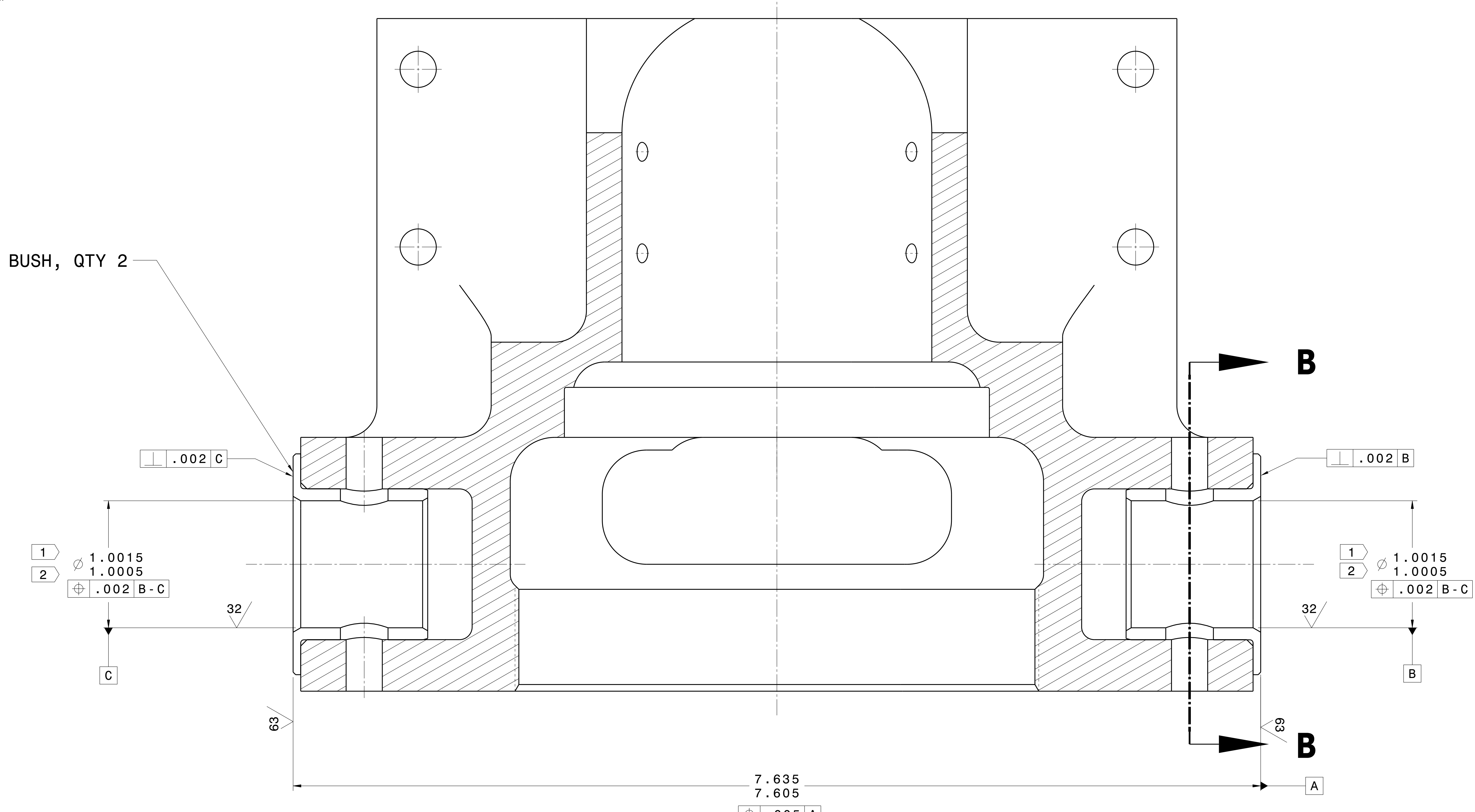

SECTION A-A

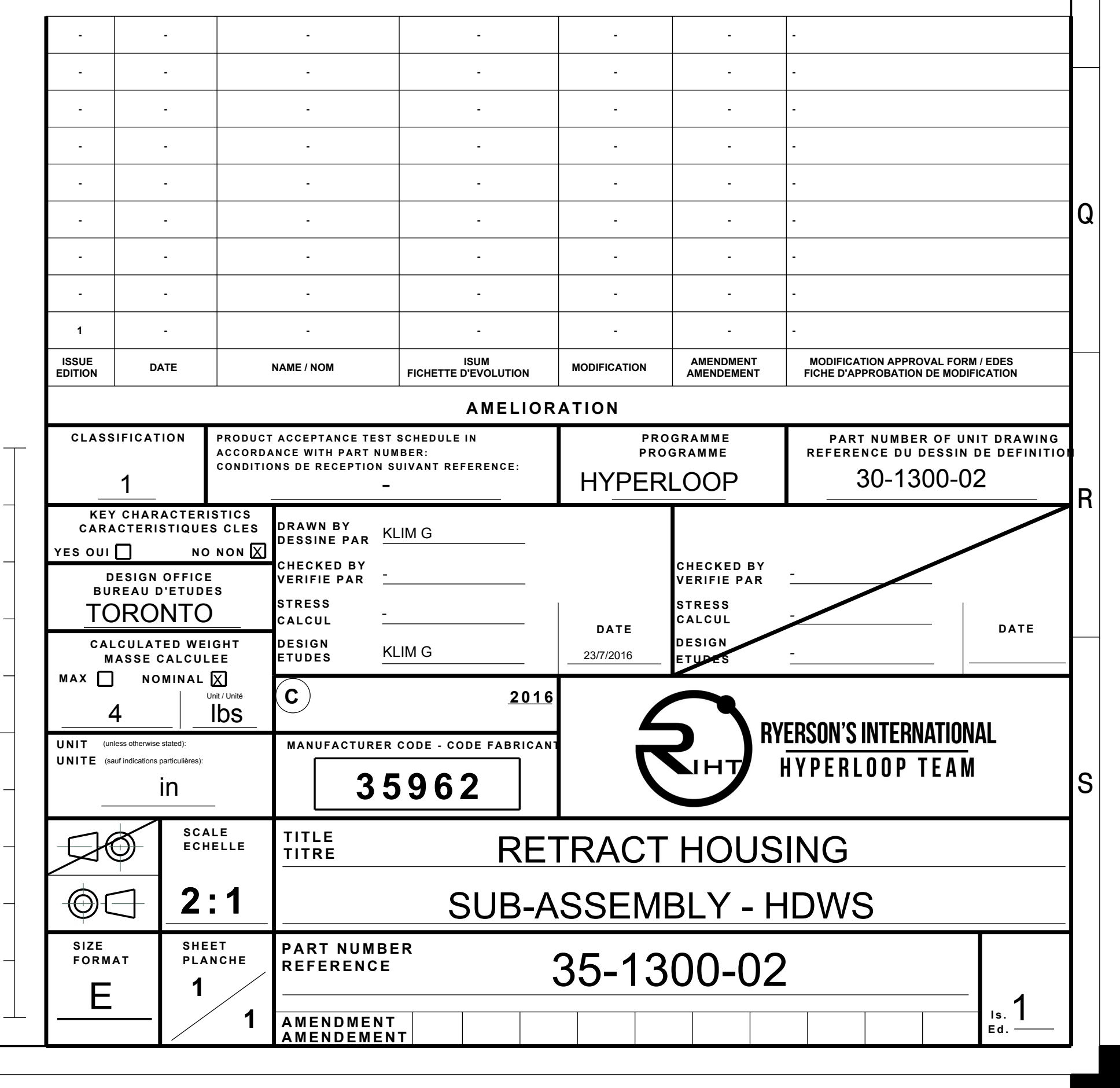




\section{NOTES:}

1 Dimension APPLIES AFTER INSTALLATION. HONE OR HAND REAM ONLY.

2 INSTALL KAMATICS BUSHINGS USING PRESS Fit OR COOLING METHOD..

3. BUSHINGS TO BE INSTALLED AFTER ANNODIZing OF MATING COMPONENT.

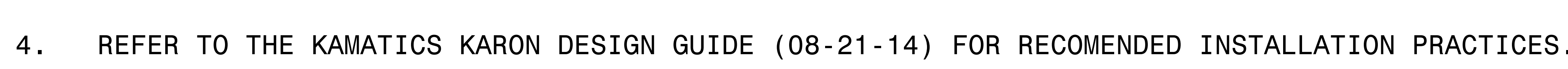

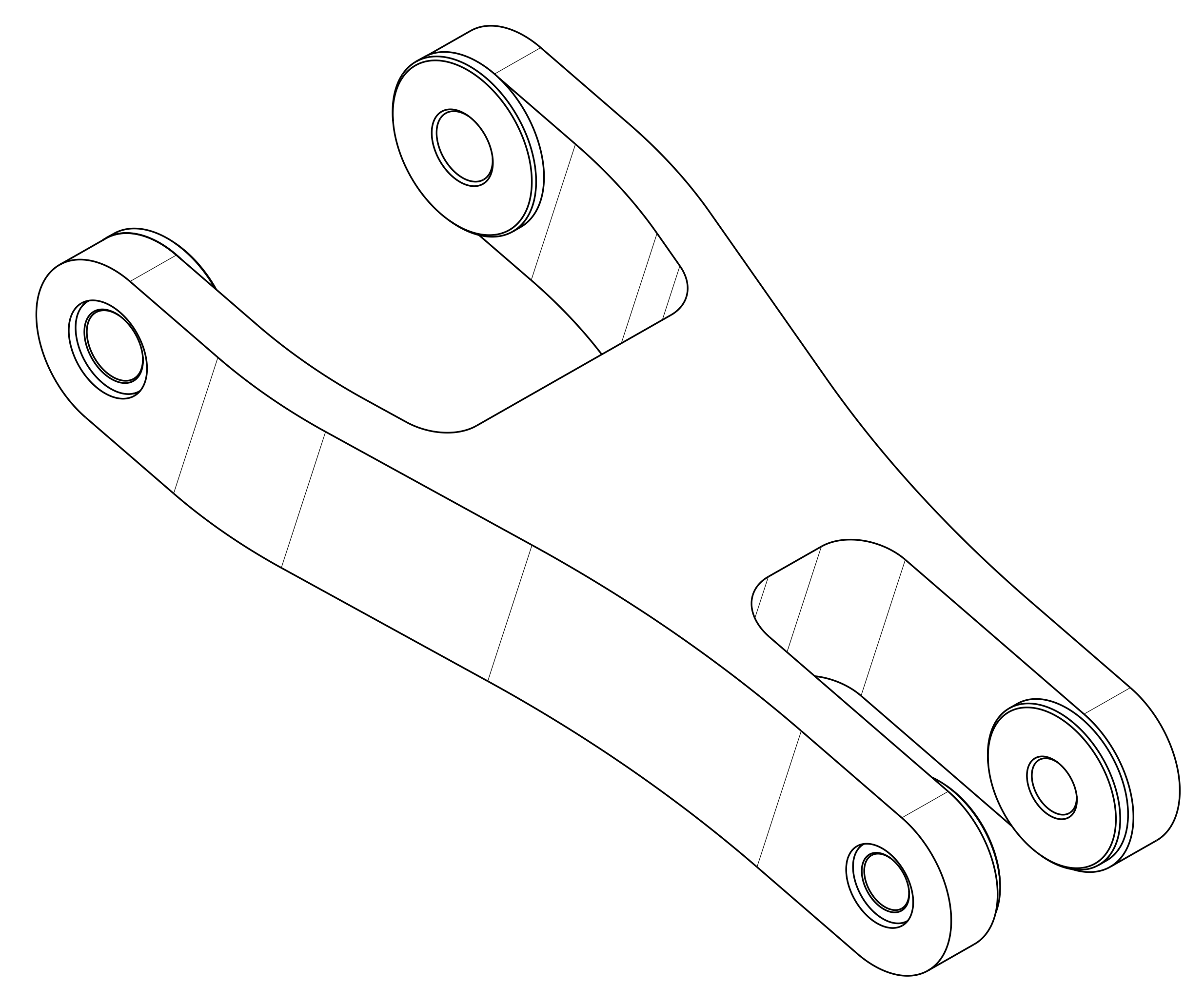

3D VIEW

SBEE OOMY
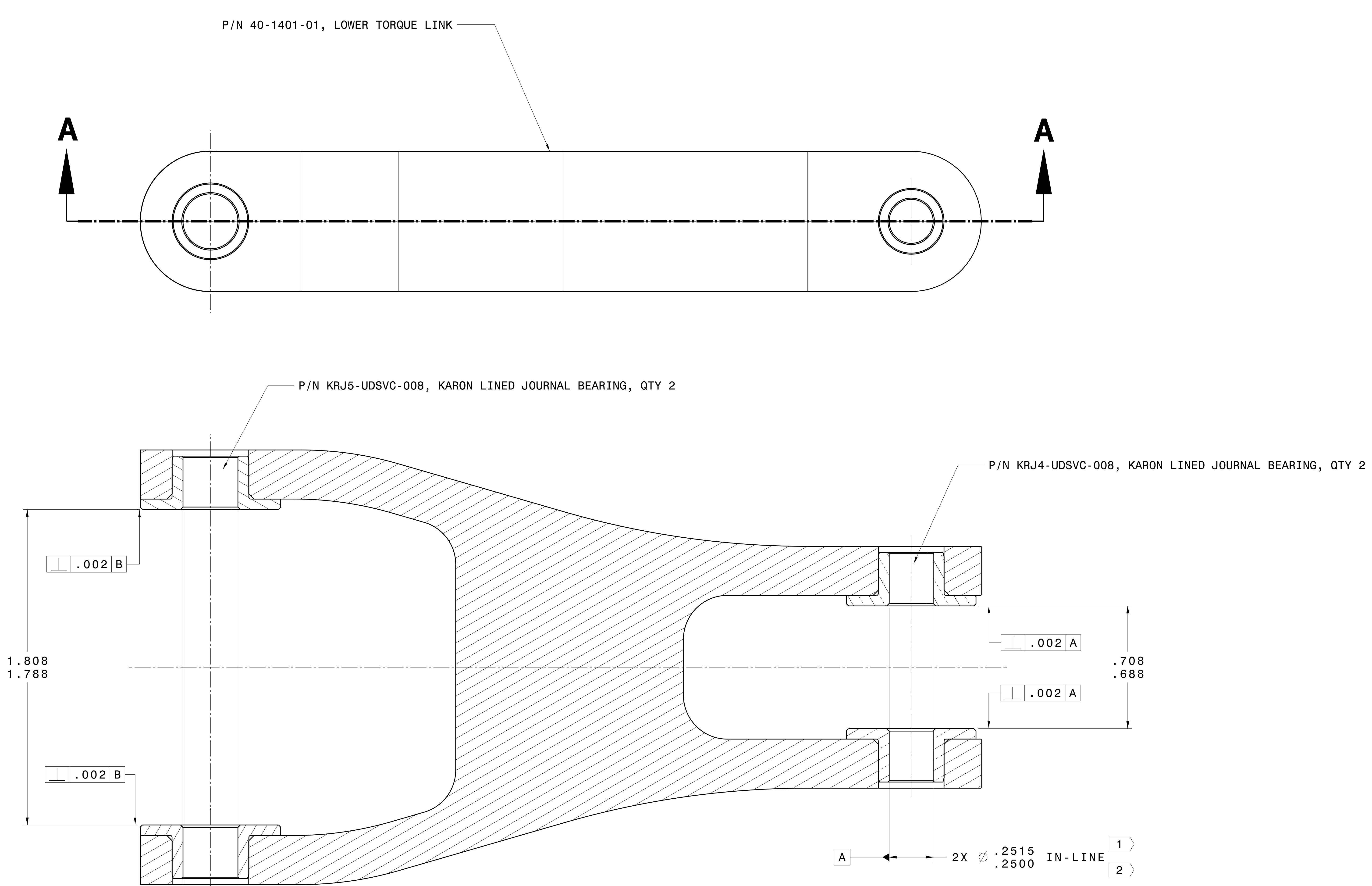

SECTION A-A

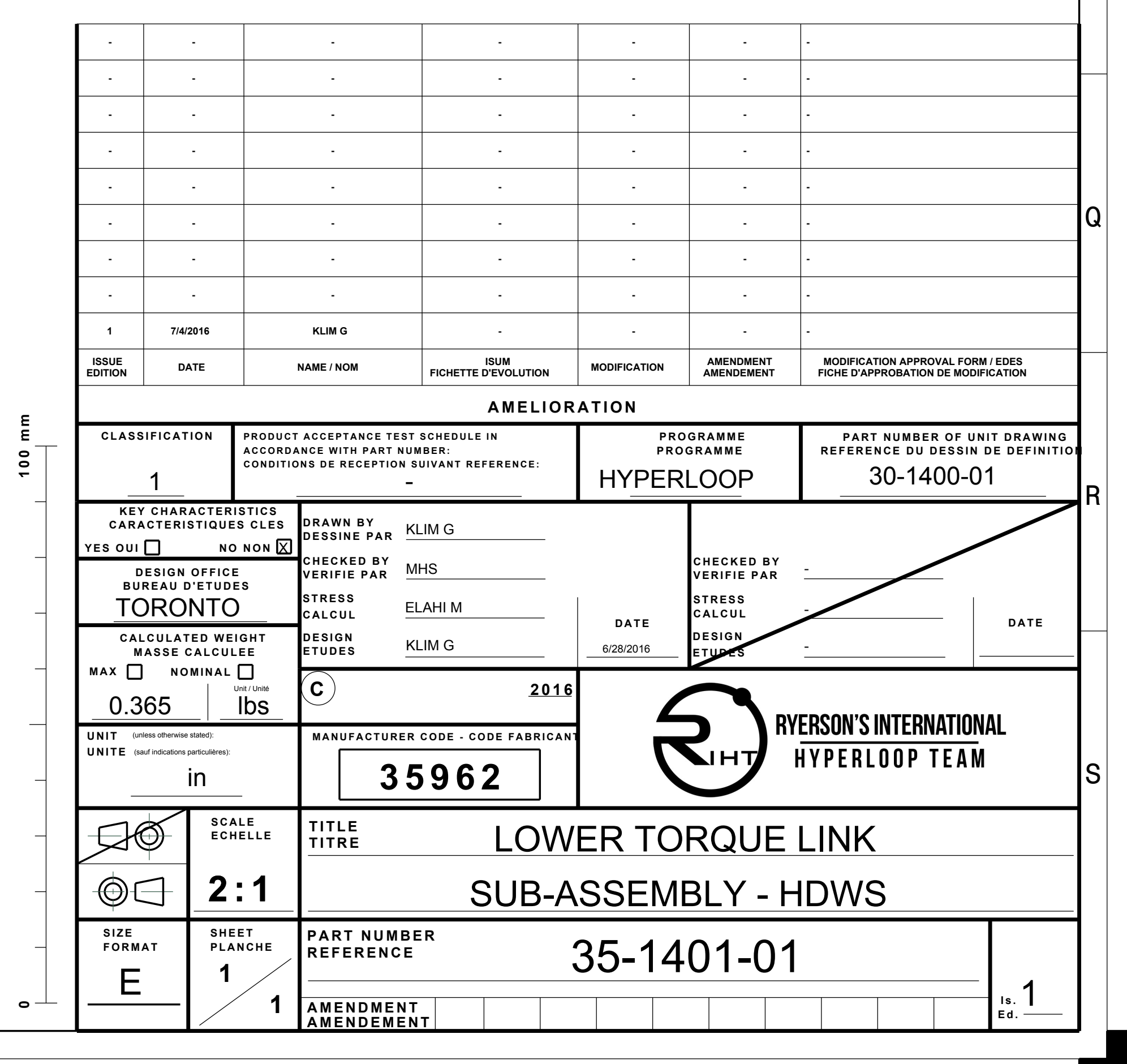




\section{NOTES:}

1 Dimension APPLIES AfTer installation. Hone OR HAND REAM ONLY.

2 INSTALL KAMATICS BUSHINGS USING PRESS Fit OR COOLING METHOD.

3. BUSHINGS TO BE INSTALLED AFTER ANNODIZING OF MATING COMPONENT.

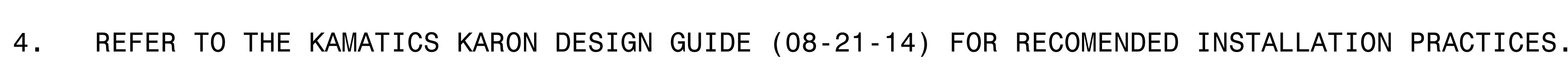

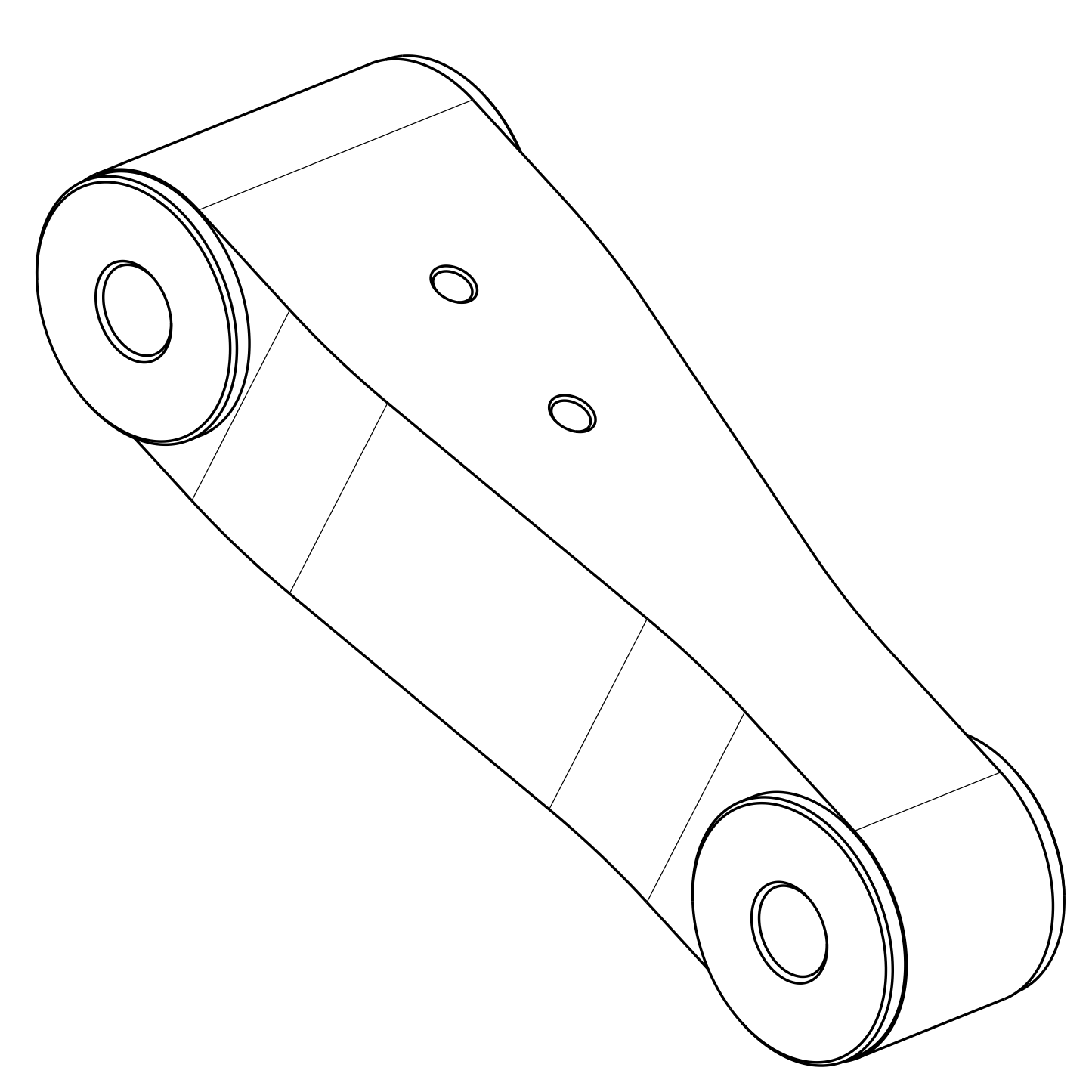

3D VIEW

SCAEF: ONLY NONE
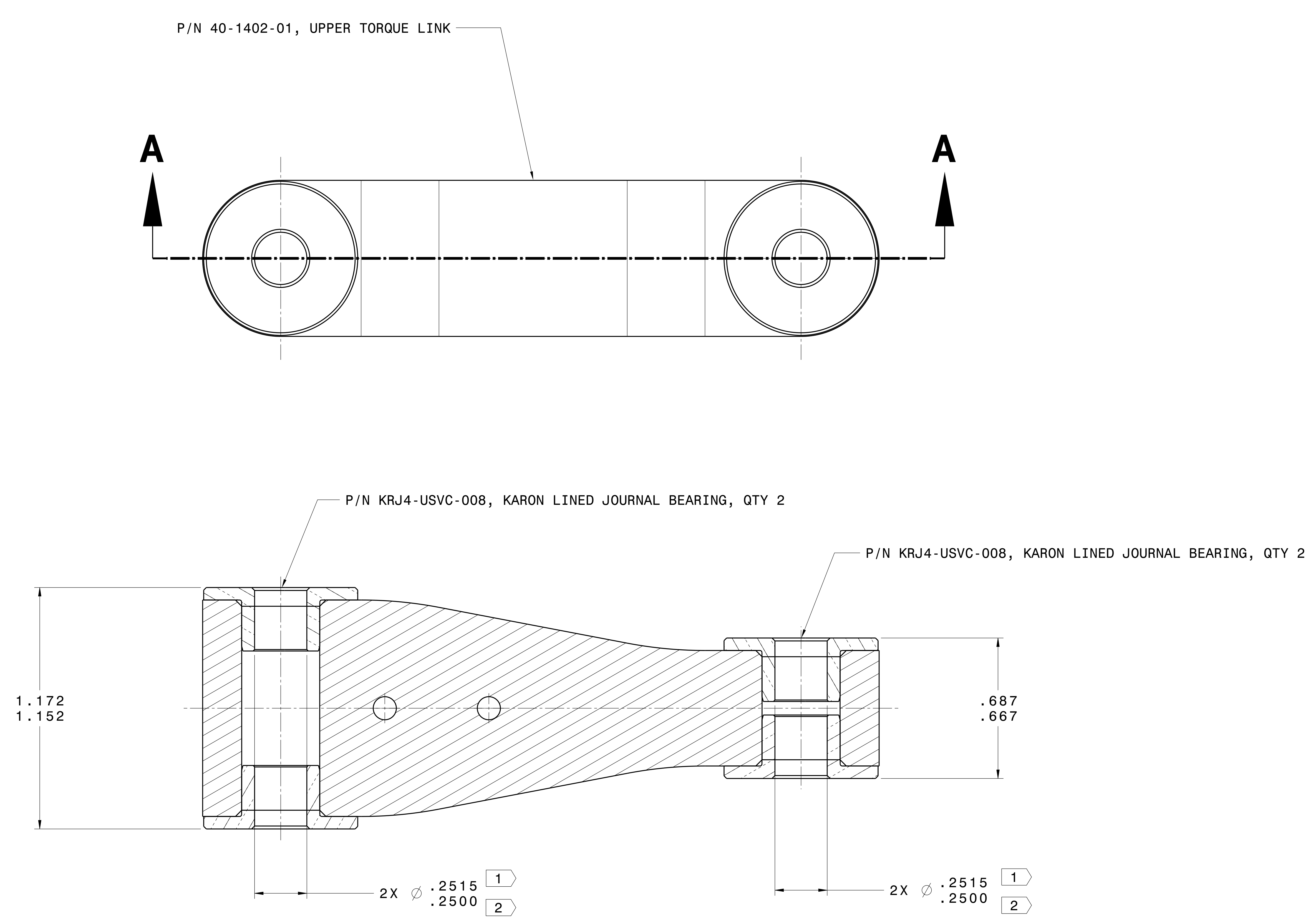

SECTION A-A

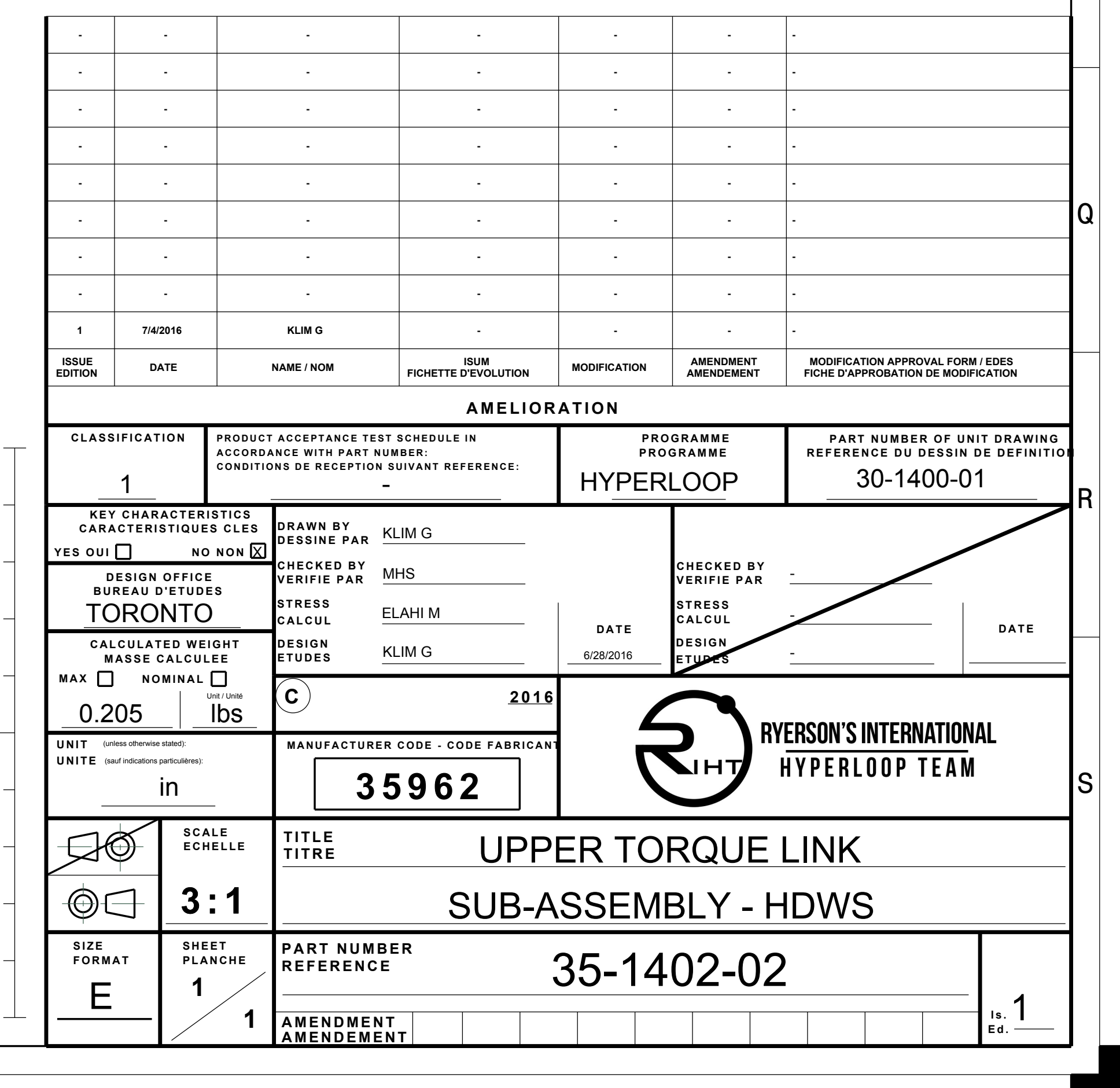




\section{NOTES:}

1. MATERIAL: ALUMINUM 6061-T6511 (BAR) PER AMS QQ-A-200/8.

2. DeburR Sharp edGes $.005 / .015$, UnLess Otherwise indicated.

3. ANODIZING ALL OVER

4 PART MARKING USING SCRIBING OR PUNCH.

5. FOR ALL AREAS NOT FULLY DEFINED ON THE FACE OF THE DRAWING,
THE CAD DATA MAY BE USED WITH A PROFILE TOLERANCE OF $\triangle .040$.

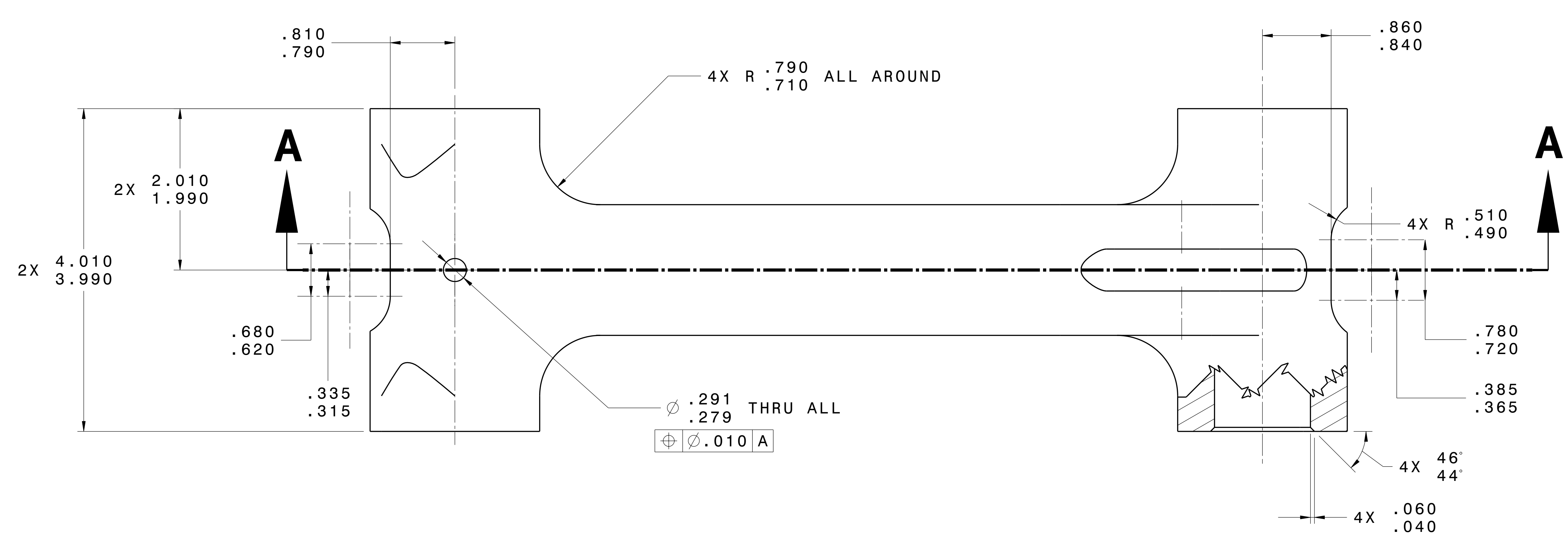

\section{$\Lambda$}
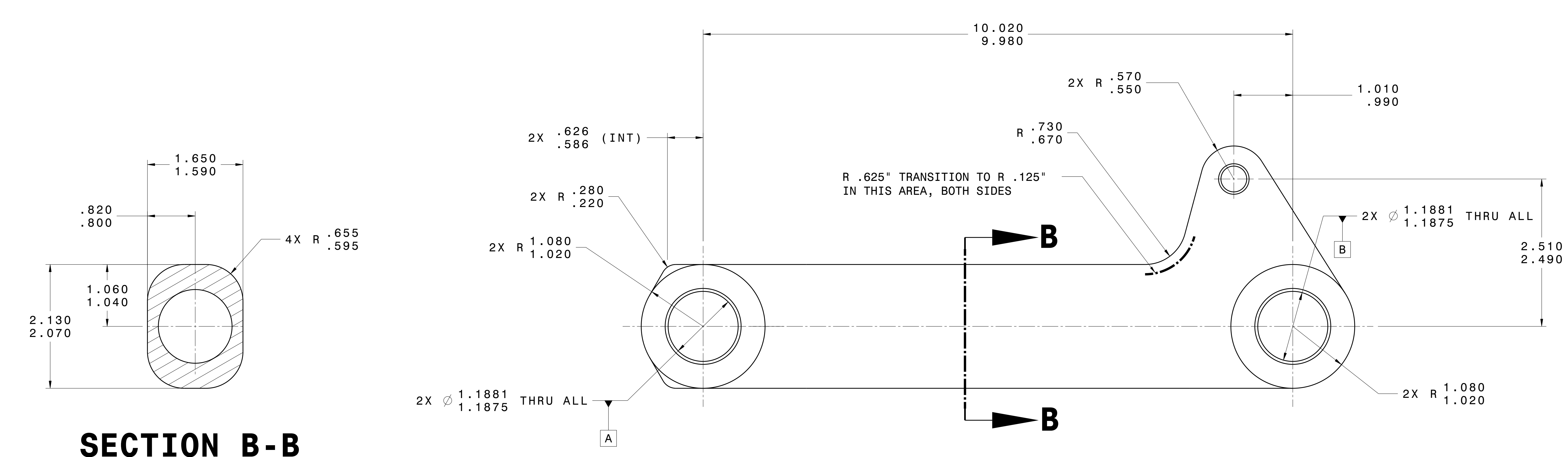

SECTION B-B

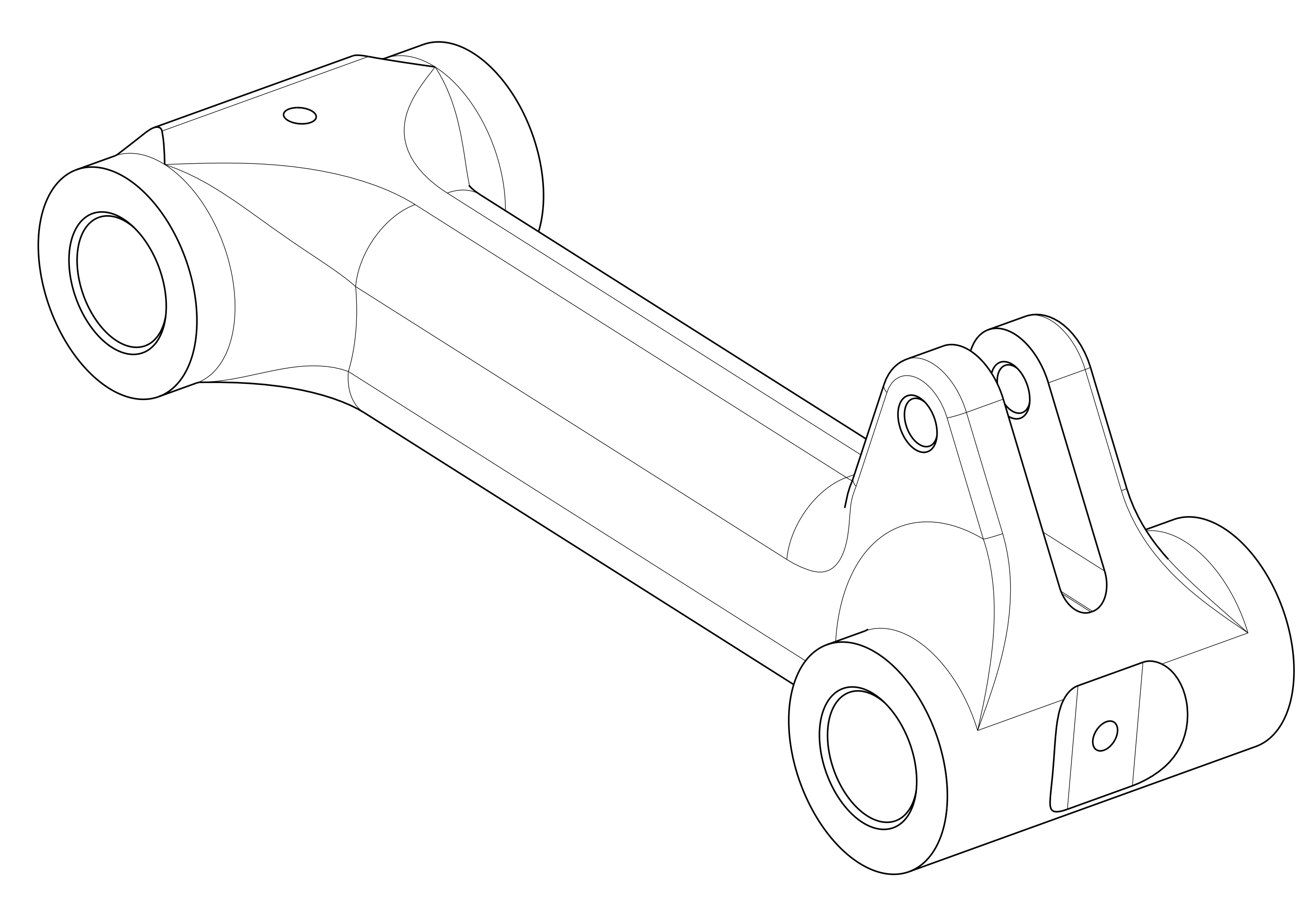

3D VIEW

SCALE: ONLY NONE
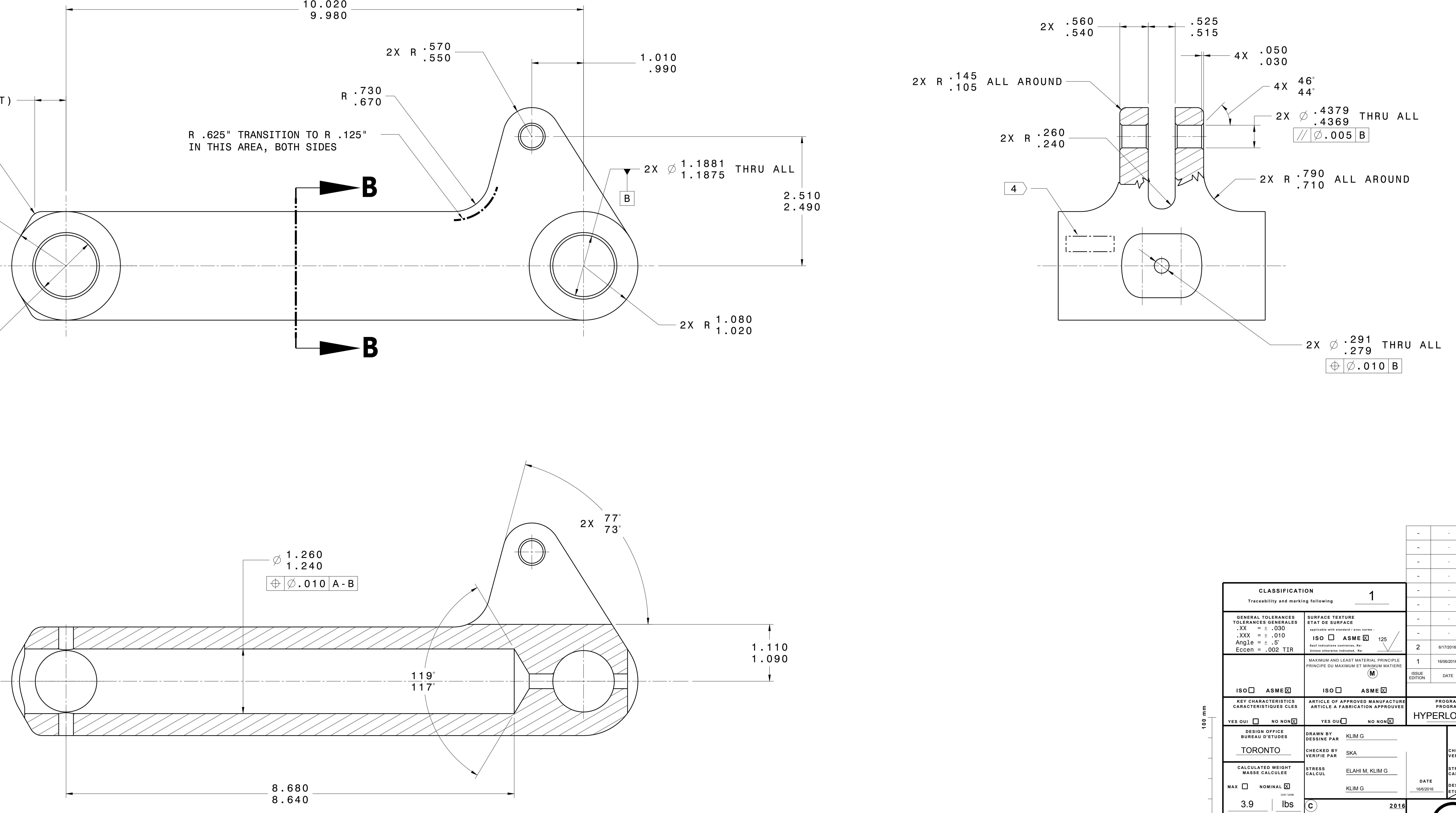

SECTION A-A

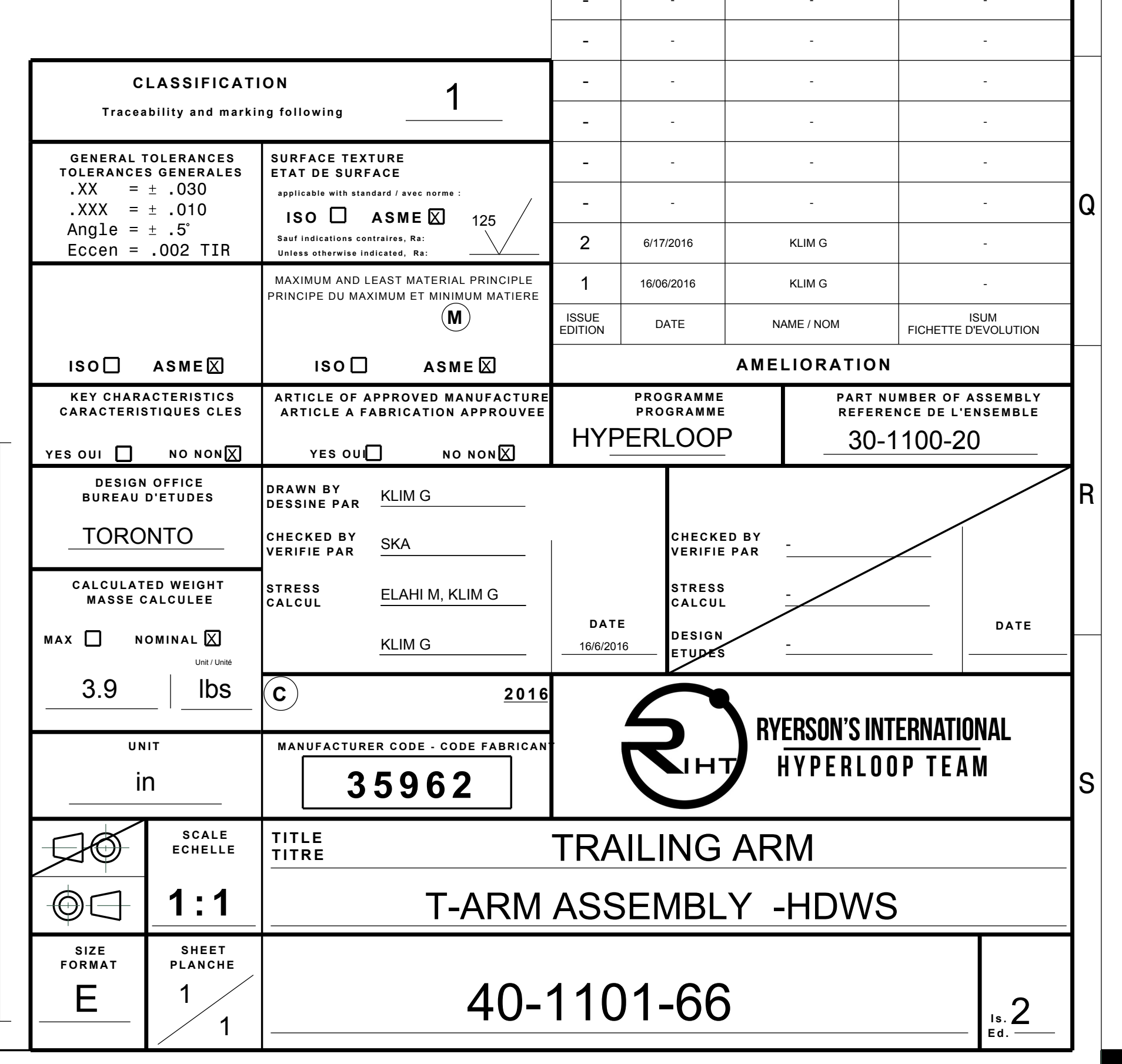




\section{NOTES:}

1. MATERIAL: CORROSION RESISTANT STEEL (303) PER ASTM A582 OR EQUiVAlENT. ALL MATERIAL TO BE IN THE ANNEALED CONDITION.

2. DEBURR SHARP EDGES $.005 / .015$, UNLESS OTHERWISE INDICATED.

3. PASSivation all OVER PER AMS 2700 METHOD 1.

4. BAG \& TAG OR FIXED TAG MARKING.

\section{D VIEW}

REF ONLY

SCALE : NONE
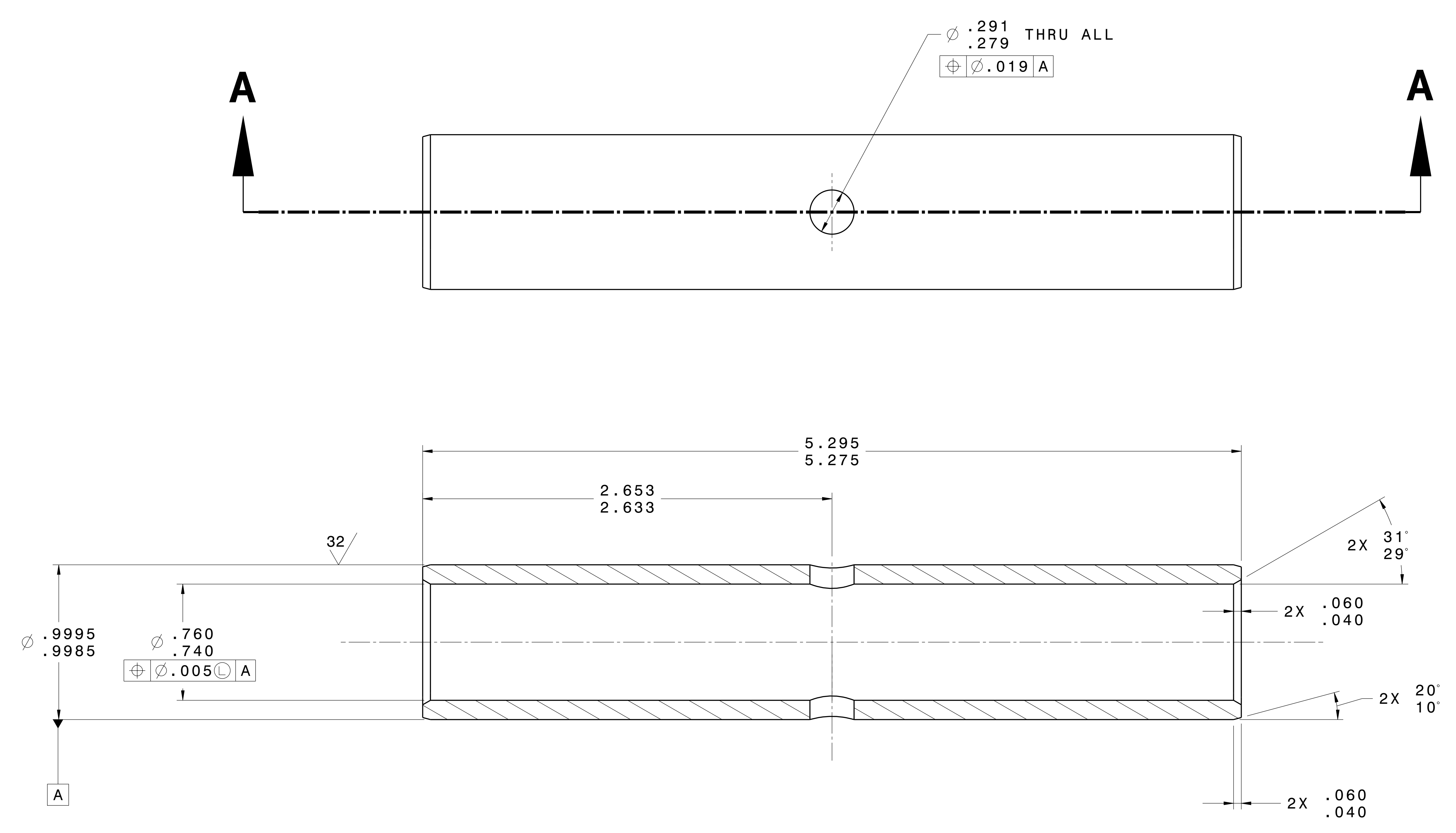

\section{SECTION A-A}

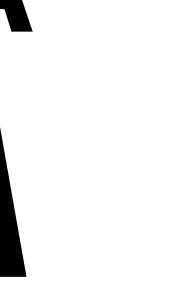
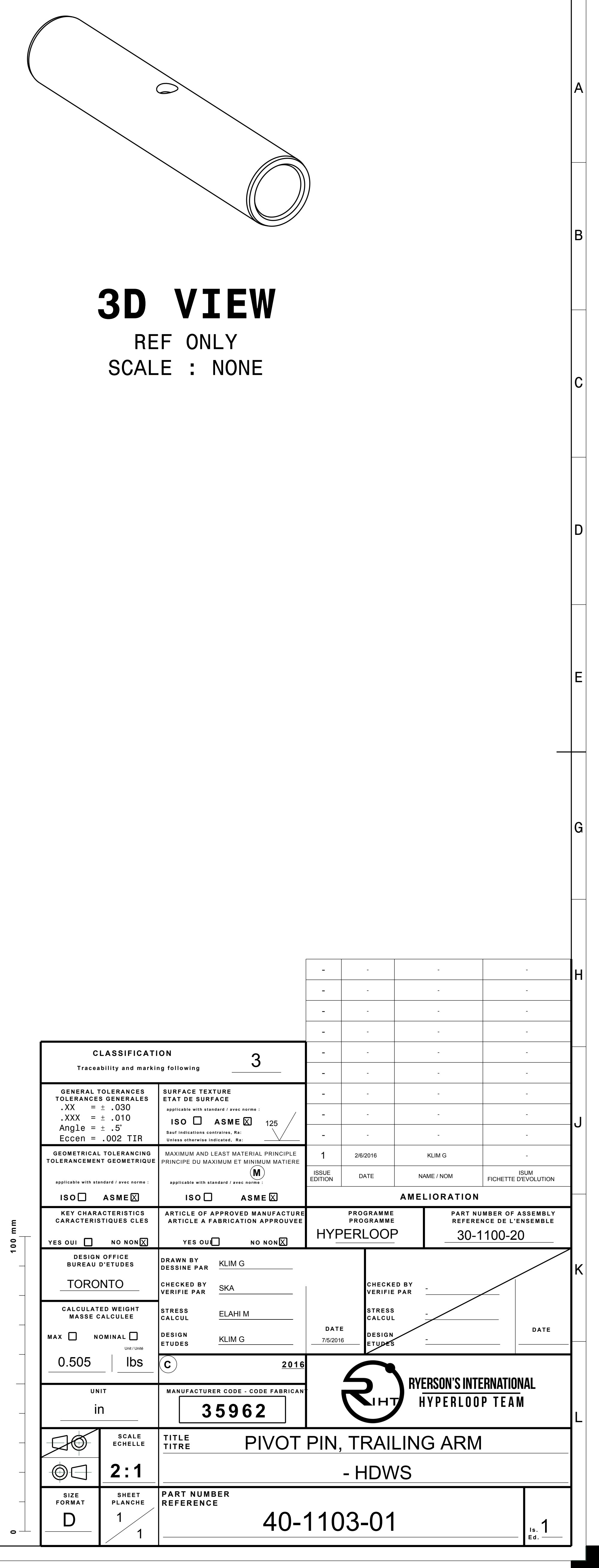


\section{NOTES:}

1. MATERIAL: AL BRONZE (C95400) PER ASTM B505 OR EQUiVALENT.

2. DEBURR SHARP EDGES $.005 / .015$, UNLESS OTHERWISE INDICATED.

3. MACHINE IN THE FULLY HEAT TREATED CONDITION.

4. PART MARKING USING BAG \& TAG.

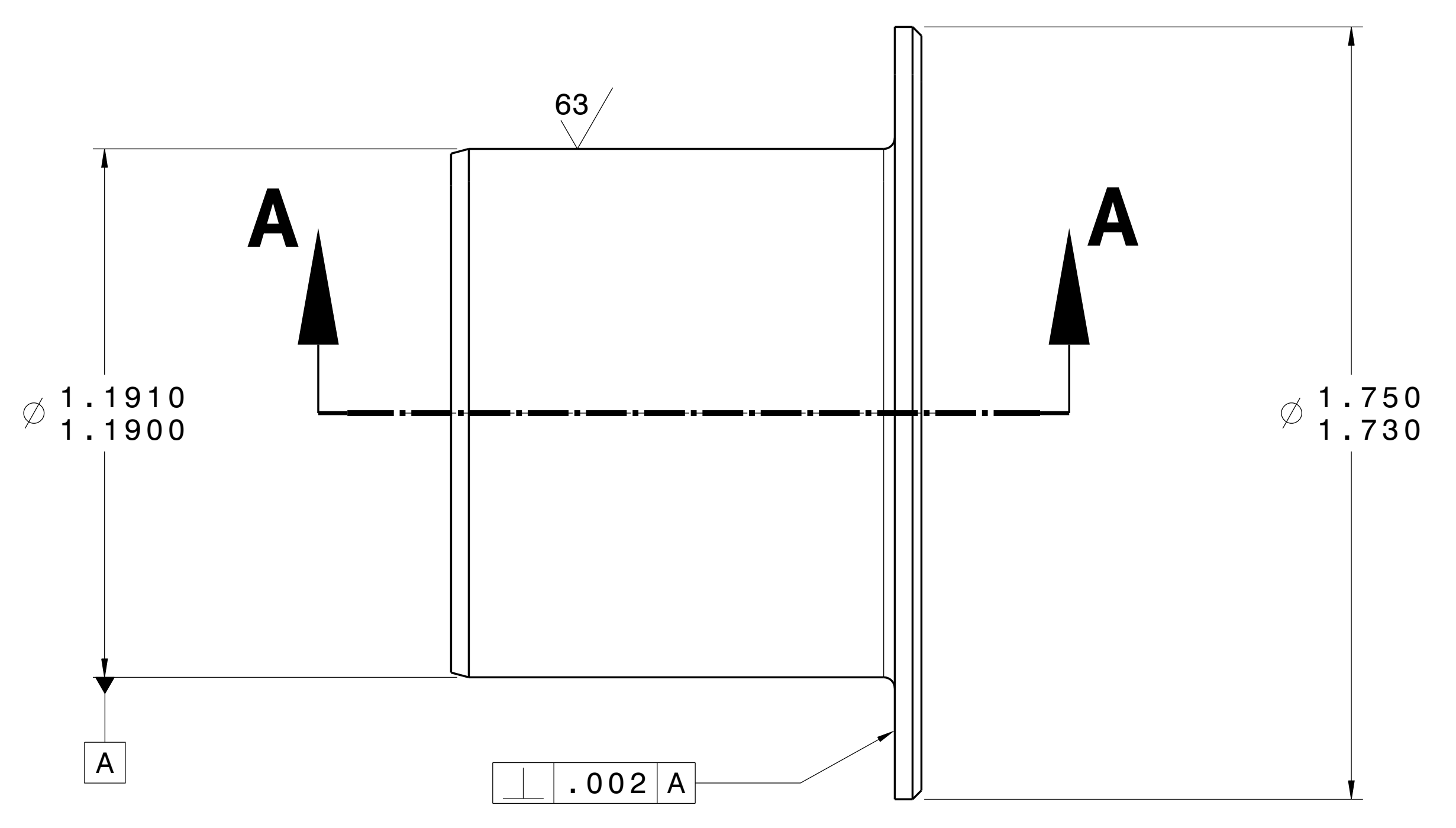

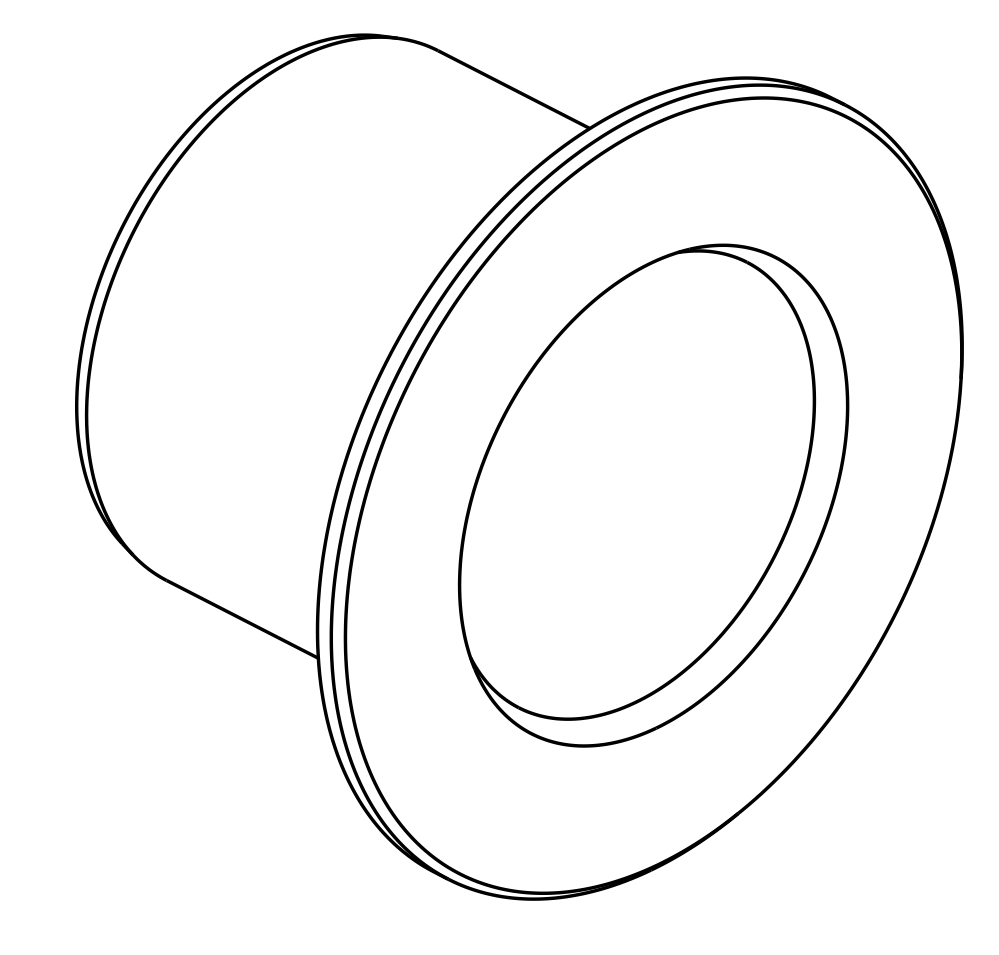

3D VIEW

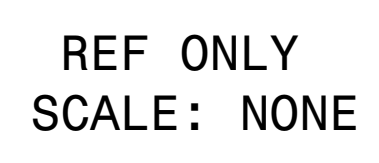

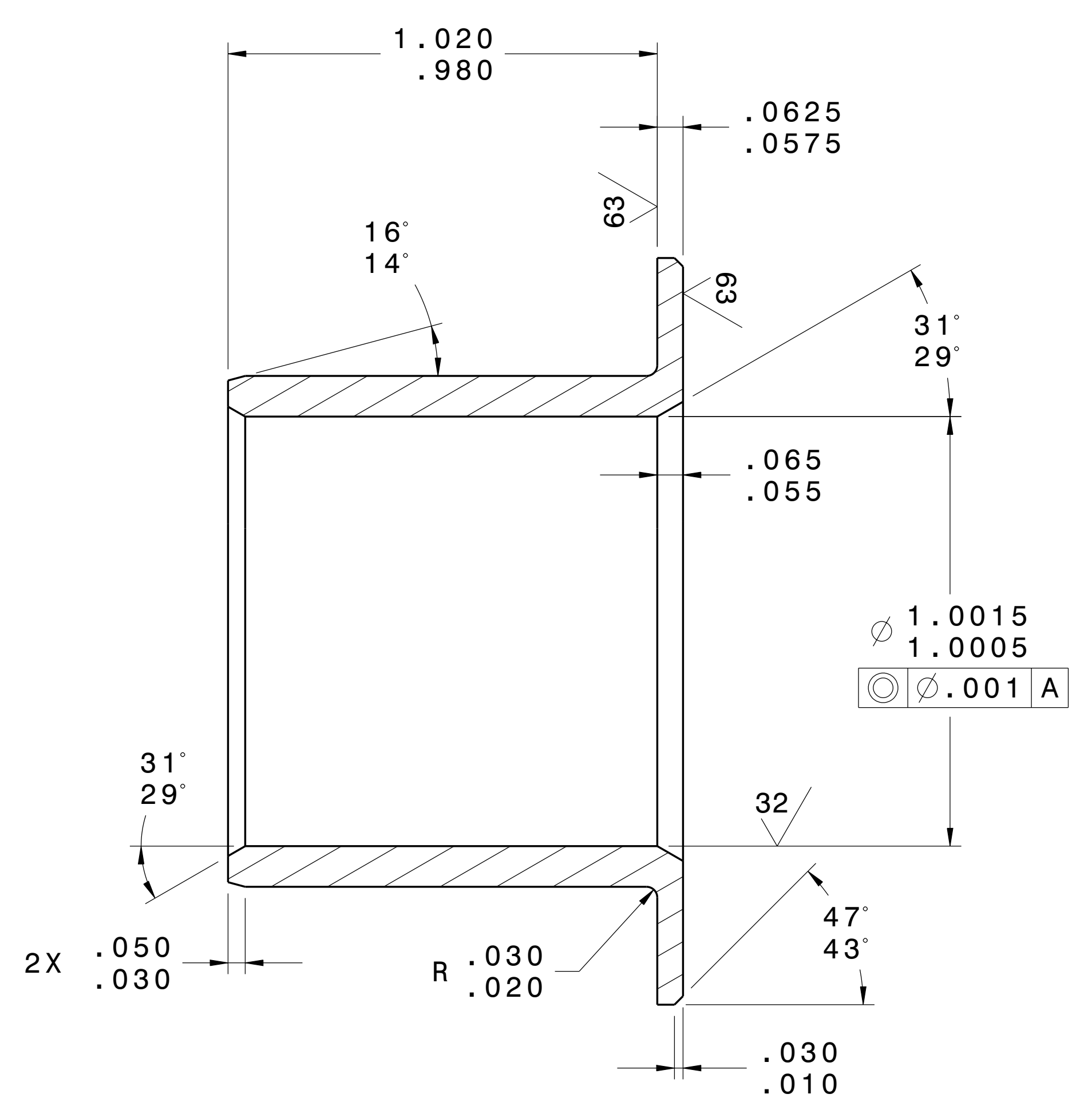

SECTION A-A

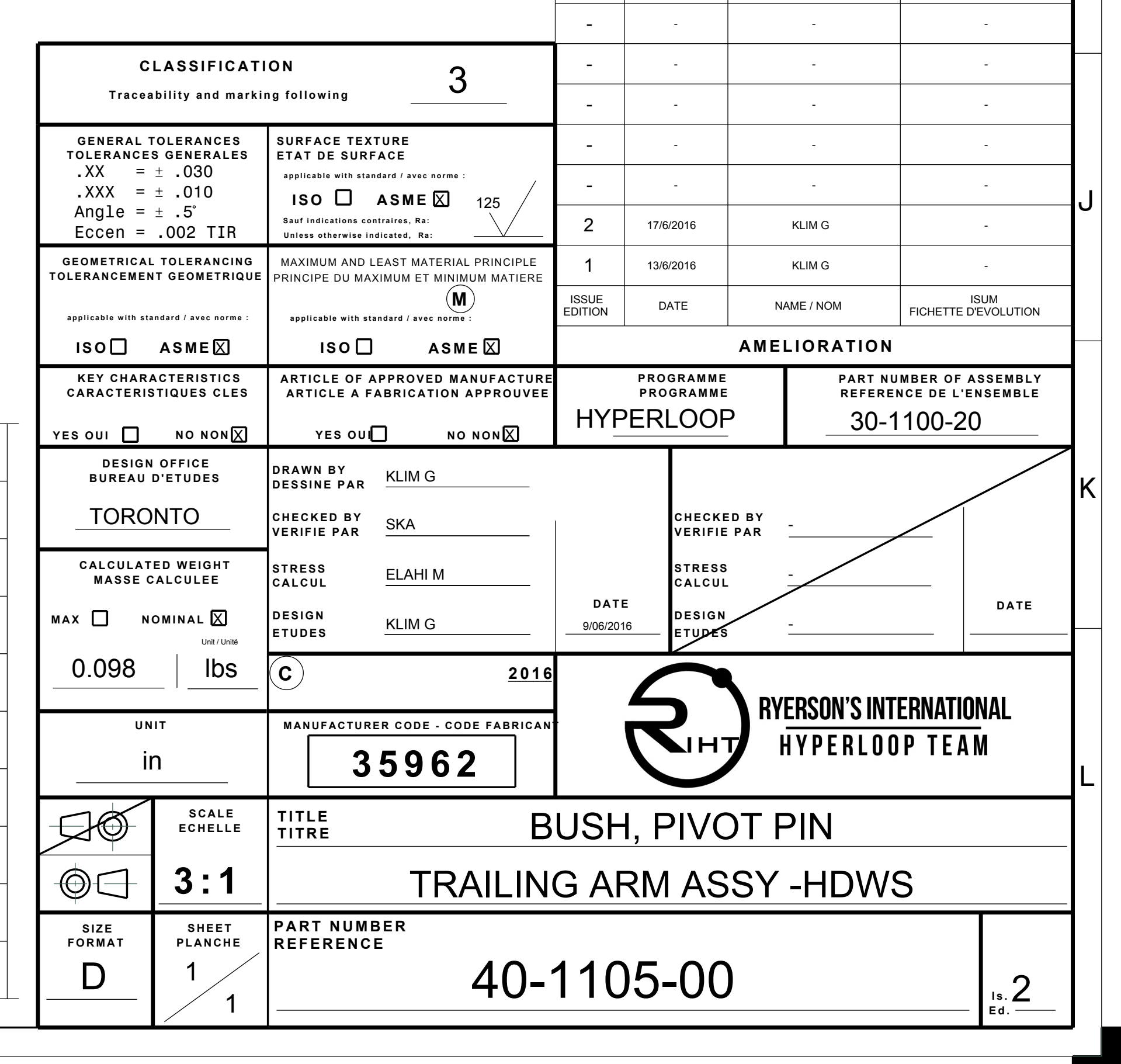




\section{NOTES:}

1. MATERIAL: AL BRONZE (C95400) PER ASTM B505 OR EQUiVALENT.

2. DEBURR SHARP EDGES $.005 / .015$, UNLESS OTHERWISE INDICATED.

3. MACHINE IN THE FULLY hEAT TREATED CONDITION.

4. PART MARKING USING BAG \& TAG.

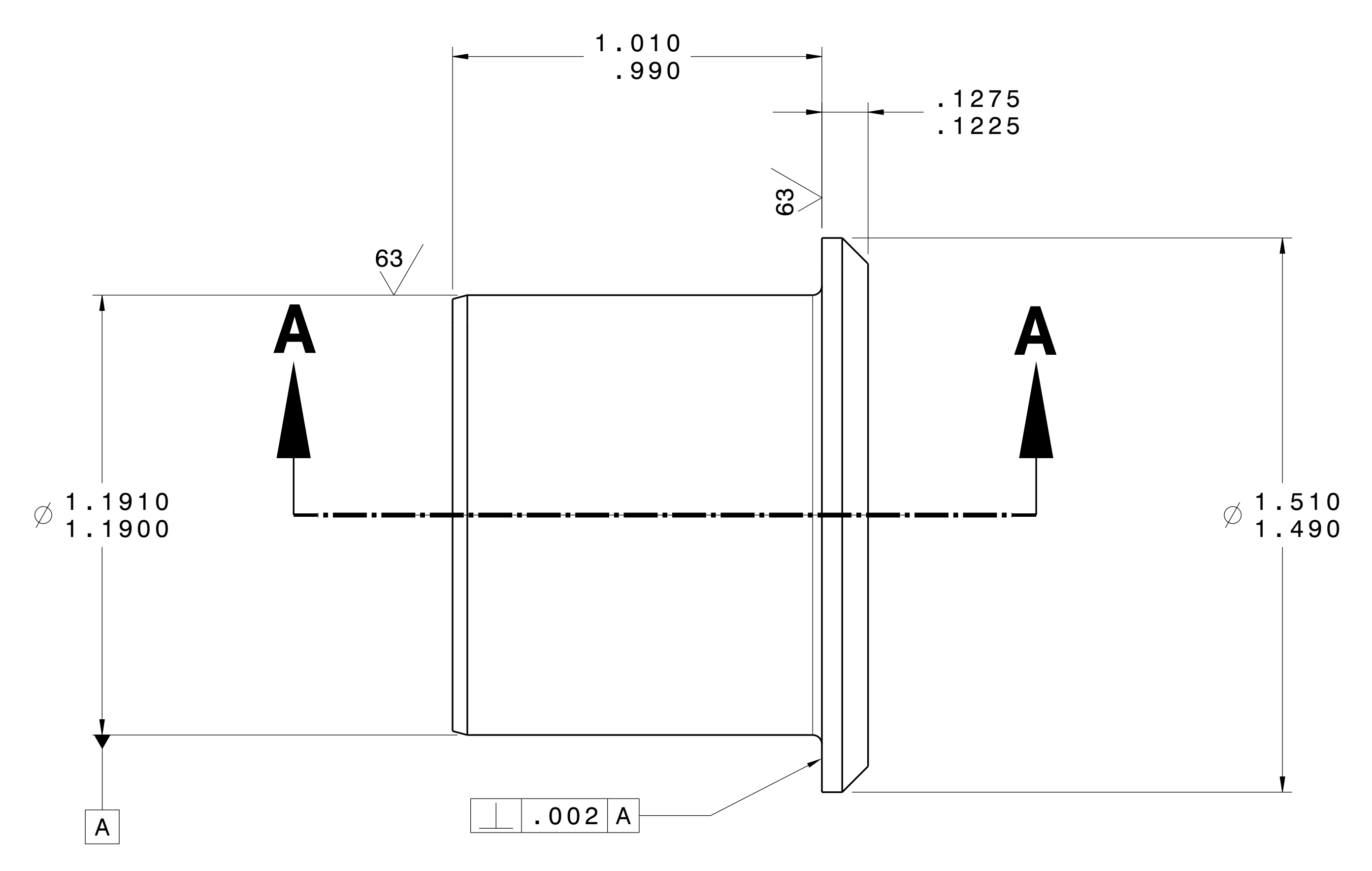

3D VIEW $\substack{\text { REE ONY } \\ \text { SCALE: NONE }}$

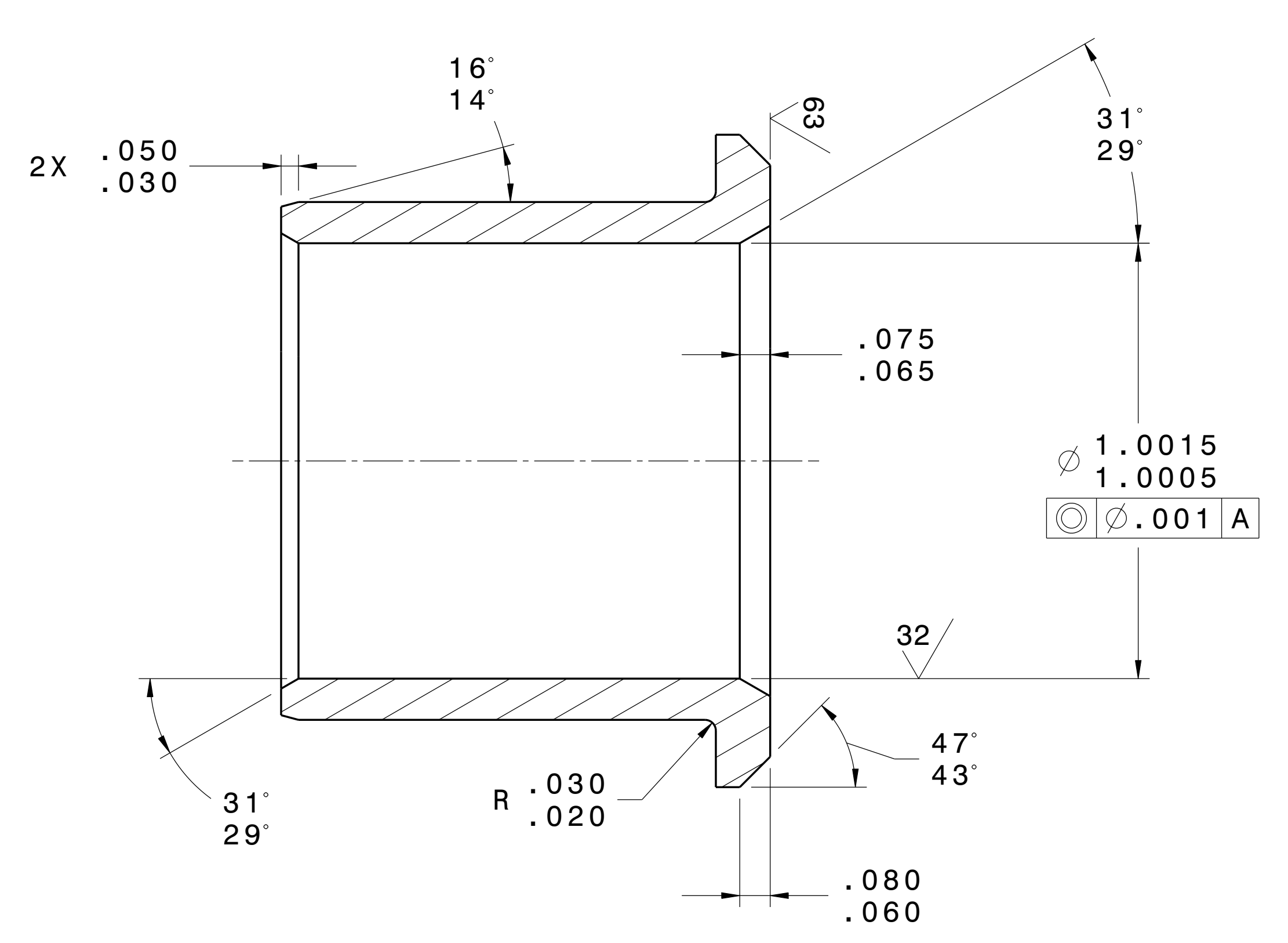

SECTION A-A

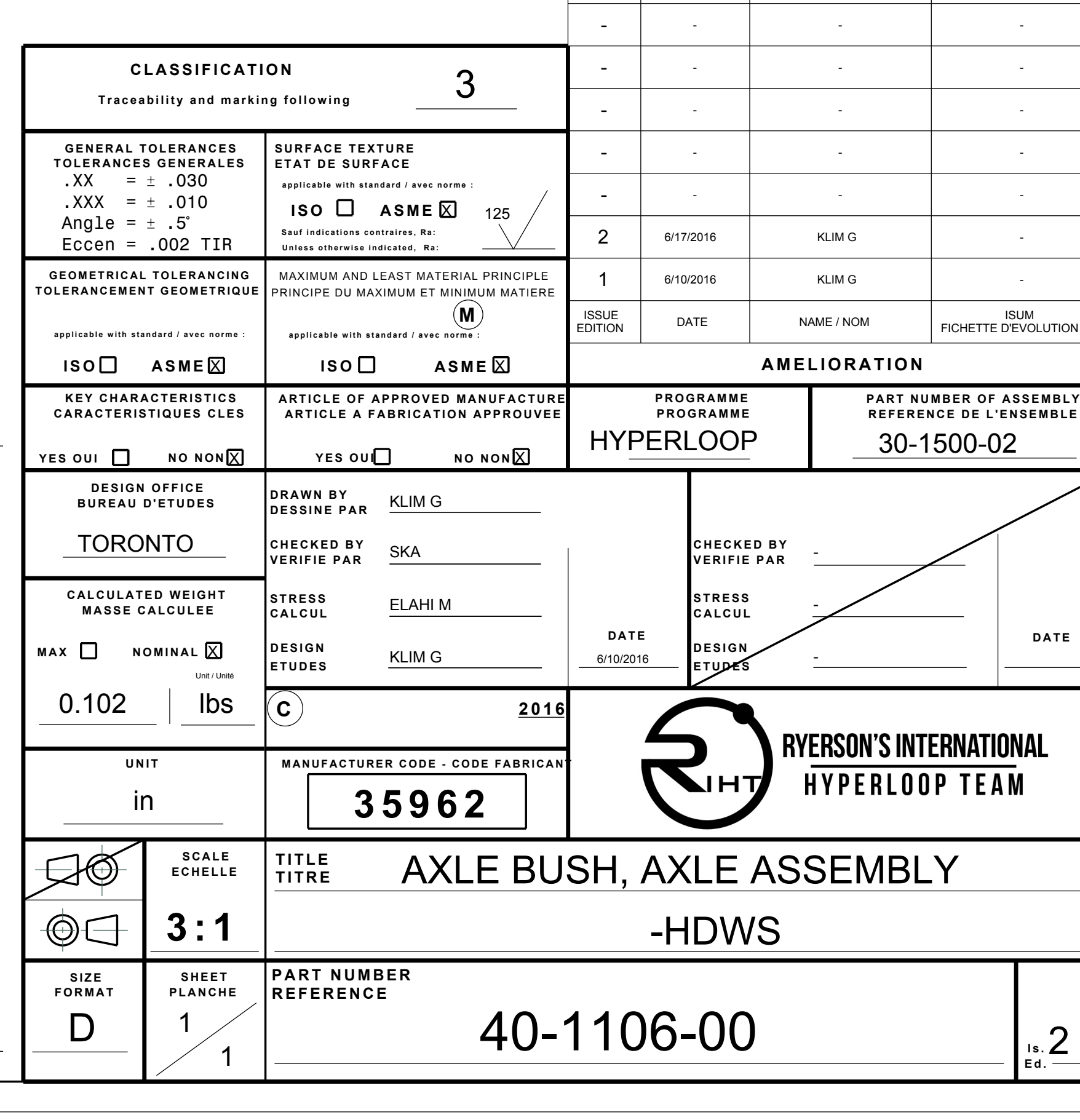




\section{NOTES:}

1. MATERIAL: ALUMinUm 6061-T6511 (BAR) PER AMS QQ-A-200/8.

2. DEBURR SHARP EDGES $.005 / .015$, UNLESS OTHERWISE INDICATED.

3. ANODIZING ALL OVER.

4 PART MARKING USING SCRIBING OR PUNCH.

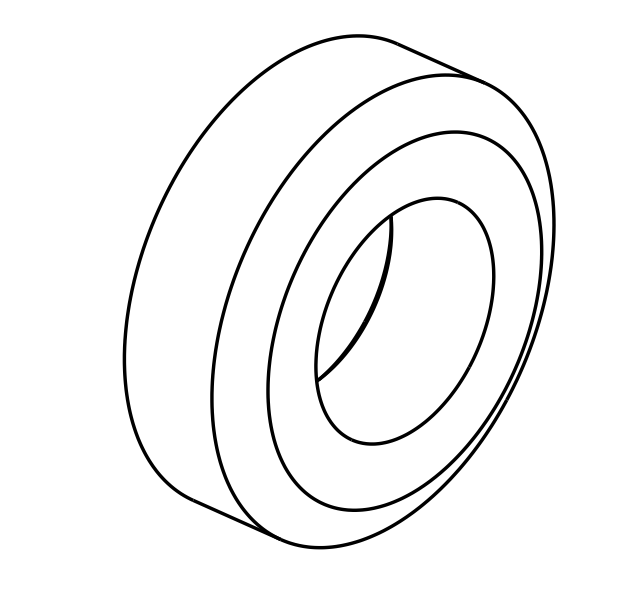

3D VIEW

REE ONI
SCALE: NONE
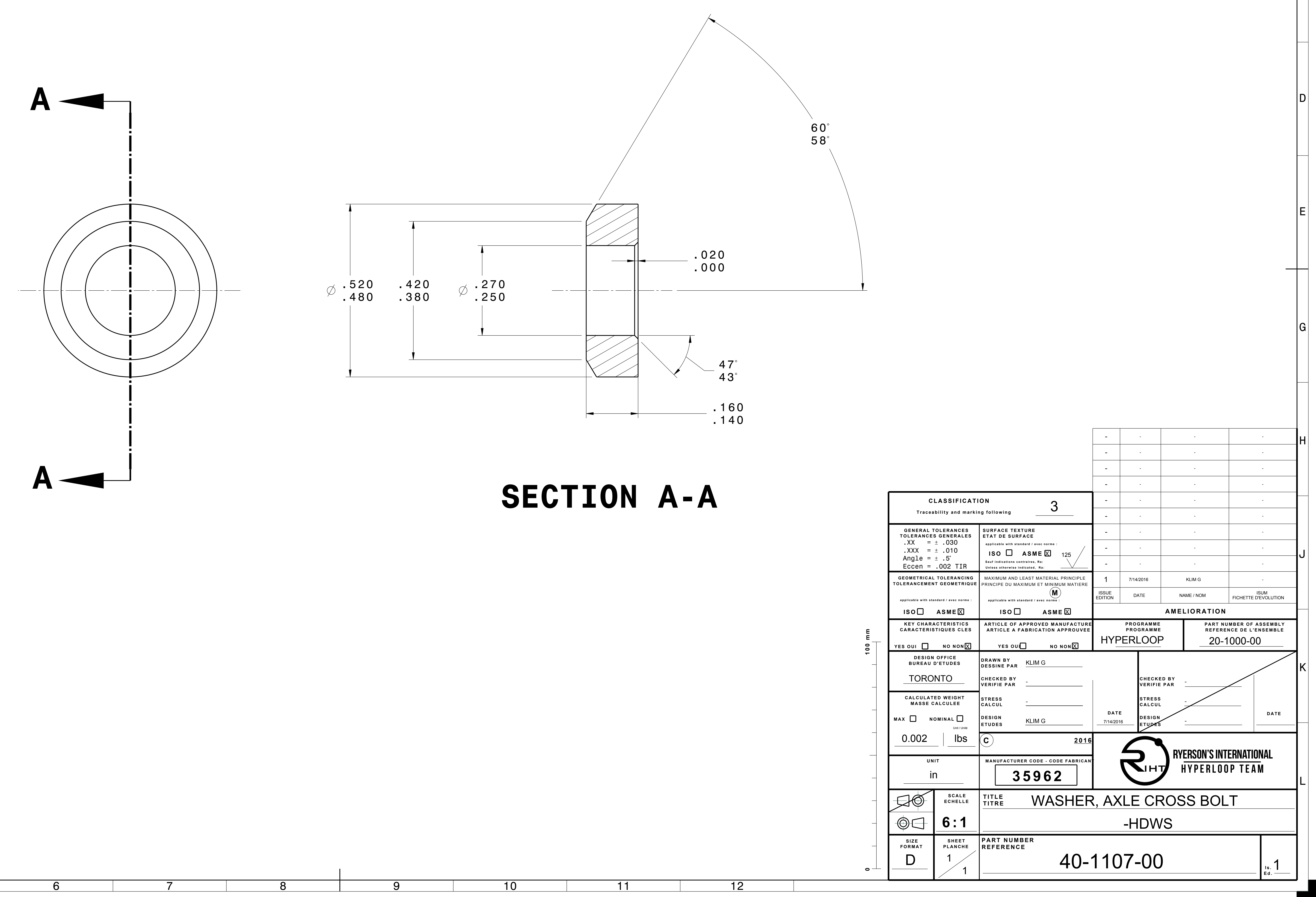


\section{NOTES:}

1. MATERIAL: BRASS SHIM STOCK.

2 MAKE QTY 20 FROM EACH OF THE FOLLOWING STOCK THICKNESSES:

$$
\begin{array}{|l|l|}
\hline \text { PART \# } & \text { THICK. } \\
\hline-01 & .005 \\
\hline-02 & .006 \\
\hline-03 & .007 \\
\hline-04 & .008 \\
\hline-05 & .010 \\
\hline-06 & .012 \\
\hline-07 & .015 \\
\hline-08 & .020 \\
\hline-09 & .025 \\
\hline-10 & .031 \\
\hline
\end{array}
$$

\section{D VIEW} REE ONY
SCALE N NONE

3. BAG AND TAG EACH STOCK THICKNESS.
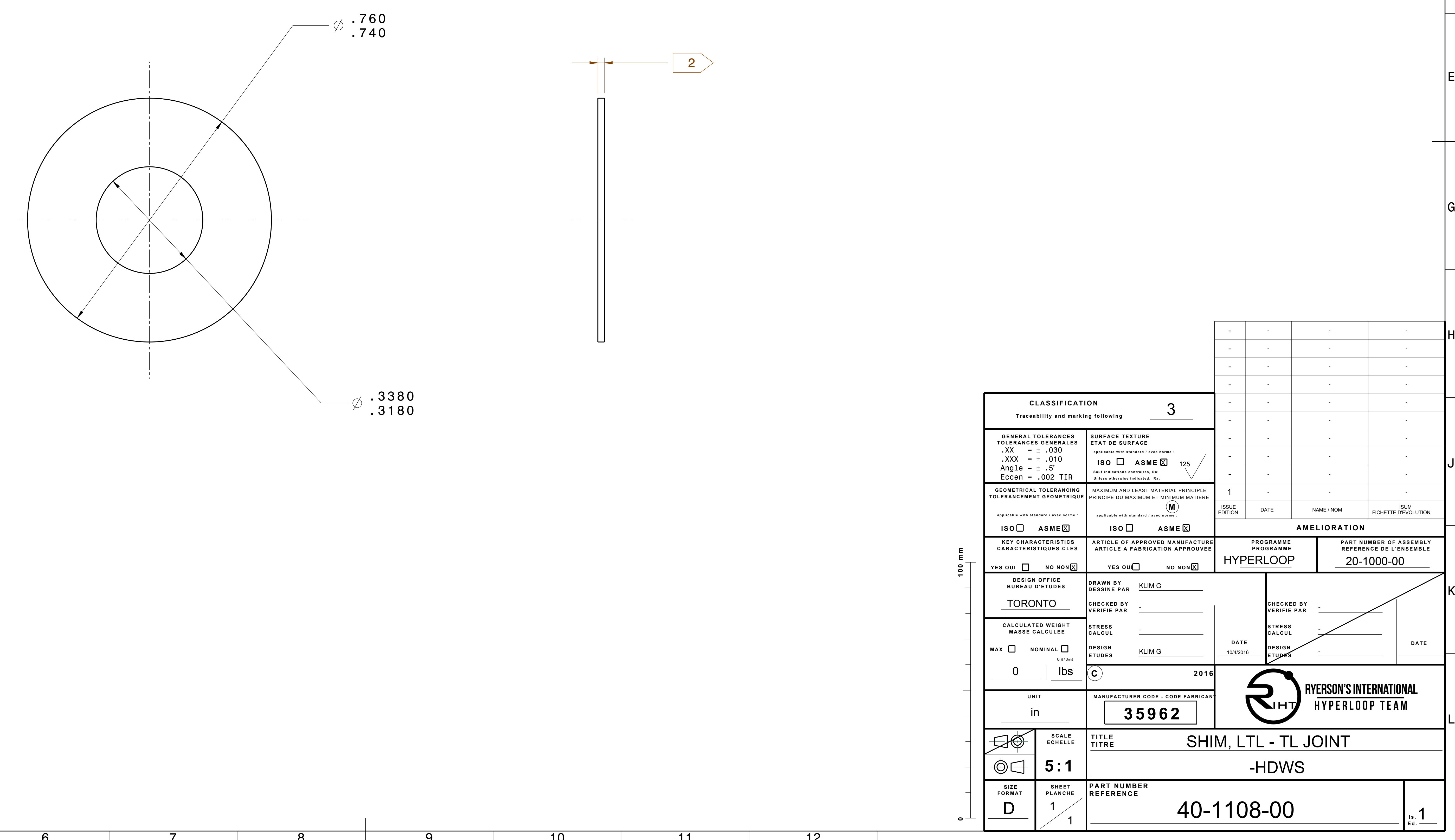
NOTES :

1. MATERIAL: CORROSION RESISTANT STEEL (303) PER ASTM A582 OR EQUiVALENT.

ALL MATERIAL TO BE IN THE ANNEALED CONDITION.

2. DEBURR SHARP EDGES $.005 / .015$, UNLESS OTHERWISE INDICATED.

3. PAssivation All OVER Per ams 2700 Method 1

4. BAG \& TAG OR FIXED TAG MARKING.
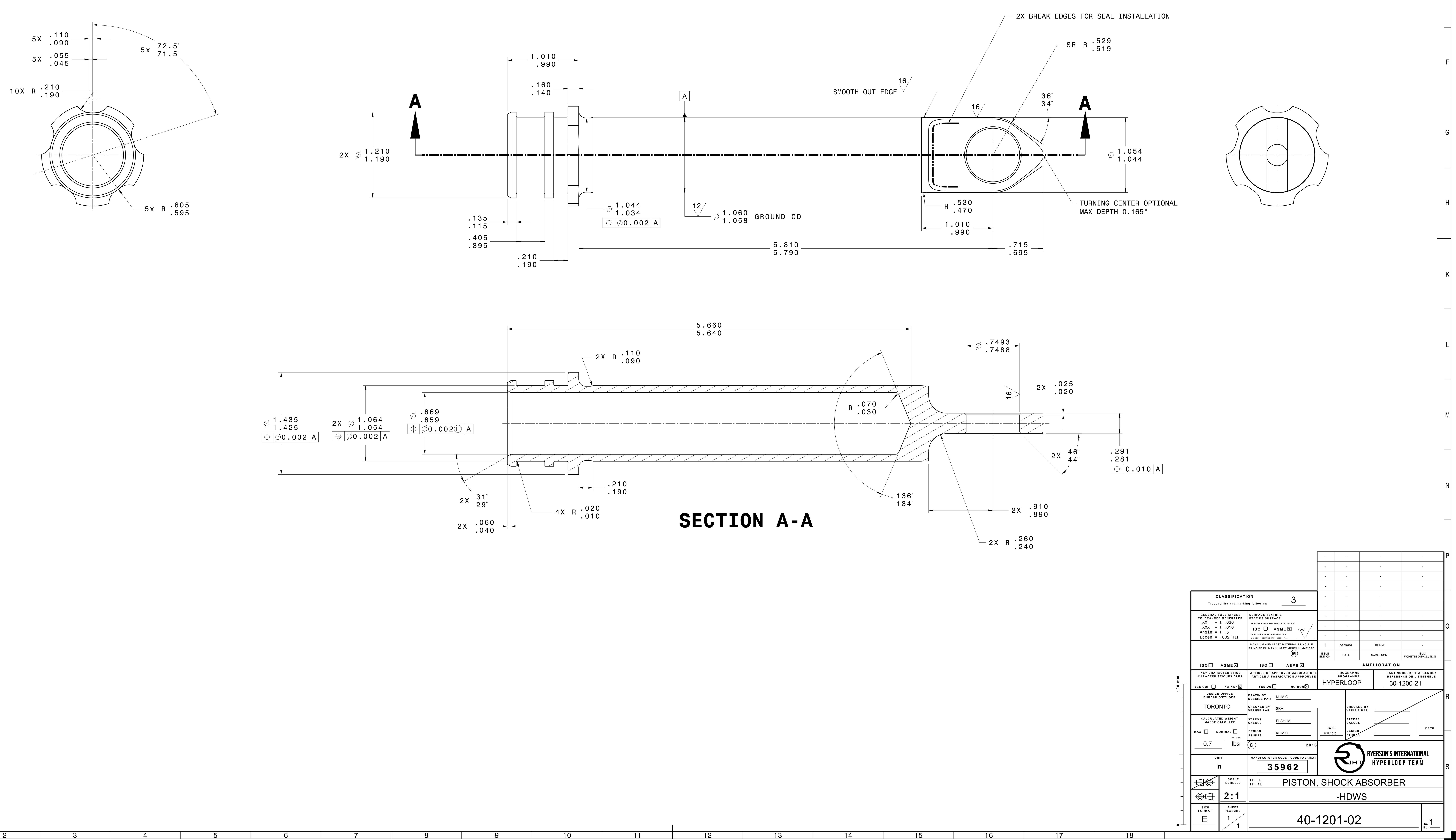


\section{NOTES:}

1. Material: aisi $12 L 14$ CArbon Steel (UNS G12144) Per AStM A108 OR EQuivalent.

2. DeBURR SHARP EDGeS $.005 / .015$, UNLESS OtHERWISE INDICATED.

3. break sharp edGes of acme thread at entry and exit to nUt.

4. MACHINE IN THE FULLY hEAT TREATED CONDition.

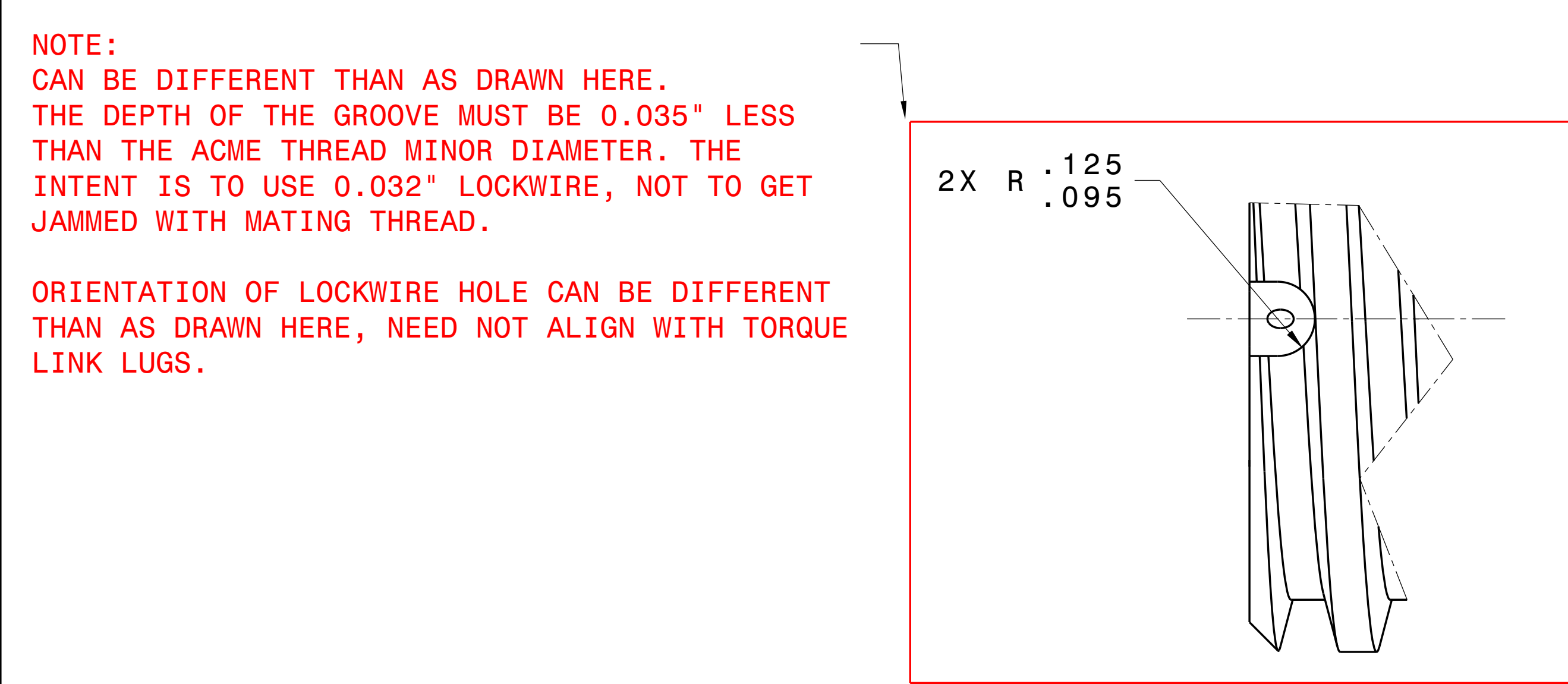

5) PART MARKING USING SCRIBING OR PUNCH.

6. THREADS PER AS8879. 7. BLACK OXIDE CORROSION PROTECTION ON ALL EXTERNAL SURFACES AND ALL THREADS.
$\varnothing .927 / .925$ AND $\varnothing 1.493 / 1.491$ BORES NOT TO HAVE BLACK OXIDE.

$2 X: 385-365-\quad$ R FULL RAD

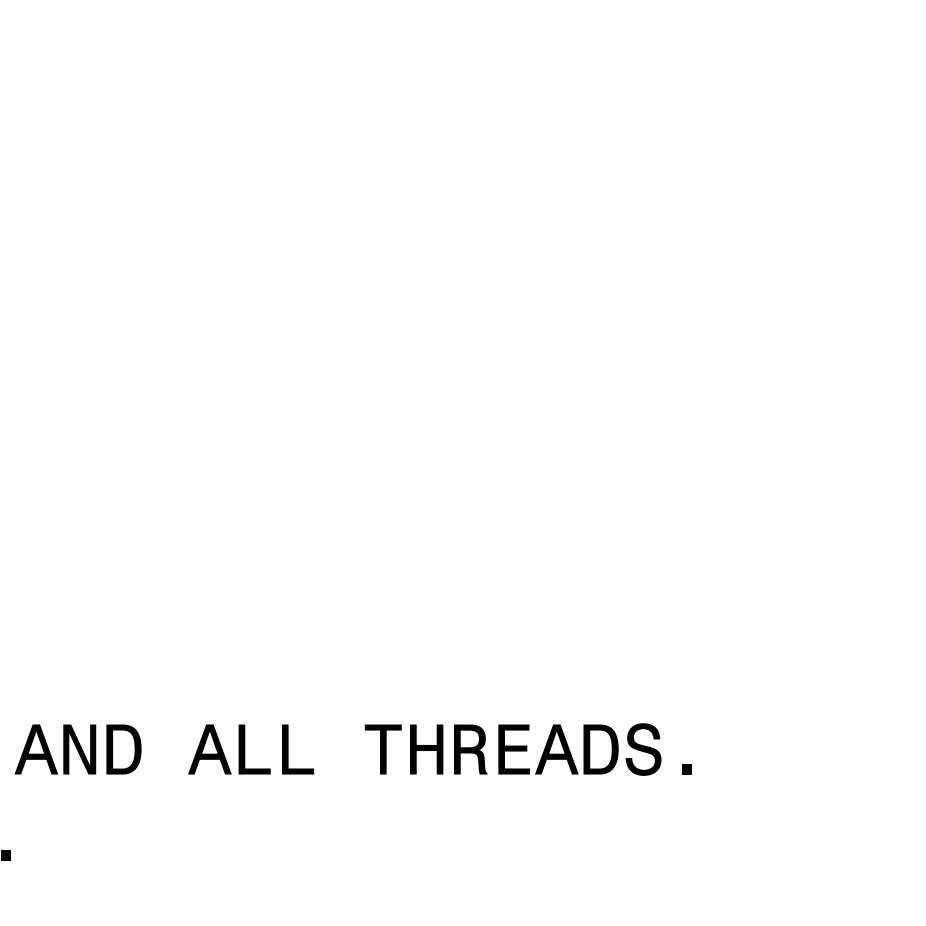

.042 ALL AROUND

$-250$

A

$\phi_{2}^{2} .250$ MAJ OR DIAMETER

(in:

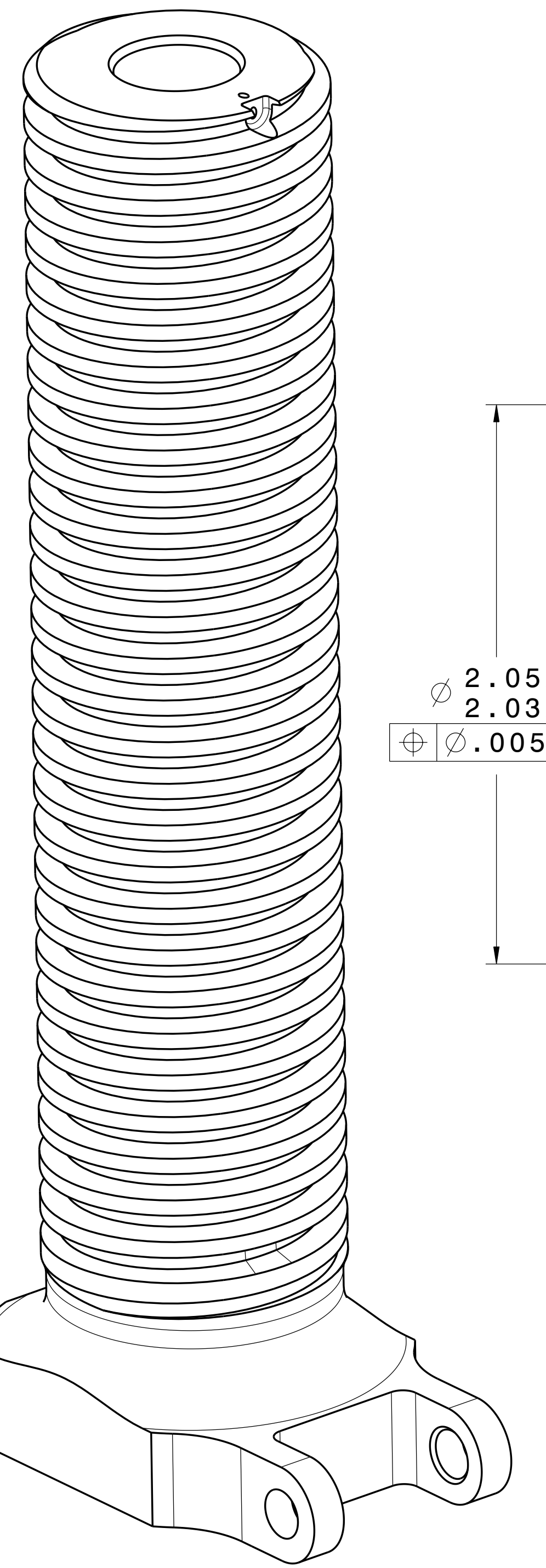

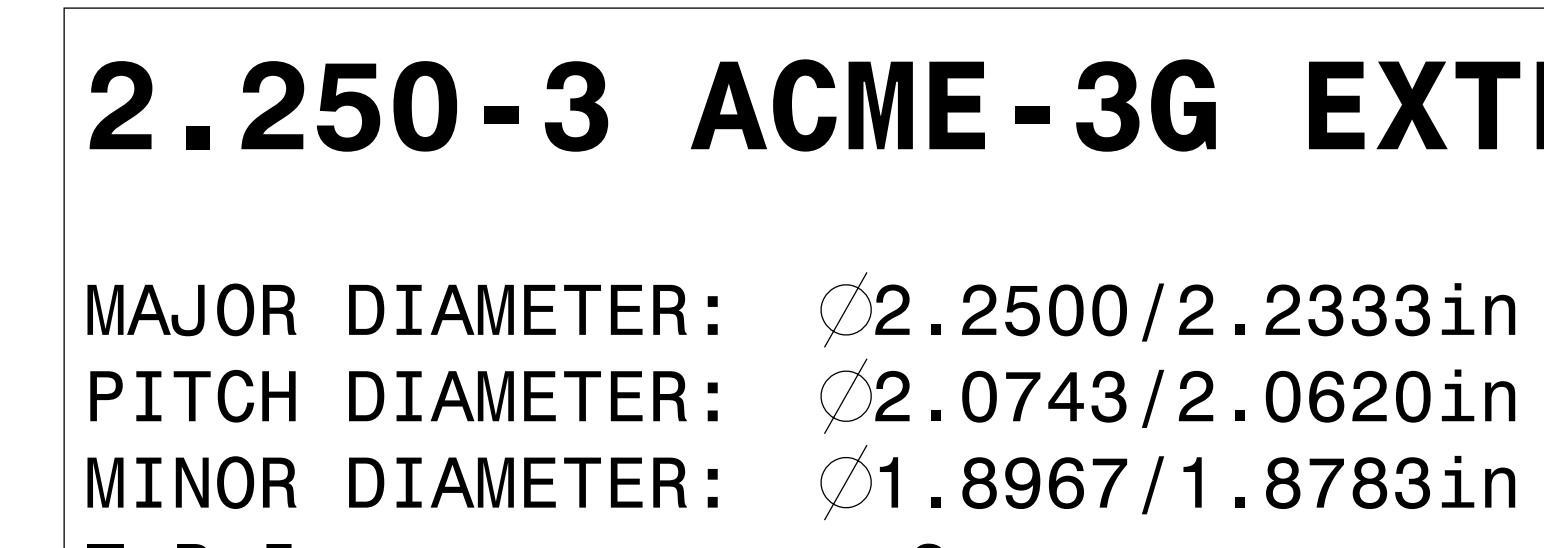 \\ T.P.I. :
PITCH: .333333}

10.280
10.220

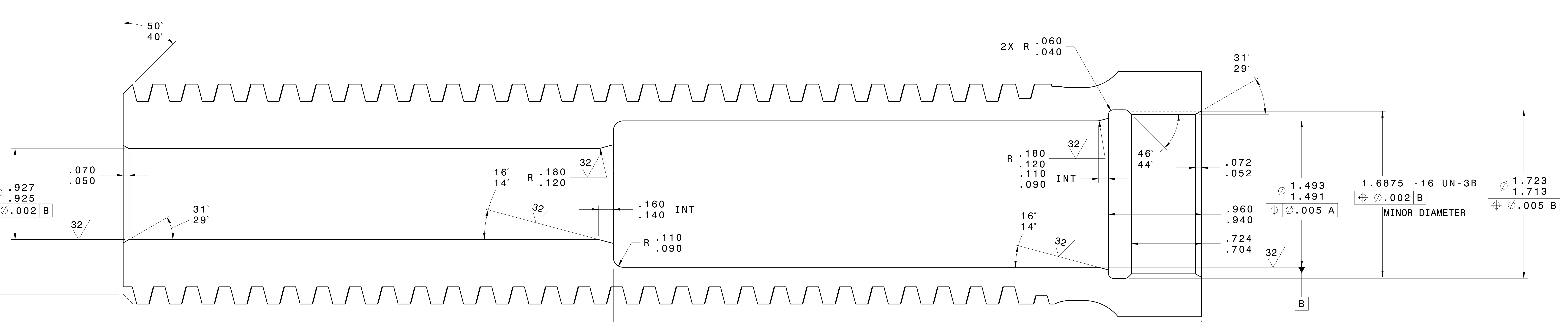

\section{D VIEW}

REF ONLY
Scale: NONE

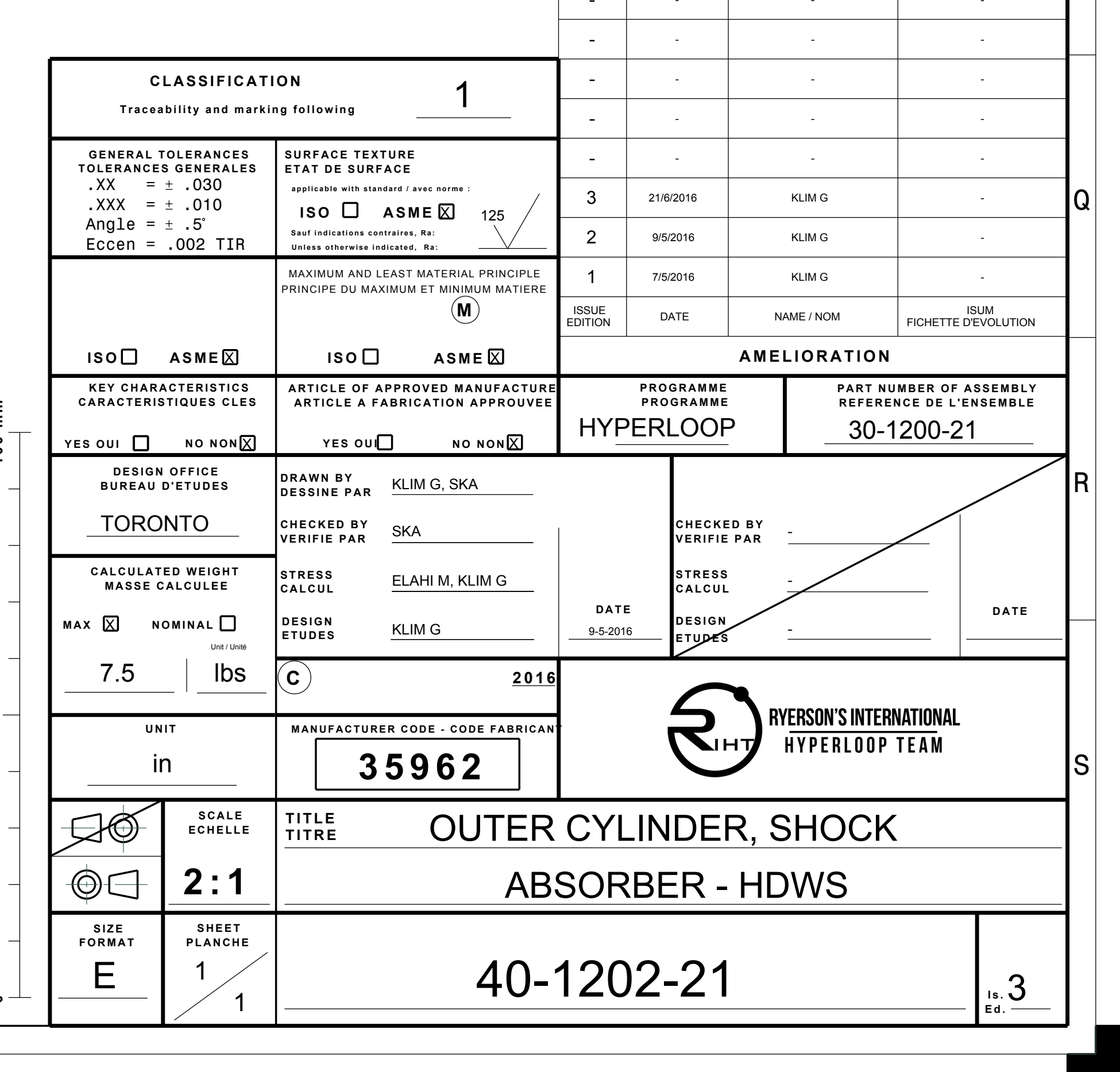




\section{NOTES:}

1. THIS DRAWING IS TO BE TREATED AS A SUPPLEMENT TO 40-1202-21. MANUFACTURE PER DRAWING 40-1202-21 EXCEPT AS SHOWN/NOTED.

2 SPECIFIES 40-1202-21A PART SPECIFIC DIMENSION.

3 DIMENSION FOR REFERENCE ONLY, REFER TO 40-1202-21.

4. $\varnothing .993 / .991$ AND $\varnothing 1.493 / 1.491$ BORES NOT TO HAVE BLACK OXIDE.

5. IDENTIFY WITH PART MARKING OF THIS DRAWING.
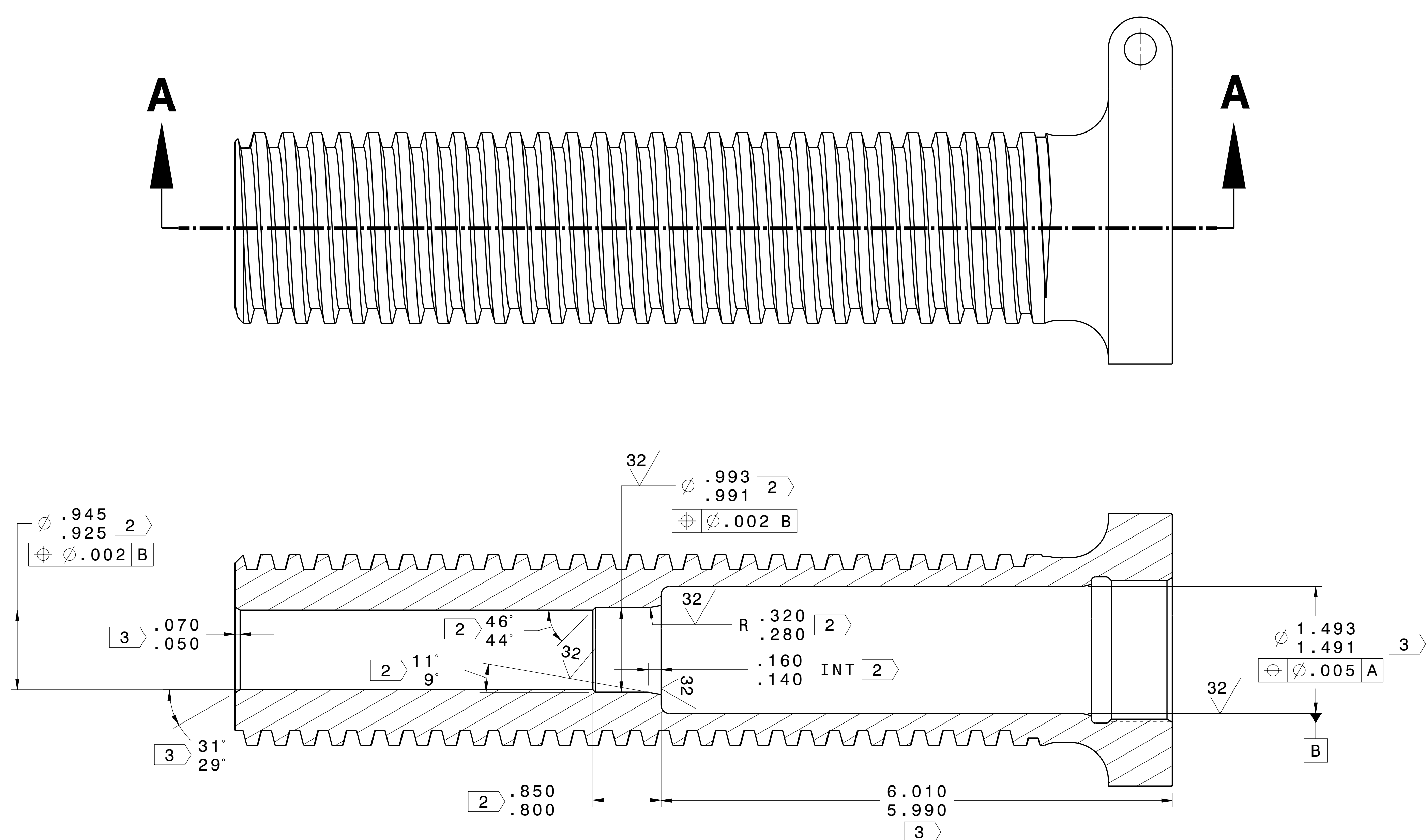

SECTION A-A

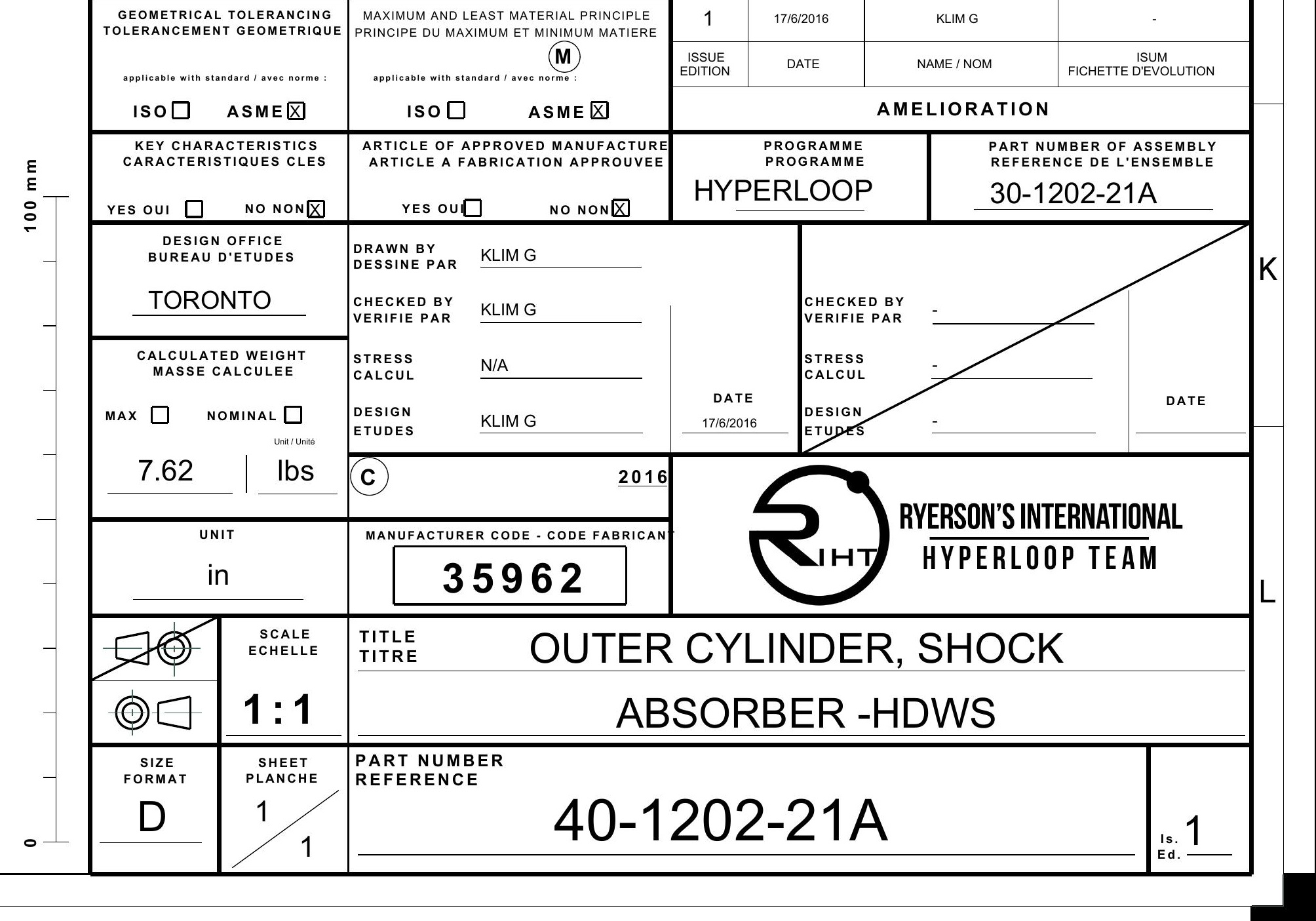




\section{NOTES:}

1. MATERIAL: AL BRONZE (C95400) PER ASTM B505 OR EQUiVALENT.

2. DEBURR SHARP EDGES $.005 / .015$, UNLESS OTHERWISE INDICATED.

3. MACHINE IN THE FULLY HEAT TREATED CONDITION.

4. BAG AND TAG OR FIXED TAG MARKING.
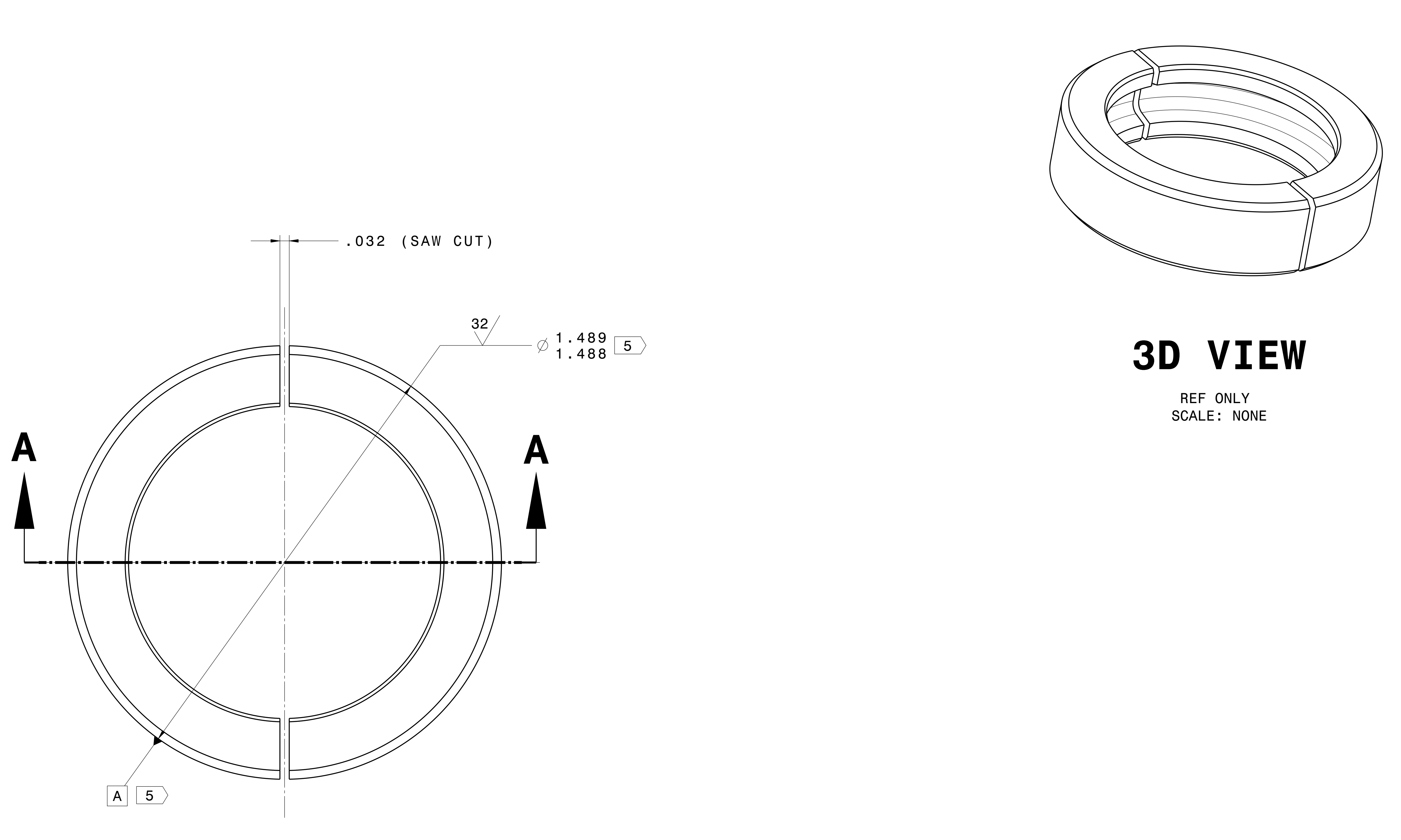

5 DIMENSION, OR DATUM, TO BE MET BEFORE OPERATION THAT RESULTS IN A DISCONTINUOUS RING.

3D VIEW

REF ONLY
SCALE: NONE

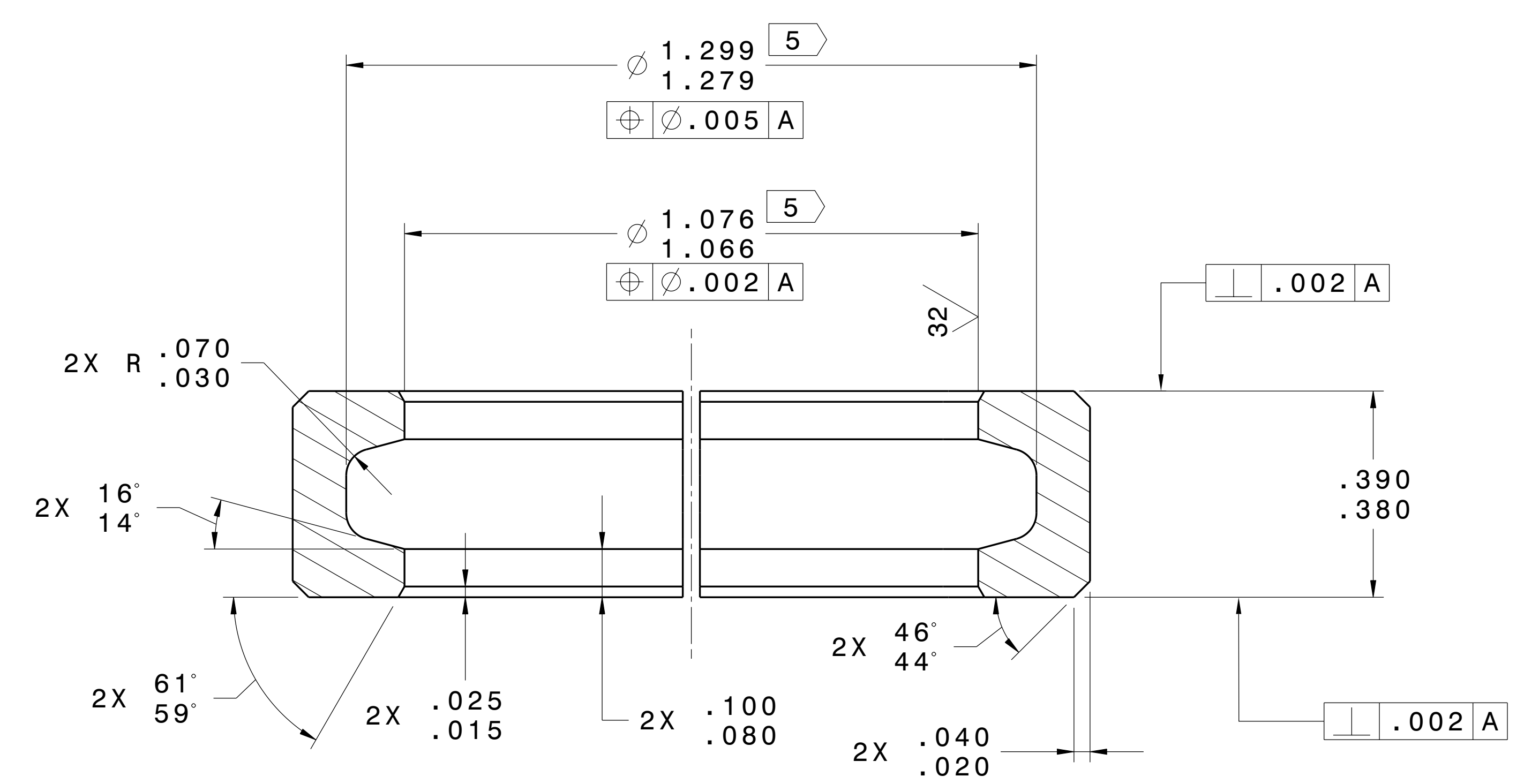

SECTION A-A 


\section{NOTES:}

1. MATERIAL: AL BRONZE (C95400) PER ASTM B505 OR EQUiVALENT.

2. DEBURR SHARP EDGES $.005 / .015$, UNLESS OTHERWISE INDICATED.

3. MACHINE IN THE FULLY hEAT TREATED CONDITION.

4. THREADS PER AS8879.

5. BAG AND TAG OR FIXED TAG MARKING.

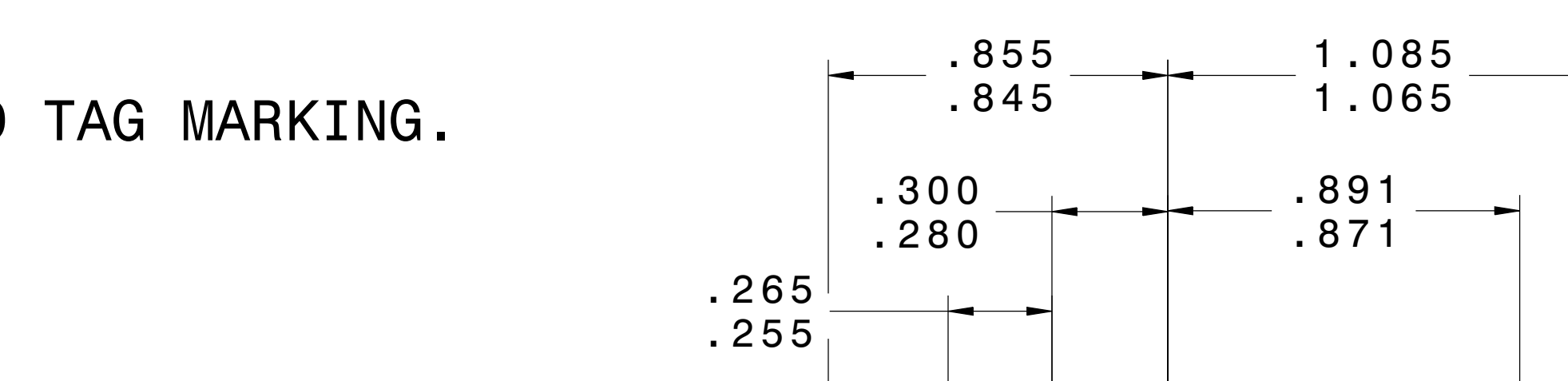

\section{D VIEW}

SCALE: NONE
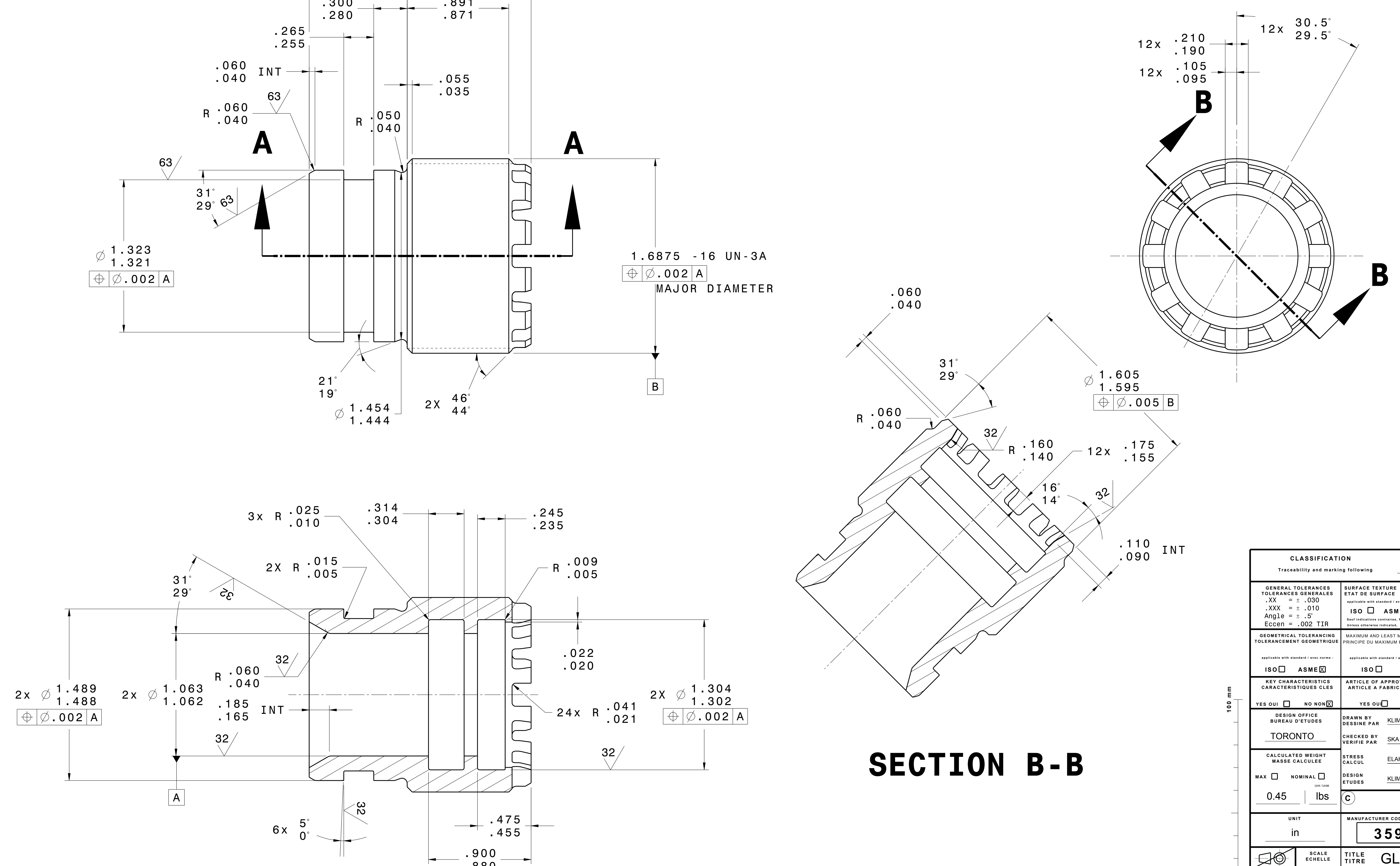

$\frac{.080}{040}$

31:

$\oplus 0.005$

R. .060

$2^{32} \times 1.160-12 \times: 175$

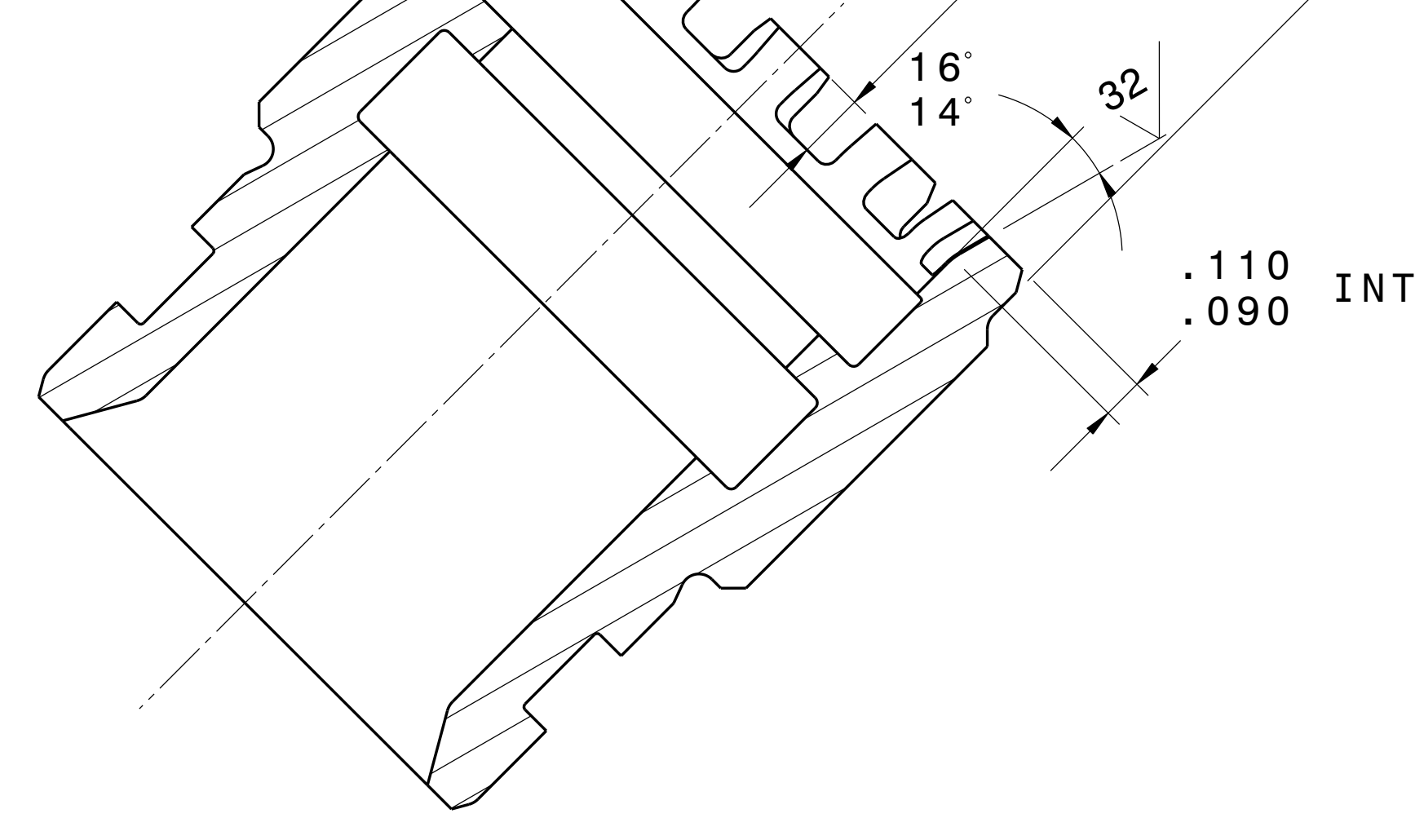

SECTION B-B

\section{SECTION A-A}

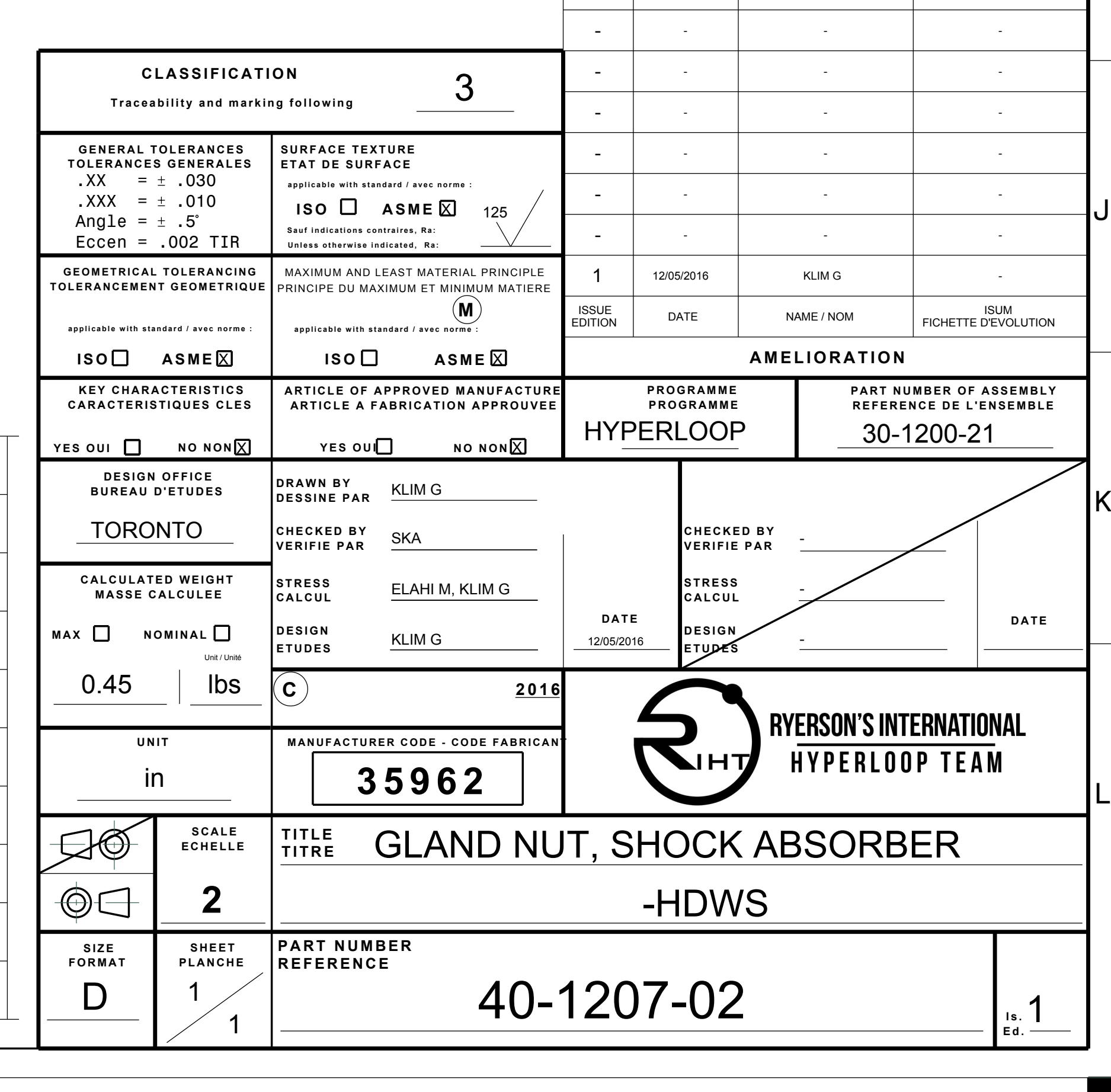




\section{NOTES:}

1. MATERIAL: MACHINe FROM AISi 1018 SteEL, COLD ROLLED, BAR STOCK.

2. DEBURR SHARP EDGES $.005 / .015$, UNLESS OTHERWISE INDICATED.

3. MACHINE IN THE FULLY HEAT TREATED CONDITION.

4 PART MARKING USING SCRIBING OR PUNCH.

5. BLACK OXIDE CORROSION PROTECTION ALL OVER.

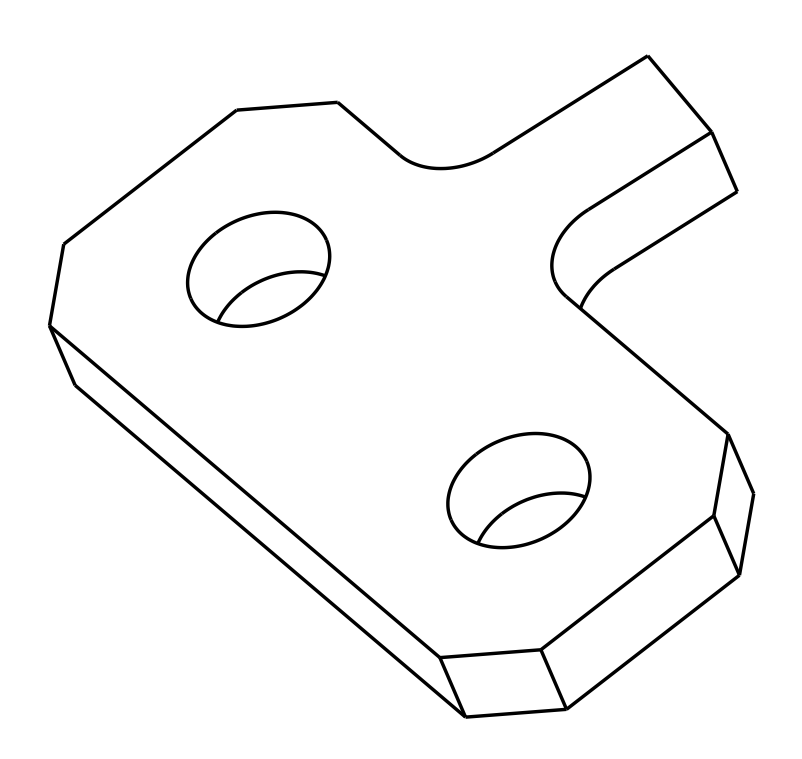

\section{D VIEW}

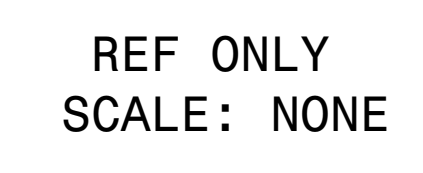
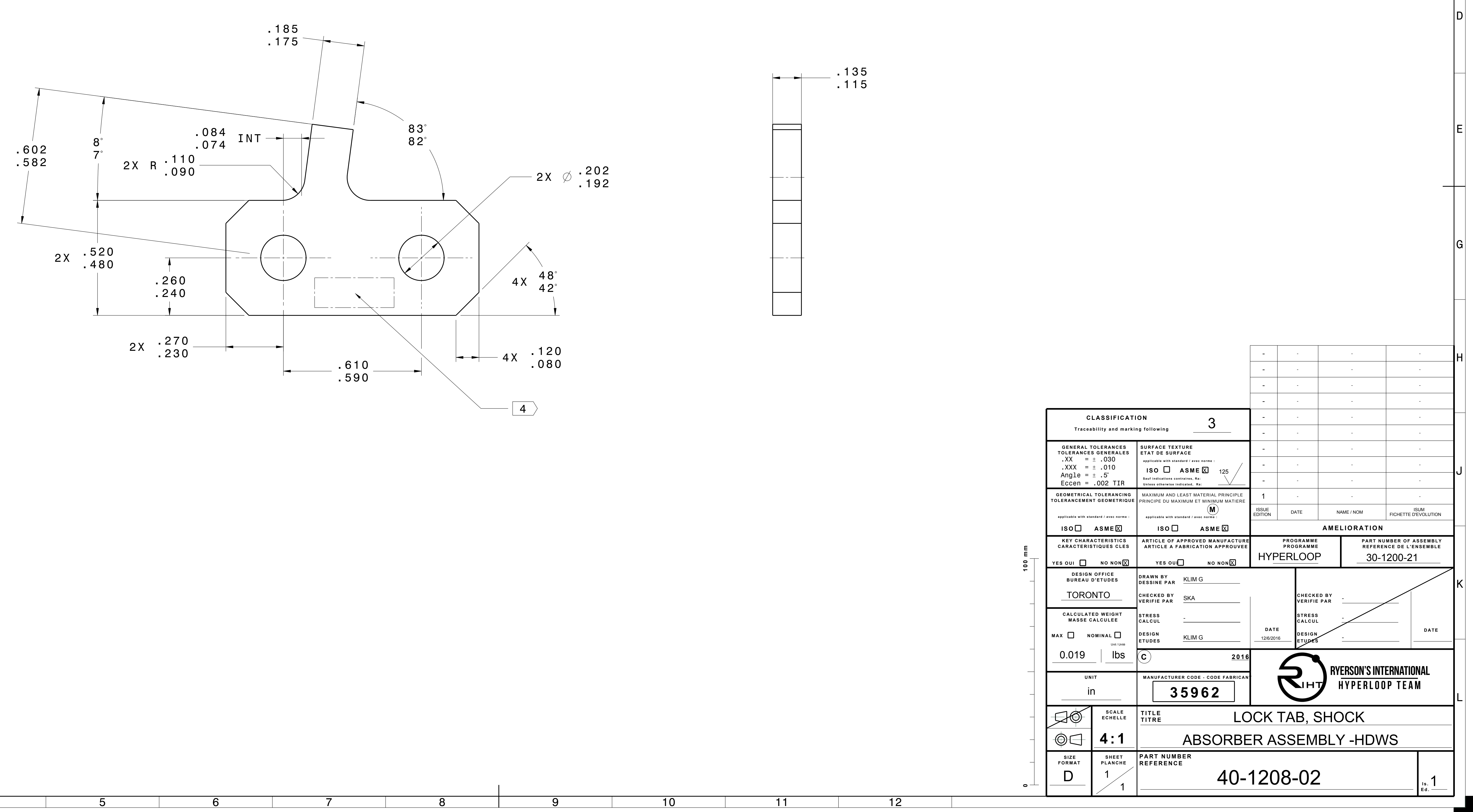


\section{NOTES:}

1. MATERial: ALUMinUm 6061-T6511 (BAR) PER AMS QQ-A-200/8.

2. DEBURR SHARP EDGES $.005 / .015$, UNLESS OTHERWISE INDICATED.

3. THREADS PER AS8879.

4. ANODIZING ALL OVER.

5. BAG AND TAG OR FIXED TAG MARKING

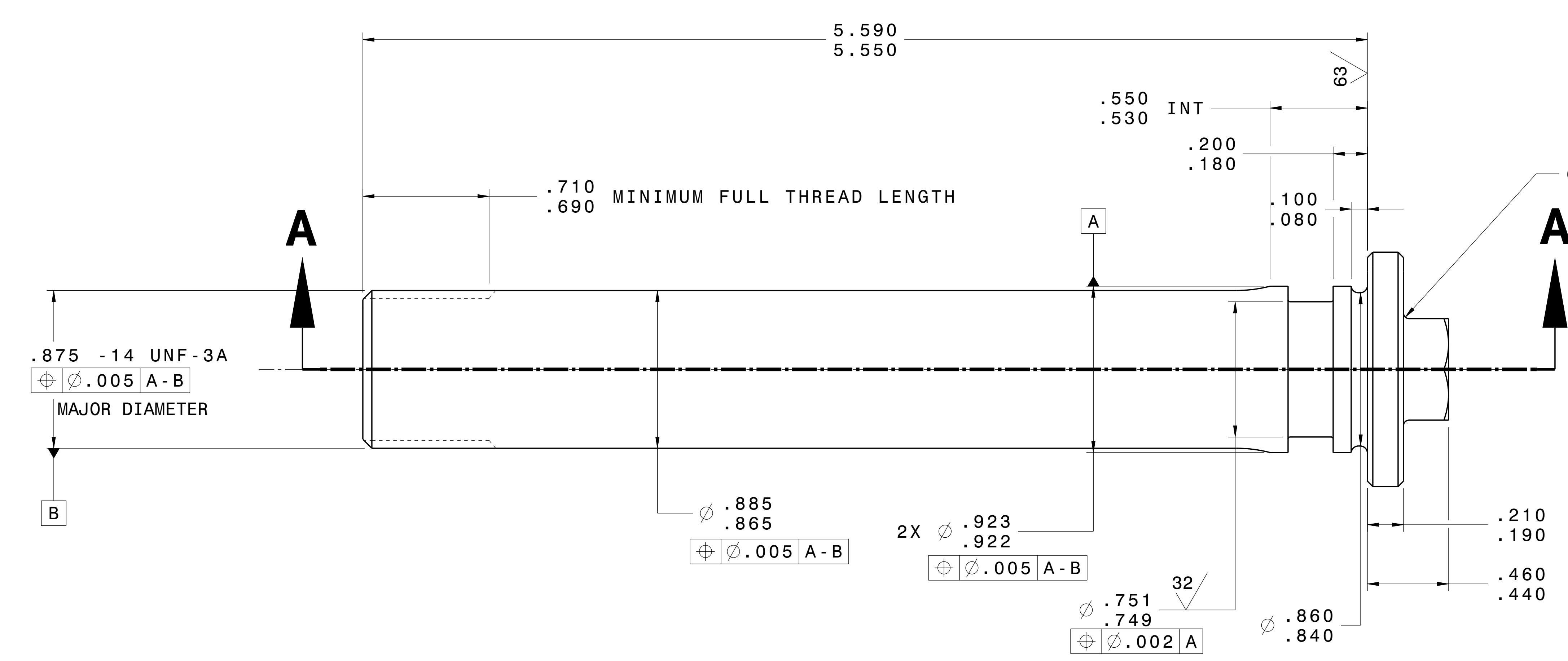

PORT PER MS33651

.585 TAP DRILL DEPTH

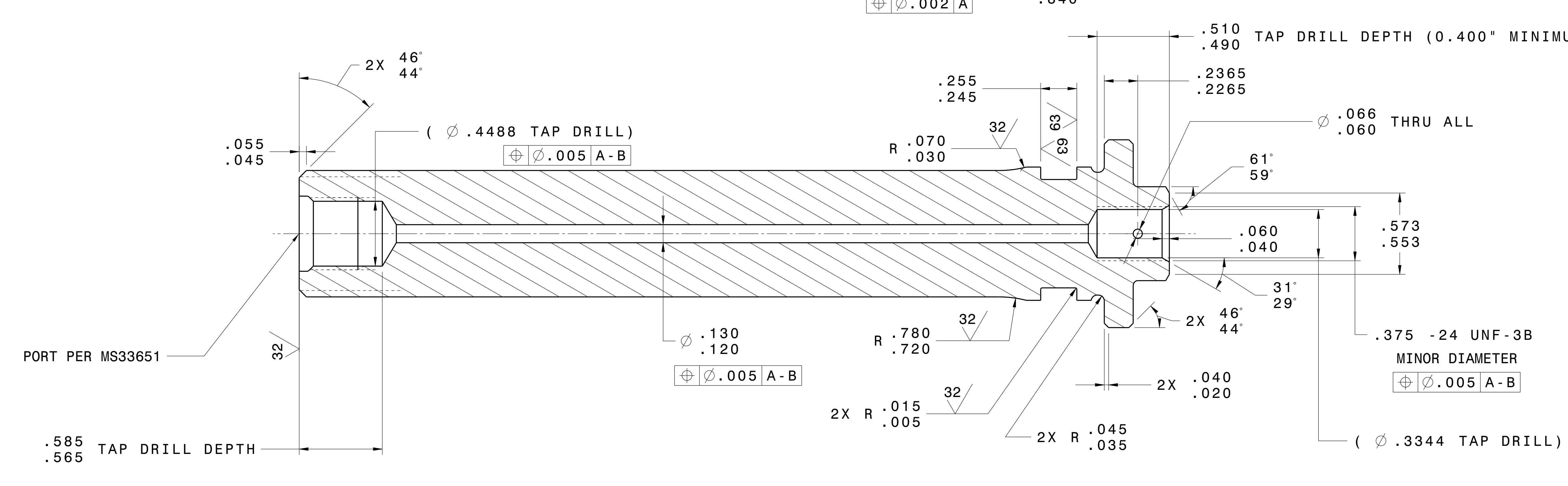

SECTION A-A
A

1
3D VIEW

SCALE: ONLY NONE

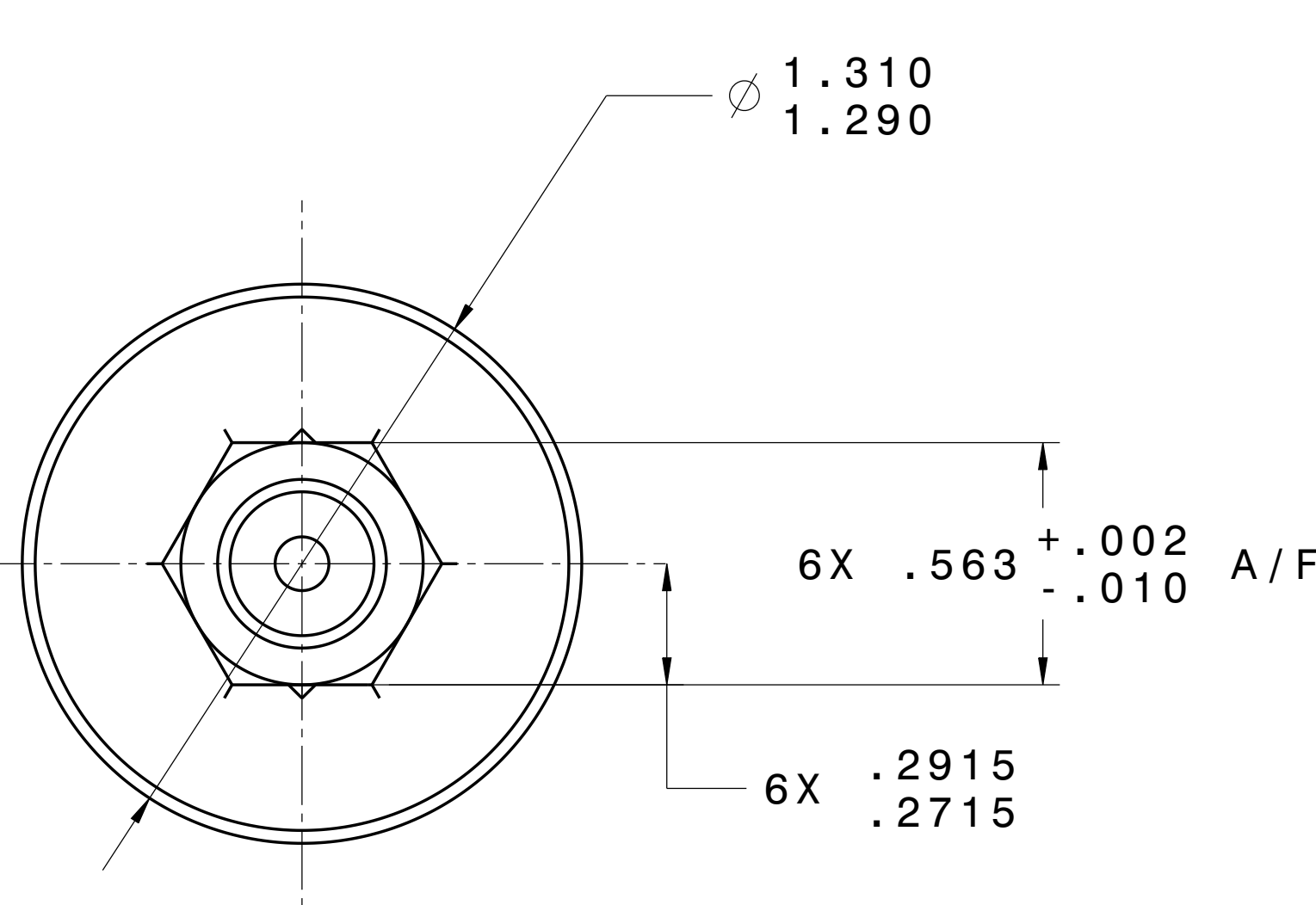

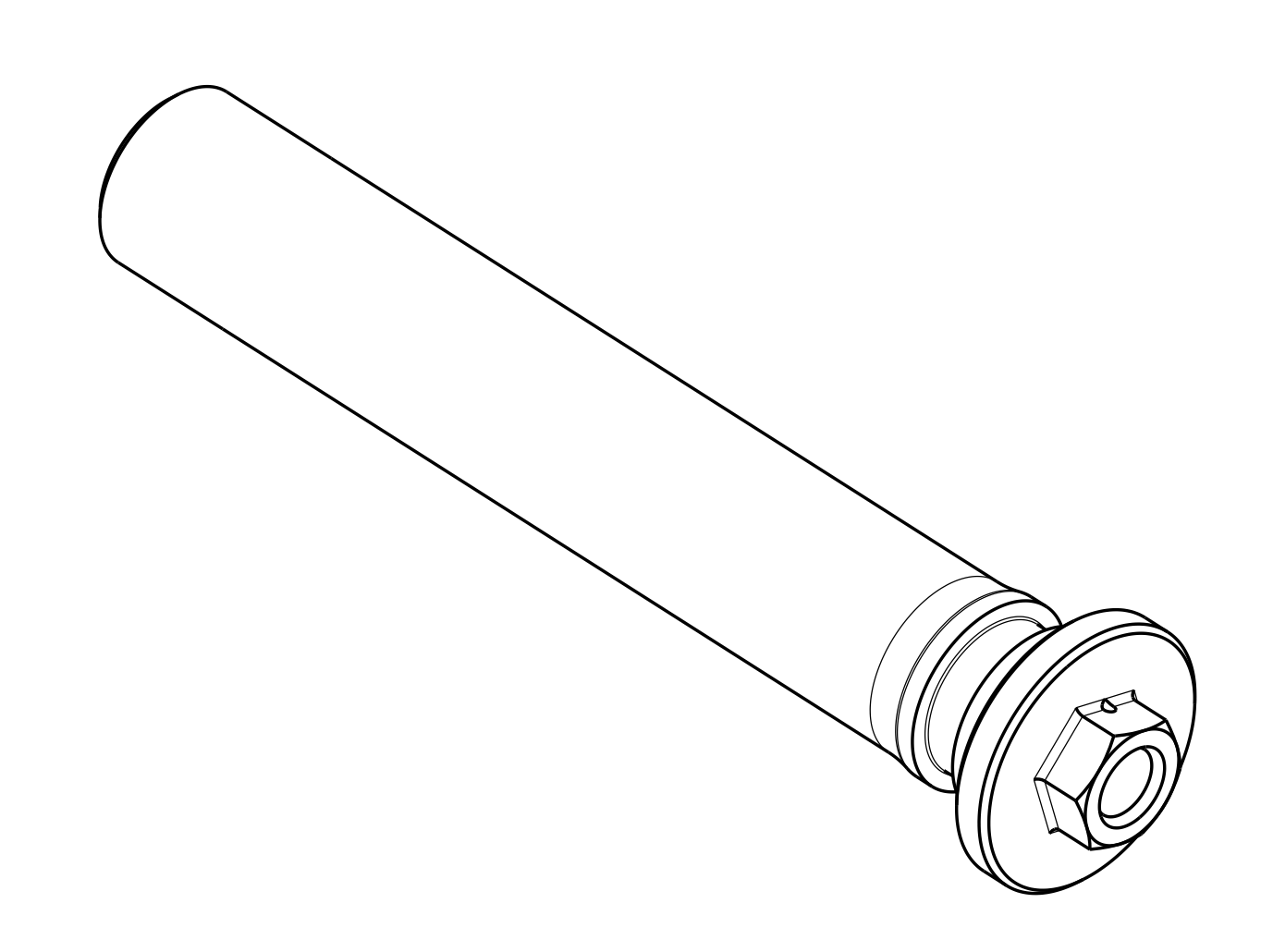

NUL THEAA DEpTH) 


\section{NOTES:}

1. THIS DRAWING IS TO BE TREATED AS A SUPPLEMENT TO 40-1202-21 ISSUE 2 AND MUST ONLY BE USED IN CONJUNCTION WITH THE AFFORMENTIONED DRAWING.

2 SPECIFIES 40-1202-21A PART SPECIFIC DIMENSION.

3 DIMENSION FOR REFERENCE ONLY, REFER TO 40-1209-00.

4. IDENTIFYU WITH PART MARKING OF THIS DRAWING.
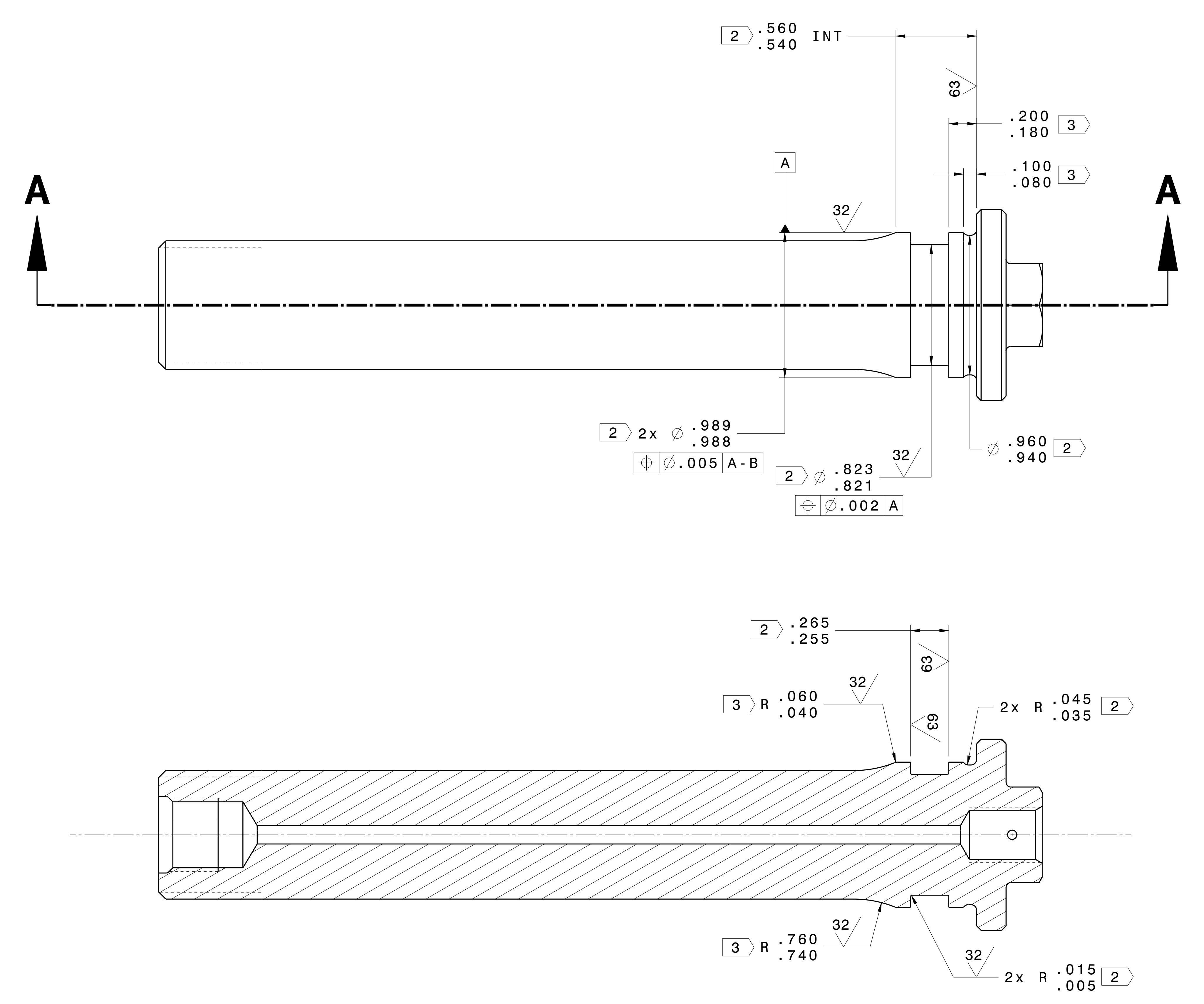

SECTION A-A

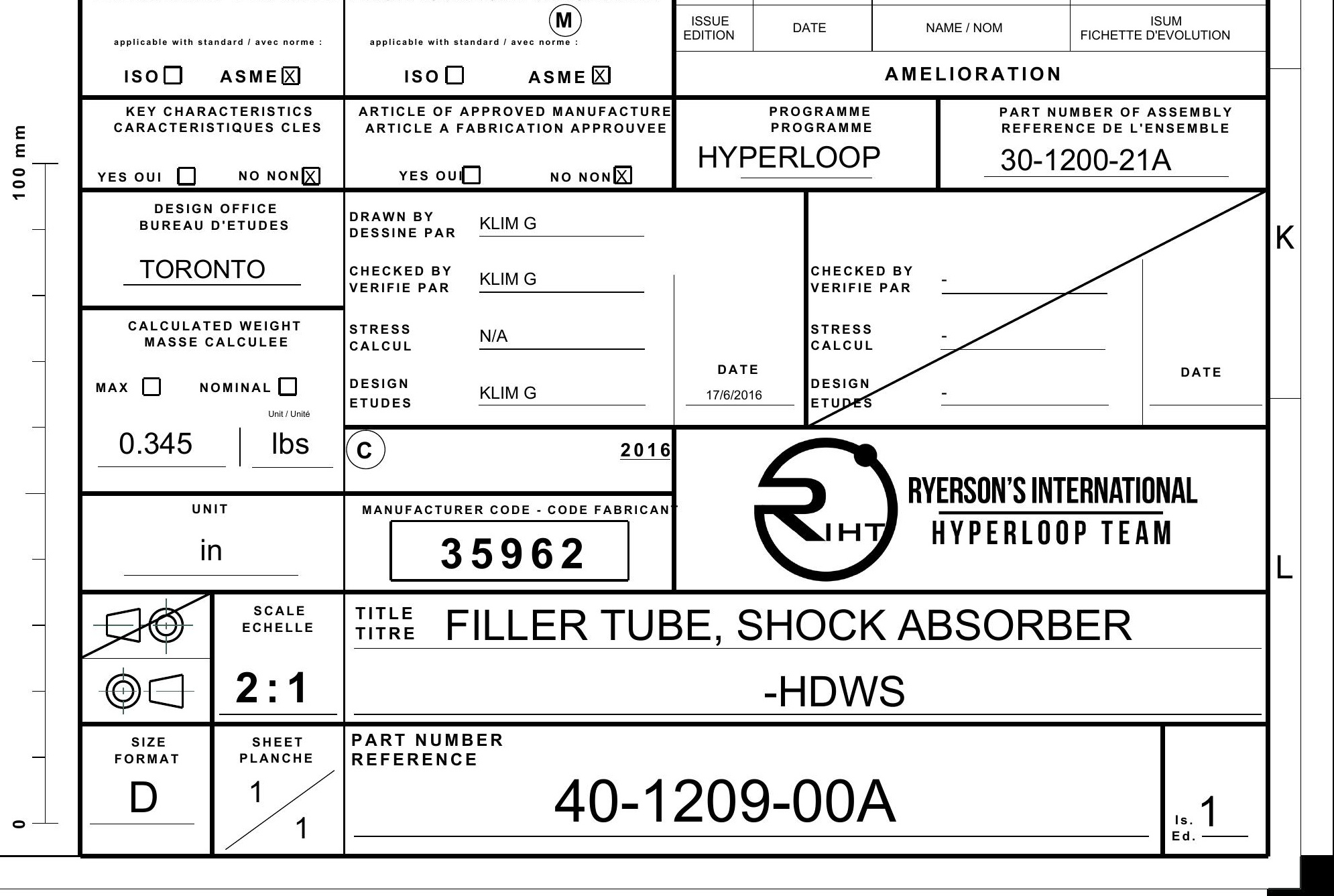




\section{NOTES:}

1. MATERIAL: BRASS SHIM STOCK.

2 MAKE QTY 20 FROM EACH OF THE FOLLOWING STOCK THICKNESSES:

$$
\begin{array}{|c|c|}
\hline \text { PART \# } & \text { THICK. } \\
\hline-01 & .005 \\
\hline-02 & .006 \\
\hline-03 & .007 \\
\hline-04 & .008 \\
\hline-05 & .010 \\
\hline-06 & .012 \\
\hline-07 & .015 \\
\hline-08 & .020 \\
\hline-09 & .025 \\
\hline
\end{array}
$$

3. BAG AND TAG EACH STOCK THICKNESS.

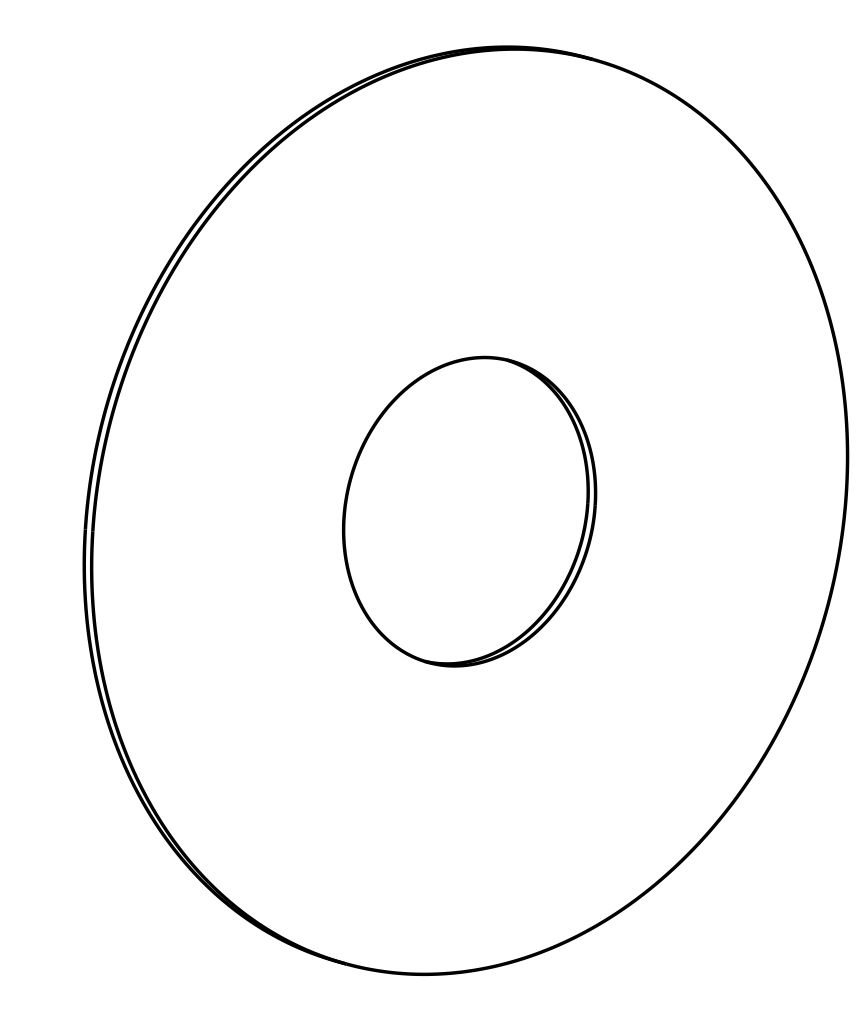

3D VIEW

REF ONLY
SCALE: NONE
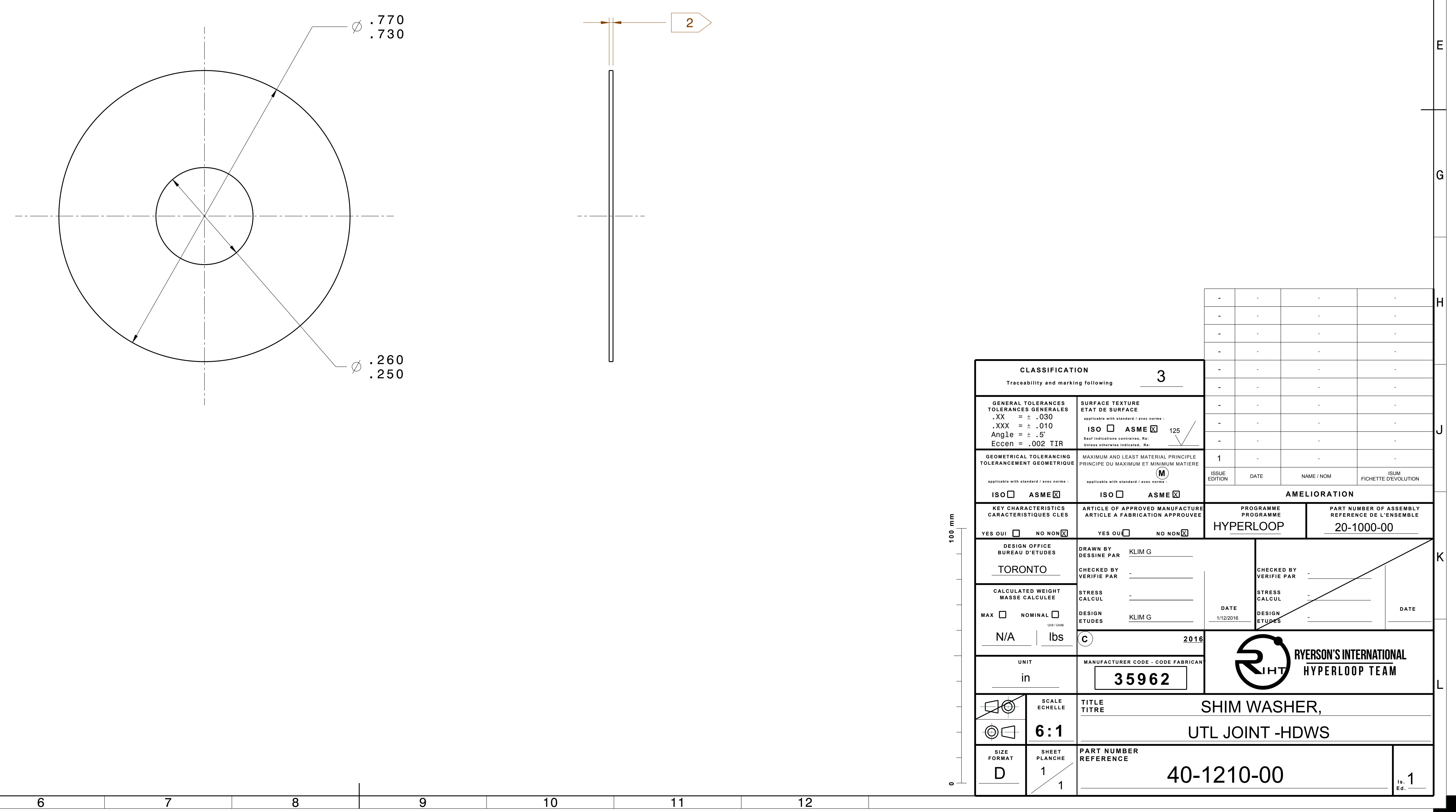


\section{NOTES:}

1. MATERIAL: AlUMinUM 6061-T6511 (BAR) PER AMS QQ-A-200/8.

2. Deburr Sharp edges $.005 / .015$, UnLess otherwise indicated.

. ANODIZING ALL OVER

4. PART MARKING USING SCRIBING OR PUNCH.

5. GENERAL ALLOWED MACHINING MISMATCH TO BE $.030 / .000$ UNLESS THERWISE INIDICATED.
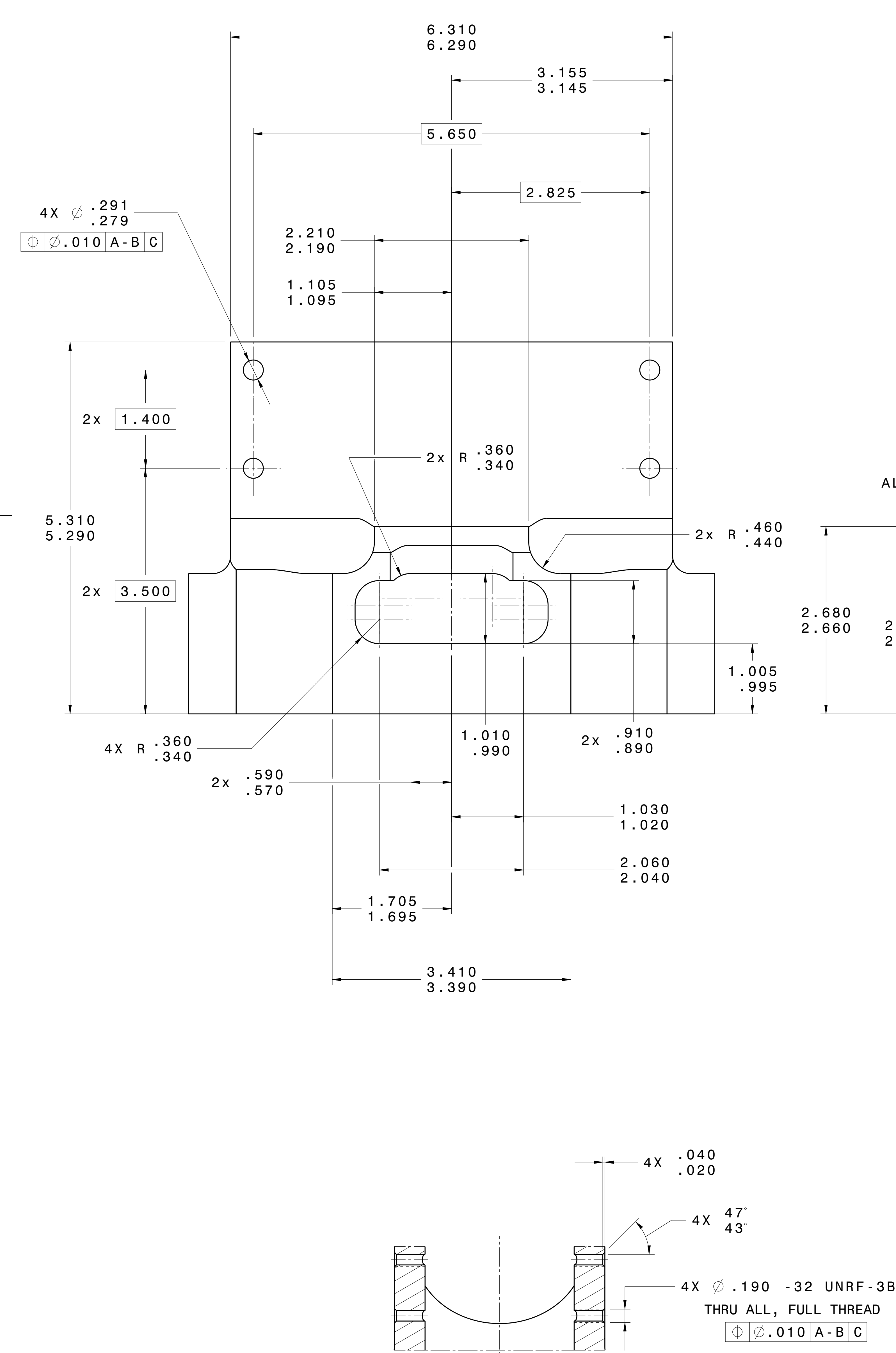

PARTIAL SECTION D-D

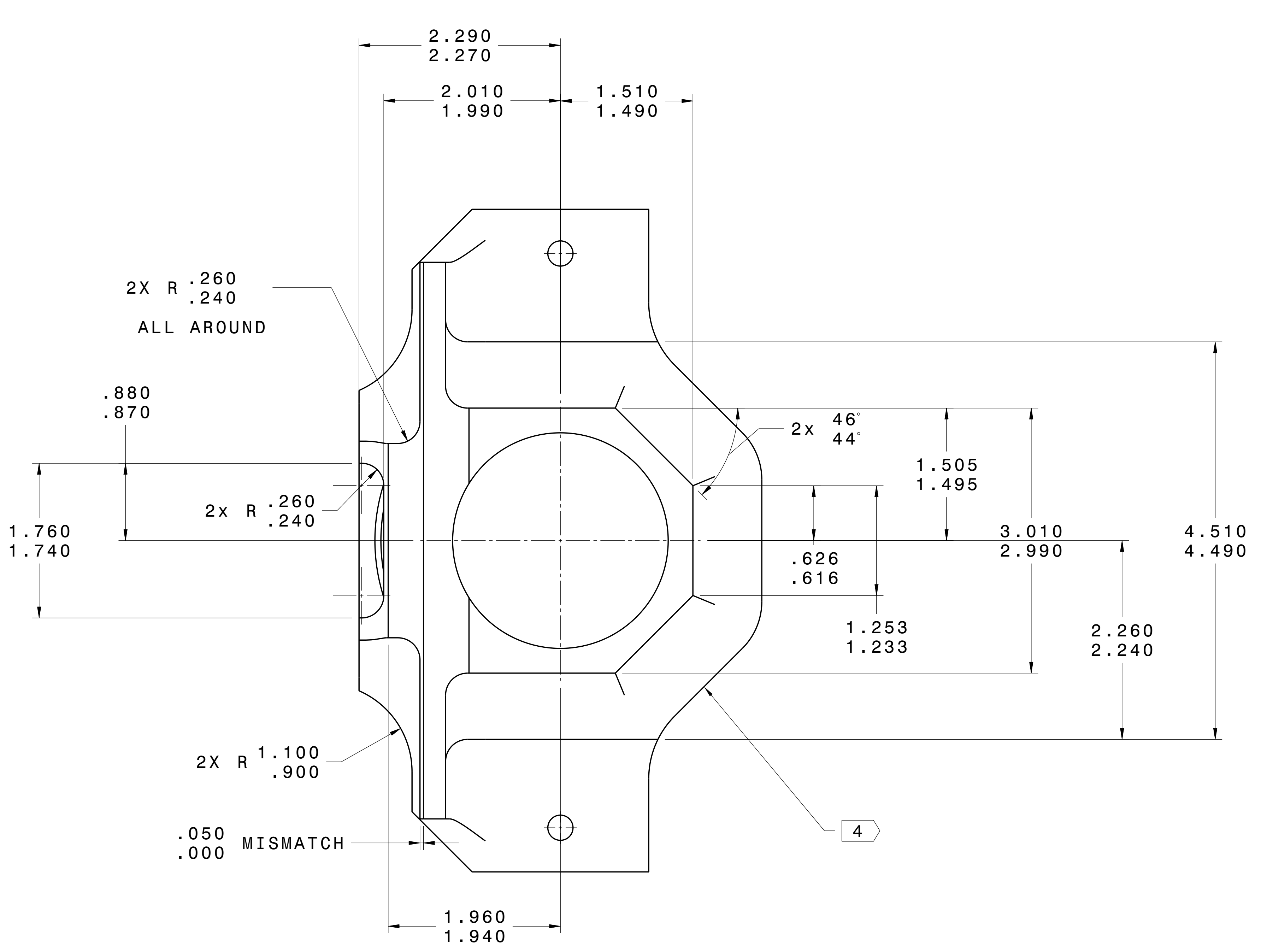

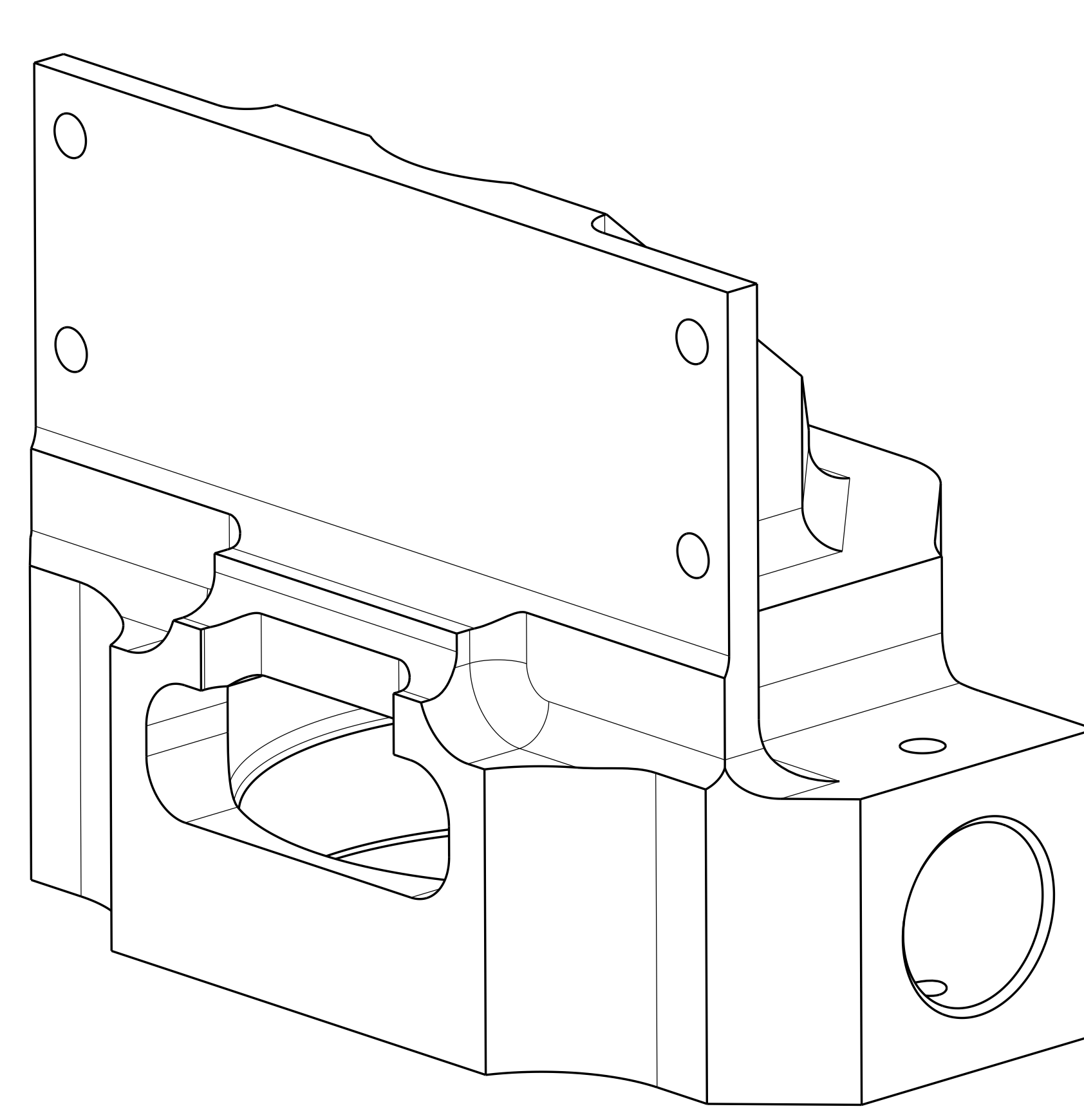

3D VIEW 1

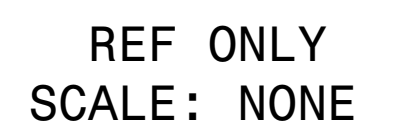

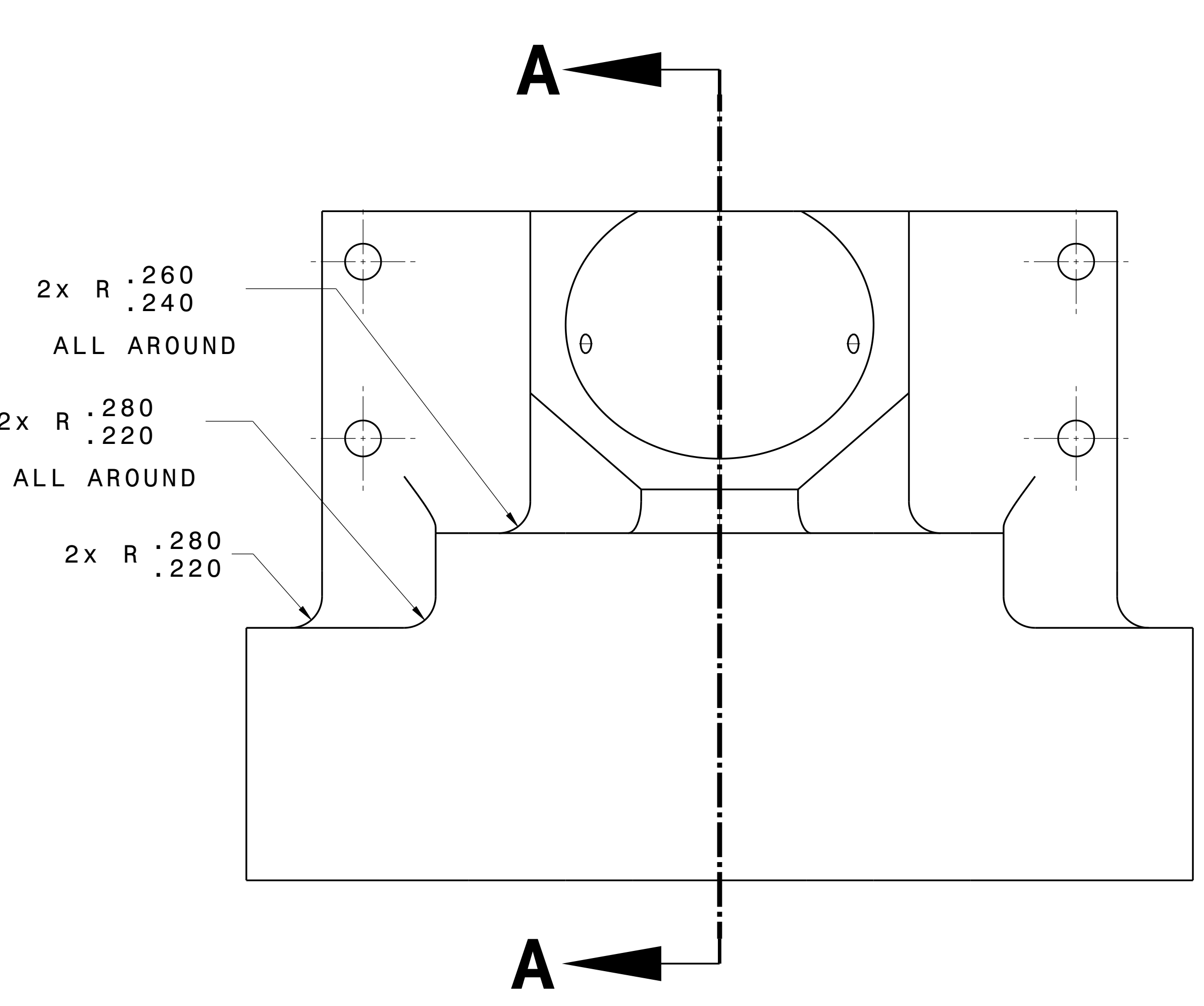

$2 \times 1: .670 \ldots \quad-2 \times 1: 090$
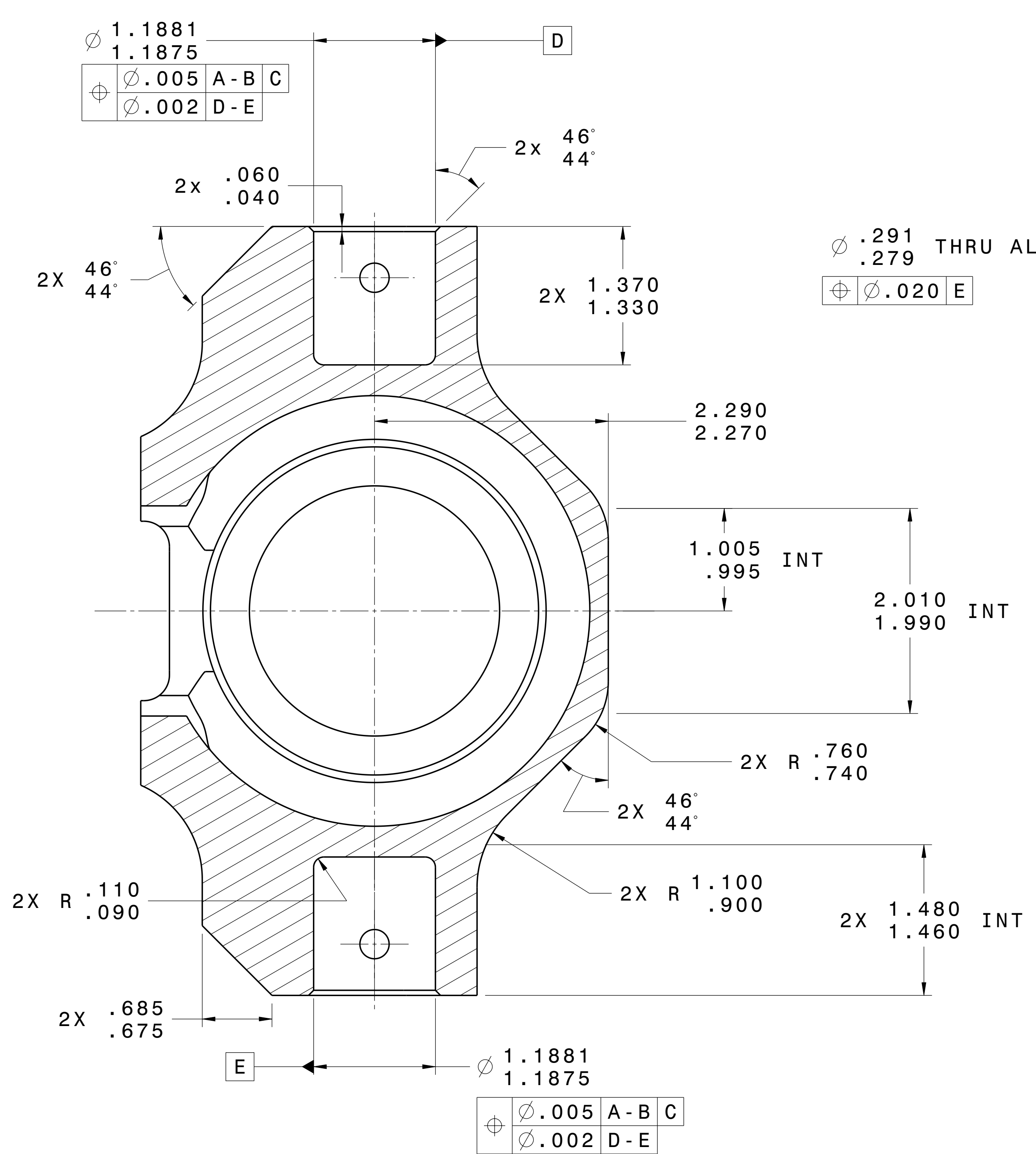

SECTION B-B

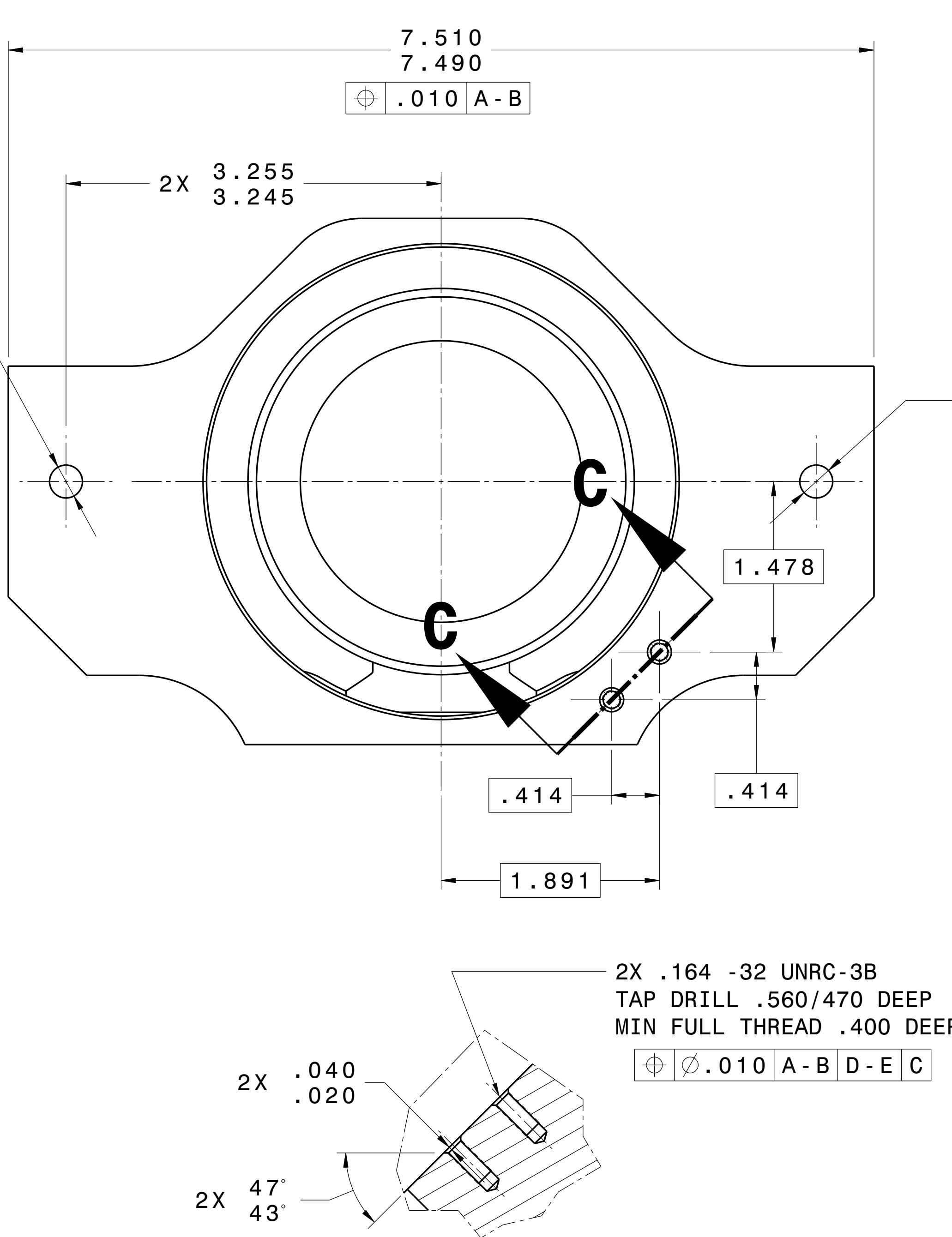

PARTIAL SECTION C-C

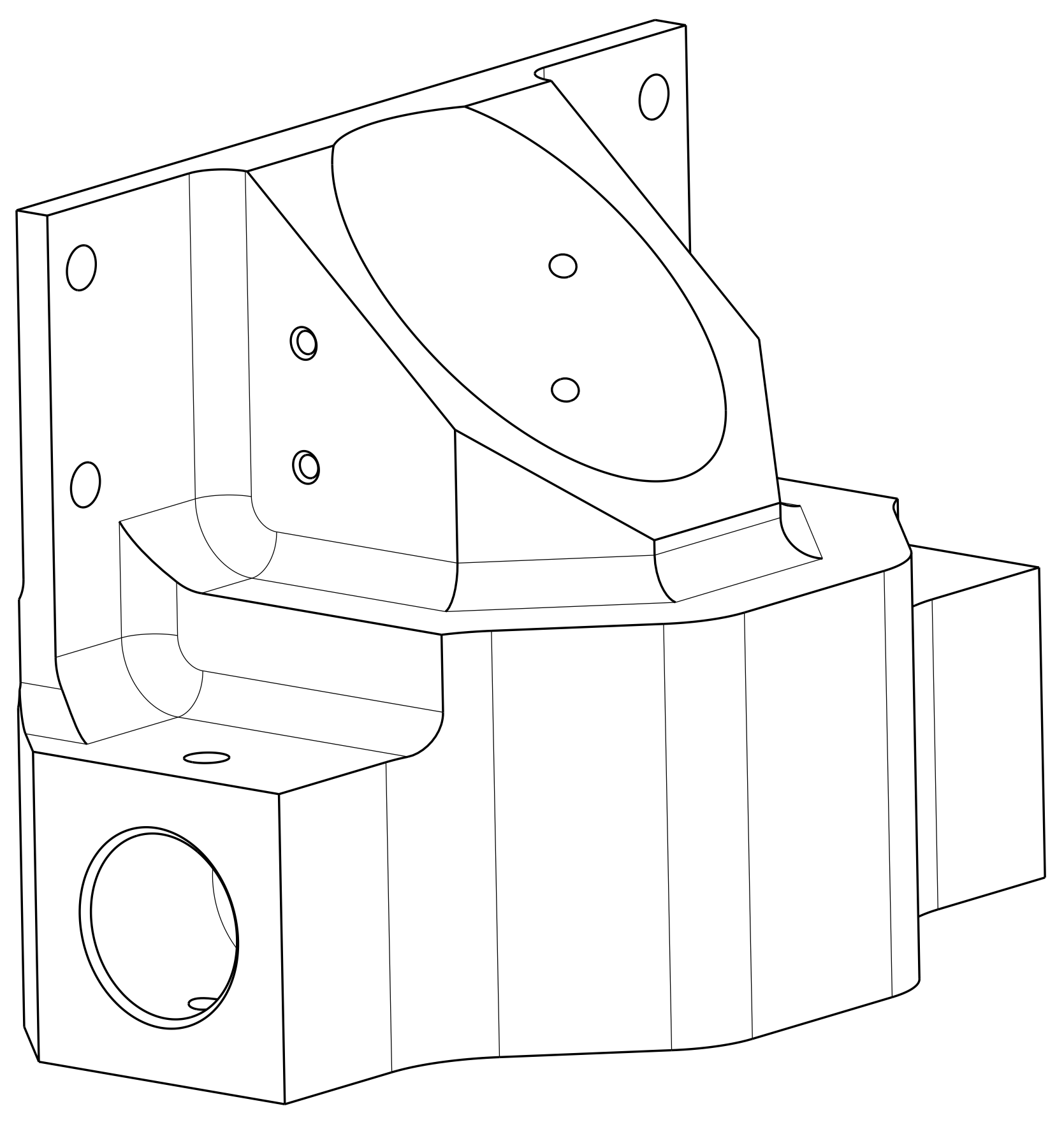

3D VIEW 2 SCAEE: ONLY NONE

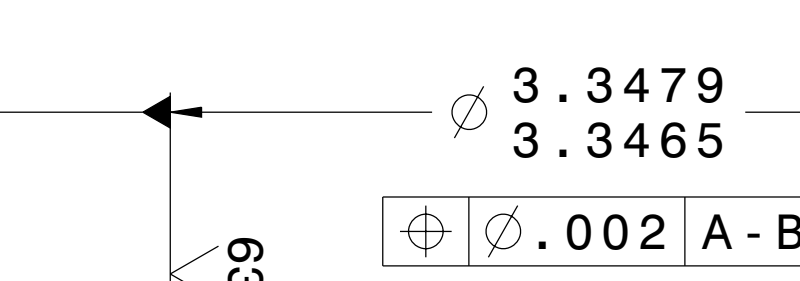

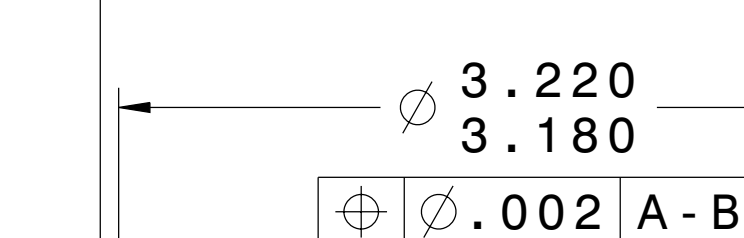

R $: 020$

.389

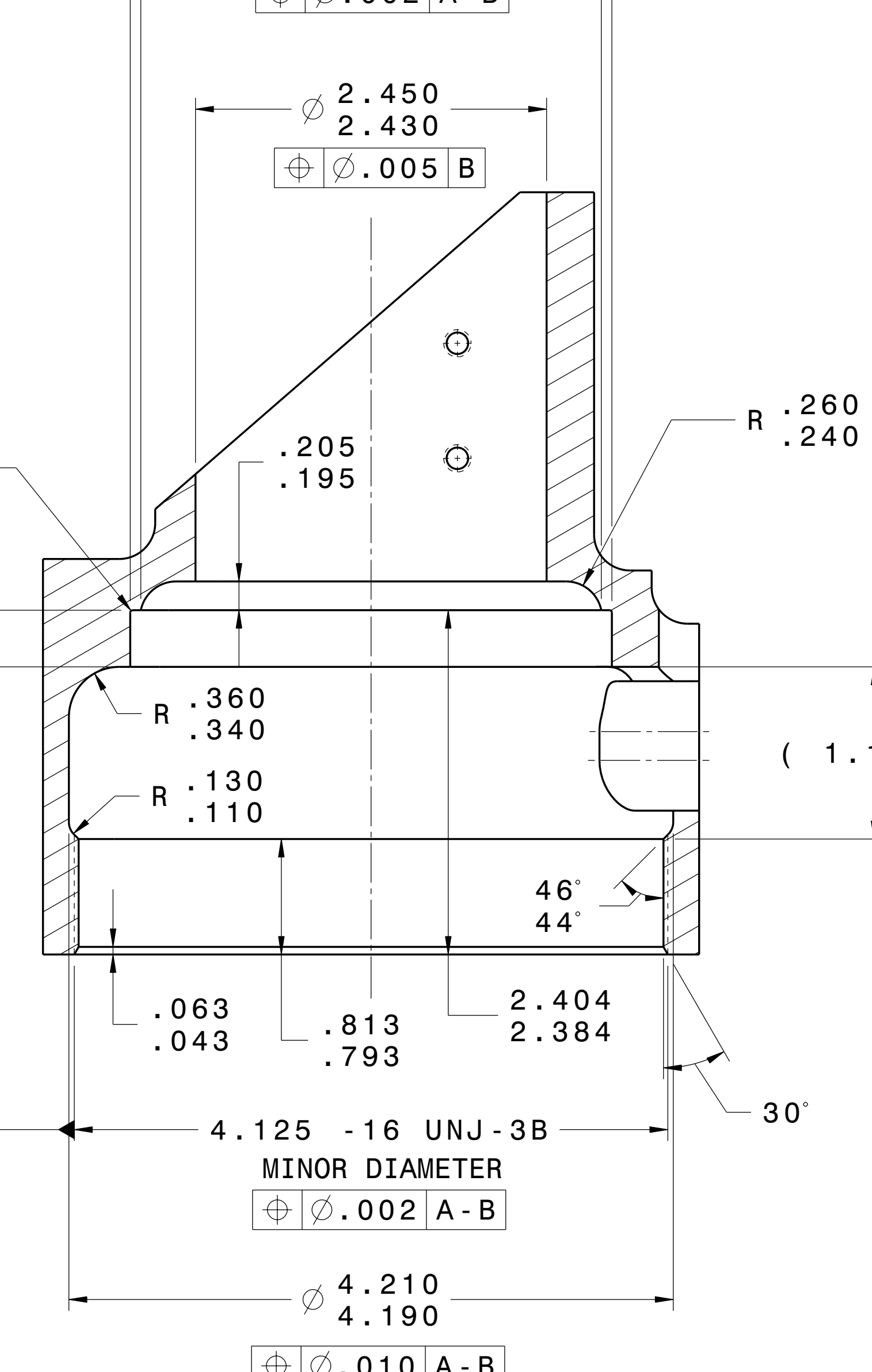

SECTION A-A

.291 THRU AL

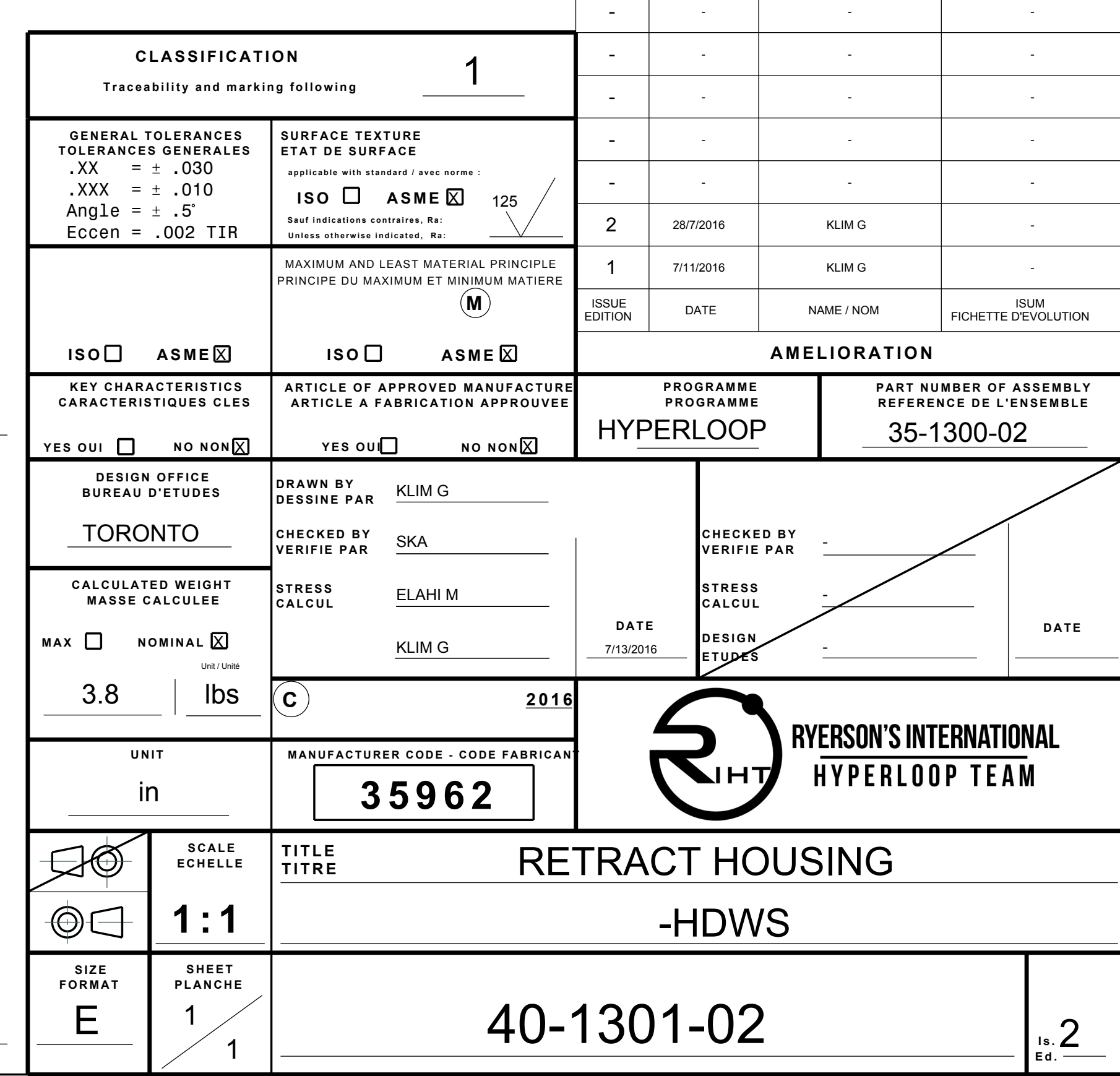




\section{NOTES :}

1. Material: AL bronze (C95400) PeR AStM B505 OR EQuivalent.

2. DEBURR SHARP EDGES $.005 / .015$, UNLESS OtHeRWISE INDICATED.

3. bReak sharp edges of acme thread at entRy and EXit to nut.

4. MACHINE IN THE FULLY HEAT TREATED CONDITION.

5 PART MARKING USING SCRIBING OR PUNCH.
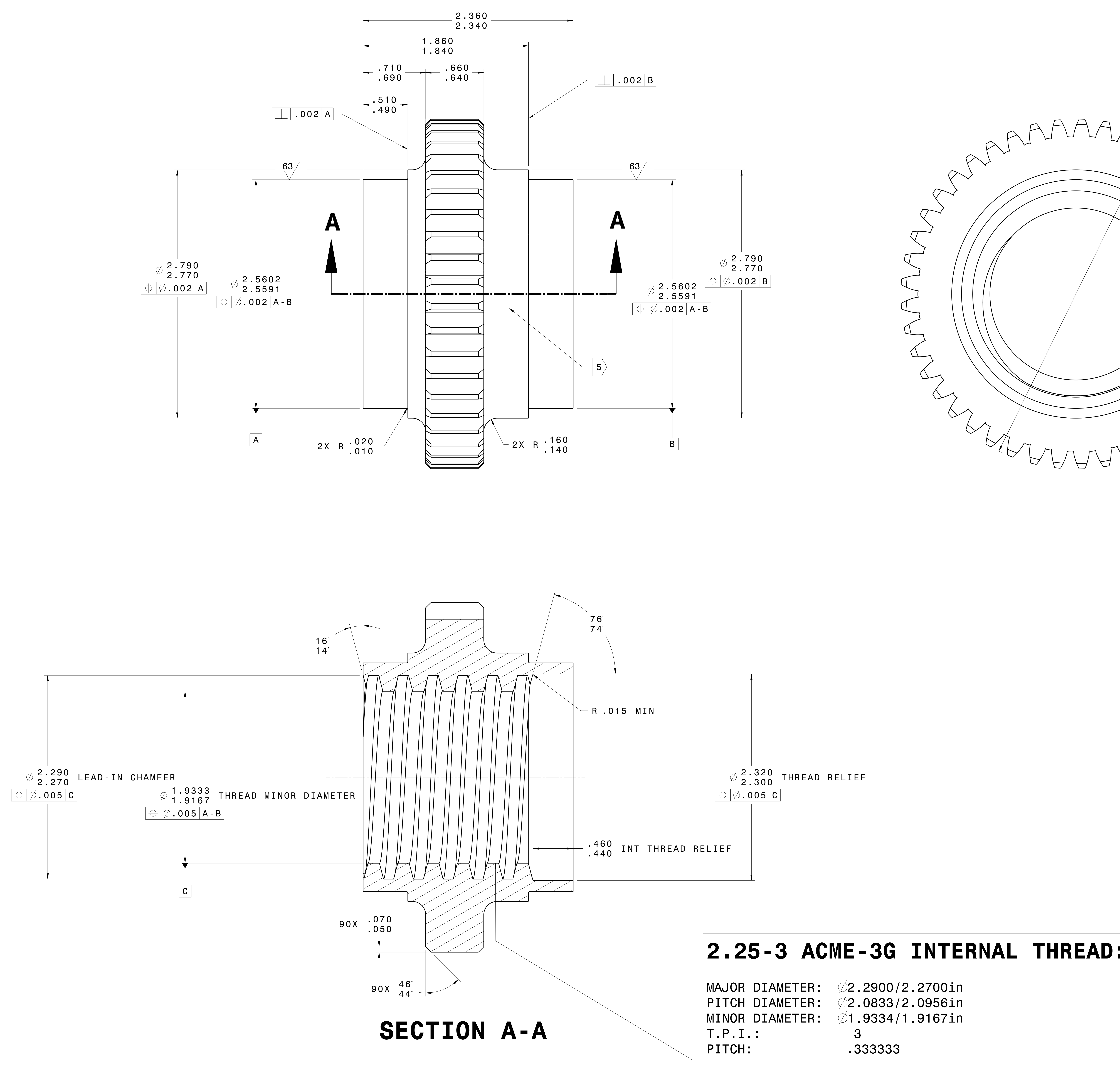

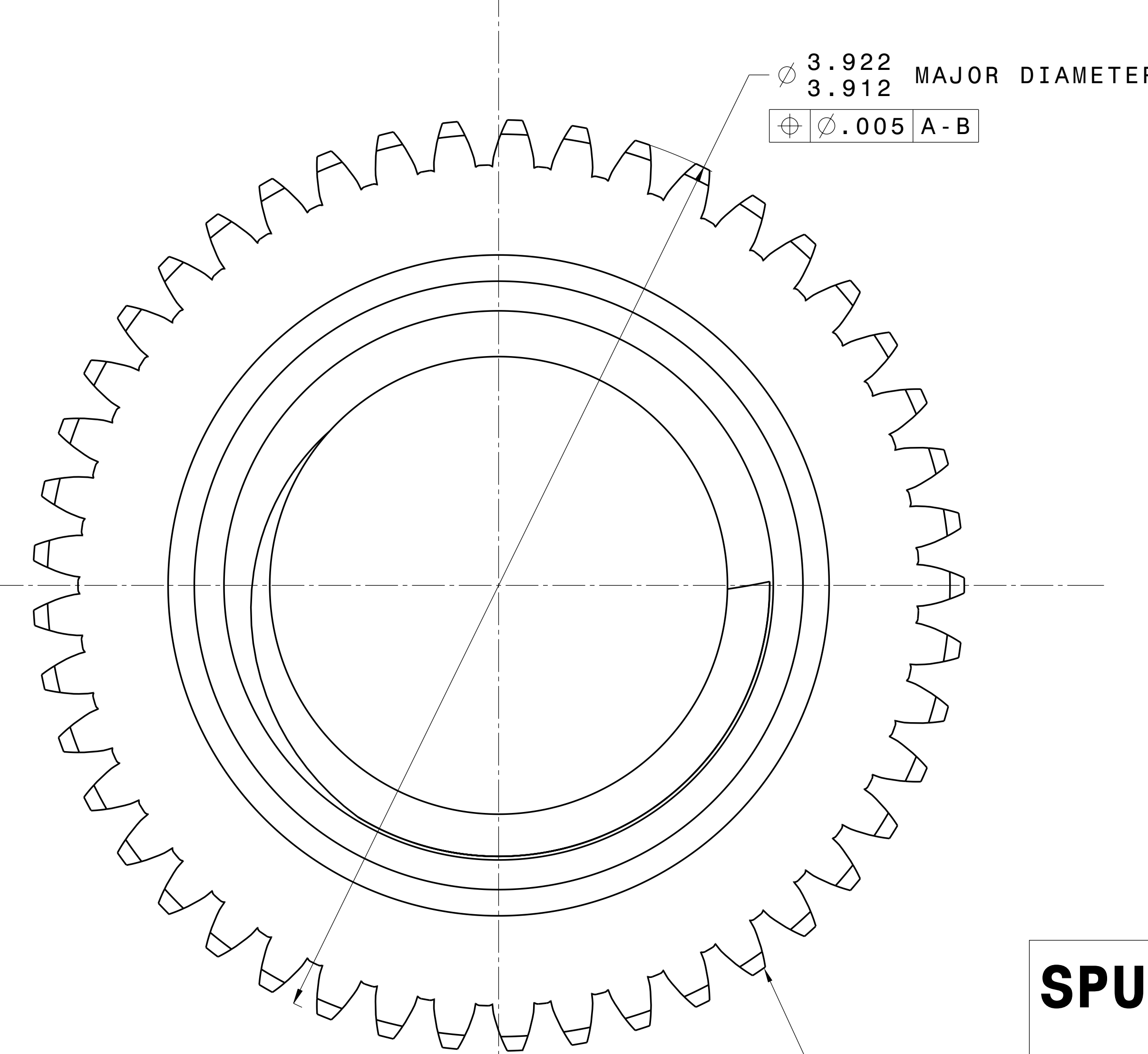

SPUR GEAR :

DIAMETRAL PITCH: No OF TEETH:

PITCH DIAMETER: BASE CIRCLE DIAMETER: CIRCULAR PITCH:

ADDENDUM:

DEDENDUM:

GEAR FILLET RADIUS: 


\section{NOTES :}

1. MAterial: Aluminum 6061-T6511 (BAR) Per AMS QQ-A-200/8

2. DebuRR SHARP EDGES $.005 / .015$, UNLESS Otherwise INDicAted,

3. THREADS PER AS8879.

4. Machine in the fully heat treated Condition.

5. BAG AND TAG OR FIXED TAG MARKING.

6. ANODIZING ALL OVER.

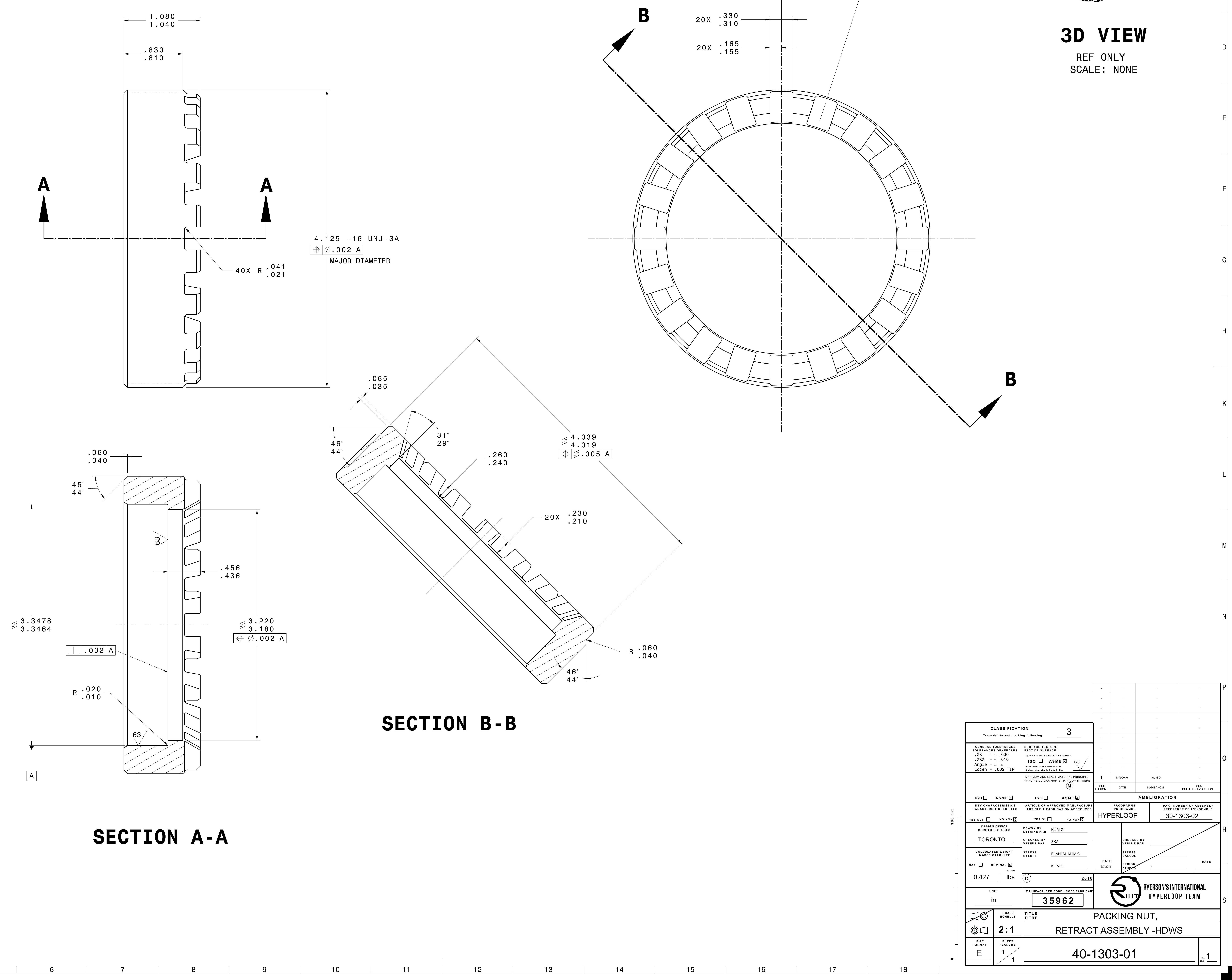




\section{NOTES:}

1. MATERIAL: MACHiNe FROM AISi 1018 STEEL, COLD ROLLED, BAR STOCK.

2. DEBURR SHARP EDGES $.005 / .015$, UNLESS OTHERWISE INDICATED.

3. MACHINE IN THE FULLY HEAT TREATED CONDITION.

4 PART MARKING USING SCRIBING OR PUNCH.

5. BLACK OXIDE CORROSION PROTECTION ALL OVER.

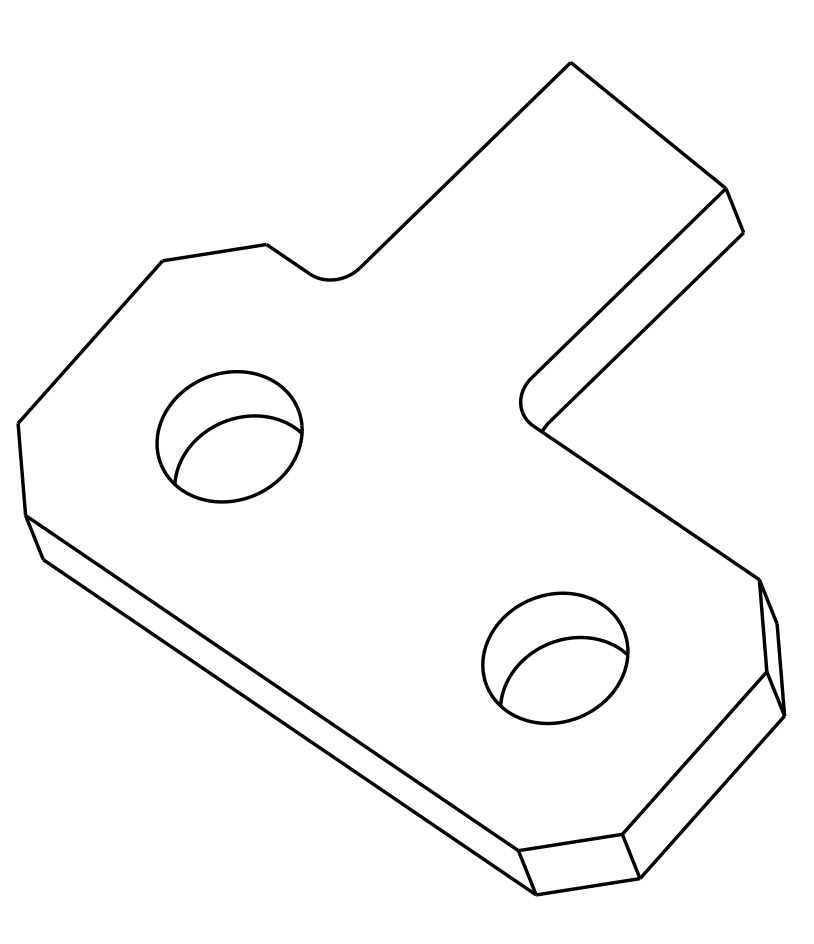

3D VIEW

AEE OOUY
ScCLE: NONE
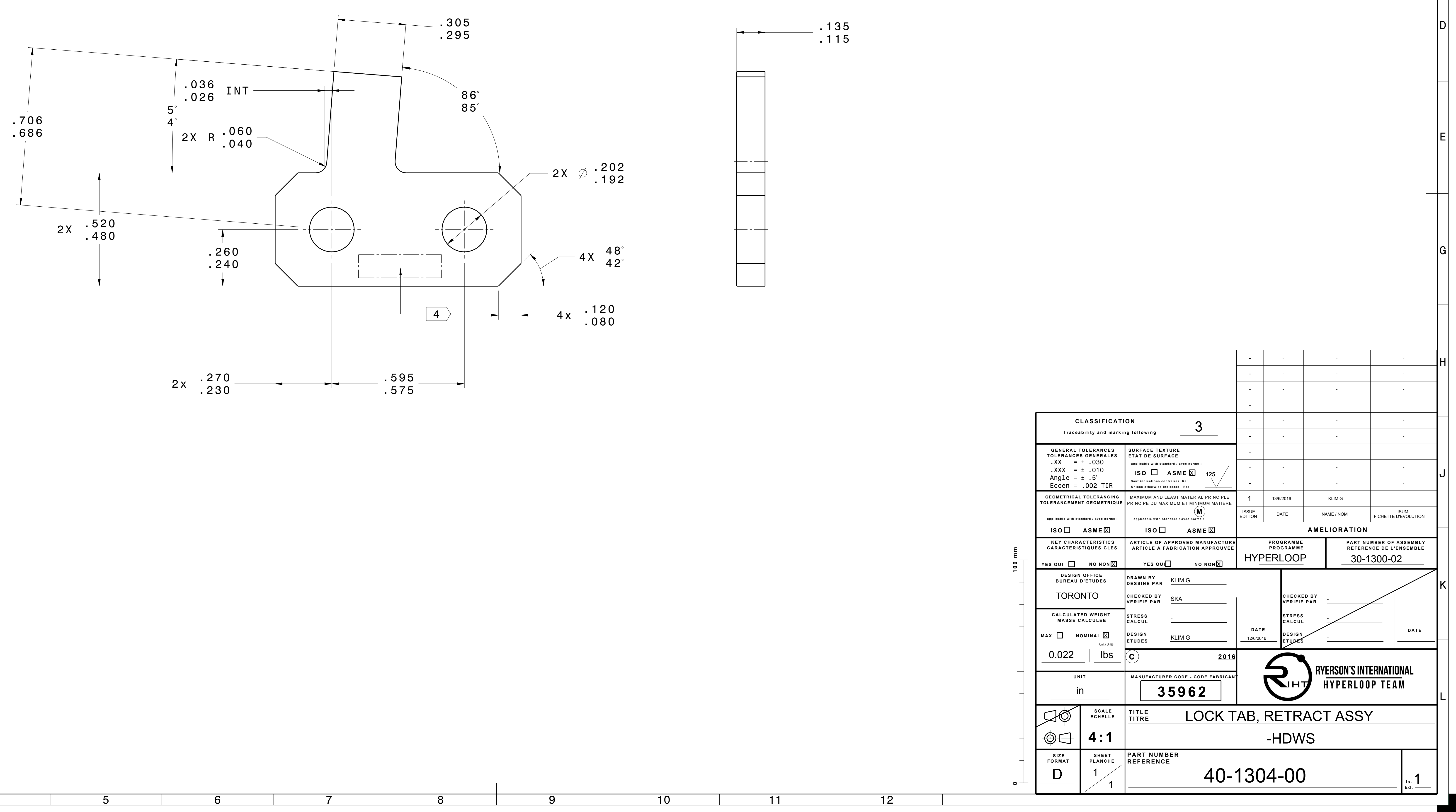


\section{NOTES:}

1. MATERIAL: CORROSION RESISTANT STEEL (303) PER ASTM A582 OR EQUIVALENT. ALL MATERIAL TO BE IN THE ANNEALED CONDITION.

2. DEBURR SHARP EDGES $.005 / .015$, UNLESS OTHERWISE INDICATED.

3. PASSivation all OVER PER AMS 2700 METHOD 1.

4. BAG \& TAG OR FIXED TAG MARKING.

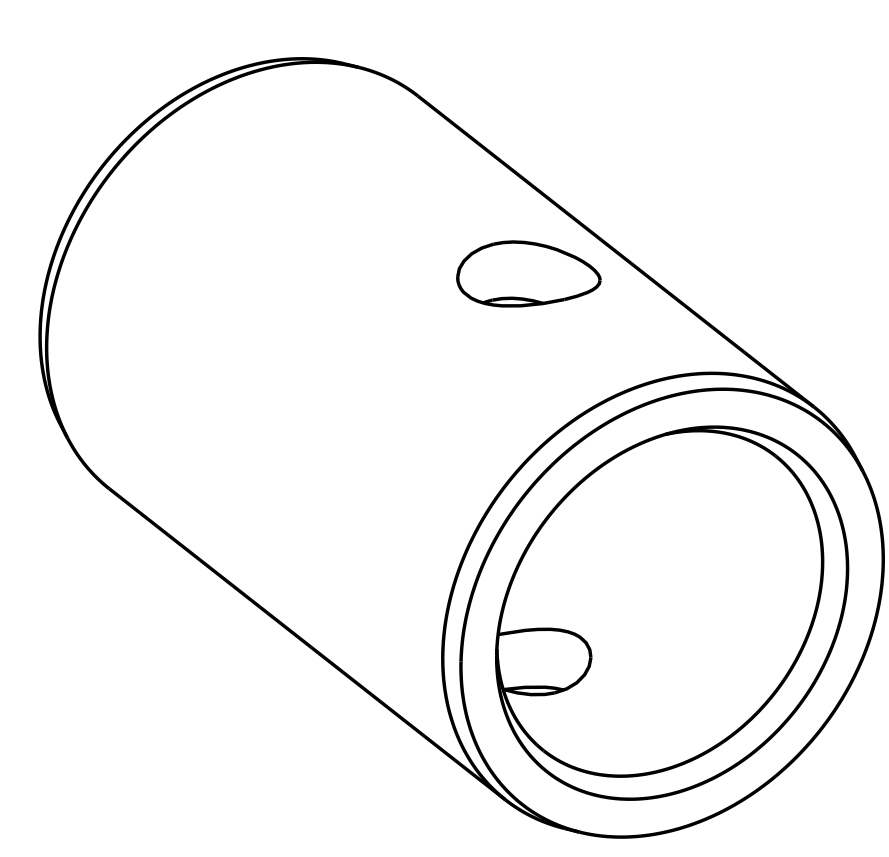

3D VIEW

REF ONLY
SCALE: NONE
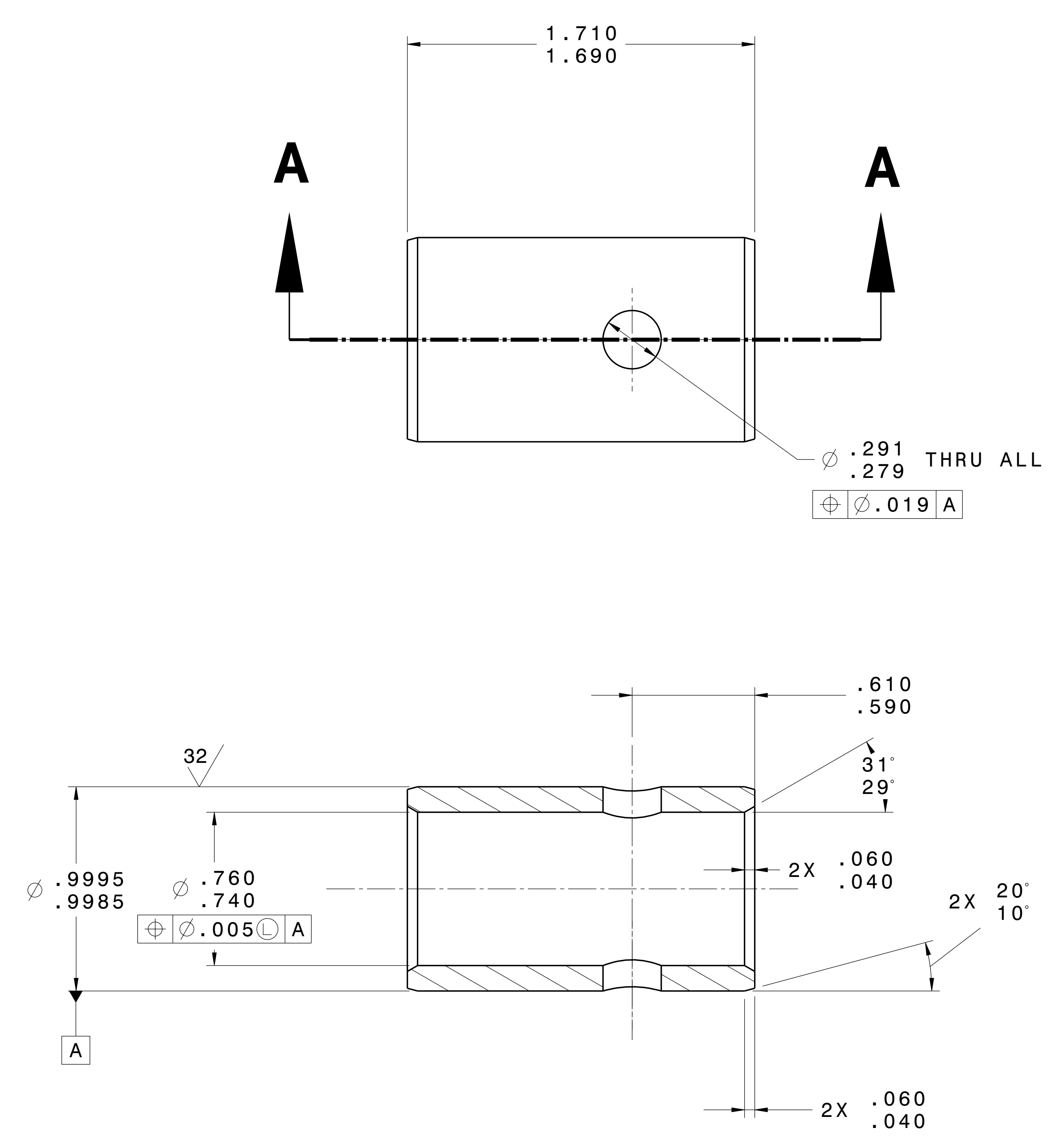

SECTION A-A

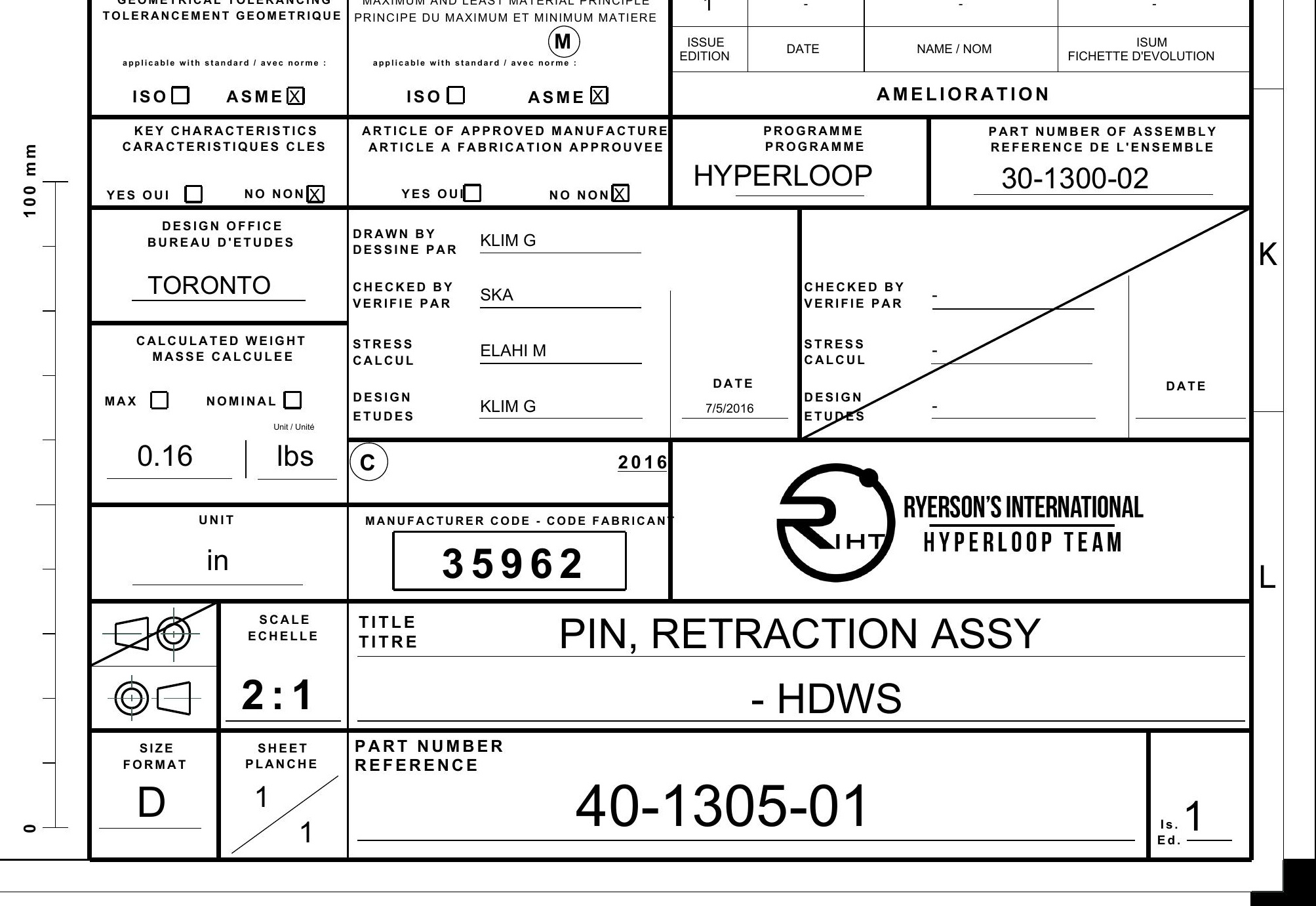




\section{NOTES:}

1. MATERIAL: AL BRONZE (C95400) PER ASTM B505 OR EQUiVALENT.

2. DEBURR SHARP EDGES $.005 / .015$, UNLESS OTHERWISE INDICATED.

3. MACHINE IN THE FULLY hEAT TREATED CONDITION.

4. PART MARKING USING BAG \& TAG.
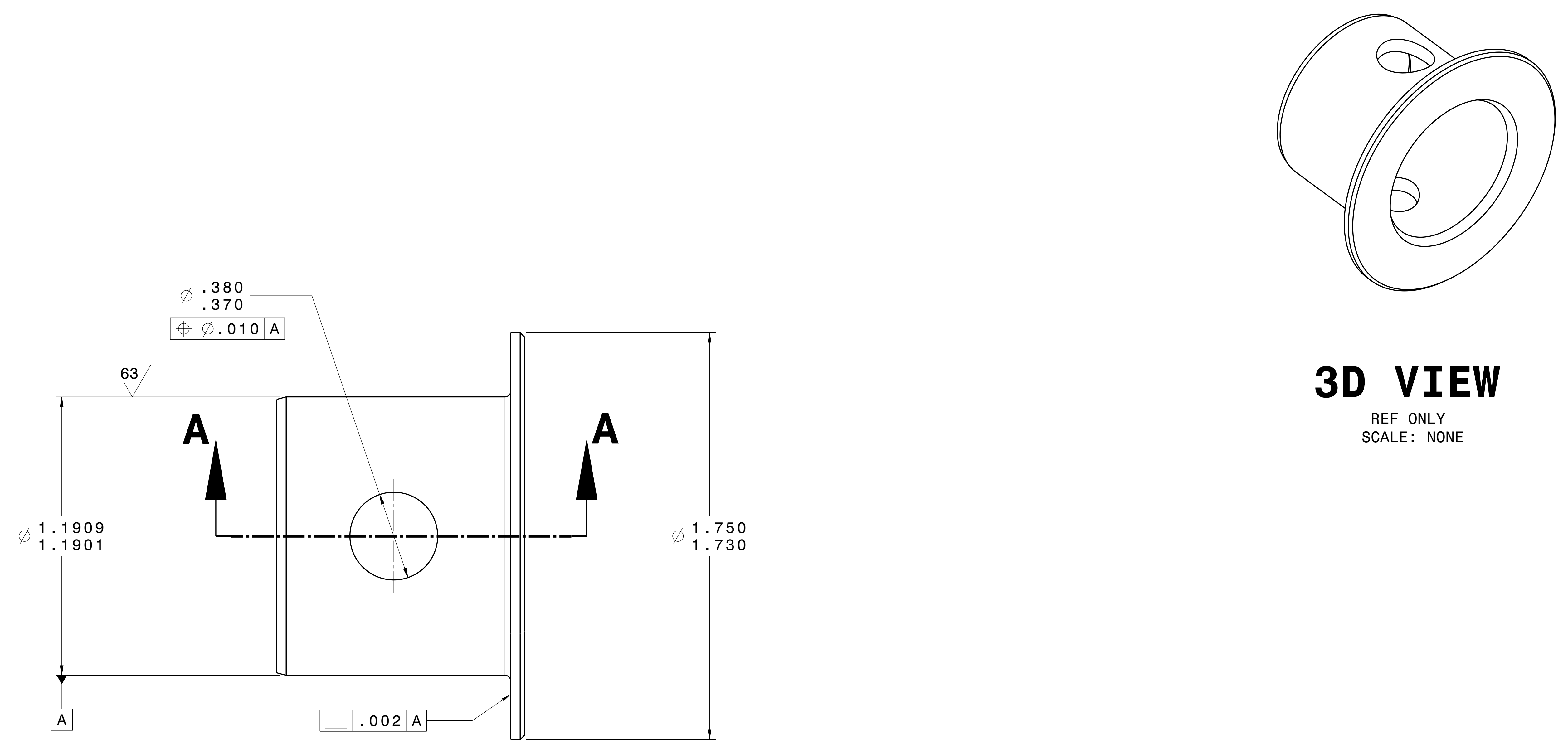

3D VIEW

REF ONYY

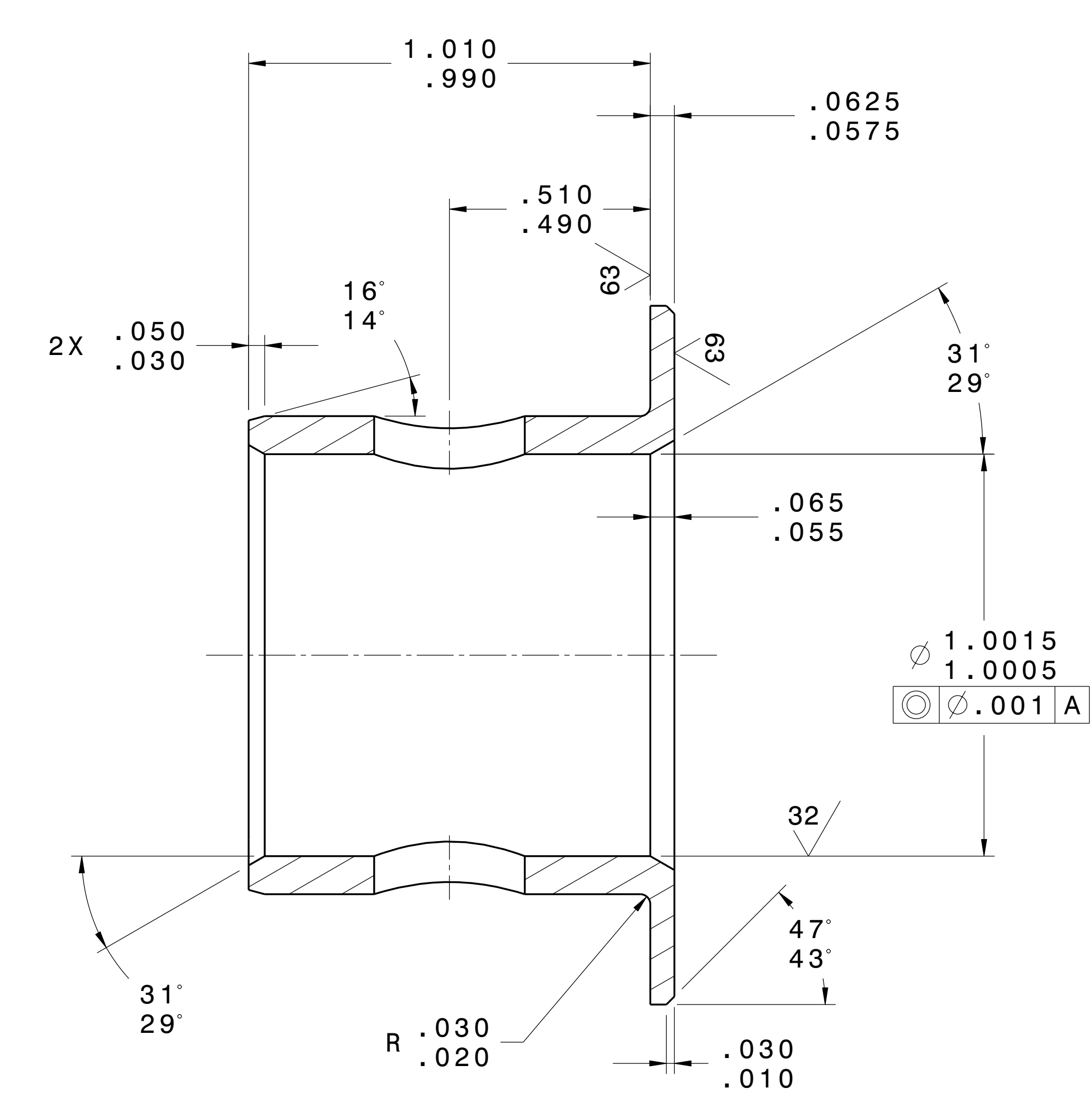

SECTION A-A

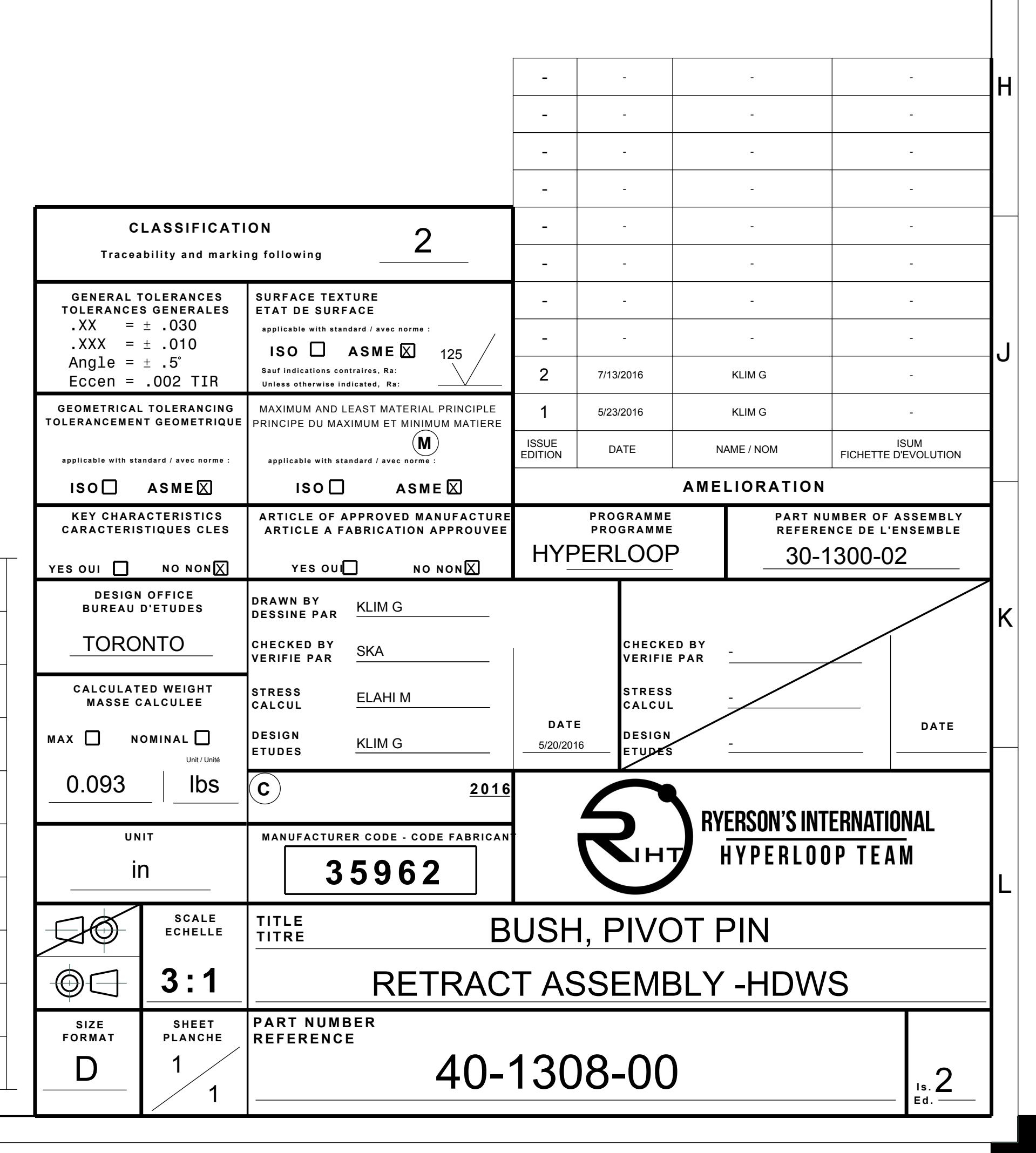




\section{NOTES:}

1. MATERIAL: C260 BRASS HALF HARD SHIM STOCK.

2 MAKE QTY 20 FROM EACH OF THE FOLLOWING STOCK THICKNESSES:

$$
\begin{array}{|c|c|}
\hline \text { PART \# } & \text { THICK. } \\
\hline-01 & .005 \\
\hline-02 & .006 \\
\hline-03 & .007 \\
\hline-04 & .008 \\
\hline-05 & .001 \\
\hline-06 & .012 \\
\hline-07 & .0015 \\
\hline-08 & .020 \\
\hline-09 & .025 \\
\hline-10 & .031 \\
\hline
\end{array}
$$

3. BAG AND TAG EACH STOCK THICKNESS.

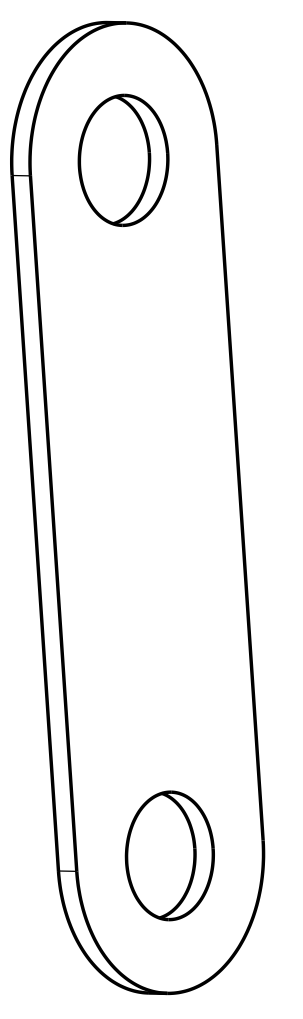

\section{D VIEW}

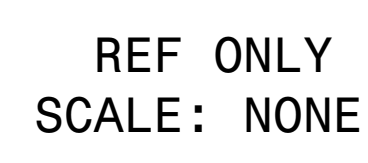
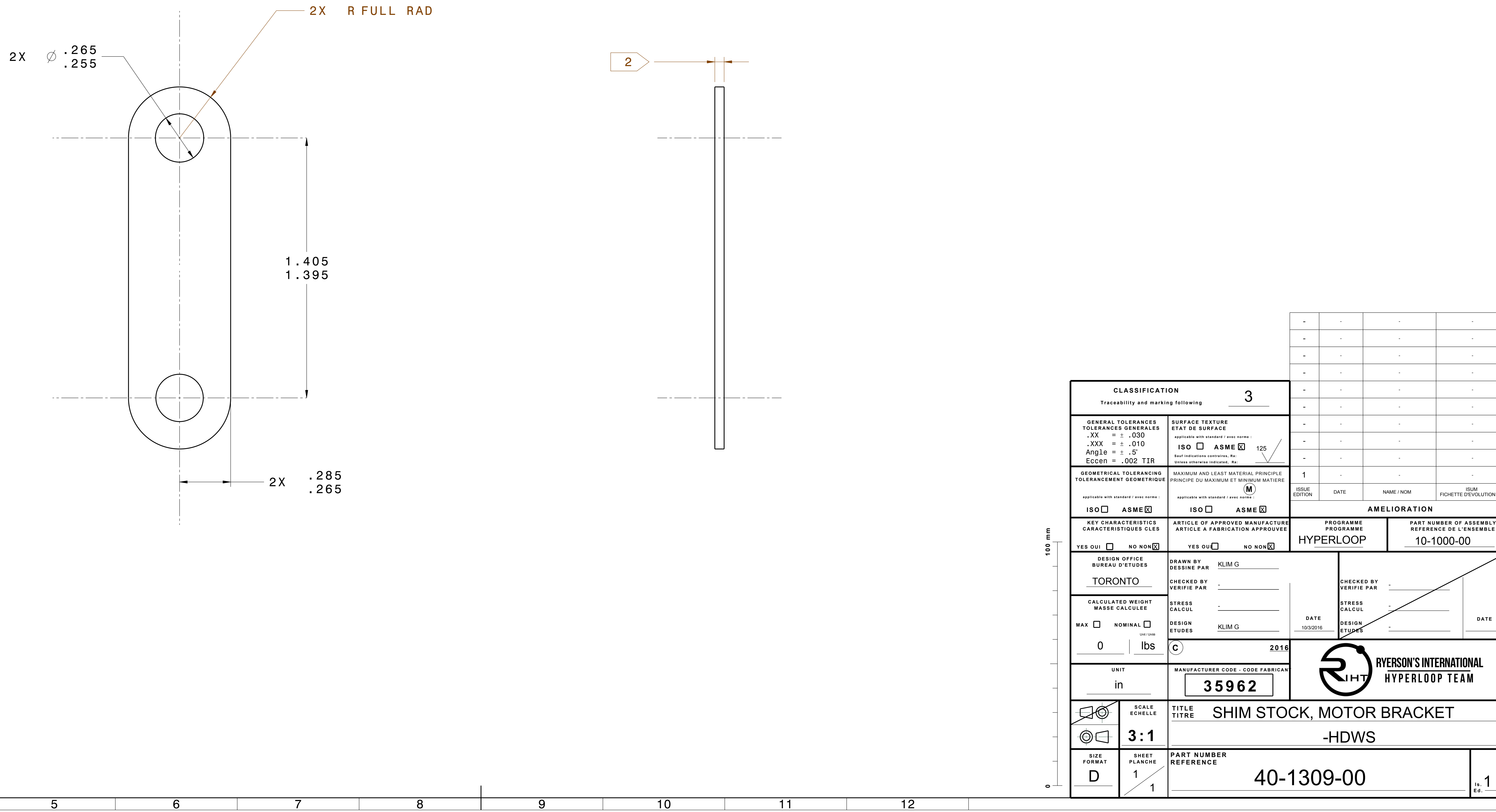


\section{NOTES:}

1. MATERial: ALUMinUm 6061-T6511 (BAR) PER AMS QQ-A-200/8.

2. DEBURR SHARP EDGES $.005 / .015$, UNLESS OTHERWISE INDICATED.

3. ANODIZING ALL OVER.

4 PART MARKING USING SCRIBING OR PUNCH.
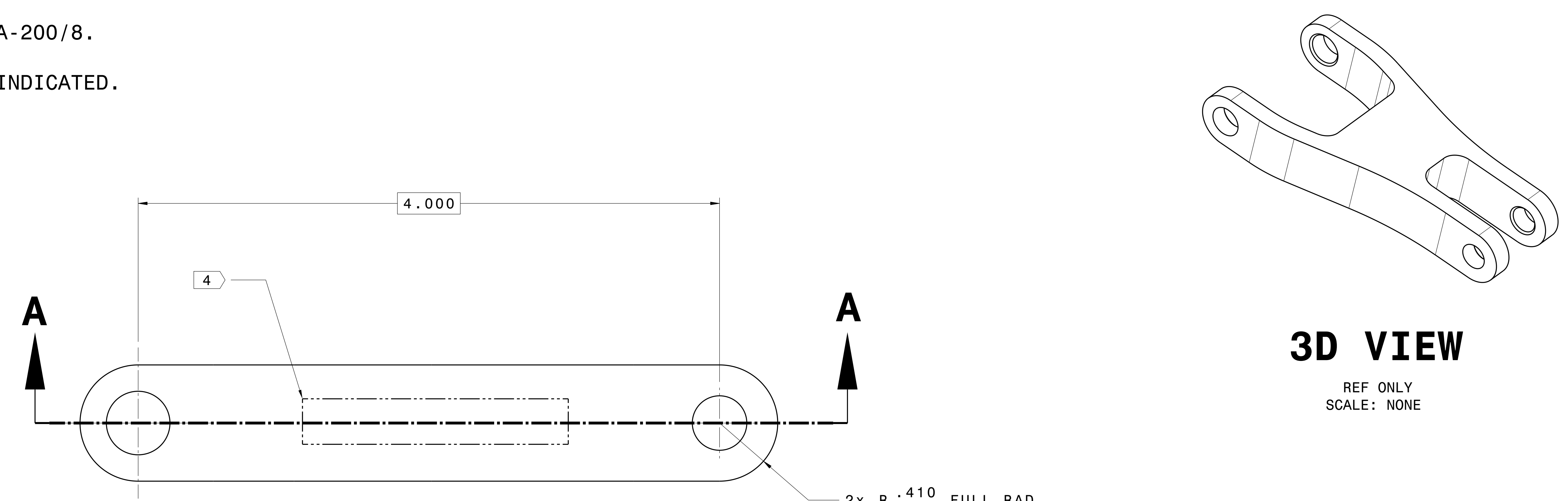

\section{D VIEW} REE ONYY
SCALE: NONE

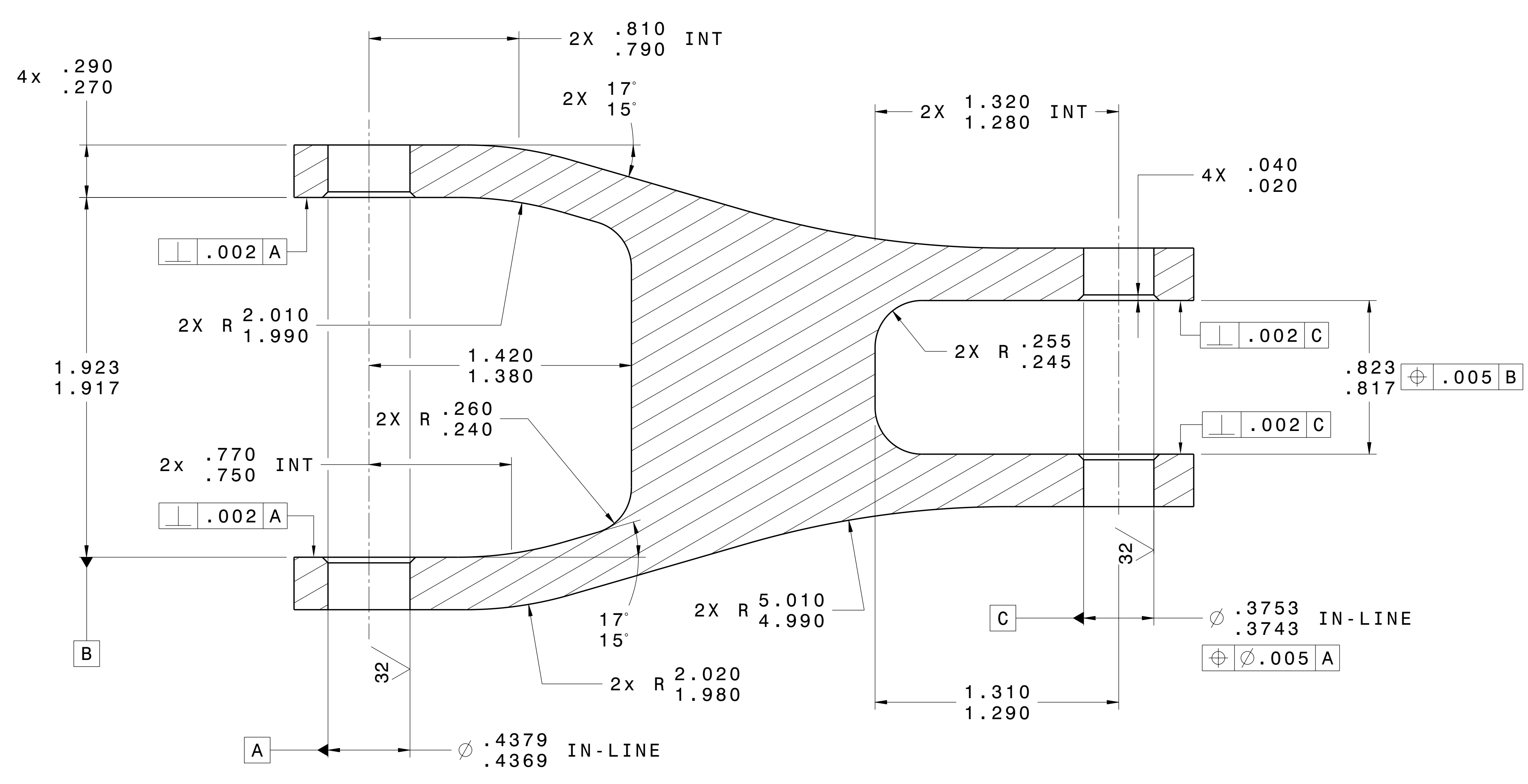

SECTION A-A

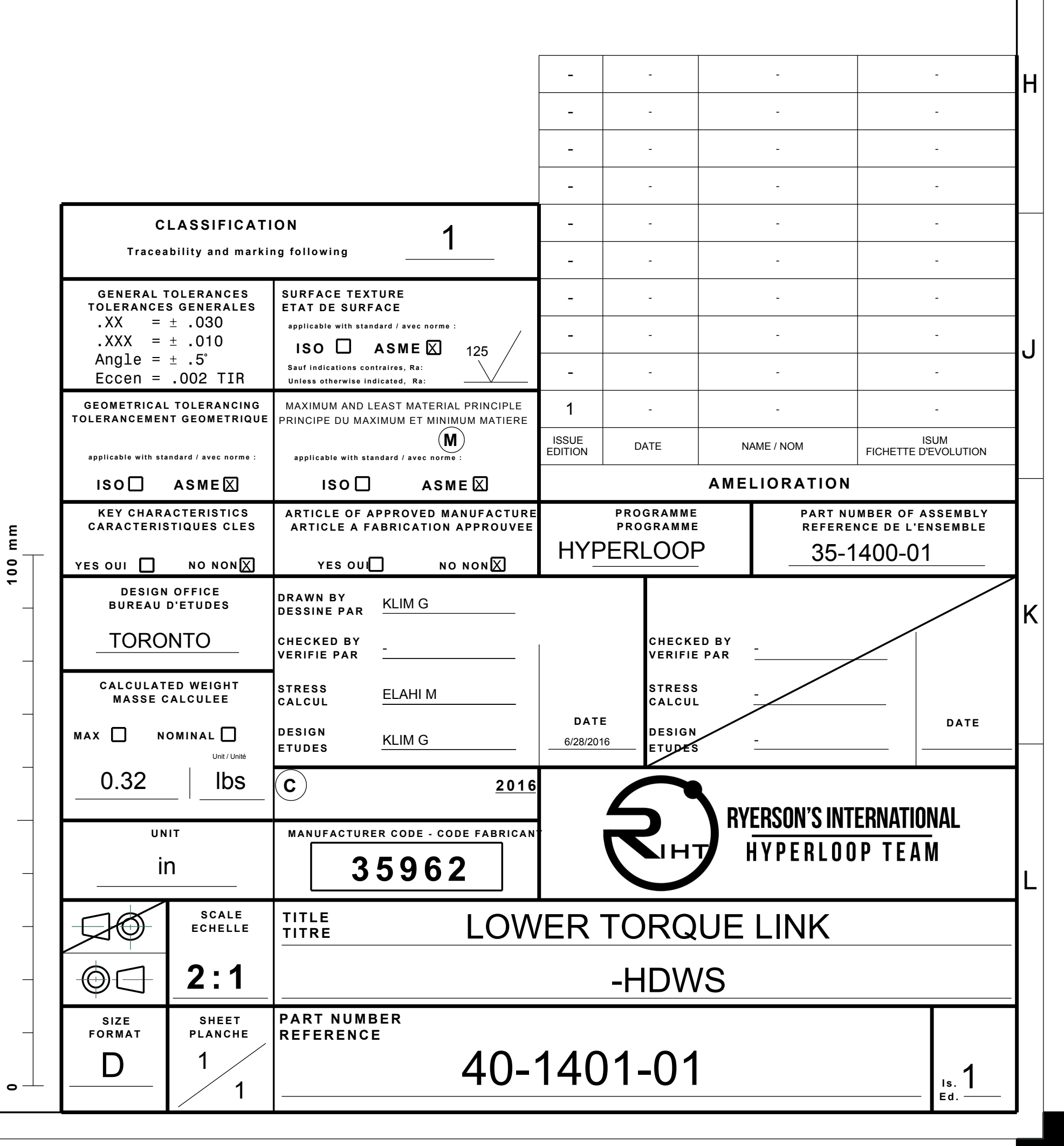




\section{NOTES:}

1. MATERIAL: ALUMinUm 6061-T6511 (BAR) PER AMS QQ-A-200/8.

2. DEBURR SHARP EDGES $.005 / .015$, UNLESS OTHERWISE INDICATED.

3. ANODIZING ALL OVER.

4. THREADS PER AS8879

5 PART MARKING USING SCRIBING OR PUNCH.
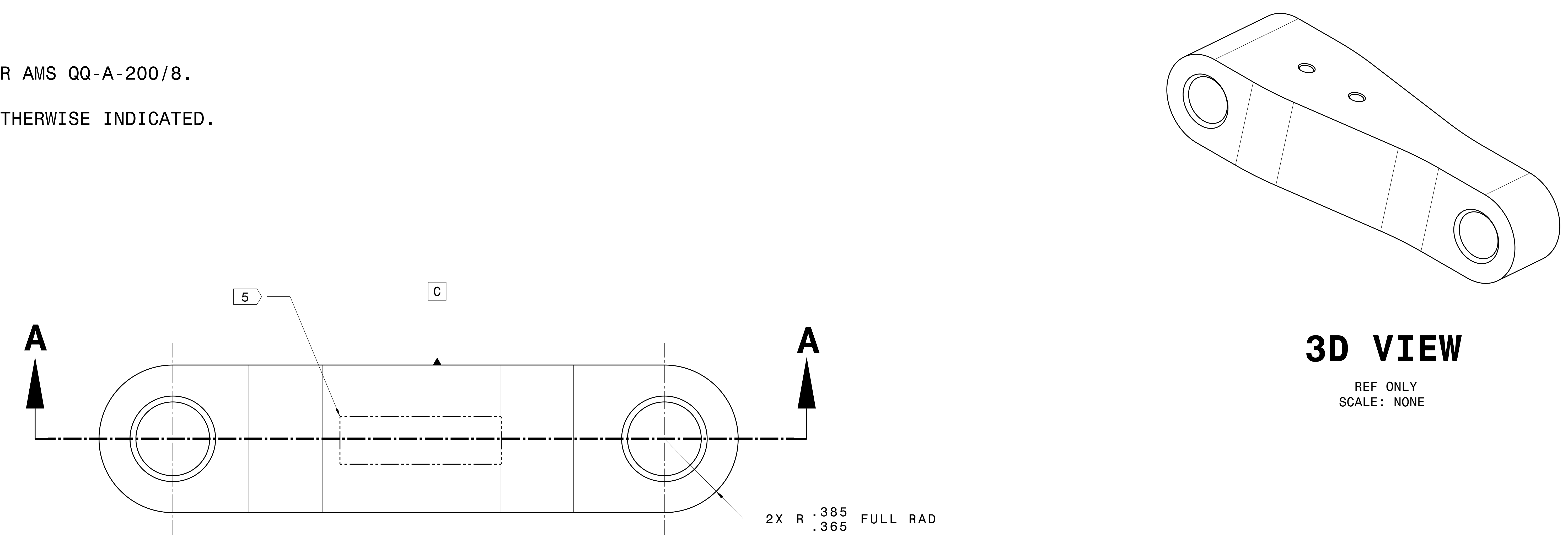

\section{D VIEW \\ REE ONLY
SCALE: NONE}

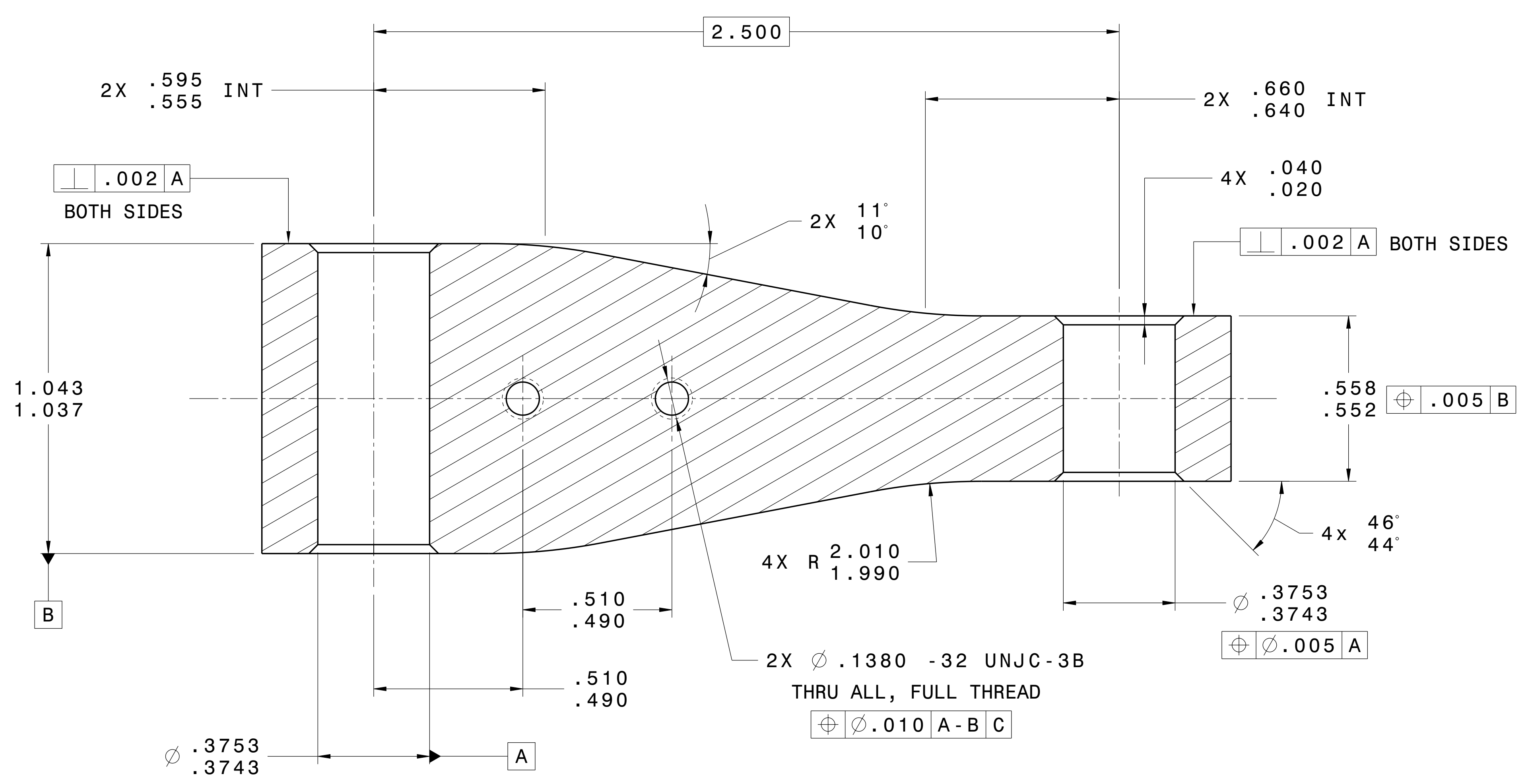

SECTION A-A

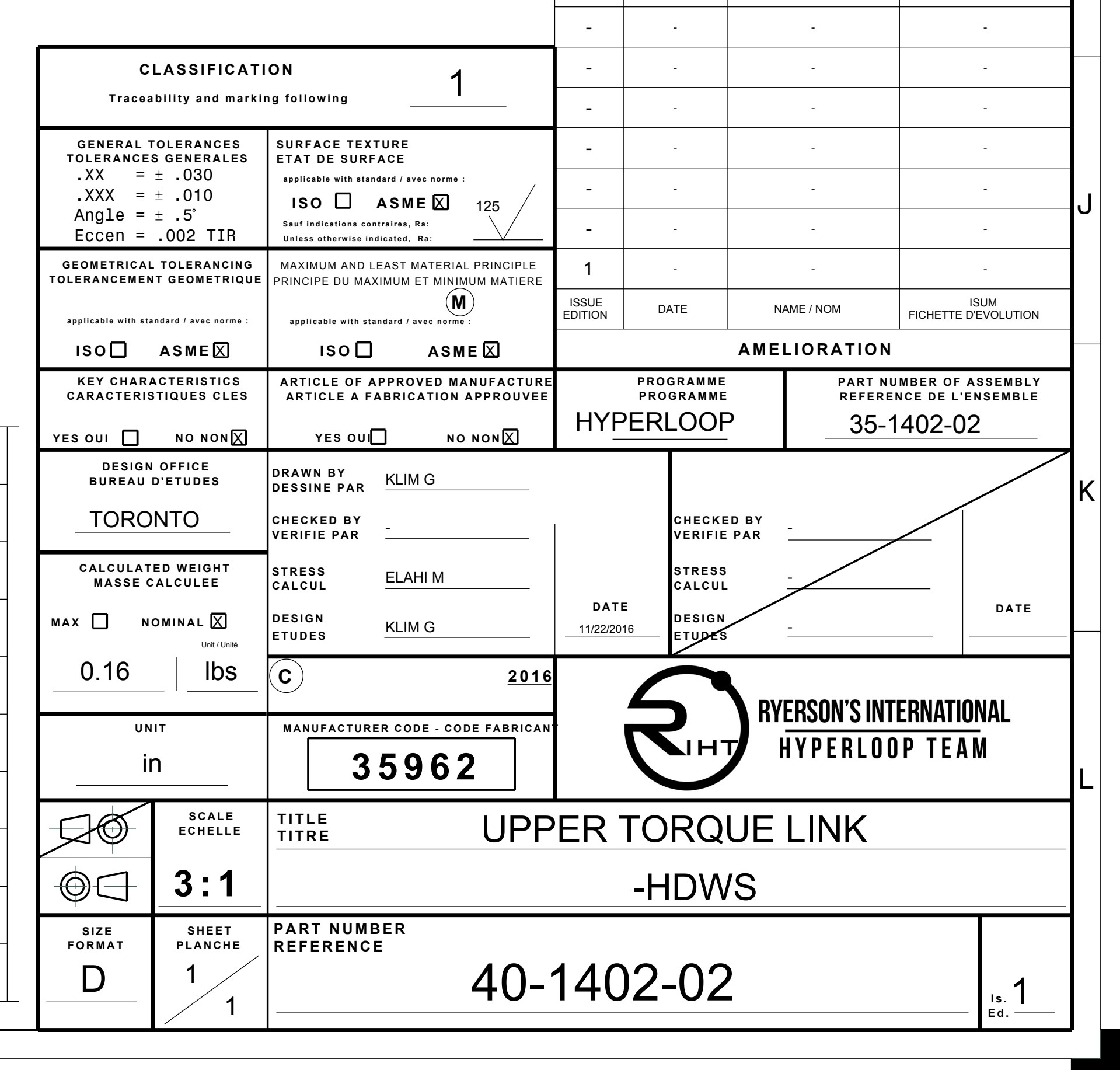


NOTES :

1. MATERIAL: CORROSiON RESistant STEEL (303) PER ASTM A582 OR EQUivalent.

ALL MATERIAL TO BE IN THE ANNEALED CONDITION.

2. DEBURR SHARP EDGES $.005 / .015$, UNLESS OTHERWISE INDICATED.

3. PAssivation All OVer per ams 2700 Method 1

4. BAG \& TAG OR FIXED TAG MARKING.
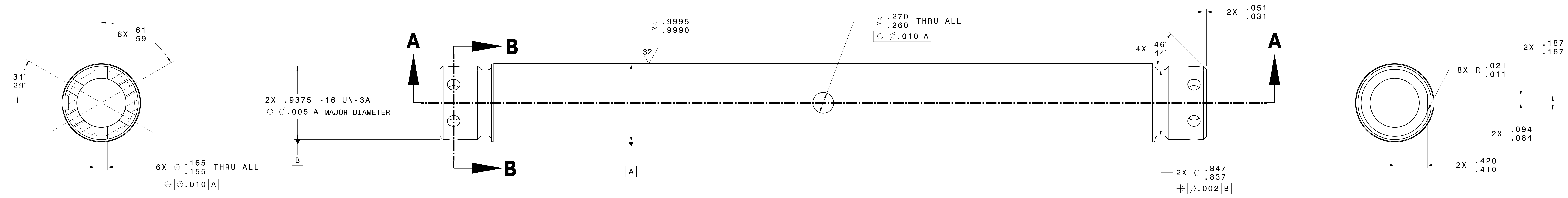

\section{SECTION B - B}

2 PLACES

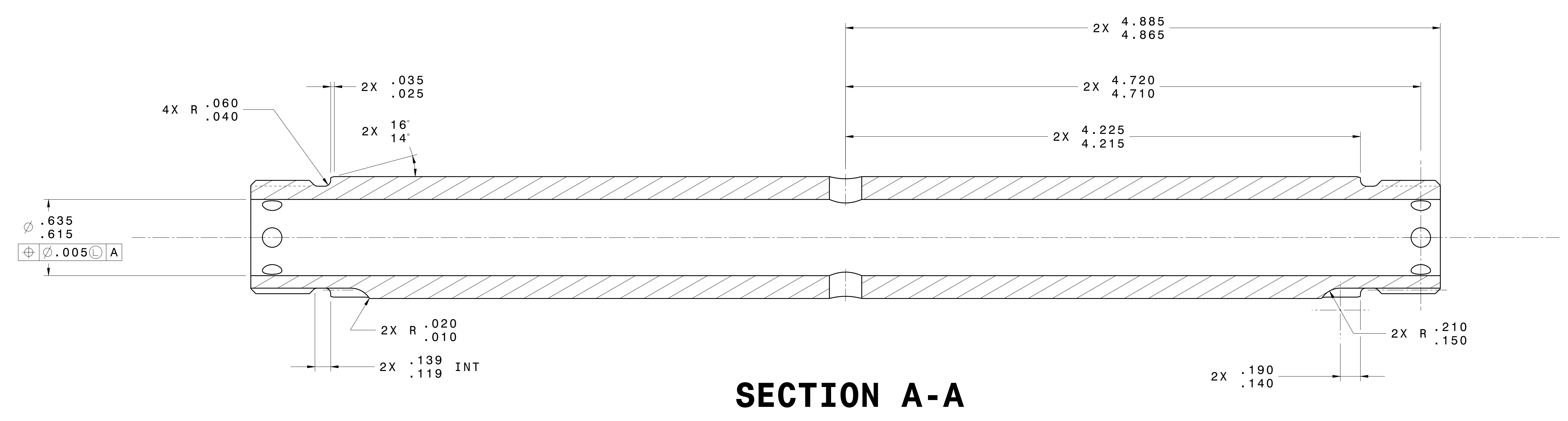

3D VIEW

REF ONYY

\section{STION A-A}

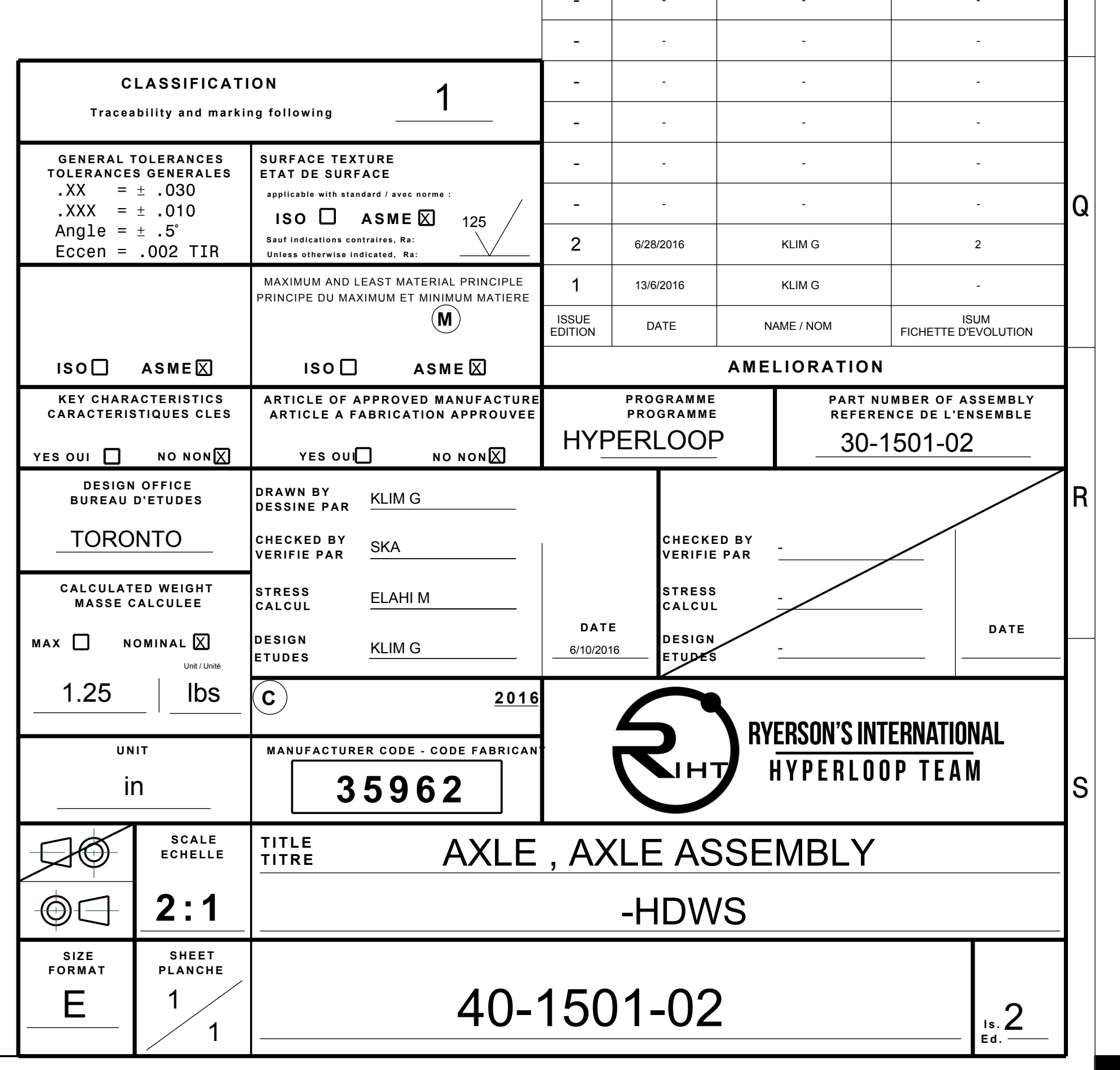




\section{NOTES:}

1. MAKE FROM MS21258C16 (CADMIUM PLATED 1018 STEEL)

2 DIMENSION TO BE MET AFTER MODIFICATION

3 BREAK SHARP EDGES $0.05 / 0.015$

4. PART MARKING USING BAG \& TAG OR FIXED TAG.

\section{D VIEW}

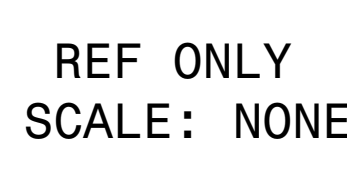

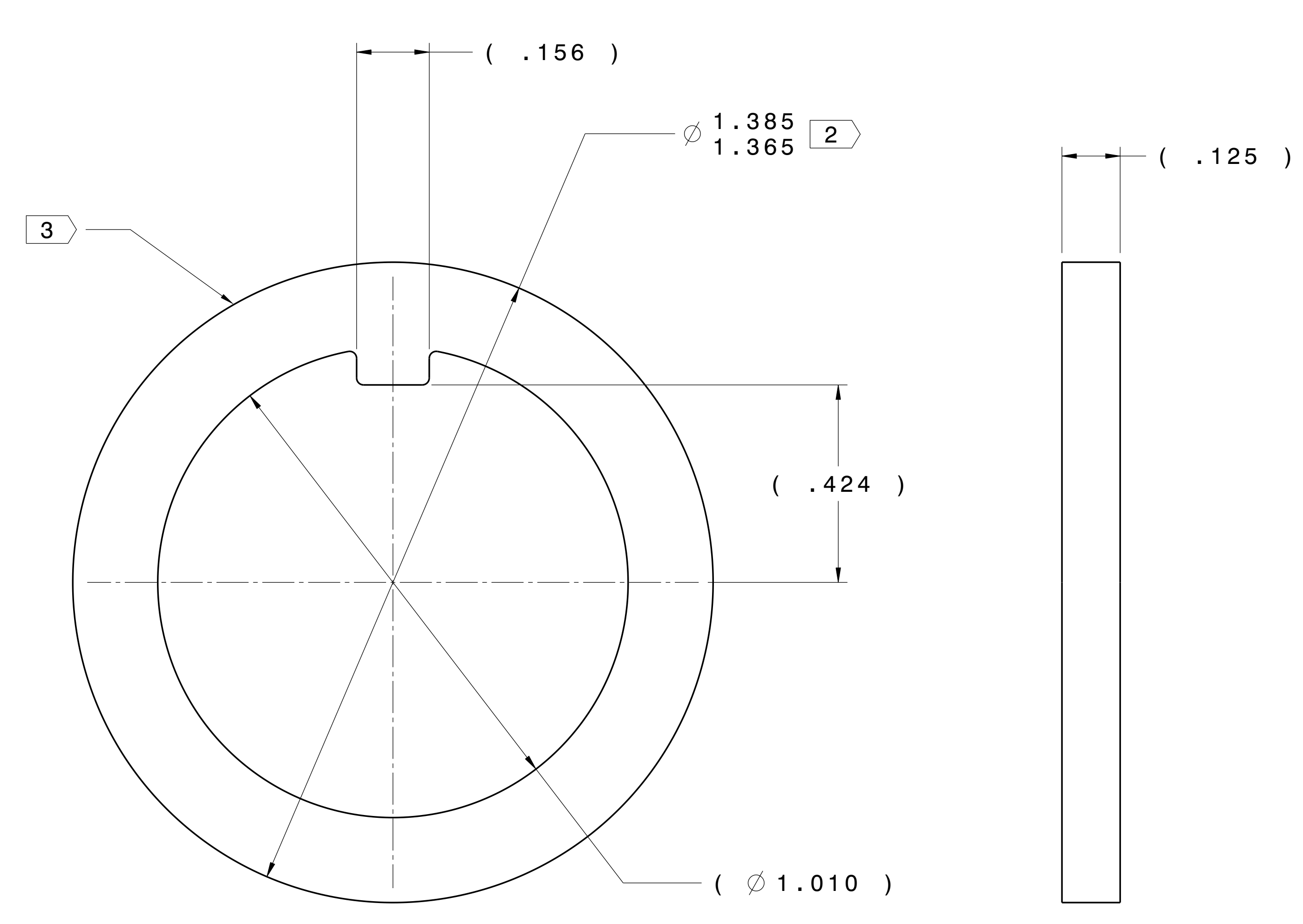




\section{NOTES:}

1. MATERIAL: 303 StAinless SteEL.

2. DEBURR SHARP EDGES $.005 / .015$, UNLESS OTHERWISE INDICATED.

3. PART MARKING USING BAG \& TAG.

\section{D VIEW}

REF ONLY
SCALE: NONE
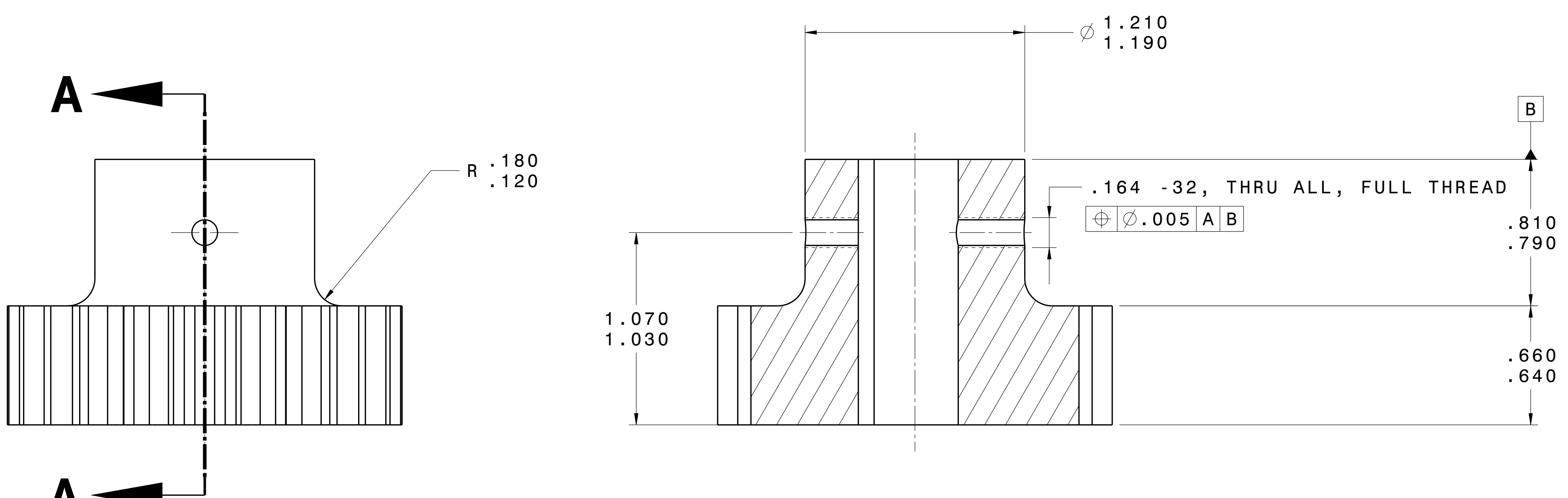

SECTION VIEW A-A

\section{SPUR GEAR :}

DIAMETRAL PITCH:

No OF TEETH:

PRESSURE ANGLE:

PITCH DIAMETER:

BASE CIRCLE DIAMETER:

CIRCULAR PITCH:

TOOTH THICKNESS:

ADDENDUM:

DEDENDUM:

GEAR FILLET RADIUS:

in

1.8794 in

.2618 in

.1309 in

.0833 in .1042 in
A $\underset{.0790}{0.0785} \because 1580$

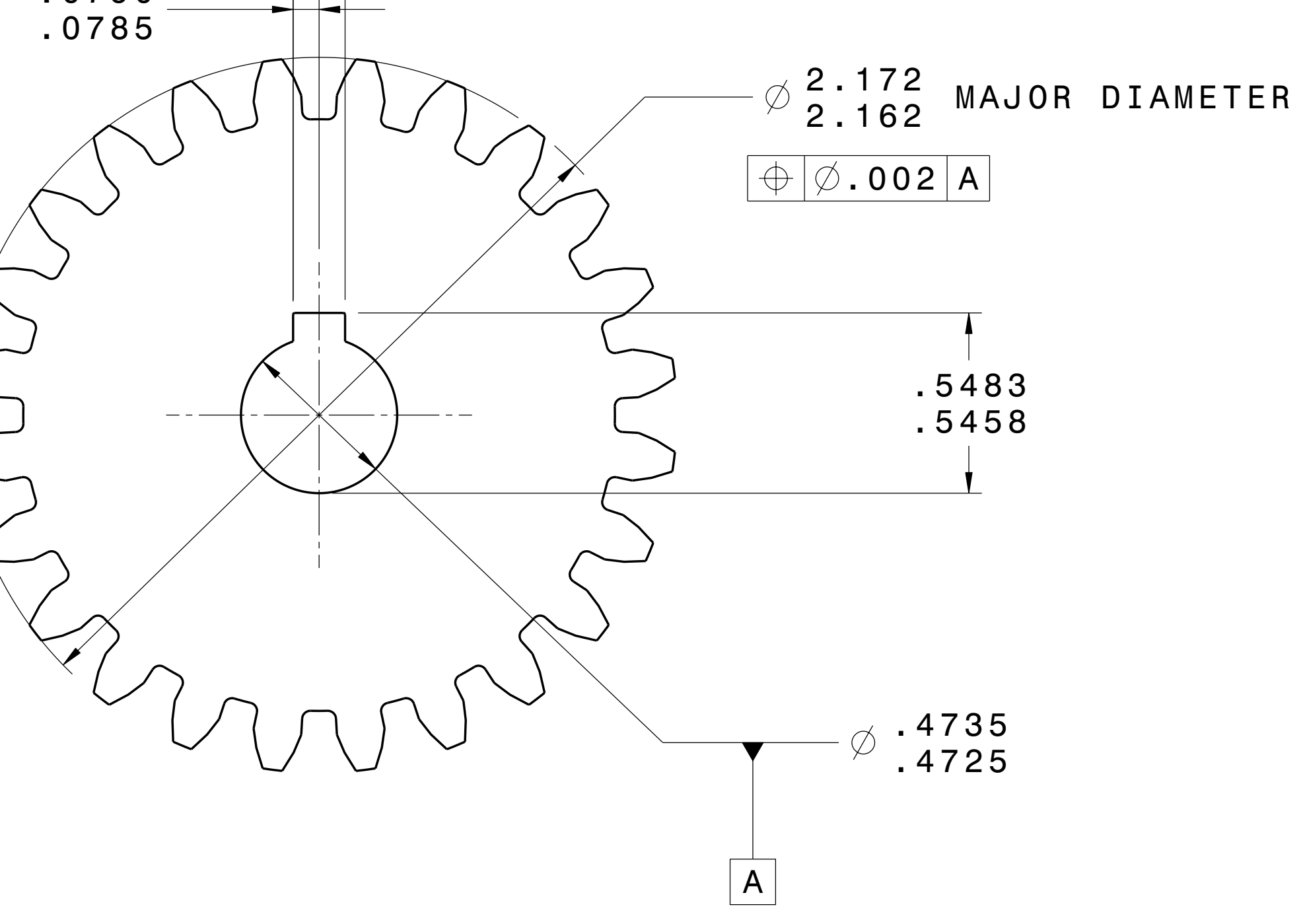

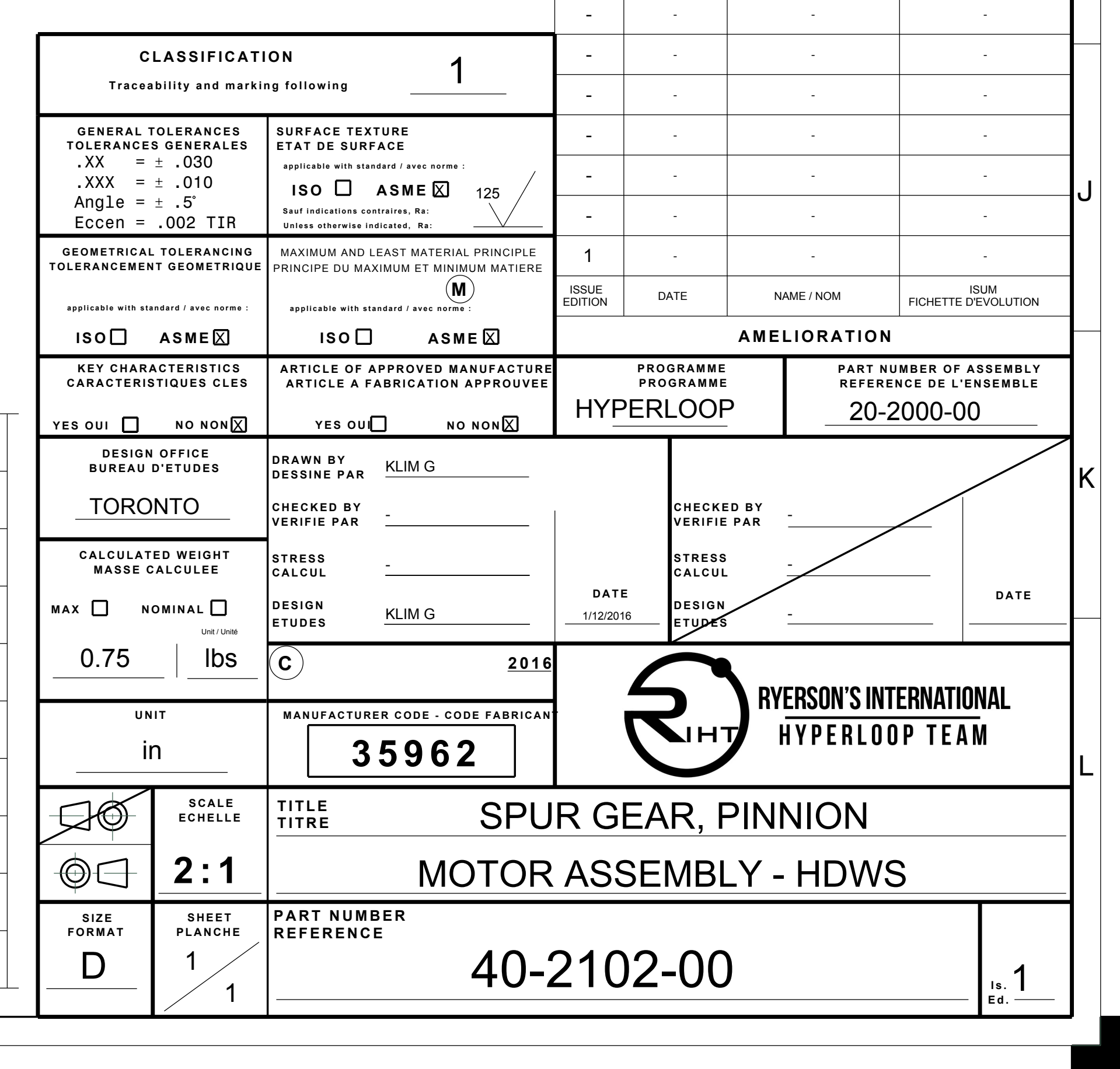


This page was intentionally left blank 


\section{Appendix B: HDWS Tooling Drawings}

This page was intentionally left blank 


\section{NOTES:}

1. MAKE FROM DELRIN.

2. DEBURR SHARP EDGES $.005 / .015$, UNLESS OTHERWISE INDICATED.

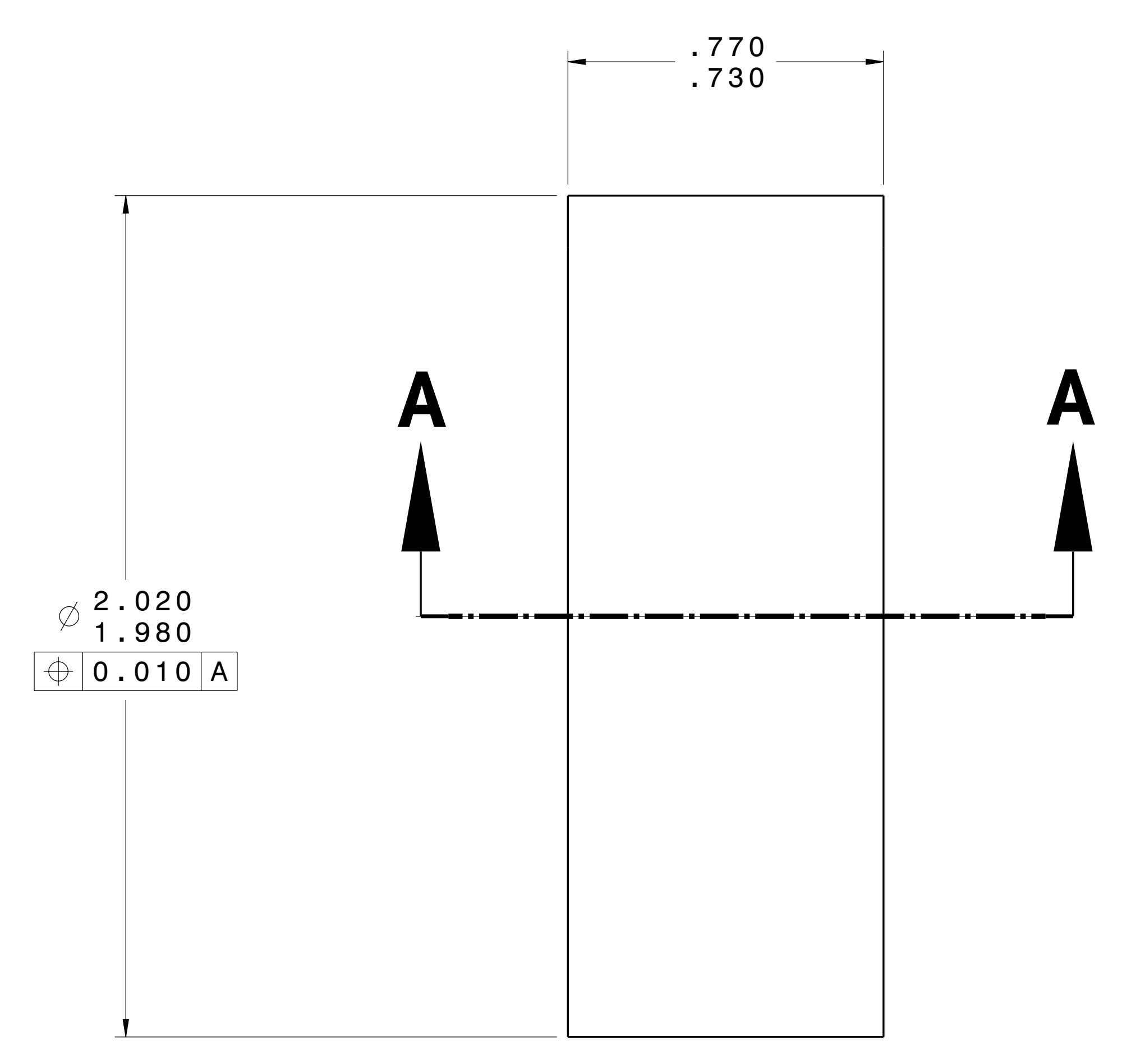

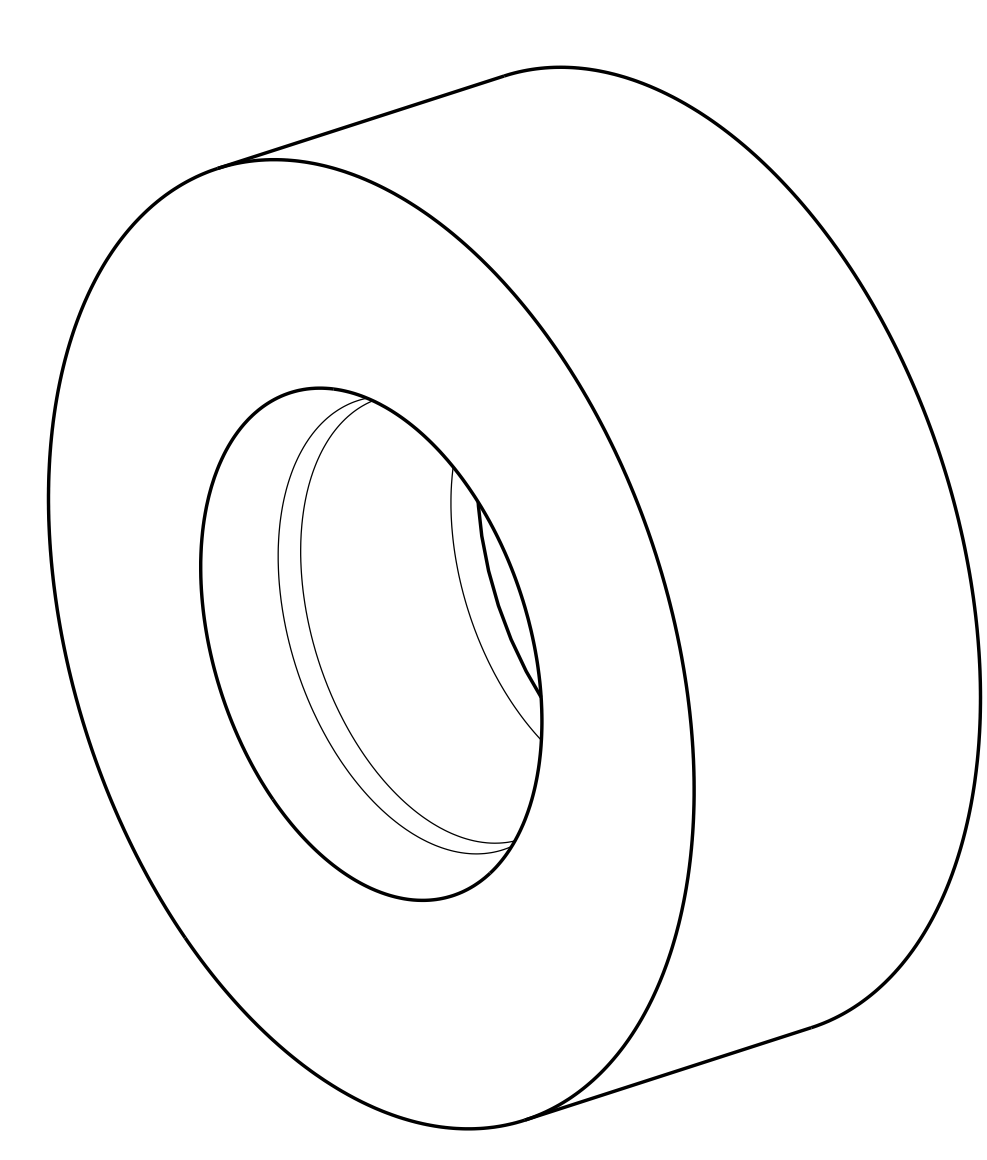

3D VIEW 1 REE ONIY
SCALE: NONE

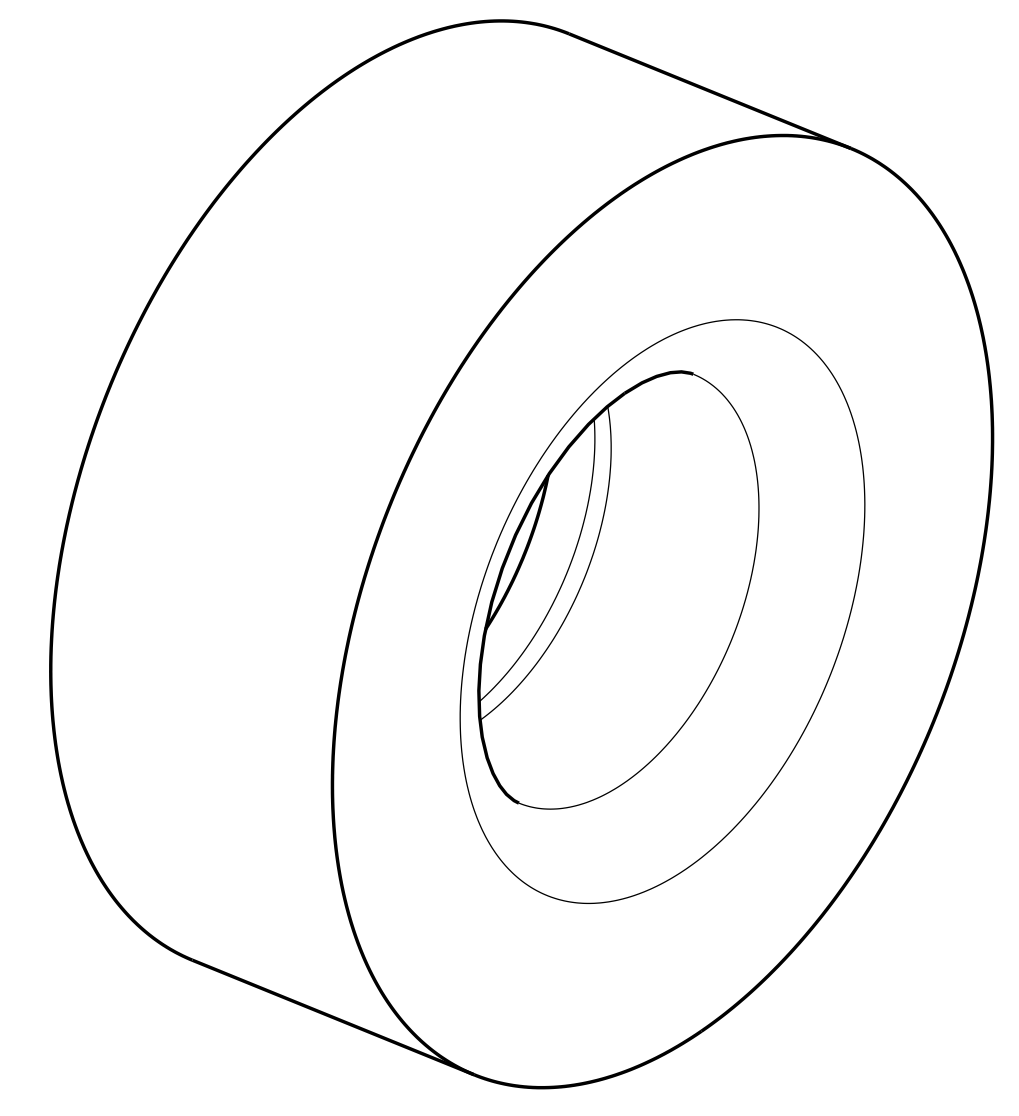

3D VIEW 2 SCAEE: ONLY

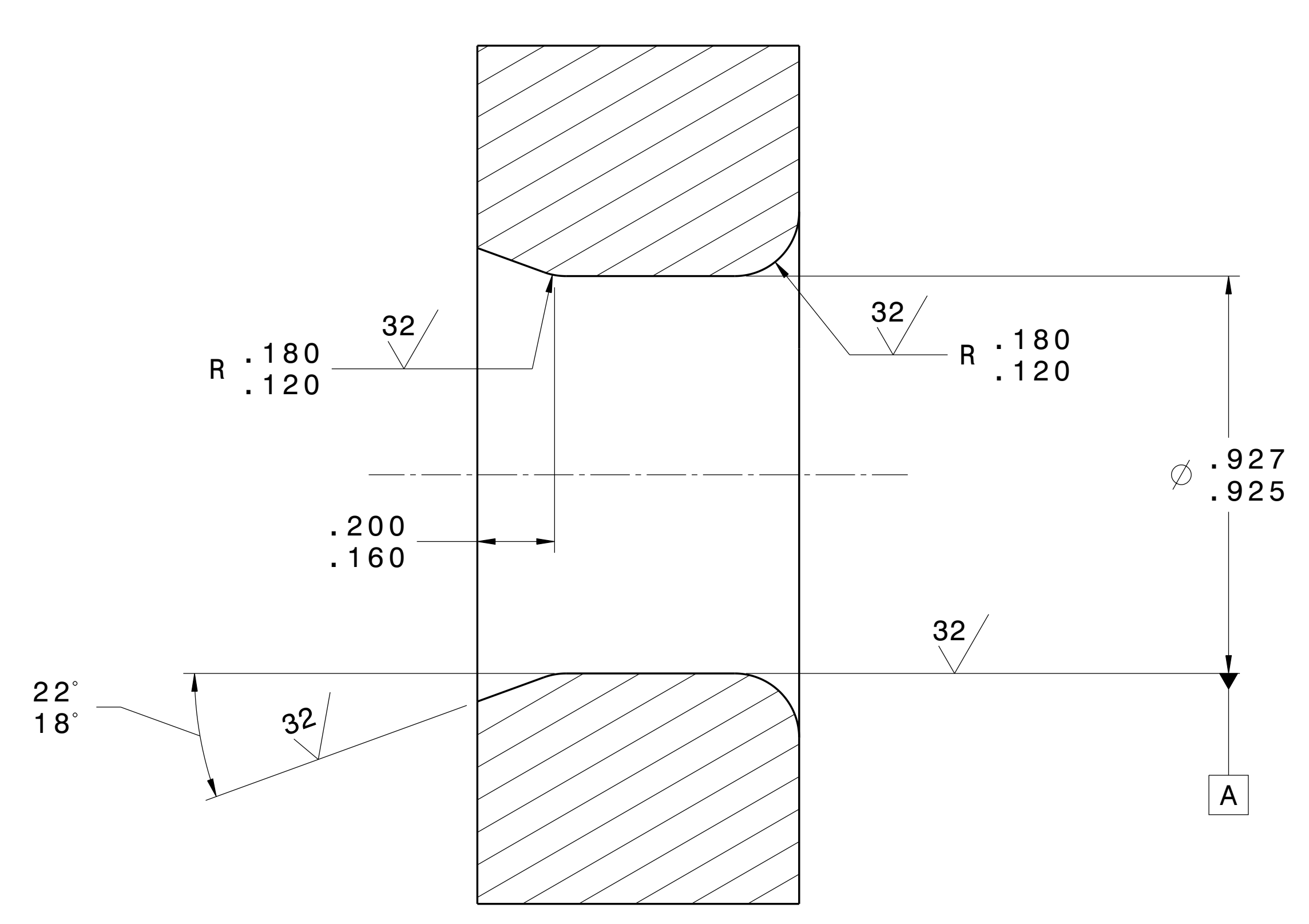

SECTION A-A

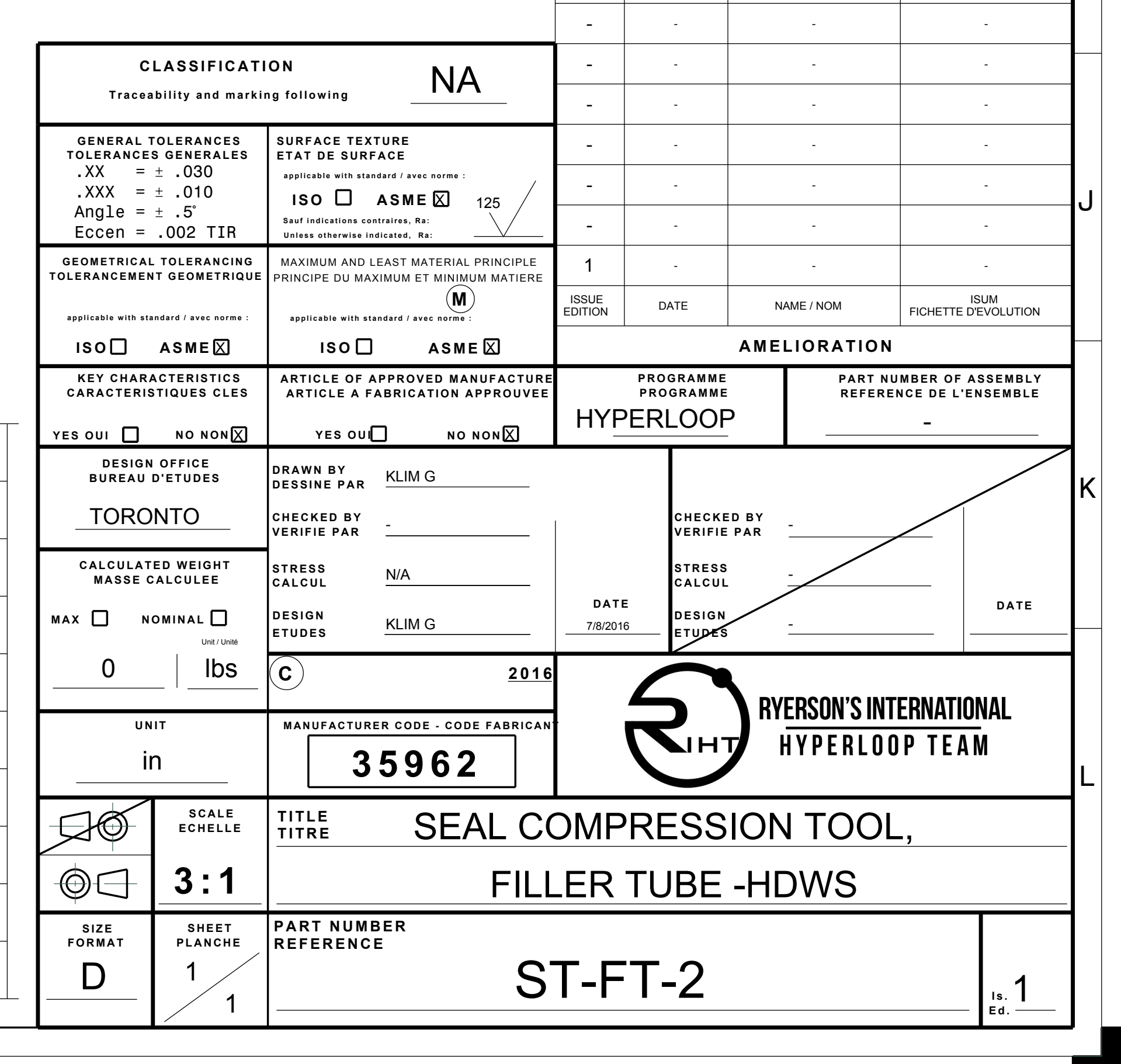




\section{NOTES:}

1. MAKE FROM DELRIN.

2. DEBURR SHARP EDGES $.005 / .015$, UNLESS OTHERWISE INDICATED.

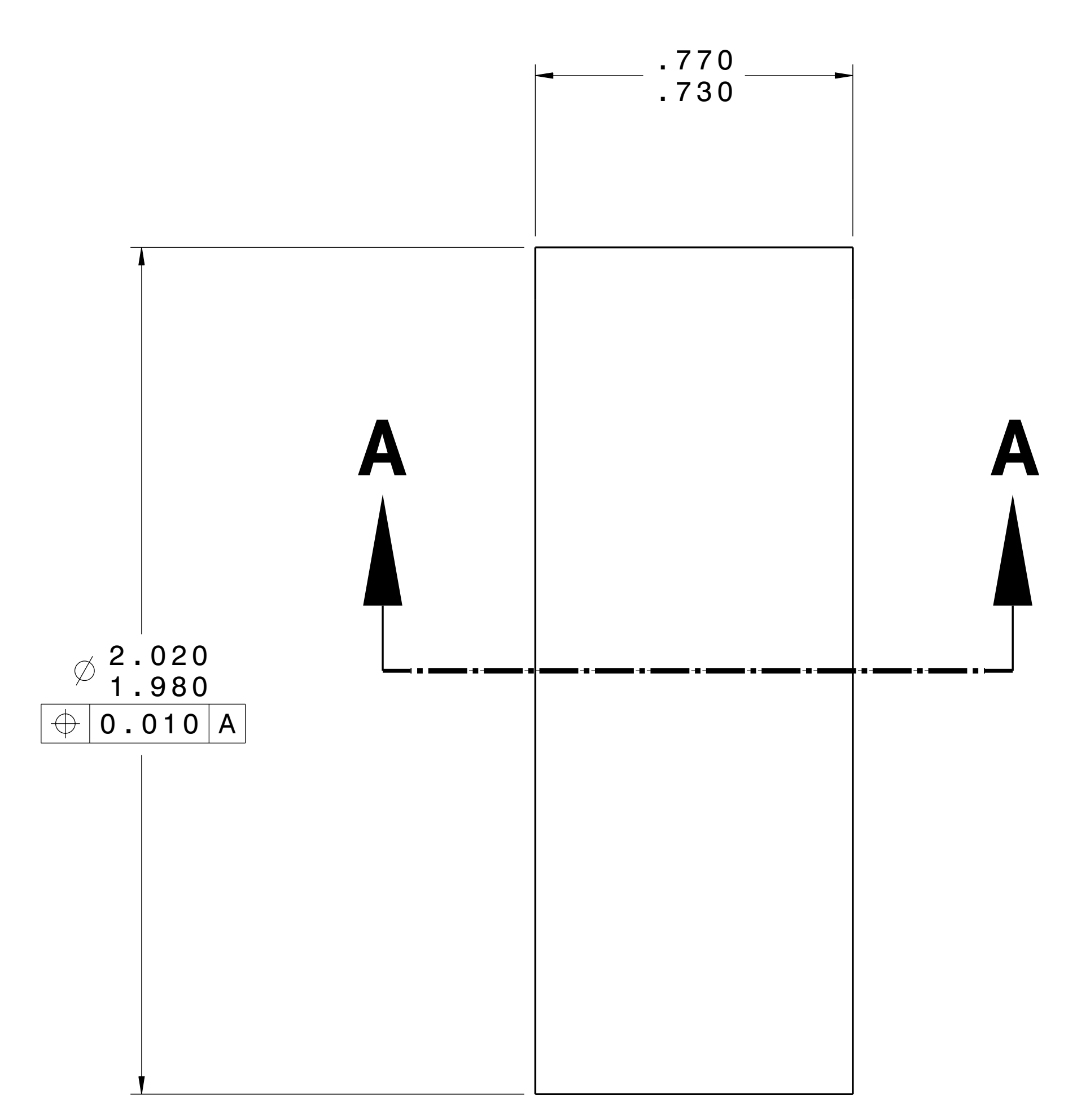

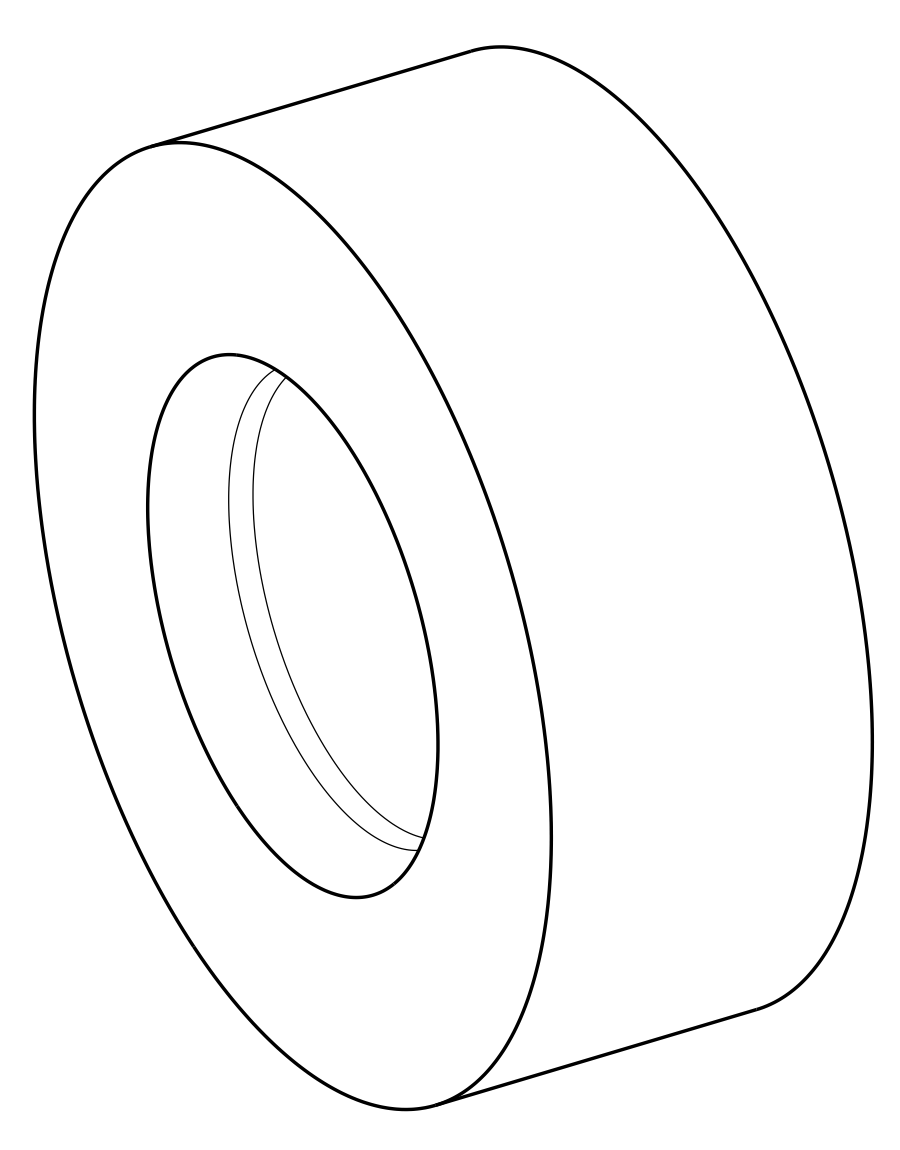

3D VIEW 1

REE ONYY
SCALE: NONE

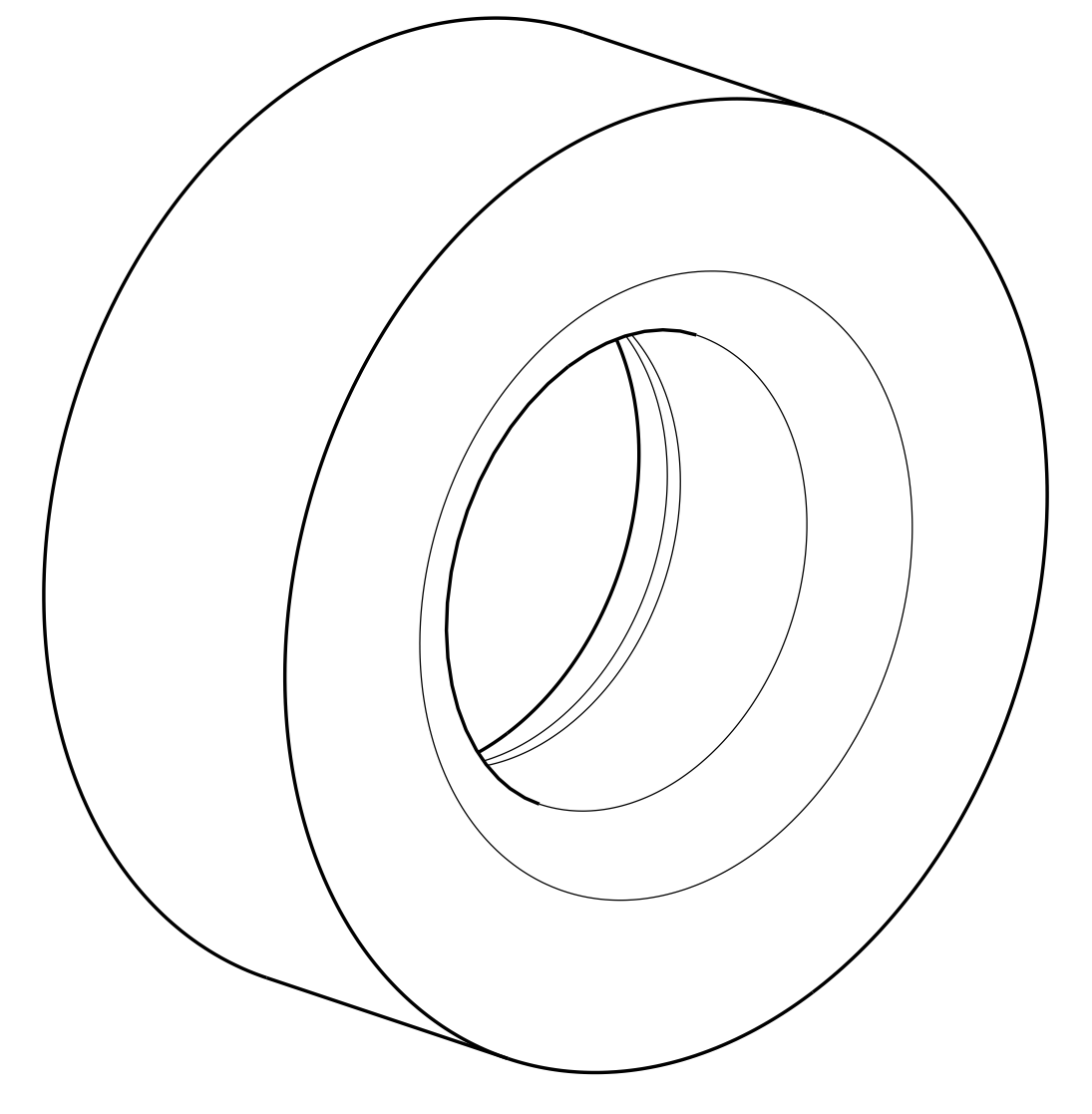

3D VIEW 2 REE ONYY
SCALE: NONE

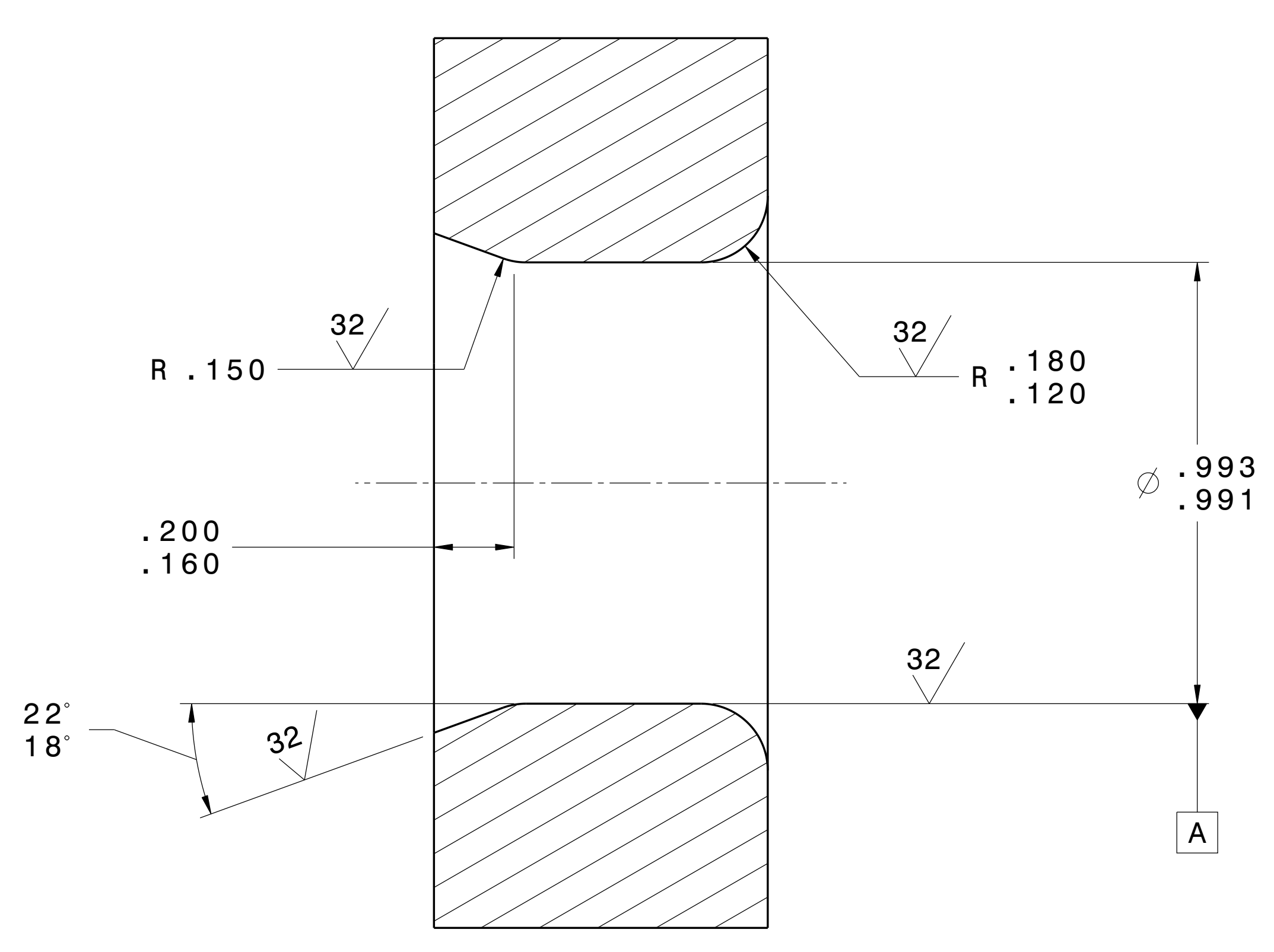

SECTION A-A

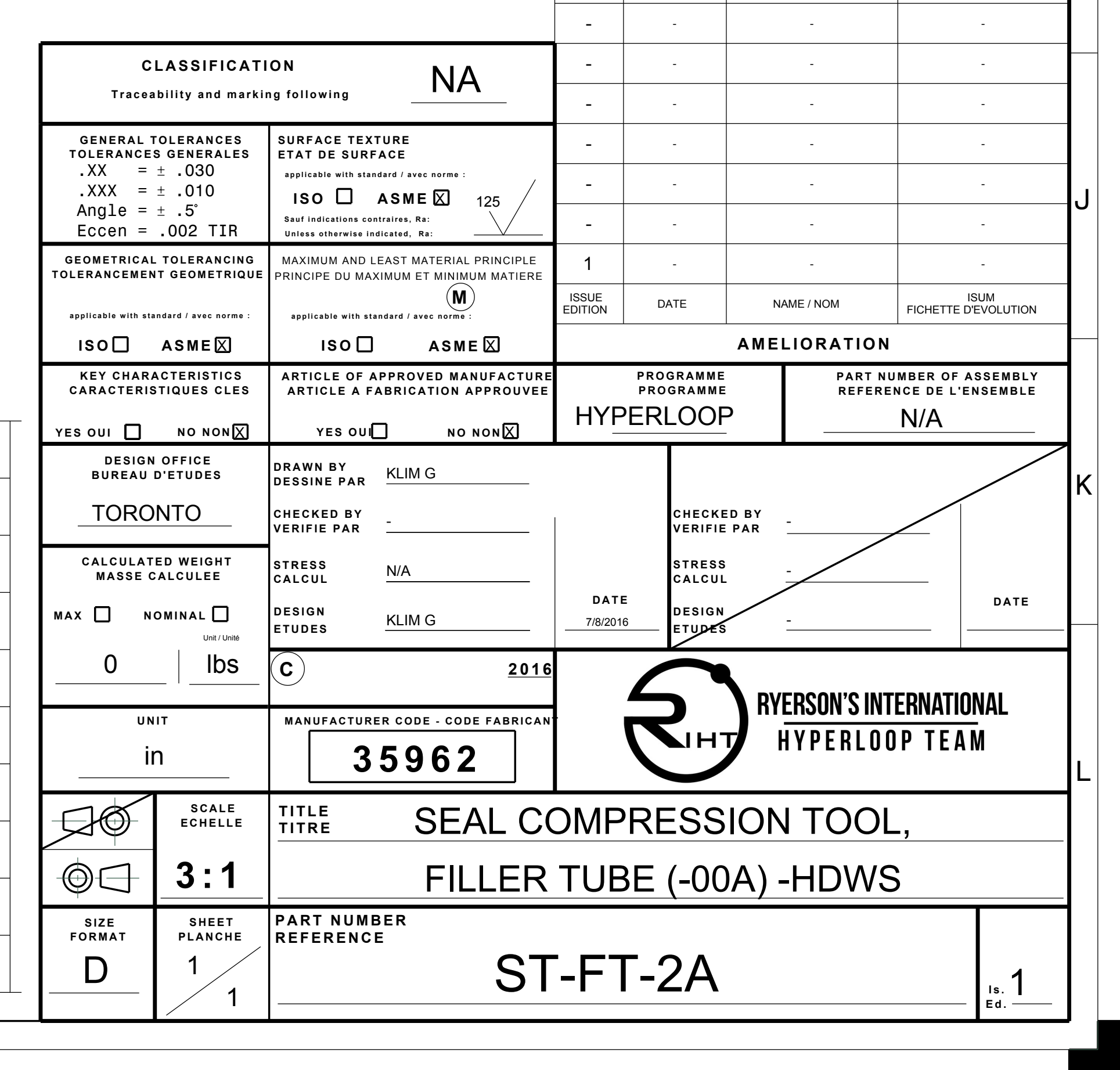




\section{NOTES:}

1. MAKE FROM DELRIN.

2. DEBURR SHARP EDGES $.005 / .015$, UNLESS OTHERWISE INDICATED.

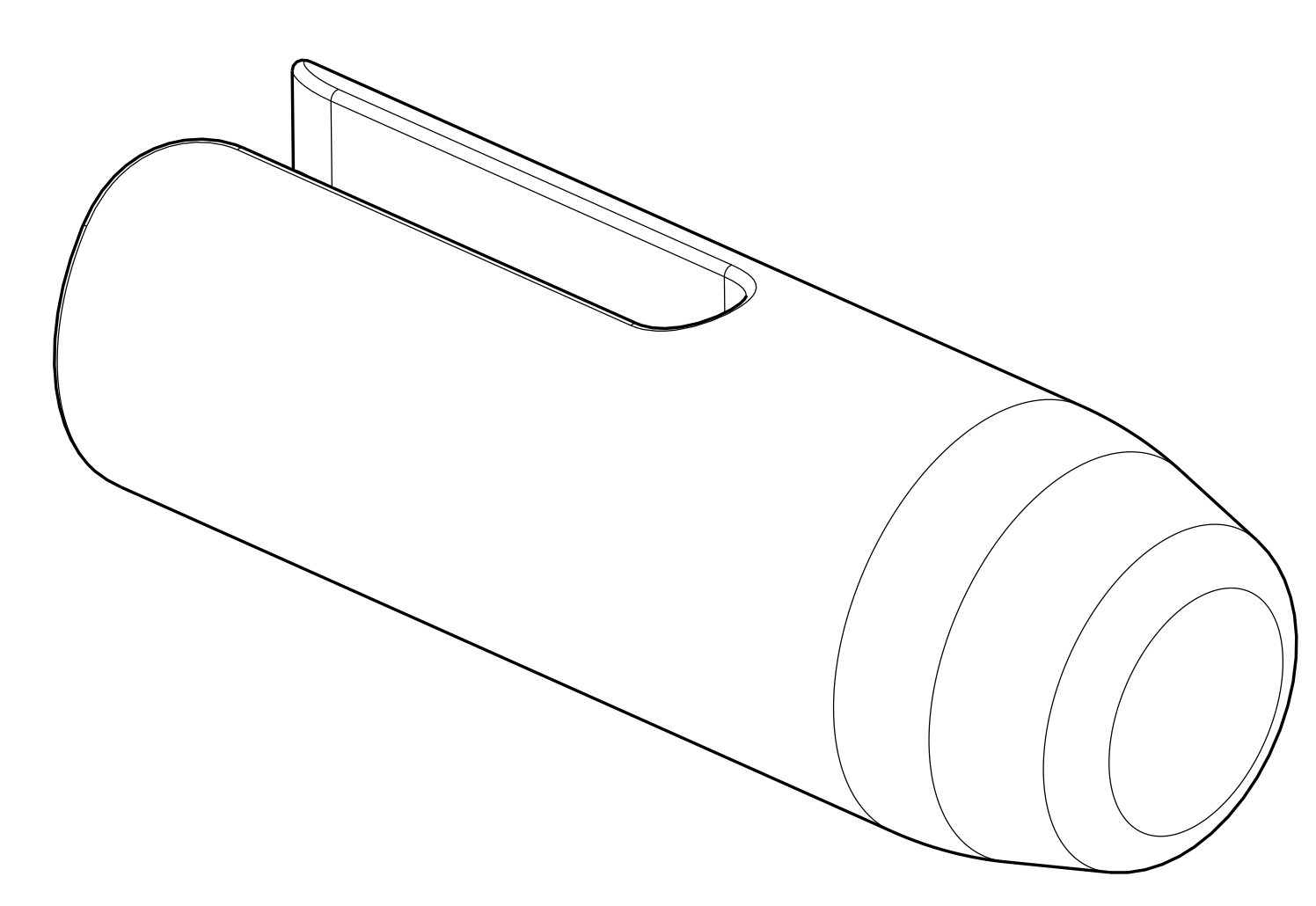

3D VIEW

Machine turning Centre as ReQuired.

REF OOLY
SCCLE: NONE

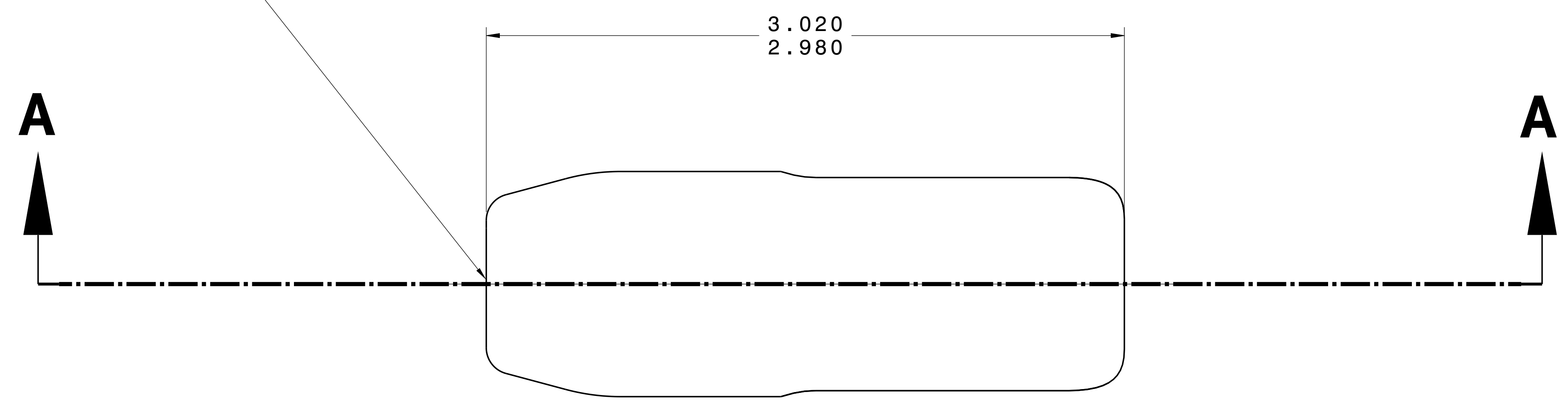

A
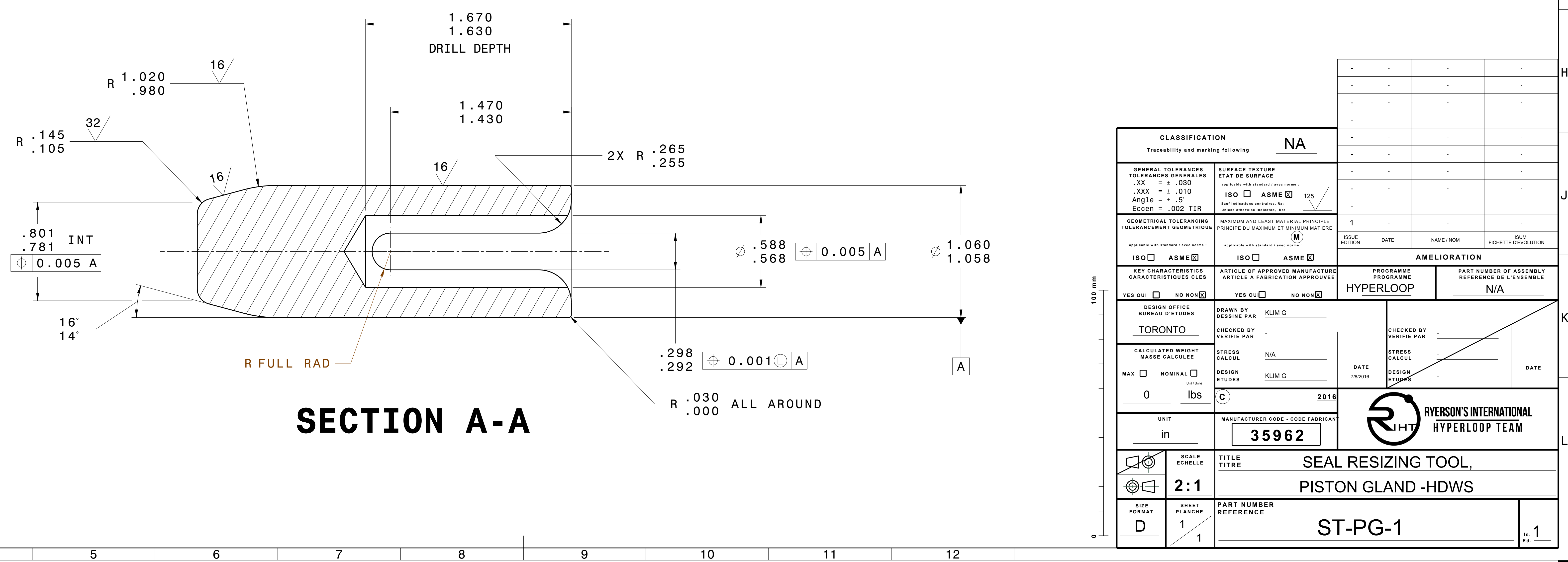


\section{NOTES:}

1. MAKE FROM DELRIN.

2. DEBURR SHARP EDGES $.005 / .015$, UNLESS OTHERWISE INDICATED.

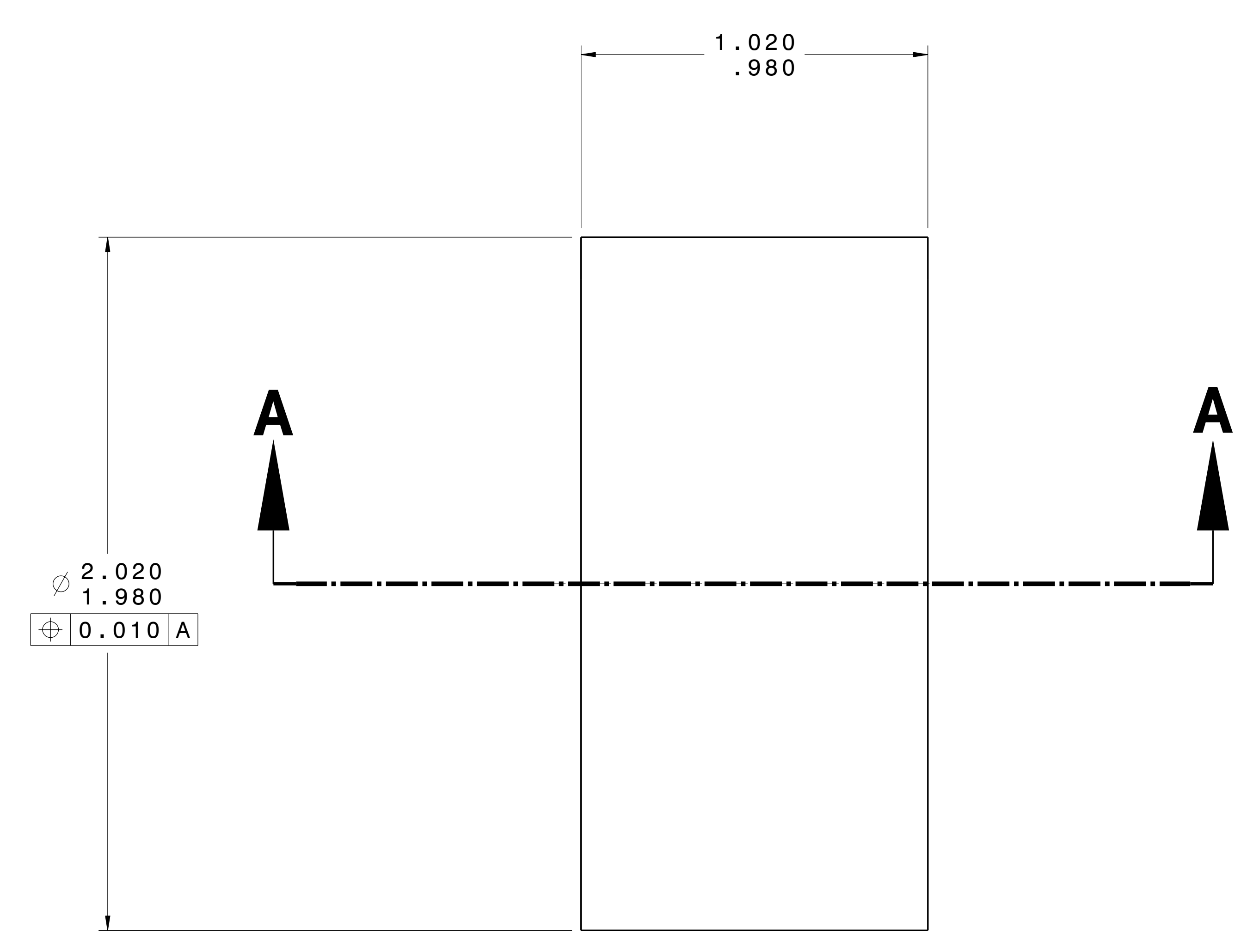

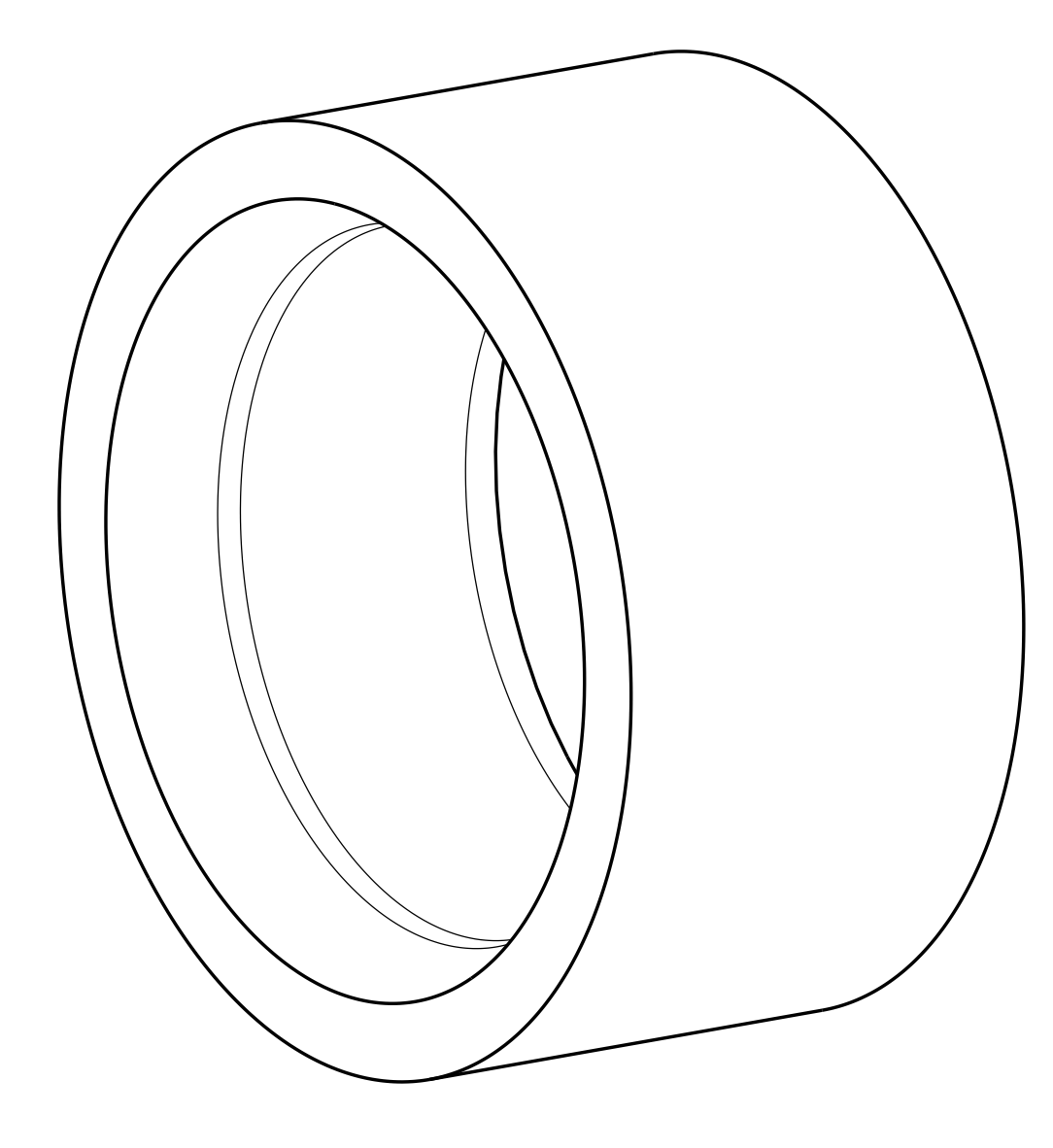

3D VIEW 1

SBEE OMLY

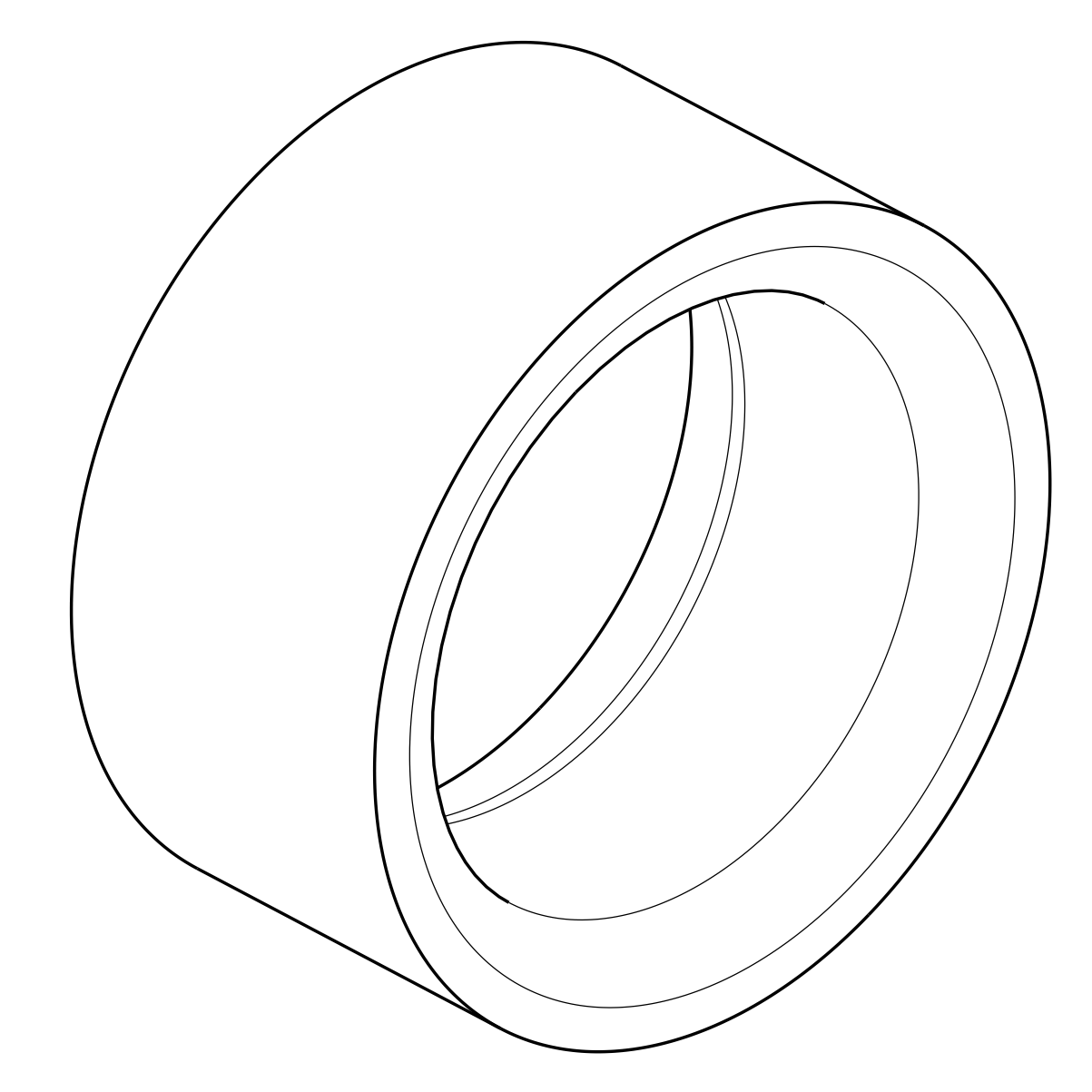

3D VIEW 2

REE ONLY
SCALE: NONE
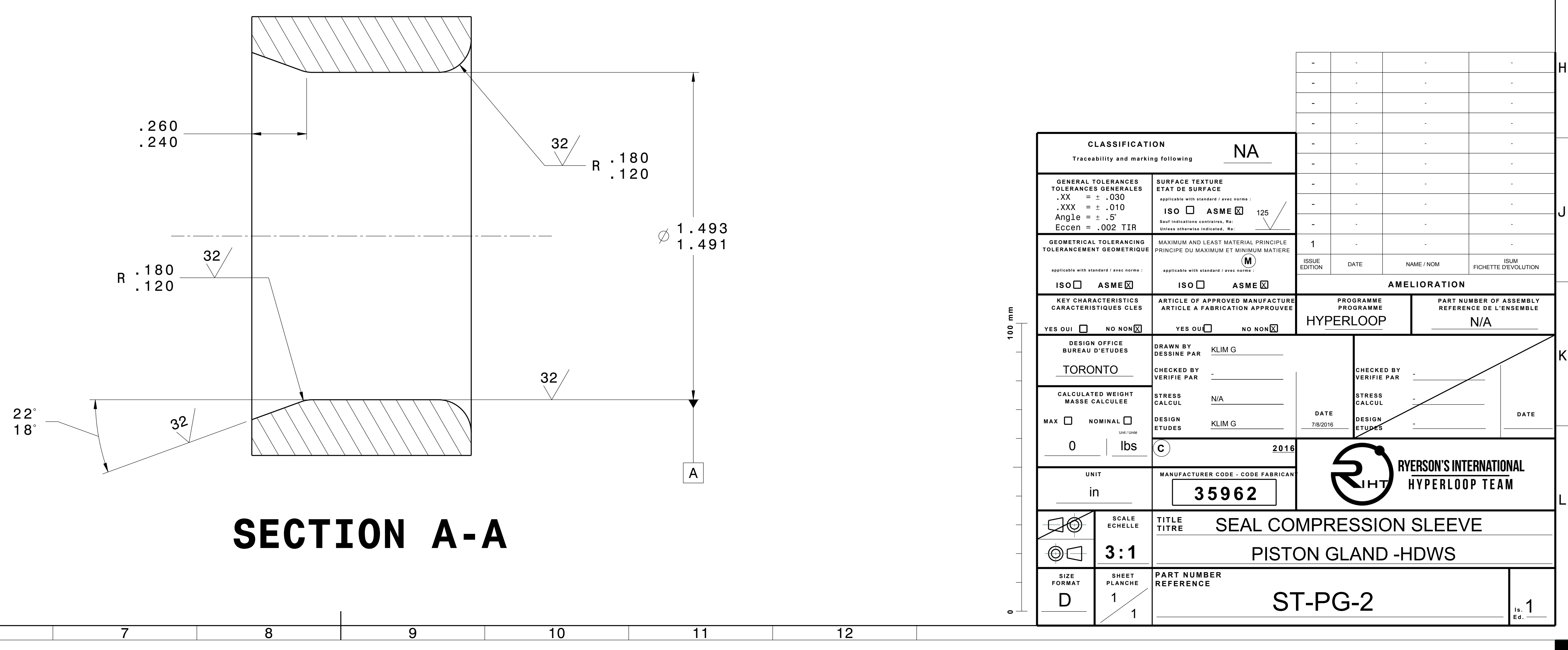

\section{SECTION A-A}




\section{Appendix C: HDWS Test Fixture Drawings}

This page was intentionally left blank 
NOTES:

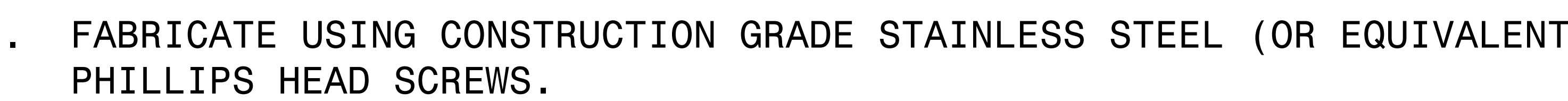

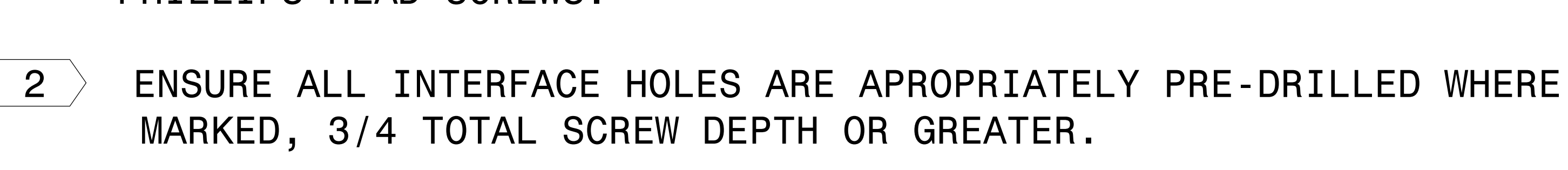

P/N BASE PLATE, TF-1101-00

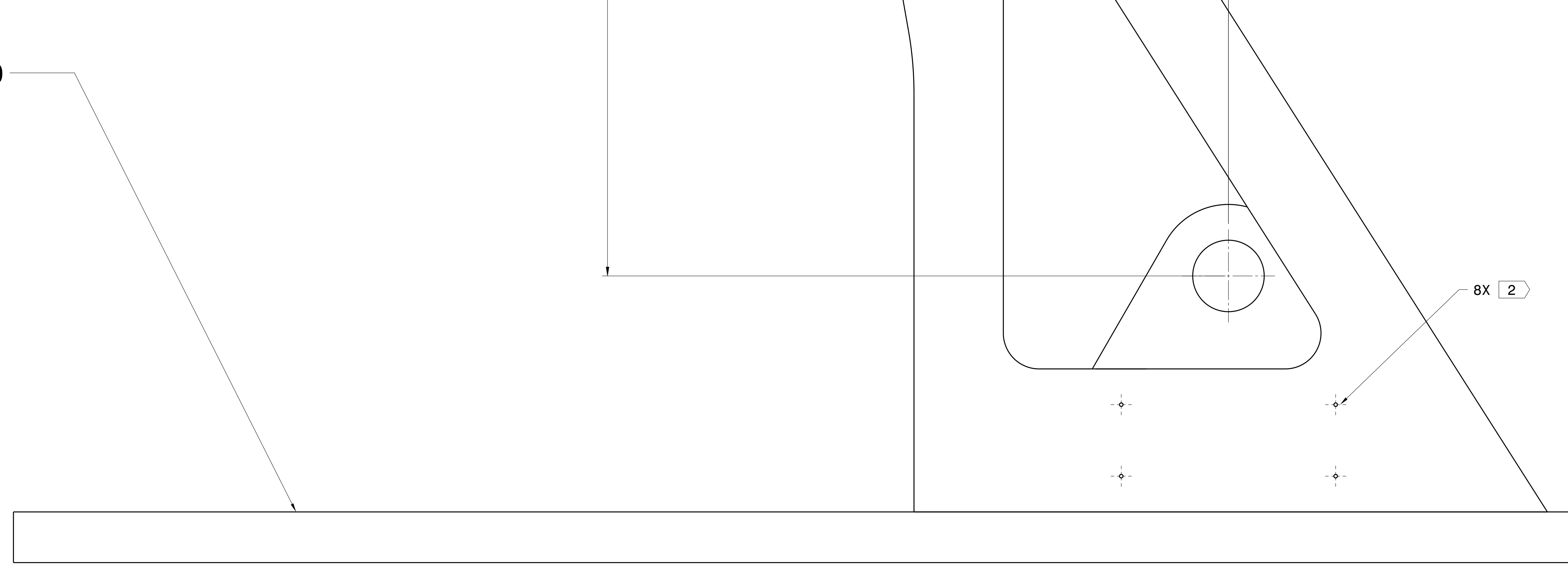

P/N HOUSING SUPPORT, TF-1102-00 (QTY 2)

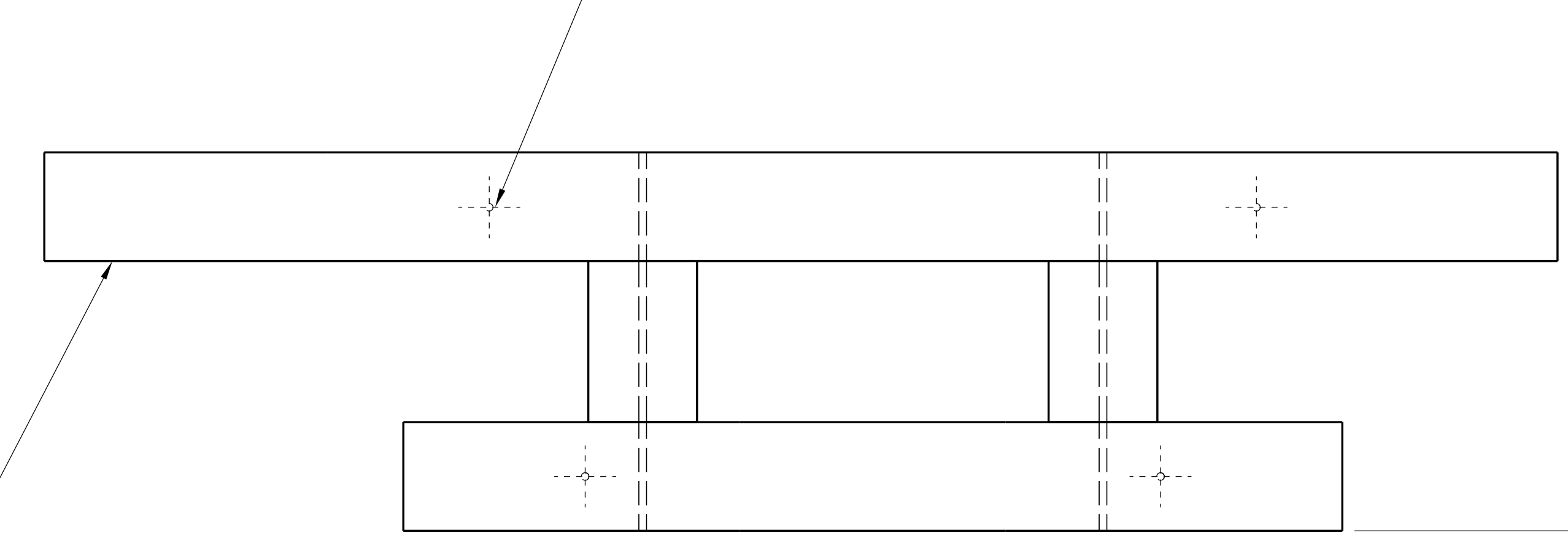

P/N T-ARM SUPPORT, TF-1103-00 (QTY 2)

P/N HORIZONTAL SUPPORT, TF-1104-00 (QTY 4)

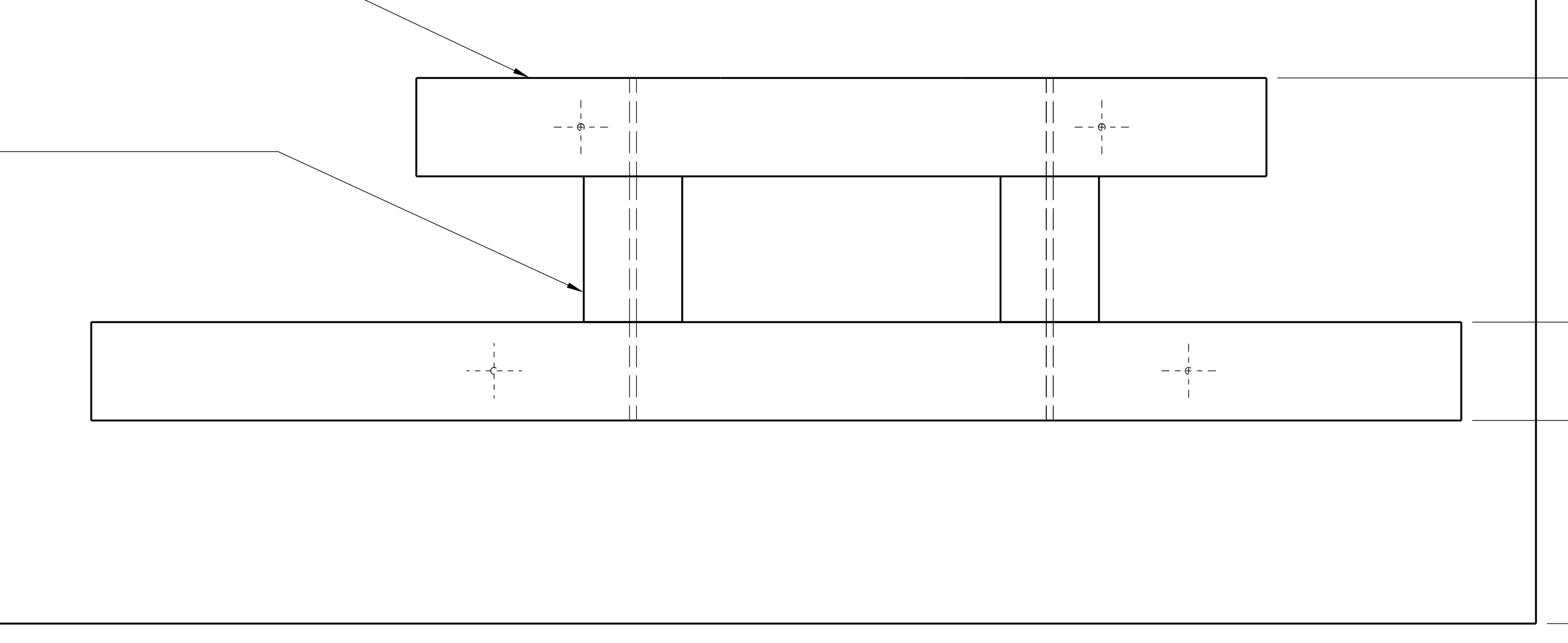

$4.14 \pm .01$ $7.66 \pm .01$
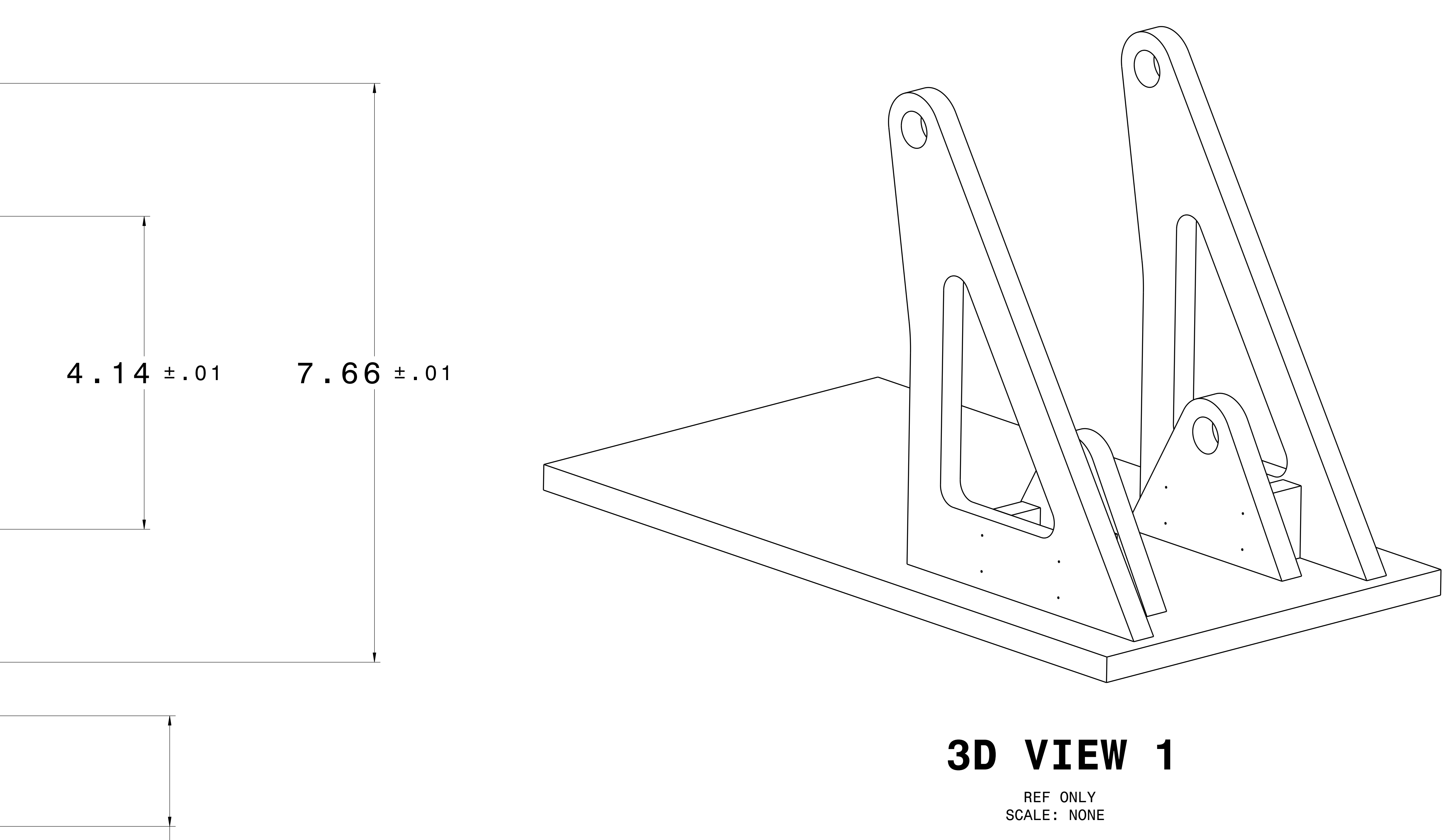

3D VIEW 1

$2 \times(1.463)$

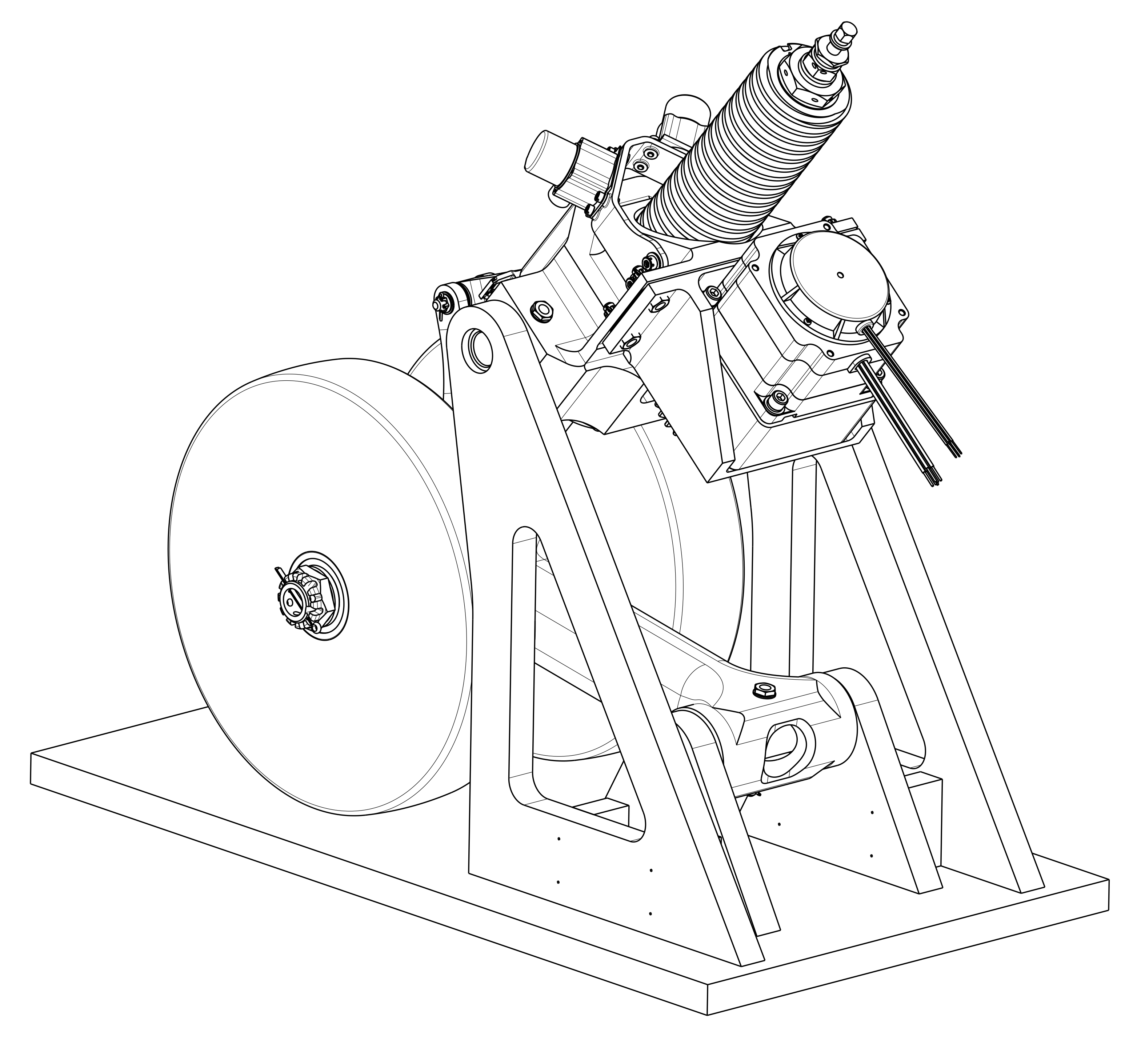

3D VIEW 2

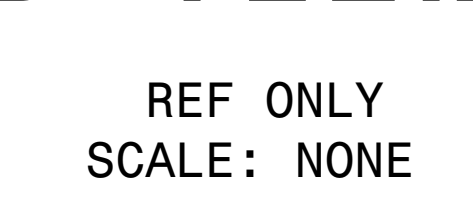

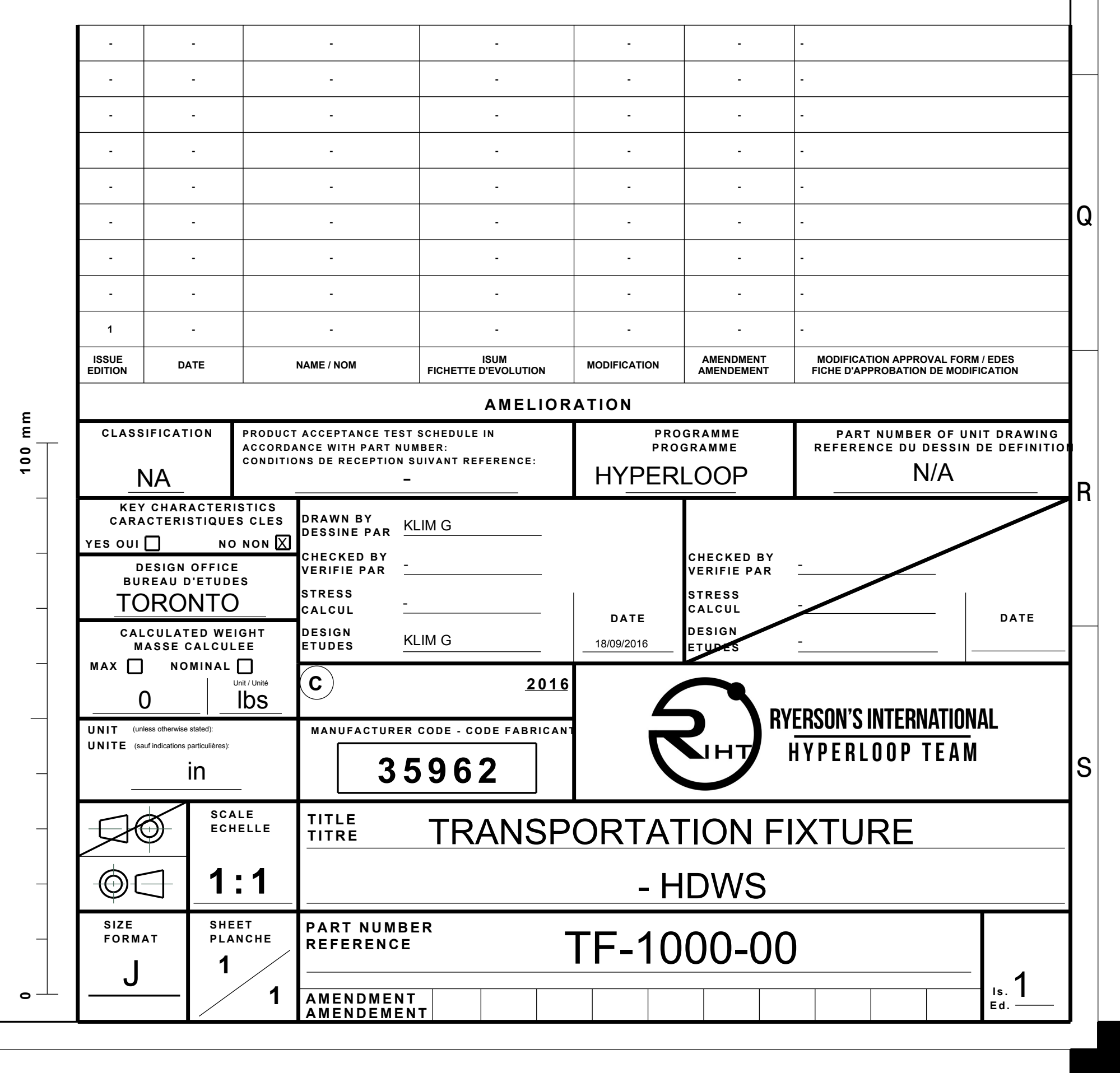




\section{NOTES:}

1. MAKE FROM 3/4" PLYWOOd, MAPLE OR BALTIC BiRCH.

2 MAY BE PRE-DRILLED AS SHOWN OR MARKED USING AN AGREED METHOD.

\section{9 (18 MM STANDARD PLY)}

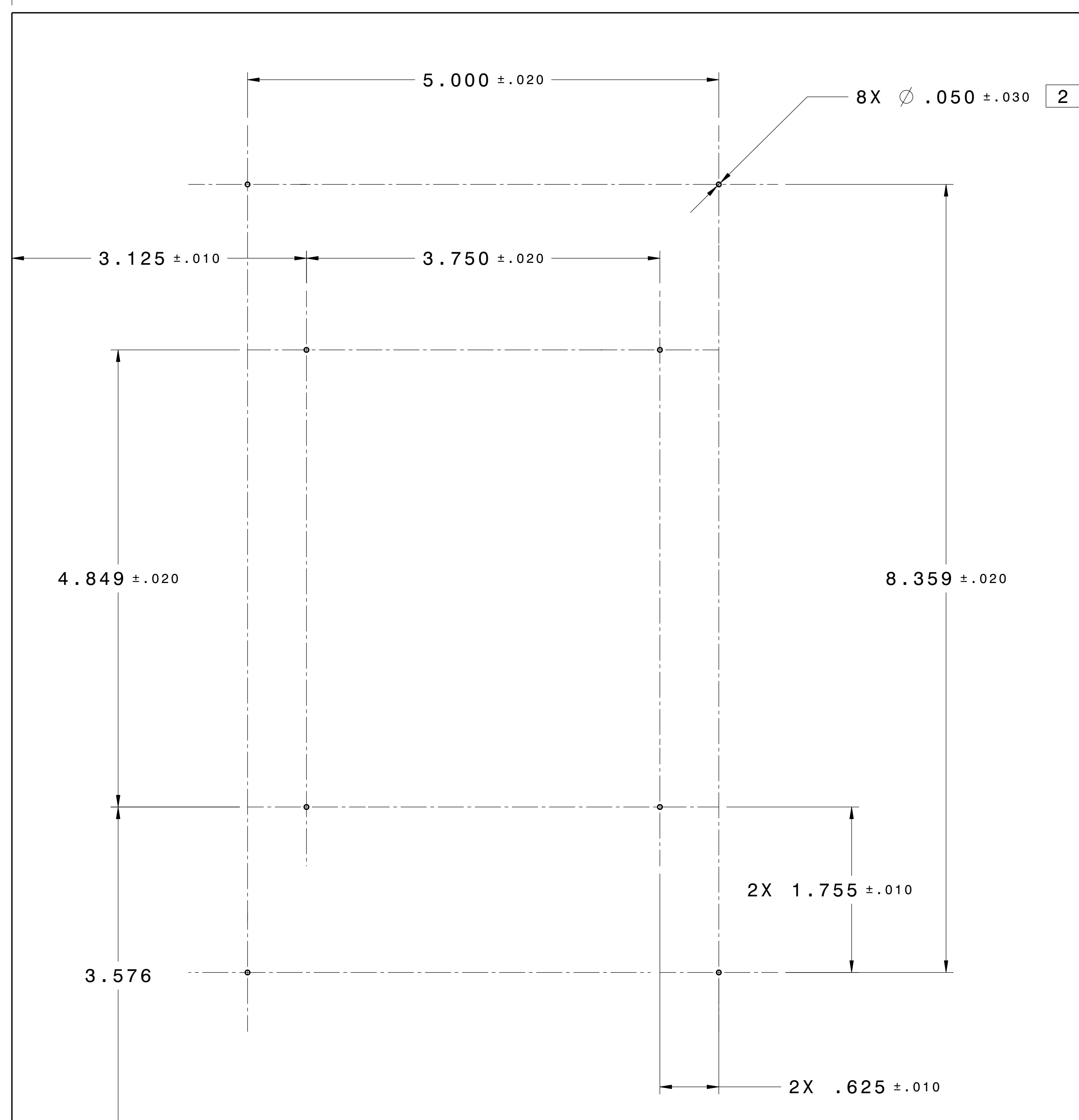

\section{D VIEW}

REE ONI
SCALE: NONE
NON

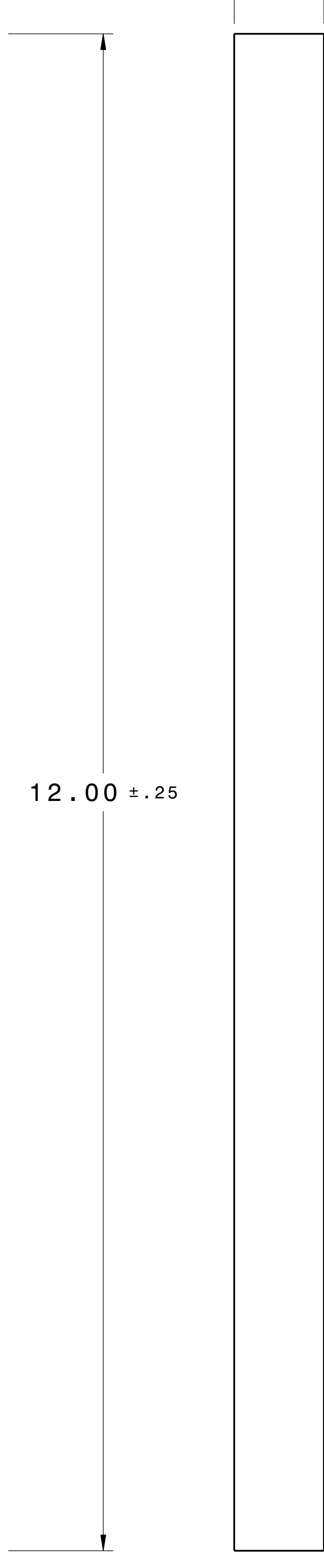

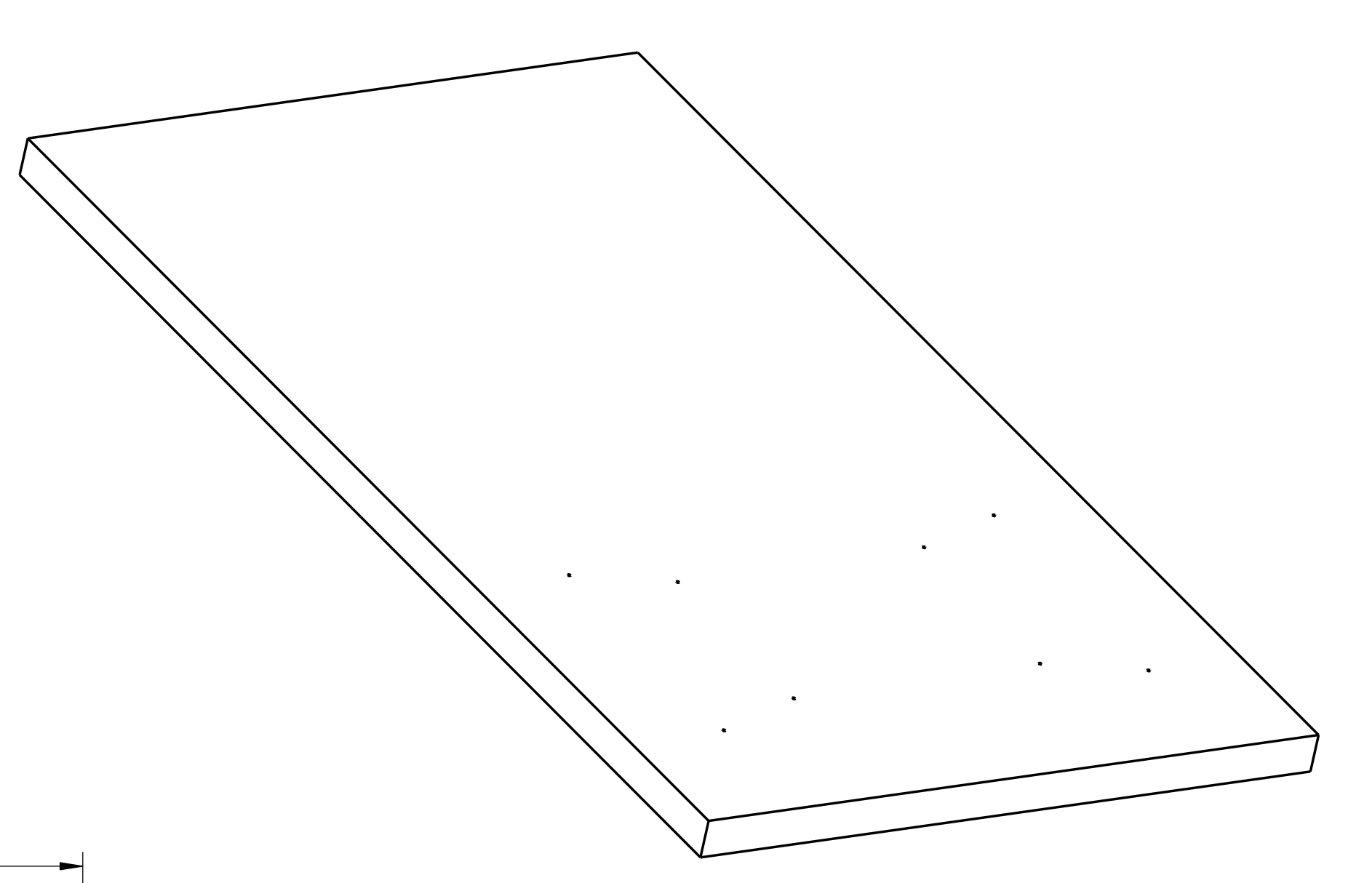

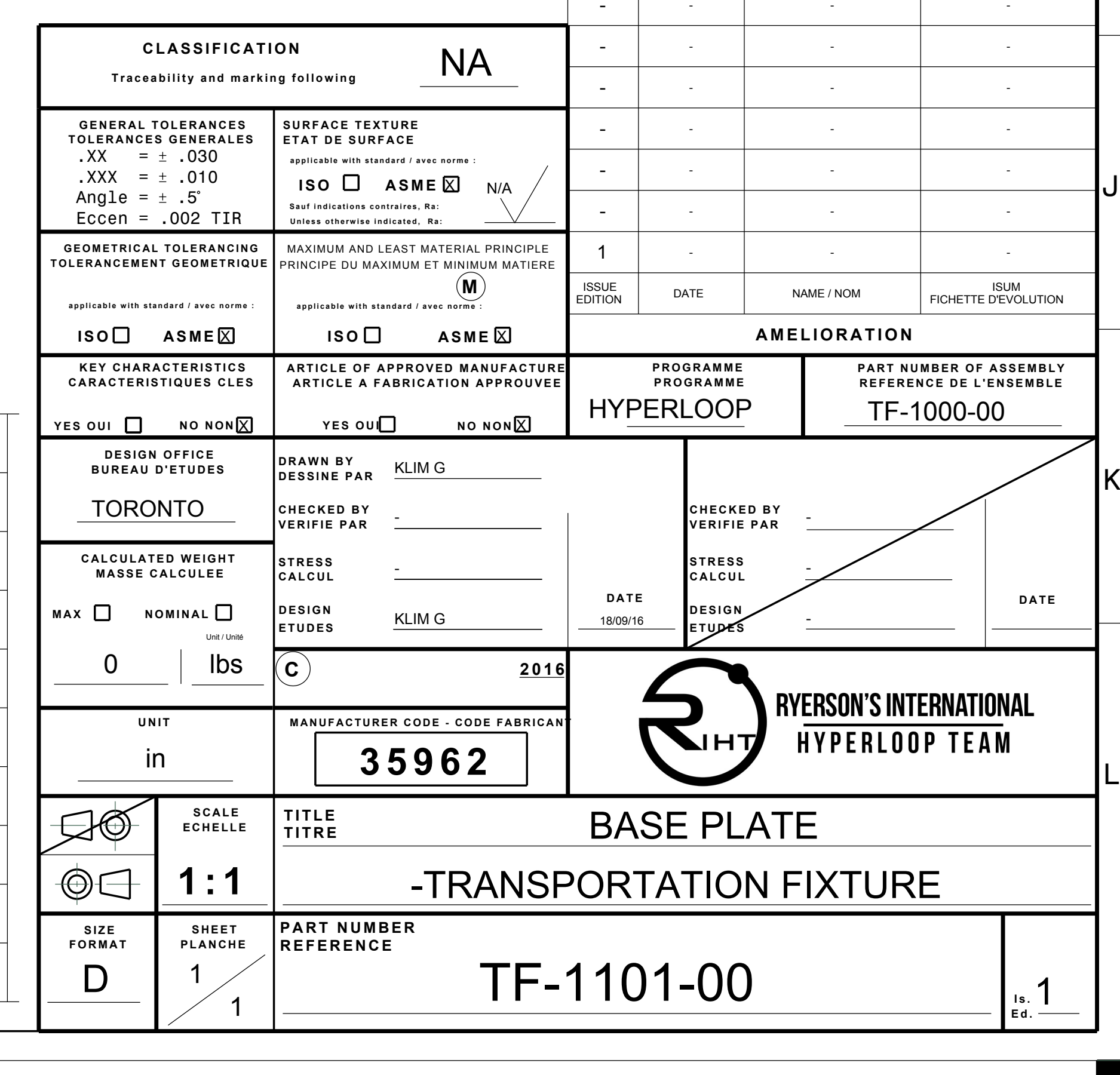




\section{NOTES:}

1. MAKE FROM 3/4" PLYWOOD, MAPLE OR BALTIC BIRCH.

2 MAY BE PRE-DRILLED AS SHOWN OR MARKED USing AN AGREED METHOD.
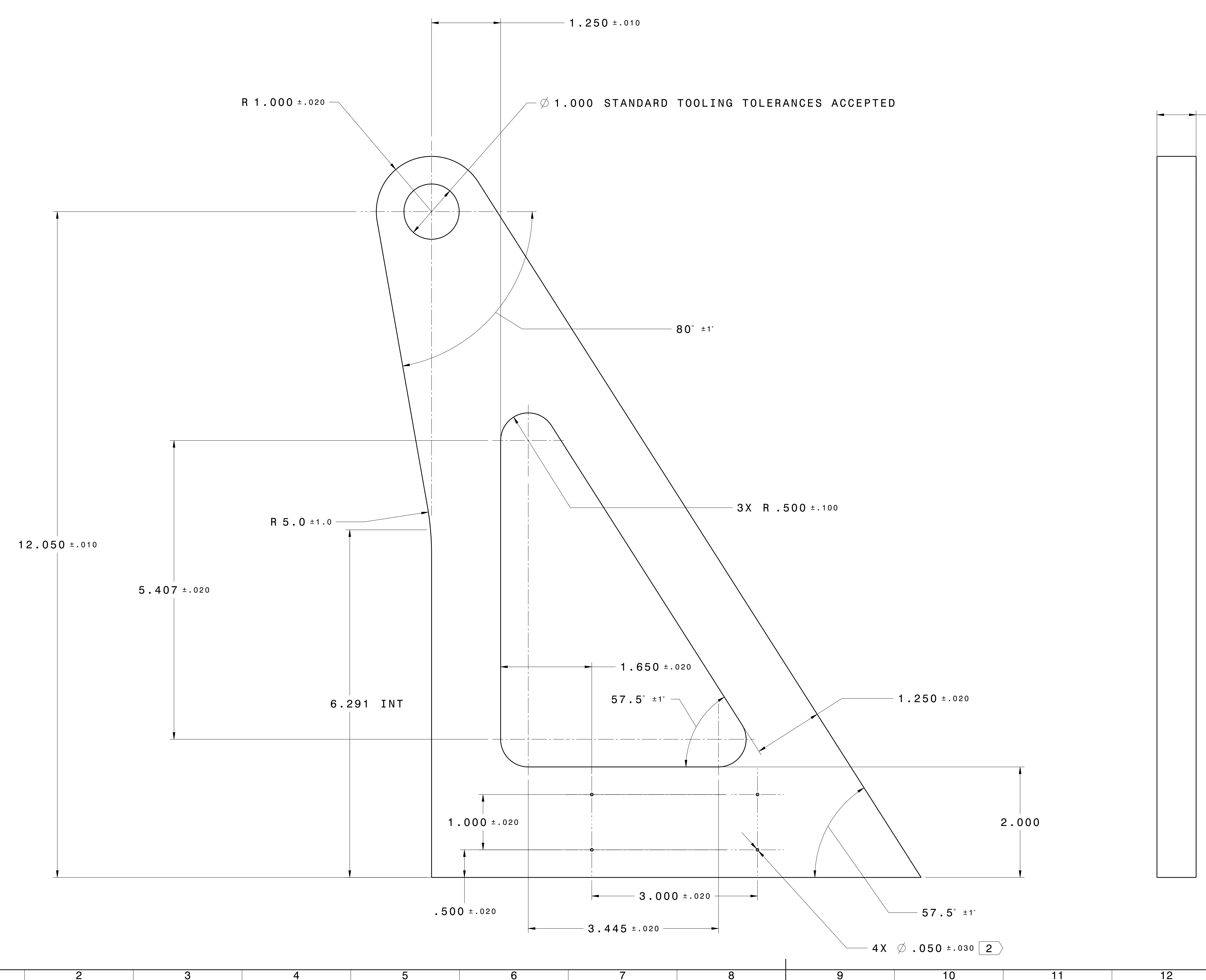

709 (18 MM STANDARD PLY)

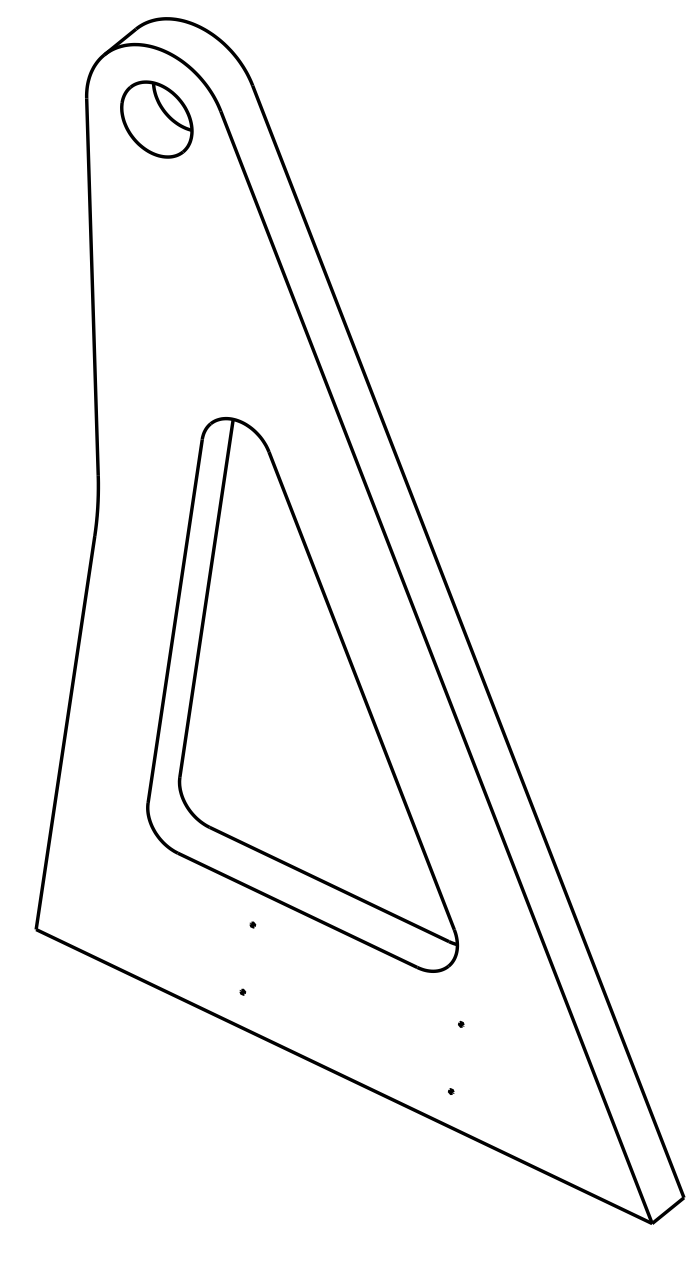

3D VIEW

REE ONLY
SCALE: NONE

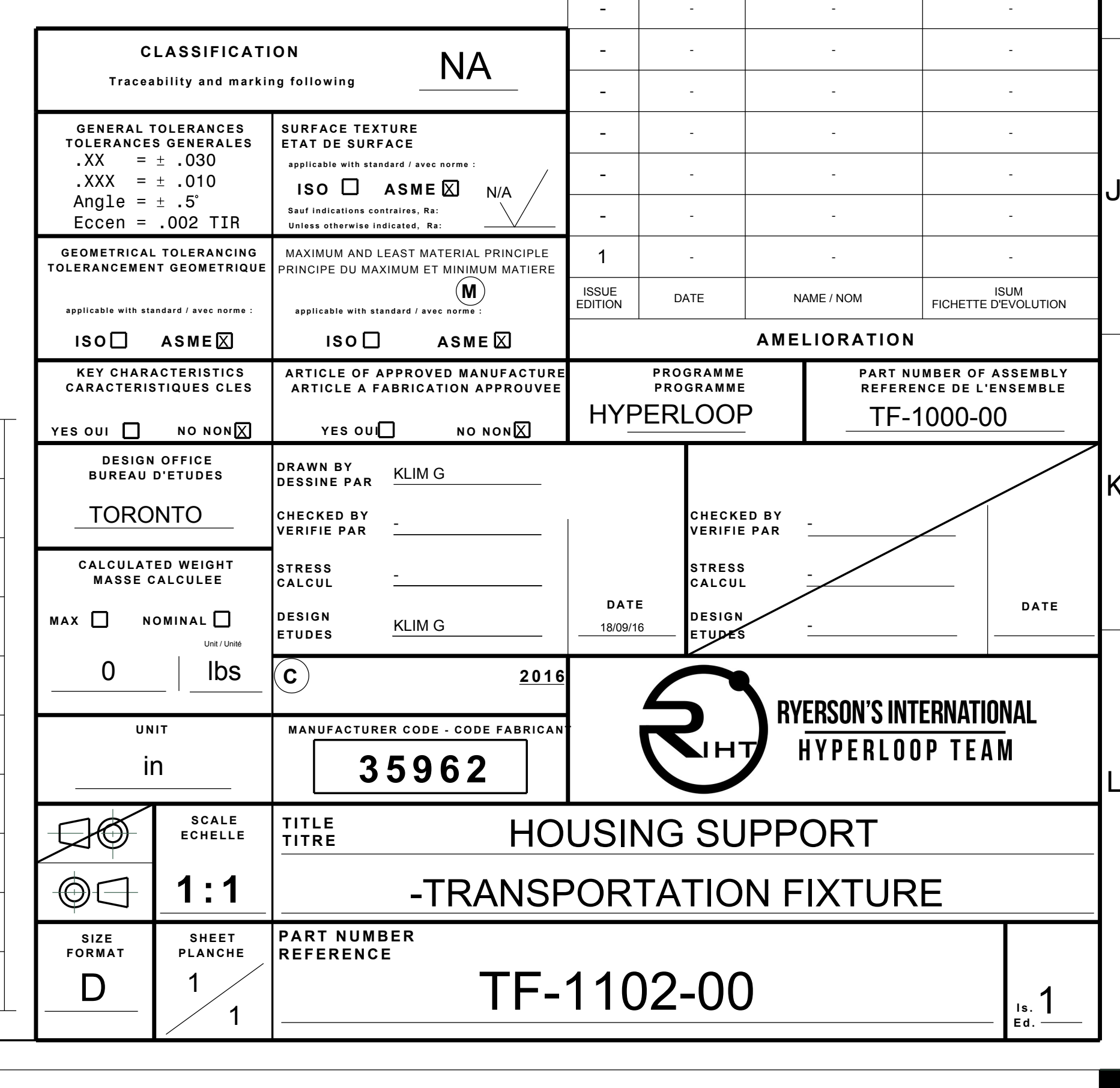




\section{NOTES:}

1. MAKE FROM 3/4" PLYWOOD, MAPLE OR BALTIC BIRCH.

2 MAY BE PRE-DRILLED AS SHOWN OR MARKED USING AN AGREED METHOD.

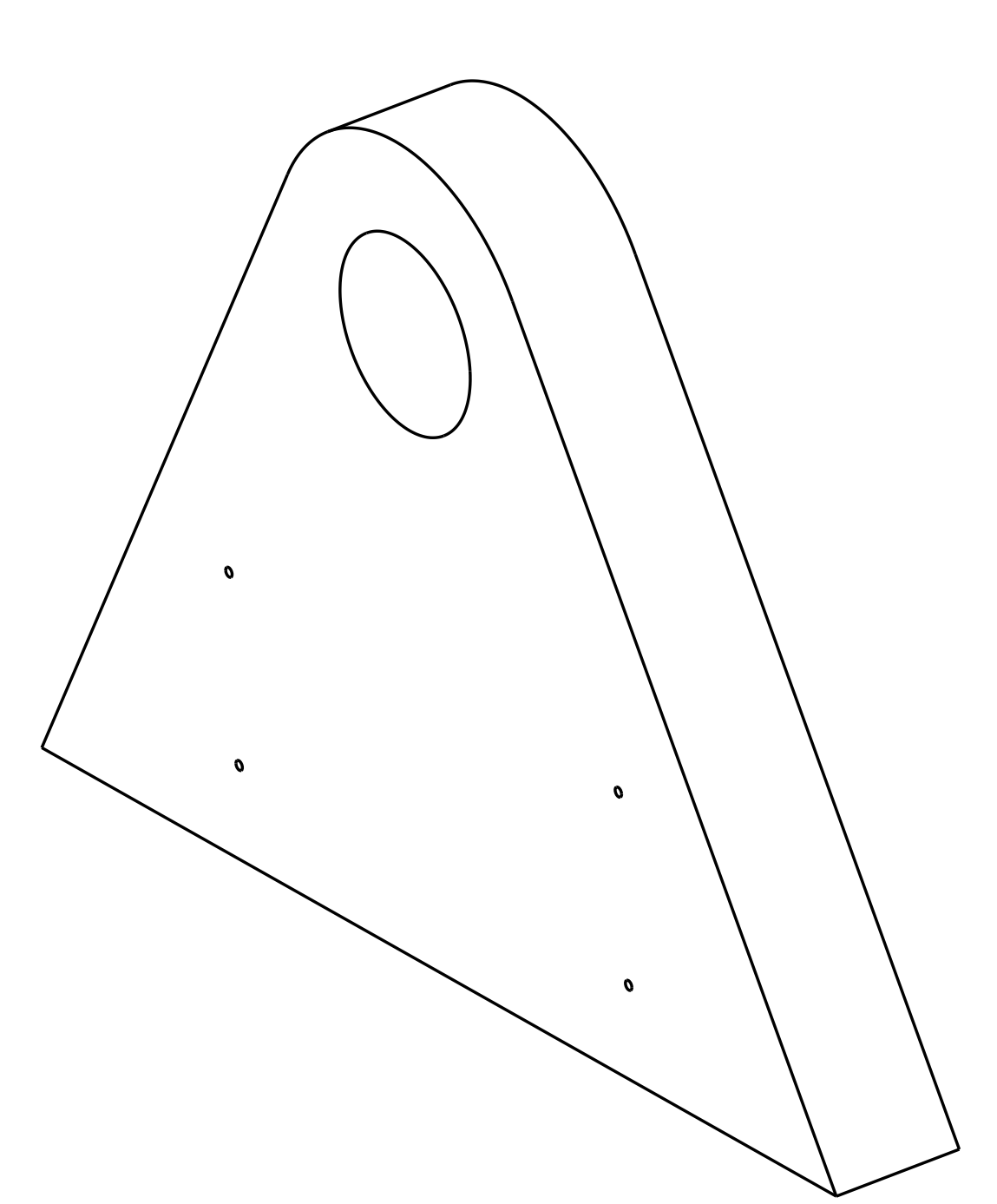

3D VIEW

REE ONLY
SCALE: NONE
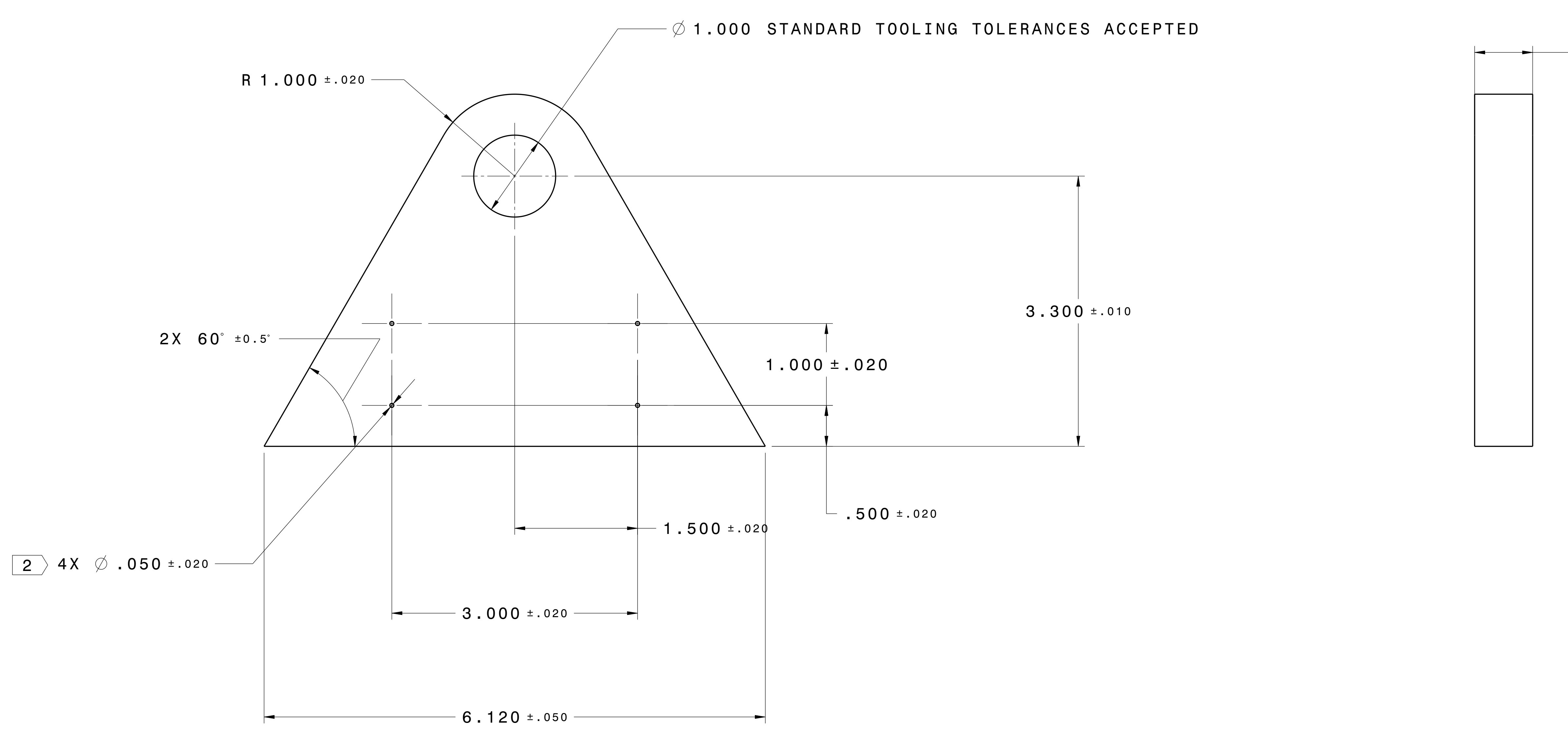

.709 (18 MM STANDARD PLY)

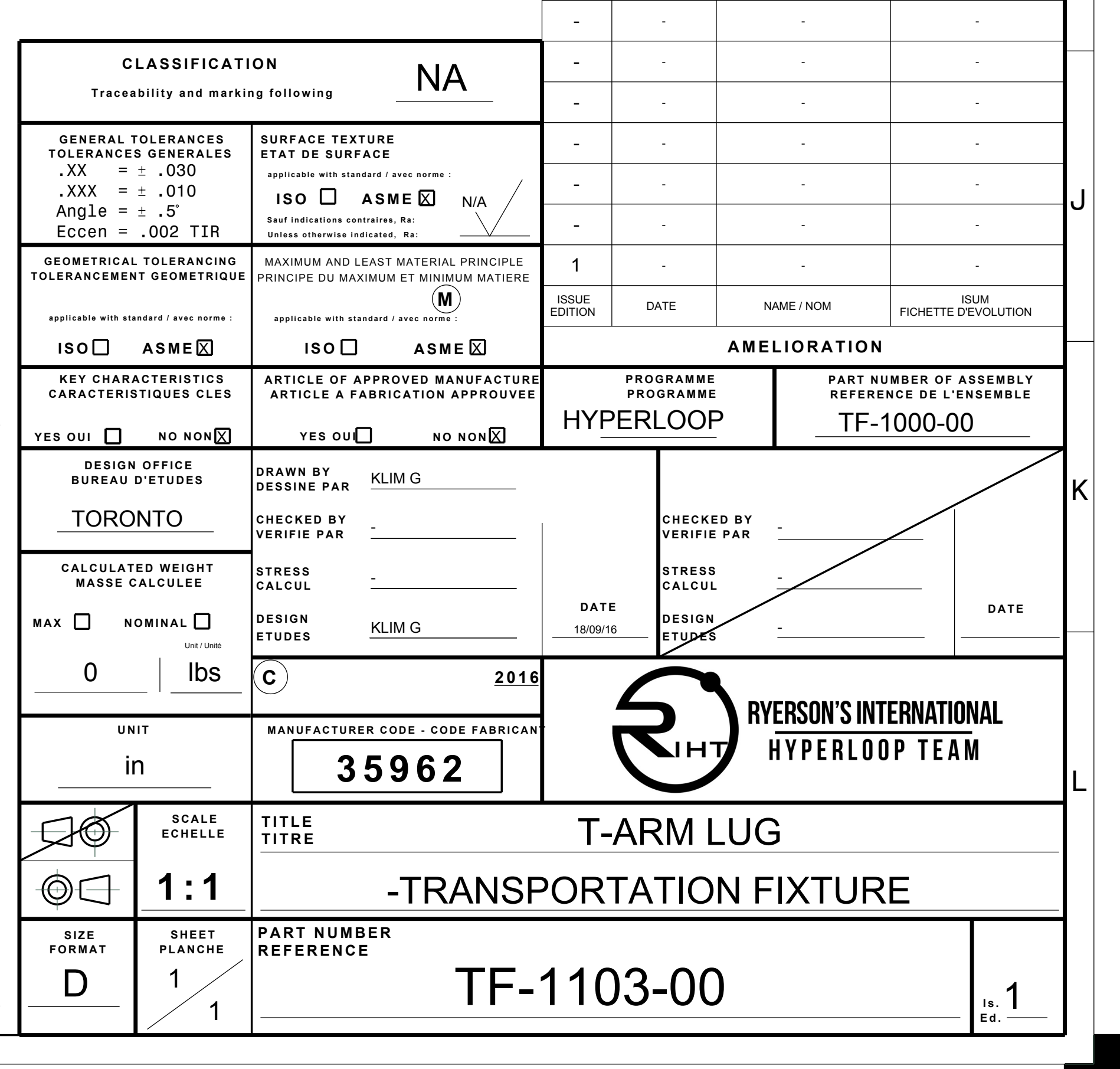




\section{NOTES:}

1. MAKE fROM 3/4" PLYWOOD, MAPLE OR BALTIC BIRCH.

2 MARKED USiNG AN AGREED METHOD, PRE-DRILL NOT REQUiRED.

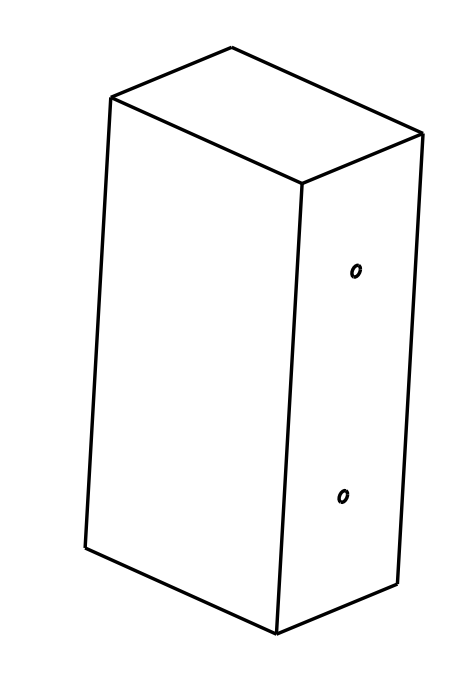

3D VIEW

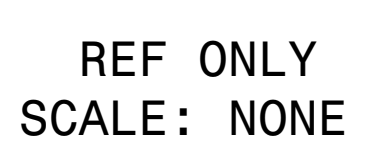

2) $4 \times \varnothing .050$ (BOTH SIDES) STANDARD TOOLING TOLERANCES ACCEPTED

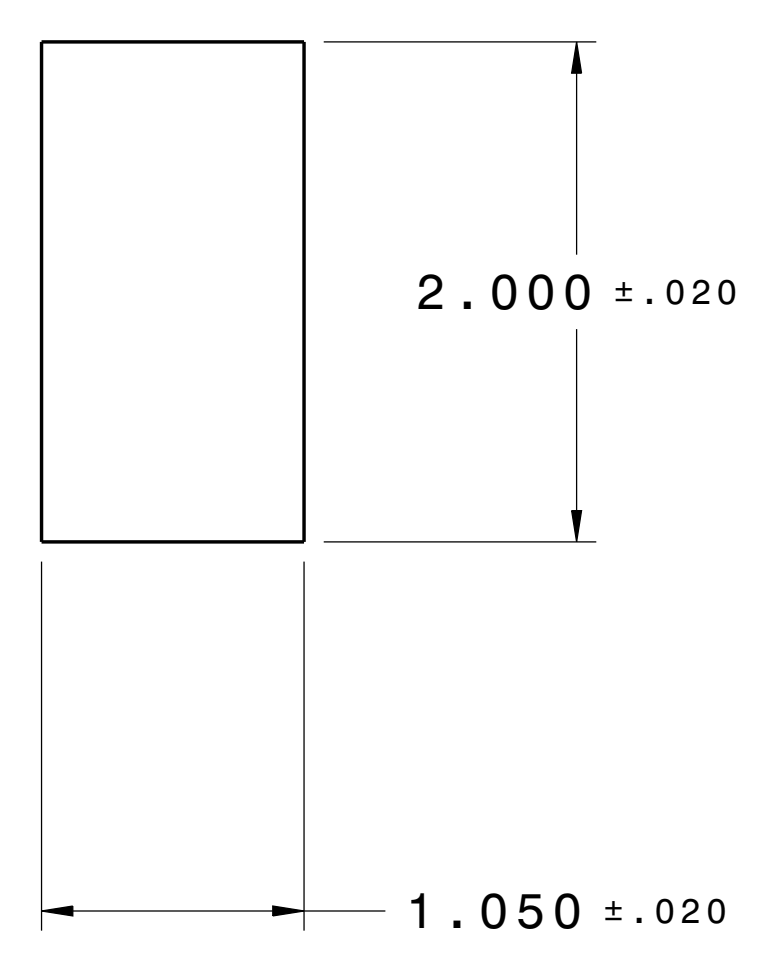

STANDARD PLY)
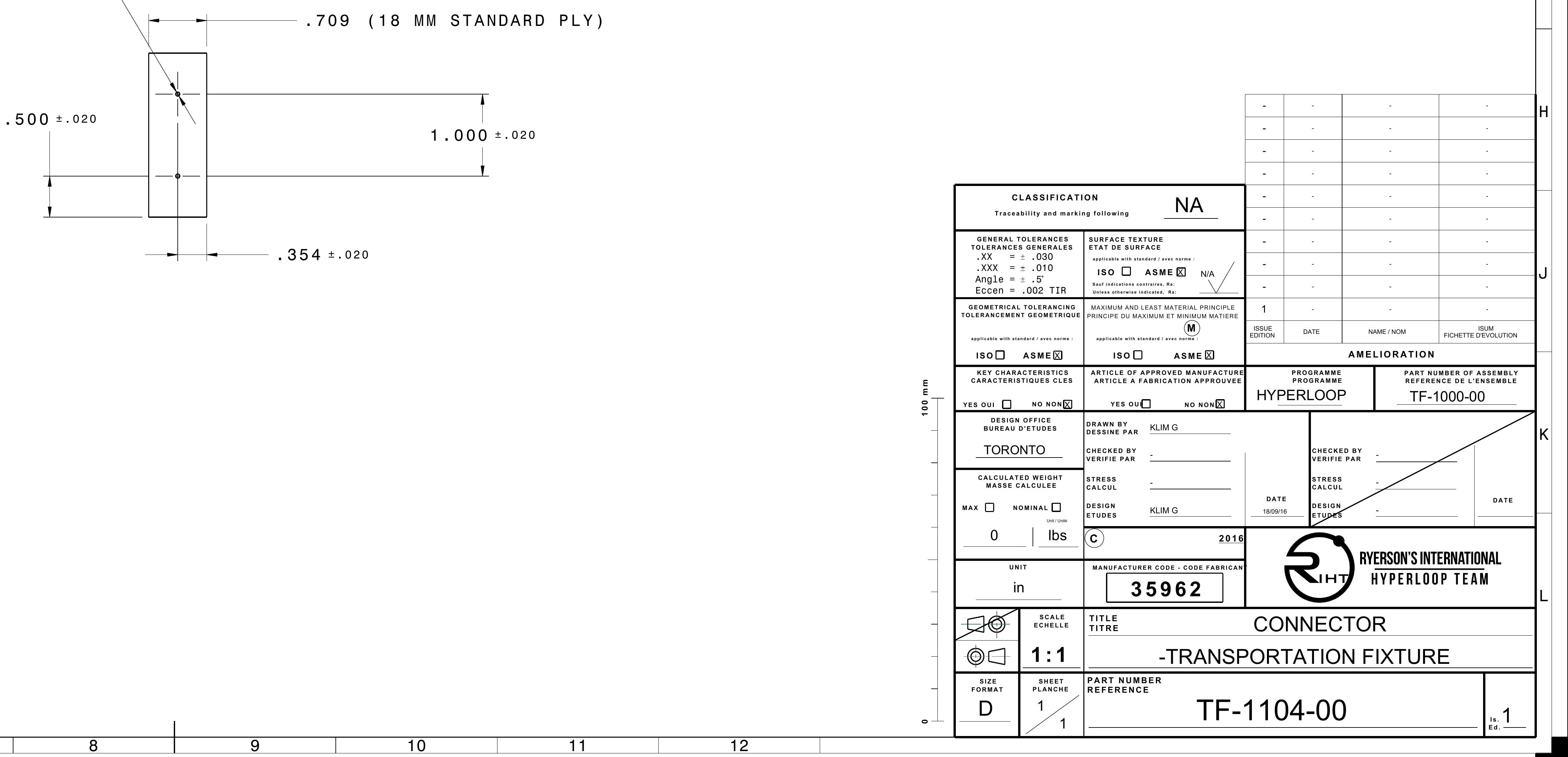


\section{P/N WTR-1105-00, DUMMY AXLE SPACERS (OTTY}

.530
.470

LoCK JOINT, COMBrinatron OF (OR EOUIVALENT)

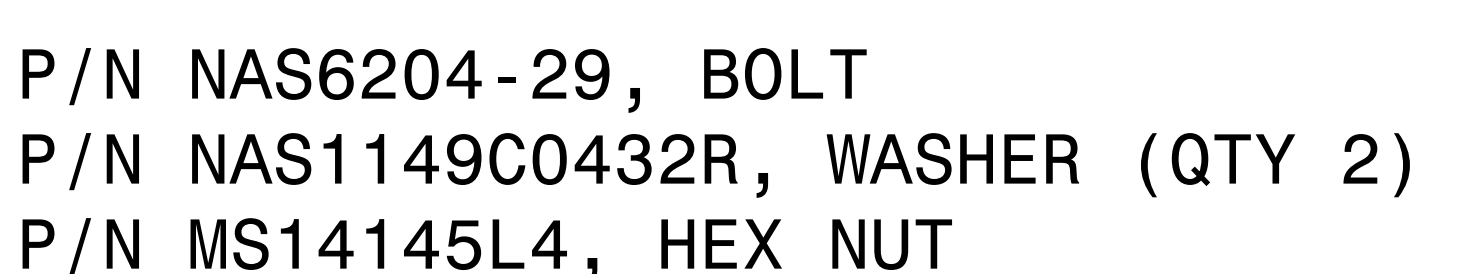

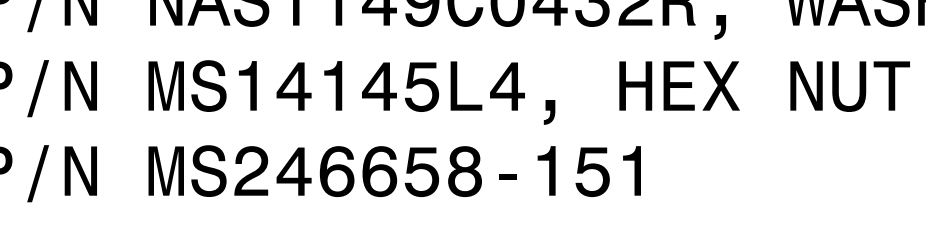

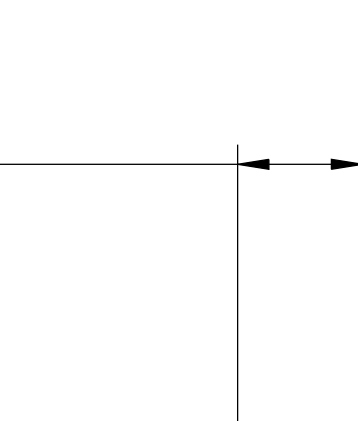

P/N WTR-1103-00, DUMMV AXLE

LOCK FLANG, MAKE FROM HS STEEL,
WATING BORE

.630

WELD JOINT AS REQUTRED

.030

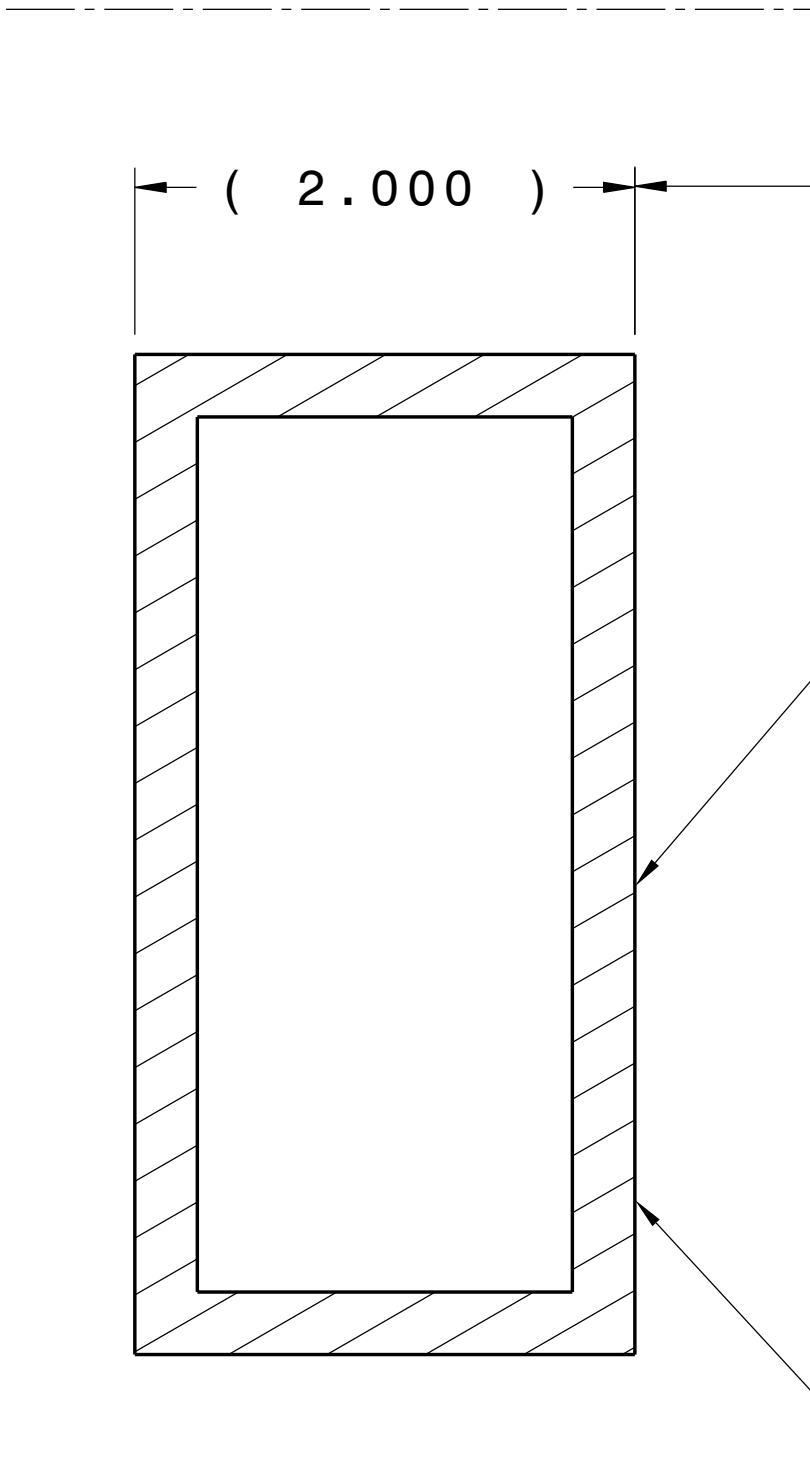

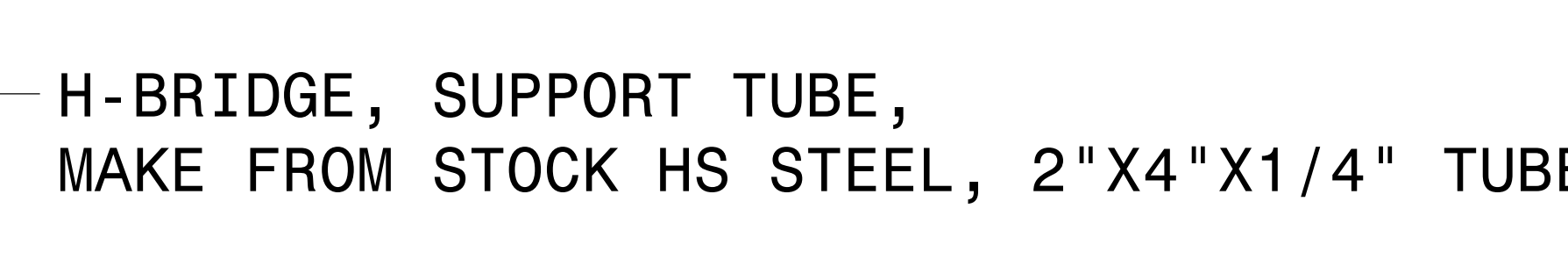

EED JOINT AS REOUURED, BOTH SIDES

\section{SECTION A-A}

PARTIAL VIEW ONLY

P/N WTR-1102-00, SUPPORT TUB

WELD JOTNT AS REOUTRED, BOTH SIDES

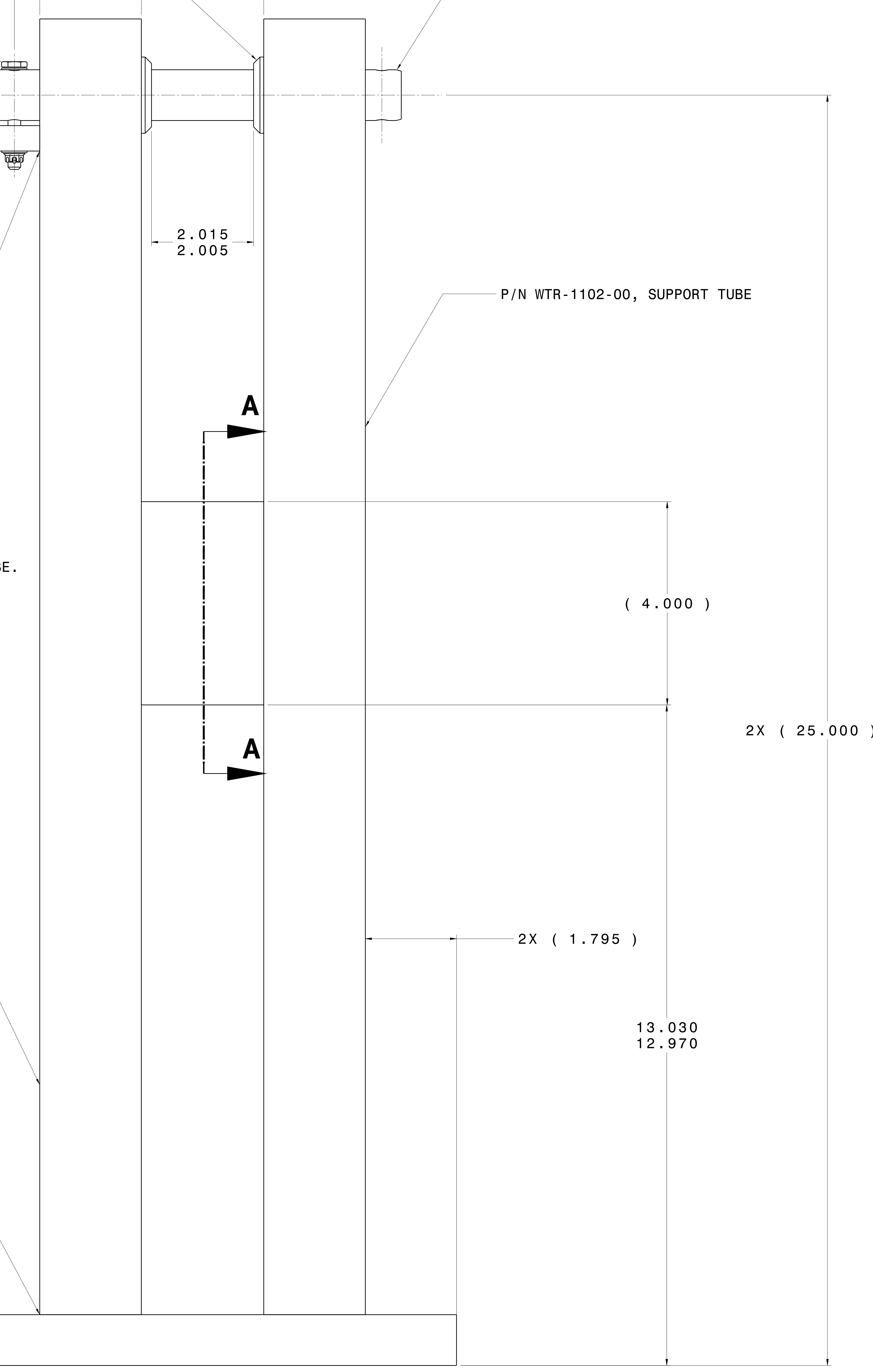

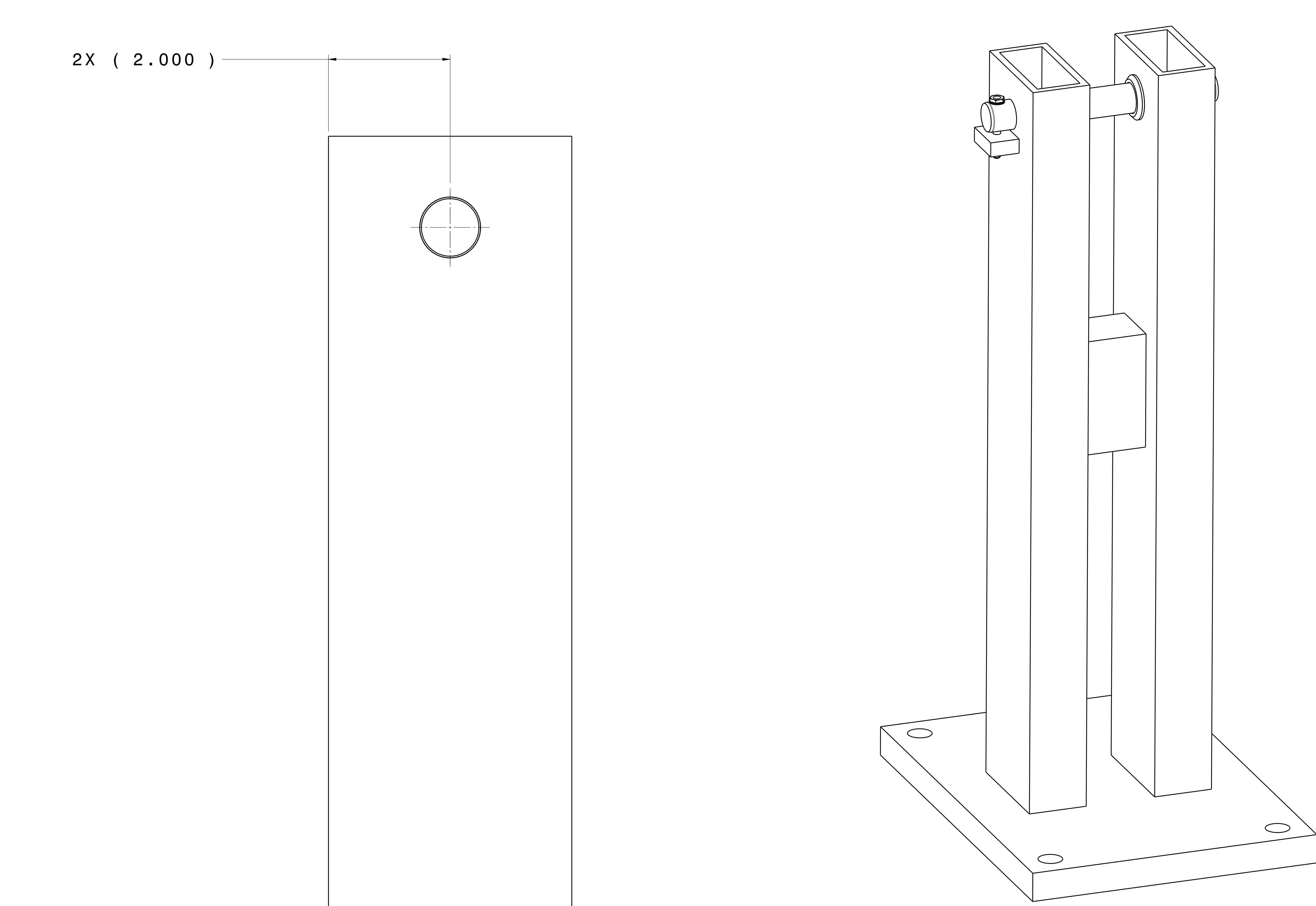

3D VIEW 1

REE ONYY
SCALE: NONE
ON

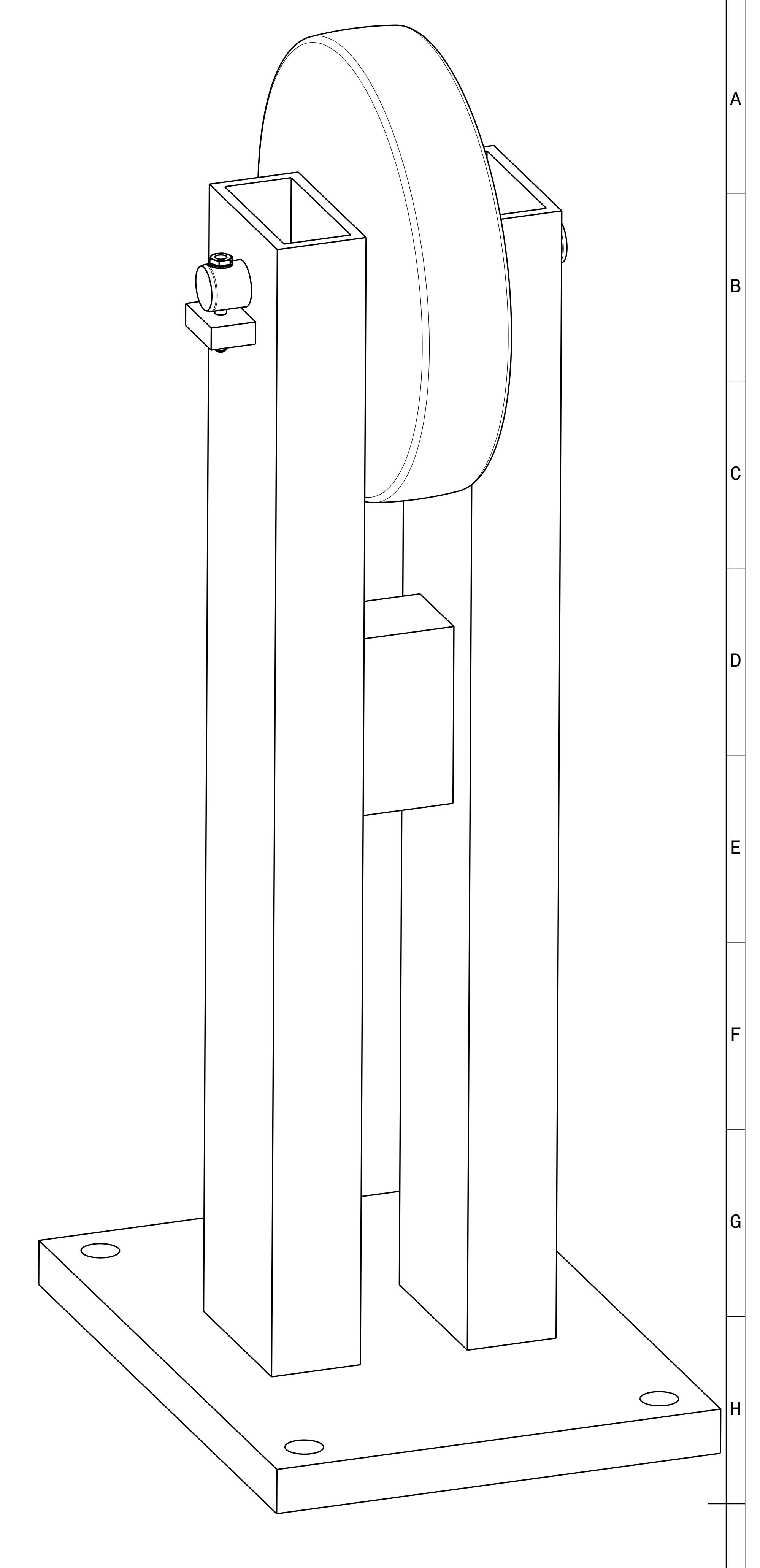

3D VIEW 2
BeE ONY
sche: NOWE 


\section{NOTES:}

1. MAKE FROM STOCK $10 \times 14836$ HOT ROLLED STEEL.

2. DEBURR SHARP EDGES $.005 / .015$, UNLESS OTHERWISE INDICATED.
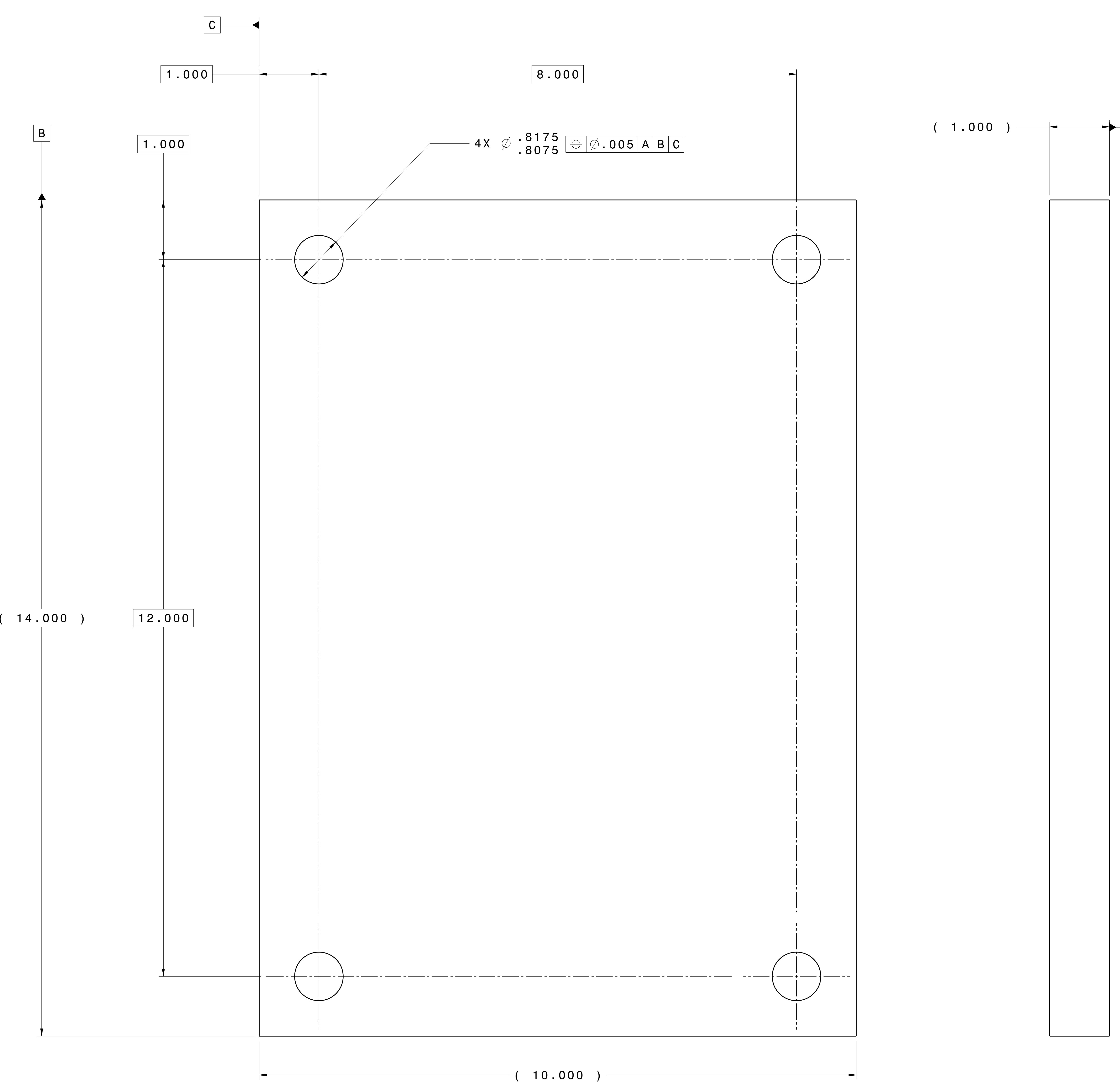

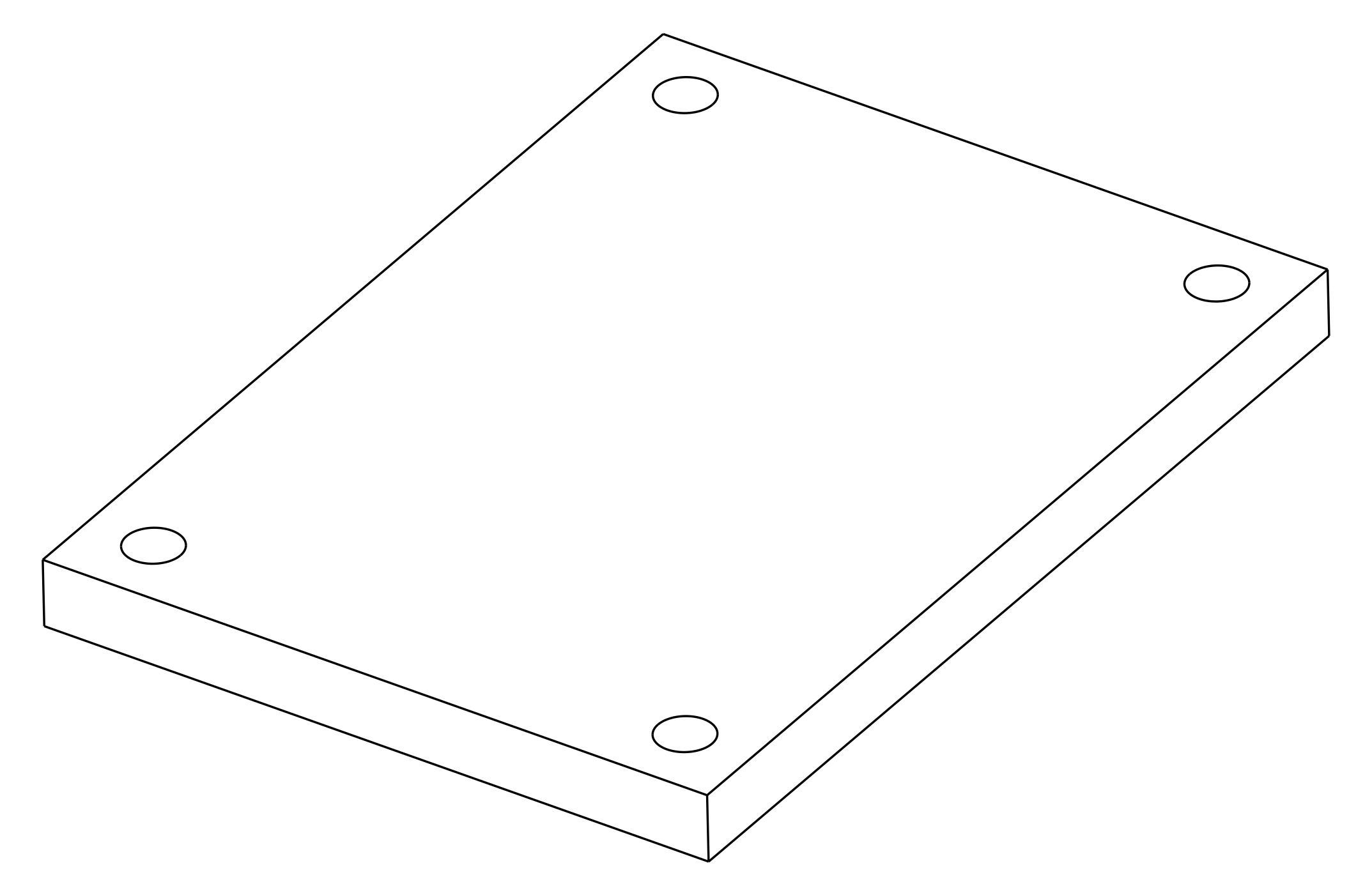

3D VIEW

BREE ONLY
SCCAL: NONE

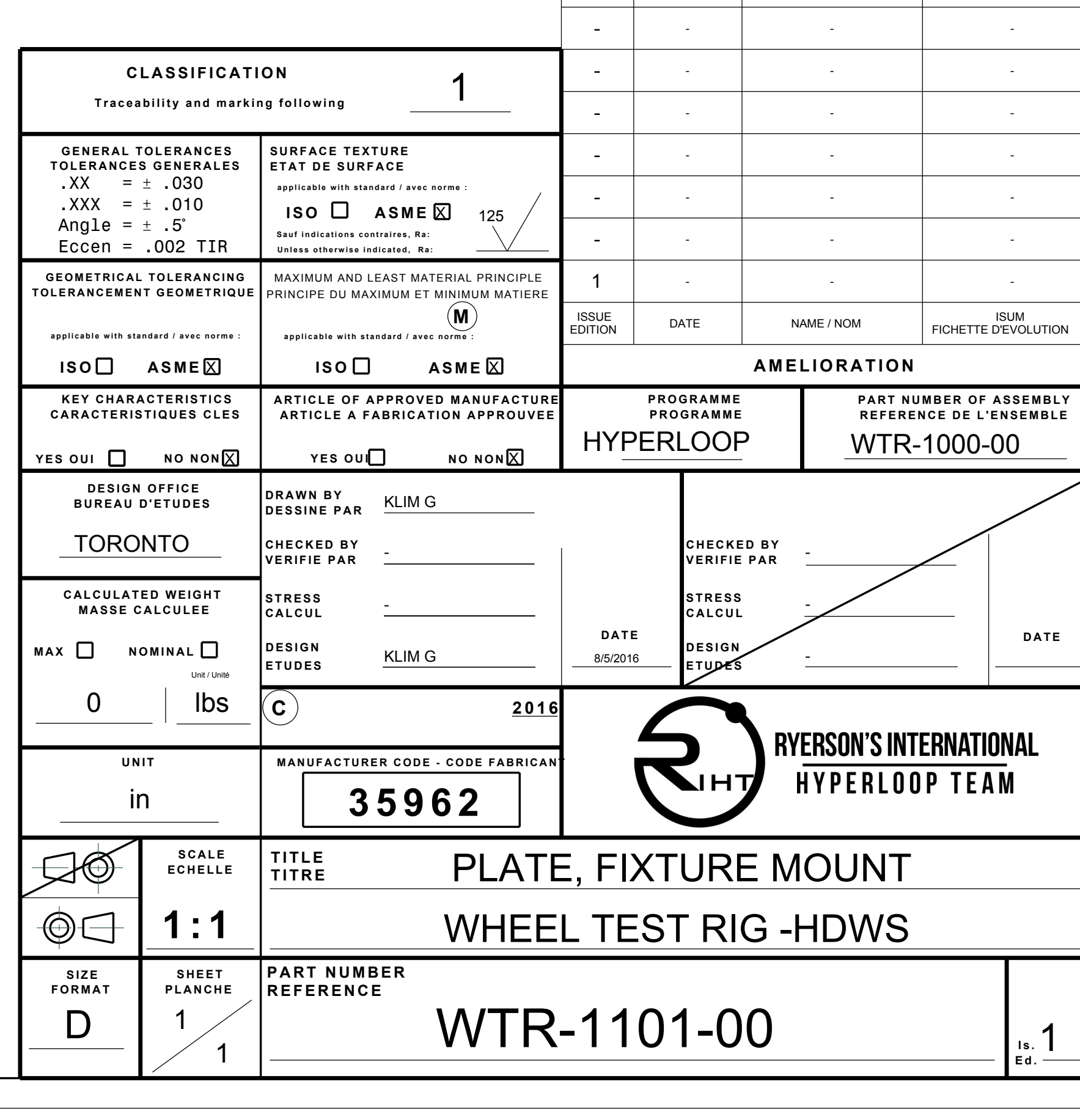


NOTES :

1. Make from Stock hS Steel, 2 " $\mathrm{X} 4$ "X1/4" tUBE.

2. DEBURR SHARP EDGES $.005 / .015$, UNLESS OTHERWISE INDICATED.
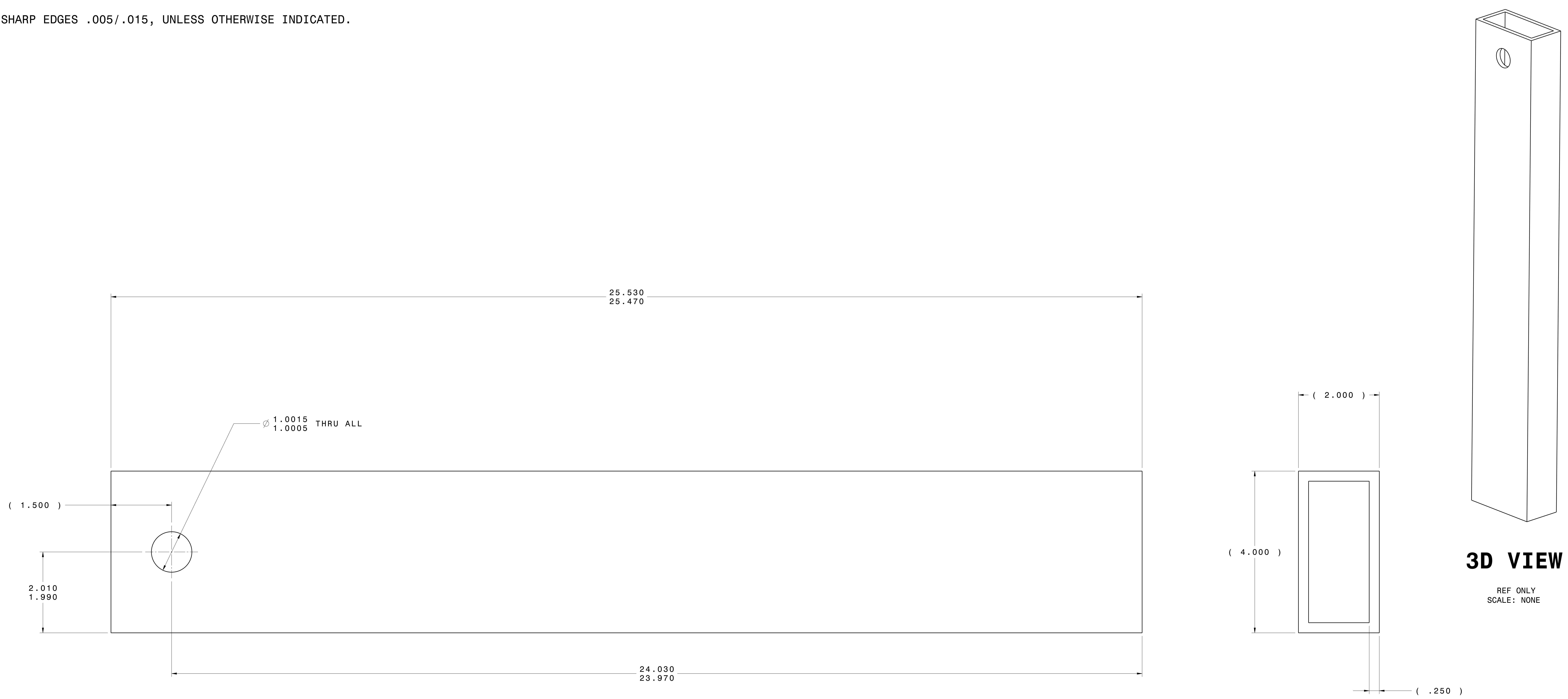

3D VIEW

SBEE ONIYY

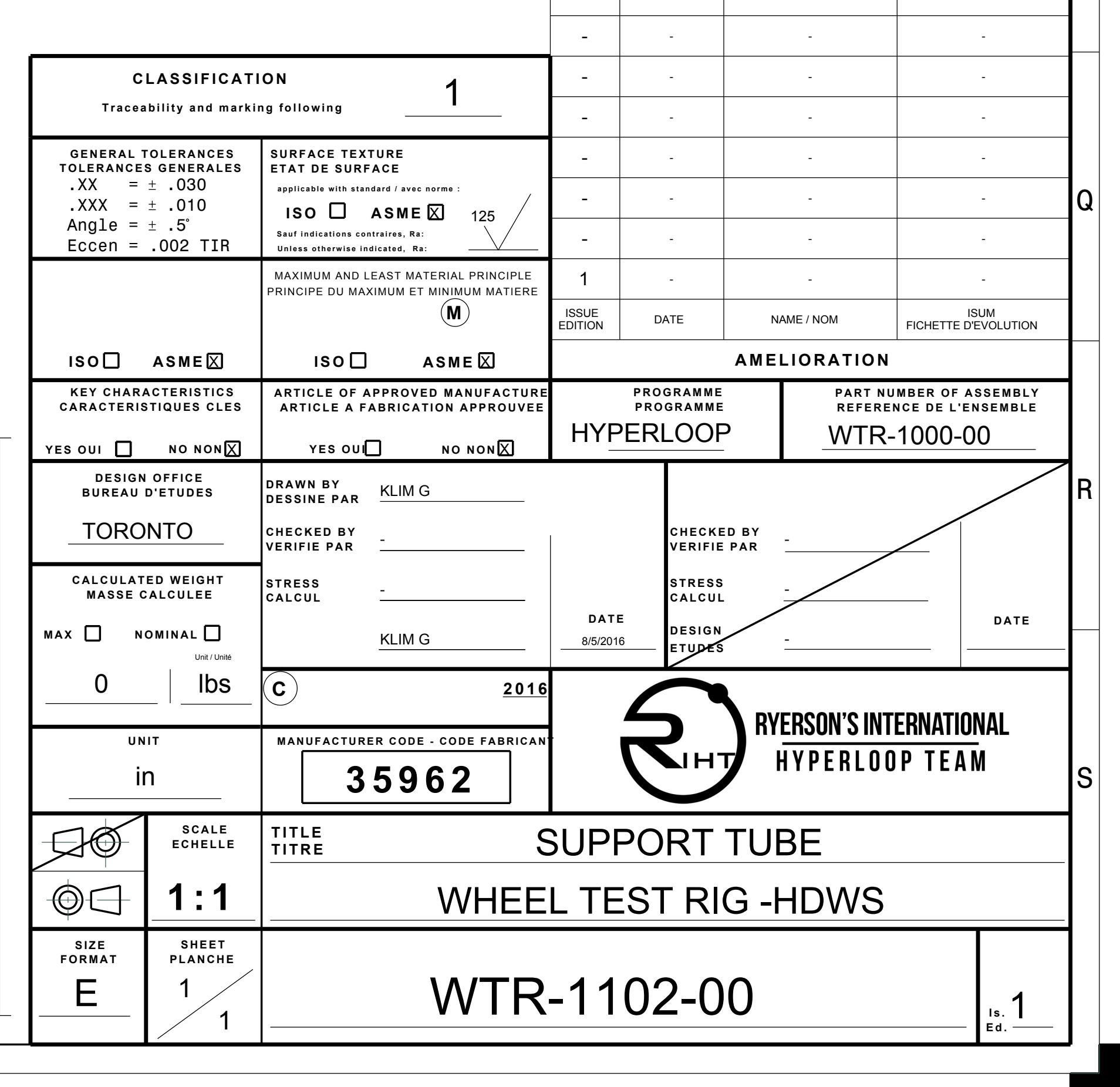




\section{NOTES:}

1. MAKe from 01 TOOL STEEL, Bar StOCK, PER AStM A681 OR EQUiVAlent.

2. DEBURR SHARP EDGES $.005 / .015$, UNLESS OTHERWISE INDICATED.

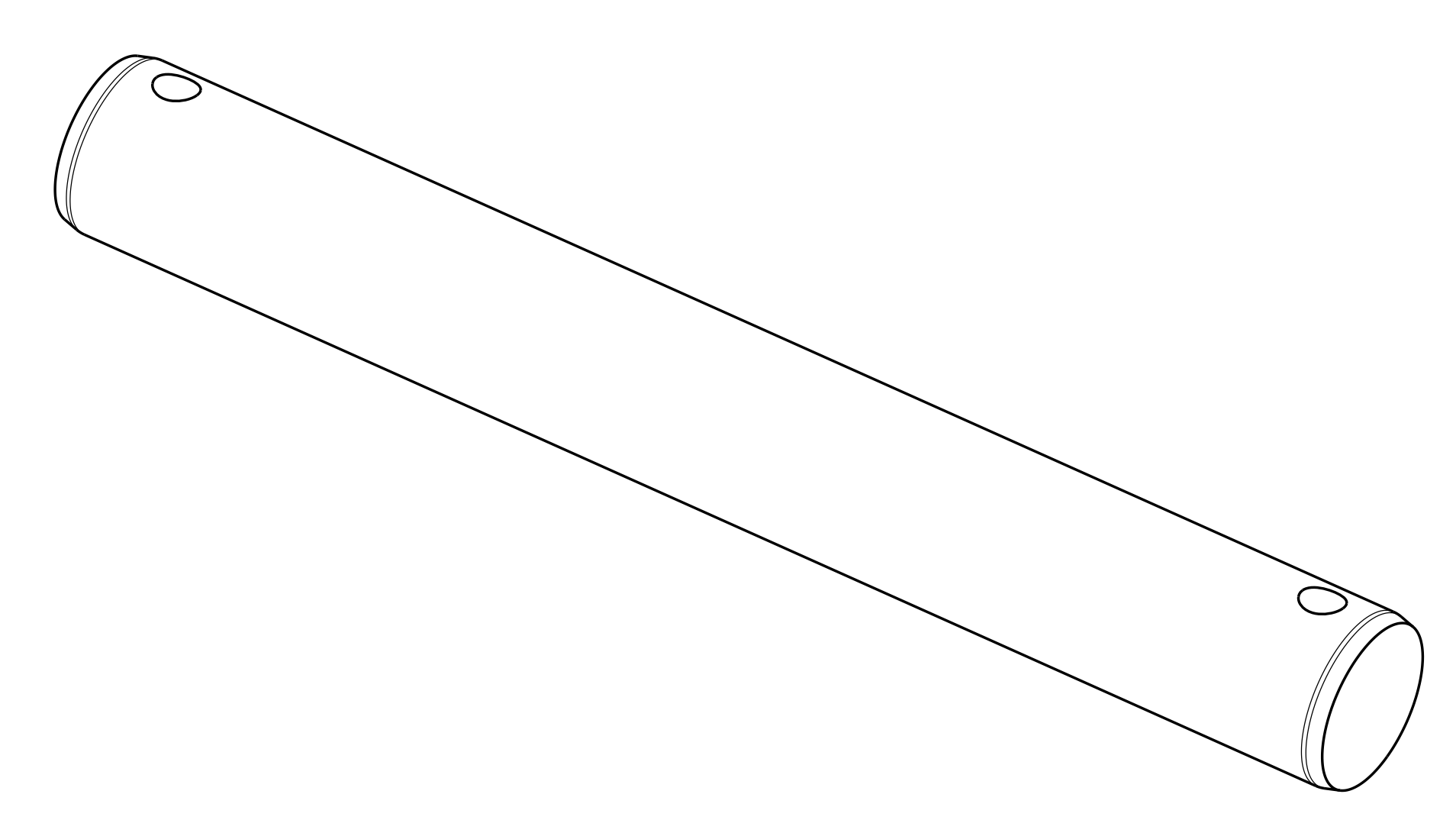

3D VIEW

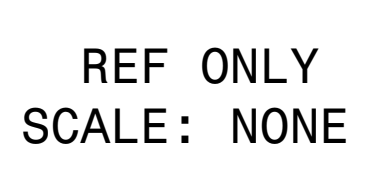
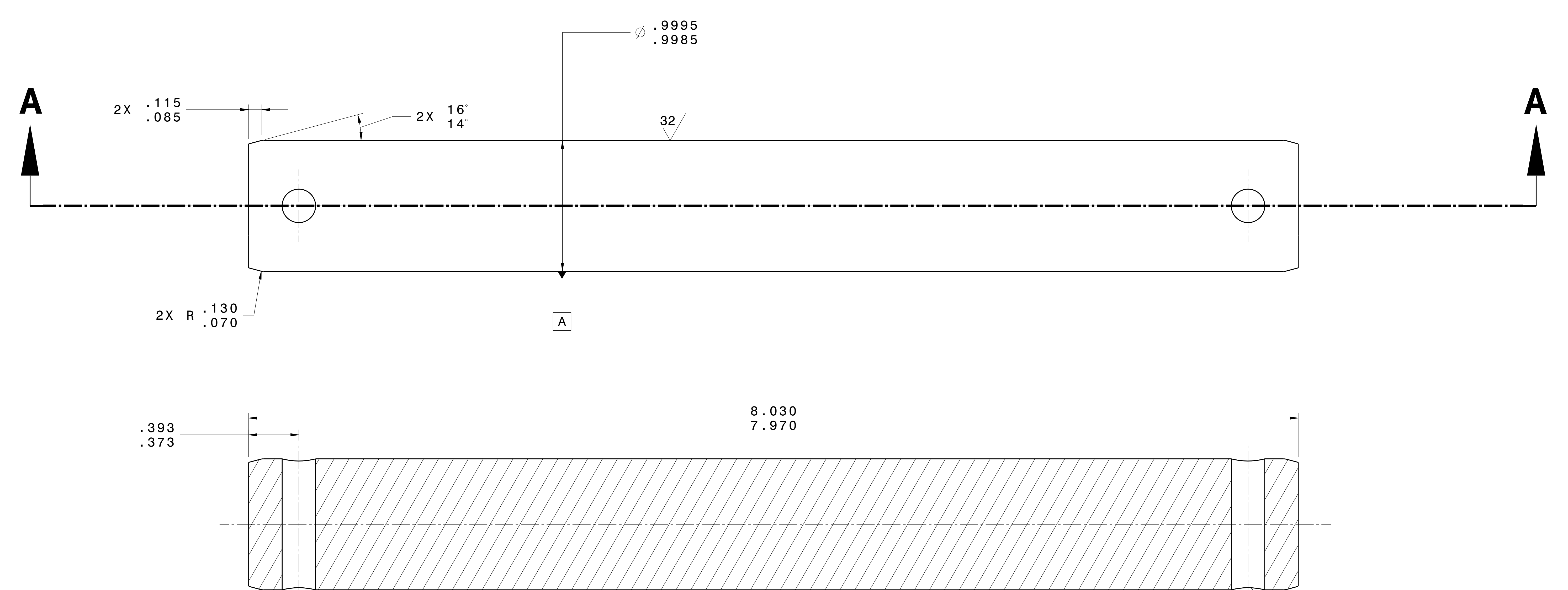

OPTIONAL SECOND BORE, NOT REQUiRED.

$\varnothing \cdot .258$ THRU AL

$\phi \not \varnothing .002 \mathrm{~A}$

SECTION A-A

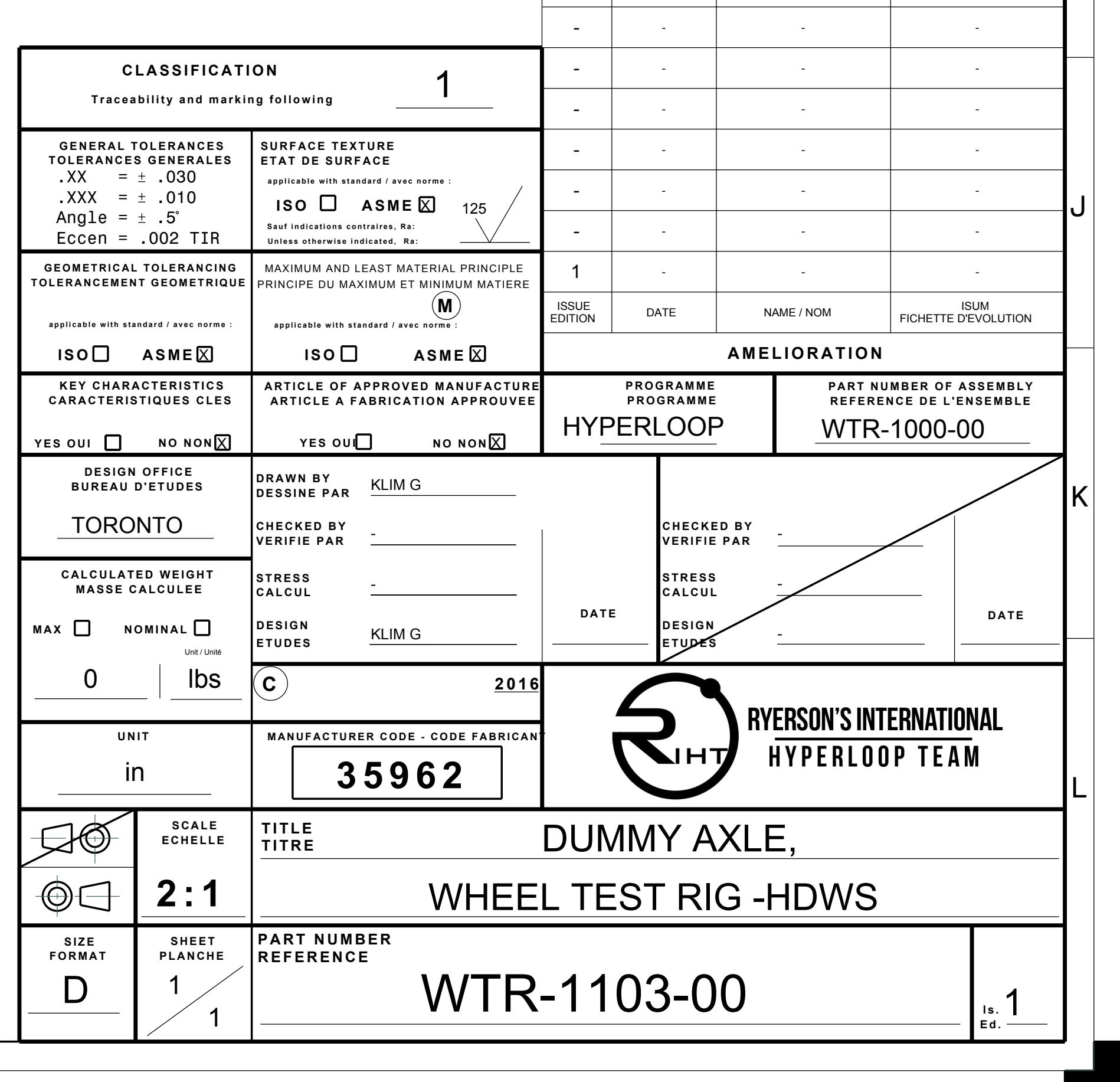




\section{NOTES:}

1. MAKE FROM DELRIN.

2. DEBURR SHARP EDGES $.005 / .015$, UNLESS OTHERWISE INDICATED.
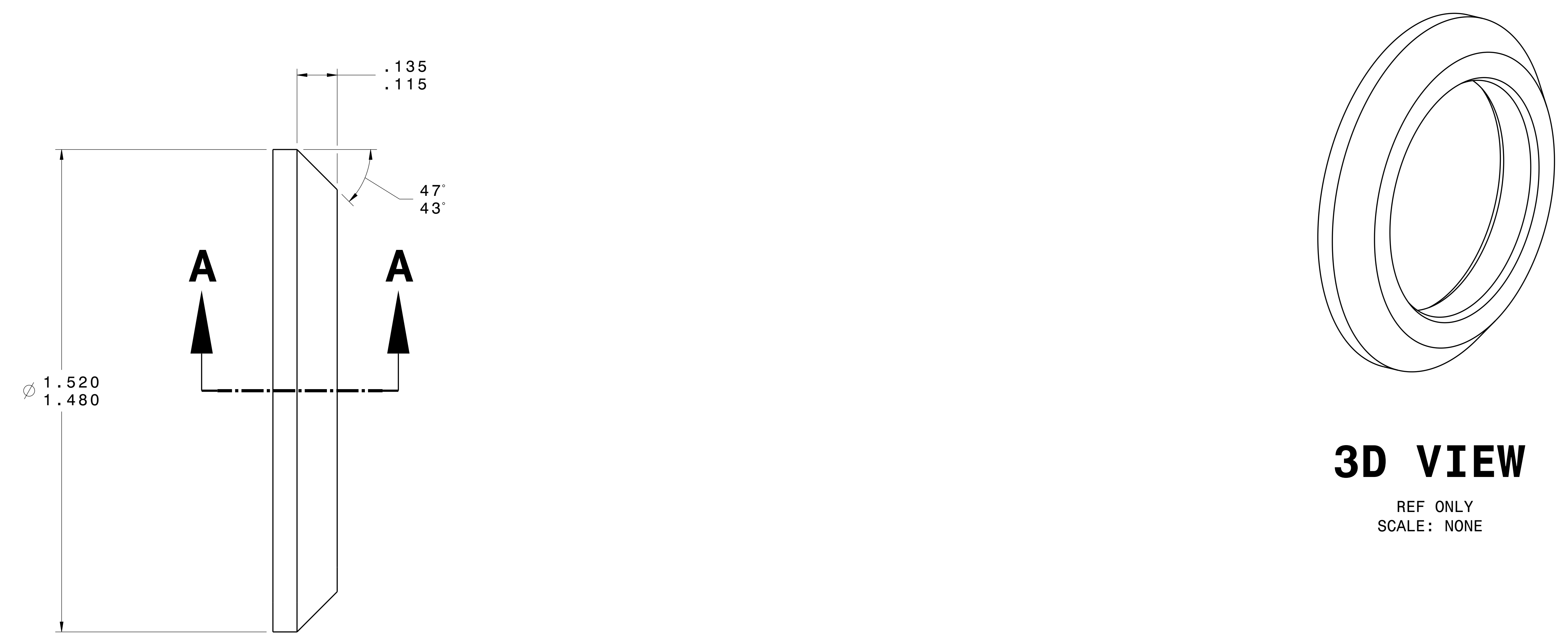

3D VIEW

REE ONLY
SCALE: NONE

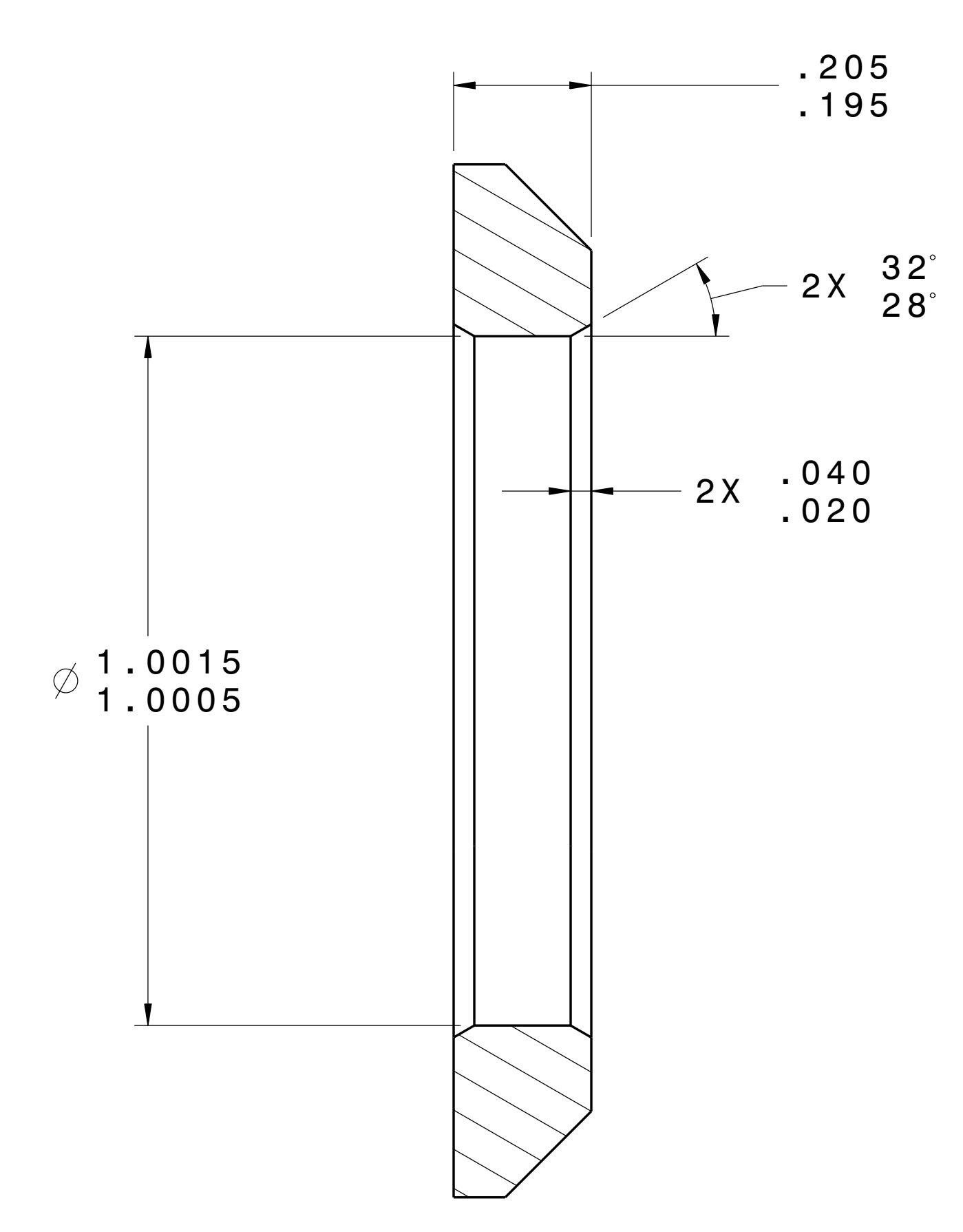

SECTION A-A

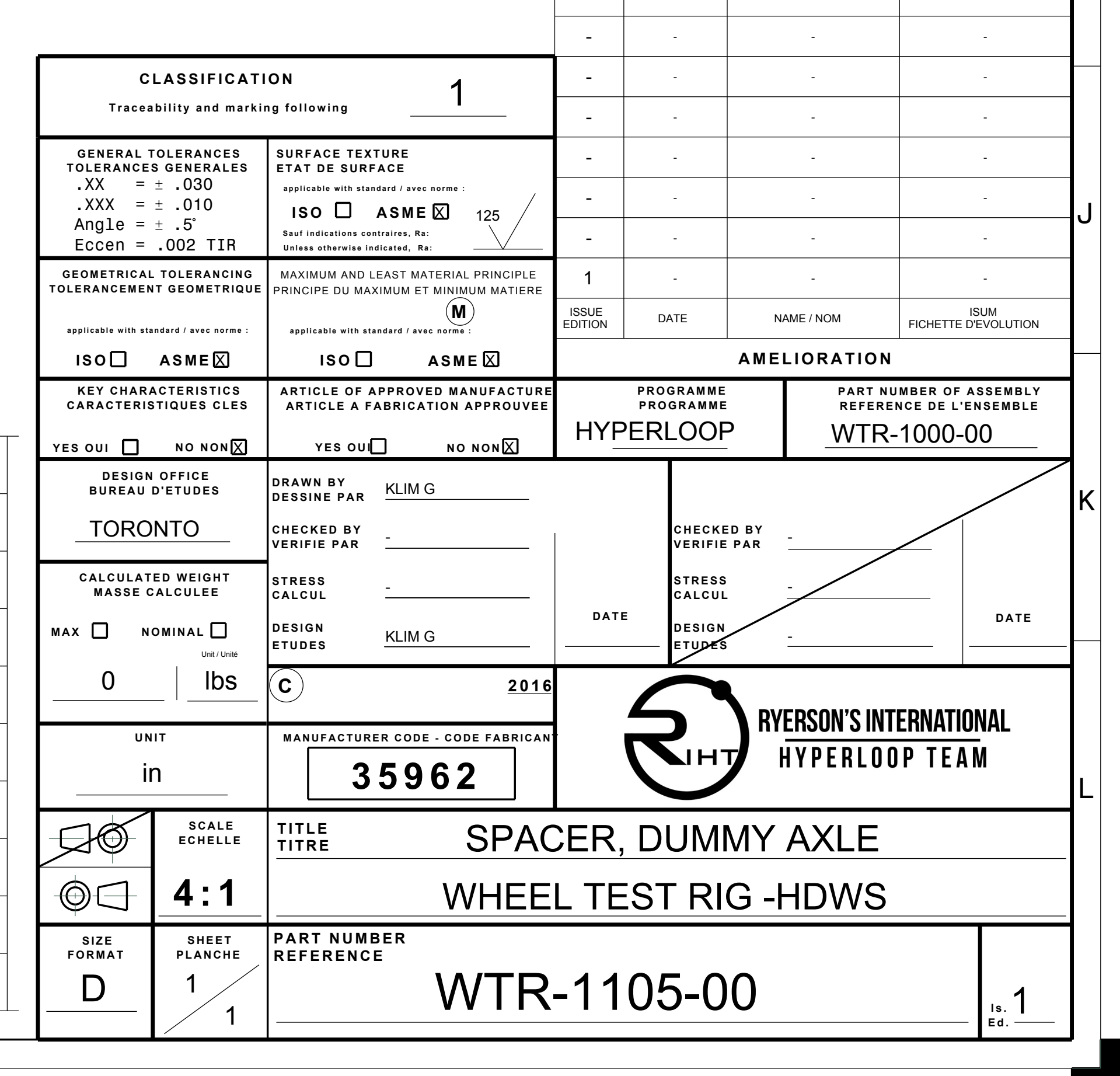




\section{Appendix D: Gas Spring Test Result Data}

The gas spring test data as recorded during testing is presented here, as recorded during the test. Two data samples are provided as recorded for HDWS \#4 and HDWS\#5 here below. For reference, the gas spring data, as calculated by Dynatool ${ }^{\odot}$ [60], is presented here with the data collected during test shown below marked in pen under the "static load column," measured at predetermined intervals as shown in the figure below. The first page for test is shown, and then only relevant pages corresponding to values recorded in the scan below are shown thereafter.

\begin{tabular}{|c|c|c|c|}
\hline Stroke & $\begin{array}{l}\text { H } 4 \\
(1 b)\end{array}$ & HDWS & $\begin{array}{l}\# 5 \\
(1 b)\end{array}$ \\
\hline 1.0 & 97 & & 103 \\
\hline 2.0 & 189 & & 186 \\
\hline 2.1 & 202 & & 205 \\
\hline 2.2 & 219 & & 222 \\
\hline $2 \cdot 3$ & 248 & & 244 \\
\hline 2.4 & 272 & & 273 \\
\hline 2.5 & 314 & & 307 \\
\hline 2.6 & 364 & & 354 \\
\hline 2.7 & 429 & & 419 \\
\hline 2.8 & 516 & & 506 \\
\hline 2.9 & 663 & & 627 \\
\hline 3.0 & 1077 & & 5 \\
\hline
\end{tabular}


Gas spring data recorded for HDWS \#4: Pg 1 of 7

\begin{tabular}{|c|c|c|c|c|c|c|c|c|}
\hline \multicolumn{9}{|c|}{$\begin{array}{l}\text { Hyperloop Preliminary GS } \\
\text { IMP }\end{array}$} \\
\hline & & & & & & Oil Volume & 6.426 & 6 in 3 \\
\hline Bottoms at & 0 & in & & & & $O D=$ & 1.059 & 9 in \\
\hline Servicing Tel & 22 & C & Operating & 22 & C & Oil Vol.(ser & 6.426 & 6 in 3 \\
\hline Cylinder:ID = & $1.492 i$ & in & $O D=$ & 1.709 & in & Piston:ID= & 0.864 & 4 in \\
\hline Servicing Inf & 60 & psi & Operating & 60 & psi & & & \\
\hline Initial Volum & 2.748 & in 3 & Initial Volı & 2.751 & in 3 & Smax $=$ & 3 & 3 \\
\hline Final Pressur & 1316.813 & psi & Final Pres: & 3445.807 & psi & Dynamic $\mathrm{n}:$ & 1.422 & \\
\hline Final Volume & 0.1546 & in 3 & Final Volu & 0.1983 & in 3 & Static $P=$ & 252.35 & \\
\hline \multirow[t]{2}{*}{$\begin{array}{l}\text { Stroke } \\
\text { (in) }\end{array}$} & $\begin{array}{l}\text { Chrome } \\
\text { (in) }\end{array}$ & $\begin{array}{l}\text { Static Pres: } \\
\text { (psi) }\end{array}$ & $\begin{array}{l}\text { Dynamic FS } \\
\text { (psi) }\end{array}$ & $\begin{array}{l}\text { Static Load } \\
\text { (lb) }\end{array}$ & $\begin{array}{l}\text { Dynamic Lc } \\
\text { (lb) }\end{array}$ & $\begin{array}{l}\text { c Vol. (static' } \mathrm{V} \\
\text { (in3) }\end{array}$ & $\begin{array}{l}\text { lol.(dynarr } \\
\text { (in3) }\end{array}$ & $\begin{array}{l}\text { Temp. } \\
\text { (C) }\end{array}$ \\
\hline & 3 & 60 & 60 & 52.8 & 52.8 & 2.751 & 2.751 & 22 \\
\hline 0.01 & 2.99 & 60.24 & 60.37 & 53.1 & 53.2 & 2.742 & 2.742 & 22.433 \\
\hline 0.02 & 2.98 & 60.48 & 60.72 & 53.3 & 53.5 & 2.734 & 2.733 & 22.834 \\
\hline 0.03 & 2.97 & 60.72 & 61.06 & 53.5 & 53.8 & 2.725 & 2.724 & 23.237 \\
\hline 0.04 & 2.96 & 60.97 & 61.41 & 53.7 & 54.1 & 2.716 & 2.715 & 523.642 \\
\hline 0.05 & 2.95 & 61.21 & 61.77 & 53.9 & 54.4 & 2.707 & 2.707 & 24.049 \\
\hline 0.06 & 2.94 & 61.46 & 62.12 & 54.1 & 54.7 & 2.699 & 2.698 & 24.457 \\
\hline 0.07 & 2.93 & 61.71 & 62.48 & 54.4 & 55 & 2.69 & 2.689 & 24.868 \\
\hline 0.08 & 2.92 & 61.96 & 62.84 & 54.6 & 55.4 & 2.681 & 2.68 & $\begin{array}{l}35.28 \\
\end{array}$ \\
\hline 0.09 & 2.91 & 62.21 & 63.21 & 54.8 & 55.7 & 2.672 & 2.671 & 25.695 \\
\hline 0.1 & 2.9 & 62.47 & 63.58 & 55 & 56 & 2.663 & 2.663 & 26.111 \\
\hline 0.11 & 2.89 & 62.72 & 63.95 & 55.2 & 56.3 & 2.655 & 2.654 & 26.529 \\
\hline 0.12 & 2.88 & 62.98 & 64.32 & 55.5 & 56.7 & 2.646 & 2.645 & 26.949 \\
\hline 0.13 & 2.87 & 63.24 & 64.7 & 55.7 & 57 & 2.637 & 2.636 & 27.372 \\
\hline 0.14 & 2.86 & 63.5 & 65.08 & 55.9 & 57.3 & 2.628 & 2.627 & 27.796 \\
\hline 0.15 & 2.85 & 63.76 & 65.46 & 56.2 & 57.7 & 2.619 & 2.619 & 28.222 \\
\hline 0.16 & 2.84 & 64.02 & 65.84 & 56.4 & 58 & 2.611 & 2.61 & 28.65 \\
\hline 0.17 & 2.83 & 64.29 & 66.23 & 56.6 & 58.3 & 2.602 & 2.601 & 29.081 \\
\hline 0.18 & 2.82 & 64.56 & 66.62 & 56.9 & 58.7 & 2.593 & 2.592 & 29.513 \\
\hline 0.19 & 2.81 & 64.83 & 67.02 & 57.1 & 59 & 2.584 & 2.583 & 29.947 \\
\hline 0.2 & 2.8 & 65.1 & 67.42 & 57.3 & 59.4 & 2.575 & 2.575 & 30.384 \\
\hline 0.21 & 2.79 & 65.37 & 67.82 & 57.6 & 59.7 & 2.567 & 2.566 & 30.823 \\
\hline 0.22 & 2.78 & 65.65 & 68.22 & 57.8 & 60.1 & 2.558 & 2.557 & 31.263 \\
\hline 0.23 & 2.77 & 65.92 & 68.63 & 58.1 & 60.5 & 2.549 & 2.548 & 31.706 \\
\hline 0.24 & 2.76 & 66.2 & 69.04 & 58.3 & 60.8 & 2.54 & 2.54 & 32.152 \\
\hline 0.25 & 2.75 & 66.48 & 69.46 & 58.6 & 61.2 & 2.531 & 2.531 & 32.599 \\
\hline 0.26 & 2.74 & 66.77 & 69.88 & 58.8 & 61.6 & 2.523 & 2.522 & 33.048 \\
\hline 0.27 & 2.73 & 67.05 & 70.3 & 59.1 & 61.9 & 2.514 & 2.513 & 33.5 \\
\hline 0.28 & 2.72 & 67.34 & 70.73 & 59.3 & 62.3 & 2.505 & 2.504 & 33.954 \\
\hline 0.29 & 2.71 & 67.63 & 71.16 & 59.6 & 62.7 & 2.496 & 2.496 & 34.411 \\
\hline 0.3 & 2.7 & 67.92 & 71.59 & 59.8 & 63.1 & 2.487 & 2.487 & 34.869 \\
\hline 0.31 & 2.69 & 68.21 & 72.03 & 60.1 & 63.4 & 2.479 & 2.478 & 35.33 \\
\hline 0.32 & 2.68 & 68.51 & 72.47 & 60.3 & 63.8 & 2.47 & 2.469 & 35.793 \\
\hline
\end{tabular}


Gas spring data recorded for HDWS \#4: Pg 3 of 7

\begin{tabular}{|c|c|c|c|c|c|c|c|c|}
\hline 0.8 & 2.2 & 85.64 & 99.21 & 75.4 & 87.4 & 2.048 & 2.047 & 61.222 \\
\hline 0.81 & 2.19 & 86.07 & 99.92 & 75.8 & 88 & 2.039 & 2.038 & 61.83 \\
\hline 0.82 & 2.18 & 86.51 & 100.63 & 76.2 & 88.6 & 2.03 & 2.03 & 62.441 \\
\hline 0.83 & 2.17 & 86.94 & 101.34 & 76.6 & 89.3 & 2.021 & 2.021 & 63.056 \\
\hline 0.84 & 2.16 & 87.39 & 102.07 & 77 & 89.9 & 2.012 & 2.012 & 63.675 \\
\hline 0.85 & 2.15 & 87.84 & 102.8 & 77.4 & 90.5 & 2.004 & 2.003 & 64.297 \\
\hline 0.86 & 2.14 & 88.29 & 103.54 & 77.8 & 91.2 & 1,995 & 1.994 & 64.924 \\
\hline 0.87 & 2.13 & 88.74 & 104.29 & 78.2 & 91.9 & 1.986 & 1.986 & 65.554 \\
\hline 0.88 & 2.12 & 89.2 & 105.05 & 78.6 & 92.5 & 1.977 & 1.977 & 66.189 \\
\hline 0.89 & 2.11 & 89.66 & 105.81 & 79 & 93.2 & 1.968 & 1.968 & 66.827 \\
\hline 0.9 & 2.1 & 90.13 & 106.58 & 79.4 & 93.9 & 1.96 & 1.959 & 67.47 \\
\hline 0.91 & 2.09 & 90.6 & 107.36 & 79.8 & 94.6 & 1.951 & 1.95 & 68.117 \\
\hline 0.92 & 2.08 & 91.08 & 108.15 & 80.2 & 95.3 & 1.942 & 1.942 & 68.767 \\
\hline 0.93 & 2.07 & 91.56 & 108.95 & 80.6 & 96 & 1.933 & 1.933 & 69.422 \\
\hline 0.94 & 2.06 & 92.04 & 109.76 & 81.1 & 96.7 & 1.925 & 1.924 & 70.081 \\
\hline 0.95 & 2.05 & 92.53 & 110.58 & 81.5 & 97.4 & 1.916 & 1.915 & 70.745 \\
\hline 0.96 & 2.04 & 93.03 & 111.4 & 81.9 & 98.1 & 1.907 & 1.907 & 71.413 \\
\hline 0.97 & 2.03 & 93.53 & 112.24 & 82.4 & 98.9 & 1.898 & 1.898 & 72.085 \\
\hline 0.98 & 2.02 & 94.03 & 113.08 & 82.8 & 99.6 & 1.889 & 1.889 & 72.761 \\
\hline 0.99 & 2.01 & 94.54 & 113.93 & 83.3 & 100.4 & 1.881 & 1.88 & 73.442 \\
\hline$\Rightarrow 1$ & 2 & 95.05 & 114.8 & 9783.7 & 101.1 & 1.872 & 1.871 & 74.128 \\
\hline 1.01 & 1.99 & 95.56 & 115.67 & 84.2 & 101.9 & 1.863 & 1.863 & 74.818 \\
\hline 1.02 & 1.98 & 96.09 & 116.56 & 84.6 & 102.7 & 1.854 & 1.854 & 75.512 \\
\hline 1.03 & 1.97 & 96.61 & 117.45 & 85.1 & 103.5 & 1.845 & 1.845 & 76.212 \\
\hline 1.04 & 1.96 & 97.15 & 118.35 & 85.6 & 104.2 & 1.837 & 1.836 & 76.916 \\
\hline 1.05 & 1.95 & 97.68 & 119.27 & 86 & 105.1 & 1.828 & 1.828 & 77.625 \\
\hline 1.06 & 1.94 & 98.22 & 120.19 & 86.5 & 105.9 & 1.819 & 1.819 & 78.339 \\
\hline 1.07 & 1.93 & 98.77 & 121.13 & 87 & 106.7 & 1.81 & 1.81 & 79.057 \\
\hline 1.08 & 1.92 & 99.32 & 122.08 & 87.5 & 107.5 & 1.802 & 1.801 & 79.781 \\
\hline 1.09 & 1.91 & 99.88 & 123.04 & 88 & 108.4 & 1.793 & 1.792 & 80.509 \\
\hline 1.1 & 1.9 & 100.45 & 124.01 & 88.5 & 109.2 & 1.784 & 1.784 & 81.243 \\
\hline 1.11 & 1.89 & 101.01 & 124.99 & 89 & 110.1 & 1.775 & 1.775 & 81.982 \\
\hline 1.12 & 1.88 & 101.59 & 125.98 & 89.5 & 111 & 1.766 & 1.766 & 82.726 \\
\hline 1.13 & 1.87 & 102.17 & 126.99 & 90 & 111.9 & 1.758 & 1.757 & 83.475 \\
\hline 1.14 & 1.86 & 102.76 & 128.01 & 90.5 & 112.8 & 1.749 & 1.748 & 84.229 \\
\hline 1.15 & 1.85 & 103.35 & 129.04 & 91 & 113.7 & 1.74 & 1.74 & 84.989 \\
\hline 1.16 & 1.84 & 103.95 & 130.09 & 91.6 & 114.6 & 1.731 & 1.731 & 85.754 \\
\hline 1.17 & 1.83 & 104.55 & 131.14 & 92.1 & 115.5 & 1.722 & 1.722 & 86.525 \\
\hline 1.18 & 1.82 & 105.16 & 132.21 & 92.6 & 116.5 & 1.714 & 1.713 & 87.302 \\
\hline 1.19 & 1.81 & 105.78 & 133.3 & 93.2 & 117,4 & 1.705 & 1.705 & 88.084 \\
\hline 1.2 & 1.8 & 106.4 & 134.39 & 93.7 & 118.4 & 1.696 & 1.696 & 88.871 \\
\hline 1.21 & 1.79 & 107.03 & 135.5 & 94.3 & 119.4 & 1.687 & 1.687 & 89.665 \\
\hline 1.22 & 1.78 & 107.67 & 136.63 & 94.8 & 120.3 & 1.679 & 1.678 & 90.464 \\
\hline 1.23 & 1.77 & 108.31 & 137.77 & 95.4 & 121.3 & 1.67 & 1.67 & 91.27 \\
\hline 1.24 & 1.76 & 108.96 & 138.92 & 96 & 122.4 & 1.661 & 1.661 & 92.081 \\
\hline 1.25 & 1.75 & 109.62 & 140.09 & 96.5 & 123.4 & 1.652 & 1.652 & 92.898 \\
\hline 1.26 & 1.74 & 110.28 & 141.27 & 97,1 & 124.4 & 1.643 & 1.643 & 93.722 \\
\hline
\end{tabular}


Gas spring data recorded for HDWS \#4: Pg 5 of 7

\begin{tabular}{|c|c|c|c|c|c|c|c|c|}
\hline 1.74 & 1.26 & 153.26 & 223.48 & 135 & 196.8 & 1.222 & 1.223 & 142.45 \\
\hline 1.75 & 1.25 & 154.47 & 225.94 & 136.1 & 199 & 1.213 & 1.214 & 143.71 \\
\hline 1.76 & 1.24 & 155.7 & 228.45 & 137.1 & 201.2 & 1.205 & 1.205 & 144.98 \\
\hline 1.77 & 1.23 & 156.94 & 231 & 138.2 & 203.5 & 1.196 & 1.197 & 146.27 \\
\hline 1.78 & 1.22 & 158.21 & 233.59 & 139.4 & 205.8 & 1.187 & 1.188 & 147.57 \\
\hline 1.79 & 1.21 & 159.49 & 236.24 & 140.5 & 208.1 & 1.178 & 1.179 & 148.88 \\
\hline 1.8 & 1.2 & 160.79 & 238.93 & 141.6 & 210.5 & 1.17 & 1.17 & 150.2 \\
\hline 1.81 & 1.19 & 162.11 & 241.67 & 142.8 & 212.9 & 1.161 & 1.162 & 151.54 \\
\hline 1.82 & 1.18 & 163.45 & 244.46 & 144 & 215.3 & 1.152 & 1.153 & 152.9 \\
\hline 1.83 & 1.17 & 164.82 & 247.3 & 145.2 & 217.8 & 1.143 & 1.144 & 154.27 \\
\hline 1.84 & 1.16 & 166.2 & 250.2 & 146.4 & 220.4 & 1.135 & 1.135 & 155.65 \\
\hline 1.85 & 1.15 & 167.6 & 253.15 & 147.6 & 223 & 1.126 & 1.127 & 157.05 \\
\hline 1.86 & 1.14 & 169.03 & 256.16 & 148.9 & 225.6 & 1.117 & 1.118 & 158.46 \\
\hline 1.87 & 1.13 & 170.48 & 259.23 & 150.2 & 228.3 & 1.108 & 1.109 & 159.89 \\
\hline 1.88 & 1.12 & 171.95 & 262.35 & 151.5 & 231.1 & 1.1 & 1.101 & 161.33 \\
\hline 1.89 & 1.11 & 173.44 & 265.54 & 152.8 & 233.9 & 1.091 & 1.092 & 162.79 \\
\hline 1.9 & 1.1 & 174.96 & 268.78 & 154.1 & 236.7 & 1.082 & 1.083 & 164.27 \\
\hline 1.91 & 1.09 & 176.5 & 272.1 & 155.5 & 239.7 & 1.073 & 1.074 & 165.76 \\
\hline 1.92 & 1.08 & 178.07 & 275.47 & 156.8 & 242.6 & 1.065 & 1.066 & 167.27 \\
\hline 1.93 & 1.07 & 179.66 & 278.92 & 158.2 & 245.7 & 1.056 & 1.057 & 168.8 \\
\hline 1.94 & 1.06 & 181.28 & 282.44 & 159.7 & 248.8 & 1.047 & 1.048 & 170.35 \\
\hline 1.95 & 1.05 & 182.93 & 286.02 & 161.1 & 251.9 & 1.038 & 1.04 & 171.91 \\
\hline 1.96 & 1.04 & 184.61 & 289.68 & 162.6 & 255.2 & 1.03 & 1.031 & 173.49 \\
\hline 1.97 & 1.03 & 186.31 & 293.42 & 164.1 & 258.4 & 1.021 & 1.022 & 175.09 \\
\hline 1.98 & 1.02 & 188.04 & 297.24 & 165.6 & 261.8 & 1.012 & 1.013 & 176.71 \\
\hline 1.99 & 1.01 & 189.8 & 301.12 & 167.2 & 265.2 & 1.003 & 1.005 & 178.35 \\
\hline 2 & 1 & 191.6 & 305.09 & 189168.8 & 268.7 & 0.995 & 0.996 & 180.01 \\
\hline 2.01 & 0.99 & 193.42 & 309.15 & 170.4 & 272.3 & 0.986 & 0.987 & 181.69 \\
\hline 2.02 & 0.98 & 195.28 & 313.29 & 172 & 275.9 & 0.977 & 0.979 & 183.39 \\
\hline 2.03 & 0.97 & 197.17 & 317.52 & 173.7 & 279.7 & 0.968 & 0.97 & 185.11 \\
\hline 2.04 & 0.96 & 199.09 & 321.84 & 175.4 & 283.5 & 0.96 & 0.961 & 186.85 \\
\hline 2.05 & 0.95 & 201.05 & 326.26 & 177.1 & 287.4 & 0.951 & 0.953 & 188.62 \\
\hline 2.06 & 0.94 & 203.05 & 330.78 & 178.8 & 291.4 & 0.942 & 0.944 & 190.4 \\
\hline 2.07 & 0.93 & 205.08 & 335.4 & 180.6 & 295.4 & 0.933 & 0.935 & 192.21 \\
\hline 2.08 & 0.92 & 207.15 & 340.13 & 182.5 & 299.6 & 0.925 & 0.927 & 194.05 \\
\hline 2.09 & 0.91 & 209.26 & 344.96 & 184.3 & 303.8 & 0.916 & 0.918 & 195,9 \\
\hline$\rightarrow 2.1$ & 0.9 & 211.41 & 349.91 & 202186.2 & 308.2 & 0.907 & 0.909 & 197.78 \\
\hline 2.11 & 0.89 & 213.6 & 354.97 & 188.1 & 312.7 & 0.899 & 0.901 & 199.69 \\
\hline 2.12 & 0.88 & 215.83 & 360.15 & 190.1 & 317.2 & 0.89 & 0.892 & 201.62 \\
\hline 2.13 & 0.87 & 218.11 & 365.46 & 192.1 & 321.9 & 0.881 & 0.883 & 203.58 \\
\hline 2.14 & 0.86 & 220.43 & 370.89 & 194.2 & 326.7 & 0.872 & 0.875 & 205.57 \\
\hline 2.15 & 0.85 & 222.8 & 376.45 & 196.2 & 331.6 & 0.864 & 0.866 & 207.58 \\
\hline 2.16 & 0.84 & 225.22 & 382.15 & 198.4 & 336.6 & 0.855 & 0.857 & 209.62 \\
\hline 2.17 & 0.83 & 227.68 & 387.99 & 200.5 & 341.7 & 0.846 & 0.849 & 211.69 \\
\hline 2.18 & 0.82 & 230.2 & 393.98 & 202.8 & 347 & 0.838 & 0.84 & 213.78 \\
\hline 2.19 & 0.81 & 232.77 & 400.11 & 205 & 352.4 & 0.829 & 0.831 & 215.91 \\
\hline$\rightarrow 2.2$ & 0.8 & 235.39 & 406.4 & 219207.3 & 358 & 0.82 & 0.823 & 218.07 \\
\hline
\end{tabular}


Gas spring data recorded for HDWS \#4: Pg 6 of 7

\begin{tabular}{|c|c|c|c|c|c|c|c|c|}
\hline 2.21 & 0.79 & 238.07 & 412.86 & 209.7 & 363.7 & 0.811 & 0.814 & 220.26 \\
\hline 2.22 & 0.78 & 240.8 & 419.48 & 212.1 & 369.5 & 0.803 & 0.806 & 222.48 \\
\hline 2.23 & 0.77 & 243.6 & 426.28 & 214.6 & 375.5 & 0.794 & 0.797 & 224.73 \\
\hline 2.24 & 0.76 & 246.45 & 433.26 & 217.1 & 381.6 & 0.785 & 0.788 & 227.02 \\
\hline 2.25 & 0.75 & 249.37 & 440.41 & 219.6 & 387.9 & 0.777 & 0.78 & 229.34 \\
\hline 2.26 & 0.74 & 252.35 & 447.76 & 222.3 & 394.4 & 0.768 & 0.771 & 231.7 \\
\hline 2.27 & 0.73 & 255.4 & 455.31 & 225 & 401 & 0.759 & 0.763 & 234.09 \\
\hline 2.28 & 0.72 & 258.52 & 463.06 & 227.7 & 407.9 & 0.75 & 0.754 & 236.52 \\
\hline 2.29 & 0.71 & 261.72 & 471.03 & 230.5 & 414.9 & 0.742 & 0.745 & 238.99 \\
\hline 2.3 & 0.7 & 264.98 & 479.23 & 233.4 & 422.1 & 0.733 & 0.737 & 241.5 \\
\hline 2.31 & 0.69 & 268.33 & 487.65 & 248236.3 & 429.5 & 0.724 & 0.728 & 244.04 \\
\hline 2.32 & 0.68 & 271.75 & 496.32 & 239.4 & 437.2 & 0.716 & 0.72 & 246.63 \\
\hline 2.33 & 0.67 & 275.26 & 505.24 & 242.4 & 445 & 0.707 & 0.711 & 249.26 \\
\hline 2.34 & 0.66 & 278.85 & 514.41 & 245.6 & 453.1 & 0.698 & 0.703 & 251.93 \\
\hline 2.35 & 0.65 & 282.53 & 523.86 & 248.9 & 461.4 & 0.69 & 0.694 & 254.65 \\
\hline 2.36 & 0.64 & 286.3 & 533.58 & 252.2 & 470 & 0.681 & 0.686 & 257.41 \\
\hline 2.37 & 0.63 & 290.17 & 543.61 & 255.6 & 478.8 & 0.672 & 0.677 & 260.22 \\
\hline 2.38 & 0.62 & 294.14 & 553.94 & 259.1 & 487.9 & 0.664 & 0.668 & 263.08 \\
\hline 2.39 & 0.61 & 298.21 & 564.59 & 262.7 & 497.3 & 0.655 & 0.66 & 265.99 \\
\hline 2.4 & 0.6 & 302.39 & 575.57 & 277266.3 & 507 & 0.646 & 0.651 & 268.94 \\
\hline 2.41 & 0.59 & 306.68 & 586.91 & 270.1 & 517 & 0.638 & 0.643 & 271.95 \\
\hline 2.42 & 0.58 & 311.08 & 598.61 & 274 & 527.3 & 0.629 & 0.634 & 275.01 \\
\hline 2.43 & 0.57 & 315.6 & 610.68 & 278 & 537.9 & 0.62 & 0.626 & 278.13 \\
\hline 2.44 & 0.56 & 320.25 & 623.13 & 282.1 & 548.9 & 0.612 & 0.618 & 281.3 \\
\hline 2.45 & 0.55 & 325.03 & 636 & 286.3 & 560.2 & 0.603 & 0.609 & 284.54 \\
\hline 2.46 & 0.54 & 329.95 & 649.3 & 290.6 & 571.9 & 0.595 & 0.601 & 287.83 \\
\hline 2.47 & 0.53 & 335 & 663.07 & 295.1 & 584 & 0.586 & 0.592 & 291.18 \\
\hline 2.48 & 0.52 & 340.21 & 677.3 & 299.7 & 596.6 & 0.577 & 0.584 & 294.6 \\
\hline 2.49 & 0.51 & 345.56 & 692.03 & 304.4 & 609.5 & 0.569 & 0.575 & 298.08 \\
\hline 2.5 & 0.5 & 351.08 & 707.27 & 3.4309 .2 & 623 & 0.56 & 0.567 & 301.62 \\
\hline 2.51 & 0.49 & 356.77 & 723.06 & 314.2 & 636.9 & 0.552 & 0.559 & 305.24 \\
\hline 2.52 & 0.48 & 362.63 & 739.39 & 319.4 & 651.3 & 0.543 & 0.55 & 308.93 \\
\hline 2.53 & 0.47 & 368.68 & 756.33 & 324.7 & 666.2 & 0.534 & 0.542 & 312.69 \\
\hline 2.54 & 0.46 & 374.92 & 773.89 & 330.2 & 681.6 & 0.526 & 0.534 & 316.52 \\
\hline 2.55 & 0.45 & 381.36 & 792.1 & 335.9 & 697.7 & 0.517 & 0.525 & 320.44 \\
\hline 2.56 & 0.44 & 388.02 & 811.01 & 341.8 & 714.3 & 0.509 & 0.517 & 324.43 \\
\hline 2.57 & 0.43 & 394.89 & 830.63 & 347.8 & 731.6 & 0.5 & 0.509 & 328.51 \\
\hline 2.58 & 0.42 & 401.99 & 850.99 & 354.1 & 749.6 & 0.492 & 0.501 & 332.67 \\
\hline 2.59 & 0.41 & 409.34 & 872.15 & 360.5 & 768.2 & 0.483 & 0.492 & 336.91 \\
\hline$\rightarrow 2.6$ & 0.4 & 416.94 & 894.15 & 364367.2 & 787.6 & 0.474 & 0.484 & 341.25 \\
\hline 2.61 & 0.39 & 424.81 & 917.03 & 374.2 & 807.7 & 0.466 & 0.476 & 345.68 \\
\hline 2.62 & 0.38 & 432.97 & 940.84 & 381.4 & 828.7 & 0.457 & 0.468 & 350.21 \\
\hline 2.63 & 0.37 & 441.42 & 965.62 & 388.8 & 850.5 & 0.449 & 0.46 & 354.83 \\
\hline 2.64 & 0.36 & 450.18 & 991.43 & 396.5 & 873.3 & 0.44 & 0.452 & 359.55 \\
\hline 2.65 & 0.35 & 459.28 & 1018.33 & 404.5 & 897 & 0.432 & 0.444 & 364.38 \\
\hline 2.66 & 0.34 & 468.73 & 1046.38 & 412.9 & 921.7 & 0.424 & 0.436 & 369.32 \\
\hline 2.67 & 0.33 & 478.54 & 1075.64 & 421.5 & 947.4 & 0.415 & 0.428 & 374.37 \\
\hline
\end{tabular}


Gas spring data recorded for HDWS \#4: Pg 7 of 7

\begin{tabular}{rrrrrrrrr}
2.68 & 0.32 & 488.74 & 1106.17 & 430.5 & 974.3 & 0.407 & 0.42 & 379.53 \\
2.69 & 0.31 & 499.36 & 1138.02 & 439.8 & 1002.4 & 0.398 & 0.412 & 384.81 \\
2.7 & 0.3 & 510.42 & 1171.28 & 429449.6 & 1031.7 & 0.39 & 0.404 & 390.21 \\
2.71 & 0.29 & 521.95 & 1206.04 & 459.7 & 1062.3 & 0.381 & 0.396 & 395.74 \\
2.72 & 0.28 & 533.96 & 1242.4 & 470.3 & 1094.3 & 0.373 & 0.388 & 401.39 \\
2.73 & 0.27 & 546.5 & 1280.43 & 481.4 & 1127.8 & 0.365 & 0.38 & 407.17 \\
2.74 & 0.26 & 559.6 & 1320.23 & 492.9 & 1162.9 & 0.356 & 0.373 & 413.09 \\
2.75 & 0.25 & 573.28 & 1361.91 & 504.9 & 1199.6 & 0.348 & 0.365 & 419.15 \\
2.76 & 0.24 & 587.61 & 1405.57 & 517.6 & 1238 & 0.34 & 0.357 & 425.35 \\
2.77 & 0.23 & 602.62 & 1451.29 & 530.8 & 1278.3 & 0.332 & 0.35 & 431.69 \\
2.78 & 0.22 & 618.34 & 1523.68 & 544.6 & 1342.1 & 0.323 & 0.343 & 437.61 \\
2.79 & 0.21 & 634.82 & 1606.65 & 559.2 & 1415.1 & 0.315 & 0.336 & 443.45 \\
\hline 2.8 & 0.2 & 652.12 & 1699.62 & 516574.4 & 1497 & 0.307 & 0.33 & 449.21 \\
2.81 & 0.19 & 670.29 & 1772.13 & 590.4 & 1560.9 & 0.299 & 0.323 & 455.66 \\
2.82 & 0.18 & 689.4 & 1817.88 & 607.2 & 1601.2 & 0.291 & 0.315 & 463.02 \\
2.83 & 0.17 & 709.51 & 1864.09 & 624.9 & 1641.9 & 0.283 & 0.308 & 470.62 \\
2.84 & 0.16 & 730.75 & 1910.7 & 643.6 & 1683 & 0.275 & 0.3 & 478.48 \\
2.85 & 0.15 & 753.18 & 1957.61 & 663.4 & 1724.3 & 0.267 & 0.293 & 486.63 \\
2.86 & 0.14 & 776.88 & 2004.73 & 684.3 & 1765.8 & 0.259 & 0.285 & 495.08 \\
2.87 & 0.13 & 801.92 & 2057.5 & 706.3 & 1812.3 & 0.251 & 0.278 & 503.69 \\
2.88 & 0.12 & 828.43 & 2135.46 & 729.7 & 1880.9 & 0.243 & 0.271 & 511.82 \\
2.89 & 0.11 & 856.5 & 2217.27 & 754.4 & 1953 & 0.235 & 0.264 & 520.14 \\
\hline 2.9 & 0.1 & 886.32 & 2303.12 & 663780.7 & 2028.6 & 0.227 & 0.258 & 528.63 \\
2.91 & 0.09 & 918 & 2393.77 & 808.6 & 2108.5 & 0.22 & 0.251 & 537.28 \\
2.92 & 0.08 & 951.68 & 2489.31 & 838.2 & 2192.6 & 0.212 & 0.245 & 546.09 \\
2.93 & 0.07 & 987.51 & 2589.65 & 869.8 & 2281 & 0.205 & 0.239 & 555.07 \\
2.94 & 0.06 & 1025.66 & 2695.13 & 903.4 & 2373.9 & 0.197 & 0.232 & 564.21 \\
2.95 & 0.05 & 1066.34 & 2805.94 & 939.2 & 2471.5 & 0.19 & 0.226 & 573.52 \\
2.96 & 0.04 & 1109.75 & 2922.22 & 977.5 & 2573.9 & 0.182 & 0.221 & 582.97 \\
2.97 & 0.03 & 1156.11 & 3044.09 & 1018.3 & 2681.3 & 0.175 & 0.215 & 592.59 \\
2.98 & 0.02 & 1206.06 & 3171.85 & 1062.3 & 2793.8 & 0.168 & 0.209 & 602.35 \\
2.99 & 0.01 & 1259.55 & 3305.68 & 1109.4 & 2911.7 & 0.161 & 0.204 & 612.26 \\
\hline 3 & 0 & 1316.81 & 3445.81 & 1079159.9 & 3035.1 & 0.155 & 0.198 & 622.3 \\
\hline & & & & & & & &
\end{tabular}

End of gas spring test data, HDWS \#4. 


\section{Appendix E: Retractable Shock Strut Concept Art}

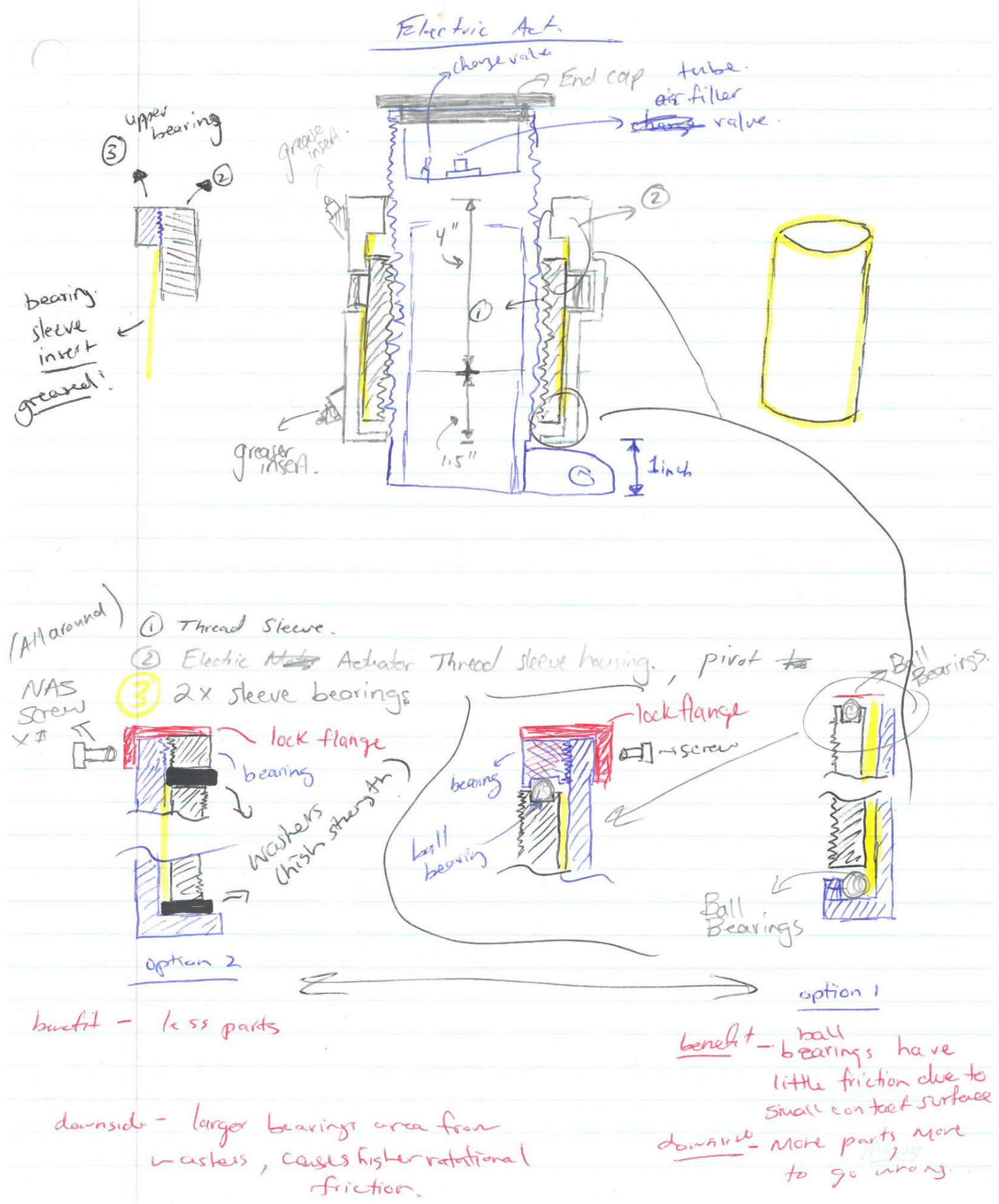




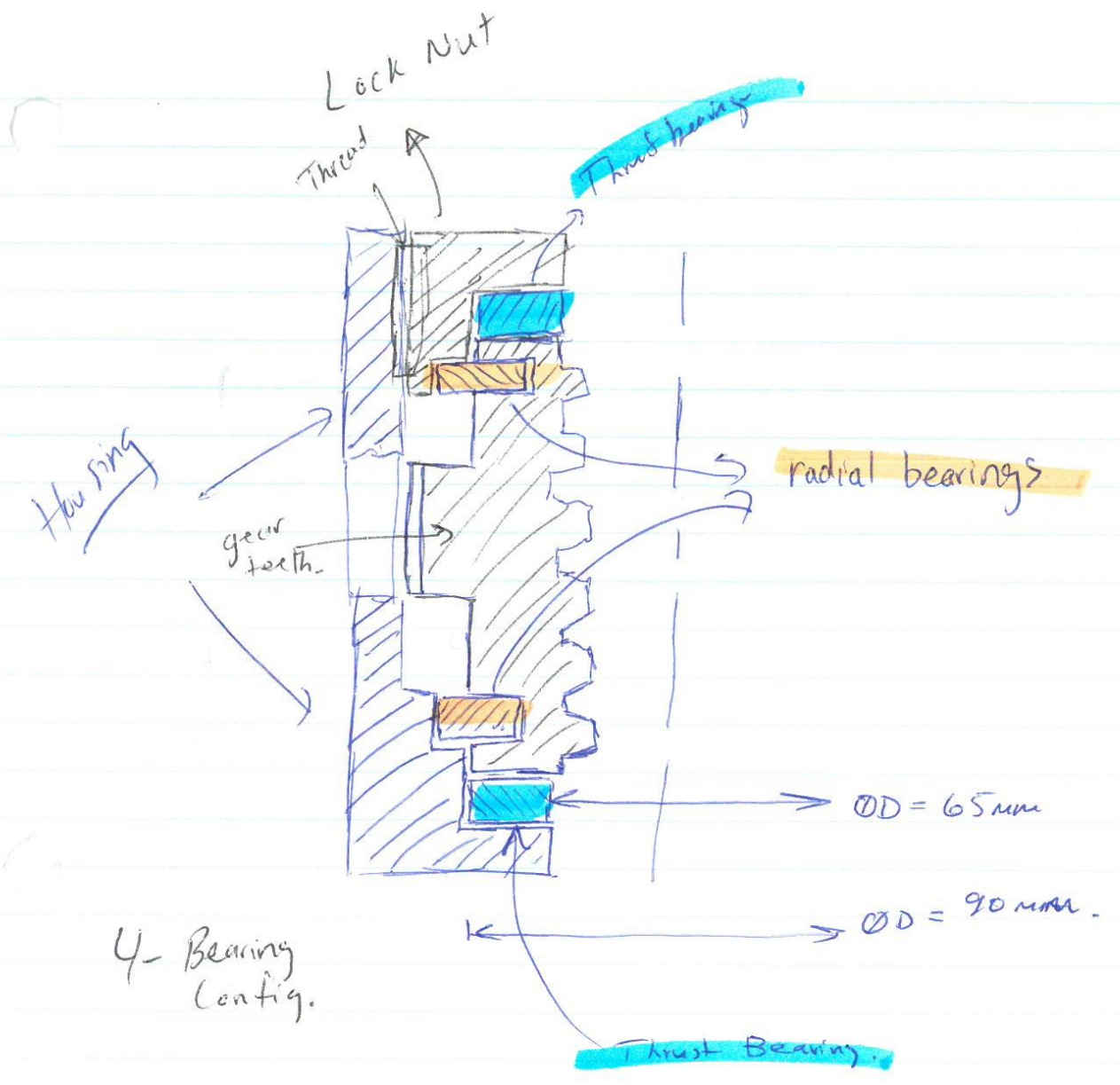




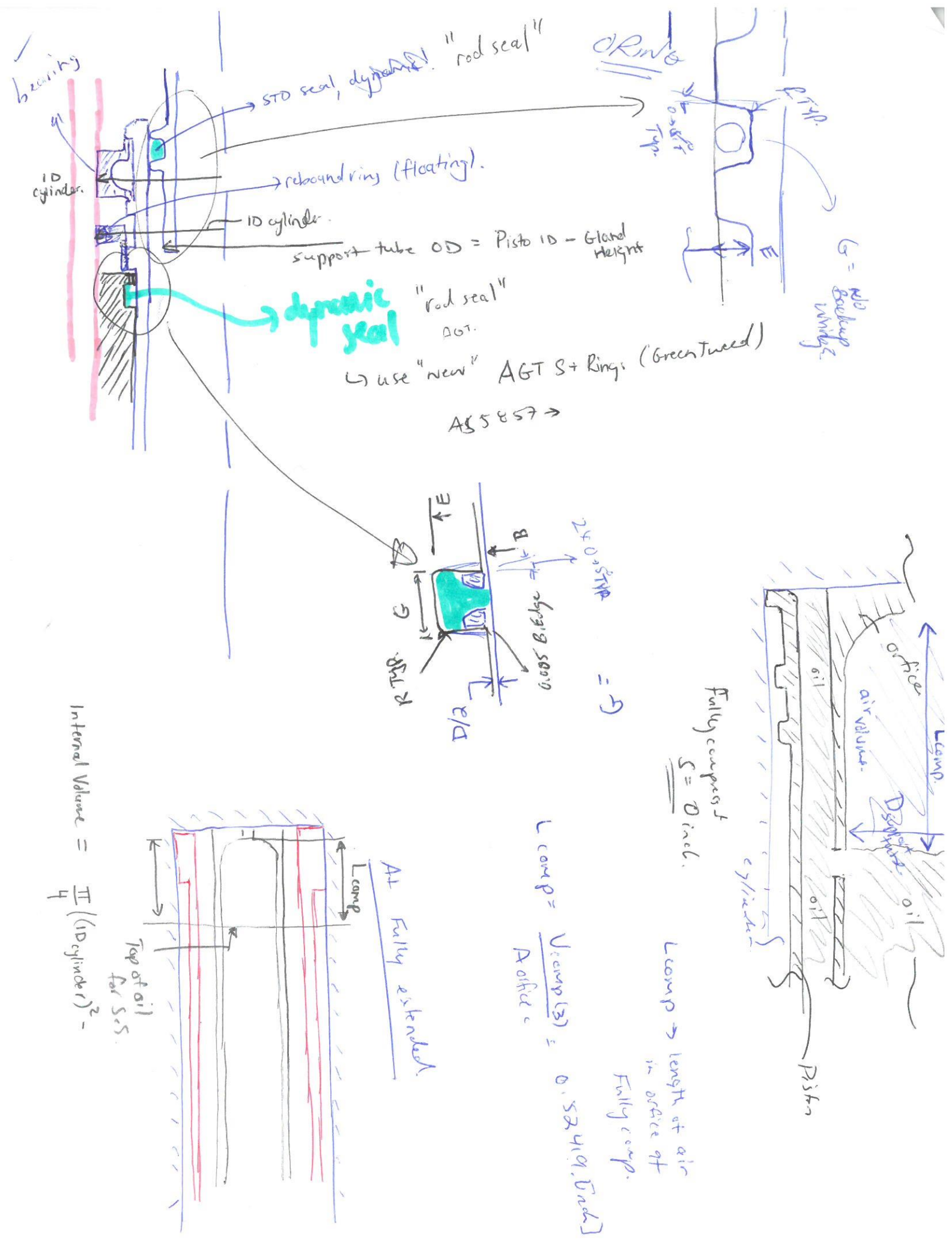




\section{Appendix F: Galvanic Chart}

Metals compatibility chart as found in the Unbrako catalogue [32]

\section{GALVANIC CORROSION}

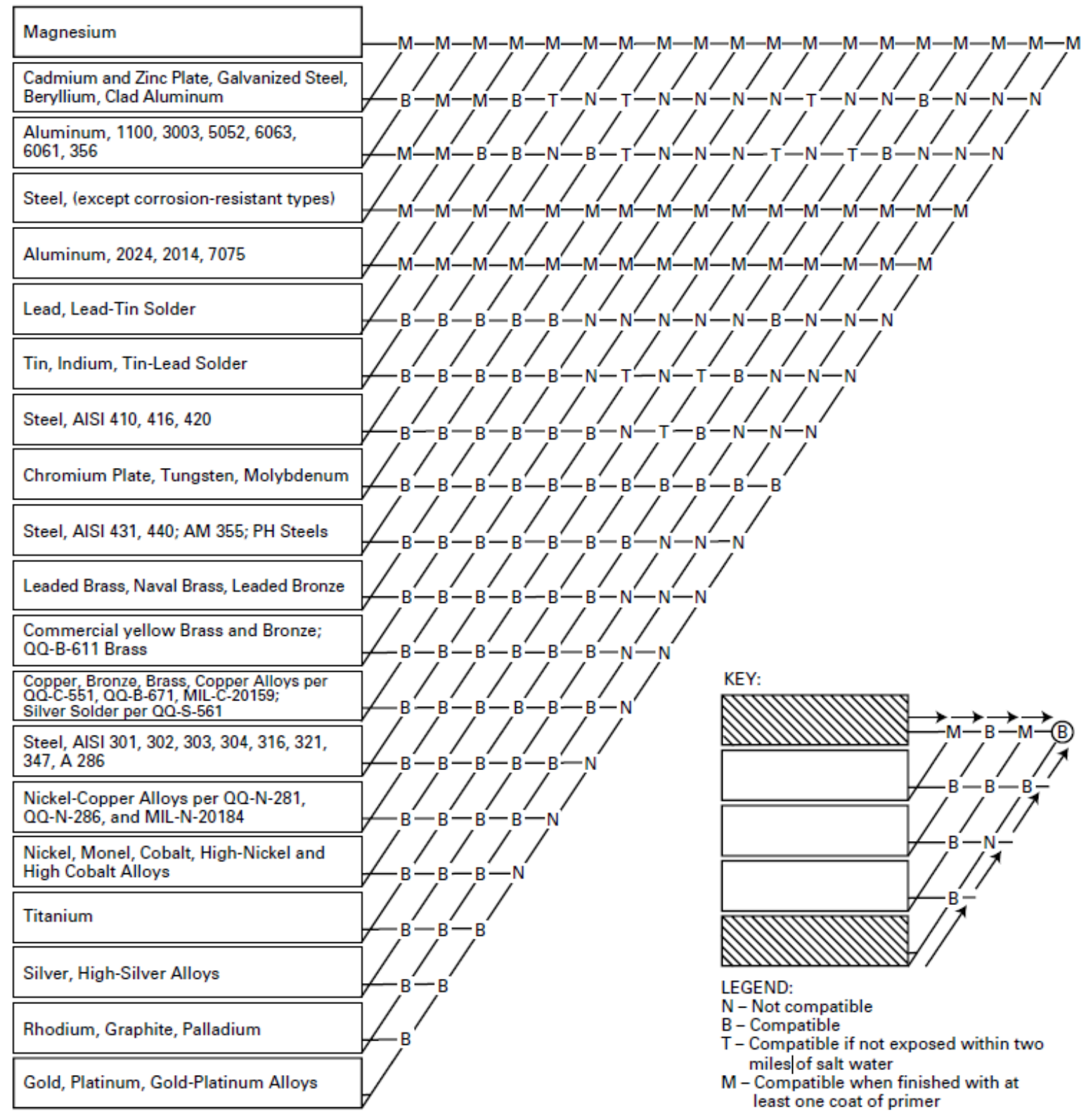

FIG. 19 - Metals compatibility chart 


\section{References}

[1] E. Musk, "Hyperloop Alpha," Space Exploration Technologies Corp., 2013.

[2] Hyperloop One, "Fact Sheet And FAQ," [Online]. Available: https://hyperloopone.com/fact-sheet-and-faq. [Accessed 288 2017].

[3] SpaceX, "Hyperloop," Space Exploration Technologies Corp., [Online]. Available: http://www.spacex.com/hyperloop. [Accessed 88 2017].

[4] SpaceX, "SpaceX Hyperloop Test-Track Specification," Space Exploration Technologies Corp., Hawthorne, 2016.

[5] N. S. Currey, Aircraft Landing Gear Design: Principles and Practices, Washington DC: AIAA, 1988.

[6] H. G. Conway, Landing Gear Design, London: Chapman \& Hall, 1958.

[7] R. C. Churchill and W. E. Luce, "Automatic Shrink Shock Strut for an Aircraft Landing Gear". United States of America Patent 5,908,174, 1 June 1991.

[8] W. M. Waide, "Aircraft Landing Gear with Integrated Extension, Retraction, and Leveling Feature". United States of America Patent 7,942,366 B2, 17 May 2011.

[9] G. P. A. Klim, M. P. Adhikari, C. Rodrigues de Souza Meireles, S. K. Amberg, M. M. Elahikahouker and S. M. Hashemi, "Deployable and Retractable Shock Strut". United States of America Patent Pat. Appl. No. 15/498,028, 26 April 2017.

[10] C. L. Taylor, D. J. Hyde and L. C. Barr, "Hyperloop Commercial Feasibility Analysis: High Level Overview," Volpe, Cambridge, MA, 2016.

[11] Hyperloop One, "Hyperloop One," [Online]. Available: https://hyperloop-one.com/. [Accessed 4 September 2017]. 
[12] Hyperloop Transportation Technologies, "Hyperloop Transportation Technologies," [Online]. Available: http://hyperlooptransp.com/\#!/. [Accessed 4 September 2017].

[13] Transpod, "Transpod," 4 September 2017. [Online]. Available: https://transpodhyperloop.com/.

[14] Hyperloop One, "Hyperloop One Goes Farther and Faster Achieving Historic Speeds," [Online]. Available: https://hyperloop-one.com/hyperloop-one-goes-farther-and-fasterachieving-historic-speeds. [Accessed 8 August 2017].

[15] J. R. Reitz, "Forces on Moving Magnets due to Eddy Currents," Journal of Applied Physics, vol. 41, no. 5, pp. 2067-2071, 1970.

[16] T. O. Shonibare, "A Kinematic Model of the Hyperloop Deployable Wheel System, Validation Using Real System Measurments," Ryerson University, Toronto, 2017.

[17] G. Klim and S. M. Hashemi, "Designing Mass-Optimized Parts Using Solidthinking Inspire with Application to the Hyperloop Deployable Wheel System," in 76th Annual SAWE International Conference, Montreal, Canada, 2017.

[18] C. J. Chin, J. S. Gray, S. M. Jones and J. J. Bertib, "Open-Source Conceptual Sizing Models for the," in 56th AIAA/ASCE/AHS/ASC Structures, Structural Dynamics, and Materials Conference, Kissimmee, 2015.

[19] SpaceX, "SpaceX Hyperloop Pod Competition II: Rules and Requirments," Space Exploration Technologies Corp., Hawthorne, 2016.

[20] NASA, "NASA Space Vehicle Design Criteria (Structures): Lubrication, Friction, and Wear," NATIONAL AERONAUTICS AND SPACE AQMfNISTRATION, Hampton, 1971.

[21] Z. Liu, Z. Long and X. Li, Maglev Trains: Key Underlying Technologies, New York: Springer-Verlag, 2015. 
[22] Federal Aviation Administration, "Aviation Maintenance Technician Handbook - Airframe, Volume 2," in Chapter 13: Aircraft Landing Gear Systems, Oklahoma City, U.S. Department of Transportation, 2012.

[23] AAIB Field Investigation, "Air accident monthly bulletins: ATR 42-320," Air Accidents Investigation Branch, 2013.

[24] W. E. Luce, "Aircraft Shock Strut and Improved Bearings Therefor". United States Patent US 2007/0164151 A1, 19 July 2007.

[25] W. Sharples, "Contractable Shock Absorber". United States of America Patent 4,291,850, 29 September 1981.

[26] J. Veaux and D. Michel, "Shock Absorber for an Aircraft Landing Gear Leg". United States of America Patent 5,310,140, 10 May 1994.

[27] N. S. Currey and J. H. Renshaw, "Landing Gear for STOL Airplanes". United States of America Patent 3,826,450, 30 July 1974.

[28] J. L. He, D. M. Rote and H. T. Coffey, "Study of Japanese electrodynamic-suspension Maglev systems," Tech. Rep. ANL/ESD-20, 1994.

[29] N. Paudel, "Dynamic Suspension Modeling of an Eddy-Current Device: An Application to Maglev," ProQuest, Charoltte, 2012.

[30] Federal Aviation Administration, Metallic Materials Properties Development and Standardization (MMPDS-07), Federal Aviation Administration, 2006.

[31] KamaticsRWG, KAron Design Guide, OKAMAN Corporation, 2017.

[32] Unbrako, "A comprehensive catalog of UNBRAKO® socket screws and related products," SPS Technologies, 1996.

[33] G. B. Hillard and D. C. Ferguson, "Anodized Aluminum as Used for Exterior Spacecraft Dielectrics," in 6th Spacecraft Charging Technology Conference, Cleveland, 2000. 
[34] Y. Goueffon, L. Arurault, C. Mabru and P. Guigue, "Black anodic coatings for space applications: study of the process parameters, characteristics and mechanical properties," Journal of Materials Processing Technology, vol. 209, no. 11, pp. 5145-5151, 2009.

[35] M. D. Griffin and J. R. French, Space Vehicle Design, Blacksburg, Virginia: American Institute of Aeronautics and Astronautics Inc., 2004.

[36] "Dow Corning® High Vacuum Grease," Dow Corning Corporation, 2017. [Online]. Available: http://www.dowcorning.com/. [Accessed 5 August 2017].

[37] Arrow Cryogenics, "Stainless Steel Passivation," [Online]. Available: http://www.arrowcryogenics.com/chemical-processing/stainless-steel-passivation. [Accessed 8 August 2017].

[38] "CATIA," Dassault Systèmes, 2017. [Online]. Available: https://www.3ds.com/. [Accessed 4 September 2017].

[39] R. Janzen, "TransPod Ultra-High-Speed Tube Transportation: Dynamics of Vehicles and Infrastructure," in X International Conference on Structural Dynamics, EURODYN 2017, Rome, 2017.

[40] G. P. Klim, M. M. Elahi, M. P. Adhikari, S. M. Hashemi, C. Rodrigues and S. Amberg, "Final Report: Hyperloop Deployable Wheel System," RIHT, Toronto, 2016.

[41] "Greene Tweed," Greene Tweed@, 2017. [Online]. Available: http://www.gtweed.com/. [Accessed 1 August 2017].

[42] "SKF," SKF Group Headquarters , [Online]. Available: http://www.skf.com/ca/en/index.html?switch=y. [Accessed 1 September 2017].

[43] "KAMAN: Specialty Bearings \& Engineering Products," @KAMAN Corporation, 2017. [Online]. Available: http://www.kaman.com/specialty-bearings-engineered-products. [Accessed 1 September 2017]. 
[44] Sunray Inc., "Sunray Inc. Polyurethane Products : Frequently Asked Questions," Sunray Inc., [Online]. Available: https://www.sunray-inc.com/faqs/. [Accessed 5 May 2016].

[45] M. M. Khan, "The Hyperloop Wheel: Design, Integration and Analysis," Ryerson University, Toronto, 2015.

[46] R. C. Hibbeler, Mechanics of Materials, 9th Edition, New York: Pearson Prentice Hall, 2014.

[47] KamaticsRWG, KAron Bearing Catalog: Spherical, Rod End and Journal Sleeve Bearings, CKAMAN Corporation, 2013.

[48] "Safran Landing Systems," (C) Safran Landing Systems, 2017. [Online]. Available: https://www.safran-landing-systems.com/fr. [Accessed 3 September 2017].

[49] "ANSYS," ANSYS, Inc. , 2017. [Online]. Available: http://www.ansys.com/. [Accessed 8 September 2017].

[50] "Inspire," solidThinking, Inc., 2017. [Online]. Available: http://www.solidthinking.com/ProductOverview.aspx?item=Inspire\%20Overview\&categor $\mathrm{y}=$ Products. [Accessed 15 August 2017].

[51] American Society of Mechanical Engineers and American National Standards Institute, "ANSI/ASME Y14.5M-1994 : Dimensioning and Tolerancing," American Society of Mechanical Engineers and American National Standards Institute, New York City, 1994.

[52] "Model Based Definition," CDassault Systèmes, 2017. [Online]. Available: https://www.3ds.com/products-services/catia/disciplines/model-based-definition/. [Accessed 1 September 2017].

[53] S. Kalpakjian and S. R. Schmid, Manufacturing Processes for Engineering Materials, 5th ed., Pearson Education, 2008.

[54] "Progressive Anodizers Inc. (C," Digital Ink Technologies Inc., 2017. [Online]. Available: http://www.progressiveanodizers.com/. [Accessed 5 September 2017]. 
[55] "Black Oxide FAQS," () Copyright 2017 Electrochemical Products, Inc., [Online]. Available: https://www.epi.com/. [Accessed 1 September 2017].

[56] T. A. Debold and J. W. Martin, "How to Passivate Stainless Steel Parts," @CRS Holdings Inc. , 2017. [Online]. Available: https://www.cartech.com/. [Accessed 10 August 2017].

[57] "Burloak Technologies Inc.," Picasso Fish, 2017. [Online]. Available: http://burloaktech.com/. [Accessed 5 September 2017].

[58] Stratasys Direct, Inc., "Direct Metal Laser Sintering," Stratasys Direct, Inc., () 2017. [Online]. Available: https://www.stratasysdirect.com/solutions/direct-metal-laser-sintering/. [Accessed 20 September 2017].

[59] Greene Tweed, "ACT® Ring Product Data," 2007. [Online]. Available: www.gtweed.com. [Accessed 1012016 ].

[60] Messier-Dowty Inc., Dynatool Program Version 2.1, Ajax: Messier-Dowty@, 2001.

[61] F. Din, Thermodynamic Functions of Gases: Volume 1, Butterworth Scientific, 1956.

[62] SAE International Standards Comittee, "SAE AIR1362B: Aerospace Hydraulic Fluids Physical Properties," SAE International, 2008.

[63] ASME/ANSI, ASME/ANSI B1.5-1997 : General Purpose Acme Thread Form, ASME/ANSI, 2009.

[64] E. Oberg, F. D. Jones, H. L. Horton and H. H. Ryffel, Machinery's Handbook 29th Edition, New York: Industrial Press, 2012.

[65] New South Whales, Technical and Further Education Comission, Manufcaturing and Engineering Educational Services, Mechanical Design Data Manual, Bankstown: New South Whales, Manufcaturing and Engineering Educational Services, TAFE Commission, 2000.

[66] Z. C. Bradford, "Actuator". United States of America Patent 2,387,713, 30 October 1945. 
This page was intentionally left blank 